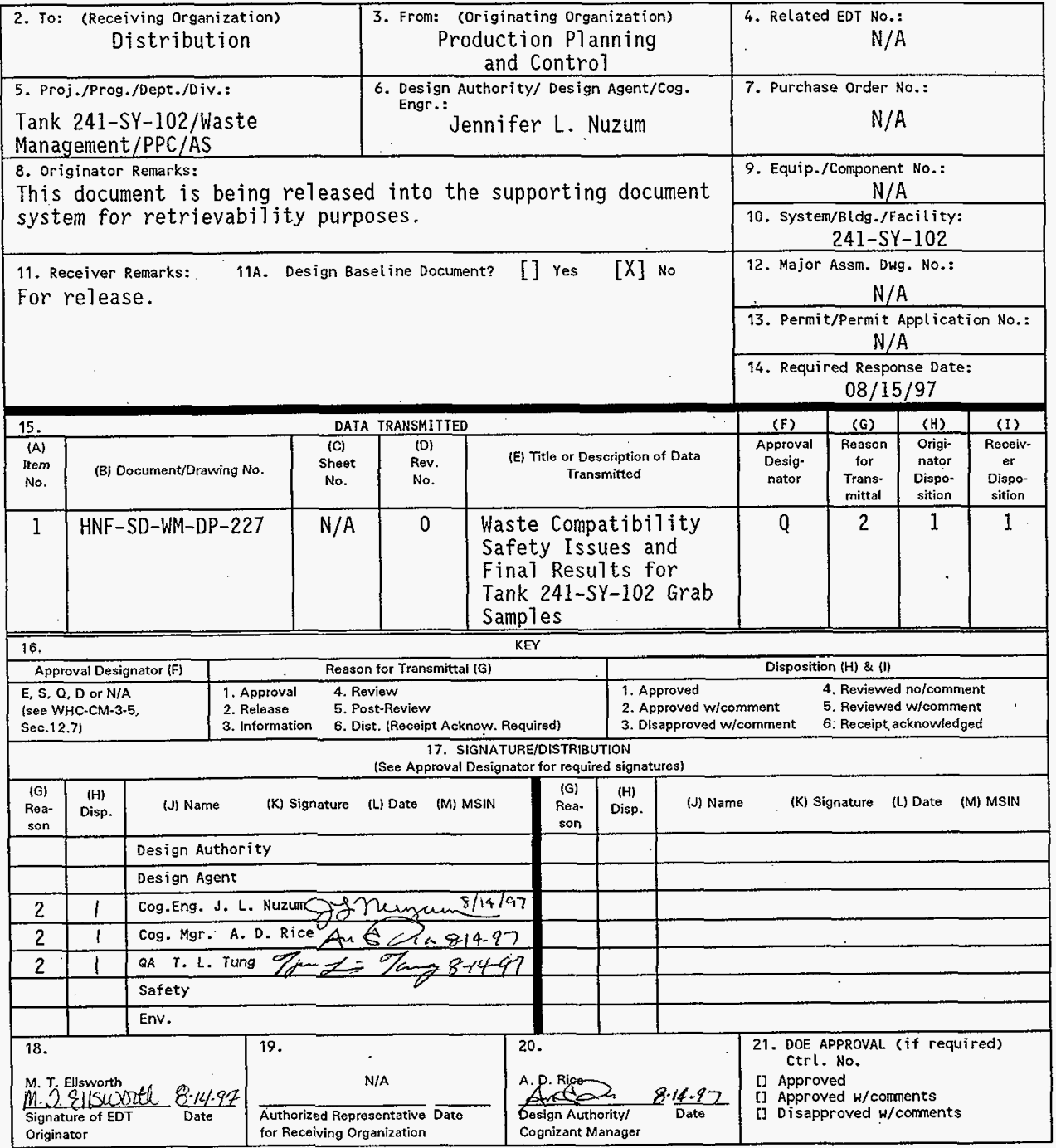




\title{
Waste Compatibility Safety Issues and Final Results for Tank 241-SY-102 Grab Samples
}

\author{
Jennifer L. Nuzum \\ Waste Management of Hanford, Inc., Richland, WA 99352 \\ U.S. Department of Energy Contract DE-AC06-96RL13200
}
EDT/ECN: EDT-620417 UC: 2070
Org Code: $7576 \mathrm{~B}$
Charge Code: MD378
B\&R Code: EW 3120074 Total Pages: 248

Key Words: Waste Compatibility, Safety Issues, Final Results, Tank 241SY-102, 241-SY-102, SY-102, Grab Samples

Abstract: $N / A$

TRADEMARK DISCLAIMER. Reference herein to any specific commercial product, process, or service by trade name, trademark, manufacturer, or otherwise, does not necessarily constitute or imply its endorsement, recommendation, or favoring by the United States Government or any agency thereof or its contractors or subcontractors.

Printed in the United States of America. To obtain copies of this document, contact: Document Control Services, P.O. Box 950, Mailstop H6-08, Richland WA 99352, Phone (509) 372-2420; Fax (509) 376-4989.
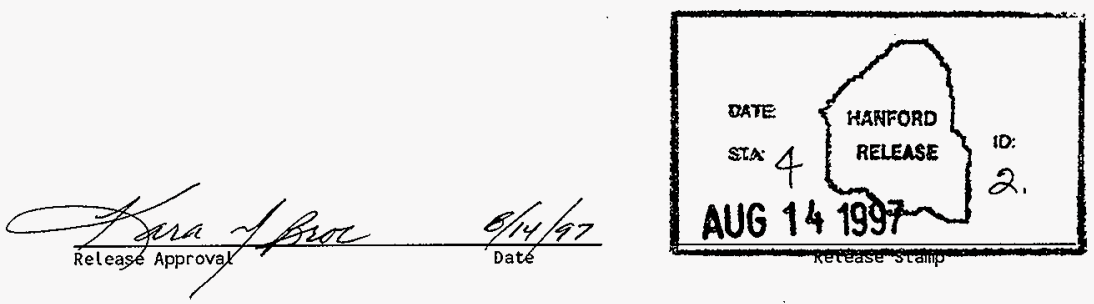

Approved for Public Release 
HNF-SD-WM-DP-227, REV. 0

\author{
ANALYTICAL SERVICES
}

\title{
WASTE COMPATIBILITY SAFETY ISSUES AND FINAL RESULTS FOR TANK 241-SY-102 GRAB SAMPLES
}

\author{
Project Coordinator: JENNIFER L. NUZUM \\ Prepared for the U.S. Department of Energy \\ Office of Environmental Restoration \\ and Waste Management \\ by \\ 222-S Laboratory \\ Waste Management of Hanford, Inc. \\ P. O. Box 700 \\ Richland, Washington
}


HNF-SD-WM-DP-227, REV. 0

THIS PAGE INTENTIONALLY LEFT BLANK 
HNF-SD-WM-DP-227, REV. 0

TABLE OF CONTENTS

Narrative . . . . . . . . . . . . . . . . 1

SY-102 Sample Breakdown (Attachment 1) . . . . . . . . . 10

Sample Data Summary . . . . . . . . . . . . . . . . . 13

Chain of Custody Forms . . . . . . . . . . . . 20

Waste Compatibility Corrosion Rules . . . . . . . . . . . . 26

Inorganic Analyses . . . . . . . . . . . . . . 28

Differential Scanning Calorimetry (DSC)

DSC Worklist \# $16284(24,26) \ldots \ldots . . . . . . . . .30$

DSC Worklist \# 16285 (27) . . . . . . . . . . . . . . 37

Thermogravimetric Analysis (TGA)

TGA Worklist \# $16282(24,26) \ldots . . . . . . . . . . . .44$

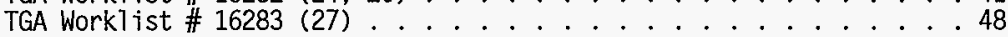

Specific Gravity Analysis ( $\mathrm{SpG}$ )

SpG Workl ist \# $16291(24,26,27) \ldots \ldots . \ldots . \ldots 52$

$\mathrm{pH}$ Analysis $(\mathrm{pH})$

pH Worklist \# $16290(24,26,27) \ldots . . . . . . . . . . .57$

Hydroxide Analysis (OH)

OH Work1 ist \# $16289(24,26,27) \ldots \ldots$. . . . . . . . . . 58

Ammonia Analysis (NH3)

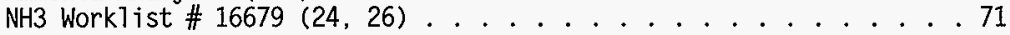

Ion Chromatographic Analysis (IC)

IC Workl ist \# $16288(24,26,27) \ldots$. . . . . . . . . . . . . . . 87

Inductively Couple P1asma Spectrographic Analysis (ICP)

ICP Workl ist \# $16306(24,26) \ldots . . . . . . . . .999$

Total Organic Analysis (TOC)

TOC Workl ist \# $16287(24,26,27) \ldots . . . . . . . .105$

Total Inorganic Analysis (TIC)

TIC Workl ist \# $16286(24,27) \ldots$. . . . . . . . . . . . . . . . . 130

TIC Work7 ist \# $16384(26)$. . . . . . . . . . . . 154 
HNF-SD-WM-DP-227, REV. 0

TABLE OF CONTENTS (Continue'd)

Radichemical Analysis . . . . . . . . . . . . . . 168

Gamma Energy Analysis (GEA)

GEA Worklist \# $16280(25,28,29) \ldots \ldots . \ldots . . . \ldots 170$

Strontium Analysis (Sr-90)

Sr-90 Worklist \#16361 $(25,28) \ldots \ldots 202$

Americium Analysis (Am-241)

Am-241 WorkTist \# $16278(25,28) \ldots . . . . . . . . .210$

Plutonium Analysis (Pu-239)

Pu-239 Worklist \# $16279(25,28) \ldots$. . . . . . . . . . . . 237

This document consists of pages 1 through 263. Pages ii, 2, 11, 14, 21, 26, 29 and 169 were intentionally left blank.

TRADEMARKS:

Perkin-Elmer is a Registered Trademark of Research and Manufacturing Company, Inc. Mettler is a Registered Trademark of Mettler Electronics. 
HNF-SD-WM-DP-227, REV. 0

NARRATIVE 
HNF-SD-WM-DP-227, REV. 0

THIS PAGE INTENTIONALLY LEFT BLANK 
HNF-SD-WM-DP-227, REV. 0

222-S ANALYTICAL SERVICES

\section{WASTE COMPATIBIITY SAFETY ISSUES AND FINAL RESULTS FOR TANK 241-SY-102 GRAB SAMPLES}

\section{Summary}

Three grab samples (2SY-96-1, 2SY-96-2, and 2SY-96-3) were taken from Riser 1A of Tank 241-SY-102 on January 14, 1997, and received by 222-S Laboratory on January 14, 1997. These samples were analyzed in accordance with Compatibility Grab Sampling and Analysis Plan (TSAP) (Sasaki, 1996) and Data Quality Objectives for Tank Farms Waste Compatibility Program (DQO) (Fowler, 1995) in support of the Waste Compatibility Program. No notifications were required based on sample results. Acetone analysis was not performed in accordance with Cancellation of Acetone Analysis for Tank 241-SY-102 Grab Samples (Schreiber, 1997).

\section{Appearance and Sample Breakdown}

Attachment 1 illustrates samples generated in the laboratory for analyses and identifies their sources. Furthermore, this reference relates tank farm identification numbers to their corresponding 222-S Laboratory Information Management System sample numbers.

Table 1 summarizes appearance information and over-the-top (OTR) dose readings performed on each sample. For each sample, two $20 \mathrm{~mL}$ subsamples were created for inorganic and radiochemical analyses. Sample 2 SY- $96-3$ was a dark brown sludge containing $99 \%$ solids. There was an insufficient amount of supernate to perform all analyses. Since there was not suitable separation between the solids and supernate, results are suspect. Results for 2SY-96-3 subsamples (S97T000027 and S97T000029) are included in the Data Summary Table (Table 2) for information only, and are not discussed in the narrative, nor are they reported with respect to the Waste Compatibility Program.

Table 1. Appearance and OTR for 241-SY-102 Liquid Grab Samples

\begin{tabular}{|c|c|c|c|c|c|c|}
\hline $\begin{array}{c}\text { Customer } \\
\text { DD }\end{array}$ & $\begin{array}{c}\text { Lab } \\
\text { ID }\end{array}$ & Color & Clarity & $\begin{array}{c}\text { Organic } \\
\text { Layer }\end{array}$ & $\begin{array}{c}\% \\
\text { Solids }\end{array}$ & $\begin{array}{c}\text { OTR } \\
\text { (MRAD/HR) }\end{array}$ \\
\hline 2 SY-96-1 & S97T000011 & Yellow & Clear & None & $<5 \%$ & 75 \\
\hline 2 SY-96-2 & S97T000012 & $\begin{array}{c}\text { Dark } \\
\text { Yellow }\end{array}$ & Clear & None & $<5 \%$ & 200 \\
\hline 2 SY-96-3 & S97T000013 & $\begin{array}{c}\text { Dark } \\
\text { Brown }\end{array}$ & Opaque & None & $99 \%$ & 1000 \\
\hline
\end{tabular}




\section{HNF-SD-WM-DP-227, REV. 0}

\section{Analytical Results}

Table 2 compiles analytical results associated with each subsample. Liquid subsamples that were prepared for analysis by an acid adjustment of the direct subsample are indicated by a " $\mathrm{D}$ " in the A\# column in Table 2.

\section{Compatibility Program Concerns}

\section{Differential Scanning Calorimetry (DSC) - Energetics Decision Rule}

For waste compatibility energetics decision concerns, the exotherm/endotherm ratio must be less than one (1). Exotherm results for all subsamples were $0.00 \mathrm{~J} / \mathrm{g}$. Thus, this requirement was satisfied for all subsamples. There were no exceptions to the quality control (QC) parameters stated in TSAP for these samples.

Nitrate (NO3-), Bydroxide (OH-), and Nitrite (NO2-) - Corrosion Decision Rule

Results with respect to waste compatibility corrosion rules are presented in Table 3 . This table presents NO3-, $\mathrm{OH}-$, and NO2- results in $\mu \mathrm{g} / \mathrm{mL}$ and molarity (M) units. The spreadsheet compares results to the concentration ranges specified in DQO. A "YES" will appear in the appropriate space for a condition that is met. Based on the NO3- concentration, only one of three sets of conditions must be met for $\mathrm{OH}$ - and NO2- concentrations. A "NO" appearing in the space is indicative of a condition not met. All conditions for corrosion control were satisfied. There were no exceptions to the QC parameters stated in TSAP for these subsamples.

\section{Specific Gravity (Sp.G) - Flammable Gas Accumulation Decision Rule}

Sp.G analysis is currently used to determine the potential the waste will cause an accumulation of flammable gases. The flammable gas decision rule requires the weighted mean Sp.G not exceed 1.41. The mean Sp.G for the two superuate subsamples was equal to 1.07 . There were no exceptions to the QC parameters stated in TSAP for these subsamples.

\section{Plutonium (239/240Pu) - Criticality Decision Rule}

All $239 / 240 \mathrm{Pu}$ results were below the criticality prevention limit of $0.80 \mu \mathrm{Ci} / \mathrm{mL}$ stated in DQO. There were no exceptions to the QC parameters stated in TSAP for these subsamples. 


\section{HNF-SD-WM-DP-227, REV. 0}

\section{Inorganic Analyses}

\section{Thermogravimetric Analysis (TGA)}

TGA results were typically determined by summing weight loss steps below $200^{\circ} \mathrm{C}$. Weight loss steps above this were not used to determine the result. More information may be obtained by examining the raw data. There were no exceptions to the QC parameters stated in TSAP for theses subsamples.

\section{$\mathbf{p H}$}

There were no exceptions to the QC parameters stated in TSAP for these subsamples.

\section{Ion Chromatography (IC)}

Only required analyte results (Cl-, F-, PO4-, SO4-, NO3-, and NO2-) were considered in this report. Other opportunistic analyte results are included in Table 2. These analytes do not have customer defined QC parameters and are not discussed.

Relative percent difference (RPD) between sample and duplicate for Subsample 2SY-96-2 (S97T000026) was $24.1 \%$ for the SO4- analyte. Poor precision is caused by sample heterogeneity. NO3- spike recovery for the same subsample was low. The spike should be considered invalid because the amount of spike added was insignificant in comparison to the sample NO3- concentration. Rerun analysis was not requested.

\section{Inductively Coupled Plasma Spectrophotometry (ICP)}

Only required analytes $(\mathrm{Al}, \mathrm{Fe}, \mathrm{Na})$ were considered in this report. Other opportunistic analytes are included in Table 2. These analytes do not have customer defined QC parameters and are not discussed.

\section{Total Organic Carbon (TOC)}

Subsample 2SY-96-1 (S97T000024) was analyzed in triplicate. The results are shown in Table 4. Spike recovery for Subsample 2SY-96-2 (S97T000026) was above the QC parameters stated in TSAP. This elevated spike recovery was due to matrix interference, and rerun analysis was not requested. 


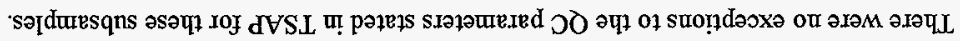

\section{(EHN) S!Sא [ॄUV e!̣ouuV}

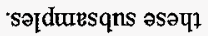

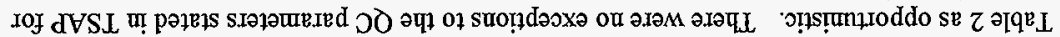

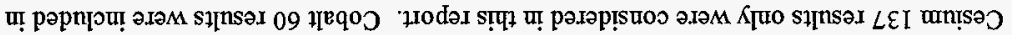

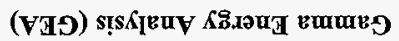

'pałsanbex łou әгәм sunuəI

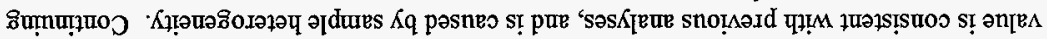

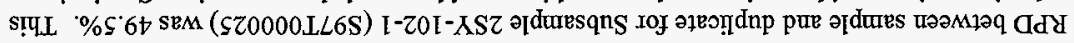

(.IS06) 06 annumo.ns

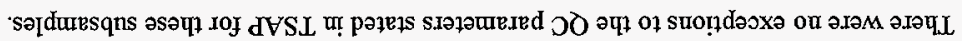

(uvItz) un!̣!xour

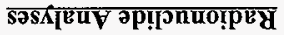

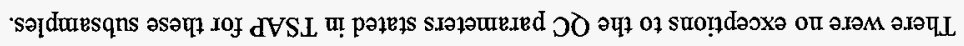

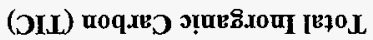

\begin{tabular}{|c|c|c|c|c|}
\hline $\mathcal{E} 0+\exists \varsigma 9^{\circ} \mathcal{E}$ & $Z 0+\exists Z \mathcal{E}^{\prime} \mathcal{E}$ & $20+3 Z L D$ & $20+906 z$ & $\nabla 20000 \mathrm{~L} L 6 \mathrm{~S}$ \\
\hline 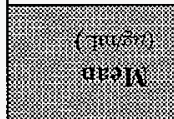 & 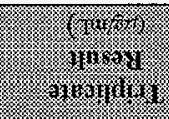 & 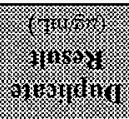 & 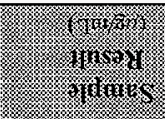 & fir \\
\hline
\end{tabular}

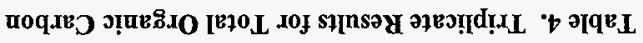




\section{HNF-SD-WM-DP-227, REV. 0}

\section{Procedures}

Table 5 lists the analytical procedures used for performing sample analyses. Abbreviations for analyses are defined in the table notes.

Table 5. Analytical Procedures

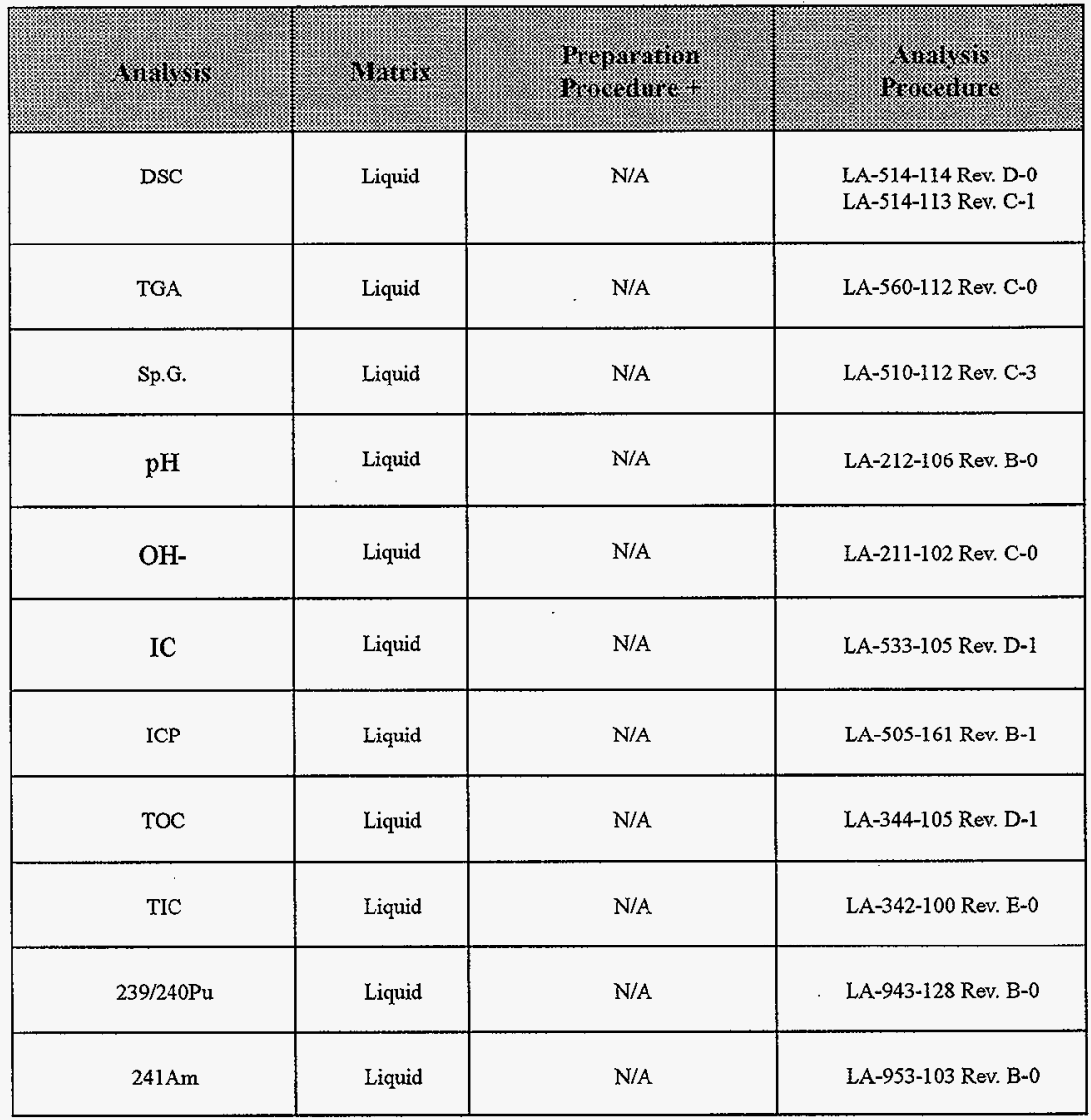


HNF-SD-WM-DP-227, REV. 0

\begin{tabular}{|c|c|c|c|}
\hline GEA & Liquid & N/A & LA-548-121 Rev. E-0 \\
\hline $90 \mathrm{Sr}$ & Liquid & N/A & LA-220-101 Rev. D-1 \\
\hline NH3 & Liquid & N/A & LA-631-001 Rev. B-2 \\
\hline
\end{tabular}

Notes:

$\begin{array}{ll}\text { N/A } & \text { not applicable (these are direct samples) } \\ \text { DSC } & \text { differential scanning calorimetry } \\ \text { TGA } & \text { thermogravimetric analysis } \\ \text { Sp.G. } & \text { specific gravity } \\ \text { OH- } & \text { hydroxide } \\ \text { IC } & \text { ion chromatography } \\ \text { ICP } & \text { inductively coupled plasma } \\ \text { TOC } & \text { total organic carbon } \\ \text { TIC } & \text { total inorganic carbon } \\ \text { 239/240Pu } & \text { plutonium } 239 / 240 \\ 241 \mathrm{Am} & \text { americium } 241 \\ \text { GEA } & \text { gamma energy analysis } \\ { }^{90} \mathrm{Sr} & \text { strontium-90 } \\ \mathrm{NH} 3 & \text { ammonia }\end{array}$




\section{HNF-SD-WM-DP-227, REV. 0}

\section{References}

Sasaki, L.M., 1996, Compatibility Grab Sampling and Analysis Plan for Fiscal Year 1997, WHC-SD-WM-TSAP-115, Revision 0-C, Westinghouse Hanford Company, Richland, WA 99352.

Fowler, K.D., 1995, Data Quality Objectives for Tank Waste Compatibility Program, WHC-SD-WM-DQO-001, Revision 1, Westinghouse Hanford Company, Richland, WA 99352.

Schreiber, R.D., 1997, Cancellation of Acetone Analysis for Tank 241-SY-102 Grab Samples, Internal Memorandum 74610-97-037, Lockheed Martin Hanford Corporation, Richland, WA 99352. 
HNF-SD-WM-DP-227, REV. 0

SY-102 SAMPLE BREAKDOWN (ATTACHMENT 1) 
HNF-SD-WM-DP-227, REV. 0

THIS PAGE INTENTIONALLY LEFT BLANK 


\section{Attachment 1 SY-102 GRAB SAMPLE BREAKDOWN}

1" Below

Zipcord

Measurement

2SY-96-1

S97T000011

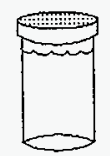

$\vec{N}$

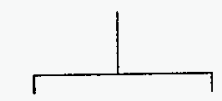

S97T000024
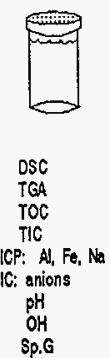

St $-\infty 0$.
Riser $1 \mathrm{~A}$

565 in.

$$
\text { 2SY-96-2 }
$$

S97T000012
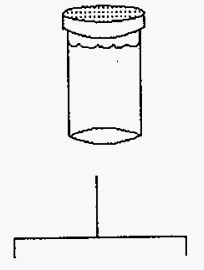

S97T000026

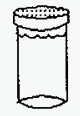

GEA: Cs-137

Pu-239/240
Am- 241
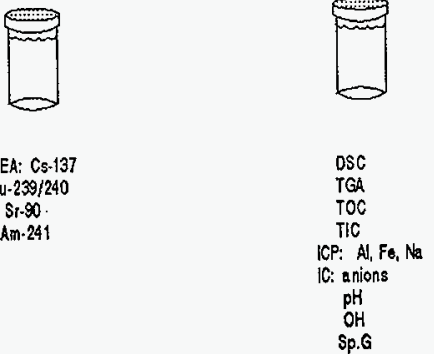

635 in.

2SY-96-3 S97T000013

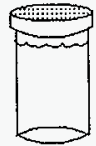

S97T000027

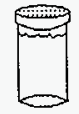

GEA: Cs-137

Pu-239/240

Sr- $\$ 0$

Am-241
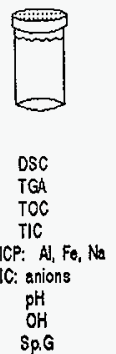
HNF-SD-WM-DP-227, REV. 0

SAMPLE DATA SUMMARY

13 
$H N F-S D \cdot W M-D P-227$, REV. 0

THIS PAGE INTENTIONALLY LEFT BLANK 
Table 2. Data Summary Table SY-102 GRAB1

CORE NUMBER: $n / a$

SEGMENT \#: 2SY-96-1

SEGMENT PORTION: Supernate

\begin{tabular}{|c|c|c|c|c|c|c|c|c|c|c|c|c|}
\hline Sample\# & $\mathrm{A} A \#$ & Analyte & Unit & Standard \% & Blank & Result & Duplicate & Average & $\mathrm{RPD} \%$ & Spk Rec \% & Det Limit & Count Err\% \\
\hline S97T000024 & & DSC Exotherm on Perkin Elmer & Joules/g & 100.3 & $n / a$ & $0.00 \mathrm{e}+00$ & $0.00 \mathrm{e}+00$ & $0.00 \mathrm{e}+00$ & 0.00 & $n / a$ & $n / a$ & $n / a$ \\
\hline S97T000024 & & Ammonia by ISE-Std Additions & $\mathrm{ug} / \mathrm{mL}$ & 88.60 & $\leq 1.000$ & $\leq \quad 1.000$ & 1.500 & $n / a$ & $\mathrm{n} / \mathrm{a}$ & 106.2 & 1.000 & $n / a$ \\
\hline$\$ 971000024$ & & $\mathrm{OH}-$ by Pot. Titration & $\mathrm{ug} / \mathrm{mL}$ & 102.2 & $<42.00$ & $1.68 \mathrm{e}+03$ & $1.61 \mathrm{e}+03$ & $1.64 \mathrm{e}+03$ & 4.26 & $\mathrm{n} / \mathrm{a}$ & 500.0 & $n / a$ \\
\hline S97T000024 & & pH Direct & pH & 100.2 & $\mathrm{n} / \mathrm{a}$ & 12.08 & 12.10 & 12.09 & 0.17 & $\mathrm{n} / \mathrm{a}$ & $1.00 \mathrm{e}-02$ & $n / a$ \\
\hline S97T000024 & & Specific Gravity & sp.G. & 99.71 & $\mathrm{n} / \mathrm{a}$ & 1.077 & 1.087 & 1.082 & 0.92 & $\mathrm{n} / \mathrm{a}$ & $1.00 \mathrm{e}-02$ & $n / a$ \\
\hline 5971000024 & & $\%$ water by TGA on Perkin Elmer & $\%$ & 99.19 & $\mathrm{n} / \mathrm{a}$ & 88.61 & 88.37 & 88.49 & 0.27 & $\mathrm{n} / \mathrm{a}$ & $n / a$ & $\mathrm{n} / \mathrm{a}$ \\
\hline S97T000024 & & TIC by Acid/Coul ometry & $u g / m L$ & 100.2 & 9.400 & $<1.46 \mathrm{e}+3$ & $1.51 \mathrm{e}+03$ & $n / a$ & $n / a$ & $n / a$ & 5.000 & $n / a$ \\
\hline 5977000024 & & Tot. Organic Carbon by coul. & ug/mL & 102.0 & $0.00 e+00$ & $2.90 \mathrm{e}+02$ & 472.0 & 381.0 & 47.8 & $\mathrm{n} / \mathrm{a}$ & 7.500 & $n / a$ \\
\hline S97T000024 & D & Silver-ICP-Acid Dil. & $\mathrm{ug} / \mathrm{mL}$ & 98.00 & $<1.00 e^{-02}$ & 2.390 & 2.510 & 2.450 & 4.90 & $\mathrm{n} / \mathrm{a}$ & 1.010 & $n / a$ \\
\hline 5977000024 & D & Aluminium-ICP-Acid Dil. & $\mathrm{ug} / \mathrm{mL}$ & 98.20 & $<5.00 e-02$ & $1.97 \mathrm{e}+03$ & $1.96 \mathrm{e}+03$ & $1.96 \mathrm{e}+03$ & 0.51 & $\mathrm{n} / \mathrm{a}$ & 5.050 & $n / a$ \\
\hline 5971000024 & D & Arsenic-ICP-Acid Dil. & $\mathrm{ug} / \mathrm{mL}$ & 103.8 & $<1.00 \mathrm{e}-01$ & $<\quad 10.10$ & $<1.01 \mathrm{e} 1$ & $n / a$ & $\mathrm{n} / \mathrm{a}$ & $\mathrm{n} / \mathrm{a}$ & 10.10 & $\mathrm{n} / \mathrm{a}$ \\
\hline $597 T 000024$ & D & Boron-ICP-Acid Dil. & ug/mL & 101.2 & $<5.00 e-02$ & 5.050 & $<5.05 \mathrm{e} 0$ & $\mathrm{n} / \mathrm{a}$ & $n / a$ & $\mathrm{n} / \mathrm{a}$ & 5.050 & $n / a$ \\
\hline 5971000024 & D & Barium-ICP-Acid Dil. & $\mathrm{ug} / \mathrm{mL}$ & 99.80 & $<5.00 e-02$ & $<5.050$ & $<5.05 e 0$ & $\mathrm{n} / \mathrm{a}$ & $n / a$ & $\mathrm{n} / \mathrm{a}$ & 5.050 & $n / a$ \\
\hline S97T000024 & ID & Beryllium-ICP-Acid Dil. & $\mathrm{ug} / \mathrm{mL}$ & 102.0 & $<5.00 e-03$ & $<5.05 e-01$ & $<5.05 \mathrm{e}-1$ & $\mathrm{n} / \mathrm{a}$ & $\mathrm{n} / \mathrm{a}$ & $\mathrm{n} / \mathrm{a}$ & $5.05 \mathrm{e}-01$ & $\mathrm{n} / \mathrm{a}$ \\
\hline$\$ 97 T 000024$ & D & Bismuth-ICP-Acid Dil. & $\mathrm{ug} / \mathrm{mL}$ & 101.2 & $<1.00 \mathrm{e}-01$ & $<\quad 10.10$ & $<1.01 \mathrm{e} 1$ & $\mathrm{n} / \mathrm{a}$ & $\mathrm{n} / \mathrm{a}$ & $n / a$ & 10.10 & $n / a$ \\
\hline$\$ 977000024$ & D & Calcium-ICP-Acid Dil. & $\mathrm{ug} / \mathrm{mL}$ & 98.40 & $<1.00 \mathrm{e}-01$ & $<10.10$ & $<1.01 \mathrm{e} 1$ & $n / a$ & $n / a$ & $\mathrm{n} / \mathrm{a}$ & 10.10 & $n / a$ \\
\hline $597 T 000024$ & $\bar{D}$ & Cadmi um-ICP-Acid Dil. & ug $/ \mathrm{mL}$ & 99.40 & $<5.00 e-03$ & $<5.05 e-01$ & $<5.05 e-1$ & $\mathrm{n} / \mathrm{a}$ & $\mathrm{n} / \mathrm{a}$ & $\mathrm{n} / \mathrm{a}$ & $5.05 e-01$ & $n / a$ \\
\hline 5971000024 & D & Cerium-ICP-Acid Dil. & $\mathrm{ug} / \mathrm{mL}$ & 99.00 & $<1.00 \mathrm{e}-01$ & $\leq \quad 10.10$ & $<1.01 \mathrm{e} 1$ & $n / a$ & $\mathrm{n} / \mathrm{a}$ & $n / a$ & 10.10 & $n / a$ \\
\hline $597 T 000024$ & D & Cobalt-ICP-Acid Dil. & $\mathrm{ug} / \mathrm{mL}$ & 100.6 & $<2.00 \mathrm{e}-02$ & 2.020 & $<2.02 \mathrm{e} 0$ & $\mathrm{n} / \mathrm{a}$ & $\mathrm{n} / \mathrm{a}$ & $\mathrm{n} / \mathrm{a}$ & 2.020 & $n / a$ \\
\hline 5971000024 & D & Chromium-ICP-Acid Dil. & $\mathrm{ug} / \mathrm{mL}$ & 99.00 & $<1.00 e-02$ & $3.69 \mathrm{e}+02$ & 368.0 & 368.5 & 0.27 & $\mathrm{n} / \mathrm{a}$ & 1.010 & $\mathrm{n} / \mathrm{a}$ \\
\hline$\$ 971000024$ & D & Copper-ICP-Acid Dil. & $\mathrm{ug} / \mathrm{mL}$ & 106.4 & $<1.00 e-02$ & $<\quad 1.010$ & $<1.01 \mathrm{e} 0$ & $n / a$ & $\mathrm{n} / \mathrm{a}$ & $\mathrm{n} / \mathrm{a}$ & 1.010 & $\mathrm{n} / \mathrm{a}$ \\
\hline$\$ 977000024$ & D & Iron-ICP-Acid Dil. & $\mathrm{ug} / \mathrm{mL}$ & 99.60 & $<5.00 \mathrm{e}-02$ & $<5.050$ & $<5.05 \mathrm{e} 0$ & $n / a$ & $n / a$ & $n / a$ & 5.050 & $n / a$ \\
\hline 5977000024 & D & Potassium-ICP-ACid Dil. & $\mathrm{ug} / \mathrm{mL}$ & 95.80 & $<5.00 e-01$ & $8.07 e+02$ & 792.0 & 799.5 & 1.88 & $\mathrm{n} / \mathrm{a}$ & 50.50 & $n / a$ \\
\hline $597 \mathrm{~T} 000024$ & D & Lanthanum-ICP-Acid Dil. & $\mathrm{ug} / \mathrm{mL}$ & 101.2 & $<5.00 e-02$ & $<\quad 5.050$ & $<5.05 \mathrm{e} 0$ & $n / a$ & $\mathrm{n} / \mathrm{a}$ & $n / a$ & 5.050 & $\mathrm{n} / \mathrm{a}$ \\
\hline 5977000024 & TD & Li thium-ICP-Acid Dil. & $\mathrm{ug} / \mathrm{mL}$ & 99.20 & $<1.00 \mathrm{e}-02$ & 1.010 & $<1.01 \mathrm{e} 0$ & $n / a$ & $\mathrm{n} / \mathrm{a}$ & $n / a$ & 1.010 & $n / a$ \\
\hline S97T000024 & D & Magnesium-ICP-Acid Dil. & $\mathrm{ug} / \mathrm{mL}$ & 99.80 & $<1.00 e-01$ & 10.10 & $<1.01 \mathrm{e} 1$ & $n / a$ & $\mathrm{n} / \mathrm{a}$ & $\mathrm{n} / \mathrm{a}$ & 10.10 & $n / a$ \\
\hline 5971000024 & D & Manganese-ICP-Acid Dil. & $\mathrm{ug} / \mathrm{mL}$ & 97.00 & $<1.00 \mathrm{e}-02$ & 1.010 & $<1.01 \mathrm{e} 0$ & $n / a$ & $n / a$ & $\mathrm{n} / \mathrm{a}$ & 1.010 & $\mathrm{n} / \mathrm{a}$ \\
\hline S97T000024 & D & Molybdenum-ICP-Acid Dil. & $\mathrm{ug} / \mathrm{mL}$ & 101.4 & $<5.00 e-02$ & $<\quad 5.050$ & $<5.05 \mathrm{e} 0$ & $n / a$ & $n / a$ & $n / a$ & 5.050 & $n / a$ \\
\hline S97T000024 & D & Sodium-ICP+Acid Dil. & $\mathrm{ug} / \mathrm{mL}$ & 102.0 & $<1.00 e-01$ & $3.49 \mathrm{e}+04$ & $3.47 \mathrm{e}+04$ & $3.48 \mathrm{e}+04$ & 0.57 & $\mathrm{n} / \mathrm{a}$ & 10.10 & $\mathrm{n} / \mathrm{a}$ \\
\hline S97T000024 & D & Neodymium-ICP-Acid Dil. & $\mathrm{ug} / \mathrm{mL}$ & 100.4 & $<1.00 e-01$ & $<\quad 10.10$ & $<1.01 \mathrm{e} 1$ & $n / a$ & $n / a$ & $n / a$ & 10.10 & $n / a$ \\
\hline $597 T 000024$ & $\bar{D}$ & Nickel-ICP-Acid Dil. & ug/mL & 99.00 & $<2.00 \mathrm{e}-02$ & $<2.020$ & $<2.02 \mathrm{e} 0$ & $n / a$ & $\mathrm{n} / \mathrm{a}$ & $\mathrm{n} / \mathrm{a}$ & 2.020 & $\mathrm{n} / \mathrm{a}$ \\
\hline $597 T 000024$ & $D$ & Phosphorus-ICP-Acid Dil. & $\underline{\mathrm{g}} / \mathrm{mL}$ & 101.2 & $<2.00 e-01$ & $3.19 \mathrm{e}+03$ & $3.19 \mathrm{e}+03$ & $3.19 \mathrm{e}+03$ & 0.00 & $\mathrm{n} / \mathrm{a}$ & 20.20 & $n / a$ \\
\hline S97T000024 & D & Lead-ICP-Acid Dil. & ug $/ \mathrm{mL}$ & 96.80 & $<1.00 e^{-01}$ & $<\quad 10.10$ & $<1.01 \mathrm{e} 1$ & $\mathrm{n} / \mathrm{a}$ & $\mathrm{n} / \mathrm{a}$ & $n / a$ & 10.10 & $n / a$ \\
\hline$\$ 971000024$ & D & Sulfur-ICP-Acid Dil. & ug/mL & 99.60 & $<1.00 \mathrm{e}-01$ & $7.47 \mathrm{e}+02$ & 751.0 & 749.0 & 0.53 & $\mathrm{n} / \mathrm{a}$ & 10.10 & $n / a$ \\
\hline 5971000024 & D & Ant imony-ICP-Acid Dil. & $\mathrm{ug} / \mathrm{mL}$ & 96.60 & $<6.00 \mathrm{e}-02$ & $\leq \quad 6.060$ & $<6.06 \mathrm{e} 0$ & $n / a$ & $n / a$ & $\mathrm{n} / \mathrm{a}$ & 6.060 & $\mathrm{n} / \mathrm{a}$ \\
\hline$\$ 977000024$ & D & Selenium-ICP-ACId Dil. & $\mathrm{ug} / \mathrm{mL}$ & 95.00 & $<1.00 \mathrm{e}-01$ & 10.10 & $<1.01 \mathrm{e} 1$ & $n / a$ & $\mathrm{n} / \mathrm{a}$ & $\mathrm{n} / \mathrm{a}$ & 10.10 & $n / a$ \\
\hline$\$ 977000024$ & D & Silicon-ICP-ACid Dil. & ug/mL & 96.60 & $<5.00 e-02$ & 8.170 & 8.310 & 8.240 & 1.70 & $n / a$ & 5.050 & $n / a$ \\
\hline S97T000024 & TD & Samarium-ICP-Acid Dil. & $\mathrm{ug} / \mathrm{mL}$ & 99.60 & $<1.00 e-01$ & 10.10 & $<1.01 \mathrm{e} 1$ & $n / a$ & $\mathrm{n} / \mathrm{a}$ & $n / a$ & 10.10 & $\mathrm{n} / \mathrm{a}$ \\
\hline $597 T 000024$ & DD & Strontium-ICP-Acid Dil. & $\mathrm{ug} / \mathrm{mL}$ & 99.60 & $<1.00 e-02$ & 1.010 & $<1.01 \mathrm{e} 0$ & $\mathrm{n} / \mathrm{a}$ & $\mathrm{n} / \mathrm{a}$ & $\mathrm{n} / \mathrm{a}$ & 1.010 & $n / a$ \\
\hline S97T000024 & D & Titanium-ICP-Acid Dil. & $\mathrm{ug} / \mathrm{mL}$ & 98.60 & $<1.00 \mathrm{e}-02$ & 1.010 & $<1.01 \mathrm{e} 0$ & $\mathrm{n} / \mathrm{a}$ & $n / a$ & $n / a$ & 1.010 & $n / a$ \\
\hline S97T000024 & ID & Thallium-ICP-Acid Dil. & $\mathrm{ug} / \mathrm{mL}$ & 95.40 & $<2.00 \mathrm{e}-01$ & 20.20 & $<2.02 \mathrm{e} 1$ & $\mathrm{n} / \mathrm{a}$ & $n / a$ & $\mathrm{n} / \mathrm{a}$ & 20.20 & $n / a$ \\
\hline 5971000024 & D & Uranium-ICP-Acid Dil. & $\mathrm{ug} / \mathrm{mL}$ & 97.60 & $<5.00 \mathrm{e}-01$ & 50.50 & $<5.05 \mathrm{e} 1$ & $\mathrm{n} / \mathrm{a}$ & $\mathrm{n} / \mathrm{a}$ & $\mathrm{n} / \mathrm{a}$ & 50.50 & $n / a$ \\
\hline 5977000024 & D & Vanadium-ICP-Acid Dil. & $\mathrm{ug} / \mathrm{mL}$ & 100.8 & $<5.00 e-02$ & 5.050 & $<5.05 \mathrm{e} 0$ & $n / a$ & $n / a$ & $\mathrm{n} / \mathrm{a}$ & 5.050 & $\mathrm{n} / \mathrm{a}$ \\
\hline 5977000024 & $D$ & Zinc-ICP-Acid Dil. & $\mathrm{ug} / \mathrm{mL}$ & 101.6 & $<7.00 \mathrm{e}-02$ & 1.010 & $<1.01 \mathrm{e} 0$ & $n / a$ & $n / a$ & $\mathrm{n} / \mathrm{a}$ & 1.010 & $n / a$ \\
\hline
\end{tabular}




\begin{tabular}{|c|c|c|c|c|c|c|c|c|c|c|c|c|}
\hline Sample\# & $\mathbf{R} \mid$ A\# & Analyte & Unit & Standard \% & Blank & Result & Dupl icate & Average & RPD \% & Spk Rec $\%$ & Det Limit & Count Err\% \\
\hline$\$ 97 T 000024$ & $D$ & Zirconiun-ICP-Acid Dil. & $\mathrm{ug} / \mathrm{mL}$ & 100.0 & $<1.00 \mathrm{e}-02$ & $<\quad 1.010$ & $<1.01 \mathrm{e} 0$ & $n / a$ & $n / a$ & $\mathrm{n} / \mathrm{a}$ & 1.010 & $\frac{n / a}{a}$ \\
\hline 5977000024 & & Fluoride-IC-Dionex $4000 / 4500$ & $4 \mathrm{~g} / \mathrm{mL}$ & 95.25 & $<1.20 e-02$ & $1.10 \mathrm{e}+03$ & $1.07 \mathrm{e}+03$ & $1.08 \mathrm{e}+03$ & 2.76 & $n / a$ & 61.81 & $n / a$ \\
\hline S97T000024 & & Chloride-IC-Dionex $4000 / 4500$ & $\mathrm{ug} / \mathrm{mL}$ & 100.5 & $<1.70 \mathrm{e}-02$ & $8.05 \mathrm{e}+02$ & 866.0 & 835.4 & 7.30 & $n / a$ & 87.57 & $\mathrm{n} / \mathrm{a}$ \\
\hline 5977000024 & & Witrite-1C - Dionex $4000 / 4500$ & $\mathrm{ug} / \mathrm{mL}$ & 108.1 & $<1.08 \mathrm{e}-01$ & $6.40 \mathrm{e}+03$ & $6.48 \mathrm{e}+03$ & $6.44 \mathrm{e}+03$ & 1.24 & $\mathrm{n} / \mathrm{a}$ & 556.3 & $n / a$ \\
\hline$\$ 97 T 000024$ & & Bromide by Ion chromatograph & $\mathrm{ug} / \mathrm{mL}$ & 94.40 & $<1.25 e^{-0} 01$ & $<6.44 \mathrm{e}+02$ & $<6.44 \mathrm{e} 2$ & $n / a$ & n/a & $n / a$ & 643.9 & $n / a$ \\
\hline 597T000024 & & Nitrate by IC-Dionex $4000 / 4500$ & $4 \mathrm{~g} / \mathrm{mL}$ & 99.16 & $3.04 \mathrm{e}-01$ & $4.25 e+04$ & $4.29 \mathrm{e}+04$ & $4.27 e+04$ & 0.94 & $n / a$ & 716.0 & $n / a$ \\
\hline S97T000024 & & Phosphate-1C-Dionex $4000 / 4500$ & $\mathrm{ug} / \mathrm{mL}$ & 98.90 & $<1.20 \mathrm{e}-01$ & $8.80 \mathrm{e}+03$ & $9.02 \mathrm{e}+03$ & $8.91 \mathrm{e}+03$ & 2.47 & n/a & 618.1 & $\mathrm{n} / \mathrm{a}$ \\
\hline$\$ 971000024$ & & Sulfate by IC-Dionex $4000 / 4500$ & $u g / m$ & 99.84 & $<1.38 \mathrm{e}-01$ & $2.40 \mathrm{e}+03$ & $2.51 \mathrm{e}+03$ & $2.45 \mathrm{e}+03$ & 4.48 & $\mathrm{n} / \mathrm{a}$ & 710.8 & $n / a$ \\
\hline $597 T 000024$ & & Dxalate-1C-Dionex $4000 / 450$ & $\mathrm{ug} / \mathrm{mL}$ & 99.62 & $\leq 1.05 e-01$ & $<5.41 \mathrm{e}+02$ & $<5.41 \mathrm{e} 2$ & $n / a$ & $n / a$ & $n / a$ & 540.9 & $n / a$ \\
\hline S97T000025 & & Strontium- $89 / 90$ High Level & $\mathrm{UC \textrm {i } / \mathrm { mL }}$ & 98.81 & $<2.39 e-04$ & $9.29 \mathrm{e}-04$ & $1.54 \mathrm{e}-03$ & $1.23 e-03$ & 49.5 & $n / a$ & $4.34 \mathrm{e}-04$ & $3.99 E+01$ \\
\hline$\$ 977000025$ & & Pu-239/240 by TRU-SPEC Resin & $\mathrm{uCi} / \mathrm{mL}$ & 99.66 & $<4.24 \mathrm{e}-06$ & $<3.81 e-06$ & $<4.21 \mathrm{E}-6$ & $n / a$ & $n / a$ & $n / a$ & $3.81 \mathrm{e}-06$ & $7.89 \mathrm{E}+00$ \\
\hline \$97T000025 & & Cobal $t-60$ by GEA & $\mathrm{UCi} / \mathrm{mL}$ & 99.69 & $<5.53 e-05$ & $<3.04 e-04$ & $<3.22 \mathrm{e}-4$ & $n / a$ & $\mathrm{n} / \mathrm{a}$ & $n / a$ & $3.04 e^{-04}$ & $n / a$ \\
\hline S97T000025 & & Ces $i$ um- $\{37$ by GEA & uCi/mL & 102.0 & $<5.62 e-05$ & 12.30 & 12.20 & 12.25 & 0.82 & $n / a$ & $n / a$ & 0.170 \\
\hline$\$ 977000025$ & & Am-241 by Extraction & $\mathrm{UC} \mathbf{i} / \mathrm{mL}$ & 85.71 & $<7.16 \mathrm{e}-06$ & $<6.87 e-06$ & $<6.17 \mathrm{E}-6$ & $n / a$ & $\mathrm{n} / \mathrm{a}$ & $\mathrm{n} / \mathrm{a}$ & $6.87 e^{-06}$ & $5.46 \mathrm{E}+00$ \\
\hline
\end{tabular}


Table 2. Data Sumnary Table \$Y-102 GRAB1

CORE NUMBER: $\mathrm{n} / \mathrm{a}$

SEGMENT \#: 2 SY-96-2

SEGMENT PORTION: Supernate

\begin{tabular}{|c|c|c|c|c|c|c|c|c|c|c|c|c|}
\hline Sample\# & $R$ Af & \# Analyte & Unit & Standard \% & Blank & Result & Duplicate & Average & RPD \% & Spk Rec \% & Det Limit & Count Err\% \\
\hline S97T000026 & & DSC Exotherm on Perkin Elmer & Joutes/g & 100.3 & $n / a$ & $0.00 \mathrm{e}+00$ & $0.00 \mathrm{e}+00$ & $0.00 \mathrm{e}+00$ & 0.00 & $n / a$ & $\mathrm{n} / \mathrm{a}$ & $n / a$ \\
\hline S97T000026 & & Ammonia by ISE-Std Additions & $\mathrm{ug} / \mathrm{mL}$ & 88.60 & $<1.000$ & 74.80 & $n / a$ & $n / a$ & $\mathrm{n} / \mathrm{a}$ & $\mathrm{n} / \mathrm{a}$ & 1.000 & $\mathrm{n} / \mathrm{a}$ \\
\hline 5971000026 & & $\mathrm{OH}$ - by Pot, Titration & $\mathrm{ug} / \mathrm{mL}$ & 102.2 & $<42.00$ & $7.98 e+03$ & $7.77 e+03$ & $7.88 e+03$ & 2.67 & $\mathrm{n} / \mathrm{a}$ & $2.50 \mathrm{e}+03$ & $n / a$ \\
\hline$\$ 9710000026$ & & ph Direct & $\mathrm{pH}$ & 100.2 & $n / a$ & 11.96 & 11.92 & 11.94 & 0.34 & $\mathrm{n} / \mathrm{a}$ & $.1 .00 \mathrm{e}-02$ & $n / a$ \\
\hline 5971000026 & & Specific Gravity & Sp.G. & 99.71 & $\mathrm{n} / \mathrm{a}$ & 1.071 & 1.083 & 1.077 & 1.11 & n/a & $1.00 \mathrm{e}-02$ & $n / a$ \\
\hline$\$ 971000026$ & & $\%$ Water by TGA on Perkin Elmer & $\%$ & 99.19 & $\mathrm{n} / \mathrm{a}$ & 85.83 & 85.94 & 85.88 & 0.13 & $n / a$ & $n / a$ & $n / a$ \\
\hline$\$ 97 T 000026$ & & IIC by Acid/Coulometry & $\mathrm{ug} / \mathrm{mL}$ & 99.17 & $3.00 e-01$ & $1.48 \mathrm{e}+03$ & $1.51 \mathrm{e}+03$ & $1.50 \mathrm{e}+03$ & 2.01 & 100.7 & 5.000 & $n / a$ \\
\hline 5975000026 & & Tot. Organic Carbon by coul. & $u g / \mathrm{mL}$ & 102.0 & $0.00 \mathrm{e}+00$ & $1.03 \mathrm{e}+03$ & $1.01 \mathrm{e}+03$ & $1.02 \mathrm{e}+03$ & 1.96 & 378.0 & 10.00 & $n / a$ \\
\hline 5971000026 & $\bar{D}$ & Silver-ICP-Acíd Dil. & $u g / m L$ & 98.00 & $<1.00 e^{-02}$ & 3.430 & 3.300 & 3.365 & 3.86 & 93.70 & 2.010 & $\mathrm{n} / \mathrm{a}$ \\
\hline 5971000026 & D & Aluminium-ICP-Acid Dil. & $u g / m L$ & 98.20 & $<5.00 e^{-02}$ & $5.12 e+03$ & $5.09 e+03$ & $5.10 \mathrm{e}+03$ & 0.59 & 95.10 & 10.10 & $n / a$ \\
\hline$\$ 977000026$ & D & Arsenic-ICP-Acid Dil. & $\mathrm{ug} / \mathrm{mL}$ & 103.8 & $<1.00 e-01$ & $<\quad 20.10$ & $<2.01 \mathrm{e} 1$ & $\mathrm{n} / \mathrm{a}$ & $n / a$ & 101.0 & 20.10 & $n / a$ \\
\hline S97ro00026 & $\frac{D}{D}$ & Boron-ICP-Acid Dil. & $\mathrm{ug} / \mathrm{mL}$ & 101.2 & $<5.00 e-02$ & 10.10 & 10.10 & $\mathrm{n} / \mathrm{a}$ & $\mathrm{n} / \mathrm{a}$ & 104.0 & 10.10 & $n / a$ \\
\hline 5977000026 & D & Barium-ICP-Acid Dill. & $\mathrm{ug} / \mathrm{mL}$ & 99.80 & $<5.00 e^{-02}$ & 10.10 & $<1.01 \mathrm{e} 1$ & $\mathrm{n} / \mathrm{a}$ & $\mathrm{n} / \mathrm{a}$ & 98.50 & 10.10 & $n / a$ \\
\hline 5971000026 & 5 & Beryl lium-ICP-Acid Dil. & $\mathrm{ug} / \mathrm{mL}$ & 102.0 & $<5.00 e-03$ & 1.010 & $<1.01 \mathrm{e} 0$ & $n / a$ & $\mathrm{n} / \mathrm{a}$ & 101.0 & 1.010 & $\mathrm{n} / \mathrm{a}$ \\
\hline$\$ 971000026$ & ID & Bismuth-ICP-ACid Dil. & $\mathrm{ug} / \mathrm{mL}$ & 101.2 & $<1.00 e-01$ & 20.10 & $<2.01 \mathrm{e} 1$ & $n / a$ & $n / a$ & 105.0 & 20.10 & $\mathrm{n} / \mathrm{a}$ \\
\hline 5977000026 & TD & Calcium-ICP-Acid Dil. & $\mathrm{ug} / \mathrm{mL}$ & 98.40 & $<1.00 e^{-01}$ & 20.10 & $<2.01 \mathrm{e} 1$ & $n / a$ & $\mathrm{n} / \mathrm{a}$ & 101.0 & 20.10 & $\mathrm{n} / \mathrm{a}$ \\
\hline 5977000026 & $\frac{T}{10}$ & Cadmium-ICP-Acid Dil. & $\mathrm{ug} / \mathrm{mL}$ & 99.40 & $<5.00 e-03$ & 1.340 & 1.390 & 1.365 & 3.66 & 99.00 & 1.010 & $\mathrm{n} / \mathrm{a}$ \\
\hline 5977000026 & D & Cerium-ICP-Acid Dil. & $\mathrm{ug} / \mathrm{mL}$ & 99.00 & $<1.00 \mathrm{e}-01$ & 20.10 & $<2.01 \mathrm{e} 1$ & $\mathrm{n} / \mathrm{a}$ & $\mathrm{n} / \mathrm{a}$ & 99.30 & 20.10 & $\mathrm{n} / \mathrm{a}$ \\
\hline 5977000026 & $\frac{10}{10}$ & Cobalt-ICP-Acid Dil. & $\mathrm{ug} / \mathrm{mL}$ & 100.6 & $<2.00 e-02$ & $<4.020$ & $<4.02 \mathrm{e} 0$ & $n / a$ & n/a & 100.0 & 4.020 & $n / a$ \\
\hline 5971000026 & $D$ & Chromium-ICP-Acid Dil. & $\mathrm{ug} / \mathrm{mL}$ & 99.00 & $<1.00 \mathrm{e}-02$ & $9.48 \mathrm{e}+02$ & 937.0 & 942.5 & 1.17 & 94.60 & 2.010 & $n / a$ \\
\hline$\$ 977000026$ & D & Copper-ICP-Acid Dil. & $\mathrm{ug} / \mathrm{mL}$ & 106.4 & $<1.00 \mathrm{e}-02$ & $<\quad 2.010$ & $<2.01 \mathrm{e} 0$ & $n / a$ & $n / a$ & 105.0 & 2.010 & $n / a$ \\
\hline S97T000026 & ID & Iron-ICP-Acid Dil. & $\mathrm{ug} / \mathrm{mL}$ & 99.60 & $<5.00 \mathrm{e}-02$ & $<10.10$ & $<1.01 \mathrm{e} 1$ & $n / a$ & $\mathrm{n} / \mathrm{a}$ & 99.00 & 10.10 & $n / a$ \\
\hline$\$ 971000026$ & D & Potassium-ICP-Acid Dil. & $\mathrm{ug} / \mathrm{mL}$ & 95.80 & $<5.00 e^{-01}$ & $2.13 e+03$ & $2.07 \mathrm{e}+03$ & $2.10 \mathrm{e}+03$ & 2.86 & 94.80 & 100.0 & $n / a$ \\
\hline 5971000026 & $\frac{D}{D}$ & Lanthanum-ICP-Acid Dil. & $\mathrm{ug} / \mathrm{mL}$ & 101.2 & $<5.00 \mathrm{e}-02$ & $<\quad 10.10$ & $<1.01 \mathrm{e} 1$ & $n / a$ & $n / a$ & 101.0 & 10.10 & $\mathrm{n} / \mathrm{a}$ \\
\hline 5971000026 & $D$ & Lithium-ICP-Acid Dil. & $\mathrm{ug} / \mathrm{mL}$ & 99.20 & $<1.00 e-02$ & 2.010 & $<2.01 e 0$ & $n / a$ & $n / a$ & 98.90 & 2.010 & $\mathrm{n} / \mathrm{a}$ \\
\hline$\$ 97 r 000026$ & D & Magnesium-ICP-Acid Dil. & $\mathrm{ug} / \mathrm{mL}$ & 99.80 & $<1.00 e-01$ & 20.10 & $<2.01 \mathrm{e} 1$ & $\mathrm{n} / \mathrm{a}$ & $\mathrm{n} / \mathrm{a}$ & 98.20 & 20.10 & $\mathrm{n} / \mathrm{a}$ \\
\hline S971000026 & $\frac{D}{D}$ & Manganese-ICP-Acid Dil. & $\mathrm{ug} / \mathrm{mL}$ & 97.00 & $<1.00 \mathrm{e}-02$ & 2.010 & $<2.01 \mathrm{e} 0$ & $\mathrm{n} / \mathrm{a}$ & $\mathrm{n} / \mathrm{a}$ & 94.70 & 2.010 & $n / a$ \\
\hline$\$ 977000026$ & $\frac{D}{D}$ & Molybdenum-ICP-Acid Dil. & $\mathrm{ug} / \mathrm{mL}$ & 101.4 & $<5.00 e-02$ & 11.50 & 10.90 & 11.20 & 5.36 & 101.0 & 10.10 & $n / a$ \\
\hline 5971000026 & D & Sodium-ICP-Acid Dil. & $\mathrm{ug} / \mathrm{mL}$ & 102.0 & $<1.00 \mathrm{e}-01$ & $4.70 \mathrm{e}+04$ & $4.68 \mathrm{e}+04$ & $4.69 \mathrm{e}+04$ & 0.43 & 97.40 & 20.10 & $\mathrm{n} / \mathrm{a}$ \\
\hline 5977000026 & D & Neodymium-ICP-Acid Dil. & $\mathrm{ug} / \mathrm{mL}$ & 100.4 & $<1.00 e^{-01}$ & $<\quad 20.10$ & $<2.01 \mathrm{e} 1$ & $n / a$ & $\mathrm{n} / \mathrm{a}$ & 100.0 & 20.10 & $\mathrm{n} / \mathrm{a}$ \\
\hline 5971000026 & D & Nickel-ICP-Acid Dil. & $\mathrm{ug} / \mathrm{mL}$ & 99.00 & $<2.00 e-02$ & $<4.020$ & $<4.02 \mathrm{e} 0$ & $\mathrm{n} / \mathrm{a}$ & $n / a$ & 97.50 & 4.020 & $n / a$ \\
\hline 5971000026 & $\frac{D}{D}$ & Phosphorus-ICP-Acid Dil. & $\mathrm{ug} / \mathrm{mL}$ & 101.2 & $<2.00 e-01$ & $1.36 \mathrm{e}+03$ & $1.34 e+03$ & $1.35 \mathrm{e}+03$ & 1.48 & 90.30 & 40.20 & $n / a$ \\
\hline S97T000026 & $\frac{5}{D}$ & Lead-ICP-Acid Dil. & $\mathrm{ug} / \mathrm{mL}$ & 96.80 & $<1.00 \mathrm{e}-01$ & $<20.10$ & $<2.01 \mathrm{e} 1$ & $\mathrm{n} / \mathrm{a}$ & n/a & 95.40 & 20.10 & $n / a$ \\
\hline$\$ 971000026$ & D & Sulfur-ICP-Acid Dil. & $\mathrm{ug} / \mathrm{mL}$ & 99.60 & $<1.00 \mathrm{e}-01$ & $5.62 e+02$ & 561.0 & 561.5 & 0.18 & 99.20 & 20.10 & $n / a$ \\
\hline 5971000026 & D & Ant imony-ICP-Acid Dil. & $\mathrm{ug} / \mathrm{mL}$ & 96.60 & $<6.00 \mathrm{e}-02$ & $<\quad 12.10$ & $<1.21 \mathrm{e} 1$ & $n / a$ & $\mathrm{n} / \mathrm{a}$ & 96.60 & 12.10 & n/a \\
\hline$\$ 971000026$ & $\bar{D}$ & Selenium-ICP-Acid Dil. & $\mathrm{ug} / \mathrm{mL}$ & 95.00 & $<1.00 \mathrm{e}-01$ & 20.10 & $<2.01 \mathrm{e} 1$ & $n / a$ & $n / a$ & 102.0 & 20.10 & $n / a$ \\
\hline $597 T 000026$ & D & Silicon-ICP-Acid Dil. & $\mathrm{ug} / \mathrm{mL}$ & 96.60 & $<5.00 \mathrm{e}-02$ & 17.70 & 16.70 & 17.20 & 5.81 & 97.20 & 10.10 & $n / a$ \\
\hline 5977000026 & D & Samarium-ICP-Acid Dil. & $\mathrm{ug} / \mathrm{mL}$ & 99.60 & $<1.00 e-01$ & 20.10 & $<2.01 \mathrm{e} 1$ & $n / a$ & $n / a$ & 98.40 & 20.10 & $n / a$ \\
\hline 5971000026 & $\frac{1}{D}$ & Strontium-ICP-Acid Dil. & $\mathrm{ug} / \mathrm{mL}$ & 99.60 & $<7.00 e^{-02}$ & 2.010 & $<2.01 \mathrm{e} 0$ & $\mathrm{n} / \mathbf{a}$ & $n / a$ & 99.10 & 2.010 & $n / a$ \\
\hline$\$ 977000026$ & D & Titaniun-ICP-Acid Dil. & $\mathrm{ug} / \mathrm{mL}$ & 98.60 & $<1.00 \mathrm{e}-02$ & 2.010 & $<2.01 \mathrm{e} 0$ & $n / a$ & $\mathrm{n} / \mathrm{a}$ & 98.40 & 2.010 & $n / a$ \\
\hline$\$ 97 T 000026$ & D & Thallium-ICP-Acid Dil. & $\mathrm{ug} / \mathrm{mL}$ & 95.40 & $<2.00 \mathrm{e}-01$ & $<\quad 40.20$ & $<4.02 \mathrm{e} 1$ & $n / a$ & $\mathrm{n} / \mathrm{a}$ & 92.50 & 40.20 & $\mathrm{n} / \mathrm{a}$ \\
\hline $597 T 000026$ & $\frac{L}{D}$ & Úranium-ICP-Acid Dit. & $\mathrm{ug} / \mathrm{mL}$ & 97.60 & $<5.00 \mathrm{e}-01$ & $<1.00 e+02$ & $<1.00 \mathrm{e} 2$ & $\mathrm{n} / \mathrm{a}$ & $\mathrm{n} / \mathrm{a}$ & 103.0 & 100.0 & $n / a$ \\
\hline 5971000026 & D & Vanadium-ICP-Acid Dil. & $\mathrm{ug} / \mathrm{mL}$ & 100.8 & $<5.00 e-02$ & $<\quad 10.10$ & $<1.01 \mathrm{e} 1$ & $n / a$ & $\mathrm{n} / \mathrm{a}$ & 100.0 & 10.10 & $n / a$ \\
\hline $597 \mathrm{~T} 000026$ & D & ZinC-ICP-Acid Dil. & $\mathrm{ug} / \mathrm{mL}$ & 101.6 & $<1.00 \mathrm{e}-02$ & 2.010 & $<2.01 e 0$ & $\mathrm{n} / \mathrm{a}$ & $n / a$ & 102.0 & 2.010 & $\mathrm{n} / \mathrm{a}$ \\
\hline
\end{tabular}




\begin{tabular}{|c|c|c|c|c|c|c|c|c|c|c|c|c|}
\hline Sample\# & A\# & Analyte & Unit & Standard \% & Blank & Result & Duplicate & Average & RPD $\%$ & Spk Rec \% & Det Limit & Count Err\% \\
\hline \$97T000026 & D & Zirconium-ICP-ACid Dil. & $\mathrm{ug} / \mathrm{mL}$ & 100.0 & $<1.00 \mathrm{e}-02$ & $<2.010$ & $<2.01 \mathrm{e} 0$ & $n / a$ & $n / a$ & 99.50 & 2.010 & $n / a$ \\
\hline $597 T 000026$ & & Fluoride-IC-Dionex $4000 / 4500$ & $\mathrm{ug} / \mathrm{mL}$ & 95.25 & $<1.20 \mathrm{e}-02$ & $6.47 \mathrm{e}+02$ & 546.0 & 596.5 & 16.9 & 95.25 & 25.45 & $\mathrm{n} / \mathrm{a}$ \\
\hline S97T000026 & & Chloride-IC-Dionex $4000 / 4500$ & $\mathrm{ug} / \mathrm{mL}$ & 100.5 & $<1.70 \mathrm{e}-02$ & $1.40 \mathrm{e}+03$ & $1.46 \mathrm{e}+03$ & $1.43 \mathrm{e}+03$ & 4.20 & 87.59 & 36.06 & $\mathrm{n} / \mathrm{a}$ \\
\hline S97T000026 & & Nitrite-IC - Dionex $4000 / 4500$ & $\mathrm{ug} / \mathrm{mL}$ & 108.1 & $<1.08 e^{-01}$ & $1.46 \mathrm{e}+04$ & $1.45 \mathrm{e}+04$ & $1.45 \mathrm{e}+04$ & 0.69 & 96.13 & 229.1 & $\mathrm{n} / \mathrm{a}$ \\
\hline \$97T000026 & & Bromide by Ion Chromatograph & $\mathrm{ug} / \mathrm{mL}$ & 94.40 & $<1.25 \mathrm{e}-01$ & $<2.65 \mathrm{e}+02$ & $<2.65 \mathrm{e} 2$ & $n / a$ & $\mathrm{n} / \mathrm{a}$ & 95.76 & 265.1 & $\mathrm{n} / \mathrm{a}$ \\
\hline $597 T 000026$ & & Nitrate by IC-Dionex $4000 / 4500$ & $\mathrm{ug} / \mathrm{mL}$ & 99.16 & $3.04 \mathrm{e}-01$ & $4.87 \mathrm{e}+04$ & $4.78 \mathrm{e}+04$ & $4.82 \mathrm{e}+04$ & 1.87 & 52.36 & 294.8 & $n / a$ \\
\hline S97T000026 & & Phosphate-IC-Dionex $4000 / 4500$ & $\mathrm{ug} / \mathrm{mL}$ & 98.90 & $<1.20 \mathrm{e}-01$ & $4.02 e+03$ & $3.96 \mathrm{e}+03$ & $3.99 e+03$ & 1.50 & 94.30 & 254.5 & $\mathrm{n} / \mathrm{a}$ \\
\hline S97T000026 & & Sulfate by IC-Dionex $4000 / 4500$ & $\mathrm{ug} / \mathrm{mL}$ & 99.84 & $<1.38 \mathrm{e}-01$ & $1.79 \mathrm{e}+03$ & $2.28 \mathrm{e}+03$ & $2.03 e+03$ & 24.1 & 95.09 & 292.7 & $\mathrm{n} / \mathrm{a}$ \\
\hline S977000026 & & Oxalate-IC-Dionex $4000 / 450$ & $\mathrm{ug} / \mathrm{mL}$ & 99.62 & $<1.05 \mathrm{e}-01$ & $<2.23 e+02$ & $<2.23 \mathrm{e} 2$ & $n / a$ & $\mathrm{n} / \mathrm{a}$ & 94.37 & 222.7 & $\mathrm{n} / \mathrm{a}$ \\
\hline S97T000028 & & Strontium-89/90 High Level & $\mathrm{uCi} / \mathrm{mL}$ & 98.81 & $<2.39 \mathrm{e}-04$ & $7.66 \mathrm{e}-03$ & $7.26 \mathrm{e}-03$ & $7.46 e-03$ & 5.36 & $n / a$ & $4.38 \mathrm{e}-04$ & $8.76 \mathrm{E}+00$ \\
\hline S97T000028 & & Pu-239/240 by TRU-SPEC Res in & $\mathrm{UCi} / \mathrm{mL}$ & 99.66 & $<4.24 \mathrm{e}-06$ & $1.53 e-05$ & $1.51 \mathrm{e}-05$ & $9.52 \mathrm{e}-05$ & 1.32 & $\mathrm{n} / \mathrm{a}$ & $4.63 e-06$ & $3.19 E+00$ \\
\hline 5977000028 & & Cobalt $t-60$ by GEA & $\mathrm{UCi} / \mathrm{mL}$ & 99.69 & $<5.53 e-05$ & $<8.86 \mathrm{e}-04$ & $<1.05 e-3$ & $\mathrm{n} / \mathrm{a}$ & $\mathrm{n} / \mathrm{a}$ & $n / a$ & $1.00 \mathrm{e}-03$ & $n / a$ \\
\hline 5977000028 & & Cesium-137 by GEA & $u \mathbf{C} \mathbf{i} / \mathrm{mL}$ & 102.0 & $<5.62 \mathrm{e}-05$ & 37.00 & 36.50 & 36.75 & 1.36 & $n / a$ & $n / a$ & 0.160 \\
\hline 5977000028 & & Am-241 by Extraction & $u C i / \mathrm{mL}^{2}$ & 85.71 & $<7.16 \mathrm{e}-06$ & $<9.16 \mathrm{e}-06$ & $<7.36 \mathrm{E}-6$ & $n / a$ & $n / a$ & $\mathrm{n} / \mathrm{a}$ & $9.16 \mathrm{e}-06$ & $7.85 E+00$ \\
\hline
\end{tabular}


Table 2. Data Summary Table SY-102 GRAB1

CORE NUMBER: $n / a$

SEGMENT \#: 2SY-96-3

SEGMENT PORIION: Supernat

\begin{tabular}{|c|c|c|c|c|c|c|c|c|c|c|c|c|}
\hline Sample\# $\quad \mathrm{R}$ & $\mathrm{R}$ A\# & Analyte & Unit & Standard \% & Blank & Result & Duplicate & Average & RPD \% & Spk Rec \% & Det Limit & Count Err\% \\
\hline S97T000027 & & DSC Exotherm using Mettler & Joules/g & 107.6 & $n / a$ & $0.00 \mathrm{e}+00$ & $0.00 \mathrm{e}+00$ & $0.00 \mathrm{e}+00$ & 0.00 & $\mathrm{n} / \mathrm{a}$ & $\mathrm{n} / \mathrm{a}$ & $n / a$ \\
\hline $597 \mathrm{r} 000027$ & & OH- by Pot. Titration & $\mathrm{ug} / \mathrm{mL}$ & 102.2 & $<42.00$ & $7.98 \mathrm{e}+03$ & $8.59 e+03$ & $8.28 \mathrm{e}+03$ & 7.36 & $\mathrm{n} / \mathrm{a}$ & $1.25 \mathrm{e}+03$ & $\mathrm{n} / \mathrm{a}$ \\
\hline$\$ 971000027$ & & pH Direct & $\mathrm{pH}$ & 100.2 & n/a & 12.39 & 12.42 & 12.41 & 0.24 & $\mathrm{n} / \mathrm{a}$ & $1.00 \mathrm{e}-02$ & $n / a$ \\
\hline S971000027 & & Specific Gravity & Sp.G. & 99.71 & $\mathrm{n} / \mathrm{a}$ & $n / a$ & $n / a$ & $n / a$ & $n / a$ & $\mathrm{n} / \mathrm{a}$ & $1.00 \mathrm{e}-03$ & $n / a$ \\
\hline$\$ 971000027$ & & \% Water by TGA using Mettler & $\%$ & 99.19 & $n / a$ & 72.02 & 72.51 & 72.27 & 0.68 & $\mathrm{n} / \mathrm{a}$ & $n / a$ & $n / a$ \\
\hline$\$ 977000027$ & & TIC by Acid/Coulometry & ug/mL & 100.2 & 9.400 & $4.19 e+03$ & $4.49 \mathrm{e}+03$ & $4.34 \mathrm{e}+03$ & 6.91 & $\mathrm{n} / \mathrm{a}$ & 5.000 & $n / a$ \\
\hline 5971000027 & & Tot. Organic Carbon by Coul. & ug/mL & 102.0 & $0.00 e+00$ & $5.65 e+03$ & $4.64 \mathrm{e}+03$ & $5.14 \mathrm{e}+03$ & 19.6 & $\mathrm{n} / \mathrm{a}$ & 55.00 & $\mathrm{n} / \mathrm{a}$ \\
\hline 5971000027 & & Fluoride-IC-Dionex $4000 / 4500$ & $\mathrm{ug} / \mathrm{mL}$ & 95.25 & $<1.20 \mathrm{e}-02$ & $4.52 \mathrm{e}+02$ & 453.0 & 452.3 & 0.22 & $n / a$ & 25.45 & $n / a$ \\
\hline 5971000027 & & Chloride-IC-Dionex $4000 / 4500$ & ug $/ \mathrm{mL}$ & 100.5 & $<1.70 \mathrm{e}-02$ & $1.10 \mathrm{e}+03$ & $1.13 e+03$ & $1.12 e+03$ & 2.69 & $n / a$ & 36.06 & n/a \\
\hline S97T000027 & & Nitrite-IC - Dionex $4000 / 4500$ & $\mathrm{ug} / \mathrm{mL}$ & 108.1 & $<1.08 \mathrm{e}-01$ & $1.54 \mathrm{e}+04$ & $1.55 \mathrm{e}+04$ & $1.54 \mathrm{e}+04$ & 0.65 & n/a & 229.1 & $n / a$ \\
\hline 5971000027 & & Bromide by Ion Chromatograph & $\mathrm{ug} / \mathrm{mL}$ & 94.40 & $<1.25 \mathrm{e}-01$ & $<2.65 \mathrm{e}+02$ & $<2.65 \mathrm{e} 2$ & $n / a$ & $\mathrm{n} / \mathrm{a}$ & $\mathrm{n} / \mathrm{a}$ & 265.1 & $n / a$ \\
\hline 5971000027 & & Nitrate by iC-Dionex $4000 / 4500$ & $\mathrm{ug} / \mathrm{mL}$ & 99.16 & $3.04 \mathrm{e}-01$ & $3.78 \mathrm{e}+04$ & $3.79 \mathrm{e}+04$ & $3.79 \mathrm{e}+04$ & 0.26 & n/a & 294.8 & $n / a$ \\
\hline S97T000027 & & Phosphate-IC-D ionex $4000 / 4500$ & $\mathrm{ug} / \mathrm{mL}$ & 98.90 & $<1.20 \mathrm{e}-01$ & $4.40 \mathrm{e}+03$ & $4.47 \mathrm{e}+03$ & $4.43 \mathrm{e}+03$ & 1.58 & $\mathrm{n} / \mathrm{a}$ & 254.5 & n/a \\
\hline $597 \mathrm{~T} 000027$ & & Sulfate by IC-D ionex $4000 / 4500$ & $\mathrm{ug} / \mathrm{mL}$ & 99.84 & $<1.38 \mathrm{e}-01$ & $2.19 e+03$ & $2.34 \mathrm{e}+03$ & $2.26 \mathrm{e}+03$ & 6.62 & n/a & 292.7 & $\mathrm{n} / \mathrm{a}$ \\
\hline \$97T000027 & & Oxalate-IC-Dionex $4000 / 450$ & ug $/ \mathrm{mL}_{\mathrm{L}}$ & 99.62 & $<1.05 \mathrm{e}-01$ & $5.79 \mathrm{e}+02$ & 553.0 & 566.0 & 4.59 & $\mathrm{n} / \mathrm{a}$ & 222.7 & $n / a$ \\
\hline $597 \mathrm{~T} 000029$ & & Stront ium- $89 / 90 \mathrm{High}$ Level & $u C \mathbf{i} / \mathrm{mL}$ & 107.1 & $<1.40 \mathrm{e}-03$ & 3.100 & 3.250 & 3.175 & 4.72 & $\mathrm{n} / \mathrm{a}$ & $2.90 e-02$ & $3.82 \mathrm{E}+00$ \\
\hline$\$ 971000029$ & & Cobal $t-60$ by GEA & $\mathrm{UCi} / \mathrm{mL}$ & 99.69 & $55.53 e-05$ & $5.31 e-03$ & $1.05 \mathrm{e}-02$ & $7.91 e^{-03}$ & 65.7 & n/a & $n / a$ & 37.1 \\
\hline$\$ 971000029$ & & Cesium-137 by GEA & $u C i / m L$ & 102.0 & $<5.62 e-05$ & 24.50 & 24.20 & 24.35 & 1.23 & $\mathrm{n} / \mathrm{a}$ & $\mathrm{n} / \mathrm{a}$ & 0.400 \\
\hline
\end{tabular}


HNF-SD-WM-DP-227, REV, 0

CHAIN OF CUSTODY FORMS 
HNF-SD-WM-DP-227, REV. 0

THIS PAGE INTENTIONALLY LEFT BLANK 


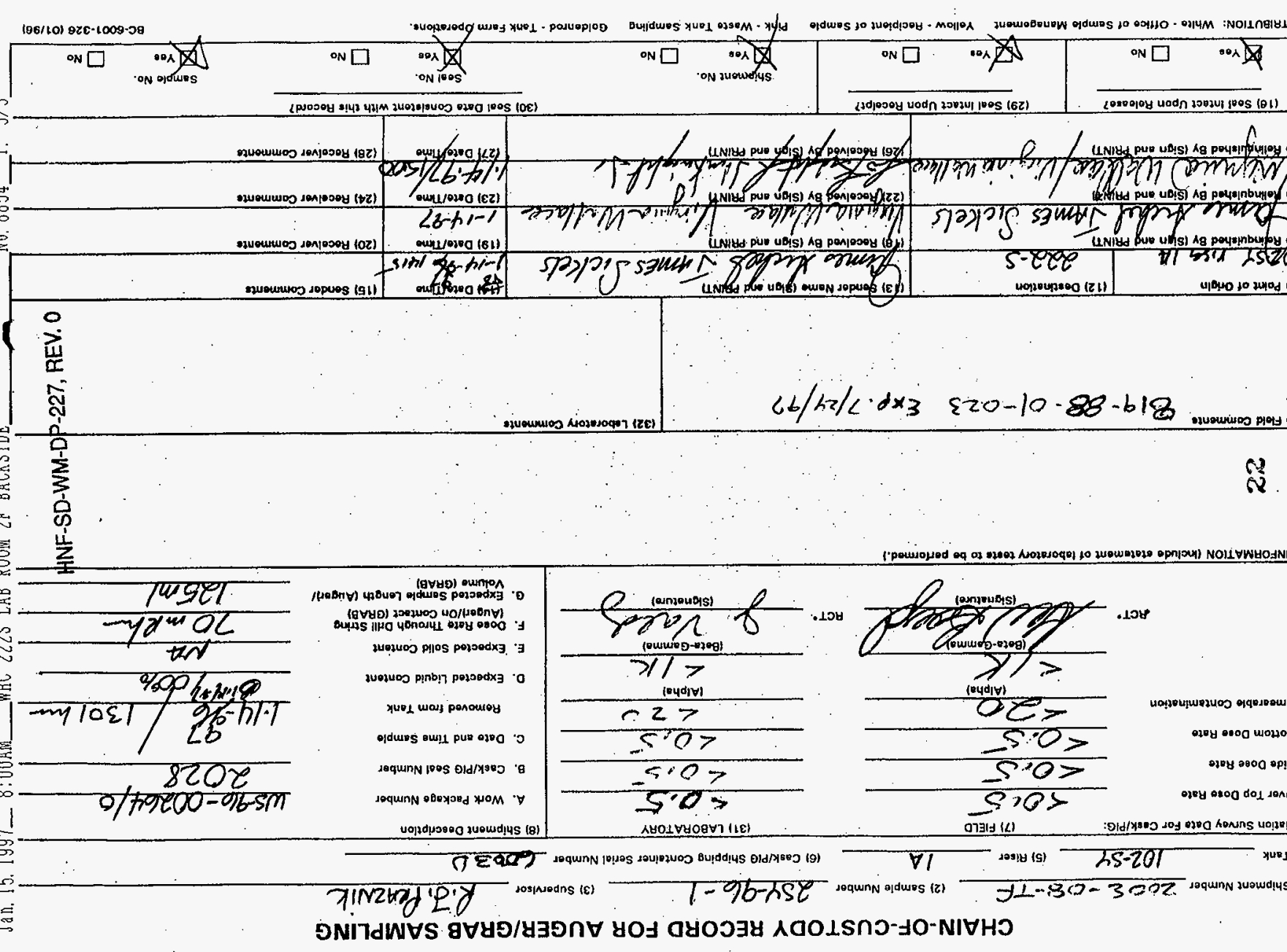




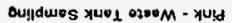

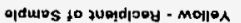

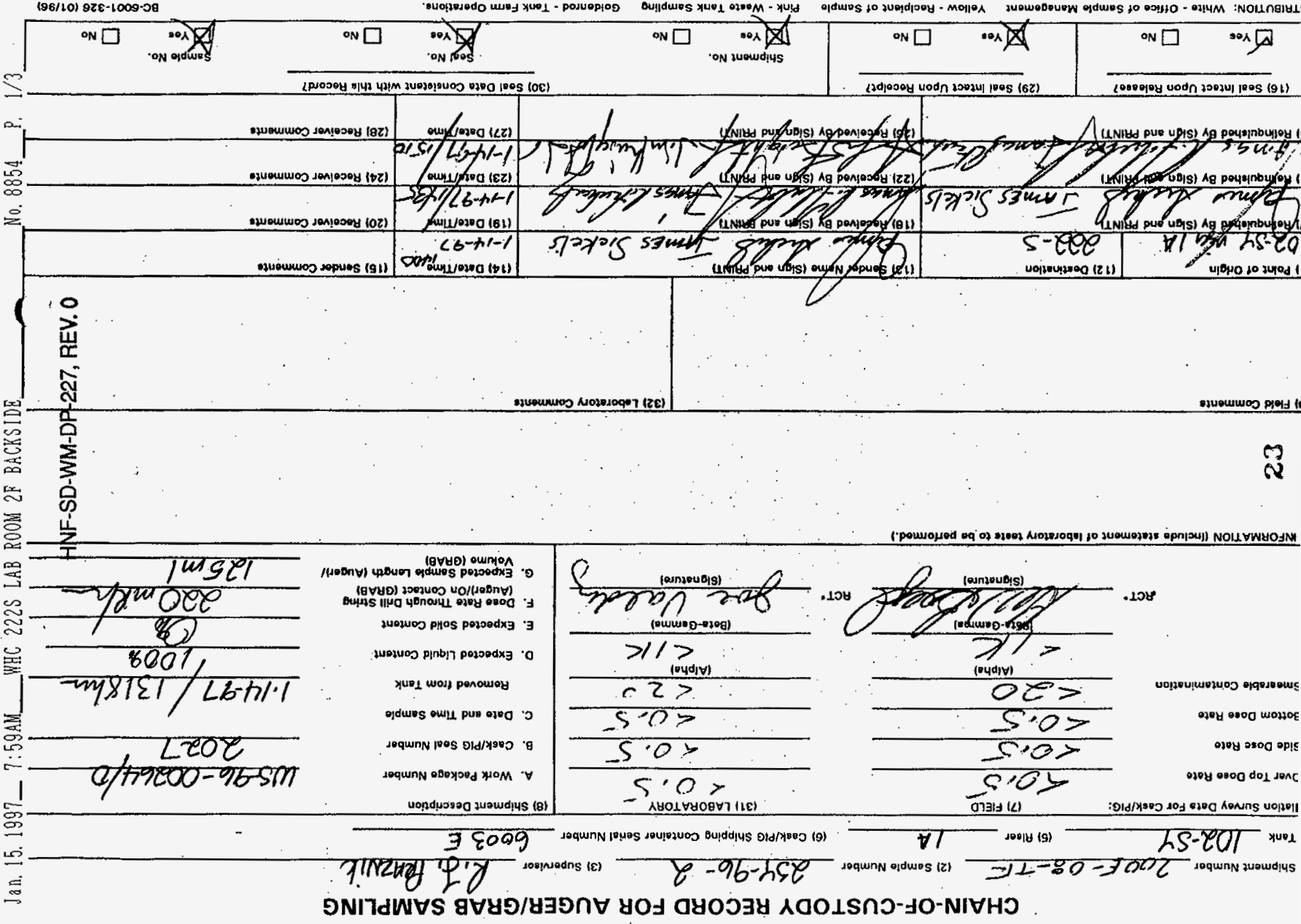


CHAIN-OF-CUSTODY RECORD FOR AUGER/GRAB SAMPLING

Shipment Number $2002-08-T F$ Tank $102-5 \%$

idiation Survoy Dato For Cosk/PIG:

Over Top Doso Rato

Sido Dose Rate

Bottom Does Rate

Smoarable Contamination

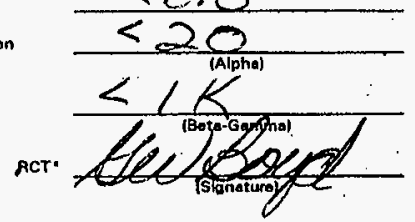
(5) Piser $\quad / A$

$<0.5$

0.7
(2) Sample Number $254-96-3$

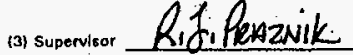
(6) CankjfIG Shipping Container Serial Number $6003 \mathrm{~B}$

(3i) LABORATORY

$\frac{0.5}{0.5}$

(8) Shipment Doscription

A. Work Peckago Number

B. Cask/Pto Sool Number

$<0,5=$

220

c. Date and Timo Samplo

Aemovod from Tank

D. Expected Liquid Content

E. Expected Solld Content

F. Dowo Rate Through Drill String

RCT:

$\angle / K$

gae Ulaldy 6. Expocted Samplo Length (Augor)/
Volumo (GRAB)

.

INFOAMATION (Inelude statement of laboratory toete to be performed.)

NS

0) Fild Commante

* Susponded solids

(32) Leboratory Commento

1) Polint of Orlain

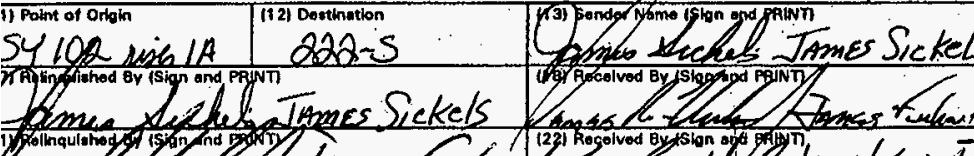

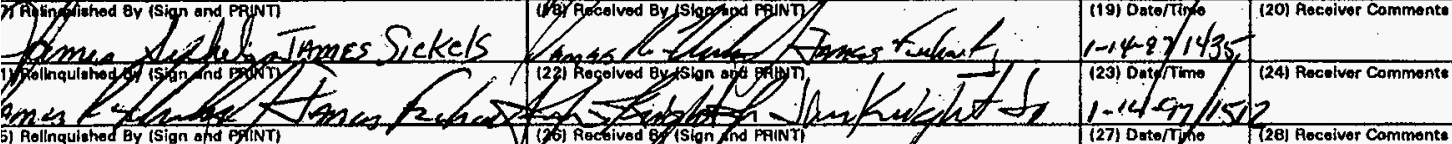

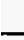

(16) Seot Intact Upan Reloge?

(29) Seal Intect Upon Rocolpt?

(30) Soal Date Conelotent whit this Rocord?

DYo: $\square$ No

$\sqrt{1}$

Shipmont No.

X Yoe $\square$ No

SeofNo.

Samplo No.

SYos

No

STRIBUTION: Whito - Offico of Samplo Management Yollow - Reclpient of Sample Pink - Wasto Tonk Sampling Goldenrod - Tenk farm Operatione.

BC-6001-326 $101 / 901$ 
HNF-SD-WM-DP-227, REV. 0

WASTE COMPATIBILITY CORROSION RULES TABLE 3 
HNF-SD-WM-DP-227, REV. 0

THIS PAGE INTENTIONALLY LEFT BLANK

26 
TABLE 3. WASTE COMPATIBILITY CORROSION RULES

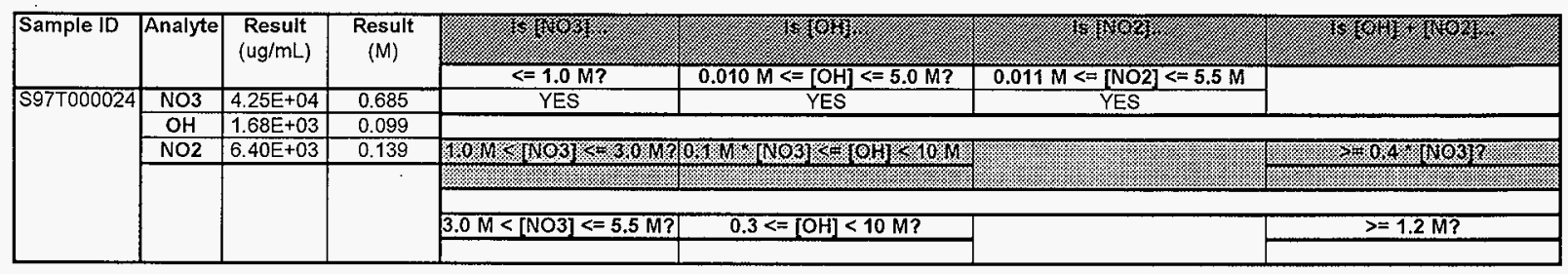

\begin{tabular}{|c|c|c|c|c|c|c|c|}
\hline \multirow[t]{2}{*}{ Sample ID } & \multirow[t]{2}{*}{ Analyte } & \multirow{2}{*}{$\begin{array}{l}\text { Result } \\
\text { (ug/mL) }\end{array}$} & \multirow{2}{*}{$\begin{array}{l}\text { Result } \\
\text { (M) }\end{array}$} & 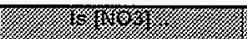 & 64 & 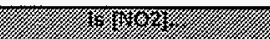 & 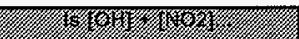 \\
\hline & & & & $<=1.0 \mathrm{M} ?$ & $0.010 \mathrm{M}<=[\mathrm{OH}]<=5.0 \mathrm{M} ?$ & $0.011 \mathrm{M}<=[\mathrm{NO} 2]<=5.5 \mathrm{M}$ & \\
\hline \multirow[t]{5}{*}{ S97T000026 } & NO3 & $4.87 E+04$ & 0.785 & YES & YES & YES & \\
\hline & $\mathrm{OH}$ & $7.98 \mathrm{E}+03$ & 0.469 & & & & \\
\hline & NO2 & $1.46 \mathrm{E}+04$ & 0.317 & \multirow{2}{*}{\multicolumn{2}{|c|}{ 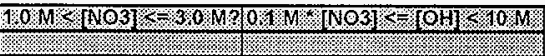 }} & 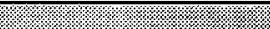 & 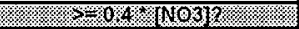 \\
\hline & & & & & & & S \\
\hline & & & & \multirow{2}{*}{\multicolumn{2}{|c|}{$3.0 \mathrm{M}<[\mathrm{NO}]<=5.5 \mathrm{M} ? \quad 0.3<=[\mathrm{OH}]<10 \mathrm{M} ?$}} & & $>=1.2 \mathrm{M} ?$ \\
\hline & & & & & & & \\
\hline
\end{tabular}


HNF-SD-WM-DP-227, REV. 0

INORGANIC ANALYSES 
HNF-SD-WM-DP-227, REV. 0

THIS PAGE INTENTIONALLY LEFT BLANK 


\section{LABCORE Data Entry Template for Worklist\# 16284}

Analyst: SMF Instrument: $\mathrm{DSCO} \stackrel{3}{3}$ Book \# 12 N14B

Method: LA-514-114 Rev/Mod D-0

Worklist Comment: Run under Nitrogen.

new

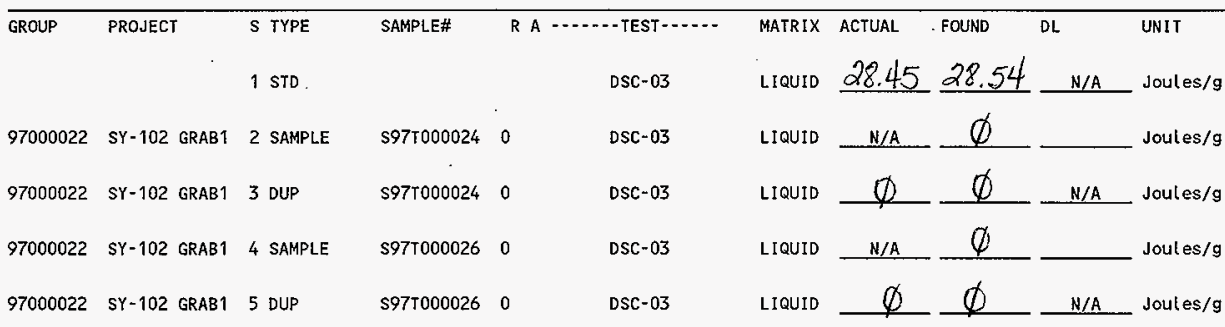

\section{Final page for worklist \# $\mathbf{1 6 2 8 4}$}
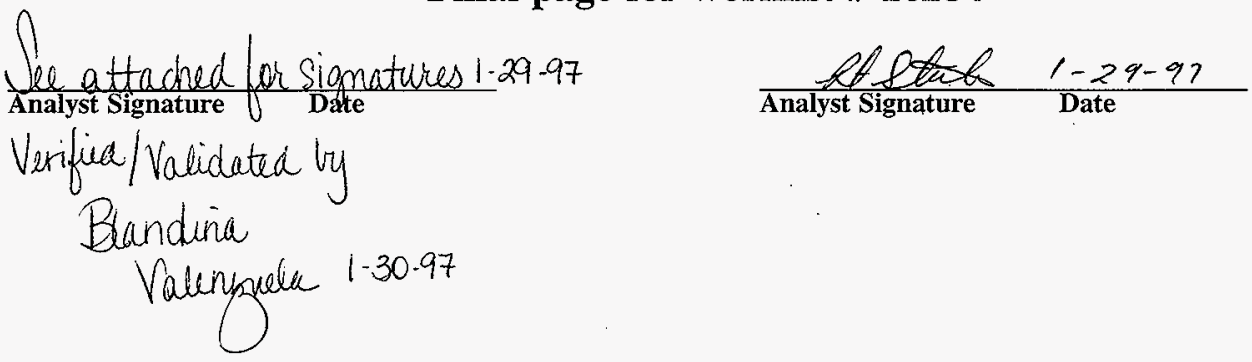

Data Entry Comments:

Units shown for $Q C$ (SPK \& STD) may not reflect the actual units. $D L=$ Detection Limit, $S=$ Worklist Slot Number,

$R=$ Replicate Number, $A=$ Aliquot Code. 


\section{LABCORE Data Entry Template for Worklist\# 16284}

Analyst: $\quad$ EXPF Instrument: DSC0 Book\# $12014 \sim B$

Method: LA-514-113 Rev/Mod

Worklist Comment: Run under Nitrogen.

new

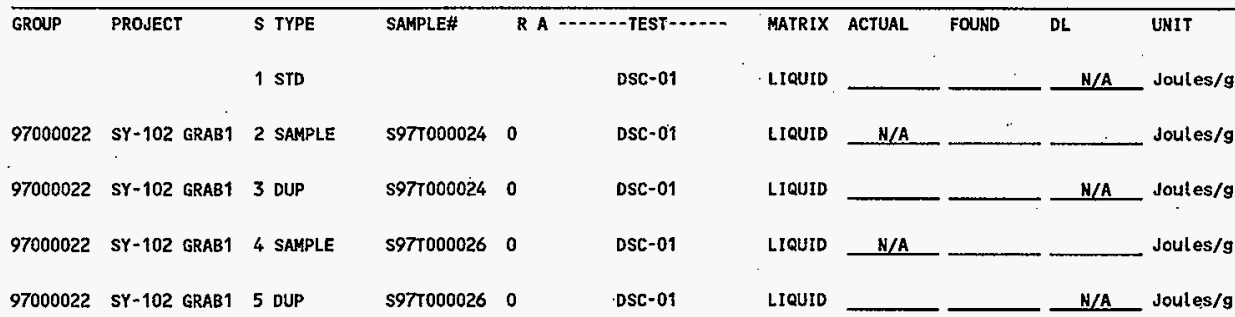

\section{Final page for worklist \# 16284}

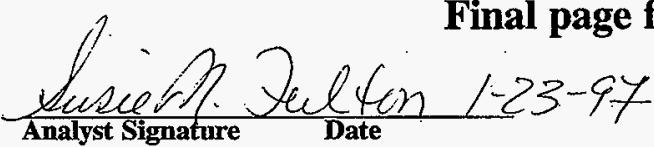

Analyst Signature Date

DSC-03 instrument was

used. $1-29.97$ Blandina

Valenzuela

Data Entry Comments:

Units shown for $Q C$ (SPK \& STD) moy not reflect the actual units. $D L=$ Detection Limit, $S=$ Worklist Slot Number, $R=$ Replicate Number, $A=$ Aliquot Code. 
Curve 1: Dosc

F11e Info: IND012301 Thu Jen 23 05: 27:02 1997.

Sample Nejght: 17.680 ag

STD 12N14-B

SIGNATURE BELOW REPRESENTS CHEMICAL TECHNOLOGIST/CHEMIST THAT COMPIETED/VERHFIED THE CALIBRATION/ANALYSIS ON PAGES 32 TO 36.

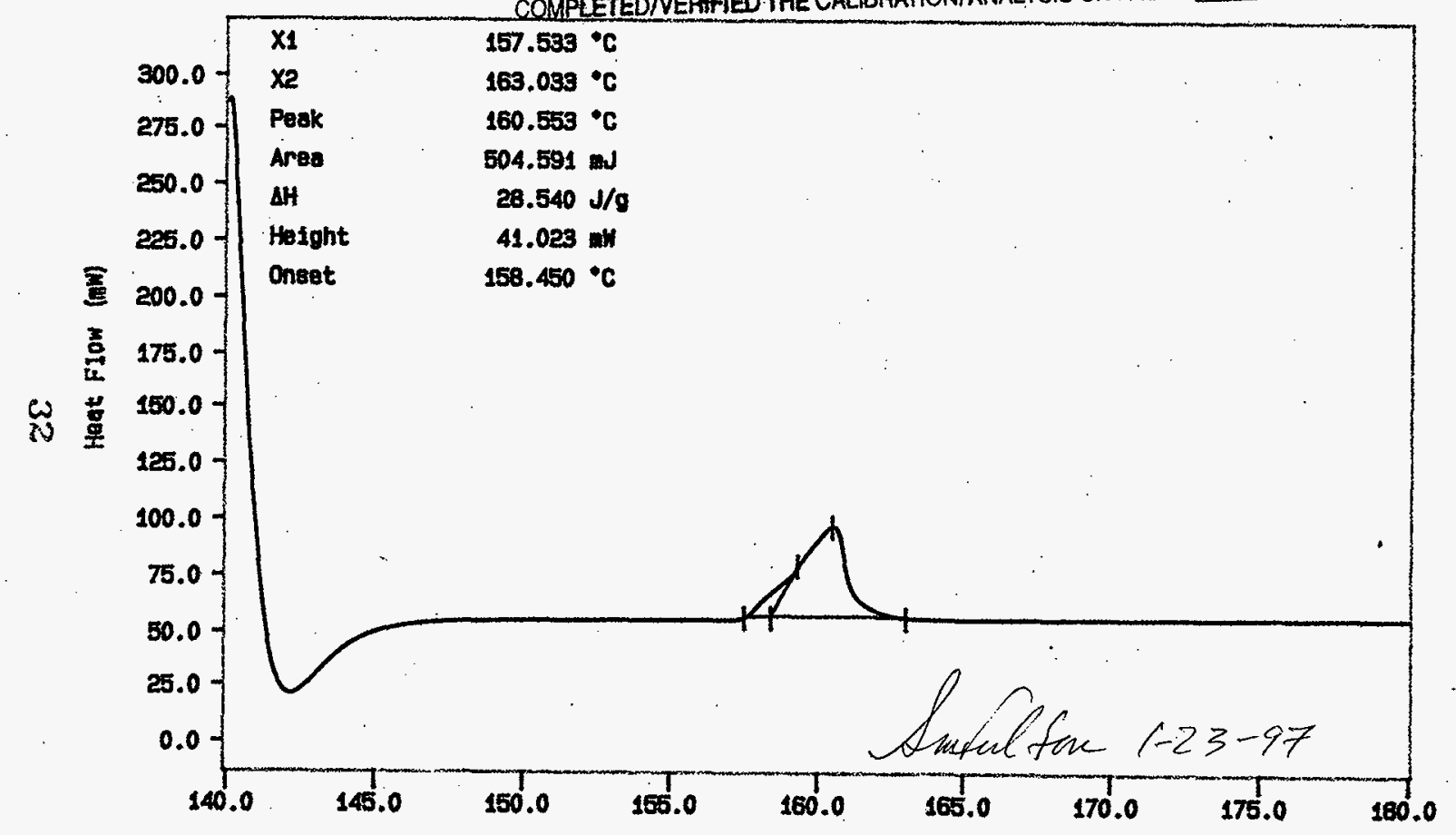

Ne EXTTERM DOWN

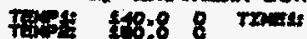
0.0 ain Matrats 10.0 o/min

Temperature $\left({ }^{\circ} \mathrm{C}\right)$

SH FULTON 
HNF-SD-WM-DP-227, REV. 0

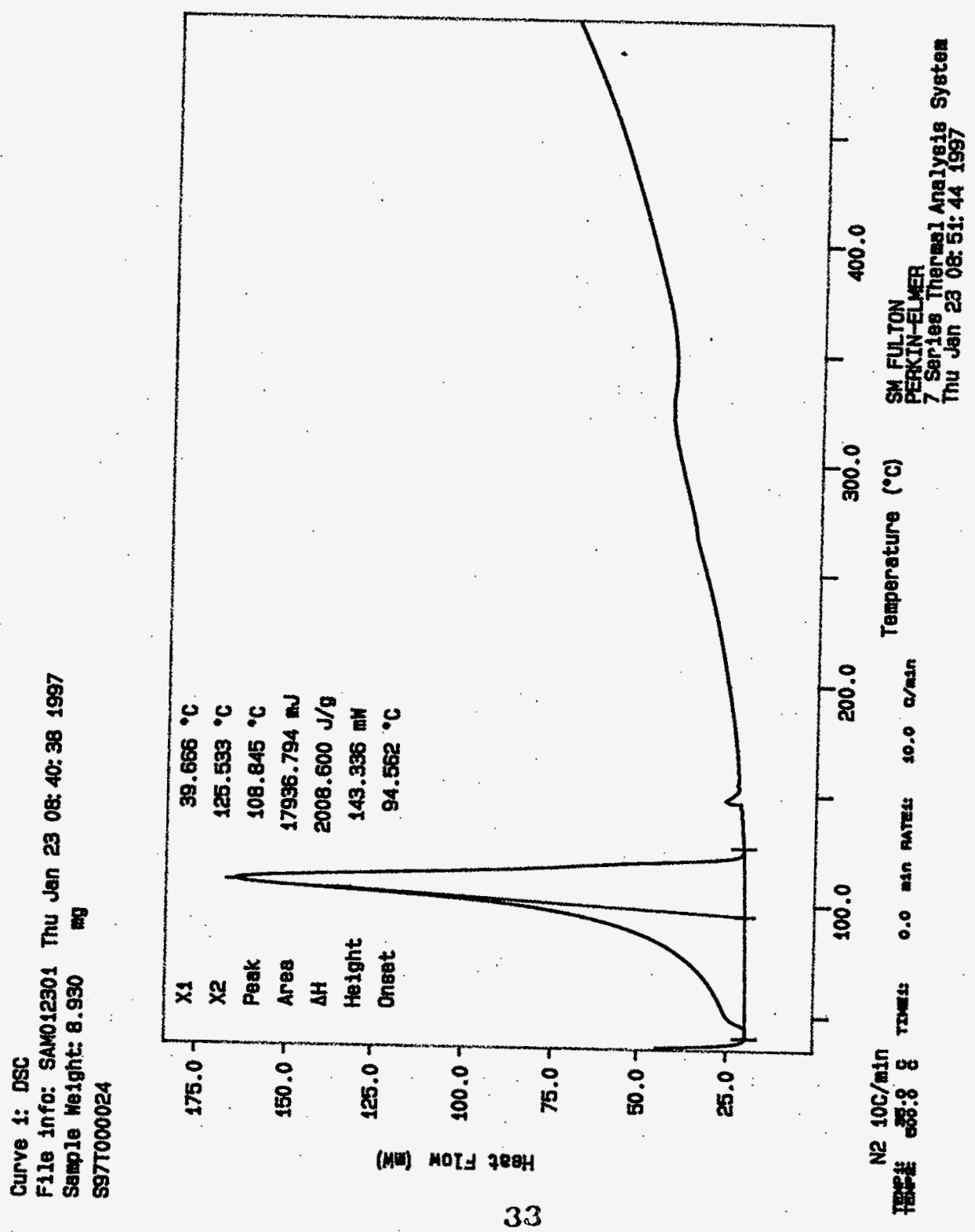


Curve 1: DSC

F1le Info: SAM012302 Thu Jan 23 10: 04: 251997

Sauple Weight: $\mathbf{8 . 5 9 0}$

ag

597T000024 DAP

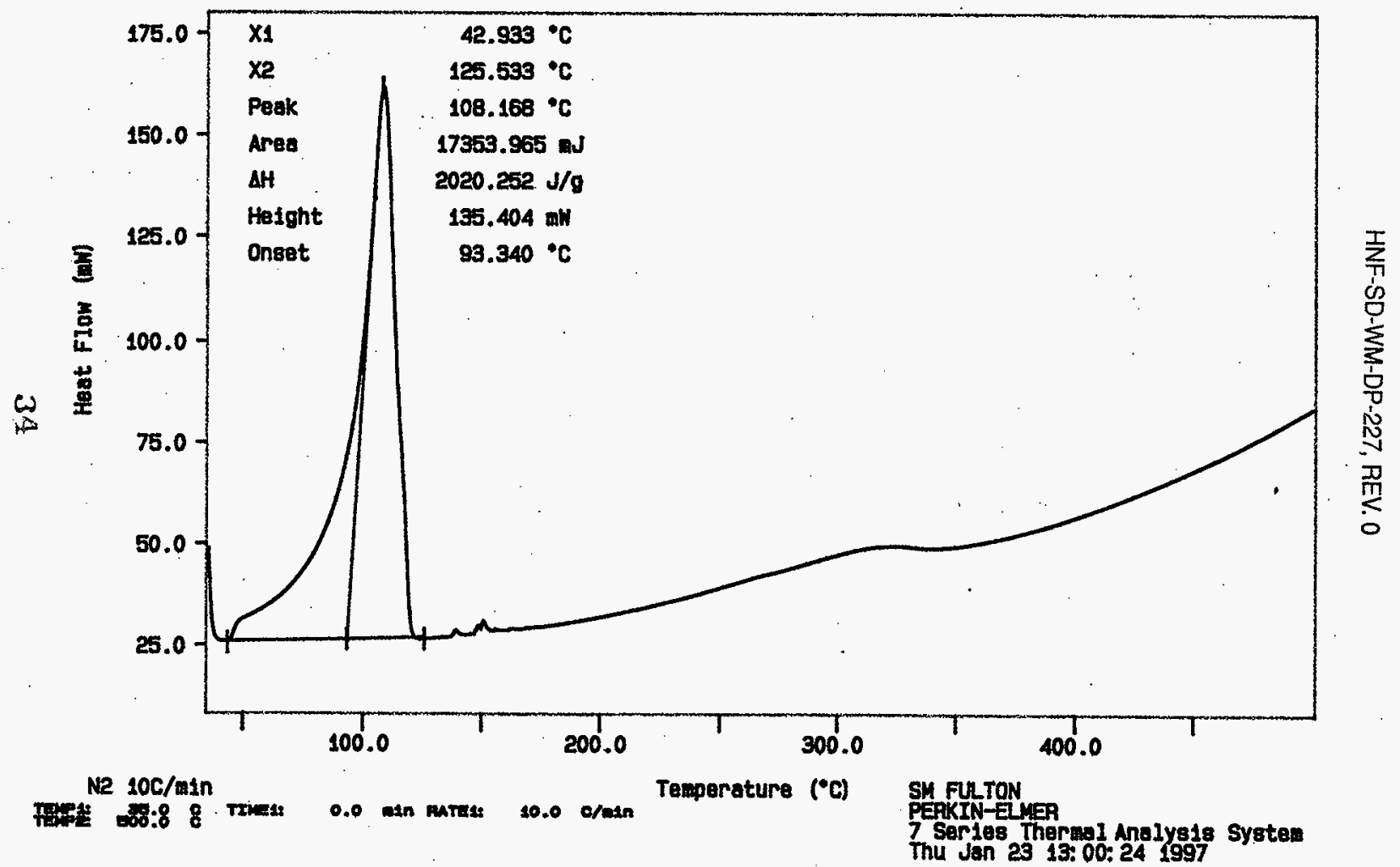


Curve 1: DSC

F1le info: SAM012303 Thu Jan 23 19:53:12 1997

Sample Weight: 11.080 ag

S97T0000276

$1-29.97 \mathrm{BN}$

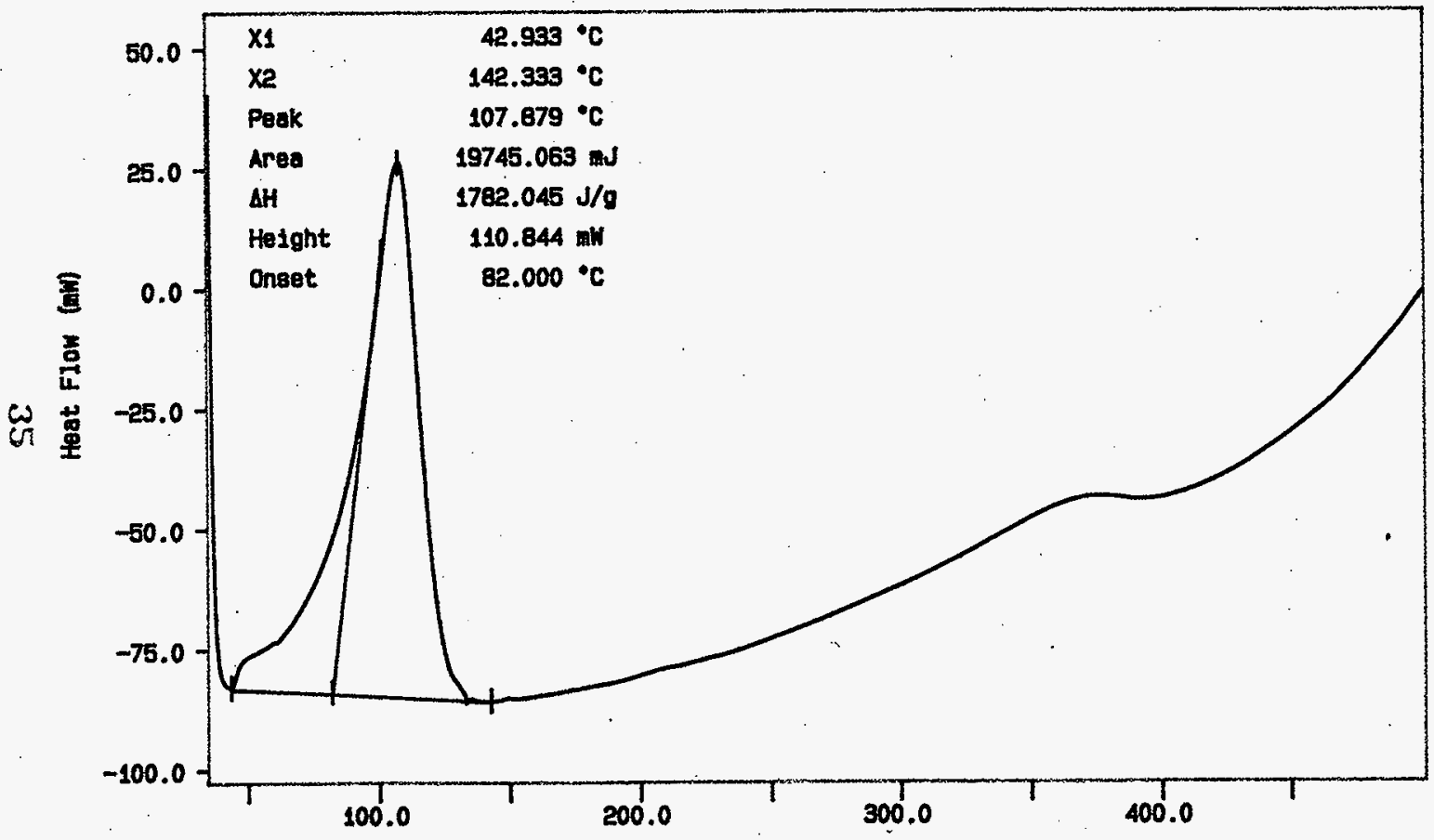

Ne 10c/min

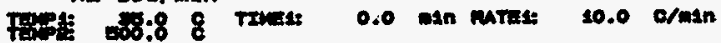

Temperature $\left({ }^{\circ} \mathrm{C}\right)$ SH FULTON

PERKIN-ELMER

7 Sertes Thernal Analysis Syatem

Thu Jan 23 14: 10:52 1997 
Curve 1: DSC

F1le info: SAM012304 Thu tan 23 15: 12: 371997

Sample NeIght: 10.280 ug

S97T000027/6 DUP

$1-29-97 \mathrm{BOH}$

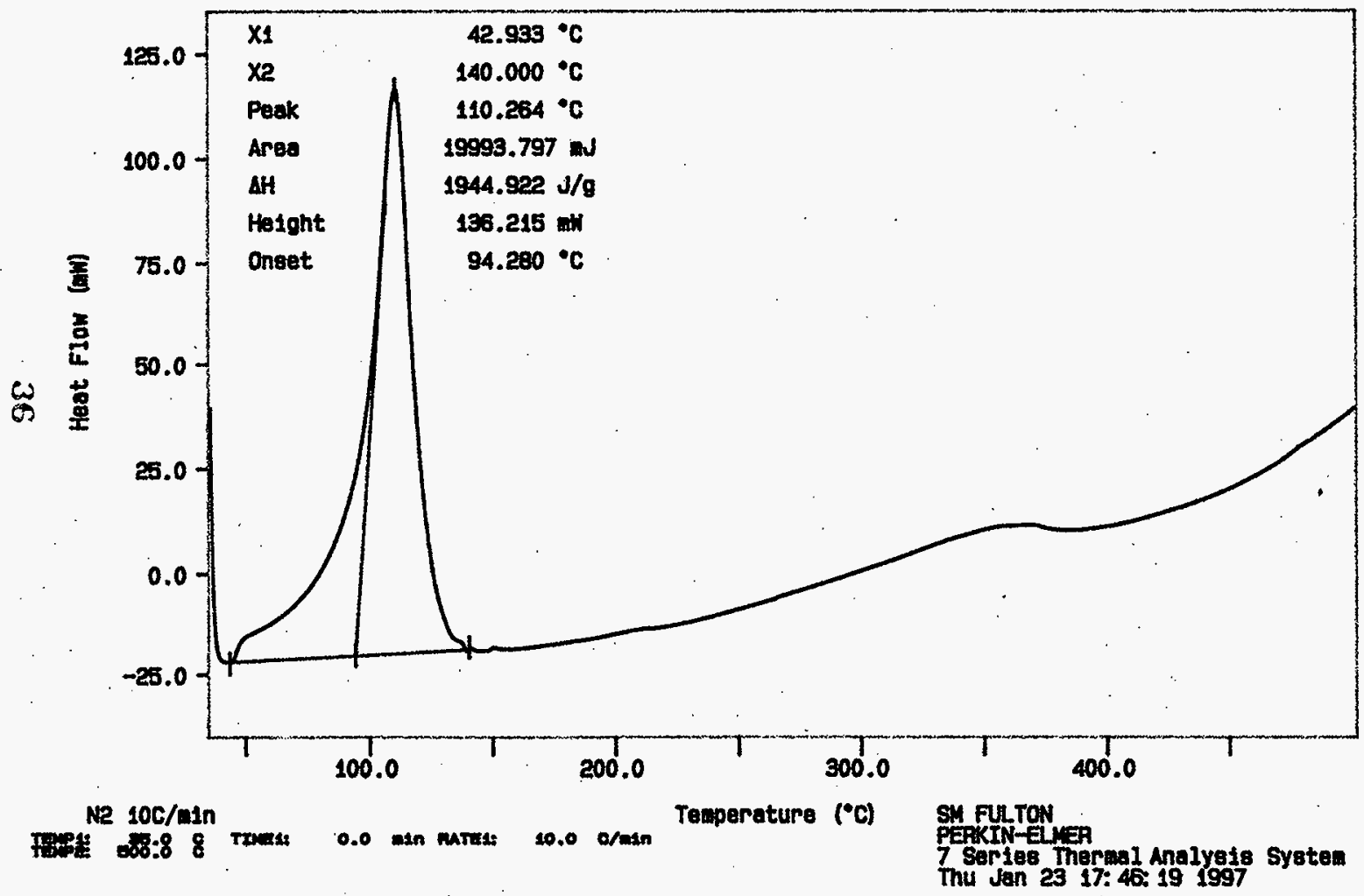


worklistrpt Version 2.1 05/15/95

HNF-SD-WM-DP-227, REV. 0

Page: $\quad 1$ $01 / 22 / 9710: 34$

LABCORE Data Entry Template for Worklist\# 16285

Analyst: $\quad B \mathrm{HF}$ Instrument: $\mathrm{DSCO} \perp$ Book\# $12 \mathrm{~N} / 4-\mathrm{B}$

Method: LA-514-113 Rev/Mod C-1

Worklist Comment: Run under Nitrogen.

new

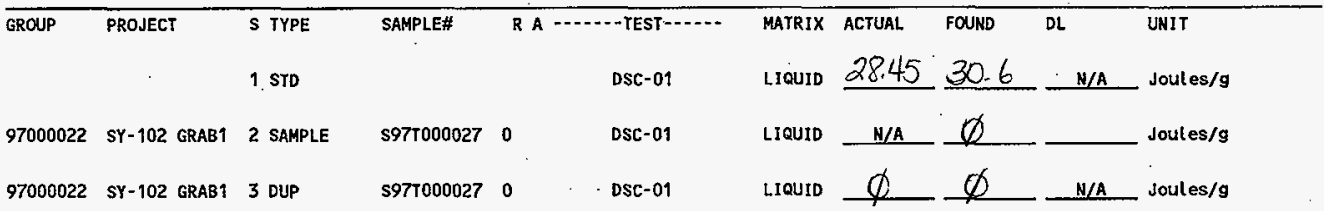

Final page for worklist \# 16285

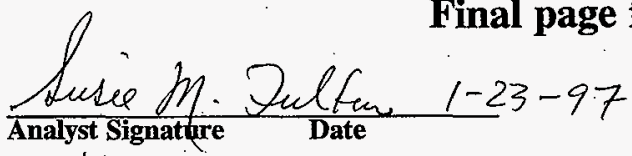

Verified/ Validated by

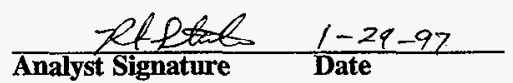

Banding

Valenzuela $1-30-97$

Data Entry Comments:

SAm S47T000027 is a dank brown slung with tiny particto (cha ylike)

Units shown for $Q C$ (SPK \& STD) may not reflect the actual units. $D L=$ Detection Limit, $S=$ Worklist Slot Number, $R=$ Replicate Number, $A=$ Aliquot Code.

37 


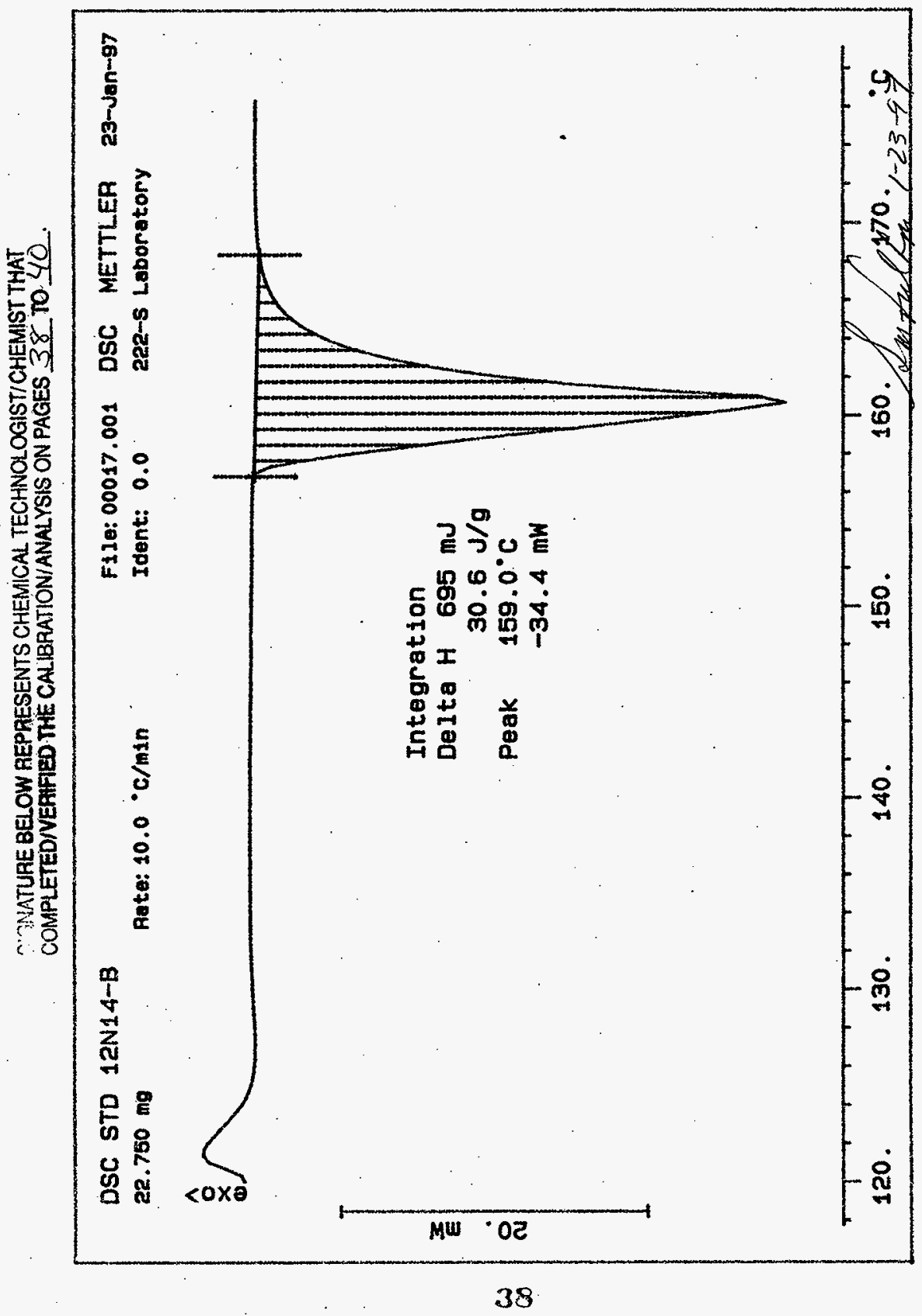




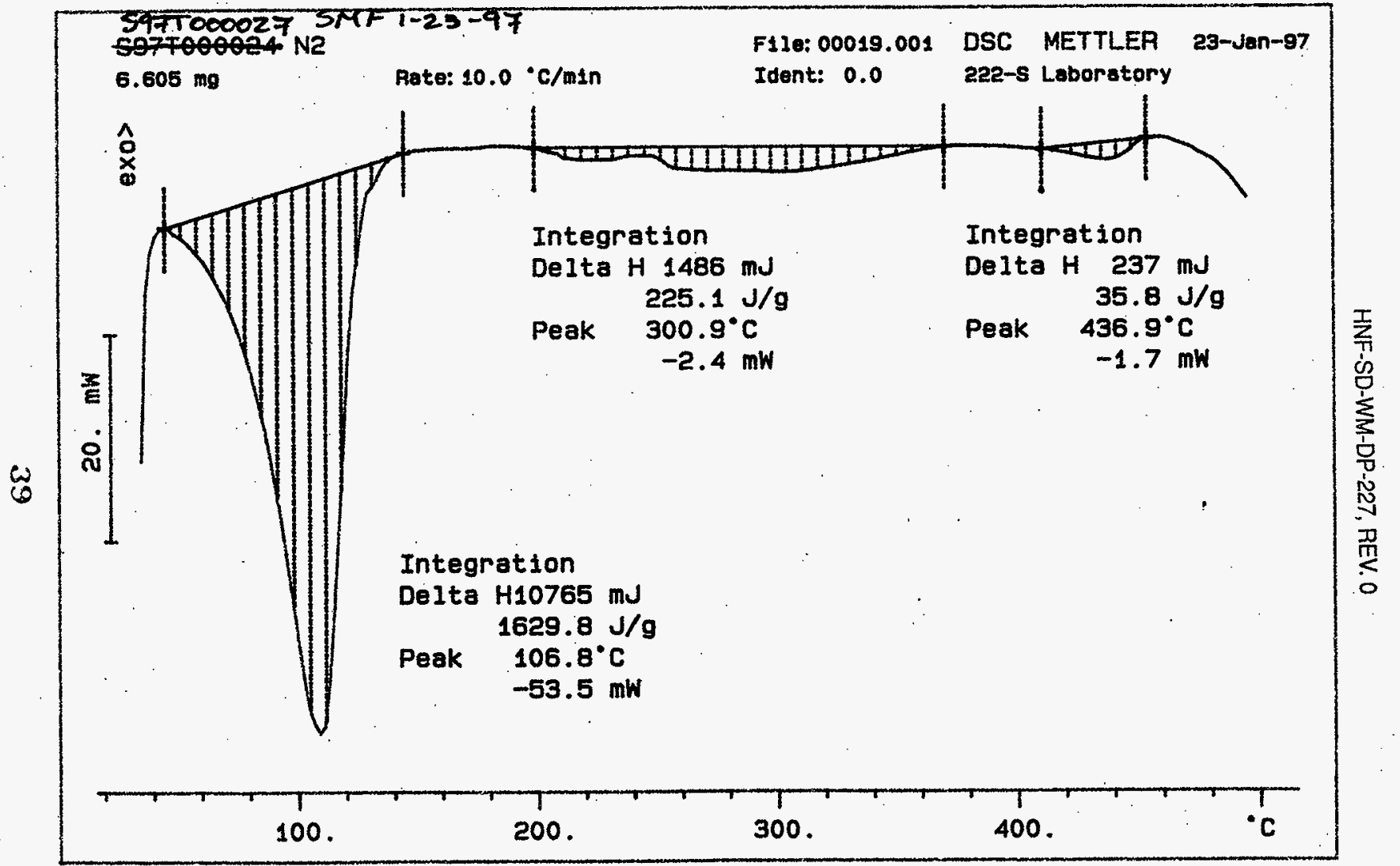




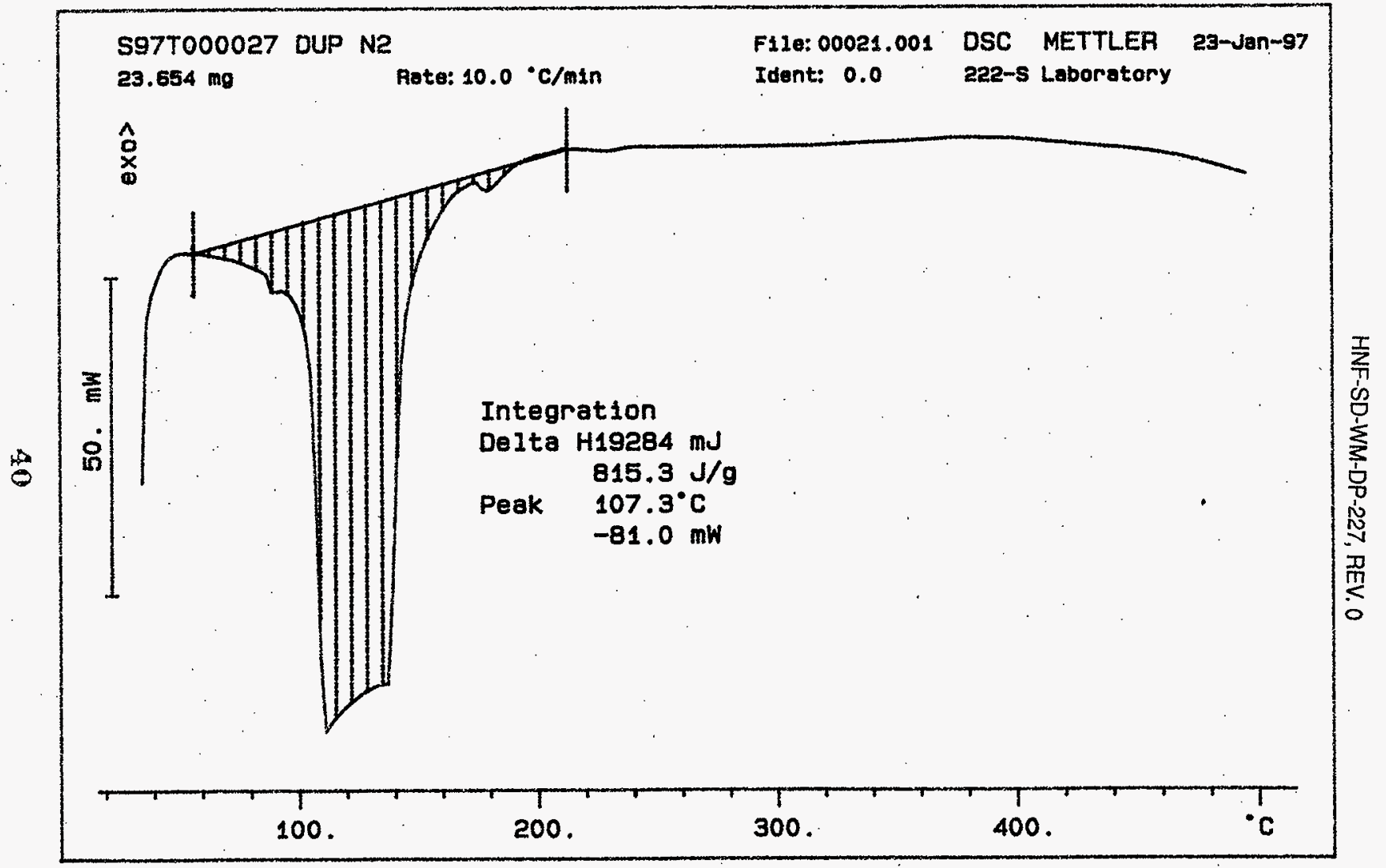




\section{LABCORE Data Entry Template for Worklist\# 16282}

Analyst: $\quad$ SMF Instrument: TGA0 3 Book 97 N8A

Method: LA-514-114 Rev/Mod D-O

Worklist Comment: Run under Nitrogen.

new

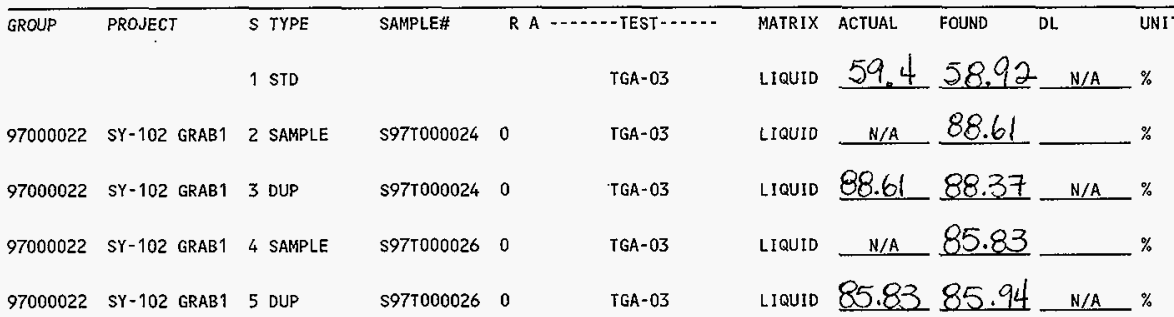

\section{Final page for worklist \# 16282}

Sec attuched.re sigmatures 1.29 .97 Analyst Signature of Date

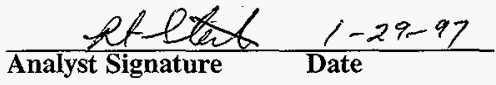

Validatid by

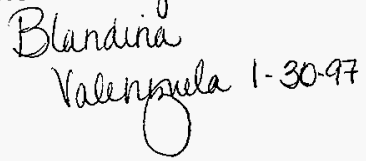

Data Entry Comments:

Units shown for QC (SPK \& STD) may not reflect the actual units. $D L=$ Detection Limit, $S=$ Worklist Slot Number, $R=$ Replicate Number, $A=$ Aliquot Code. 


\section{LABCORE Data Entry Template for Worklist\# 16282}

Analyst: $\quad$ SME $\quad$ Instrument: TGA0 Book\# $9708-A$

Method: LA-560-112 Rev/Mod

Worklist Comment: Run under Nitrogen.

new

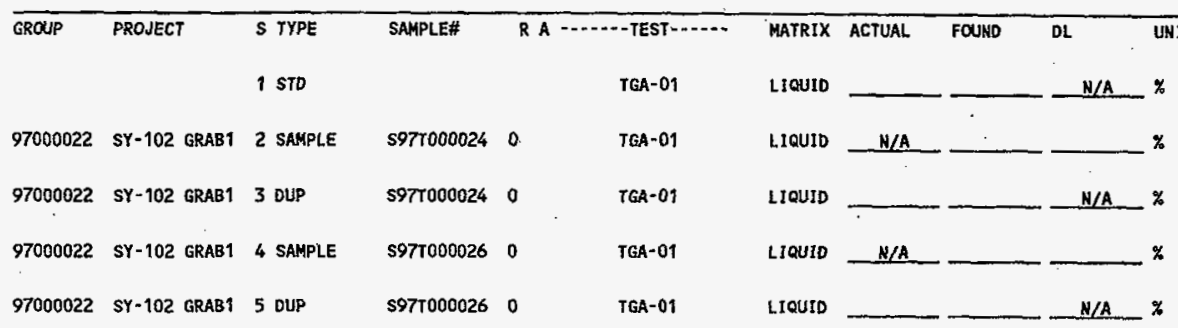

\section{Final page for worklist \# 16282}

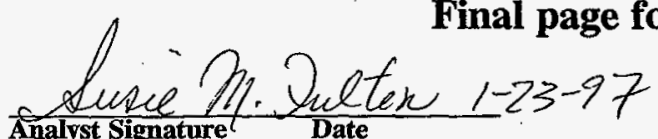

$$
\begin{aligned}
& \text { TGA-03 instrument } \\
& \text { was used. }
\end{aligned}
$$

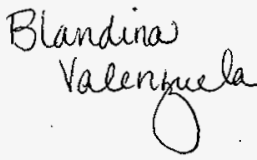

Data Entry Comments:

Units shown for $Q C$ (SPK \& STD) may not reflect the actual units. $D L=$ Detection Limit, $S=$ Worklist Slot Number, $R=$ Replicate Number, $A=$ Aliquot Code. 
Curve 1: TEA

F118 Info: TER012301 Thu Jan 23 05: 53:59 1997

Sample helght: 15.973 og

TGA STD 97NB-A : SIGNATURE BELOW REPRESENTS CHEMICAL TECHNOLOGIST/CHEMIST THAT COMPLETEDNERHFIED THE CALIBRATION/ANALYSIS ON PAGES $\% 3$ TO 47.

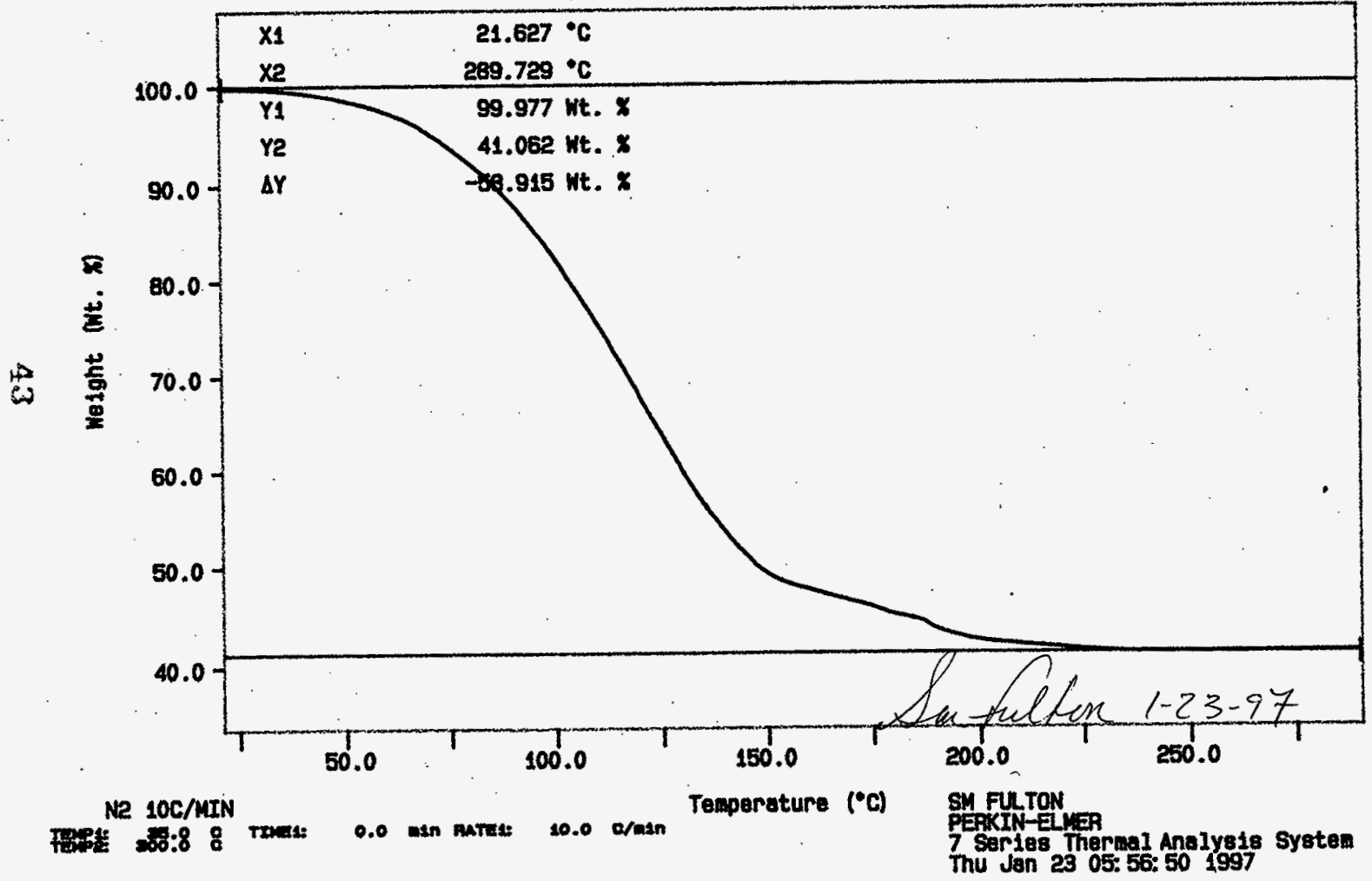


Curve 1: TGA

F1le info: SAM012301 Thu Jan 23 08: 4241997

Somple Velght: 10.050 ag

\$97T000024

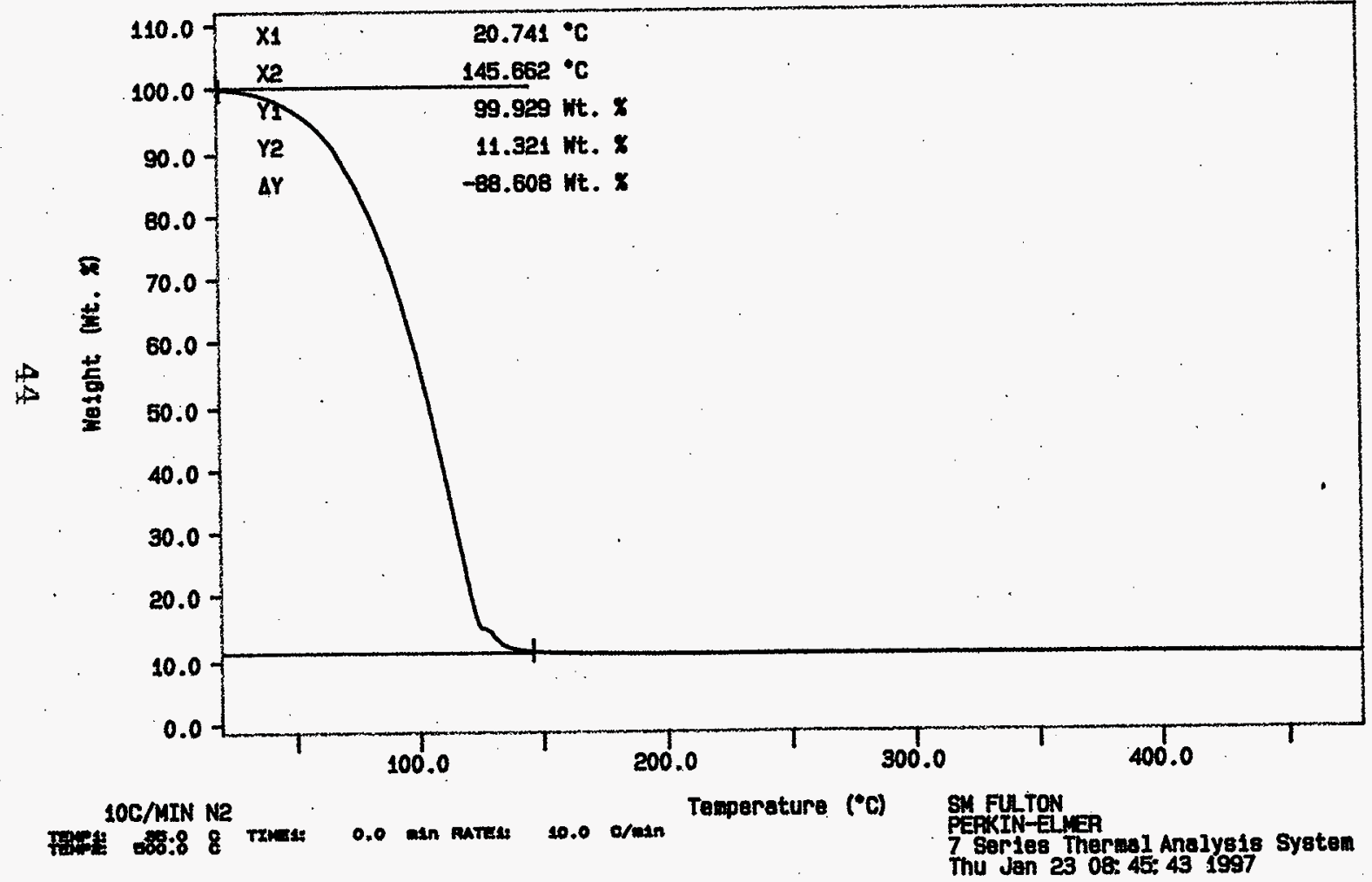


Curve 1: TBA

F11e info: SAk012302 Thu Jan 23 10: 06: 481997

Sample Vejght: 10.097 an

597T000024 DUP

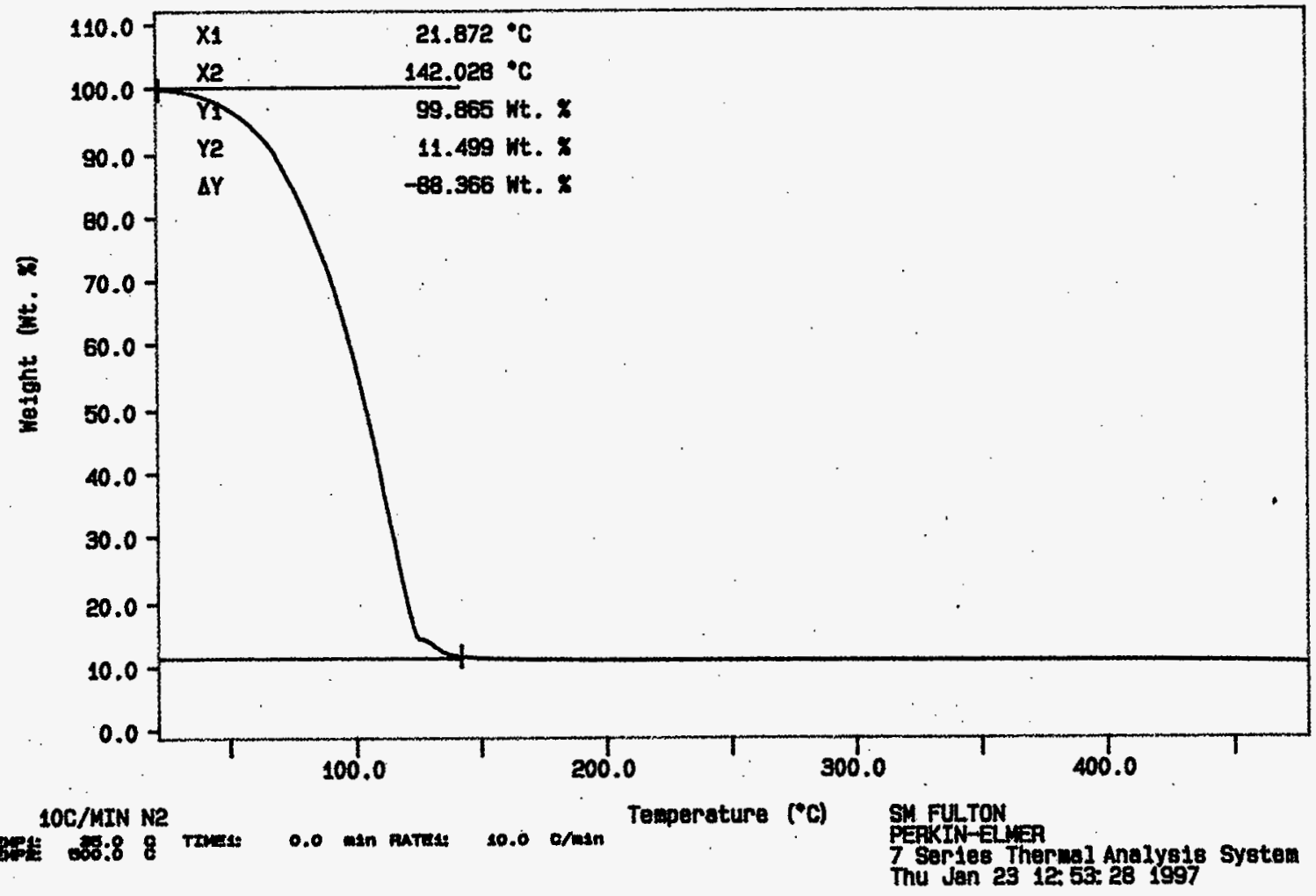


Cunve 1: TEA

F118 Info: SAM012303 Thu Jan 23 13:54: 471997

Sanple Weight: 10.685

s97T000028

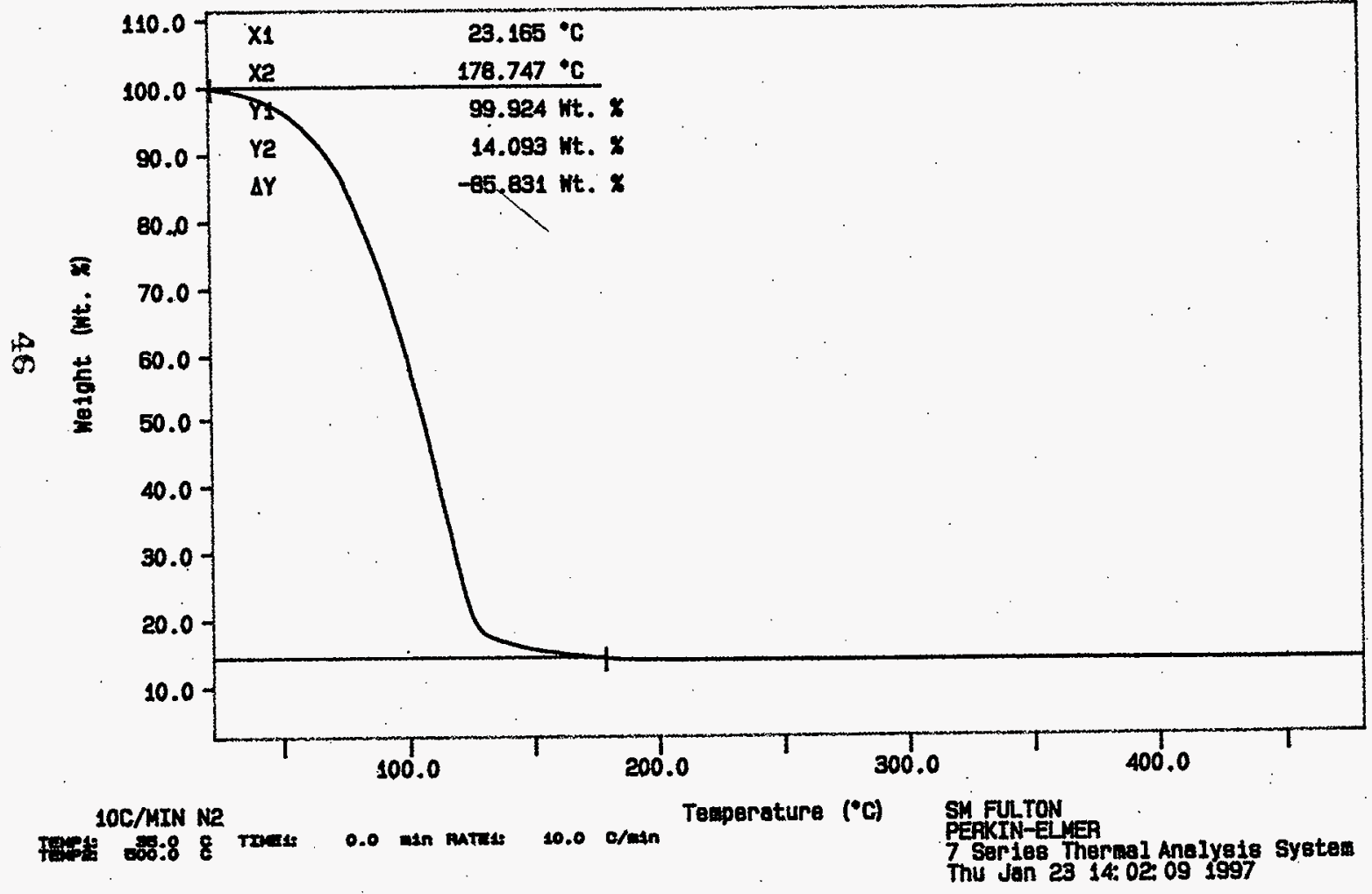


Curve 1: TEA

F11e info: SAM012004 Thu Jan $2315: 15: 401997$

Sample We1ght: 10.108 ang

S97T000026 Dip

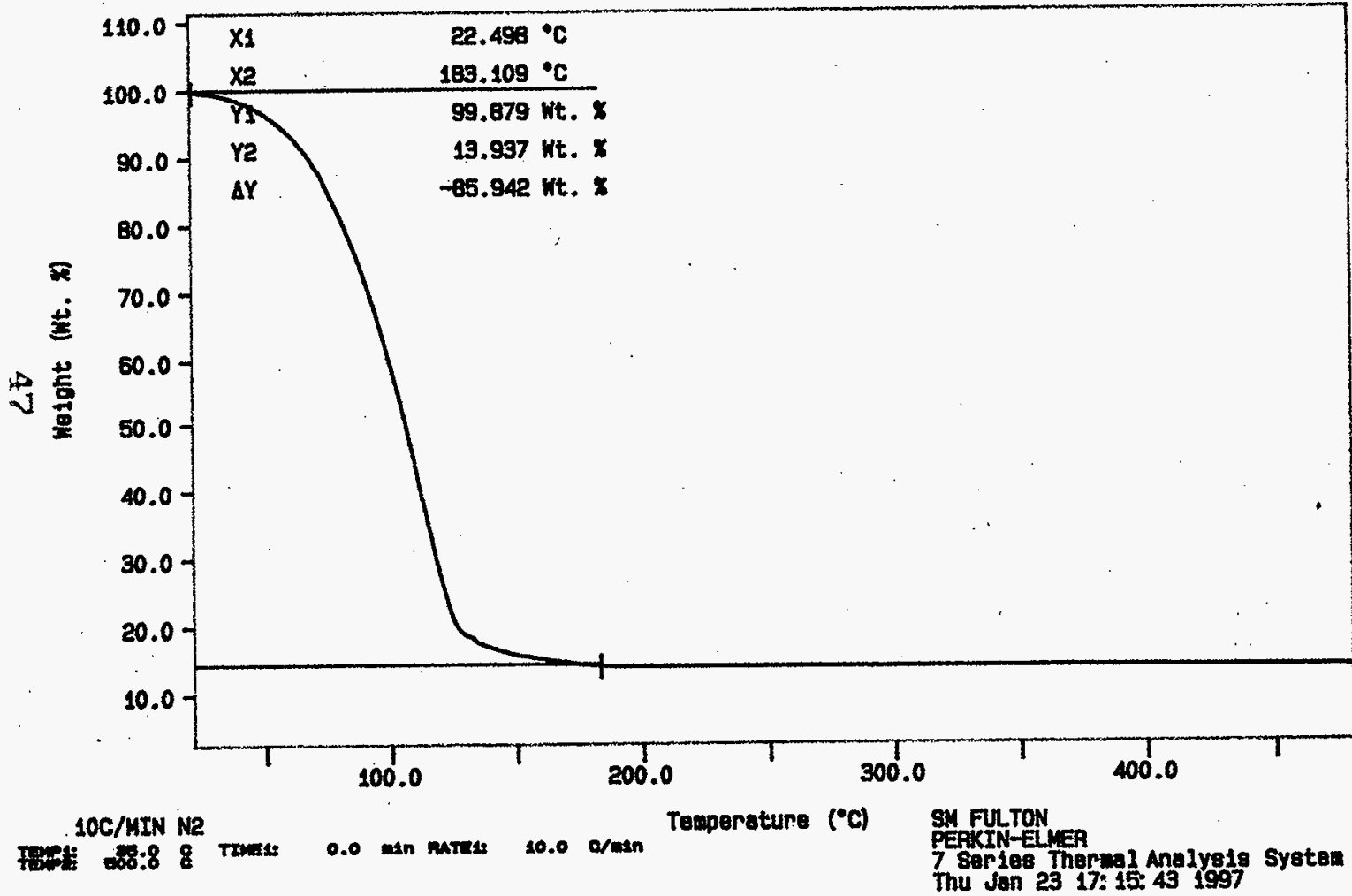




\section{LABCORE Data Entry Template for Worklist\# 16283}

Analyst:

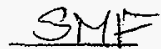

Instrument: TGA0

Book $99108-A$

Method: LA-560-112 Rev/Mod $\mathrm{C}-\mathrm{O}$

Worklist Comment: Run under Nitrogen.

new

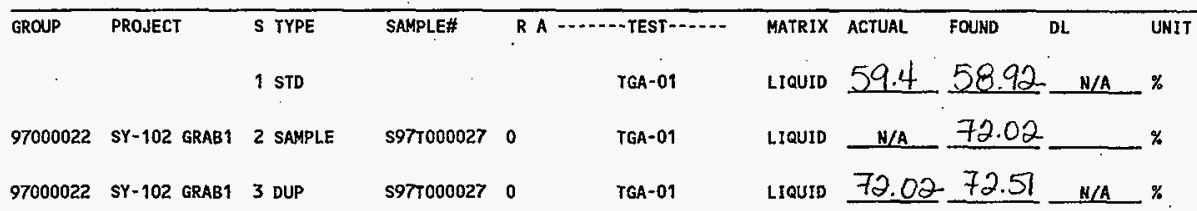

Hual 17.2 Hinal page for

Verifua /Validatea by
Bundina
Valengula $1-30.97$

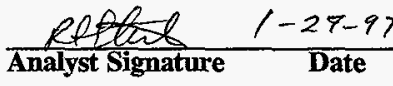




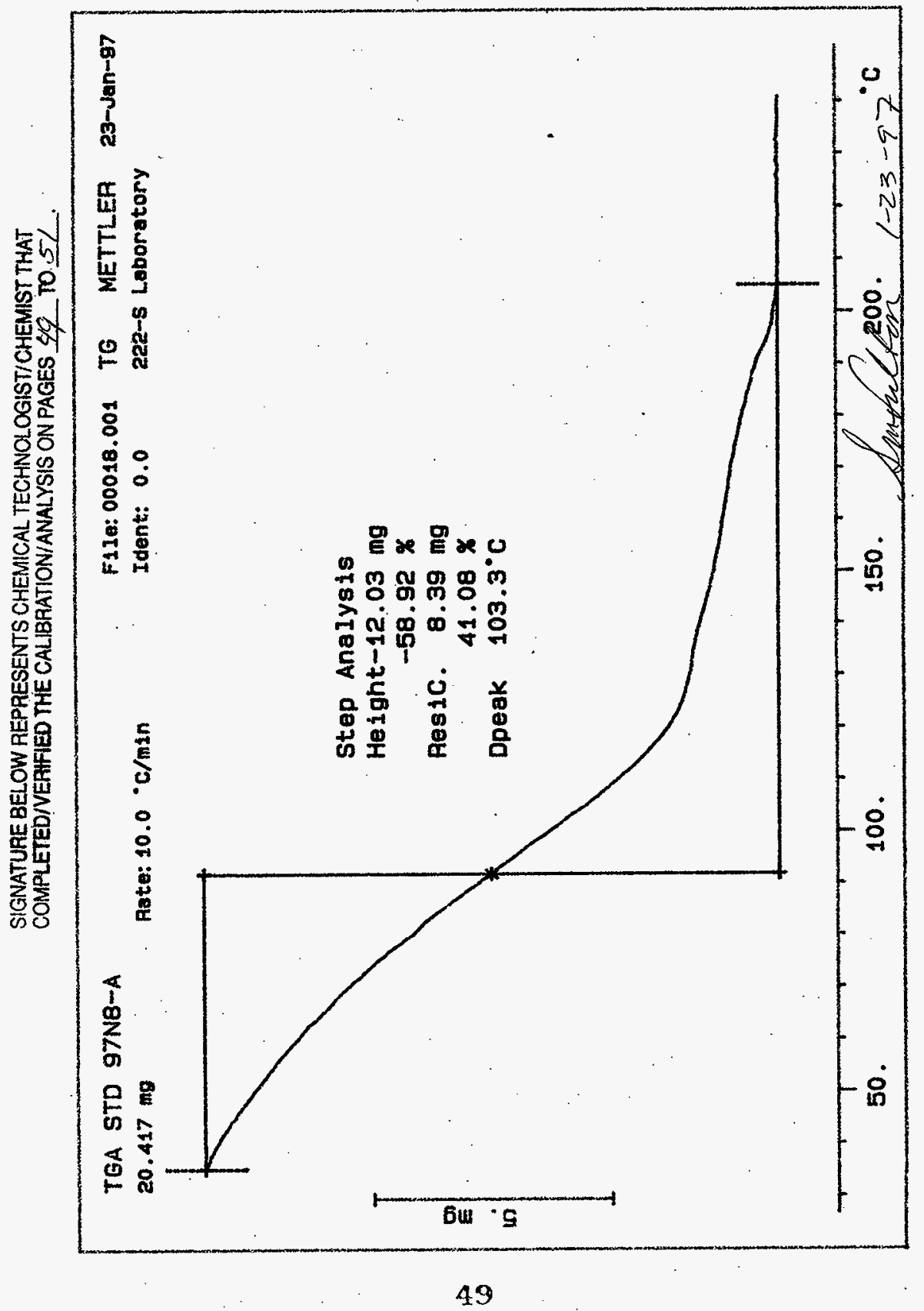




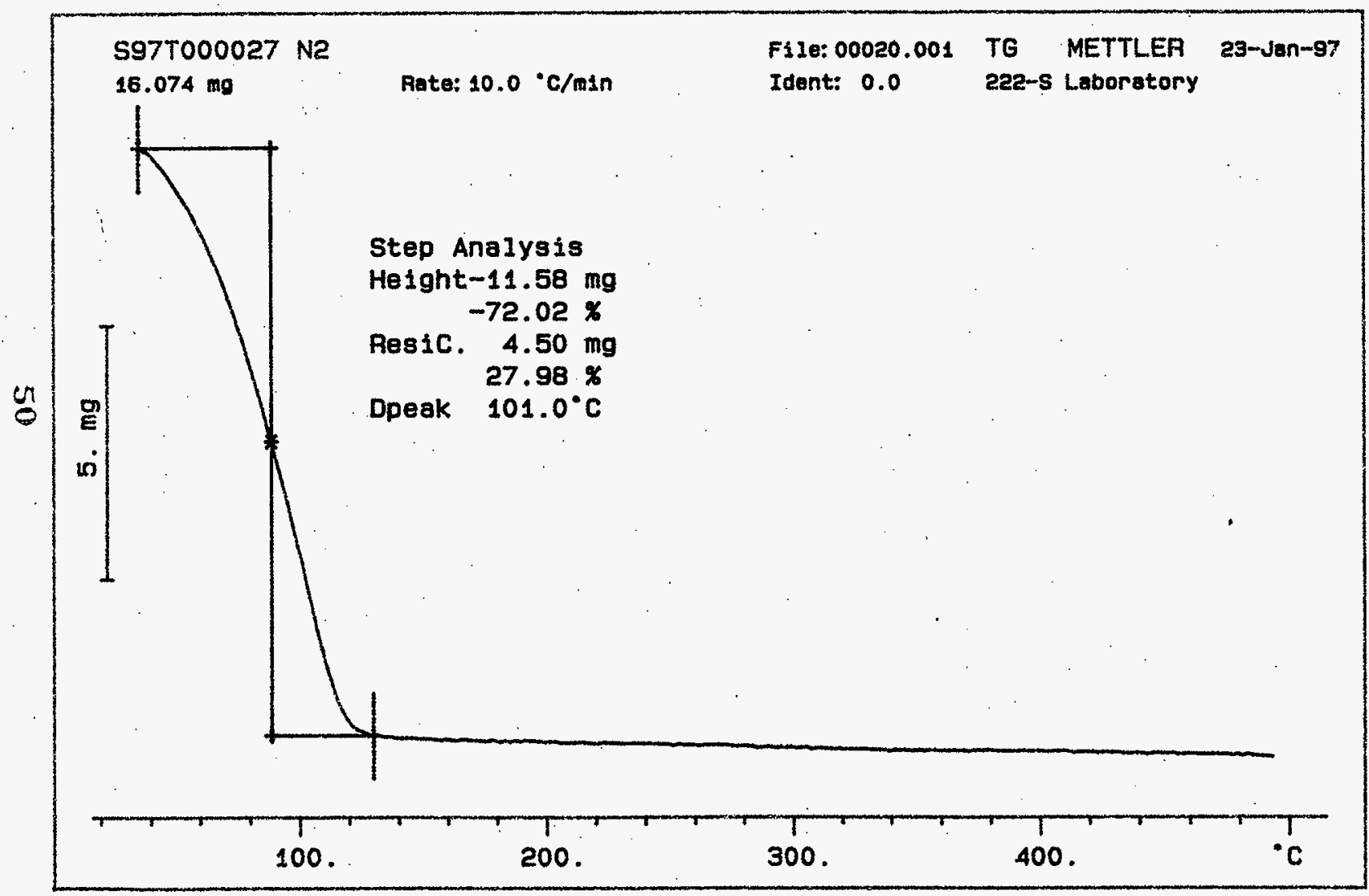




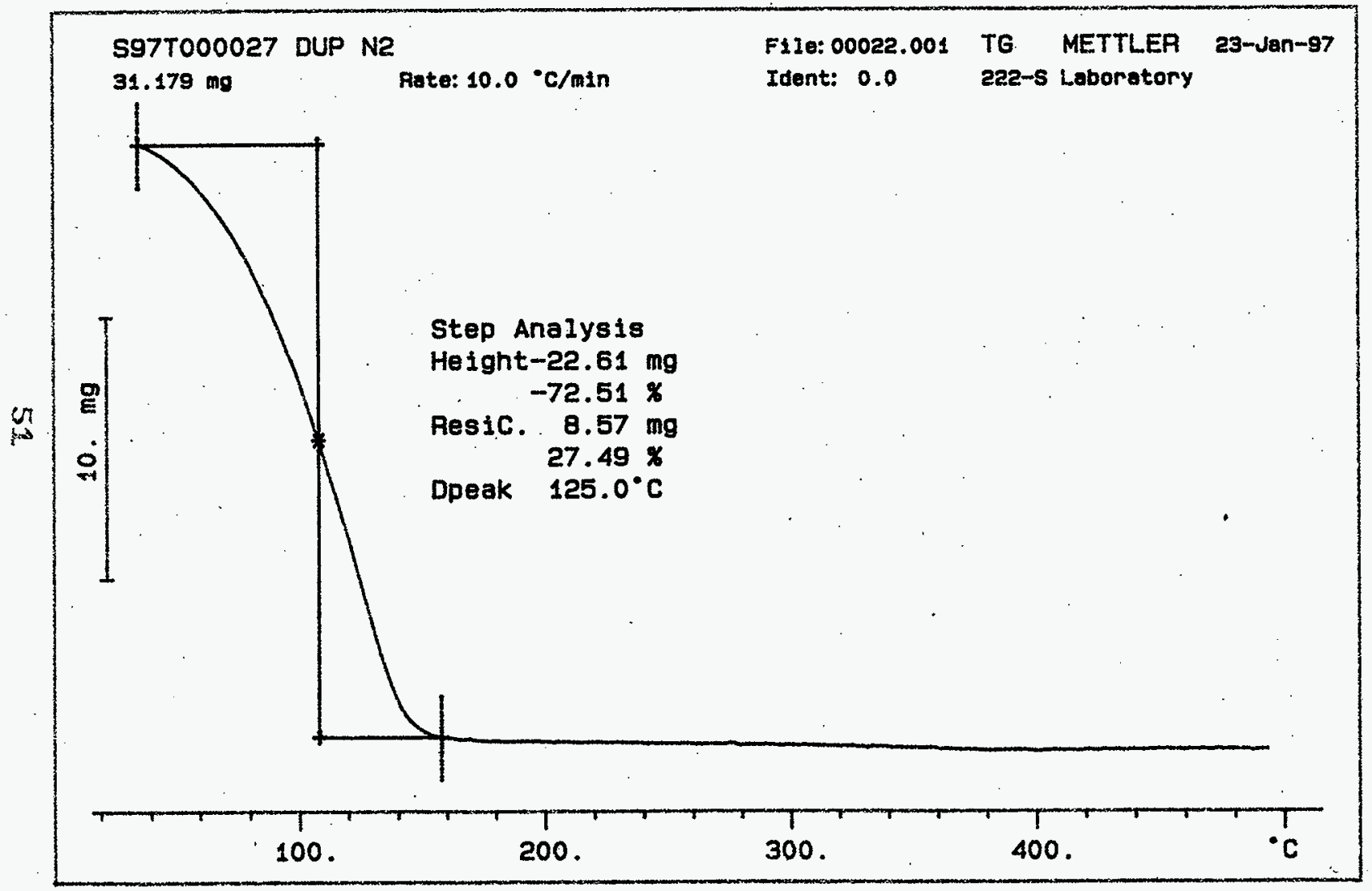




\title{
LABCORE Completed Worklist Report for Worklist\# 16291
}

Analyst: dcd

Instrument: BA001

Method:LA 510-112 Rev/Mod $\mathrm{C}-3$
Book\#

new

\begin{tabular}{|c|c|c|c|c|c|c|c|c|}
\hline Seq Type & Sample & R A & Test & Matrix & Actual & Found & DL or Yield Unit & \\
\hline $1 \% \mathrm{STO}$ & & 0 & 50001 & ITOUTD & 1098 & $1 \times 394$ & $1 \% 9 \% 11 \%$ ororovor) & 1\%, \\
\hline 2 SAMPLE & $\$ 97 T 000024$ & 0 & SPG-01 & LIOUID & $N / A$ & 1.077 & $1.000-002$ Sp.G. & \\
\hline $3 \mathrm{ppp} / \mathrm{k}$ & 5971000074 & 0 & spo 01 & Houtp & 1077 & 1007 & $14002 \%$ SpD & mוk \\
\hline 4 SAMPLE & $597 T 000026$ & 0 & SpG-01 & LIQUID & $\mathbf{N} / \mathbf{A}$ & 1.071 & $1.000-002 \quad s_{p} \cdot G$ & \\
\hline $500 \%$ & 897000026 & 0 & sPa 01 & $1+10070$ & 1017 & 1083 & $\% 1,14 \% \% p$ & \\
\hline 6 SAMPLE & $\$ 972000027$ & 0 & SPG-01 & LIOUID & $\mathbf{N} / \mathbf{A}$ & $n / a$ & $1.000-003 \quad s_{p . G}$ & \\
\hline 7 Dop & $59190007 \%$ & 0 & 80001 & 110020 & $1+\%$ q & $11 \%$ & $1 \% 1 / \mathrm{RPD} /$ & \\
\hline
\end{tabular}

\section{Comments Section:}

Comments for sample\# S97T000027 and test SPG-01 .

S97T000027 is a thick brown paste.Sample is too thick too obtain an accurate spg weight. new

\section{Final page for worklist\# 16291}

\author{
Analyst Signature Date \\ Date

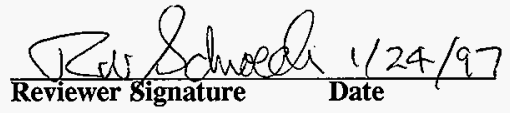 \\ Customer regiested SPG-02 test for 5977000027. \\ Rus $1 / 27 / 97$
}




\section{LABCORE Data Entry Template for Worklist\# 16291}

Analyst: $\quad$ DCD $\quad$ Instrument: BA001_ Book \# /33N/6-A

Method: LA-510-112 Rev/Mod

Worklist Comment: SY-102 Grab. Use CAL PIPETTE.

new

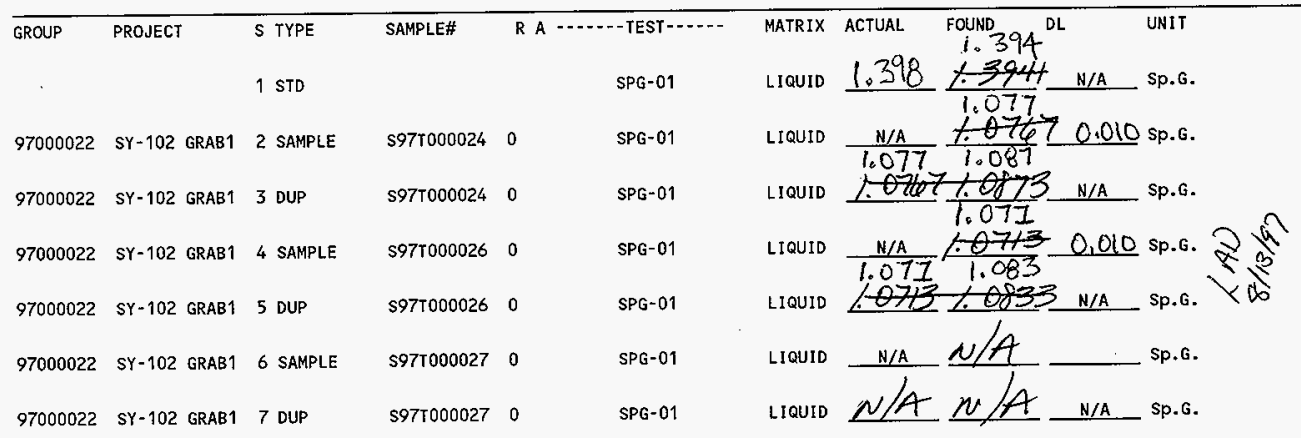

Final page for worklist \# 16291

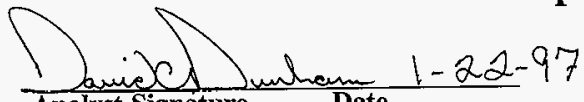

Analyst Signature

\section{Date}

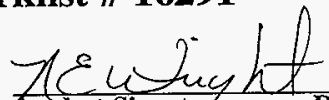

Analyst Signature 
HNF-SD-WM-DP-227, REV. 0

PLACE ANALYTICAL CARD IN BOX BELOW OR ATTACH TRAVELER

SPECIFIC GRAVITY : LA-510-112 (C-3)

\begin{tabular}{|c|c|c|c|}
\hline (5) & & STD & REPLICATE \\
\hline STD & Gross Weight (W2) & 1.9556 & 1.9471 \\
\hline FWorkist & Tare Weight (W1) & 1.8503 & 1.8429 \\
\hline 16291 & Weight of Solution (W2-W1) & 0.1053 & 0.1042 \\
\hline 610st Code & Volume of Solution $\mu \mathrm{L}$ & 75.1400 & 75.1400 \\
\hline SPG-01 & Specific Gravity & 1.4014 & 1.3867 \\
\hline 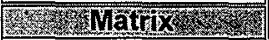 & Specific Gravity (Average) & 1.3941 & \\
\hline
\end{tabular}

LIQUID

S.

133 N16A

Instrumpnt Gode Gross Weight (W2) $=$ Wt. of vial + cap + cotton + solution

BA001

2.7. Analvst

DCD

W.

$01 / 22 / 97$

Tare Weight $(W 1)=$ Wt. of vial + cap + cotton

Specific Gravity $=[(W 2-W 1) * 1000 \mu \mathrm{L} / \mathrm{mL}] /[$ Vol. of Solution $\mu \mathrm{L} * 1.000 \mathrm{~g} / \mathrm{mL}]$

1.7\%

v RESULT $v$

11:30 PM

Specific Gravity Average $=$

1.394

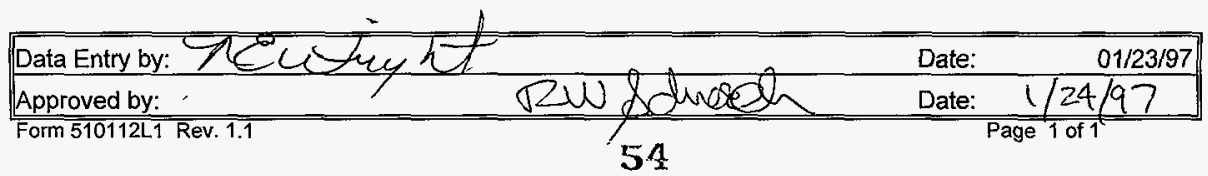




\section{SPECIFIC GRAVITY : LA-510-112 (C-3)}

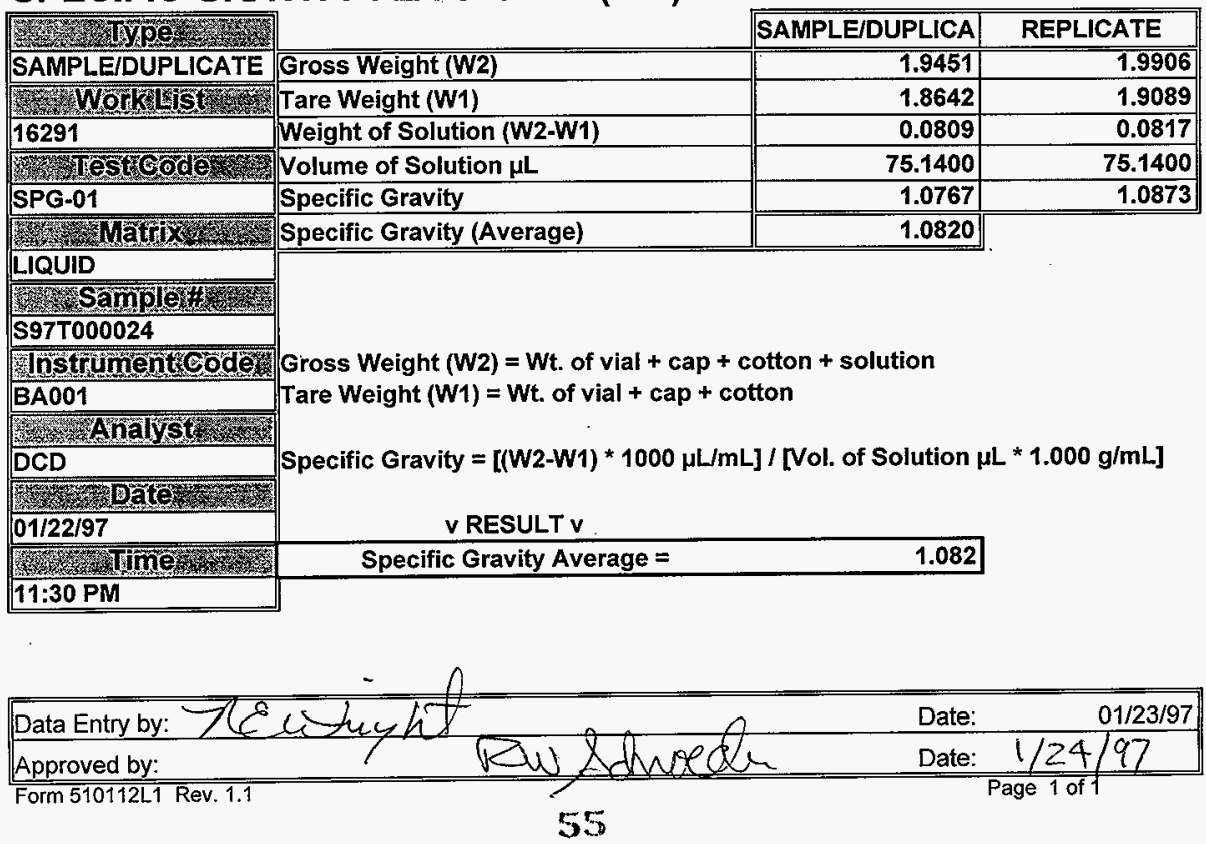




\section{SPECIFIC GRAVITY : LA-510-112 (C-3)}

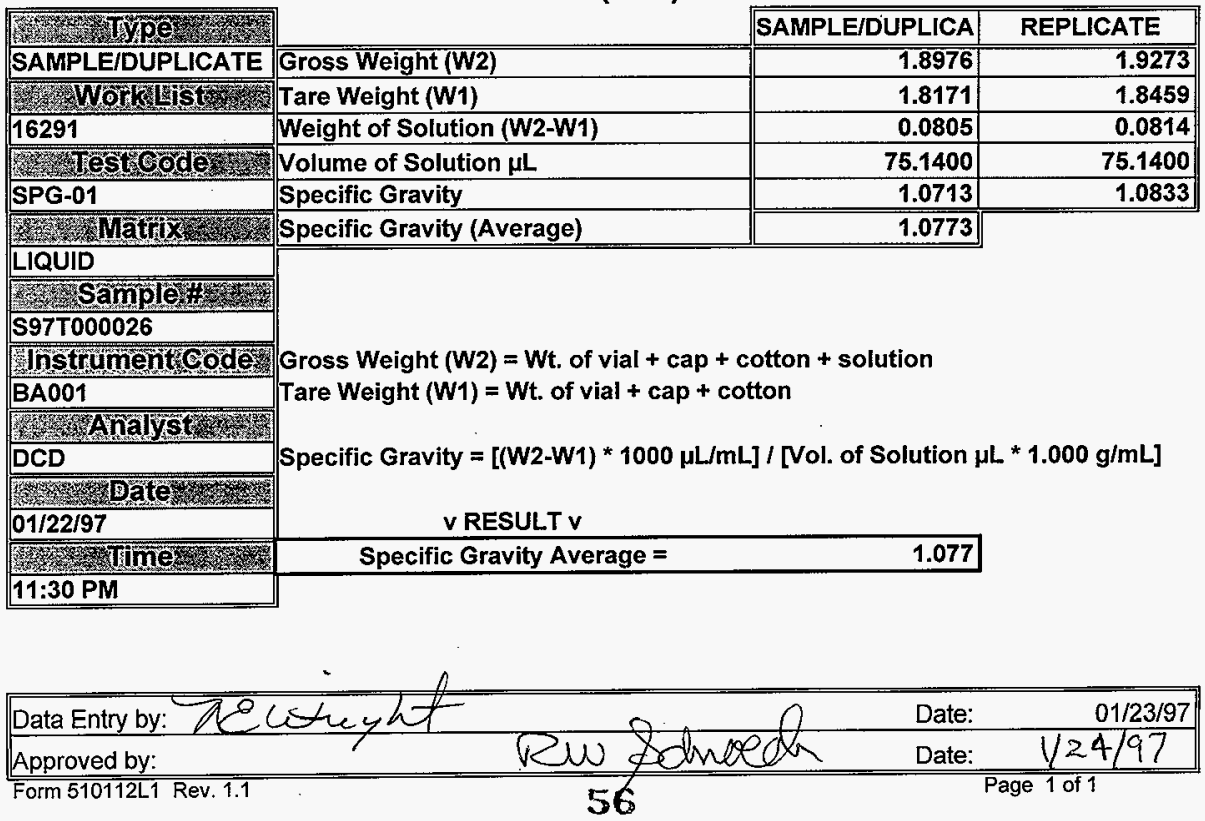




\section{LABCORE Data Entry Template for Worklist\# 16290}

Analyst: $\quad$ Instrument: $\mathrm{PH} 01 \longrightarrow$ Book \# 18 N19-D

Method: LA-212-106 Rev/Mod B-O

Worklist Comment: SY-102 Grab. Std: DIRECT.

new

\begin{tabular}{|c|c|c|c|c|c|c|c|c|c|c|c|}
\hline \multirow[t]{2}{*}{ GROUP } & \multirow{2}{*}{\multicolumn{2}{|c|}{ PROJECT }} & $S$ TYPE & SAMPLE\# & \multicolumn{2}{|c|}{ R A $\cdots$ - TEST $\cdots$} & MATRIX & ACTUAL & FOUND & $\mathrm{DL}$ & UNIT \\
\hline & & & 1 STDPH & & & PH-01 & LIQUID & 8.00 & 8.02 & N/A & pH \\
\hline 97000022 & $s Y-102$ & GRAB1 & 2 SAMPLE & S97T000024 & 0 & PH-01 & LIQUID & N/A & 12.08 & 0.01 & pH \\
\hline 97000022 & $s \gamma-102$ & GRAB1 & 3 DUP & S97r000024 & 0 & PH- 01 & LIQUID & 12.08 & 12.10 & N/A & pH \\
\hline 97000022 & SY-102 & GRAB1 & 4 SAMPLE & $\$ 97 T 000026$ & 0 & $\mathrm{PH}-01$ & LIQUID & N/A & 11.96 & 0.01 & $\mathrm{pH}$ \\
\hline 97000022 & sY-102 & GRAB1 & 5 DUP & $\$ 977000026$ & 0 & $\mathrm{PH}-01$ & LIQUID & 11.94 & 11.92 & N/A & pH \\
\hline 97000022 & SY-102 & GRAB1 & 6 SAMPLE & $\$ 97 T 000027$ & 0 & $\mathrm{PH}-01$ & LIQUID & N/A & 12.39 & 0.01 & $\mathrm{pH}$ \\
\hline 97000022 & $\$ Y-102$ & GRABT & 7 DUP & $\$ 97 T 000027$ & 0 & PH-01 & LIQUID & 1239 & 12.42 & N/A & pH \\
\hline
\end{tabular}

\section{Final page for worklist \# 16290}
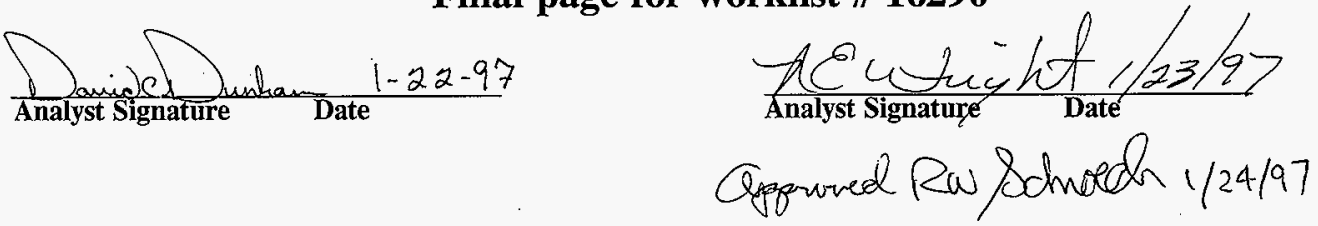

Data Entry Comments:

Units shown for $Q C$ (SPK \& STD) may not reflect the actual units. $D L=$ Detection Limit, $S=$ Worklist Slot Number, $R=$ Replicate Number, $A=$ Aliquot Code. 


\section{LABCORE Completed Worklist Report for Worklist\# 16289}

Analyst: slh

Instrument: $\mathrm{PH} 01$

$211-102$

Method: $L A 342-+00$ Rev/Mod $80-1 / 28 / 97$

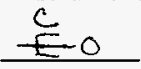

Book\#

new

Worklist Comment: SY-102 Grab. Std: $0.050 \mathrm{ml}$.

\begin{tabular}{lllllll}
\hline Seq Type & Sample\# R A & Test & Matrix & Actual & Found DL or Yield Unit
\end{tabular}

$1 \mathrm{BLK} / \mathrm{k}+\%$

2 STD

$0+01$

roup

LIQUID

अ.

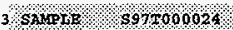

4 DUP S97T000024

OH-01 1 $010 \mathrm{D}$

LIOUID

2.66404 अ०

$0 \mathrm{H} 01$

OH- 01

io 1010

LIQUID

$\mathrm{OH}-01$

6 DOP S97T000025

or 01 Iout 1.7004 1010 trt

7 Snut t 5971000027

OH-OI

LIQUID

$\frac{\mathrm{NA}}{1.6803}+1.6103$

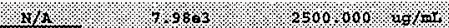

$7.9803 \quad 7.7703$

$2.667 \mathrm{RPD}$

8 DUP

$597 T 0000270$

Final page for worklist\# 16289

\section{Analyst Signature}

Date

Analyst Signature

Date

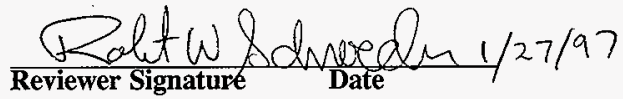




\section{LABCORE Data Entry Template for Worklist\# 16289}

\section{Analyst: SCH Instrument: PH01 _ Book\# 79N8}

Method: LA-211-102 Rev/Mod C-O

Worklist Comment: SY-102 Grab. Std: 0.050ml.

new

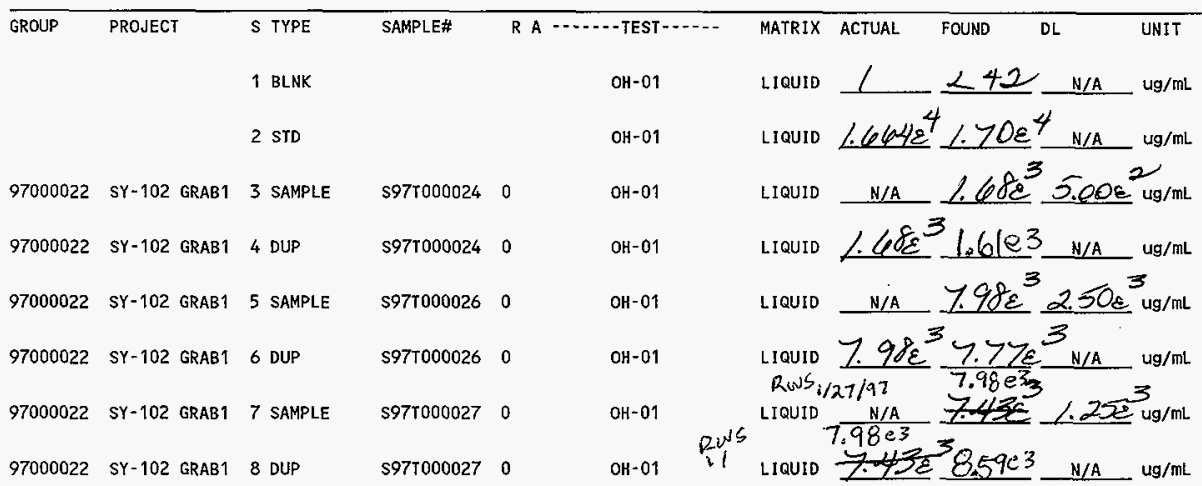

Final page for worklist \# 16289

\section{?}

androdtood

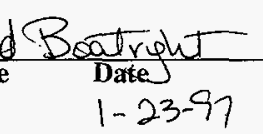

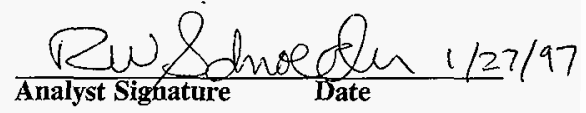

Data Entry Comments:

Units shown for $Q C$ (SPK \& $S T D$ ) may not reflect the actual units. $D L=$ Detection Limit, $S=$ Worklist Slot Number,

$R=$ Replicate Number, $A=$ Aliquot Code. 


\section{HNF-SD-WM-DP-227, REV. 0}
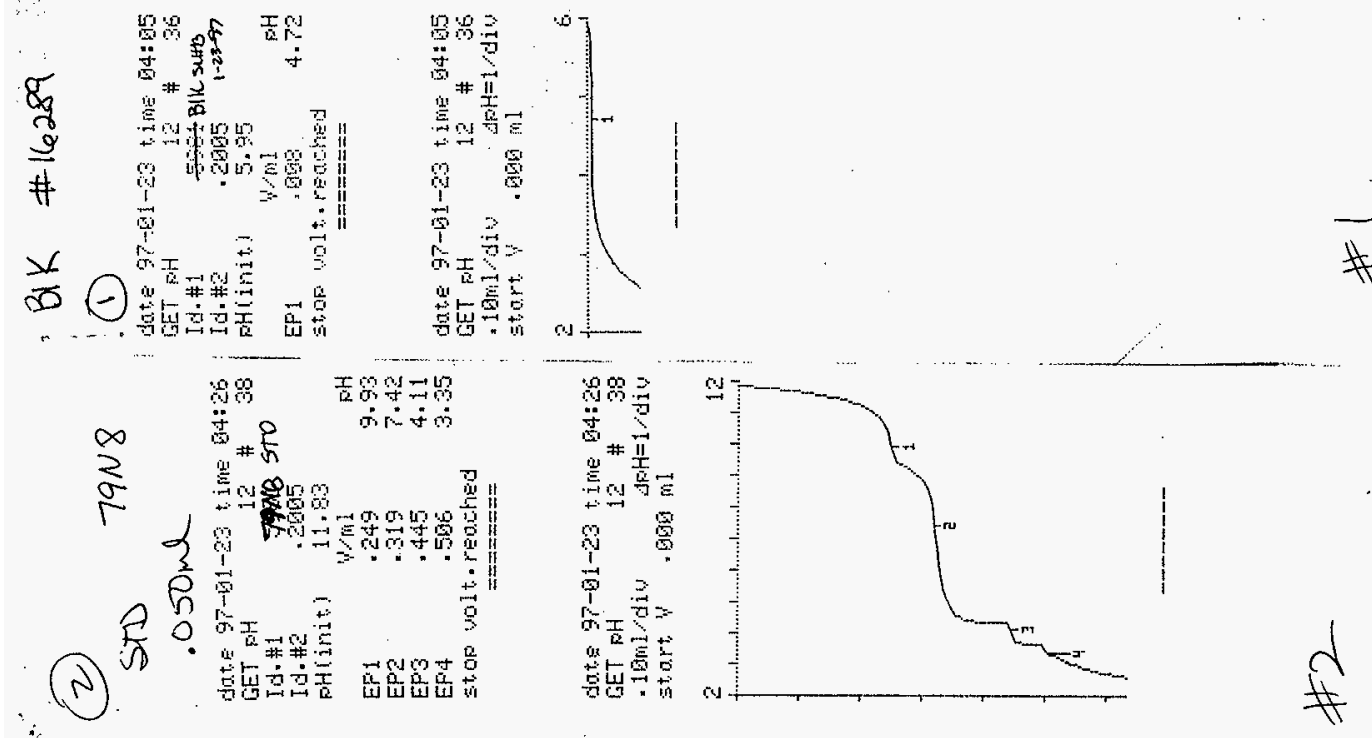


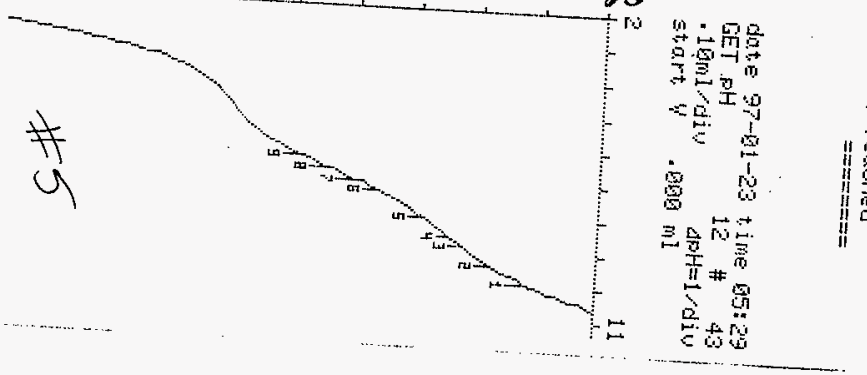

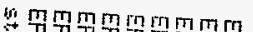

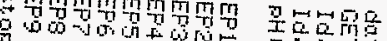

$=$

$\underset{z}{2}$

0

$\frac{6}{1} \cdot \therefore \therefore . .$.

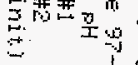

0

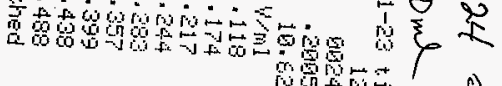

9.

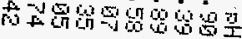
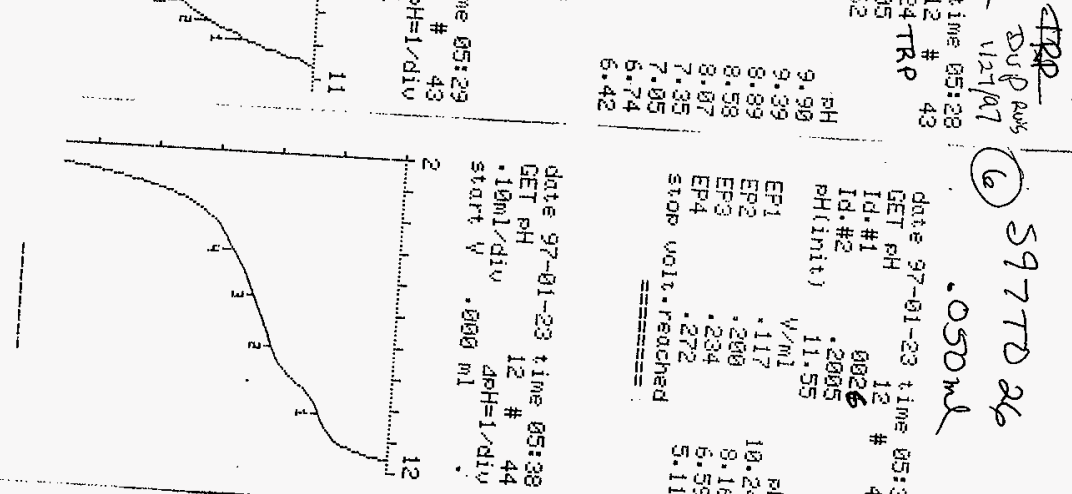

$\stackrel{m}{\mathrm{~m} T} \mathrm{~m}$

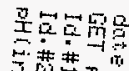

(6)

$\#$

O)

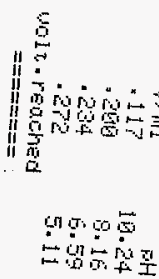

范柴

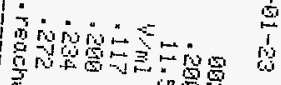

O 0
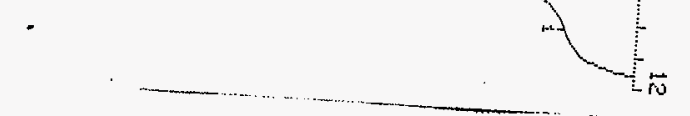
$\leftrightarrow \lim _{0}$ $\lim _{i}$
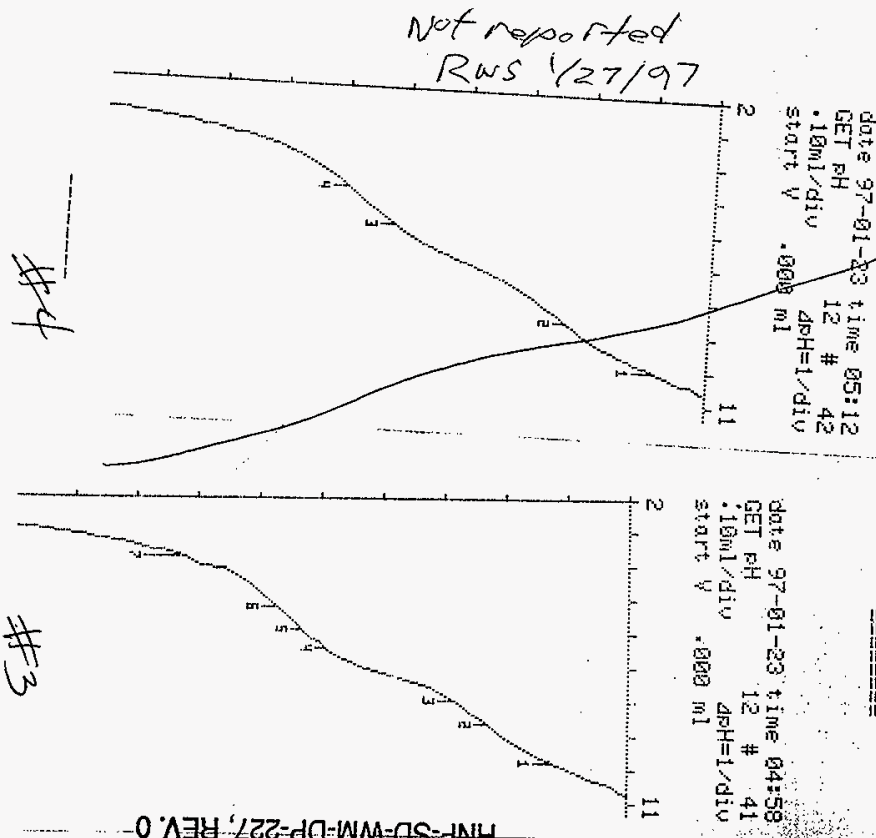

(ङ) 
. S97T027TRP. S97T027

(10). $100 \mathrm{ml}$

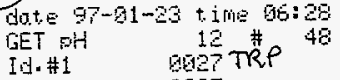

I $\mathrm{u}^{2} \# 2$

oH(init) 11.75

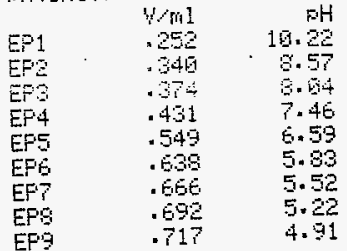

montal stop

$===:===$

O) date $97-61-23$ time $05: 29$

2 COET $\mathrm{PH}$ 12 \# 48 - ibrilis de start $V$. 6 Eg m1

4

12

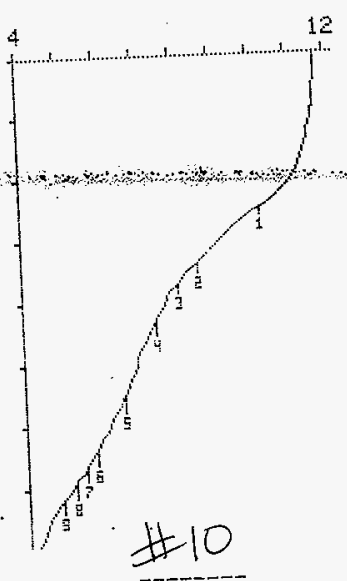

(9) Dup

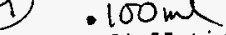

dote $97-131-23$ time $96: 15$ CET FH 12 \#. 47 Id.\#1 01027 Dup $1 \mathrm{~d}=\# 2$. 2065 Hinit 11.82

PHtime W/ml FH

EF1 $\quad .243 \quad 10.25$

EF $\quad .326 \quad 8.60$

EF3 $\quad .433 \quad 7.35$

EF4 $\quad .654 \quad 5.31$

stop y renched

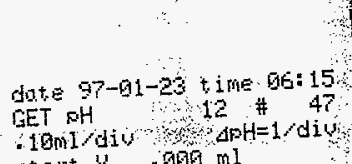
start $Q \quad .000 \mathrm{~m}$

3

12

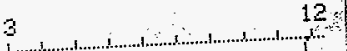

$3+\ldots+1$

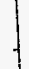

.

\section{7}

(8) $.100 \mathrm{ml}$

dot.e $97-01-23$ time $06: 62$ GET Fil $12 \# 46$

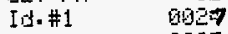
Id. \#2 . 2005

oH(init) 11.80

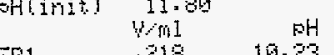

EF1 $1210 \quad 10.23$

EF2 $\quad .295 \quad 9.68$

$\begin{array}{lll}\text { EF4 } & .472 & 5.93 \\ \text { EFS } & .590 & 5.67\end{array}$

atop 4 renched

$== \pm====$

dnte $97-61-23$ t, ime $06: 92$ SET BH $12 \# 46$ *tonizdiu " ApH=1/di start $V$. $900 \mathrm{ml}$

3

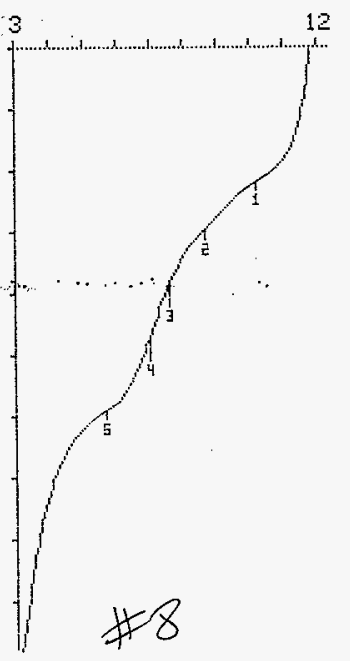

- SqZTO26 DUP

7

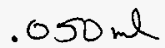

dote $97-61-39$ time $05: 48$ GET FH 12 \# 45

Id. \#

$\begin{array}{llr} & W \mathrm{ml} & \mathrm{PH} \\ \mathrm{EP} 1 & .114 & 10.20\end{array}$

EFe $\quad 187$ 9.58

EF3 $.250 \quad 5.34$

stop wolt. rectied

$======$

date $97-61-23$ time $05: 48$ GET $\mathrm{PH}$ $12 \ldots \# 45$

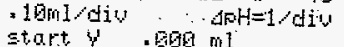
$.60 \mathrm{ml}$

2

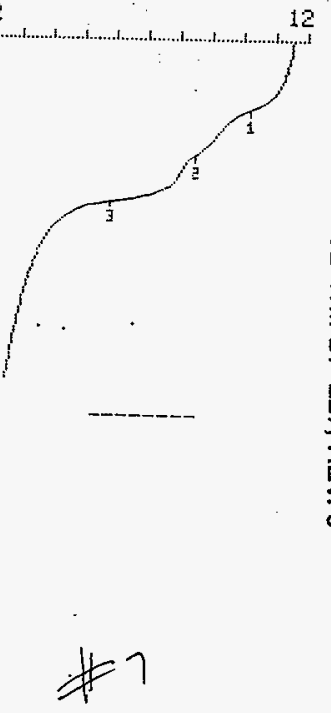




\begin{tabular}{|c|c|c|}
\hline \multicolumn{2}{|c|}{ OH (AUTO) : LA-211-102 (C-0) } & BLK \\
\hline 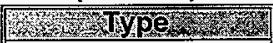 & Sample Size $(\mathrm{mL})$ SS & 3.000 \\
\hline BLK & Concentration of $\mathrm{HNO}_{3}$ (Molarity) & 0.2005 \\
\hline Whorkisist & HNO3 Titrant at $\mathrm{OH}$ end-point in $\mathrm{mL}$ & 0.008 \\
\hline 16289 & Dilution Factor DF & 1 \\
\hline Wriestoded & Concentration of $\mathrm{OH}$ in Sample (Molarity) & $5.35 \mathrm{E}-04$ \\
\hline $\mathrm{OH}-01$ & $\mathrm{OH}$ in Sample in $\mu \mathrm{g} / \mathrm{mL}$ (PPM) & $9.09 \mathrm{E}+00$ \\
\hline Notrix & \multirow{4}{*}{ Detection Limit $=125 \mu \mathrm{g} / \mathrm{SS}$ * DF } & \\
\hline LIQUID & & \\
\hline Sample\# & & \\
\hline BLANK & & \\
\hline linstrumenteoden & Detection Limit $(\mu \mathrm{g} / \mathrm{mL})$ & 4.17E+01 \\
\hline PH01 & \multirow{5}{*}{\multicolumn{2}{|c|}{$\begin{array}{l}\mathrm{OH} \text { Molarity }=\left((\mathrm{mL} H N O 3)^{*}(\mathrm{M} \mathrm{HNO})\right) / \text { Sample Size in } \mathrm{mL} * \text { Dilution Factor } \\
\mathrm{OH} \text { in } \mu \mathrm{g} / \mathrm{mL}=(\mathrm{OH} \text { MOLARITY })^{*}(17.0 \mathrm{~g} / \mathrm{mole})^{*}((1000000 \mu \mathrm{g} / \mathrm{g}) /(1000 \mathrm{~mL} / \mathrm{L}))\end{array}$}} \\
\hline rexályst & & \\
\hline SLH & & \\
\hline 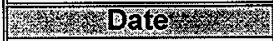 & & \\
\hline $01 / 23 / 96$ & & \\
\hline NThers & & 3LK \\
\hline \multirow[t]{2}{*}{$05: 30 \mathrm{AM}$} & Concentration of $\mathrm{OH}$ in Sample (Molarity) & 5.35E-04 \\
\hline & $\mathrm{OH}$ in Sample in $\mu \mathrm{g} / \mathrm{mL}$ (PPM) & $<42$ \\
\hline
\end{tabular}

The Result is < Detection Limit

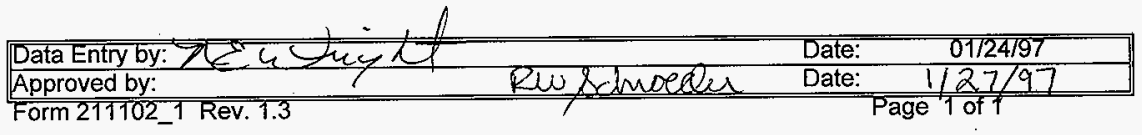




\begin{tabular}{|c|c|c|}
\hline \multicolumn{2}{|c|}{ OH (AUTO) : LA-211-102 (C-0) } & STD \\
\hline 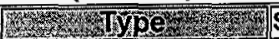 & Sample Size $(\mathrm{mL})$ SS & 0.050 \\
\hline STD & Concentration of HNO3 (Molarity) & 0.2005 \\
\hline 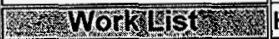 & HNO3 Titrant at $\mathrm{OH}$ end-point in $\mathrm{mL}$ & 0.249 \\
\hline 16289 & Dilution Factor DF & \\
\hline Testeode & Concentration of $\mathrm{OH}$ in Sample (Molarity) & $9.98 \mathrm{E}-01$ \\
\hline $\mathrm{OH}-01$ & $\mathrm{OH}$ in Sample in $\mathrm{\mu g} / \mathrm{mL}$ (PPM) & $1.70 \mathrm{E}+04$ \\
\hline \multirow{2}{*}{$\begin{array}{l}\text { Malrix } \\
\text { LIQUID }\end{array}$} & \multirow{4}{*}{ Detection Limit $=125 \mu \mathrm{g} / \mathrm{SS} * \mathrm{DF}$} & \\
\hline & & \\
\hline Sainded & & \\
\hline \multicolumn{2}{|c|}{$79 \mathrm{~N} 8$} & \\
\hline Instrumancode & Detection Limit $(\mu \mathrm{g} / \mathrm{mL})$ & $2.50 \mathrm{E}+03$ \\
\hline PHO1 & \multirow{4}{*}{\multicolumn{2}{|c|}{$\begin{array}{l}\mathrm{OH} \text { Molarity }=\left((\mathrm{mL} \text { HNO3 })^{*}(\mathrm{M} H \mathrm{HO})\right) / \mathrm{Sample} \text { Size in } \mathrm{mL} \text { Dilution Factor } \\
\mathrm{OH} \text { in } \mu \mathrm{g} / \mathrm{mL}=(\mathrm{OH} \text { MOLARTTY })^{*}(17.0 \mathrm{~g} / \mathrm{mole})^{*}((1000000 \mu \mathrm{g} / \mathrm{g}) /(1000 \mathrm{~mL} / \mathrm{L}))\end{array}$}} \\
\hline & & \\
\hline SLH & & \\
\hline \multirow{2}{*}{$01 / 23 / 96$} & & \\
\hline & & STD \\
\hline \multirow[t]{2}{*}{$05: 30$ AM } & Concentration of $\mathrm{OH}$ in Sample (Molarity) & $9.98 \mathrm{E}-01$ \\
\hline & $\mathrm{OH}$ in Sample in $\mu \mathrm{g} / \mathrm{mL}$ (PPM) & $1.70 E+04$ \\
\hline
\end{tabular}

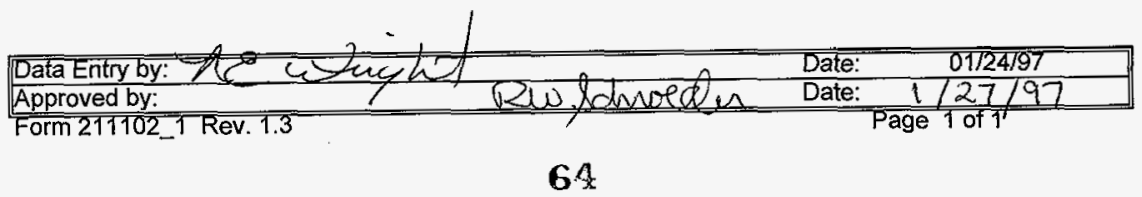


HNF-SD-WM-DP-227, REV. 0

PLACE ANALYTICAL CARD IN BOX BELOW OR ATTACH TRAVELER

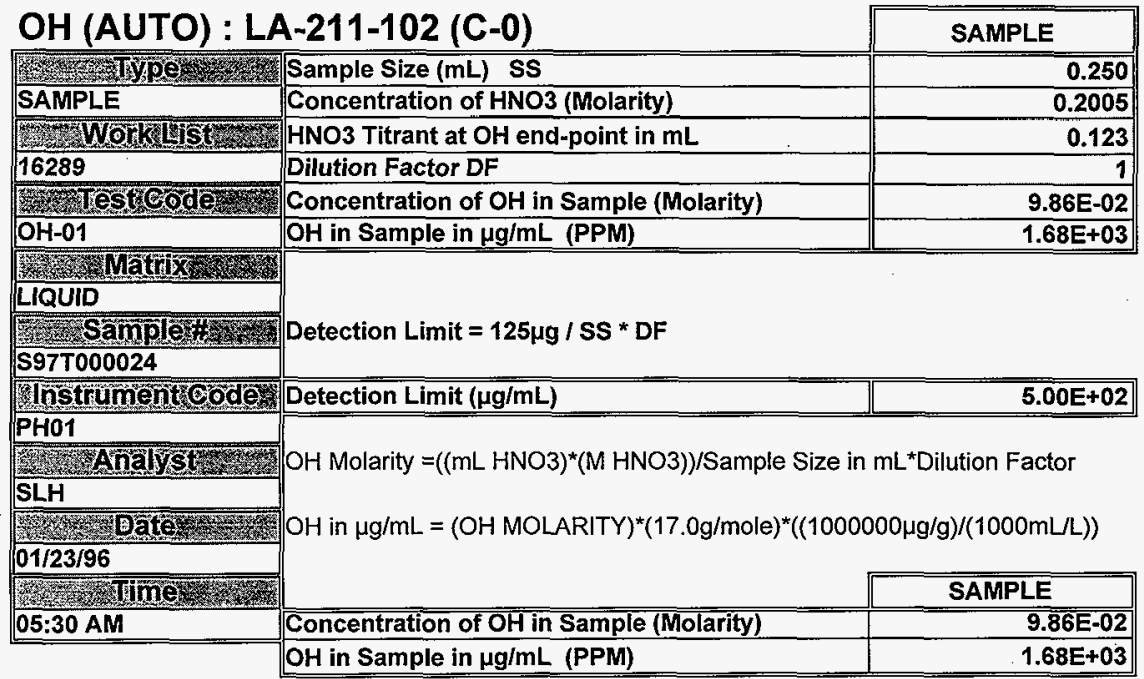

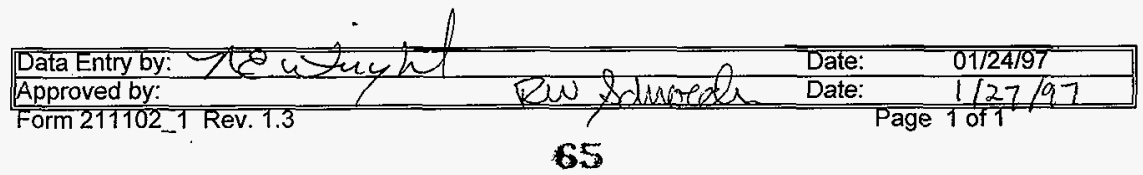




\section{-211-102 (C-0)}

Sample Size $(\mathrm{mL})$ SS

Concentration of HNO3 (Molarity)

HNO3 Titrant at OH end-point in $\mathrm{mL}$

Dilution Factor DF

Concentration of $\mathrm{OH}$ in Sample (Molarity)

$\mathrm{OH}$ in Sample in $\mu \mathrm{g} / \mathrm{mL}$ (PPM)
TRIPLICATE.

Detection Limit $=125 \mu \mathrm{g} / \mathrm{SS}$ * DF

Detection Limit $(\mu \mathrm{g} / \mathrm{mL})$

$5.00 E+02$

$\mathrm{OH}$ Molarity $=\left((\mathrm{mL} H N O 3)^{\star}(\mathrm{M} \mathrm{HNO} 3)\right) /$ Sample Size in $\mathrm{mL}{ }^{*}$ Dilution Factor $\mathrm{OH}$ in $\mu \mathrm{g} / \mathrm{mL}=(\mathrm{OH} \text { MOLARITY })^{*}\left(17.0 \mathrm{~g} / \mathrm{mole}^{*}((1000000 \mu \mathrm{g} / \mathrm{g}) /(1000 \mathrm{~mL} / \mathrm{L}))\right.$

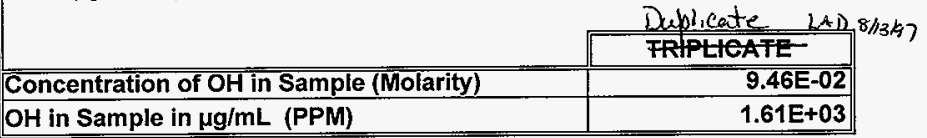

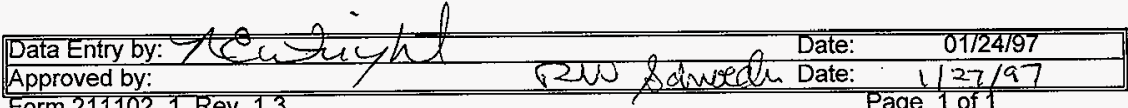




\begin{tabular}{|c|c|c|}
\hline \multirow{2}{*}{\multicolumn{2}{|c|}{ OH (AUTO) : LA-211-102 (C-0) }} & \multirow[b]{2}{*}{ SAMPLE } \\
\hline & & \\
\hline 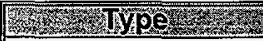 & Sample Size $(\mathrm{mL})$ SS & 0.050 \\
\hline SAMPLE & Concentration of $\mathrm{HNO} 3$ (Molarity) & 0.2005 \\
\hline 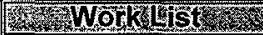 & HNO3 Titrant at $\mathrm{OH}$ end-point in $\mathrm{mL}$ & 0.117 \\
\hline 16289 & Dilution Factor DF & 1 \\
\hline V-TIEsteode & Concentration of $\mathrm{OH}$ in Sample (Molarity) & 4.69E-01 \\
\hline $\mathrm{OH}-01$ & OH in Sample in $\mu \mathrm{g} / \mathrm{mL}$ (PPM) & $7.98 \mathrm{E}+03$ \\
\hline \multirow{2}{*}{$\begin{array}{l}\text { Matrix } \\
\text { LQUID }\end{array}$} & \multirow{4}{*}{ Detection Limit $=125 \mu \mathrm{g} / \mathrm{SS}$ * DF } & \\
\hline & & \\
\hline \multirow{2}{*}{$\begin{array}{l}\text { Sampledt } \\
\text { S97T000026 } \\
\end{array}$} & & \\
\hline & & \\
\hline Instrumenteode & Detection Limit $(\mu \mathrm{g} / \mathrm{mL})$ & $2.50 E+03$ \\
\hline \multirow{2}{*}{ PH01 } & \multirow{5}{*}{\multicolumn{2}{|c|}{$\begin{array}{l}\mathrm{OH} \text { Molarity }=\left((\mathrm{mL} H N O 3)^{*}(\mathrm{M} H \mathrm{HO})\right) / \text { Sample Size in } \mathrm{mL}{ }^{*} \text { Dilution Factor } \\
\mathrm{OH} \text { in } \mu \mathrm{g} / \mathrm{mL}=(\mathrm{OH} \text { MOLARITY })^{*}(17.0 \mathrm{~g} / \mathrm{mole})^{*}((1000000 \mu \mathrm{g} / \mathrm{g}) /(1000 \mathrm{~mL} / \mathrm{L}))\end{array}$}} \\
\hline & & \\
\hline \multirow{2}{*}{ SLH } & & \\
\hline & & \\
\hline $01 / 23 / 96$ & & \\
\hline \multirow{3}{*}{$\begin{array}{l}\text { 05:30 AM } \\
\end{array}$} & & SAMPLE \\
\hline & Concentration of $\mathrm{OH}$ in Sample (Molarity) & 4.69E-01 \\
\hline & $\mathrm{OH}$ in Sample in $\mu \mathrm{g} / \mathrm{mL}$ (PPM) & 7.98E+03 \\
\hline
\end{tabular}

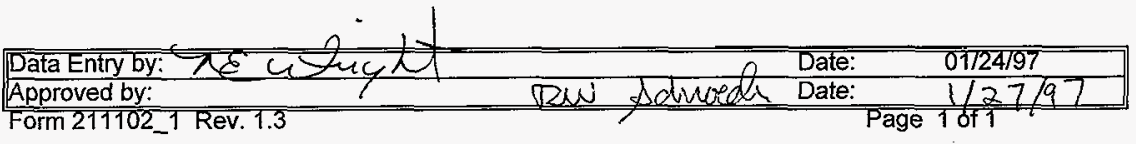




\begin{tabular}{|c|c|c|}
\hline OH (AUTO) : LA & $A-211-102(C-0)$ & DUPLICATE \\
\hline Fipe & Sample Size $(\mathrm{mL})$ SS & 0.050 \\
\hline DUPLICATE & Concentration of $\mathrm{HNO} 3$ (Molarity) & 0.2005 \\
\hline SWork & HNO3 Titrant at $\mathrm{OH}$ end-point in $\mathrm{mL}$ & 0.114 \\
\hline 16289 & Dilution Factor DF & \\
\hline Eritestoodo & Concentration of $\mathrm{OH}$ in Sample (Molarity) & 4.57E-01 \\
\hline $\mathrm{OH}-01$ & $\mathrm{OH}$ in Sample in $\mu \mathrm{g} / \mathrm{mL}$ (PPM) & $7.77 \mathrm{E}+03$ \\
\hline Nuatrix & & \\
\hline LIQUID & & \\
\hline S Sample & Detection Limit $=125 \mu \mathrm{g} / \mathrm{SS}$ * DF & \\
\hline S97T0000026 & & \\
\hline Instrumentrede & Detection Limit $(\mathrm{\mu g} / \mathrm{mL})$ & $2.5 \overline{0 E+03}$ \\
\hline PHO1 & & \\
\hline Analyst & OH Molarity $=\left((\mathrm{mL} \text { HNO3 })^{*}(\mathrm{M} \mathrm{HNO})\right) / \mathrm{Samp}$ & "Dilution Factor \\
\hline SLH & & \\
\hline Daten & $\mathrm{OH}$ in $\mu \mathrm{g} / \mathrm{mL}=(\mathrm{OH} \text { MOLARITY })^{\star}(17.0 \mathrm{~g} / \mathrm{mole}$ & $\mathrm{g} / \mathrm{g}) /(1000 \mathrm{~mL} / \mathrm{L}))$ \\
\hline $01 / 23 / 96$ & & \\
\hline 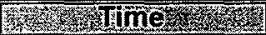 & & DUPLICATE \\
\hline $05: 30 \mathrm{AM}$ & Concentration of $\overline{\mathrm{OH}}$ in Sample (Molarity) & $4.57 \mathrm{E}-01$ \\
\hline & $\mathrm{OH}$ in Sample in $\mu \mathrm{g} / \mathrm{mL}$ (PPM) & 7.77E+03 \\
\hline
\end{tabular}

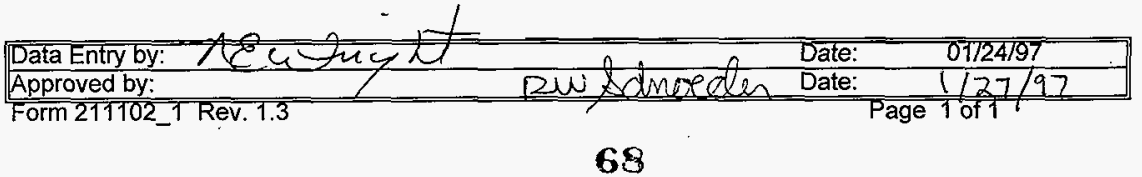




\begin{tabular}{|c|c|c|}
\hline \multicolumn{2}{|c|}{ OH (AUTO) : LA-211-102 (C-0) } & \multirow{2}{*}{ DUPLICATE } \\
\hline 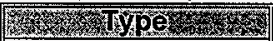 & Sample Size (mL) SS & \\
\hline QUPLIGATE SaMPle & Concentration of $\mathrm{HNO}$ (Molarity) & 0.2005 \\
\hline WorkList & HNO3 Titrant at $\mathrm{OH}$ end-point in $\mathrm{mL}$ & 0.234 \\
\hline 16289 & Dilution Factor DF & 1 \\
\hline resteoder & Concentration of $\mathrm{OH}$ in Sample (Molarity) & 4.69E-01 \\
\hline $\mathrm{OH}-01$ & $\mathrm{OH}$ in Sample in $\mu \mathrm{g} / \mathrm{mL}$ (PPM) & $7.98 \mathrm{E}+03$ \\
\hline \multirow{2}{*}{$\begin{array}{l}\text { LIQUID } \\
\text { LItro }\end{array}$} & \multirow{4}{*}{ Detection Limit $=125 \mu \mathrm{g} / \mathrm{SS}$ * DF } & \\
\hline & & \\
\hline Samplet & & \\
\hline S97T000027 & & \\
\hline Instrumenteoden & Detection Limit $(\mu \mathrm{g} / \mathrm{mL})$ & $1.25 \mathrm{E}+03$ \\
\hline PH01 & \multirow{5}{*}{\multicolumn{2}{|c|}{$\begin{array}{l}\mathrm{OH} \text { Molarity }=\left((\mathrm{mL} H N O 3)^{*}(\mathrm{M} H \mathrm{NO})\right) / \text { Sample Size in } \mathrm{mL}^{*} \text { Dilution Factor } \\
\mathrm{OH} \text { in } \mu \mathrm{g} / \mathrm{mL}=(\mathrm{OH} \text { MOLARITY })^{*}(17.0 \mathrm{~g} / \mathrm{mole})^{*}((1000000 \mu \mathrm{g} / \mathrm{g}) /(1000 \mathrm{~mL} / \mathrm{L}))\end{array}$}} \\
\hline \multirow{2}{*}{$\begin{array}{l}\text { Shalyst } \\
\text { SLH }\end{array}$} & & \\
\hline & & \\
\hline \multirow{2}{*}{$\begin{array}{l}01 / 23 / 96 \\
0\end{array}$} & & \\
\hline & & \\
\hline \multirow{3}{*}{$\begin{array}{l}\text { 05:30 AM } \\
\text { 05es }\end{array}$} & & DUPLICATE \\
\hline & Concentration of $\mathrm{OH}$ in Sample (Molarity) & 4.69E-01 \\
\hline & $\mathrm{OH}$ in Sample in $\mu \mathrm{g} / \mathrm{mL}$ (PPM) & $7.98 \mathrm{E}+03$ \\
\hline
\end{tabular}

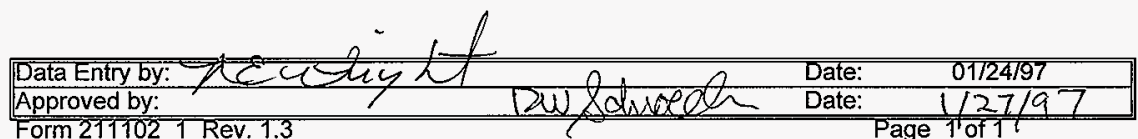




\section{OH (AUTO) : LA-211-102 (C-0)}

TRIPLICATE

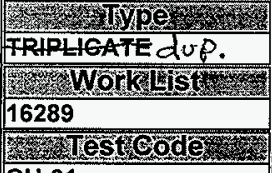

$\mathrm{OH}-01$

Sample Size (mL) SS

Concentration of $\mathrm{HNO} 3$ (Molarity)

$\mathrm{HNO}$ Titrant at $\mathrm{OH}$ end-point in $\mathrm{mL}$

Concentration of $\mathrm{OH}$ in Sample (Molarity)

$\mathrm{OH}$ in Sample in $\mu \mathrm{g} / \mathrm{mL}$ (PPM)

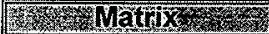

LIQUID

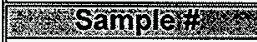

S97T000027

1"strumenticode Detection Limit ( $\mu \mathrm{g} / \mathrm{mL})$

$1.25 \mathrm{E}+03$

PH01

1.2. Analyst

SLH

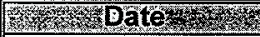

$01 / 23 / 96$

15.

05:30 AM

Detection Limit $=125 \mu \mathrm{g} / \mathrm{SS}$ * DF

Detection Limit ( $\mu$ g/mL)

$\mathrm{OH}$ Molarity $=\left((\mathrm{mL} H N O 3)^{*}(\mathrm{M} H N O 3)\right) /$ Sample Size in $\mathrm{mL}^{*}$ Dilution Factor

$\mathrm{OH}$ in $\mu \mathrm{g} / \mathrm{mL}=(\mathrm{OH} \text { MOLARITY })^{*}(17.0 \mathrm{~g} / \mathrm{mole})^{*}((1000000 \mu \mathrm{g} / \mathrm{g}) /(1000 \mathrm{~mL} / \mathrm{L}))$

\begin{tabular}{|l|c|}
\cline { 2 - 2 } \multicolumn{1}{c|}{} & TRIPLICATE \\
\hline Concentration of $\mathrm{OH}$ in Sample (Molarity) & $5.05 \mathrm{E}-01$ \\
\hline $\mathrm{OH}$ in Sample in $\mu \mathrm{g} / \mathrm{mL}$ (PPM) & $8.59 \mathrm{E}+03$ \\
\hline
\end{tabular}

Data Entry by: 7 ecsuy hal

Form 211102_1 Rev. 1.3 


\section{LABCORE Completed Worklist Report for Worklist\# 16679}

Analyst: raw

Instrument: NH301

Method: $44-631-001$ Rev/Mod B-2

Worklist Comment: NH3-01 NH3STD 1ML LMH

\begin{tabular}{lllllll}
\hline Seq Type & Sample\# R A & Test & Matrix & Actual & Found DL or Yield Unit
\end{tabular}

\begin{tabular}{|c|c|c|c|c|c|c|c|}
\hline $10 \mathrm{BH}$ & & 6 & Mr3 03 & $1+010$ & 1 & $4700=0$ & moOHo/m \\
\hline 2 STD & & 0 & NA3 -01 & IIQOTD & $3.86 \mathrm{~B}+02$ & $3.80 \mathrm{E}+2$ & $98.446 \&$ Recovery \\
\hline 3 SAMPLA & 5974000024 & 0 & $\mathrm{NH} 30 \mathrm{~s}$ & GICUID & \%14. & $1000 \mathrm{p}+00$ & 28000 , 19/min \\
\hline $4 \mathrm{DUP}$ & 5972000024 & 0 & NH3 -01 & IIOUID & $<1.008+0$ & $1.50 \mathrm{~B}+0$ & $40 \% 2$ RPD \\
\hline 558 & $597+00002$ & 0 & $4+3,01$ & OToTo & $8 \times 68+02$ & $810 \mathrm{~s}+02$ & $106-26 \% 10-0 \times 4$ \\
\hline 6 SAMPLB & $\$ 97 T 000026$ & 0 & NH3-01 & IIQUID & $N / A$ & $7.488+01$ & 1.000 \\
\hline $7 \mathrm{sTD}$ & & 0 & $143-01$ & IIQOID & $3 \% 868+02$ & $3 \mathbf{4 2 8} \times 2$ & $68601 \%<-00 \% 14$ \\
\hline
\end{tabular}

Final page for worklist\# 16679
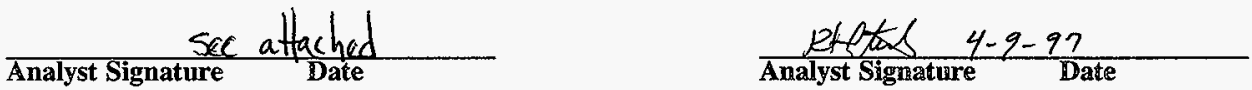

validated into Labcore by 


\section{LABCORE Data Entry Template for Worklist\# 16679}

Page: 1

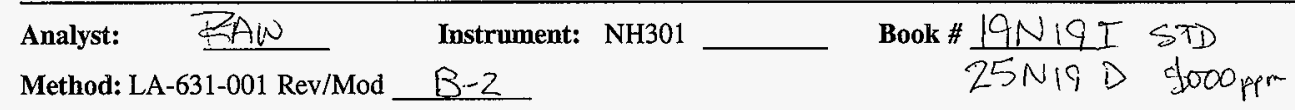

Worklist Comment: NH3-01 NH3STD 1ML LMH

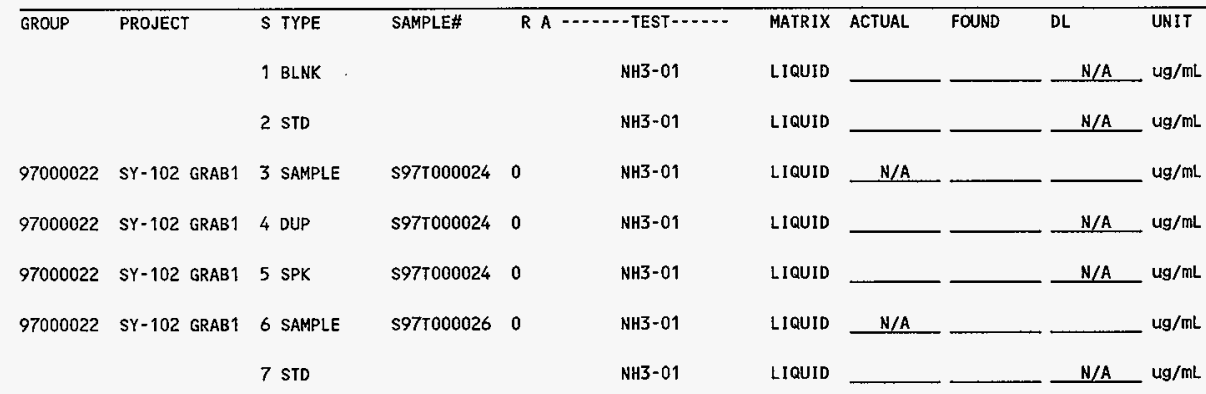

Final page for worklist \# 16679

\section{Analyst Signature Date}

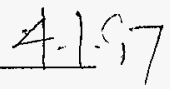

Analyst Signature Date

Data Entry Comments:

Units shown for QC (SPK \& STD) may not reflect the actual units. $D L=$ Detection Limit, $S=$ Worklist Slot Number, $R=$ Replicate Number, $A=$ Aliquot Code. 


\section{HNF-SD-WM-DP-227, REV. 0}

DOUELE KNOWN AODTTION SELECTED

AT $13: 47, \quad 04-01-97$

SAMPLE VOL= 25.000 AT $13: 48.04-01-97$

ENTERED

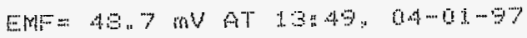

$E M F=49.0 \mathrm{mV}$ AT $13: 49,04-01-97$

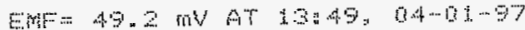

ENTEFED

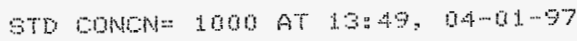
ENTEPED

STO VOL= . 25000 AT 13 II, $04-0 \mathrm{~L}-97$

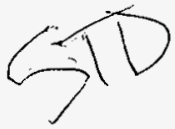

ENTEFED

EMF:= 37.0 nV AT $13: 53: 04-01-97$

EMF= $36.9 \mathrm{mV}$ AT 13: $1,94-01-97$

EMF 36.9 mV AT $13: 51,94-01-97$

ENTEFEO

STD VOL:= 2.500I AT $13 n 54,04-01-97$

ENTEFED

EMF $=-2,0$ mV AT 1.345\%, 04-01-97

$E M F=-2,1$ mU AT $1355,4,-01-97$

$E M=-2.0 \mathrm{mV}$ AT $1315 \%$. 0.-01-97

ENTERED

An NHS SIOFE=-60 9 mV/DEt

AT $13: 53: 04-01-97$

1.NH3 GONCN:: 1.5 .5

OOUBLE KNOWN ADTITIUN SELECTED

AT 13:54:04-01-57 
SAMPLE VOL:: 25, 000 AT $13: 54,04-04-97$

ENTEFED

$E$ ENE $=111,3$ mV AT $13 n 57,04-01-97$

EMr $=111,9$ nV AT $13050,04-01-97$

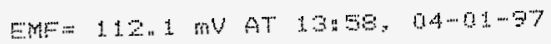

ENTEFED

6TO CONDN= 1000 AT $13 \times 50,04-01 \cdots 97$

ENTEREO

STD VOL $=.25000$ AT $13456,04-01-97$

ENTEFET

EMrF $=57.2$ mV AT $14: 00:$ 04 - $01-97$

$E M F=57.1 \mathrm{WW} A T$ 14:00, 04-01-97

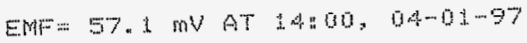

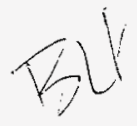

ENTEFED

BTD vOL $=2,5030$ AT LAn 01 . 04-01-67

ENTEFED

$E F E=1.1 \mathrm{nV}$ ar $14: 02,04-01-97$

ENF $=0.9 \mathrm{mV}$ AT $14.02,04-01-97$

ENTEFED

1. NHS SLOFE=-5\%.0 mV/OEC

AT 14:82, 04:-01-97

in $\mathrm{NHS}$ CONON= 1 . 31

DOUBLE WOWN ADDTTION SEIELTED

AT 1.4:02, 04-01-97 


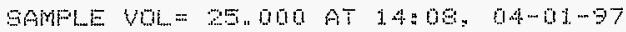

ENTEFED

EWF $=$ I5.1 mV AT 44:11, 0.4-01-97

EMF= $=115,3$ mV AT $14: 12,014-01-97$

EIF $=145,3$ mV AT $14,12,0 A-61-97$

ENTEREO

BTO CONEN= 1000 AT $14: 32, \quad 34 \cdots 01-57$

ENTEFES

STO VOL= .25000 AT $14: 12:$ BA-61-97

ENTEFEX

EMF= 57.5 mU AT $14: 13,04-01-57$

ENF $=57,4$ пU AT म4. $14,04-61-97$

EME= 57, 3 nV AT $14.14,04-01-97$

ENTEFED

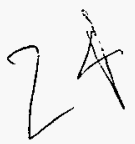

STO VOL= 2.5000 AT $14.44,04-01 .-97$

ENTERED

EMF = 0.9 InV AT $1415,04-10,-97$

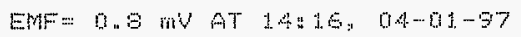

ENTEFEY

1. NHS SLOPE=-..59, D mU/DEO

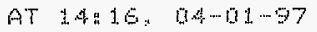

I. NH3 CONCN= 1 15

DOUEIE KNOWN ADOTTION BELEITEL

AT 14:17: 04-0:-67 


\section{HNF-SD-WM-DP-227, REV. 0}

SAMFLE VOL= 2\%, 100 AT 1.417: 04-11-97

ENTERED
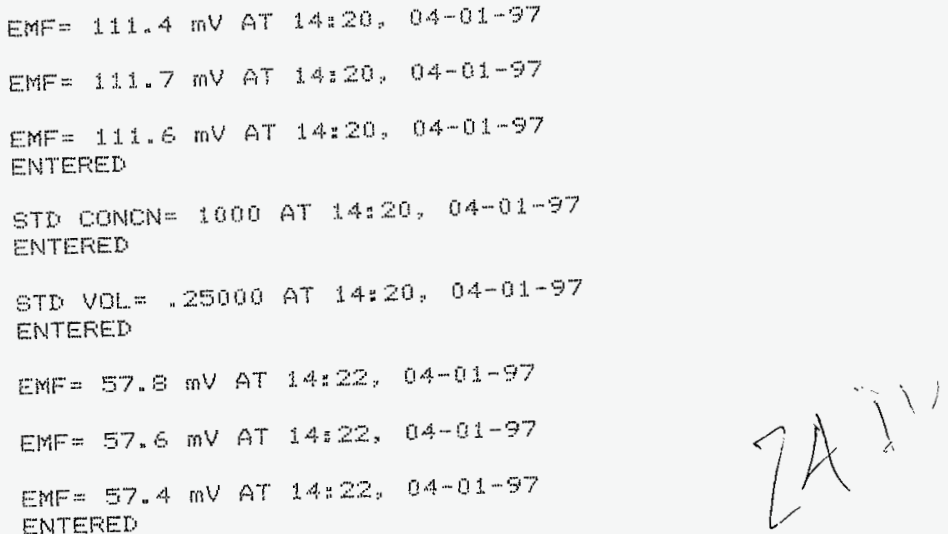

STD VOL= 2, 5000 AT 14.23, 04-01-97

ENTEFEO

EWF $=1.2$ mV AT $14,24,04-01-97$

EMF $=1.1$ mV AT $14=24,04-01-97$

ENTERED

1.MHS SLDPE $=-59.3 \mathrm{nV} / \mathrm{DEO}$

AT 1.42, $04-01-97$

$1:$ NHB CONDN= 1,37

DOUBLE FNOWN ADTTTON SELEOTED

AT 4 4 $25,04-01-97$ 
$\angle G-70-10$ "Ze a

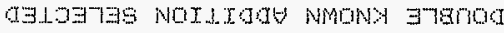

$$
\begin{aligned}
& \angle " \angle I=N O N D O E H N T \\
& \angle E-T 0-100 \text { :TE:Tा IH }
\end{aligned}
$$

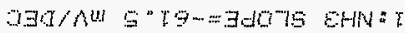

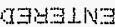

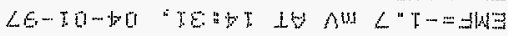

$$
\begin{aligned}
& \angle E-T a-70 \text { "TE а }
\end{aligned}
$$

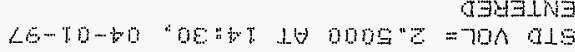

$$
\begin{aligned}
& \angle 6-10 \cdots+0 \text { "os }
\end{aligned}
$$

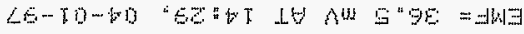

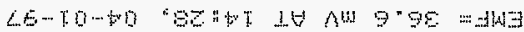

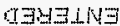

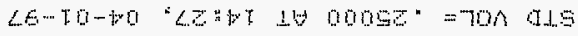

$$
\begin{aligned}
& 93431 \mathrm{~N} 3
\end{aligned}
$$

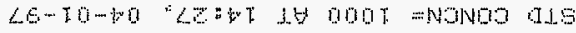

$$
\begin{aligned}
& \angle 6-10+00 \text { "Le: }
\end{aligned}
$$

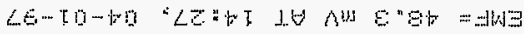

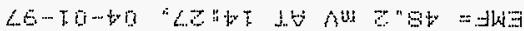

$$
\begin{aligned}
& \text { gYGIN }
\end{aligned}
$$

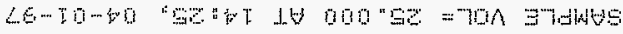


SAMPLE VOL= 23, 000 AT $1433,04-01-97$

ENTERED

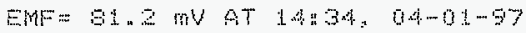

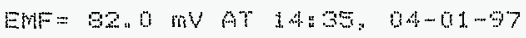

EmF $=02.2$ mV AT $\{4: 36,04-01-97$

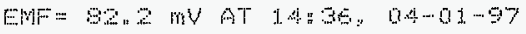

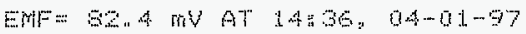

$E M F=E 2.4$ mV AT $14 a 36, \quad 04-61-97$

ENTEFED

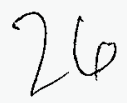

5TD CONCN= 2000 AT 44.37, 04-01-97

ENTEFEO

STD VQL = "2500U AT $14237,04-01-97$

ENTEFEO

EMF= 51,8 mV AT $14535,04-01-97$

EWF:= 31.7 in कT $14: 39,04-01-97$

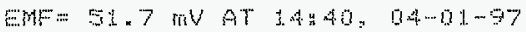

$E M=51.7$ mV AT $14: 43,04-61-97$

ENTEFEO

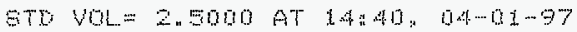

ENTEREO

EMF= 0.6 mV AT $14,42, \quad 04-01-97$

EMF $=0.6$ mV AT $14 n 42,04-01-97$

ENTEREO

1.NHS BLOFE =-59.3 $\mathrm{MV} / \mathrm{DEC}$

AT $14: 42,04-01-97$

1: NHS DONCN: 4.30 


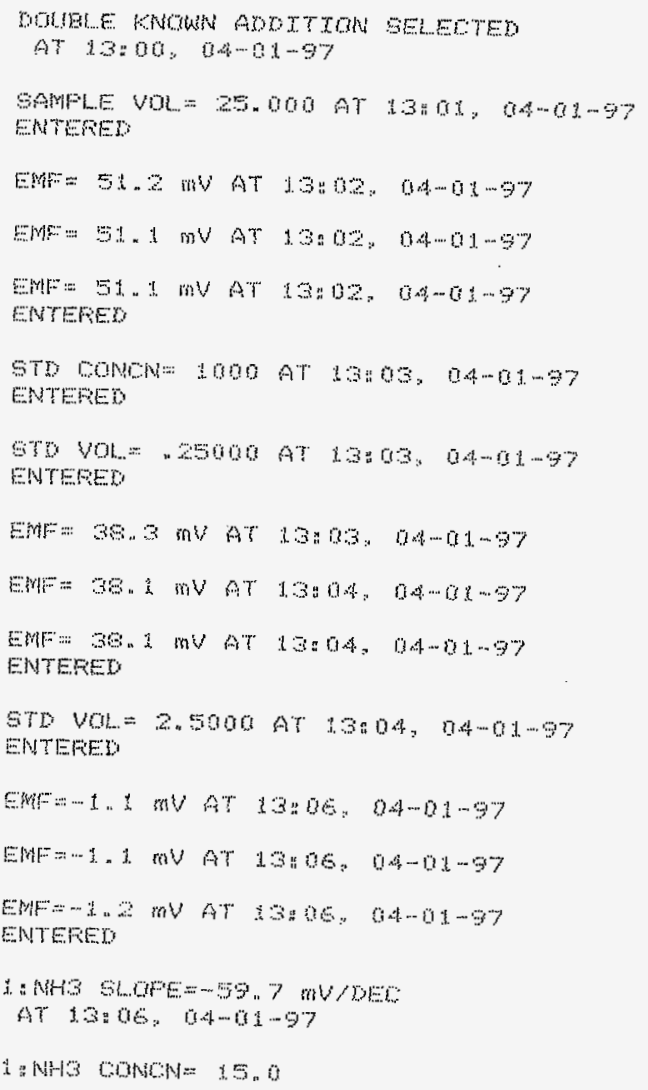

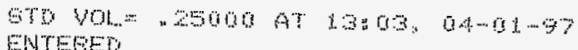

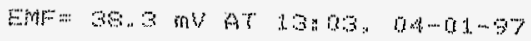

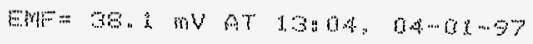

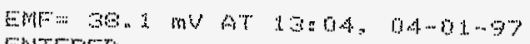

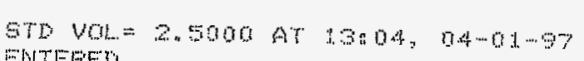
ENTERET

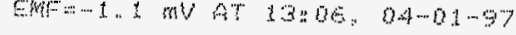

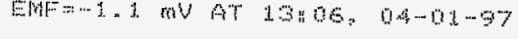

EVF-3.

E. Thentory

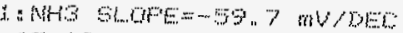

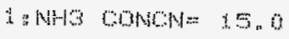

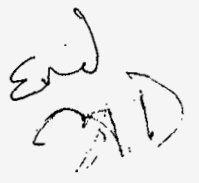


HNF-SD-WM-DP-227, REV. 0

WORKBOOK PAGE: STD2

AMMONIA (NH3) : LA-631-001 (B-2)

LIQUIDSISOLIDS

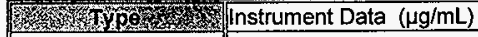

STD Blank Result from the Instrument $(\mu \mathrm{g} / \mathrm{mL})$

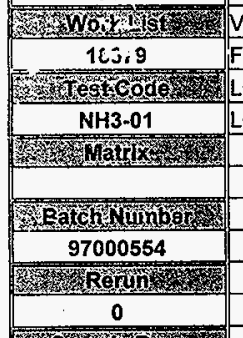

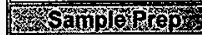

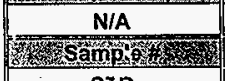
Vol of Sample for Dilution ( $\mathrm{mL}$ ) or Vol of Sample Direct $(\mathrm{mL})$

Final Vol of Dilution $(\mathrm{mL})$ or Vol of Sample Direct $(\mathrm{mL})$

LCS Standard Book Number

LCS Standard Concentration $(\mu \mathrm{g} / \mathrm{mL})$

$\mathrm{NH} 3$ Concentration $(\mu \mathrm{g} / \mathrm{mL})$

$\mathrm{NH} 3 \mathrm{CONC}$

STD

ID

BR

VSAM

FVOL LCS

STD VAL

Method Detection Limit ( $\mathrm{g} / \mathrm{mL})$

1.00 ]

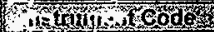

Nrisjor

RTS

NH3 Concentration $(\mu \mathrm{g} / \mathrm{mL})=($ ID-BR $) *(F V O L /$ VSAM $)$

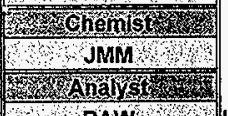

RAW

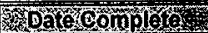

$04 / 09 / 97$

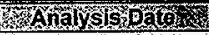

$04 / 01 / 97$

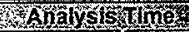

Sanp 01 Totn 4 NH3 Concentration $(\mu \mathrm{g} / \mathrm{mL})$

$98 \%$

SY 102

Analyst:

Signature of Chemist:

SAMPLE.WB1 REVXX
LCS Standard \% Recovery 
HNF-SD-WM-DP-227, REV. 0

WORKBOOK PAGE: BLANK1

AMMONIA (NH3) : LA-631-001 (B-2)

通

LIQUIDS/SOLIDS

BLNK

Blank Result from the Instrument $(\mu \mathrm{g} / \mathrm{mL})$

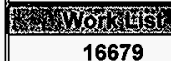

Vol of Sample for Dilution $(\mathrm{mL})$ or Vol of Sample Direct $(\mathrm{mL})$

Final Vol of Dilution $(\mathrm{mL})$ or Vol of Sample Direct $(\mathrm{mL})$

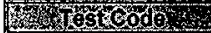

NH3-01

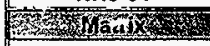

LIQUID

Baccionomo

97000554

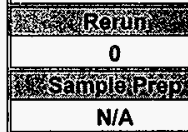

$\mathrm{NH} 3$ Concentration $(\mu \mathrm{g} / \mathrm{mL})$

NH3 CONC

$<$

$1.00 E+00$

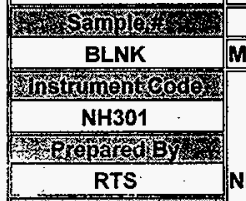

Method Detection Limit $(\mu \mathrm{g} / \mathrm{mL})$

1.00

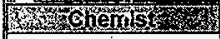

$\mathrm{JMM}$

-

RAW

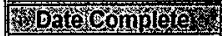

04/09/97

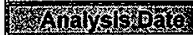

04/01/97

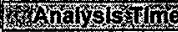

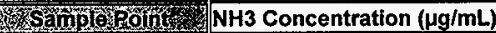

$<1.00 E+00$

SY-102

NH3 Concentration $(\mu \mathrm{g} / \mathrm{mL})=(\mathrm{BR}) \cdot(\mathrm{FVOL} / \mathrm{VSAM})$

Analyst:

Signature of Chemist:

SAMPLE.WB1 REV XX
RAW Date: 04/09/97

JMM Date: 04/io/a7 
HNF-SD-WM-DP-227, REV. 0

WORKBOOK PAGE: SAM3

\begin{tabular}{|c|c|c|c|}
\hline \multicolumn{3}{|c|}{ AMMONIA (NH3) : LA-631-001 (B-2) } & SAMPLE \\
\hline 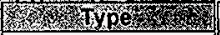 & instrument Data $(\mu \mathrm{g} / \mathrm{mL})$ & ID & 8.150 \\
\hline SAMPLE & Blank Result from the Instrument $(\mu \mathrm{g} / \mathrm{mL})$ & BR & 1.3100 \\
\hline Wod 15 & Vol of Sample for Dilution $(\mathrm{mL})$ or Vol of Sample Direct $(\mathrm{mL})$ & VSAM & 1.0 \\
\hline 16679 & Final Vol of Dilution $(\mathrm{mL})$ or Vol of Sample Direct $(\mathrm{mL})$ & FVOL & 25.0 \\
\hline \multicolumn{4}{|c|}{ 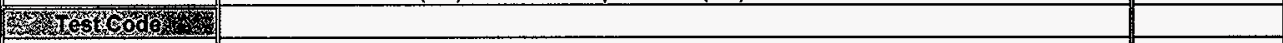 } \\
\hline \multicolumn{4}{|l|}{\begin{tabular}{|c|}
$\mathrm{NH301}$ \\
$\mathrm{N} 301$
\end{tabular}} \\
\hline \multicolumn{4}{|l|}{ 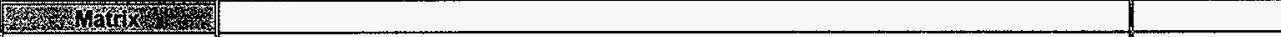 } \\
\hline \multicolumn{4}{|l|}{ LIQUID } \\
\hline \multicolumn{4}{|l|}{ 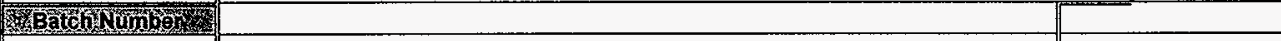 } \\
\hline \multicolumn{4}{|l|}{97000554} \\
\hline \multicolumn{4}{|l|}{ 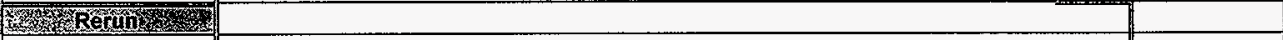 } \\
\hline \multicolumn{4}{|l|}{0} \\
\hline \multicolumn{4}{|l|}{ S Sanderisen } \\
\hline N/A & $\mathrm{NH} 3$ Concentration $(\mathrm{\mu g} / \mathrm{mL})$ & $\mathrm{NH} 3 \mathrm{CONC}$ & $1.00 E+00$ \\
\hline \multicolumn{4}{|c|}{ 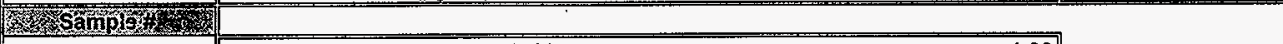 } \\
\hline S97T000024 & Method Detection Limit ( $\mu \mathrm{g} / \mathrm{mL}$ ) & 1.00 & \\
\hline \multicolumn{4}{|c|}{ 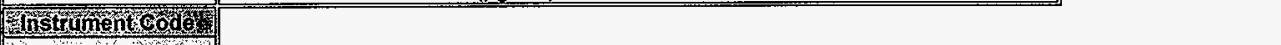 } \\
\hline \multicolumn{4}{|l|}{$\mathrm{NH} 301$} \\
\hline \multicolumn{4}{|l|}{ Whepared By } \\
\hline \multicolumn{4}{|c|}{ RTS $N H 3$ Concentration $(\mu \mathrm{g} / \mathrm{mL})=(\mathrm{ID}-\mathrm{BR}) *(\mathrm{FVOL} / \mathrm{VSAM})$} \\
\hline \multicolumn{4}{|c|}{ 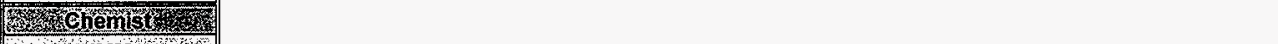 } \\
\hline \multicolumn{4}{|l|}{ JMMM } \\
\hline \multicolumn{4}{|l|}{ 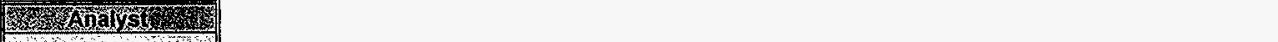 } \\
\hline \multicolumn{4}{|l|}{ RAW } \\
\hline \multicolumn{4}{|l|}{ 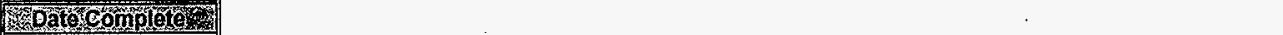 } \\
\hline \multicolumn{4}{|l|}{$04 / 09 / 97$} \\
\hline \multicolumn{4}{|l|}{ Yhalys tsoge } \\
\hline \multicolumn{4}{|l|}{$04101 / 97$} \\
\hline \multicolumn{4}{|l|}{ 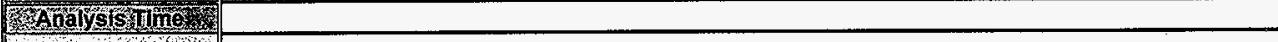 } \\
\hline \multicolumn{4}{|c|}{ 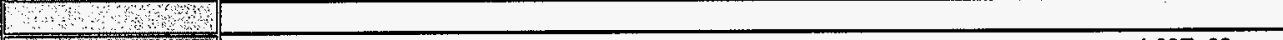 } \\
\hline STampletroin & NH3 Concentration $(\mu \mathrm{g} / \mathrm{mL})$ & & $<1.00 E+00$ \\
\hline
\end{tabular}

\begin{tabular}{|lll|}
\hline Analyst: & RAW Date: & $04 / 09 / 97$ \\
\hline Signature of Chemist: & JMM Date: $04 / 10 / 97$ \\
\hline SAMPLE.WB1 REV XX
\end{tabular}


WORKBOOK PAGE: DUP4

\section{AMMONIA (NH3) : LA-631-001 (B-2)}

LIQUIDSISOLIDS

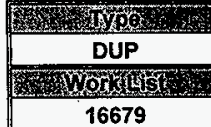

instrument Data $(\mu \mathrm{g} / \mathrm{mL})$

Blank Result from the Instrument $(\mu \mathrm{g} / \mathrm{mL})$

Vol of Sample for Dilution $(\mathrm{mL})$ or Vol of Sample Direct $(\mathrm{mL})$

Final Vol of Dilution $(\mathrm{mL})$ or Vol of Sample Direct $(\mathrm{mL})$

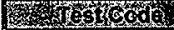

3. That

LIQUID

3a

Sample Concentration $(\mu \mathrm{g} / \mathrm{mL})$

97000554

19.

0

$\frac{0}{\text { N/A }}$

\begin{tabular}{|c|c|} 
N/A \\
\hline S97T000024
\end{tabular}

$\mathrm{NH} 3$ Concentration $(\mu \mathrm{g} / \mathrm{mL})$

NH3 CONC

$1.50 \mathrm{E}+00$

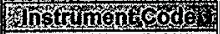

$\mathrm{NH} 30 \%$

Thenared 3 ,

RTS

Method Detection Limit $(\mu \mathrm{g} / \mathrm{mL})$

1.00

RPD (DUP \& SAM) $=$ Absolute Val. $(($ NH3 CONC - SAM CONC $) /(($ NH3 CONC + SAM CONC $) / 2)) * 10$

$\mathrm{NH} 3$ Concentration $(\mu \mathrm{g} / \mathrm{mL})=(\mathrm{ID}-\mathrm{BR}) *(\mathrm{FVOL} / \mathrm{VSAM})$

JMM

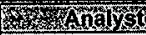

RAW 6 :

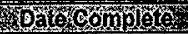

$04109 / 97,3$

Thank

$04 / 01 / 97$

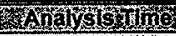

RPD (DUP \& SAM)

NH3 Concentration $(\mu \mathrm{g} / \mathrm{mL})$

$40 \%$

SBMplog

SY. 102

Analyst:

Signature of Chemist:

SAMPLE.WB1 REV XX

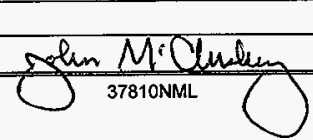

RAW Date: 04/09/97

JMM Date: o4/10 97 
WORKBOOK PAGE: SPIKE5

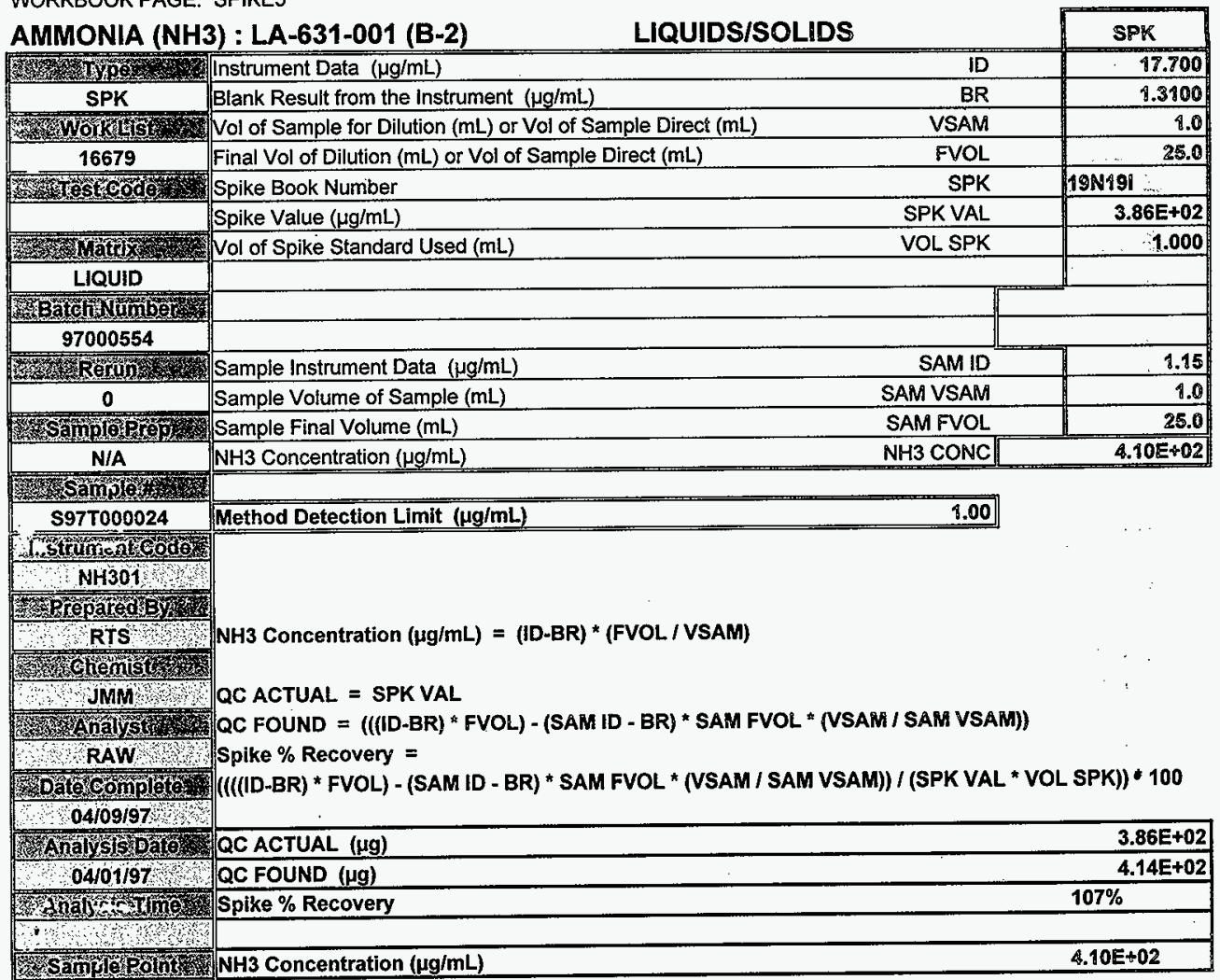

SY -102

\begin{tabular}{|c|c|c|c|}
\hline Analyst: & RAW & Date: & $04 / 09 / 97$ \\
\hline Signature of Chemis: & JMM & Date: & 0410197 \\
\hline
\end{tabular}


WORKBOOK PAGE: SAM6

AMMONIA (NH3) : LA-631-001 (B-2)

LIQUIDS/SOLIDS

SAMPLE

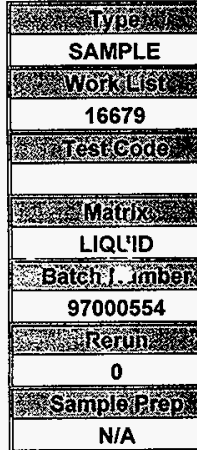

Instrument Data $(\mu \mathrm{g} / \mathrm{mL})$

Blank Result from the Instrument $(\mu \mathrm{g} / \mathrm{mL})$

Vol of Sample for Dilution $(\mathrm{mL})$ or Vol of Sample Direct $(\mathrm{mL})$

Final Vol of Dilution $(\mathrm{mL})$ or Vol of Sample Direct $(\mathrm{mL})$

$\mathrm{NH} 3$ Concentration $(\mu \mathrm{g} / \mathrm{mL})$

NH3 CONC

$7.48 E+01$

Sanoleres.

S97T000026

Method Detection Limit $(\mu \mathrm{g} / \mathrm{mL})$

$\$ .00$

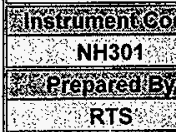

NH3 Concentration $(\mu \mathrm{g} / \mathrm{mL})=($ ID-BR $) *(F V O L /$ VSAM $)$

Jisin

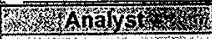

RAW

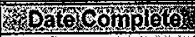

$04 / 09 / 97$

C.

$04 / 01 / 97$

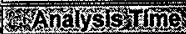

-

$7.48 E+0$ \&

SY-102

\begin{tabular}{|l}
\hline Analyst: ' \\
Signature of Chemist: \\
SAMPLE.WB1 REV XX
\end{tabular}

RAW Date: 04/09/97

JMM Date: 04/10/97 
WORKBOOKPAGE: ST_END7

\begin{tabular}{|c|c|c|c|}
\hline \multicolumn{4}{|c|}{ (1) - } \\
\hline AMMONIA (NH: & 3) : LA-631-001 (B-2) & & STD \\
\hline (1) & Instrument Data $(\mu \mathrm{g} / \mathrm{mL})$ & ID & 15.000 \\
\hline STD & Blank Result from the Instrument $(\mu \mathrm{g} / \mathrm{mL})$ & BR & 8.3100 \\
\hline Wodn & Vol of Sample for Dilution $(\mathrm{mL})$ or Vol of Sample Direct $(\mathrm{mL})$ & VSAM & $\{.0$ \\
\hline 16679 & Final Vol of Dilution $(\mathrm{mL})$ or Vol of Sample Direct $(\mathrm{mL})$ & FVOL & 25.0 \\
\hline Her & LCS Standard Book Number & LCS & 19N191 \\
\hline & LCS Standard Concentration ( $\mu \mathrm{g} / \mathrm{mL})$ & STD VAL & $3.86 E+02$ \\
\hline 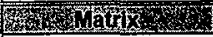 & & & \\
\hline & & & \\
\hline 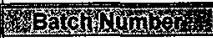 & & & \\
\hline 97000554 & & & \\
\hline Swank & & & \\
\hline 0 & & & \\
\hline W & & & \\
\hline N/A & $\mathrm{NH} 3$ Concentration $(\mu \mathrm{g} / \mathrm{mL})$ & $\mathrm{NH} 3 \mathrm{CONC}$ & $3.42 E+02$ \\
\hline 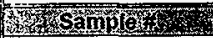 & & & \\
\hline & Method Detection Limit $(\mu \mathrm{g} / \mathrm{mL})$ & 1.00 & \\
\hline 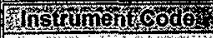 & & & \\
\hline $\mathrm{NH} 301$ & & & \\
\hline Spropardos & & & \\
\hline HRTS & NH3 Concentration $(\mu \mathrm{g} / \mathrm{mL})=(\mathrm{ID}-\mathrm{BR}) *(\mathrm{FVOL} / \mathrm{VSAM})$ & & \\
\hline - & & & \\
\hline DUMM JM & & & \\
\hline 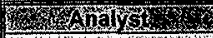 & & & \\
\hline RAW & LCS Standard \% Recovery $=(\mathrm{NH} 3$ CONC / STD VAL $) \cdot 100$ & & \\
\hline 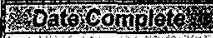 & & & \\
\hline $04 / 09 / 97$ & & & \\
\hline Aralissonto & & & \\
\hline $04 / 01 / 97$ & & & \\
\hline Whass & LCS Standard \% Recovery & & $89 \%$ \\
\hline किस, & $\cdot$ & & \\
\hline 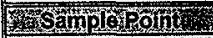 & NH3 Concentration $(\mu \mathrm{g} / \mathrm{mL})$ & & $3.42 ㅌ ㅏ ㄴ+02$ \\
\hline
\end{tabular}

SY $=102$

\begin{tabular}{|c|c|c|c|c|}
\hline Analyst: & & RAW & Date: & $04 / 09 / 97$ \\
\hline Signature of Chemist: & 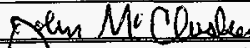 & JMM & Date: & $04 / 10 / 17$ \\
\hline
\end{tabular}




\section{LABCORE Completed Worklist Report for Worklist\# 16288}

Analyst: eal

Instrument: ICO2

Book $\# 36$ N2OD

Method: LA533/OS Rev/Mod D-1

Worklist Comment: Run per procedure.

new

\begin{tabular}{|c|c|c|c|c|c|c|c|c|c|}
\hline Seq Type & Samplet & $\mathbf{R A}$ & & Test & Matrix & Actual & Found DL or Yield & Unit & \\
\hline $10 \mathrm{cos}$ & & 0 & $20 \mathrm{Co}$ & \% & $\alpha c$ & 1 & $4200 \%$ & aq 10 & \\
\hline $1 \mathrm{CCB}$ & & 0 & arc-oc & CL & $Q C$ & 1 & $<1.700-2$ & $\mathrm{ug} / \mathrm{mat}$ & \\
\hline $18 \mathrm{se}$ & & 0 & $0+000$ & roz & oc & 1 & 410800 & $49 / m_{1}$ & \\
\hline $1 \mathrm{CCB}$ & & 0 & arc-QC & $\mathrm{BR}$ & QC & 1 & $<1.25 e-1$ & ug/mis & \\
\hline $1 \mathrm{cs}$ & & 0 & $010-0 c$ & $\mathrm{No3}$ & oc & 1 & 304001 & wg/m & \\
\hline $1 \mathrm{CCB}$ & & 0 & GIC-QC & PO4 & $Q C$ & 1 & $<1.20 e-1$ & $\mathrm{ug} / \mathrm{ml}$ & \\
\hline 140 & & 0 & $6100 \mathrm{c}$ & 804 & oc & 1 & $<1,3801$ & $101=$ & \\
\hline $1 \mathrm{CCB}$ & & 0 & arc-Qc & OXALATB2 & $\mathrm{Qc}$ & 1 & $<1.05 a-1$ & ug/m & \\
\hline $2, \mathrm{eq}$ & & 80 & $0 x-0$ & $8 \% 1 \%$ & $8 c$ & 5,800001 & $5620+01$ & 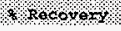 & \\
\hline $2 \mathrm{ccv}$ & & 0 & IC-QC & cr & ec & 7.900001 & $7.94 e+01$ & \& Recovery & \\
\hline 200 & & 0 & 010,0 & $\$ 0 \%$ & oc & 5,20002 & $6.660+02$ & 6 Reoloys & \\
\hline $2 \mathrm{ccv}$ & & 0 & arc-oc & $\mathrm{BR}$ & $\mathrm{QC}$ & $5.8900 \mathrm{e} 2$ & $5.56 e+02$ & \& Recovery & \\
\hline $2 \mathrm{ccd}$ & & 0 & $0 \mathrm{rc}-\mathrm{CO}$ & 403 & oc & 5,140002 & $58890+02$ & $8 / 0000 \% x$ & \\
\hline $2 \mathrm{ecv}$ & & 0 & arc-ec & PO4 & $Q c$ & 5.440002 & $5.38 e+02$ & \& Recovery & \\
\hline $2 \mathrm{cct}$ & & 0 & 6100 & 800 & 00 & 610000 & $0300+02$ & $8 R+000 x y$ & ঋt \\
\hline $2 \mathrm{CeV}$ & & 0 & $\theta \pi c-\theta c$ & OXALARE2 & $Q c$ & $5.3300 e 2$ & $5.318+02$ & \& Recovery & \\
\hline $3 \mathrm{SAPPD}$ & 597000028 & 0 & $0 \times 0-01$ & $P-02$ & 10UTh & $\mathrm{N} / \mathrm{A}$ & $1009 / 03$ & $401 \mathrm{mr} /$ & \\
\hline 3 SAMPLE & 5975000024 & 0 & OIC-01 & $C L-02$ & IIQUID & $3 / 2$ & $8.047 e+02$ & $\mathrm{ug} / \mathrm{mL}$ & \\
\hline $3 \mathrm{ssmpt}$ & 5972000024 & 0 & 010,01 & $102-02$ & $1000 \pi$ & N/2 & 6405603 & $60 / 61$ & \\
\hline 3 SAMPLE & $597 T 000024$ & 0 & $0 I C-01$ & $\mathrm{BR}-02$ & LIQJID & $\mathrm{N} / \mathrm{A}<$ & $6.4390+02$ & $\mathrm{Hg} / \mathrm{mL}$ & \\
\hline $3 \% 4 \mathrm{PDB}$ & 807000024 & 0 & $810 \% 01$ & 20302 & atoUTh & vata & $42540+04$ & 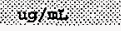 & \\
\hline 3 SAMPLE & $\$ 977000024$ & 0 & DIC-01 & $204-02$ & IIQUID & $N / A$ & $8.802 e+03$ & $u g / m L$ & \\
\hline 3 SAMPLE & 8021000024 & o & $0 x c-01$ & $504-02$ & $4-10 \mu 10$ & $\mathrm{x} / \mathrm{A}$ & $2 \times 3990+03$ & $10 / 7 \mathrm{mt}$ & \\
\hline 3 SAMPLE & $597 \mathrm{~T} 000024$ & 0 & IC-01 & OXALATE2 & IIQUID & $\mathrm{N} / \mathrm{A}<$ & $5.4090+02$ & $\mathrm{ug} / \mathrm{mL}$ & \\
\hline $4 \mathrm{Dup}$ & 5978000024 & 0 & $0 I C-01$ & $1002 \%$ & $1,0,110$ & $110 \times+03$ & $1000+03$ & $\mathrm{Rop}$ & \\
\hline 4 DUP & $597 \pi 000024$ & 0 & GIC-01 & $\mathrm{CL}-02$ & $\triangle I Q U L D$ & $8.05 e+02$ & $8.66 e+02$ & RPD & \\
\hline $4 \mathrm{Dop}$ & 5972000024 & 8 & 01001 & 00202 & 470010 & 640803 & $6 \times 8040 s$ & ApD & \\
\hline 4 DUP & 5972000024 & 0 & eIc-01 & $B R-02$ & LIQUID & $<6.44 e 2$ & $<6.44 \mathrm{e} 2$ & RPD & \\
\hline $40 \pm$ & 89700002 & 0 & oIc- 01 & $\mathrm{Nos} 02$ & 1,0010 & $4250+04$ & $4.290+04$ & $\mathrm{KPD}$ & \\
\hline 4 DUP & 5977000024 & 0 & axc-01 & PO4-02 & LIQUID & $8.800+03$ & $9.02 \theta+03$ & $\mathrm{RPD}$ & \\
\hline 4008 & 897000024 & 0 & $0 \times 0 \times 1$ & 60400 & 400010 & $2000+03$ & $2 / 51000$ & $\mathrm{RpD}$ & \\
\hline 4 DUR & $597 \mathrm{r} 000024$ & 0 & QIC-01 & OXALATE2 & IIOUID & $<5.41 \mathrm{e} 2$ & $<5.4102$ & RPD & \\
\hline $5 S A M P U$ & $807 \times 000026$ & 0 & $01 \mathrm{~L}-01$ & $8+02$ & HodTn & N/A & $64718+42$ & volot & १॥ \\
\hline 5 SAMPLE & $597 \mathrm{~T} 000026$ & 0 & QIC-01 & CL -02 & IIQUID & $\mathrm{N} / \mathrm{A}$ & $1.405 e+03$ & $v g / 2 n$ & \\
\hline $5,8 \mathrm{APDP}_{2}$ & 89,71000026 & 10 & $0 \mathrm{r} \sigma-01$ & $10 \times, 02$ & -100tD & $\mathrm{N} / \mathrm{A}$ & $1,4590-04$ & $0196 \mathrm{hr}$ & \\
\hline 5 SAMPLB & s975000026 & 0 & arc-01 & $\mathrm{BR}-02$ & IIQUID & $N / A<$ & $2.651 a+02$ & $\mathrm{ug} / \mathrm{mr}$ & \\
\hline 5 SN0p & 8204000026 & 0 & $6 \times c-0$ & 203002 & $3100+1$ & $\mathrm{~s}+\mathrm{x}$ & $48690+04$ & ug $/ 4$ & \\
\hline 5 SAMPLE & $597 T 000026$ & 0 & aIC-0I & $804-02$ & IIOUID & $N / A$ & $4.018 \theta+03$ & $\mathrm{ug} / \mathrm{sac}$ & \\
\hline $5 / 8 x+12$ & 89,7000026 & 0 & 01001 & 80402 & Lotrip & 912 & $17880 \times 03$ & $\mathrm{xg}_{\mathrm{m}}$ & \\
\hline 5 SAMPLE & 5971000026 & 0 & OIC-0I & OXALATE2 & IIQUID & $N / A<$ & $2.227 e+02$ & $\mathrm{ug} / \mathrm{mL}$ & \\
\hline $600 P /$ & 8971000026 & 0 . & $010-01$ & $1002 \%$ & $4+00+0$ & $6410+02$ & $5,46 \%+02$ & RpD & \\
\hline
\end{tabular}

Units shown for $Q C(B L K / B K G)$ may not reflect the actual units. 


\section{LABCORE Completed Worklist Report for Worklist\# 16288}

\begin{tabular}{|c|c|c|c|c|c|c|c|c|c|}
\hline Seq Type & Sample & $\mathbf{R}$ & & Test & Matrix & Actual & Found DL or Yield & Unit & \\
\hline 6 DUP & S97T000026 & 0 & QIC-01 & $\mathrm{CL}-02$ & LIQUID & $1.400+03$ & $1.46 e+03 \quad 4.196$ & RPD & \\
\hline 6 DUP & S97T000026 & 0 & $0 \mathrm{IC}-01$ & $\mathrm{NO2}-02$ & IIQOID & $1.46 \theta+04$ & $2.45 e+04$ & RPD & \\
\hline $6 \mathrm{Dup}$ & 8971000026 & 0 & $0+0,01$ & $\mathrm{BR} 0 \mathrm{O}$ & LOUID & 226504 & 22,6522 & $\mathrm{RPO}$ & \\
\hline 6 DUP & S97T000026 & 0 & IC-01 & NO3 -02 & ITQUID & $4.870+04$ & $4.780+04$ & $R P D$ & \\
\hline 6 bup & 8971000026 & 0 & $6+0-01$ & $204-02$ & Hooto & $4029+03$ & $3.960+03$ & Xo & \\
\hline 6 DUP & $S 972000026$ & 0 & eIc-01 & $504-02$ & LIQUID & $1.79 e+03$ & $2.28 \theta+03$ & RPD & \\
\hline $6 \%$ Ut & 5971000076 & $\%$ & 01001 & $0 \times-412$ & 140019 & $<2,230$ & 42302 & Rop & \\
\hline 7 SPR & s97T000026 & 0 & IC-01 & $\mathrm{P}-02$ & IIQUID & 5.900001 & $5.62 e+01$ & \& Recovery & \\
\hline $7 \mathrm{spx}$ & 8974000026 & 0 & $01 c 01$ & $\mathrm{ct} 02$ & IOUID & 7000001 & $6-920+01$ & $\gamma / 0 \circ-0 \times p \times$ & \\
\hline 7 SPR & S97T000026 & 0 & GIC-01 & $\mathrm{NO} 2=02$ & IIQUID & 5.420002 & $5.21 e+02$ & \& Recovery & \\
\hline $7 \mathrm{SPK}$ & 897000026 & $\%$ & 01001 & Benoz & Erout & $5 \times 890002$ & $5,640 \times 02$ & $1 p_{0}-\gamma_{0} y$ & \\
\hline 7 SPK & $S 97 T 000026$ & 0 & aIC-01 & $\mathrm{NO3-02}$ & LIQUTD & $5.9400 e 2$ & $3.11 e+02$ & \& Recovery & \\
\hline $7 \mathrm{SPK}$ & 5978000026 & 0 & 01601 & 804,02 & LIOUD & 5440002 & $5130+00$ & $64000802 y$ & \\
\hline 7 SPR & \$97T000026 & 0 & IC-01 & SO4=02 & ITOUTD & 6.310002 & $6.000+02$ & \% Recovery & \\
\hline $78 \mathrm{xx}$ & 8971000026 & 0 & 6100 & $00 \times 4 \times x 2$ & IoUle & $5 \times 30002$ & 503602 & $8 \mathrm{Recopes}$ & \\
\hline 8 SAMPLE & S97T000027 & 0 & aIC-01 & $\mathrm{F}-02$ & LIQUID & $\mathbf{N} / \mathbf{A}$ & $4.516 e+02$ & ug/mL & \\
\hline 8 SAYPLE & 597000027 & 0 & $0+c-01$ & $\mathrm{Cl}-02$ & YLQTD & $3 / 2$ & 101050003 & og/ 1 & \\
\hline 8 SAMPLE & 5975000027 & 0 & $O I C-01$ & $\mathrm{NO}-02$ & IIQUID & $N / A$ & $1.5370+04$ & $\mathrm{ug} / \mathrm{mL}$ & \\
\hline 8 SANHE & 5971000027 & 0 & $6+01$ & $\mathrm{Bg}-2$ & LOUTD & $\mathrm{x} / \mathrm{A}=\mathrm{\alpha}$ & $26510+02$ & $49 / 7$ & \\
\hline 8 SAMPLE & S975000027 & 0 & aIC-02 & NO3-02 & IIQUID & $N / A$ & $3.783 \mathrm{e}+04$ & $\mathrm{ug} / \mathrm{mL}$ & \\
\hline 8 SAMPAB & $1597500002 \%$ & 0 & $0+0-0$ & $104-02$ & LIOUID & $1 / 1$ & $4,3960+03$ & $10 / \mathrm{m}$ & \\
\hline 8 SAMPLE & $597 \div 000027$ & 0 & $O I C-0 I$ & $504-02$ & IIQUID & $N / 2$ & $2.188 \theta+03$ & $\mathrm{ug} / \mathrm{mL}$ & \\
\hline 8 SAMPRA & $\$ 990000027$ & 0 & QLOHL & OXA1AR2 & HIOUID & $\mathrm{N} / \mathrm{a} / \mathrm{m}$ & $5 \% 00+02$ & toglmol & \\
\hline 9 DUP & $\$ 97 T 000027$ & 0 & -IC-01 & F-02 & IIQOID & $4.52 e+02$ & $4.53 \theta+02$ & RPD & \\
\hline 900 & 897000027 & 0 & 01001 & $\mathrm{c}-0 \mathrm{~d}$ & Yroutp & $1-100+03$ & $1,130+03$ & Rep & \\
\hline 9 DUP & 597 T000027 & 0 & OIC-OI & $302-02$ & IIQUID & $1.54 a+04$ & $1.55 e+04$ & RPD & \\
\hline $9 \mathrm{DoR}$ & 897000002 & 0 & $0+0+01$ & $\mathrm{BR}-\mathrm{O} 2$ & $x+Q 0+0$ & 826542 & 42,6562 & $\mathrm{RPO}$ & \% \\
\hline 9 DUP & \$97T000027 & 0 & OIC-OI & $\mathrm{HO3}-02$ & LIQUID & $3.78 a+04$ & $3.790+04$ & RPD & \\
\hline 9000 & 597000027 & 0 & $010-01$ & $104-02$ & tjooth & $4-400-03$ & $4,40-03$ & $\mathrm{EP}$ & \\
\hline 9 DUP & s97T000027 & 0 & ac- 02 & $504-02$ & LIQUID & $2.19 a+03$ & $2.340+03$ & RPD & \\
\hline $9 \mathrm{DUP}$ & 3912000027 & 0 & 010.03 & $0 \times 1 / 4152$ & 3,000 & $5790 \cdot 02$ & $5,536 \times 0$ & SPD & \\
\hline
\end{tabular}

\section{Final page for worklist\# 16288}

Analyst Signature Date
\[ \begin{array}{l}\text { Analyst Signature Date } \\ \text { Reviewer Signature }\end{array} \]
Validated $1.28 .97 \mathrm{Hea}$
Date $1-28.97$

Units shown for $Q C(B L K / B K G)$ may not reflect the actual units. 


\section{LABCORE Data Entry Template for Worklist\# 16288}

Analyst: EAL Instrument: ICO TCO2 Book\#36N20.D

Method: LA-533-105 Rev/Mod D./

Worklist Comment: Run per procedure.

new

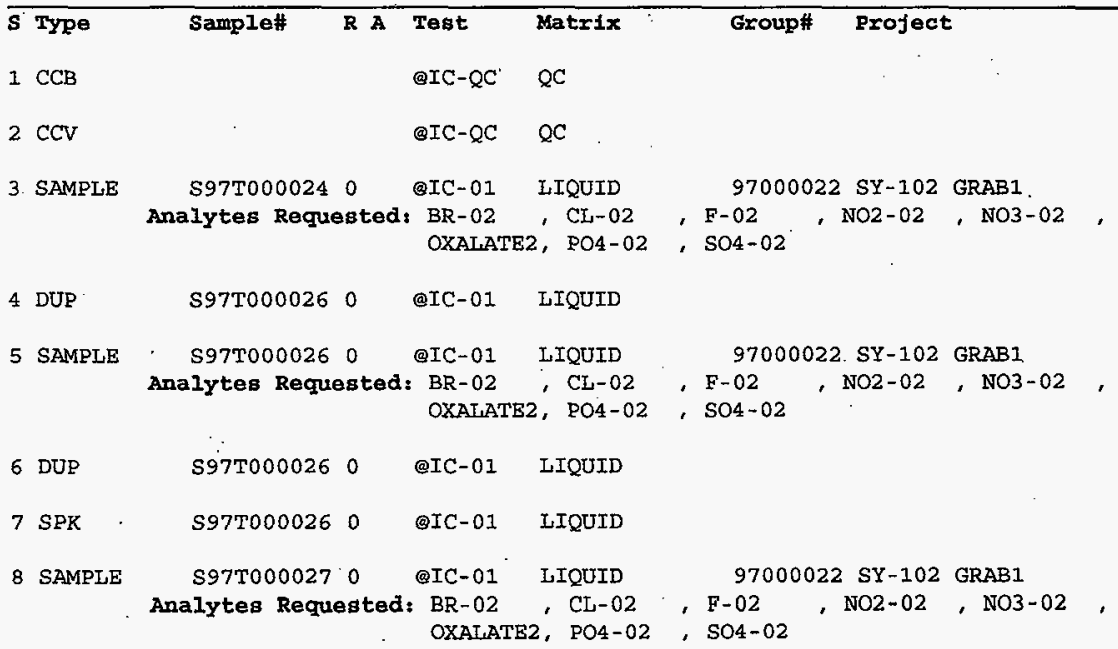

9 DUP S97T0000270 QIC-01 LIQUID

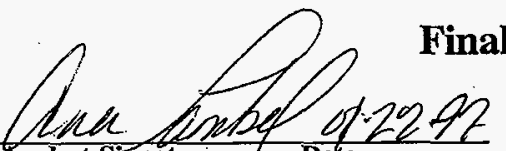

Analyst Signature 7 Date

Analyst Signature Date

$$
\begin{aligned}
& \text { 16288JA.CSV uploaded 1-28.97 Hea } \\
& \text { validated 1-28.97 Hea }
\end{aligned}
$$

Data Entry Comments:

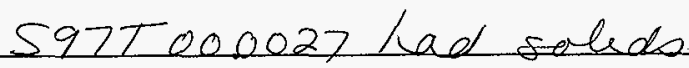
jout the higind uraio 
Data Reprocessed on 01/28/1997 09:56:34

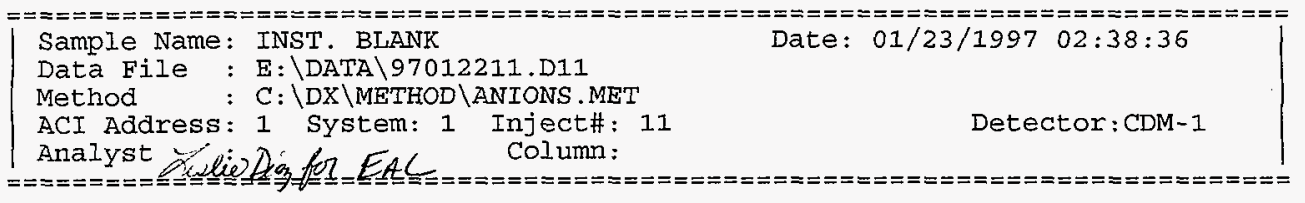

Calibration Volume Dilution Points Rate start stop Area Reject

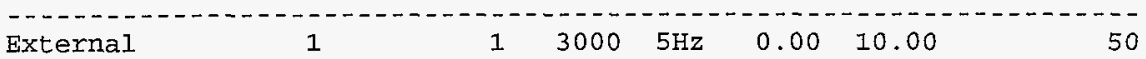

$* * * * * * * * * * * * * * * * * * * * * * * * * * *$ Peak Report : All Peaks $* * * * * * * * * * * * * * * * * * * * * * * * * * * *$

$\begin{array}{lrrr}\text { Pk. Ret Component } & \text { Concentration } & \text { Height } & \text { Area Bl. } \\ \text { Num } & \text { Timelta } & \text { Code }\end{array}$

10.87

$2 \quad 1.42$

31.56 CHLORIDE

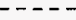

3.28 NITRATE

6.42 SULFATE

\begin{tabular}{rrrr} 
& 0.000 & 71 & 480 \\
0.000 & 34 & 156 \\
0.008 & 54 & 187 \\
0.304 & 156 & 1098 \\
& 0.113 & 29 & 358 \\
\hline Totals & 0.425 & 344 & 2279
\end{tabular}

File: 97012211.D11 Sample: INST. BLANK

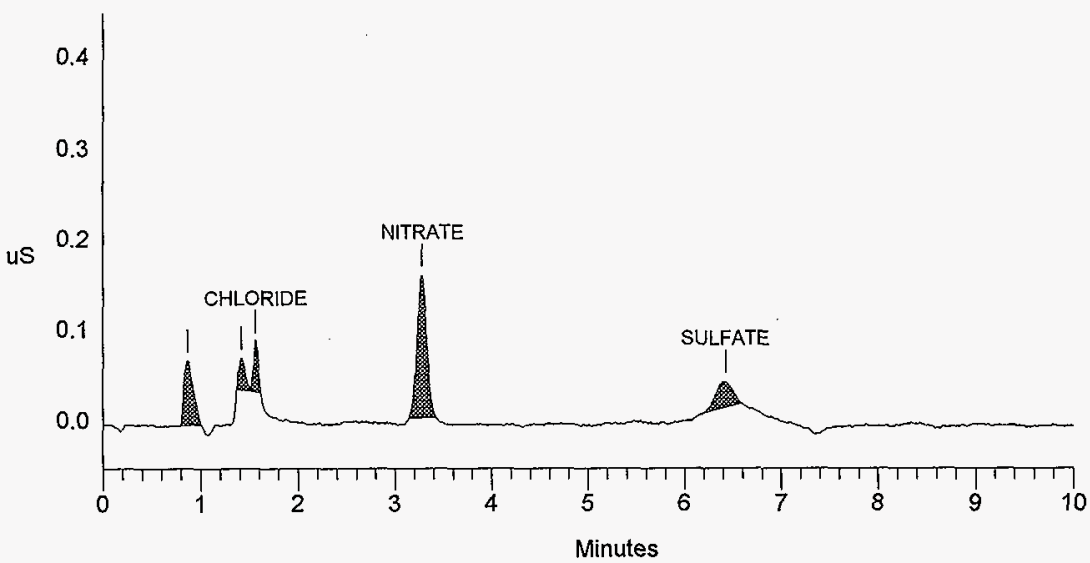

SIGNATURE ABOVE REPRESENTS CHEMICAL TECHNOLOGIST/CHEMIST THAT COMPLETEDNERIFIED THE CALIBRATIONIANALYSIS ON PAGES 90 TO 98 


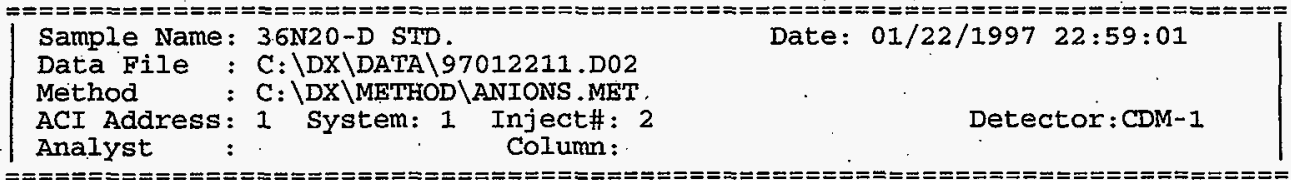

Calibration Volume Dilution Points Rate start Stop Area Reject

Cal

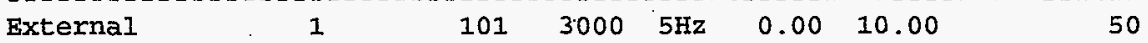

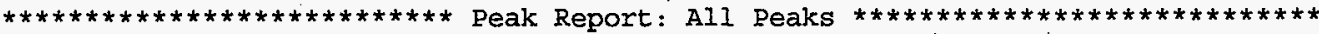

Pk. Ret Component Concentration Height Area Bl. $\%$ Delta

Num Time Name $\mathrm{ug} / \mathrm{ml}$ Code

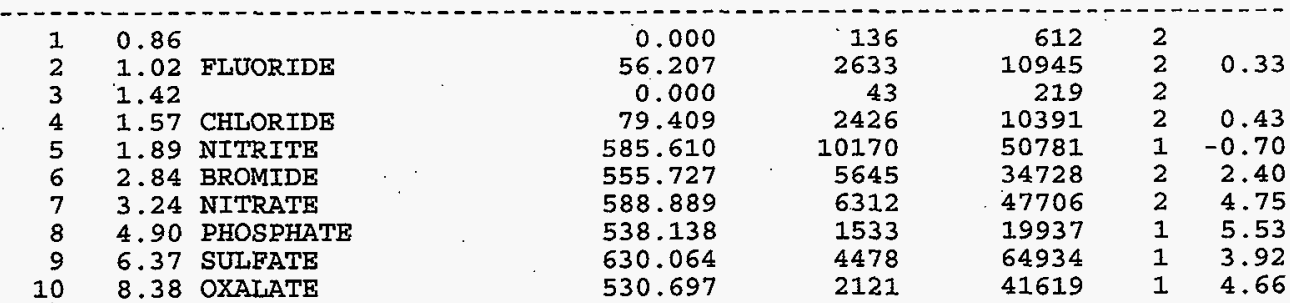

Totals $3564.741 \quad 35498 \quad 281873$

File: 97012211.D02 Sample: 36N20-D STD.

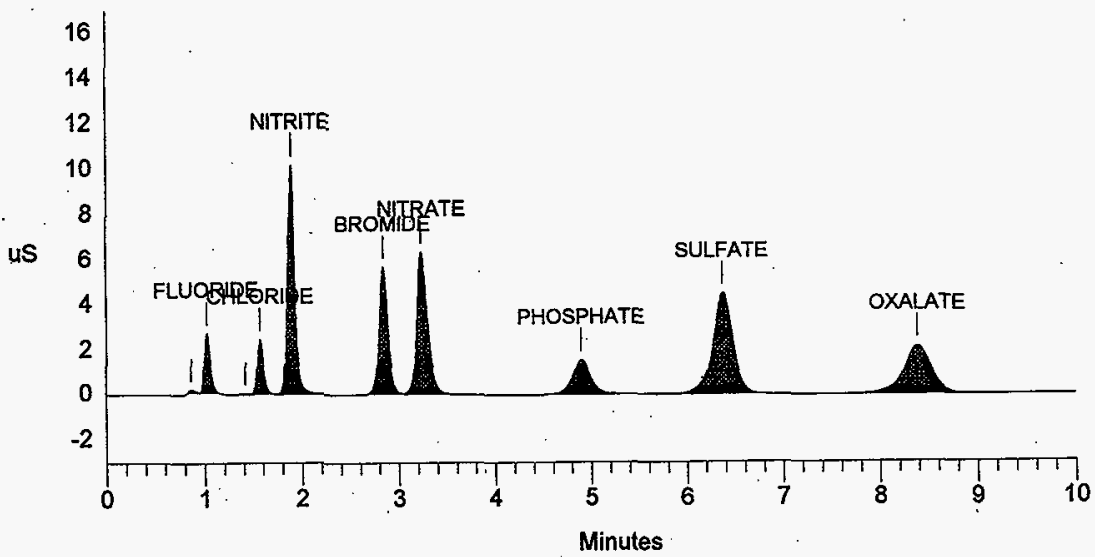




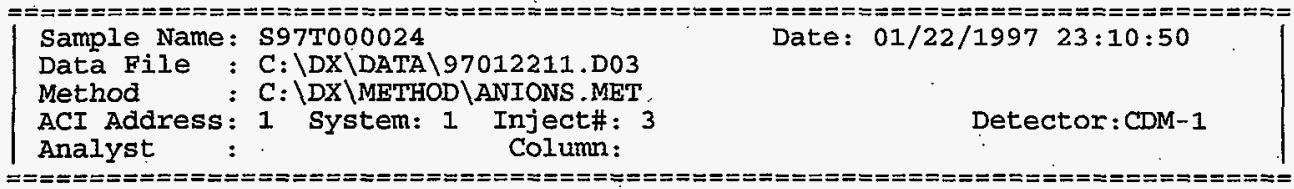

Calibration Volume Dilution Points Rate start stop Area Reject

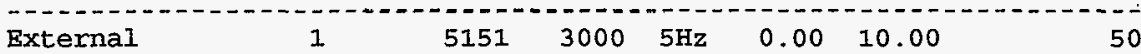

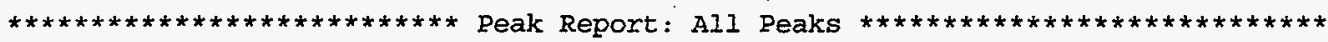

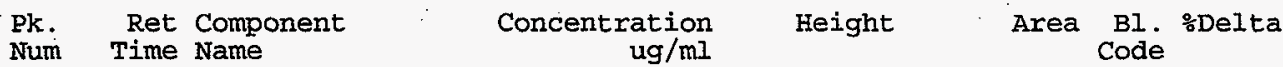

$\begin{array}{lll}1 & 0.87 & \\ 2 & 1.02 & \text { FIUUORIDE } \\ 3 & 1.56 & \text { CHLORIDE } \\ 4 & 1.88 & \text { NITRITE } \\ 5 & 3.22 & \text { NITRATE } \\ 6 & 4.90 & \text { PHOSPHATE } \\ 7 & 6.40 & \text { SULFATE }\end{array}$

\begin{tabular}{|c|c|c|c|c|c|}
\hline & $\begin{array}{r}0.000 \\
1096.719 \\
804.691 \\
6405.237 \\
42539.178 \\
8801.757 \\
2399.064\end{array}$ & $\begin{array}{r}85 \\
952 \\
476 \\
2022 \\
9071 \\
470 \\
281\end{array}$ & $\begin{array}{r}390 \\
4111 \\
2106 \\
10343 \\
68597 \\
5992 \\
4020\end{array}$ & $\begin{array}{l}2 \\
2 \\
1 \\
1 \\
1 \\
1 \\
1\end{array}$ & $\begin{array}{r}-0.33 \\
0.00 \\
-1.05 \\
4.31 \\
5.68 \\
4.35\end{array}$ \\
\hline Totals & 62046.646 & 13358 & 95560 & & \\
\hline
\end{tabular}

\section{File: 97012211.D03 Sample: S97T000024}

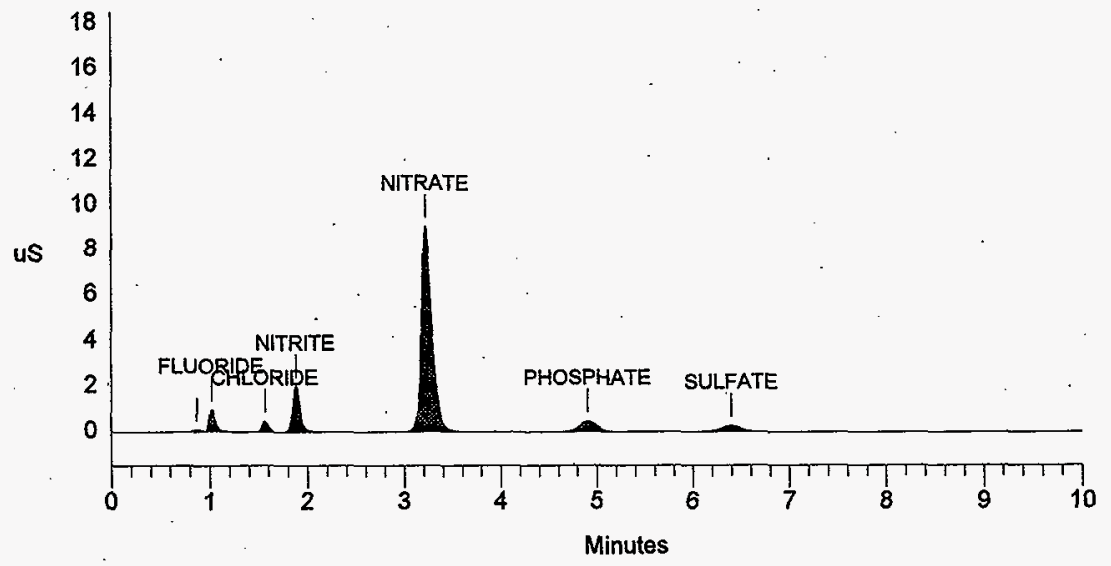




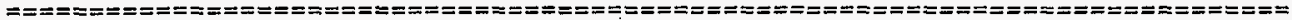

Calibration Volume Dilution Points Rate start stop Area Reject

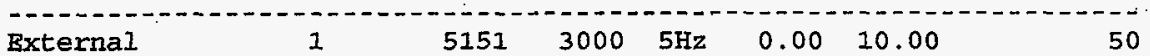

$\star * * * * * * * * * * * * * * * * * * * * * * * * * *$ Peak Report: All Peaks $* * * * * * * * * * * * * * * * * * * * * * * * * * * *$

Pk. Ret Component Concentration Height Area Bl. ㅁDelta Num Time Name $\mathrm{ug} / \mathrm{ml}$ Code

$\begin{array}{lll}1 & 0.86 \\ 2 & 1.02 & \text { FLUORIDE } \\ 3 & 1.56 & \text { CHLORIDE } \\ 4 & 1.88 & \text { NITRITE } \\ 5 & 3.22 & \text { NITRATE } \\ 6 & 4.91 & \text { PHOSPHATE } \\ 7 & 6: 40 & \text { SULFATE }\end{array}$

$\begin{array}{rr}0.000 & 83 \\ 1073.237 & 956 \\ 866.048 & 500 \\ 6482.721 & 2028 \\ 42914.226 & 9168 \\ 9017.254 & 477 \\ 2510.839 & 292\end{array}$

$-$

Totals

62864.326

13504

$\begin{array}{rrr}374 & 2 & \\ 4020 & .2 & 0.33 \\ 2261 & 1 & 0.00 \\ 10476 & 1 & -1.05 \\ 69228 & 1 & 4.10 \\ 6151 & 1 & 5.82 \\ 4246 & 1 & 4.35\end{array}$

\section{File: 97012211.D04 Sample: S97T000024 DUP}

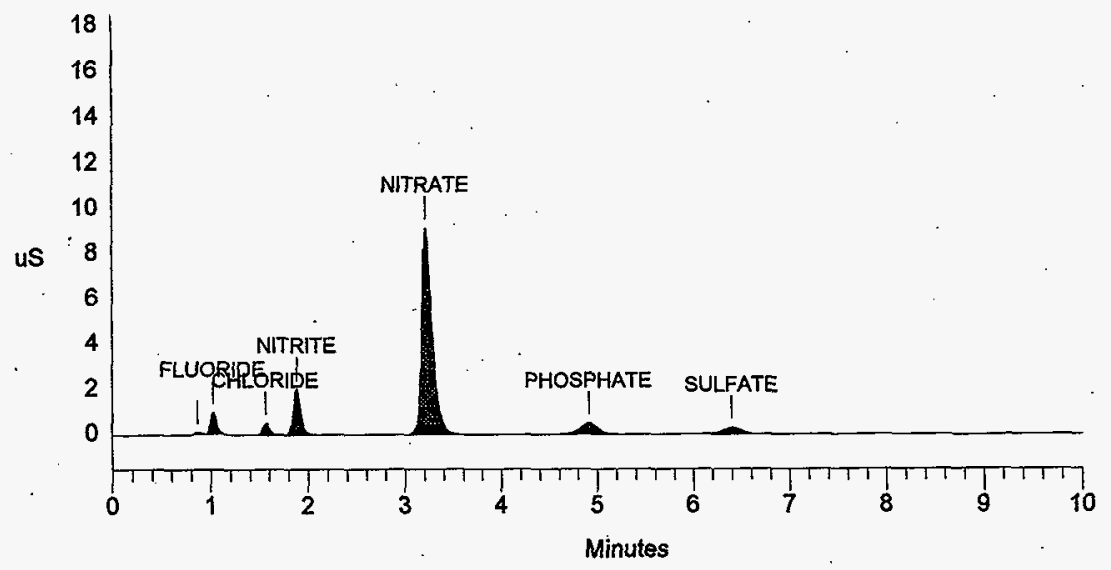


HNF-SD-WM-DP-227, REV. 0

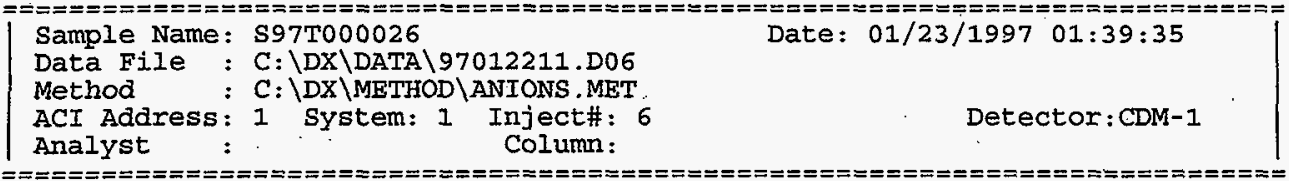

Calibration Volume Dilution Points Rate start stop Area Reject

External

External

$\begin{array}{lllll}2.121 & 3000 & 5 \mathrm{~Hz} & 0.00 & 10.00\end{array}$

50

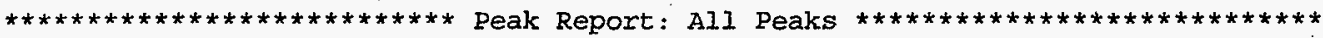

Pk. Ret Component Concentration Height Area Bl: $\%$ Delta

Num Time Name ug/ml

$\begin{array}{lll}1 & 0.87 \\ 2 & 1.02 & \text { FLUORIDE } \\ 3 & 1.56 & \text { CHLORIDE } \\ 4 & 1.89 & \text { NITRITE } \\ 5 & 3.17 & \text { NITRATE } \\ 6 & 4.91 & \text { PHOSPHATE } \\ 7 & 6.40 & \text { SULFATE }\end{array}$

$\begin{array}{rrrrr}0.000 & 230 & 1103 & 2 & \\ 647.056 & 1462 & 5938 & 2 & 0.33 \\ 1404.946 & 1881 & 8748 & 2 & 0.00 \\ 14587.311 & 11714 & 60414 & 2 & -0.70 \\ 48689.117 & 24257 & 201186 & 1 & 2.59 \\ 4017.957 & 515 & 6699 & 1 & 5.82 \\ 1787.926 & 537 & 7939 & 1 & 4.35\end{array}$

Totals

71134.314

40595

292027

File: 97012211.D06 Sample: S97T000026

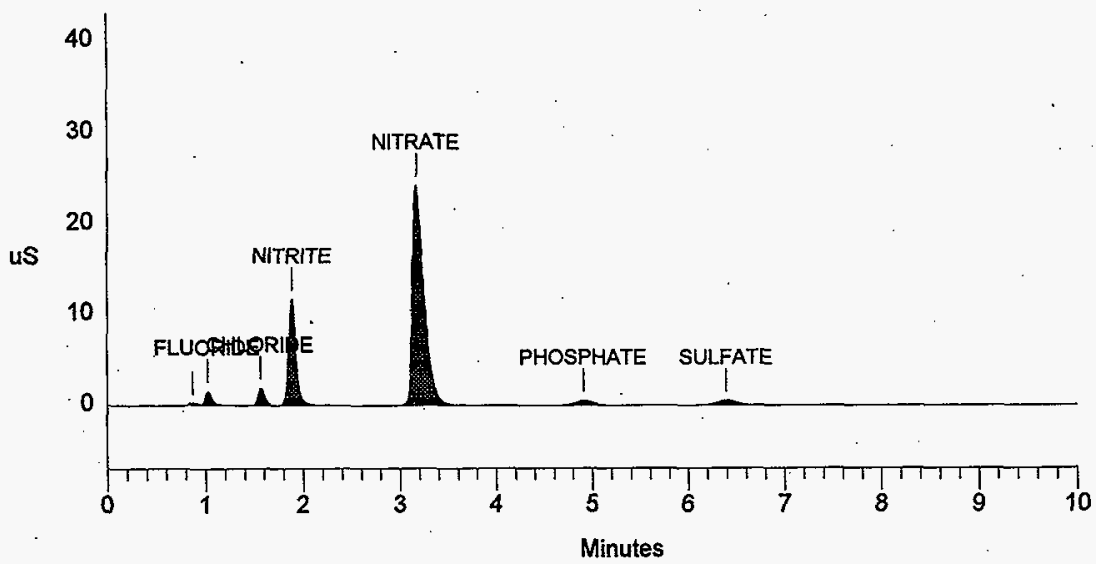




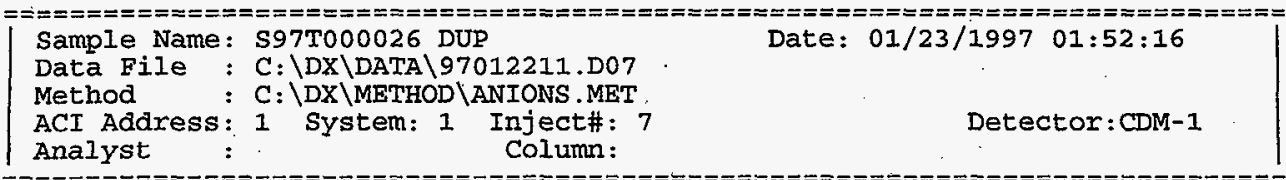

Calibration Volume Dilution Points Rate Start Stop Area Reject

External $\quad 1 \quad 2121 \quad 3000 \quad 5 \mathrm{~Hz} 0.00 \quad 10.00 \quad 50$

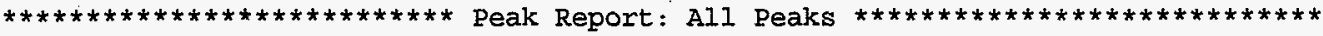

Pk. Ret Component $\begin{gathered}\text { Concentration } \\ \text { ug/ml Height Time Name }\end{gathered}$
Num Area BI.
Code

$\begin{array}{lll}1 & 0.87 & \\ 2 & 1.02 & \text { FLUORIDE } \\ 3 & 1.56 & \text { CHLORIDE } \\ 4 & 1.89 & \text { NITRITE } \\ 5 & 3.16 & \text { NITRATE } \\ 6 & 4.91 & \text { PHOSPHATE } \\ 7 & 6.38 & \text { SULFATE }\end{array}$

\begin{tabular}{|c|c|c|c|}
\hline & $\begin{array}{r}0.000 \\
545.572 \\
1456.309 \\
14534.866 \\
47835.104 \\
3959.326 \\
2283.849\end{array}$ & $\begin{array}{r}208 \\
1303 \\
1909 \\
11704 \\
24291 \\
515 \\
580\end{array}$ & $\begin{array}{r}949 \\
4989 \\
9069 \\
60193 \\
197400 \\
6593 \\
10372\end{array}$ \\
\hline 20 & 70615.025 & 40510 & 289566 \\
\hline
\end{tabular}

\section{File: 97012211.D07 Sample: S97T000026 DUP}

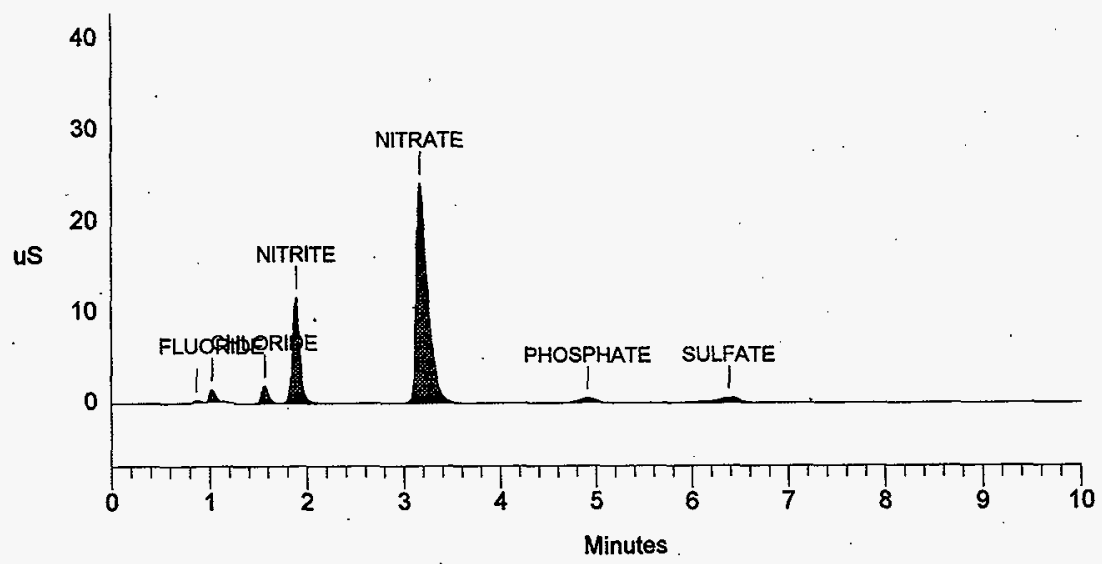




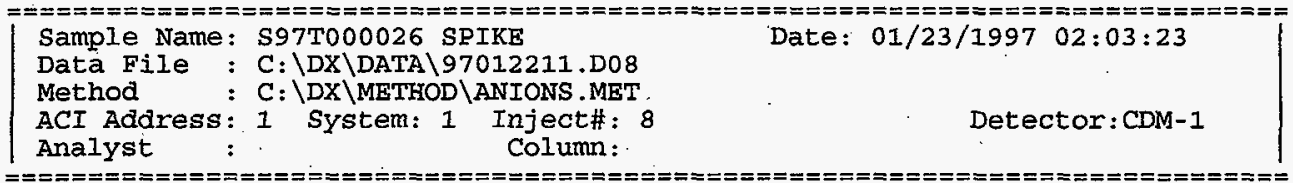

Calibration Volume Dilution Points Rate start Stop Area Reject

External $\quad 1 \quad 21.21 \quad 3000 \quad 5 \mathrm{~Hz} 0.0010 .00 \quad 50$

$\star * * * * * * * * * * * * * * * * * * * * * * * * * *$ Peak Report: All Peaks $* * * * * * * * * * * * * * * * * * * * * * * * * * * *$

Pk. Ret Component Concentration Height Area Bl. $\%$ Delta Num Time Name ug/mI Code

$\begin{array}{lll}1 & 0.87 & \\ 2 & 1.02 & \text { FLUORIDE } \\ 3 & 1.56 & \text { CHLORIDE } \\ 4 & 1.89 & \text { NITRITE } \\ 5 & 2.83 & \text { BROMIDE } \\ 6 & 3.15 & \text { NITRATE } \\ 7 & 4.89 & \text { PHOSPHATE } \\ 8 & 6.37 & \text { SULFATE } \\ 9 & 8.37 & \text { OXALATE }\end{array}$

$\begin{array}{rrrr} & 0.000 & 253 & 1142 \\ 1782.217 & 3780 & 16636 \\ 2802.394 & 3862 & 17574 \\ 25102.953 & 20326 & 104910 \\ 11395.591 & 5625 & 33875 \\ & 54970.628 & 27408 & 229330 \\ & 14386.086 & 1962 & 25644 \\ & 13918.586 & 4754 & 68415 \\ & 10158.439 & 1987 & 37872 \\ \text { Totals } & 134516.895 & 69959 & 535397\end{array}$

File: 97012211.D08 Sample: S97T000026 SPIKE

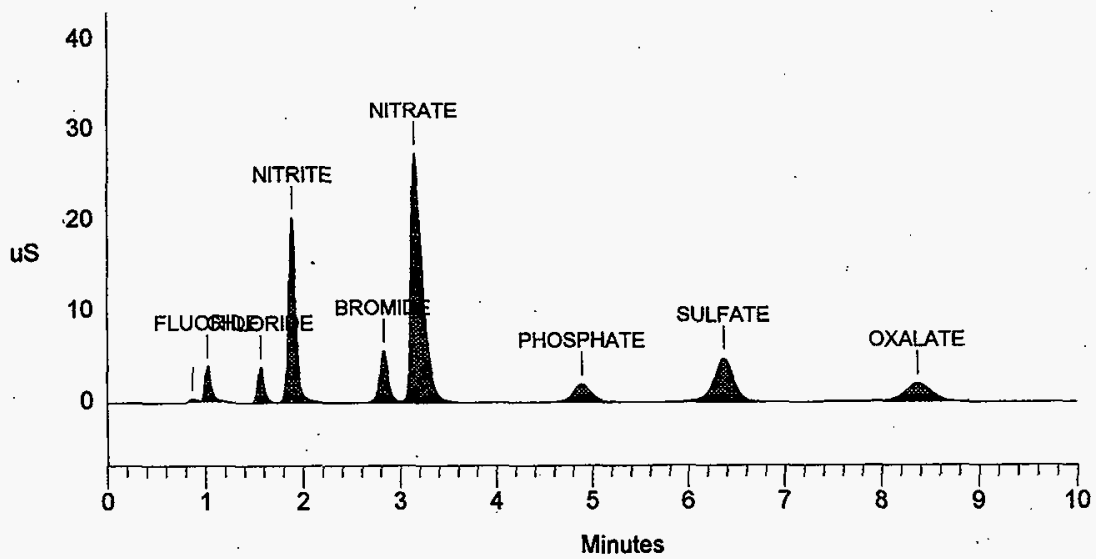


HNF-SD-WM-DP-227, REV. 0

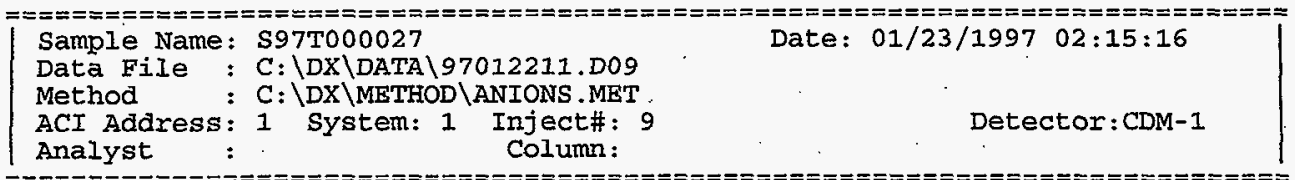

Calibration Volume Dilution Points Rate start stop Area Reject

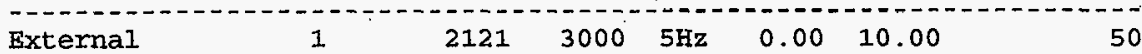

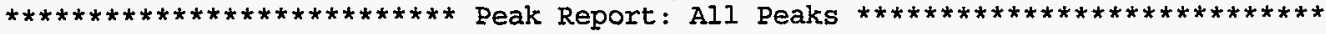

$\begin{array}{lrrr}\text { Pk. Ret Component } & \text { Concentration } & \text { Height } & \text { Area Bl. } \\ \text { Num } / \mathrm{ml} & \text { Time Name } & \text { Code }\end{array}$

$\begin{array}{rrlrrrr}1 & 0.87 & 0.000 & 175 & 896 & 2 \\ 2 & 1.02 \text { FLUORIDE } & 451.643 & 944 & 4111 & 2 & -0.33 \\ 3 & 1.14 & 0.000 & 440 & 2621 & 2 & \\ 4 & 1.56 \text { CHLORIDE } & 1104.610 & 1502 & 6879 & 2 & 0.00 \\ 5 & 1.88 \text { NITRITE } & 15370.304 & 12409 & 63713 & 2 & -1.05 \\ 6 & 3.18 \text { NITRATE } & 37834.767 & 19195 & 153745 & 1 & 2.80 \\ 7 & 4.90 \text { PHOSPHATE } & 4396.259 & 571 & 7379 & 1 & 5.68 \\ 8 & 6.38 \text { SULFATE } & 2188.107 & 696 & 9902 & 1 & 4.13 \\ 9 & 8.42 \text { OXALATE } & 578.994 & 96 & 1674 & 1 & 5.16\end{array}$

Totals $\quad 61924.684 \quad 36028 \quad 250919$

\section{File: 97012211.D09 Sample: S97T000027}

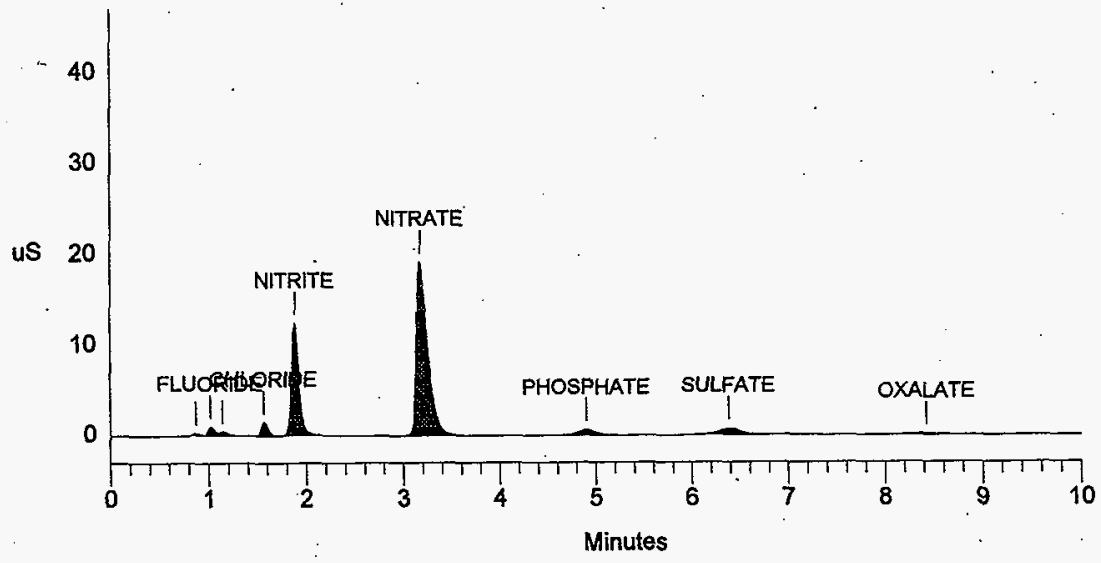


Sample Name: S97T000027 DUP

Data File: C: \DX\DATA 97012211. D10

Method : C: \DX\METHOD \ANIONS.MET .

ACI Address: 1 System: 1 Inject\#: 10

Detector: CDM-1

Analyst Column:

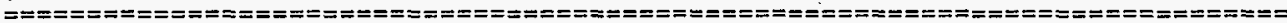

Calibration Volume Dilution Points Rate start stop Area Reject

External $11213000.5 \mathrm{~Hz} 0.0010 .00 \quad 50$

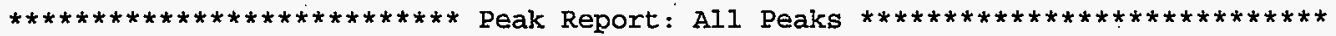

Pk. Ret Component . Concentration Height Area Bl. \&Delta

Num Time Name $\mathrm{ug} / \mathrm{ml}$

Code

$\begin{array}{lrlrrrr}1 & 0.87 & & 0.000 & 171 & 836 & 2 \\ 2 & 1.02 \text { FLUORIDE } & 452.663 & 946 & 4121 & 2 & -0.33 \\ 3 & 1.14 & 0.000 & 452 & 2523 & 2 & \\ 4 & 1.56 \text { CHLORIDE } & 1125.444 & 1534 & 7008 & 2 & 0.00 \\ 5 & 1.88 \text { NITRITE } & 15522.483 & 12468 & 64354 & 2 & -1.05 \\ 6 & 3.18 \text { NITRATE } & 37912.212 & 19219 & 154078 & 1 & 2.80 \\ 7 & 4.90 \text { PHOSPHATE } & 4469.596 & 573 & 7511 & 1 & 5.68 \\ 8 & 6.38 \text { SULFATE } & 2339.205 & 719 & 10644 & 1 & 4.13 \\ 9 & 8.42 \text { OXALATE } & 553.302 & 93 & 1578 & 1 & 5.16 \\ & & \text { Totals } & 62374.904 & 36177 & 252653 & \end{array}$

\section{File: 97012211.D10 Sample: S97T000027 DUP}

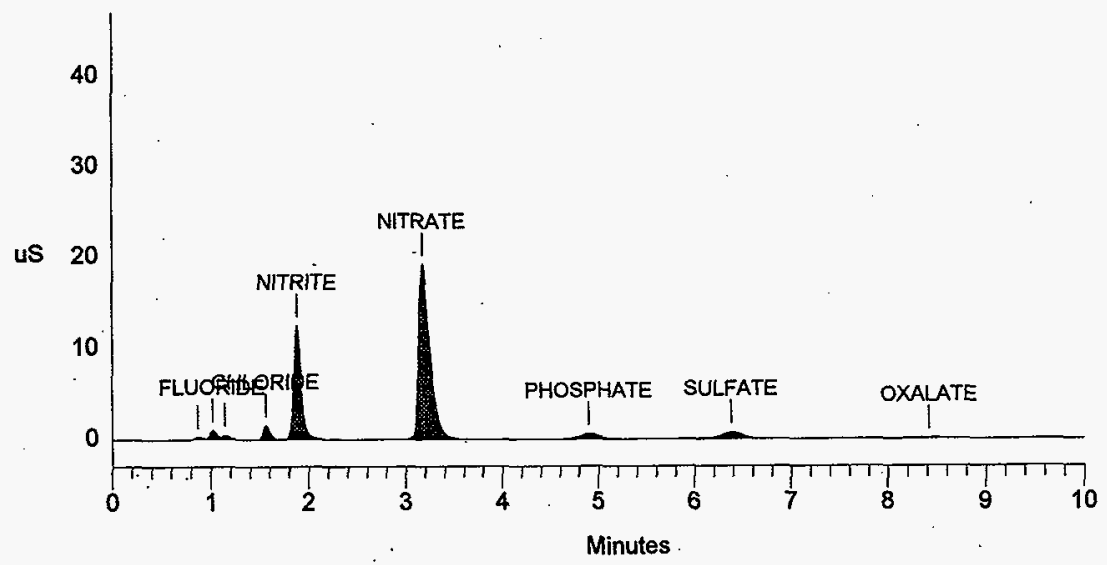




\section{LABCORE Data Entry Template for Worklist\# 16306}

Analyst: $\quad \not K$ S \& I

Method: LA-505-151/161 Rev/Mod $B-1$ 1.27-97

Worklist Comment: SY-102 DIRECT

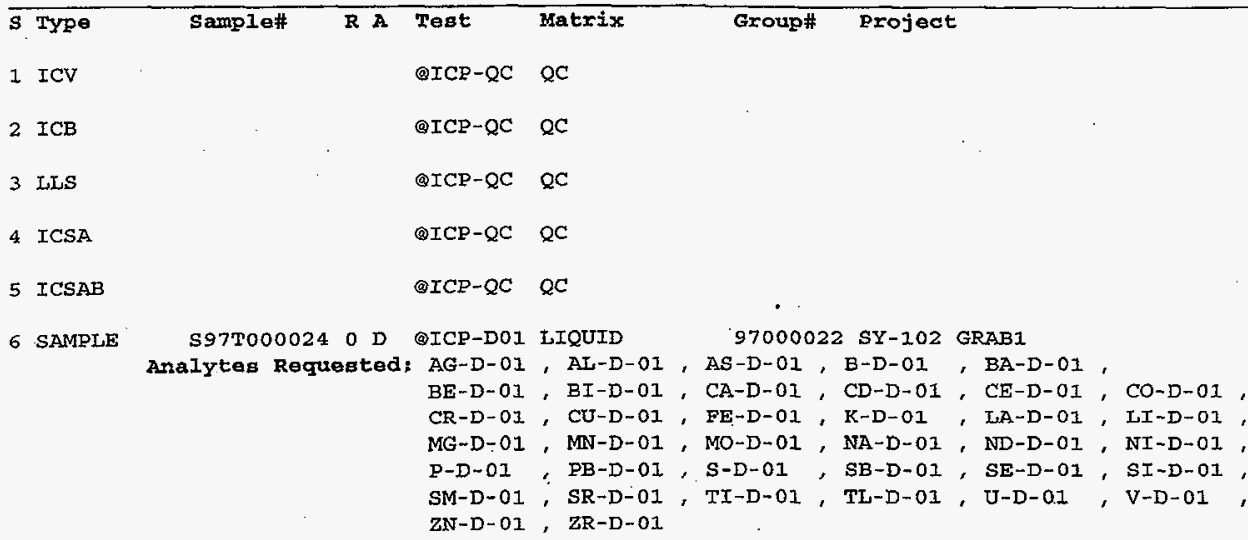

7 DUP S97T000024 O D OICP-D0I LIQUID

\&ed $1.27-47$

8 SERDIL S97T000026 O D QICP-D01 LIQUID

9 SAMPLE S97T000026 O D OICP-D01 LIQUID Analytes Requested: $A G-D-01, A L-D-01, A S-D-01, B-D-01, B A-D-01$, BE-D-01， BI-D-01，CA-D-01，CD-D-01，CE-D-01，CO-D-01 , CR-D-0I , CU-D $-01, F E-D-01, K-D-01$, IAA-D-01， LIT-D-01 , MG-D-01， MN-D-01， MO-D-01， NA-D-01 , ND-D-01， NI-D-01 , P-D-01 , PB-D-01，S-D-01，SB-D-0I，SE-D-01，SI-D-01 , SM-D-01，SR-D-01，TI-D-0I，TL-D-01，U-D-01，V-D-01， ZN-D-01, ZR-D-01

10 DUP S97T000026 O D BICP-D01 LIQUID

11 SPK $/ \mathrm{PPM}^{-}$S97T000026 O D @ICP-D01 LIOUID $\operatorname{spk} 10 \mathrm{ppm}$, 遮 12.7 .47 -12 eer

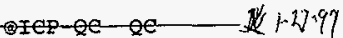

Data Entry Comments:

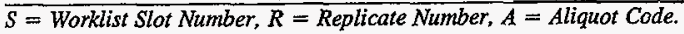




\section{HNF-SD-WM-DP-227, REV. 0}

\begin{tabular}{|c|c|c|c|c|c|c|c|c|c|}
\hline Ana & lysis Report & Sum & mary & & Mon 01-27- & $9702: 2$ & $9: 39$ & $P M$ & pag \\
\hline$\#$ & Sample Name & & $\begin{array}{l}\text { File } \\
\end{array}$ & Method & $\begin{array}{l}\text { Date } \\
----\end{array}$ & Time & $O P I D$ & & Mode \\
\hline $\begin{array}{l}1 \\
2 \\
3 \\
4 \\
5 \\
6 \\
7 \\
8 \\
9 \\
10 \\
11 \\
12 \\
13 \\
14 \\
15 \\
16 \\
17 \\
18\end{array}$ & $\begin{array}{l}I C V \\
I C B \\
I L S \\
I C S A \\
I C S A B \\
S 97 T 000024 \\
S 97 T 000024 D D \\
C C V \\
C C B \\
S 97 T 000026-L \\
S 97 T 000026- \\
S 97 T 000026-D \\
S 97 T 000026-A \\
S 97 T 000026-A X \\
I C S A \\
I C S A B \\
C C V I \\
C C B-I\end{array}$ & & $\begin{array}{l}970127 D \\
970127 D \\
970127 D \\
970127 D \\
970127 D \\
970127 D \\
970127 D \\
970127 D \\
970127 D \\
970127 D \\
970127 D \\
970127 D \\
9701270 \\
970127 D \\
970127 D \\
970127 D \\
970127 D \\
9701270\end{array}$ & $\begin{array}{l}I C P 2 \\
I C P 2 \\
I C P 2 \\
I C P 2 \\
I C P 2 \\
I C P 2 \\
I C P 2 \\
I C P 2 \\
I C P 2 \\
I C P 2 \\
I C P 2 \\
I C P 2 \\
I C P 2 \\
I C P 2 \\
I C P 2 \\
I C P 2 \\
I C P 2 \\
I C P 2\end{array}$ & $\begin{array}{l}01 / 27 / 97 \\
01 / 27 / 97 \\
01 / 27 / 97 \\
01 / 27 / 97 \\
01 / 27 / 97 \\
01 / 27 / 97 \\
01 / 27 / 97 \\
01 / 27 / 97 \\
01 / 27 / 97 \\
01 / 27 / 97 \\
01 / 27 / 97 \\
01 / 27 / 97 \\
01 / 27 / 97 \\
01 / 27 / 97 \\
01 / 27 / 97 \\
01 / 27 / 97 \\
01 / 27 / 97 \\
01 / 27 / 97\end{array}$ & $\begin{array}{l}13: 22 \\
13: 25 \\
13: 28 \\
13: 31 \\
13: 34 \\
13: 40 \\
13: 44 \\
13: 49 \\
13: 53 \\
13: 57 \\
14: 80 \\
14: 93 \\
14: 86 \\
14: 11 \\
14: 16 \\
14: 19 \\
14: 23 \\
14: 26\end{array}$ & $\begin{array}{l}D K S \\
D K S \\
D K S \\
D K S \\
D K S \\
D K S \\
D K S \\
D K S \\
D K S \\
D K S \\
D K S \\
D K S \\
D K S \\
D K S \\
D K S \\
D K S \\
D K S \\
D K S\end{array}$ & $\begin{array}{l}Q \\
Q \\
Q \\
Q \\
Q \\
S \\
S \\
Q \\
Q \\
S \\
S \\
S \\
S \\
S \\
Q \\
Q \\
Q\end{array}$ & $\begin{array}{l}\text { CONC } \\
\text { CONC } \\
\text { CONC } \\
\text { CONC } \\
\text { CONC } \\
\text { CONC } \\
\text { CONC } \\
\text { CONC } \\
\text { CONC } \\
\text { CONC } \\
\text { CONC } \\
\text { CONC } \\
\text { CONC } \\
\text { CONC } \\
\text { CONC } \\
\text { CONC } \\
\text { CONC } \\
\text { CONC }\end{array}$ \\
\hline
\end{tabular}

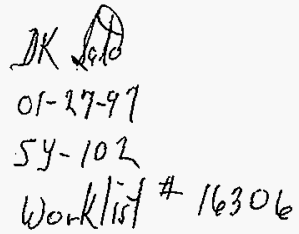

5977000024

5971000026

SIGNATURE ABOVE REPRESENTS CHEMICAL TECHNOLOGIST/CHEMIST THAT COMPLETEDNERIFIED THE CALIBRATIONANALYSIS ON PAGES 101 TO $10 \%$. 
HNF-SD-WM-DP-227, REV. 0 Analysis Report

Averages

Mon 01-27-97 02:29:39 PM page 2

\begin{tabular}{|c|c|c|c|c|c|c|c|}
\hline$\#$ & Sample Name & & $A 1$ & As & $B$ & $B a$ & Be \\
\hline $\begin{array}{l}1 \\
2 \\
3 \\
4 \\
5 \\
6 \\
7 \\
8 \\
9 \\
10 \\
111 \\
12 \\
13 \\
144 \\
15 \\
16 \\
17 \\
18\end{array}$ & $\begin{array}{l}I C V \\
I C B \\
L L S \\
I C S A \\
I C S A B \\
S 97 T 000024 \\
S 97 T 000024 D D \\
C C V \\
C C B \\
S 97 T 000026-L \\
S 97 T 000026- \\
S 97 T 000026-D \\
S 97 T 000026-A \\
S 97 T 000026-A X \\
I C S A \\
I C S A B \\
C C V I 1 \\
C C B-I\end{array}$ & $\begin{array}{l}4.902 \\
.0003 \\
.0220 \\
.0016 \\
.9627 \\
2.386 \\
2.509 \\
4.903 \\
.0006 \\
3.068 \\
3.433 \\
3.303 \\
191.8 \\
19870 \\
.0119 \\
.9626 \\
4.914 \\
.0003\end{array}$ & $\begin{array}{l}4.908 \\
.0088 \\
.1112 \\
242.8 \\
242.6 \\
1966 . \\
1962 . \\
4.894 \\
5066 \\
5192 . \\
5124 . \\
5094 . \\
5315 \\
24710 \\
242.8 \\
242.2 \\
4.901 \\
.0091\end{array}$ & $\begin{array}{l}5.190 \\
.0123 \\
.2094 \\
-.0281 \\
-.0513 \\
-1.832 \\
-1.221 \\
5.196 \\
.0120 \\
-6.637 \\
-7.220 \\
-5.680 \\
203.7 \\
20840 \\
-.0270 \\
-.0442 \\
5.174 \\
.0131\end{array}$ & $\begin{array}{l}5.062 \\
.0014 \\
.1034 \\
.0031 \\
=.0007 \\
4.090 \\
4.187 \\
5.051 \\
=.0033 \\
12.91 \\
9.959 \\
10.05 \\
209.6 \\
19940 \\
.0002 \\
=.0031 \\
5.050 \\
.0033\end{array}$ & $\begin{array}{l}4.993 \\
-.0001 \\
.1010 \\
.0003 \\
.4798 \\
-.0148 \\
.0076 \\
4.984 \\
-.0001 \\
-.0668 \\
.0068 \\
.0134 \\
198.0 \\
20610 \\
.0004 \\
.4810 \\
5.018 \\
. .0000\end{array}$ & $\begin{array}{l}5.104 \\
.0003 \\
.0104 \\
.0002 \\
.4798 \\
.0165 \\
.0035 \\
5.085 \\
.0007 \\
.2565 \\
.0846 \\
.0674 \\
202.7 \\
20780 \\
.0004 \\
.4770 \\
5.059 \\
.0003\end{array}$ \\
\hline 非 & Sample Name & $B i$ & $\mathrm{Ca}$ & & $\mathrm{Ce}$ & & $C r$ \\
\hline $\begin{array}{l}1 \\
2 \\
3 \\
4 \\
5 \\
6 \\
7 \\
8 \\
9 \\
10 \\
11 \\
12 \\
13 \\
14 \\
15 \\
16 \\
17 \\
18\end{array}$ & $\begin{array}{l}\text { ICV } \\
\text { ICB } \\
\text { LLS } \\
\text { ICSA } \\
\text { ICSAB } \\
\text { S97T000024 } \\
\text { S97T000024_D } \\
C C V \\
C C B \\
S 97 T 000026 \quad L \\
S 97 T 000026 \\
S 97 T 000026 \quad D \\
S 97 T 000026-A \\
S 97 T 000026-A X \\
I C S A \\
I C S A B \\
C C V \quad 1 \\
C C B-1\end{array}$ & $\begin{array}{l}5.062 \\
.0048 \\
.2390 \\
.0026 \\
.0161 \\
5.405 \\
3.546 \\
5.088 \\
.0138 \\
33.95 \\
10.23 \\
12.06 \\
211.1 \\
20420 \\
.0402 \\
=.0009 \\
5.048 \\
.0235\end{array}$ & $\begin{array}{l}4.917 \\
-.0001 \\
2573 \\
247.9 \\
248.4 \\
2.182 \\
2.500 \\
4.922 \\
-.0008 \\
3.217 \\
6.056 \\
4.263 \\
203.6 \\
20090 \\
249.2 \\
248.8 \\
4.950 \\
.0018\end{array}$ & $\begin{array}{l}4.967 \\
.0014 \\
.0116 \\
.0003 \\
.9343 \\
.3044 \\
.2993 \\
4.960 \\
.0001 \\
1.751 \\
1.342 \\
1.387 \\
20013 \\
19910 \\
.0000 \\
.9342 \\
4.971 \\
.0004\end{array}$ & $\begin{array}{l}4.951 \\
-.0060 \\
.2022 \\
.0139 \\
.0087 \\
-1.271 \\
.0830 \\
4.936 \\
-.0086 \\
-5.308 \\
-.3657 \\
. .8136 \\
199.5 \\
20280 \\
.0121 \\
.0087 \\
4.940 \\
-.0004\end{array}$ & $\begin{array}{l}5.027 \\
-.0001 \\
.0420 \\
.0001 \\
.4650 \\
.3212 \\
.2232 \\
5.016 \\
-.0011 \\
.1008 \\
.1745 \\
.2950 \\
201.1 \\
31.13 \\
-.0005 \\
.4660 \\
5.034 \\
-.0007\end{array}$ & $\begin{array}{l}4.951 \\
.0005 \\
.0212 \\
.0085 \\
.4809 \\
368.7 \\
368.0 \\
4.949 \\
9.0003 \\
960.6 \\
948.2 \\
936.9 \\
1138 \\
21030 \\
.0084 \\
4813 \\
4.961 \\
.0005\end{array}$ \\
\hline \# & Sample Name & $\mathrm{Cu}$ & $E$ u & & $K$ & $\begin{array}{l}L a \\
-\ldots .\end{array}$ & $\begin{array}{l}L \dot{I} \\
-\ldots . . .\end{array}$ \\
\hline $\begin{array}{l}1 \\
2 \\
3 \\
4 \\
5 \\
6 \\
7 \\
8 \\
9\end{array}$ & $\begin{array}{l}I C V \\
I C B \\
L L S \\
I C S A \\
I C S A B \\
S 97 T 000024 \quad \\
S 97 T 000024 \quad D \\
C C V \\
C C B\end{array}$ & $\begin{array}{l}5.320 \\
.0007 \\
.0218 \\
.0096 \\
.4838 \\
.2130 \\
.2571 \\
5.306 \\
-.0002\end{array}$ & $\begin{array}{l}=.0021 \\
-.0008 \\
=.0002 \\
-.0212 \\
-.0191 \\
=.0980 \\
-.0799 \\
-.0016 \\
-.0008\end{array}$ & $\begin{array}{l}4.979 \\
-.0023 \\
.1030 \\
93.04 \\
92.92 \\
.3139 \\
.4398 \\
4.954 \\
-.0031\end{array}$ & $\begin{array}{r}4.793 \\
-.0966 \\
Q .6518 \\
.4494 \\
1665 \\
807.2 \\
792.1 \\
4.829 \\
. .0404\end{array}$ & $\begin{array}{l}5.064 \\
-.0009 \\
.1039 \\
=.0047 \\
=.0044 \\
-.1065 \\
.0871 \\
5.051 \\
-.0003\end{array}$ & $\begin{array}{l}4.965 \\
-.0010 \\
.0205 \\
.0019 \\
1.029 \\
-.0715 \\
.0244 \\
4.952 \\
-.0008\end{array}$ \\
\hline
\end{tabular}


HNF-SD-WM-DP-227, REV. 0

Analysis Report

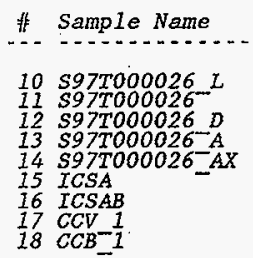

\# Sample Name

1 ICV

2 I $C B$

3 $2 L S$

5 ICSAB

6 S97T000024

7 S97T000024_D

$8 \mathrm{CCV}$

$9 \mathrm{CCB}$

10 S97T000026 L

11 S97T000026

12
13 S97T000026 D

14 S97T000026-AX

15 ICSA

16 ICSAB

$\begin{array}{ll}17 & C C V \\ 18 & C C B-1\end{array}$

非 Sample Name

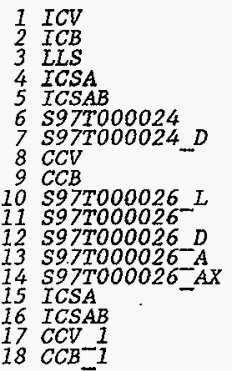

Averages

Cu

Eu

1.055
.3197
2338
211.8
-8.489
-.0100
.4818
5.292
.0000

$\mathrm{Mg}$

4.994
.0039
2122
252.4
251.7
-.2891
.3841

4.976

.0049

.8944

0505

197.4

20320 .

252.4

251.29

.0043

$P$

5.063

$-.0081$

.0115

.0029

3186 .

3186 :

5.088

$-.0207$

1348 .

1358 .

1341 .

1539.

$22080^{\circ}$

$-.0020$

$-.0024$

.0042

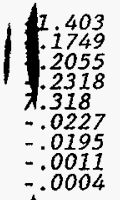

Mr

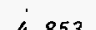

4.853

$-.0002$

.0194

.4419

$-.0438$

$-.0410$

4.854

$-.0002$

$-.3286$

$-.0447$

$-.0875$

190.3

19870.

$-.0065$

4.864

$-.0000$

$P b$

4.843

$-.0139$

.1886

.0044

.9663

$-2.669$

4.849

- 0190

$-10.20$

$-1.103$

$-5.996$

191.8

20450 .

.0276

49781

$-.0056$
Mon 01-27-97 02:29:39 PM

page 3
La

$\begin{array}{ll}-.1753 & 2154 . \\ 1.704 & 2127 . \\ 1.255 & 2071 . \\ 199.1 & 2318 \\ 19930 . & 21510 \\ 92.95 & .3842 \\ 92.69 & .2248 \\ 4.947 & 4.882 \\ -.0018 & .0660\end{array}$

Mo

$\begin{array}{ll}5.071 & 5.104 \\ -.0023 & -.0066 \\ .0987 & .2023 \\ -.0077 & 194.9 \\ -.0105 & 195.2 \\ 3.798 & 34920 \\ 3.815 & 34700 . \\ 5.064 & 5.070 \\ 1.0035 & -.0062 \\ 12.26 & 47620 . \\ 11.46 & 47010 \\ 10.94 & 46770 \\ 215.3 & 47210 . \\ 19740 & 67250 \\ -.0112 & 194.1 \\ 5.0081 & 1943 \\ 5.090 & 5.059 \\ -.0004 & -.0029\end{array}$

$S b$

4.977

$-.0070$

.2041

$-.0367$

$-.0399$

747.2

4.964

$-.0104$

573.2

561.7

761.1

20710.

$-.0443$

$-.0330$

4.967

.0074

$$
\begin{aligned}
& 4.831 \\
& .0003 \\
& .1060 \\
& =.0095 \\
& =.0061 \\
& . .7030 \\
& =.6933 \\
& 4.793 \\
& i 0032 \\
& 1.295 \\
& .1 .117 \\
& .6508 \\
& 194.1 \\
& 20020 \\
& .0202 \\
& . .0010 \\
& 4.844 \\
& .0017
\end{aligned}
$$

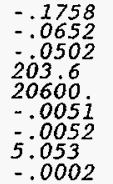

$-1.639$

$-1653$

- 3060

198.8

20600 .

.0021

i. 027

4.931

$-.0002$

$N d$

$\mathrm{Ni}$

$\begin{array}{ll}5.015 & 4.947 \\ .0007 & .0066 \\ .2011 & .0455 \\ .0019 & -.0066 \\ .0035 & .9132 \\ -.2129 & .2418 \\ .0142 & .4628 \\ 5.017 & 4.955 \\ .0012 & -.0072 \\ -.3364 & -3.208 \\ -.4018 & -1.692 \\ .3570 & -.3265 \\ 201.9 & 195.9 \\ 20490 . & 19830 \\ .0025 & -.0008 \\ .0046 & .9157 \\ 5.003 & 4.937 \\ -.0000 & -.0037\end{array}$

Se

Si

$\begin{array}{ll}4.750 & 4.830 \\ .0196 & -.0059 \\ .2338 & .1282 \\ -.0158 & -.0130 \\ 5.0734 & -.0114 \\ 5.049 & 8.172 \\ 5.890 & 8.307 \\ 4.753 & 4.831 \\ .0301 & -.0100 \\ 31.82 & 9.213 \\ 9.864 & 17.72 \\ 7.349 & 16.73 \\ 204.2 & 213.0 \\ 19530 & 20070 \\ -.0083 & -.0105 \\ -.0188 & -.0123 \\ 4.729 & 4.832 \\ .0293 & -.0064\end{array}$




\begin{tabular}{|c|c|c|c|c|c|c|c|}
\hline Ana & Iysis Report & rages & & Mon $01-27$ & $-9702: 29$ & $: 39$ PM & page 4 \\
\hline 非 & Sample Name & $S m$ & $S r$ & Th & $r_{i}$ & $T 1$ & $U$ \\
\hline $\begin{array}{l}1 \\
2 \\
3 \\
4 \\
5 \\
6 \\
7 \\
8 \\
9 \\
10 \\
11 \\
12 \\
13 \\
13 \\
15 \\
16 \\
17 \\
18\end{array}$ & $\begin{array}{l}I C V \\
I C B \\
L L S \\
I C S A \\
I C S A B \\
S 97 T 000024 \\
S 97 T 000024 D D \\
C C V \\
C C B \\
S 97 T 000026 L L \\
S 97 T 000026-D \\
S 97 T 000026 D D \\
S 97 T 000026-A \\
S 97 T 000026-A X \\
I C S A \\
I C S A B \\
C C V I I \\
C C B-1\end{array}$ & $\begin{array}{l}4.979 \\
-.0090 \\
.2035 \\
-.0066 \\
-.0072 \\
-1.150 \\
-.7689 \\
4.954 \\
-.0090 \\
-15.83 \\
-1.296 \\
-1.687 \\
197.7 \\
20040 \\
-.0026 \\
-.0073 \\
4.960 \\
-.0026\end{array}$ & $\begin{array}{l}4.976 \\
-.0002 \\
.0204 \\
.0018 \\
.0018 \\
.0916 \\
.0966 \\
4.961 \\
.0003 \\
.1572 \\
.4396 \\
4397 \\
19911 \\
20010 \\
.0018 \\
.0018 \\
4.954 \\
-.0001\end{array}$ & $\begin{array}{l}.0512 \\
.0032 \\
.0015 \\
.0117 \\
.0169 \\
.0904 \\
.3845 \\
.0536 \\
.0021 \\
4.205 \\
.6433 \\
.6010 \\
2.251 \\
96.36 \\
.0090 \\
.0107 \\
.0456 \\
.0011\end{array}$ & $\begin{array}{l}4.933 \\
-.0002 \\
.0200 \\
.0007 \\
.0008 \\
-.0328 \\
.0164 \\
4.915 \\
-.0005 \\
.7276 \\
.0850 \\
.0357 \\
197.9 \\
18290 \\
.0007 \\
.0007 \\
4.929 \\
. .0005\end{array}$ & $\begin{array}{l}4.769 \\
-.0771 \\
.3868 \\
-.0190 \\
-.0784 \\
-2.186 \\
-2.940 \\
4.799 \\
-.0288 \\
-26.29 \\
-1.681 \\
-3.484 \\
185.9 \\
19050 \\
-.0031 \\
.0210 \\
4.758 \\
-.0293\end{array}$ & $\begin{array}{l}9.761 \\
-.0454 \\
.4923 \\
.0158 \\
-.0044 \\
14.54 \\
75.53 \\
9.728 \\
-.0444 \\
.36 .38 \\
21.45 \\
21.42 \\
413.2 \\
1496 \\
.0206 \\
.0145 \\
9.721 \\
-.0200\end{array}$ \\
\hline 非 & Sample Name & $V$ & $\begin{array}{l}Y \\
-\ldots-\ldots\end{array}$ & $Z n \quad 1$ & $Z x$ & & \\
\hline $\begin{array}{r}1 \\
2 \\
3 \\
4 \\
5 \\
6 \\
7 \\
8 \\
9 \\
10 \\
11 \\
12 \\
13 \\
14 \\
15 \\
16 \\
17 \\
18\end{array}$ & $\begin{array}{l}\text { ICV } \\
\text { ICB } \\
\text { LLS } \\
\text { ICSA } \\
\text { ICSAB } \\
\text { S97T000024 } \\
\text { S97T000024_D } \\
C C V \\
\text { CCB } \\
\text { S97T000026_L } \\
\text { S97T000026 } \\
\text { S97T000026 D } \\
\text { S97TO00026-A } \\
\text { S97TO00026_AX } \\
\text { ICSA } \\
\text { ICSAB } \\
C C V 1 \\
C C B-I\end{array}$ & $\begin{array}{l}5.041 \\
-.0025 \\
.1019 \\
-.0020 \\
.4686 \\
=.2328 \\
=.1256 \\
5.036 \\
=.0025 \\
=3.607 \\
=.2014 \\
=.3462 \\
201.44 \\
12.16 \\
=.0009 \\
5681 \\
5.042 \\
=.0011\end{array}$ & $\begin{array}{l}.0054 \\
-.0007 \\
.0001 \\
.0064 \\
.0064 \\
=.0933 \\
-.0779 \\
.0057 \\
-.0007 \\
.1 .009 \\
-.1352 \\
-.2140 \\
.1080 \\
28.52 \\
.0068 \\
.0067 \\
.0056 \\
-.0004\end{array}$ & $\begin{array}{l}5.080 \\
.0007 \\
.0237 \\
.0046 \\
.9536 \\
.2140 \\
.1150 \\
5.083 \\
.0005 \\
1.182 \\
.9866 \\
.6604 \\
205.4 \\
.63 .25 \\
.0047 \\
.9541 \\
5.097 \\
.0005\end{array}$ & $\begin{array}{l}4.996 \\
-.0023 \\
.0206 \\
-.0053 \\
-.0054 \\
-.2308 \\
-.1839 \\
4.985 \\
-.0022 \\
-3.073 \\
-.2812 \\
-.4873 \\
200.00 \\
20390 \\
-.0044 \\
-.0053 \\
4.993 \\
-.0008\end{array}$ & & \\
\hline
\end{tabular}




\section{LABCORE Completed Worklist Report for Worklist\# 16287}

Analyst: knt

Instrument: TOC01

Book\# 24NizF

Method: 4 - $344-105$ Rev/Mod D-1

Worklist Comment: SY-102 Grab. Std: TOCSTD 0.200ml. REWORKED BY RTS

\begin{tabular}{llllll}
\hline Seq Type & Sample\# R A & Test & Matrix Actual Found DL or Yield Unit
\end{tabular}

\begin{tabular}{|c|c|c|c|c|c|c|c|c|}
\hline 1 Btok & & 8 & 10001 & trouto & 1 & 0 & $0 \% 000$ & ug/ \\
\hline $2 \operatorname{STD}$ & & 0 & TOC-01 & IIQUID & 3.00003 & $3.06 \mathrm{E3}$ & 102.000 & \& Recovery \\
\hline 3 SpHLL & 8972000024 & 0 & Toc 01 & $14 Q T X D$ & $N / 3$ & $2,90 \mathrm{E}_{2}$ & 7500 & ug/pht \\
\hline 4 DUP & S97T000024 & 0 & TOC-01 & IIQUID & 2.9082 & $4.72 \mathrm{E} 2$ & 47.769 & RPD \\
\hline $5+2+1$ & 8970000024 & 0 & $x \circ 01$ & Houro & 200082 & $3 \times 382$ & 13.505 & RPD \\
\hline 6 SAMPLB & S97T000026 & 0 & TOC-0I & LIQUID & $N / A$ & $1.03 \mathrm{B3}$ & 10.000 & $\mathrm{ug} / \mathrm{sir}$ \\
\hline $7 \mathrm{DOR}$ & $89 \% 2000026$ & 0 & $\mathrm{roc}-\mathrm{l}$ & LoUrp & 100383 & 10183 & 1961 & Ppo \\
\hline B SPR & \$97T000026 & 0 & roc- 01 & IIQUID & 100 & 378.0 & 378.000 & \& Recovery \\
\hline $9 \mathrm{SXOP}$ & 8971000027 & 0 & $100-1$ & $4+0+1$ & s/4 & 56513 & $\$ 5000$ & ug $/ \mathrm{mL}$ \\
\hline DUP & \$97T000027 & 0 & TOC-01 & IIQUID & $5.65 \mathrm{B3}$ & $4.64 \mathrm{B3}$ & 19.631 & RPD \\
\hline $\mathrm{RRTLL}$ & $\$ 97000027$ & 0 & $x 00-01$ & LIOUID & 5.6583 & 6.5983 & 15.359 & Ppo \\
\hline
\end{tabular}

\section{Final page for worklist\# 16287}

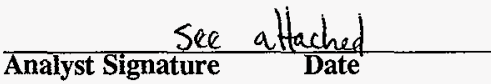

$\frac{\text { sec atfached }}{\text { Analyst Signature }}$ validated into Labcore by selm MCOluebey oz/05/97
Reviewer signatyre Date

note: Spike frilure accepted due to tharee reasons

1) liquid sample was muddy (therefore nonhomogenous) and I believic a verun would abs fail spoke recovory

2) Std, blank, and baseline were porfect

3) Sample and dup are near identical, therefore acceptable jmm $02 / 05 / 97$ 


\section{LABCORE Data Entry Template for Worklist\# 16287}

Analyst: KNT Instrument: TOC01_ Book\# PUNIZF

Method: LA-344-105 Rev/Mod D-I

Worklist Comment: SY-102 Grab. Std: TOCSTD 0.200ml. REWORKED BY RTS

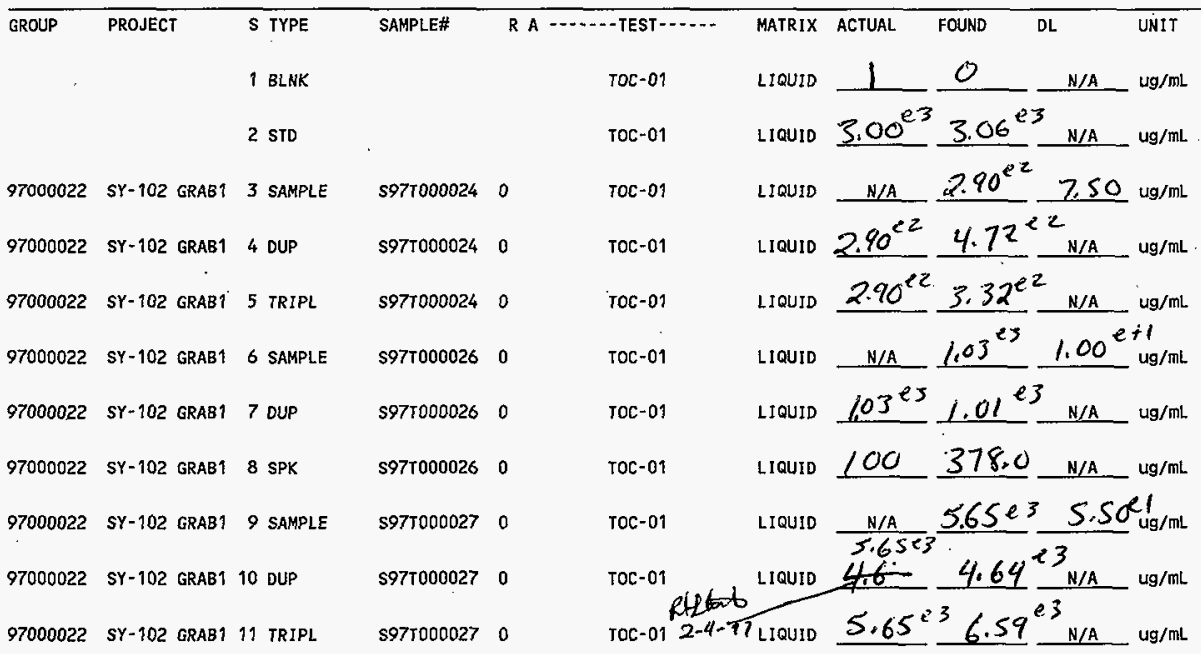

\section{Final page for worklist \# 16287}

Pflles for Kin themes 1-24-97

Analyst Signature Date

$$
2-4-97
$$

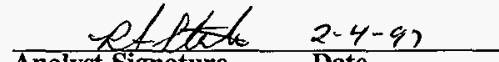

Analyst Signature
Date

\section{Data Entry Comments:}

Units shown for QC (SPK \& STD) may not reflect the actual units. $D L=$ Detection Limit, $S=$ Worklist Slot Number, $R=$ Replicate Number, $A=$ Aliquot Code. 
HNF-SD-WM-DP-227, REV. 0

TOC- TOTAL ORGANIC CARBON ANALYSIS REPORT

TICTOC REV 2.0

$\ll$ BLANK ANALYSIS $\gg>$

Sample: BASE

Sample Size $=200 \mathrm{uL}$

Dil Factor $=1$

Blank ID \# = BASE

Blank value $=\mathrm{N} / \mathrm{A}$
Date: $01 / 27 / 97$

Time: $02: 20: 49$

$$
\begin{array}{ll}
\text { Analyst : } & \text { KN THOMAS } \\
\text { Min Readings }= & 14 \\
\text { Max Readings }= & 14 \\
\% \text { Difference }= & 10
\end{array}
$$

BLANK VALUE $=2.6$ micrograms carbon

BIANK FACTOR $=2.6 / 7.003998=$
0.20
0.30

0.50

0.70

1.00

1.30

1.50

1. 70

1.90

2.00

2.20

2.30

2.50

2.60
0.00
33.33
40.00
28.57
30.00
23.08
13.33
11.76
10.53
5.00
9.09
4.35
8.00
3.85 


\section{HNF-SD-WM-DP-227, REV. 0}

TOC- TOTAI ORGANIC CARBON ANALYSIS REPORT TICTOC REV 2.0

Sample: $24 \mathrm{~N} 12-\mathrm{F}$

Sample Size $=200 \mathrm{uI}$

Dil Factor $=11$

Blank ID \# = BASE

Blank Value $=.3712166 \mathrm{ug} / \mathrm{minute} \mathrm{C}$
Time: 02:41:11

$\begin{array}{ll}\text { Analyst : } & \text { KN THOMAS } \\ \text { Min Readings }= & 14 \\ \text { Max Readings }= & 14 \\ \% \text { Difference }= & 10\end{array}$

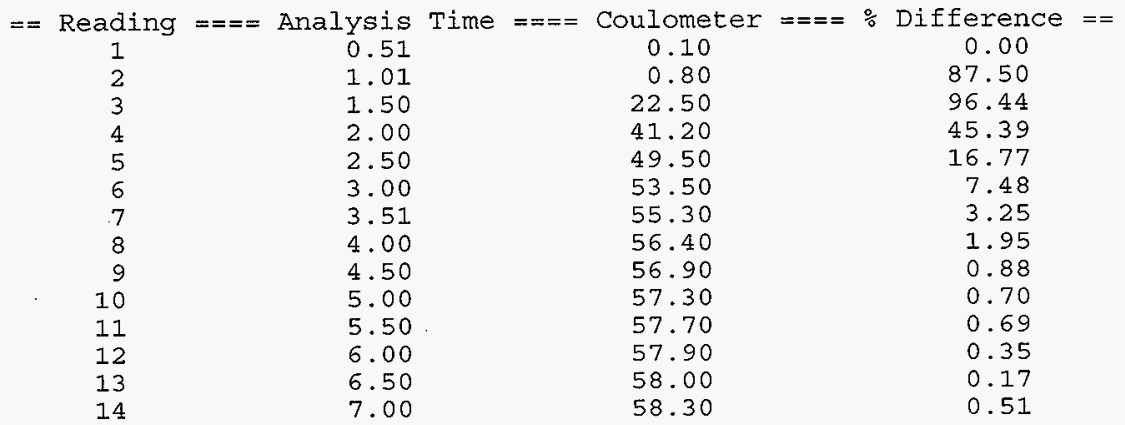

BLANK VALUE $=2.6$ micrograms carbon BLANK FACTOR $=2.6 / 7.003998=+3.7 \mathrm{E}-01$ ug $/ \mathrm{min}$ Carbon

SAMPLE RESULTS:

$\left(\begin{array}{l}58.3-2.599938)(11) /(200) \\ 58.3-2.599938)(11) /(200)(12)=\end{array}\right.$

$+3.06 \mathrm{E}+00 \quad \mathrm{~g} / \mathrm{L}$ Carbon

$+2.55 \mathrm{E}-01$ Molar Carbon

Sample Run By: 


\section{TOC- TOTAL ORGANIC CARBON ANALYSIS REPORT \\ TICTOC REV 2.0 \\ $\ll$ BLANK ANALYSIS $\gg>$}

Sample: BLNK

Sample Size $=200 \mathrm{uI}$

Dil Factor $=1$

Blank ID \# = BLNK

Blank value $=\mathrm{N} / \mathrm{A}$
Date: $01 / 27 / 97$

Time: 03:04:08

$$
\begin{array}{ll}
\text { Analyst : } & \text { KN THOMAS } \\
\text { Min Readings }= & 14 \\
\text { Max Readings }= & 14 \\
\% \text { Difference }= & 10
\end{array}
$$

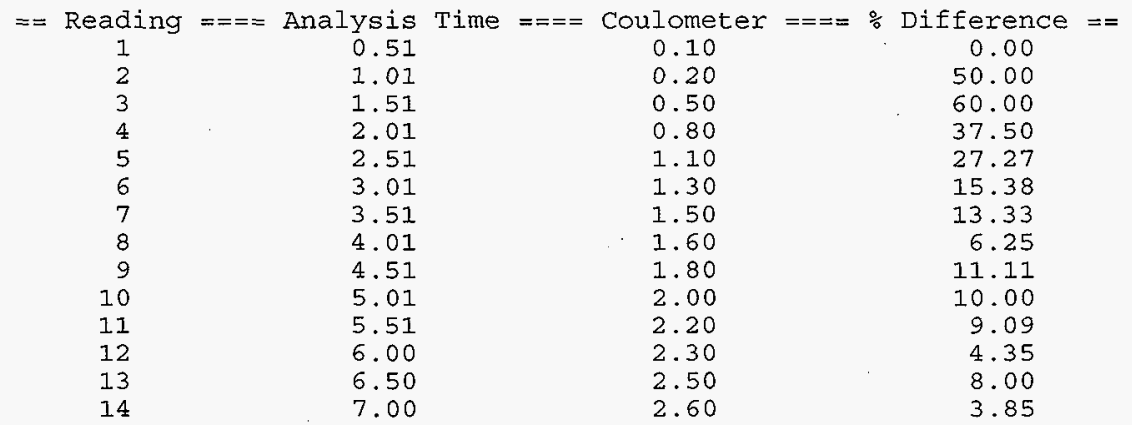

BLANK VALUE $=2.6$ micrograms carbon

BLANK FACTOR $=2.6 / 7.00383=$

$+3.7 \mathrm{E}-01 \mathrm{ug} / \mathrm{min}$ Carbon

Sample Run By: 


\section{HNF-SD-WM-DP-227, REV. 0}

TOC- TOTAL ORGANIC CARBON ANALYSIS REPORT

TICTOC REV 2.0

Sample: S97T000024

Sample Size $=200$ uL

Dil Factor $=1.5$

Blank ID \# = BINK

Blank Value $=.3712254 \mathrm{ug} /$ minute $\mathrm{C}$
Date: $01 / 27 / 97$

Time: 05:09:56

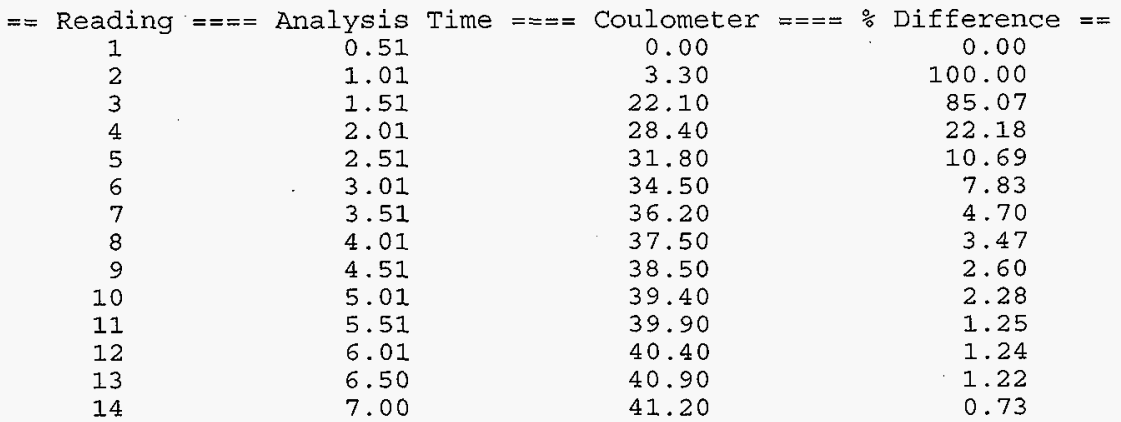

BLANK VALUE $=2.6$ micrograms carbon

BLANK FACTOR $=2.6 / 7.00383=+3.7 \mathrm{E}-01$ ug $/ \mathrm{min}$ Carbon

SAMPLE RESULTS:
$(41.2-2.600368$
$(41.2-2.600368)(1.5) /(200)(12)=$
$+2.89 \mathrm{E}-01$
g/L Carbon
$+2.41 \mathrm{E}-02$ Molar Carbon

Sample Run By:

KN THOMAS 00000


Sample: S97T000024DUP Date: 01/27/97

Sample Size $=200 \mathrm{uI}$

Dil Factor $=1.5$

Blank ID \# = BLNK

Blank Value $=.3712254 \mathrm{ug} /$ minute $\mathrm{C}$
Time: 05:19:09

Analyst : KN THOMAS

Min Readings $=14$

Max Readings $=14$

: Difference $=10$

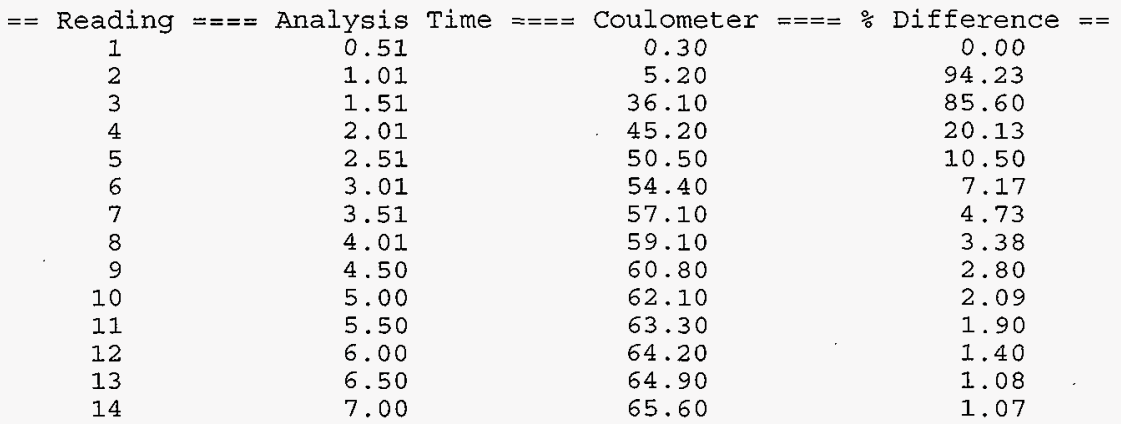

BLANK VALUE $=2.6 \mathrm{micrograms}$ carbon

BLANK FACTOR $=2.6 / 7.00383=$

$+3.7 \mathrm{E}-01 \quad \mathrm{ug} / \mathrm{min}$ Carbon

SAMPLE RESULTS :

$(65.6-2.600062)(1.5) /(200)=$

$+4.72 \mathrm{E}-01$

$+3.94 \mathrm{E}-02$

9/L Carbon

Molar Carbon

Sample Run By:

KN THOMAS

00000

\section{1}


Sample: S97T00024 TRP Date: 01/27/97

Sample Size $=200$ ut

Dil Factor $=1.5$

Blank ID \# = BLK

Blank Value $=1.727374 \mathrm{ug} /$ minute $\mathrm{C}$
Time: $17: 29: 10$

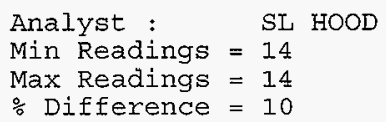

$==$ Reading $===$ Analysis Time $====$ C

$\begin{array}{rr}1 & 0.51 \\ 2 & 1.01 \\ 3 & 1.51 \\ 4 & 2.01 \\ 5 & 2.51 \\ 6 & 3.01 \\ 7 & 3.51 \\ 8 & 4.01 \\ 9 & 4.50 \\ 10 & 5.00 \\ 11 & 5.50 \\ 12 & 6.00 \\ 13 & 6.51 \\ 14 & 7.00\end{array}$

$\begin{array}{cc}\text { Coulometer }===\text { Difference }== \\ 0.20 & 0.00 \\ 5.60 & 96.43 \\ 23.50 & 76.17 \\ 30.00 & 21.67 \\ 34.30 & 12.54 \\ 37.10 & 7.55 \\ 39.50 & 6.08 \\ 41.20 & 4.13 \\ 42.40 & 2.83 \\ 43.70 & 2.97 \\ 44.50 & 1.80 \\ 45.50 & 2.20 \\ 46.30 & 1.73 \\ 46.90 & 1.28\end{array}$

BLANK VALUE $=12.1$ micrograms carbon

$$
\text { BLANK FACTOR }=12.1 / 7.004852=0+1.7 \mathrm{E}+00 \mathrm{ug} / \mathrm{min} \text { Carbon }
$$




\section{HNF-SD-WM-DP-227, REV. 0}

TOC- TOTAL ORGANIC CARBON ANALYSIS REPORT TICTOC REV 2.0

Sample: S97T000026

Sample Size $=200 \mathrm{uL}$

Dil Factor $=2$

Blank ID \# = BIK

Blank Value $=1.727374 \mathrm{ug} / \mathrm{minute} \mathrm{C}$
Date: $01 / 27 / 97$

ug/minute $C$
Time: $05: 57: 48$

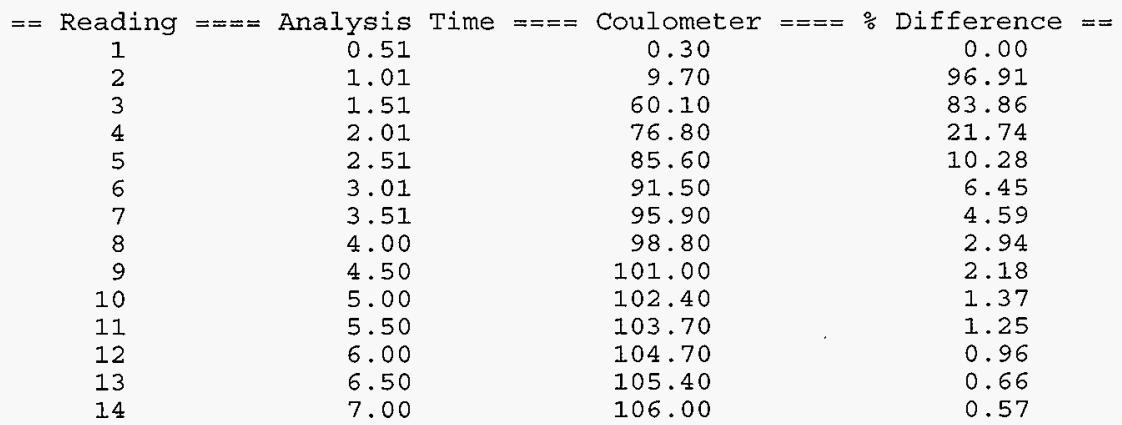

BLANK VALUE $=12.1$ micrograms carbon

BLANK FACTOR $=12.1 / 7.004852=+1.7 \mathrm{E}+00 \quad \mathrm{ug} / \mathrm{min}$ Carbon

SAMPLE RESULTS :

$\begin{array}{rrl}(106-12.09989)(2) /(200)= & +9.390 \mathrm{E}-01 & \mathrm{~g} / \mathrm{L} \text { Carbon } \\ (106-12.09989)(2) /(200)(12)= & +7.825 \mathrm{E}-02 & \text { Molar Carbon }\end{array}$

Sample Run By:

KN THOMAS 00000 


\section{TOC- TOTAL ORGANIC CARBON ANALYSIS REPORT} TICTOC REV 2.0

Sample: S97T00026 DUP

$$
\begin{aligned}
\text { Sample Size } & =200 \mathrm{uL} \\
\text { Dil Factor } & =2 \\
\text { Blank ID \# } & =\text { BLK }
\end{aligned}
$$$$
\text { Blank Value }=1.727374 \mathrm{ug} / \text { minute } \mathrm{C}
$$

Time: $08: 22: 08$

$$
\begin{array}{ll}
\text { Analyst : } & \text { KN THOMAS } \\
\text { Min Readings }= & 14 \\
\text { Max Readings }= & 14 \\
\% \text { Difference }= & 10
\end{array}
$$

$\begin{array}{cccc}\text { Reading }===\text { Analysis } & \text { Time }=== & \text { Coulometer }=== & \text { Difference }== \\ 1 & 0.51 & 0.20 & 0.00 \\ 2 & 1.01 & 2.80 & 92.86 \\ 3 & 1.51 & 54.30 & 94.84 \\ 4 & 2.01 & 73.30 & 25.92 \\ 5 & 2.51 & 82.50 & 11.15 \\ 6 & 3.01 & 88.70 & 6.99 \\ 7 & 3.51 & 93.10 & 4.73 \\ 8 & 4.01 & 96.10 & 3.12 \\ 9 & 4.51 & 98.40 & 2.34 \\ 10 & 5.01 & 100.00 & 1.60 \\ 11 & 5.51 & 101.40 & 1.38 \\ 12 & 6.01 & 102.30 & 0.88 \\ 13 & 6.51 & 103.00 & 0.68 \\ 14 & 7.00 & 103.80 & 0.77\end{array}$

BLANK VALUE $=12.1$ micrograms carbon

BLANK FACTOR $=12.1 / 7.004852=$ $+1.7 \mathrm{E}+00 \mathrm{ug} / \mathrm{min}$ Carbon SAMPLE RESULTS:

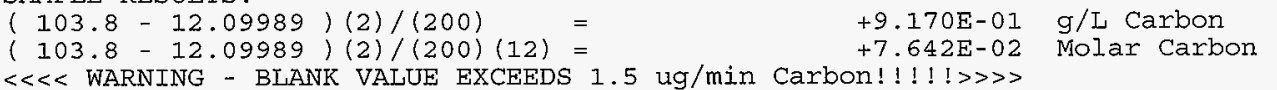


Sample: S97T00026 SPK Date: 01/27/97 Time: 13:35:43

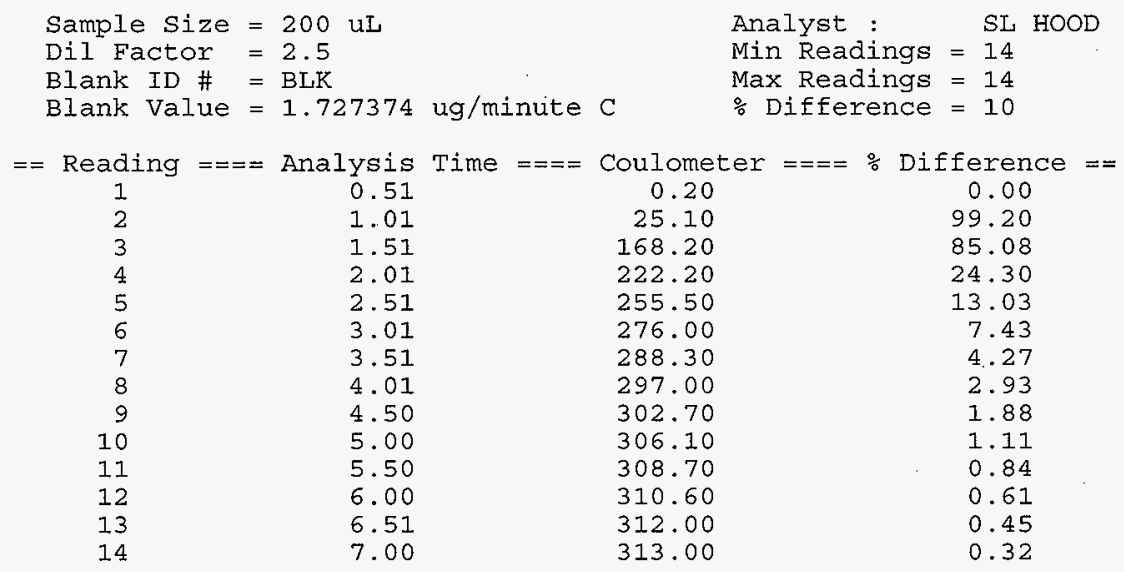

BLANK VALUE $=12.1$ micrograms carbon

BLANK FACTOR $=12.1 / 7.004852=+1.7 \mathrm{E}+00 \mathrm{ug} / \mathrm{min}$ Carbon

SAMPLE RESULTS:

$\begin{array}{llll}(313-12.09995)(2.5) /(200) & & +3.761 \mathrm{E}+00 & \mathrm{~g} / \mathrm{L} \text { Carbon } \\ (313-12.09995)(2.5) /(200)(12)= & +3.134 \mathrm{E}-01 & \text { Molar Carbon }\end{array}$

$\ll<$ WARNING - BLANK VALUE EXCEEDS $1.5 \mathrm{ug} / \mathrm{min}$ Carbon!!!!!! > >

Sample Run By:

$\overline{\text { SL HOOD }} 00000$ 
HNF-SD-WM-DP-227, REV. 0

TOC- TOTAL ORGANIC CARBON ANALYSIS REPORT TICTOC REV 2.0

Sample: $597 T 0027$

Date: $01 / 27 / 97$

Time: $14: 38: 12$

Sample Size $=200 \mathrm{uL}$

Dil Factor $=11$

Blank ID \# = BLK

Blank Value $=1.727374 \mathrm{ug} /$ minute $\mathrm{C}$

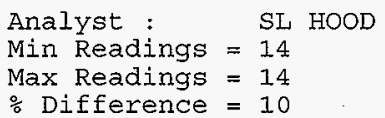

$==$ Reading $====$ Analysis

$\begin{array}{rr}1 & 0.51 \\ 2 & 1.01 \\ 3 & 1.51 \\ 4 & 2.01 \\ 5 & 2.51 \\ 6 & 3.01 \\ 7 & 3.51 \\ 8 & 4.01 \\ 9 & 4.51 \\ 10 & 5.01 \\ 11 & 5.51 \\ 12 & 6.01 \\ 13 & 6.51 \\ 14 & 7.00\end{array}$

Time $====$ Coulometer

0.20
14.90
64.80
79.50
87.50
92.90
96.70
99.20
101.10
102.40
103.30
104.10
104.80
105.30

$\because$ Difference $==$

1.01

1.51

2.01

2.51

3.01

3.51

4.01

4.51

5.01

5.51

6.51

105.30

\begin{abstract}
0.00
98.66

77.01

18.49

9.14

5.81

3.93

2.52

1.88

1.27

0.87

0.77

0.67

0.47
\end{abstract}

BLANK VALUE $=12.1$ micrograms carbon

BLANK FACTOR $=12.1 / 7.004852=+1.7 \mathrm{E}+00 \mathrm{ug} / \mathrm{min}$ Carbon

SAMPLE RESULTS:

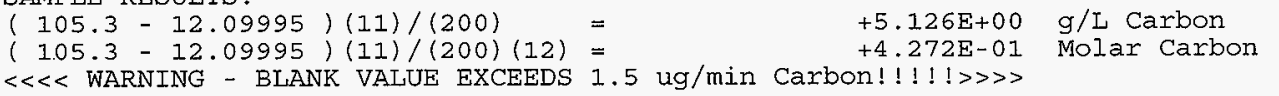

Sample Run By:

\begin{tabular}{ll}
\hline SL HOOD & 00000
\end{tabular} 
Sample: S97T00027DUP

$$
\begin{aligned}
\text { Sample Size } & =200 \mathrm{uI} \\
\text { Dil Factor } & =11 \\
\text { Blank ID \# } & =\text { BLK } \\
\text { Blank Value } & =1.727374 \mathrm{ug} / \text { minute } \mathrm{C}
\end{aligned}
$$

Time: $15: 37: 56$

$$
\begin{array}{ll}
\text { Analyst : } & \text { SL HOOD } \\
\text { Min Readings }= & 14 \\
\text { Max Readings }= & 14 \\
: \text { Difference }= & 10
\end{array}
$$

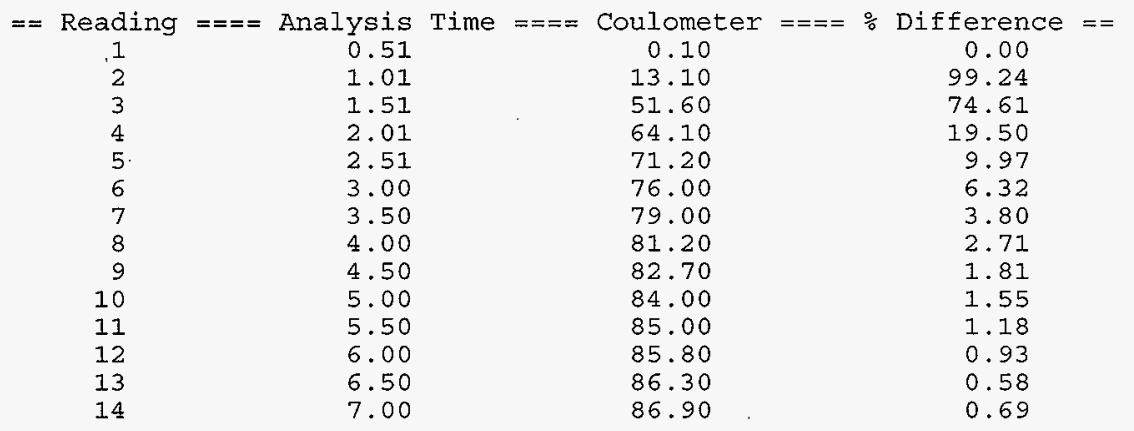

BLANK VALUE $=12.1$ micrograms carbon

BLANK FACTOR $=12.1 / 7.004852=+1.7 \mathrm{E}+00 \mathrm{ug} / \mathrm{min}$ Carbon

SAMPLE RESULTS:

$\left(\begin{array}{llll}(86.9-12.09678)(11) /(200) & = & +4.11 \mathrm{E}+00 & \mathrm{~g} / \mathrm{I} \text { Carbon } \\ (86.9-12.09678)(11) /(200)(12)= & +3.43 \mathrm{E}-01 & \text { Molar Carbon }\end{array}\right.$

« WARNING - BLANK VALUE EXCEEDS $1.5 \mathrm{ug} / \mathrm{min}$ Carbon!!!!!! ! >>

Sample Run By:

SL HOOD 00000 
Sample: S97T00027 TRP Date: 01/27/97 Time: 16:51:33

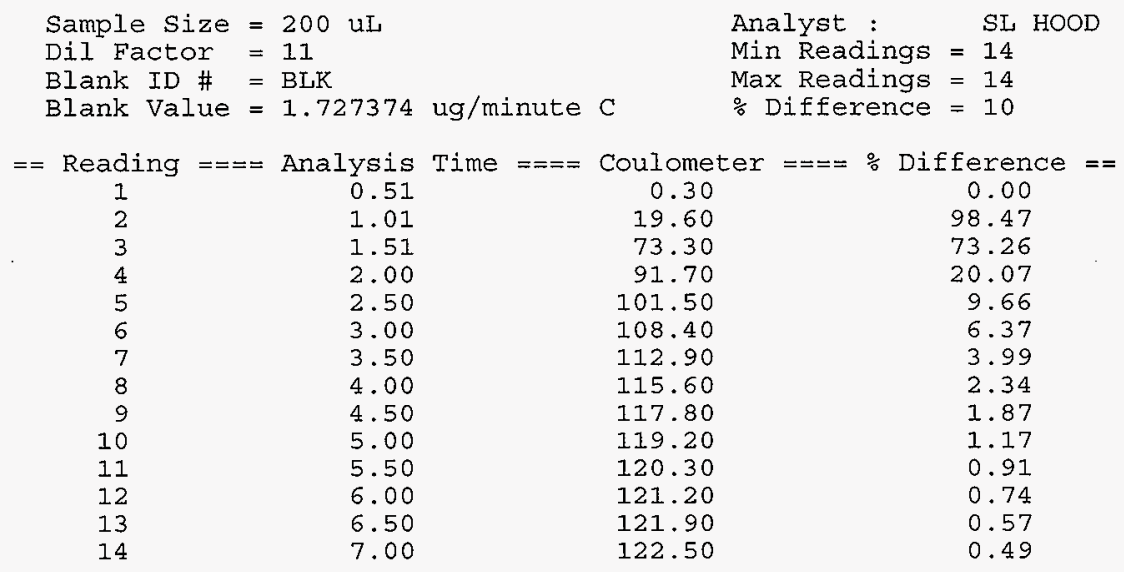

BLANK VALUE $=12.1$ micrograms carbon

BLANK FACTOR $=12.1 / 7.004852=+1.7 \mathrm{E}+00 \mathrm{ug} / \mathrm{min}$ Carbon

SAMPLE RESULTS:

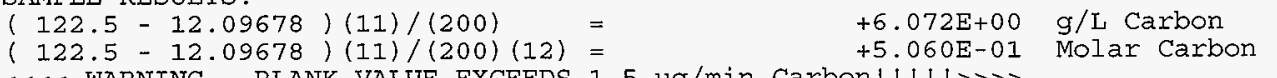

« WARNING - BLANK VALUE EXCEEDS $1.5 \mathrm{ug} / \mathrm{min}$ Carbon!!!!!! $\gg \gg>$

Sample Run By:

\begin{tabular}{ll}
\hline SL HOOD & 00000
\end{tabular} 


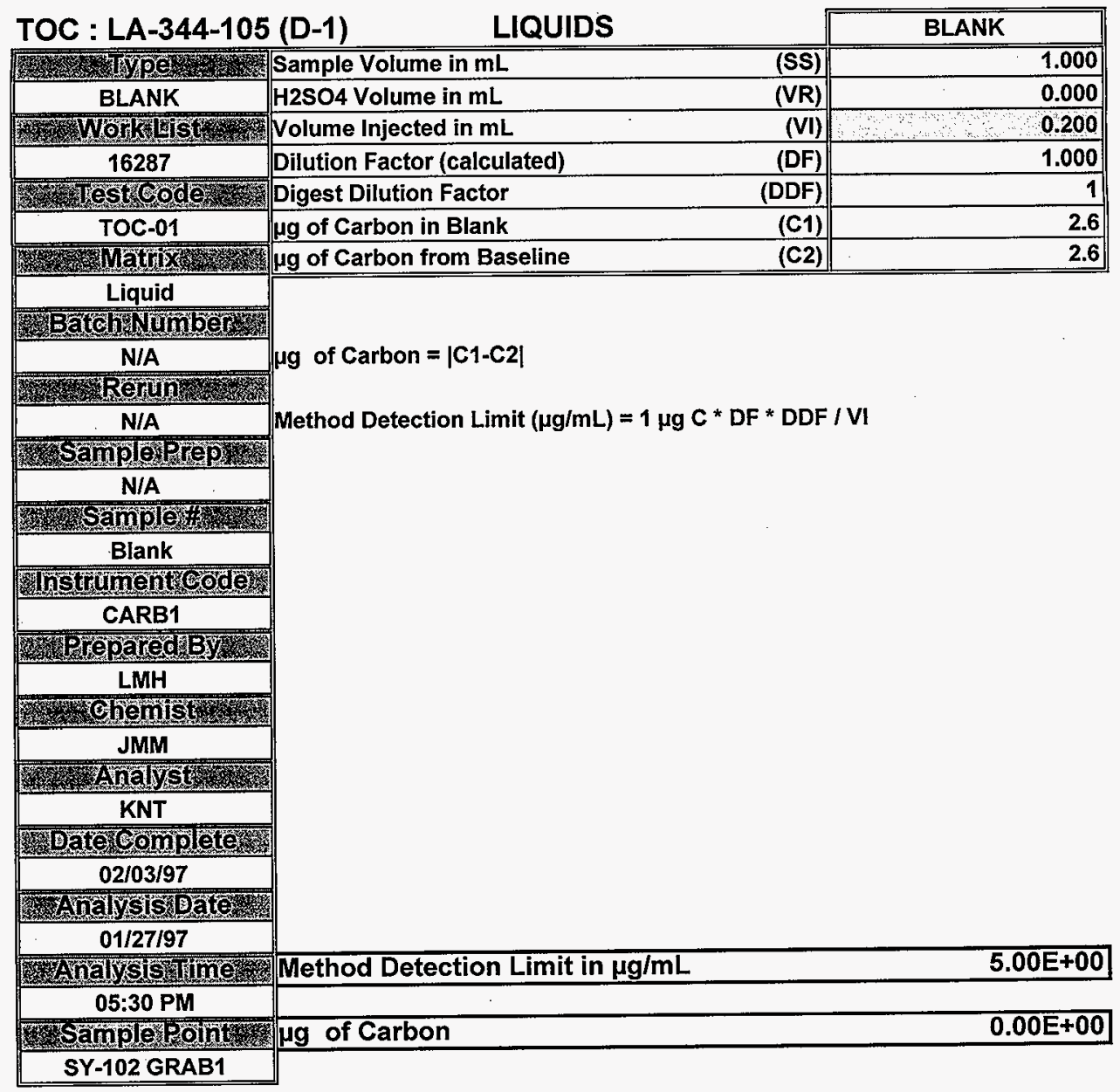

\begin{tabular}{|c|c|c|c|}
\hline Data Entered By: & LMH & Date: & $02 / 03 / 97$ \\
\hline Signature of Chemist: & Nolun McChuben & Date: & $02 / 03 / 97$ \\
\hline
\end{tabular}




\begin{tabular}{|c|c|c|}
\hline TOC : LA-344-105 & LIQUIDS & STANDARD \\
\hline 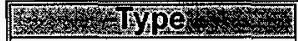 & Sample Volume in $\mathrm{mL}$ & 0.200 \\
\hline STANDARD & $\mathrm{H} 2 \mathrm{SO} 4$ Volume in $\mathrm{mL}$ & 2.000 \\
\hline 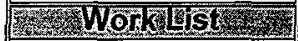 & Volume Injected in $\mathrm{mL}$ & 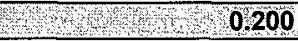 \\
\hline 16287 & Dilution Factor (calculated) & 11.000 \\
\hline mesteodo & Digest Dilution Factor & -1 \\
\hline TOC-01 & $\mu \mathrm{g}$ of Carbon Found & 58.3 \\
\hline Y. Matrix & ug of Carbon from Baseline & 2.6 \\
\hline LIQUID & Standard Book Number & 24N12F \\
\hline BatchNomber & Standard Value $(\mu \mathrm{g} / \mathrm{ml})$ & 3000 \\
\hline N/A & & \\
\hline 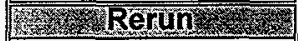 & & \\
\hline N/A & Hg of Carbon $/ \mathrm{mL}=\left(\mathrm{C}_{1-C 2}\right)^{*} \mathrm{DF}{ }^{*} \mathrm{DDF} / \mathrm{VI}$ & \\
\hline Sampleprep & & \\
\hline N/A & Method Detection Limit $(\mu \mathrm{g} / \mathrm{mL})=1 \mu \mathrm{g} \mathrm{C} * \mathrm{DF} * \mathrm{DDF} /$ & \\
\hline Sampleftris & & \\
\hline Instument Code & & \\
\hline CARB1 & & \\
\hline Prepared B & & \\
\hline LMH & & \\
\hline 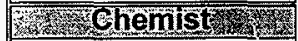 & & \\
\hline RWS & & \\
\hline Analysts & & \\
\hline KNT & & \\
\hline Datresoletes & & \\
\hline $01 / 28 / 97$ & & \\
\hline Analysispater & & \\
\hline $01 / 27 / 97$ & & \\
\hline Analysis & Method Detection Limit in $\mu \mathrm{g} / \mathrm{mL}$ & $5.50 \mathrm{E}+01$ \\
\hline 05:30 PM & QC Actual in $\mu \mathrm{g} / \mathrm{mL}$ & $3.00 E+03$ \\
\hline Samplexpont & QC Found in $\mu \mathrm{g} / \mathrm{mL}$ & $3.06 \mathrm{E}+03$ \\
\hline SY-102 GRAB1 & Percent Standard Recovery & 102.1 \\
\hline
\end{tabular}

\begin{tabular}{|c|c|c|c|}
\hline Data Entered By: & $\mathrm{LMH}$ & Date: & $01 / 28 / 97$ \\
\hline Signature of Chemist: & $\mathrm{glm}$ & Date: & $02 / 03 / 97$ \\
\hline
\end{tabular}




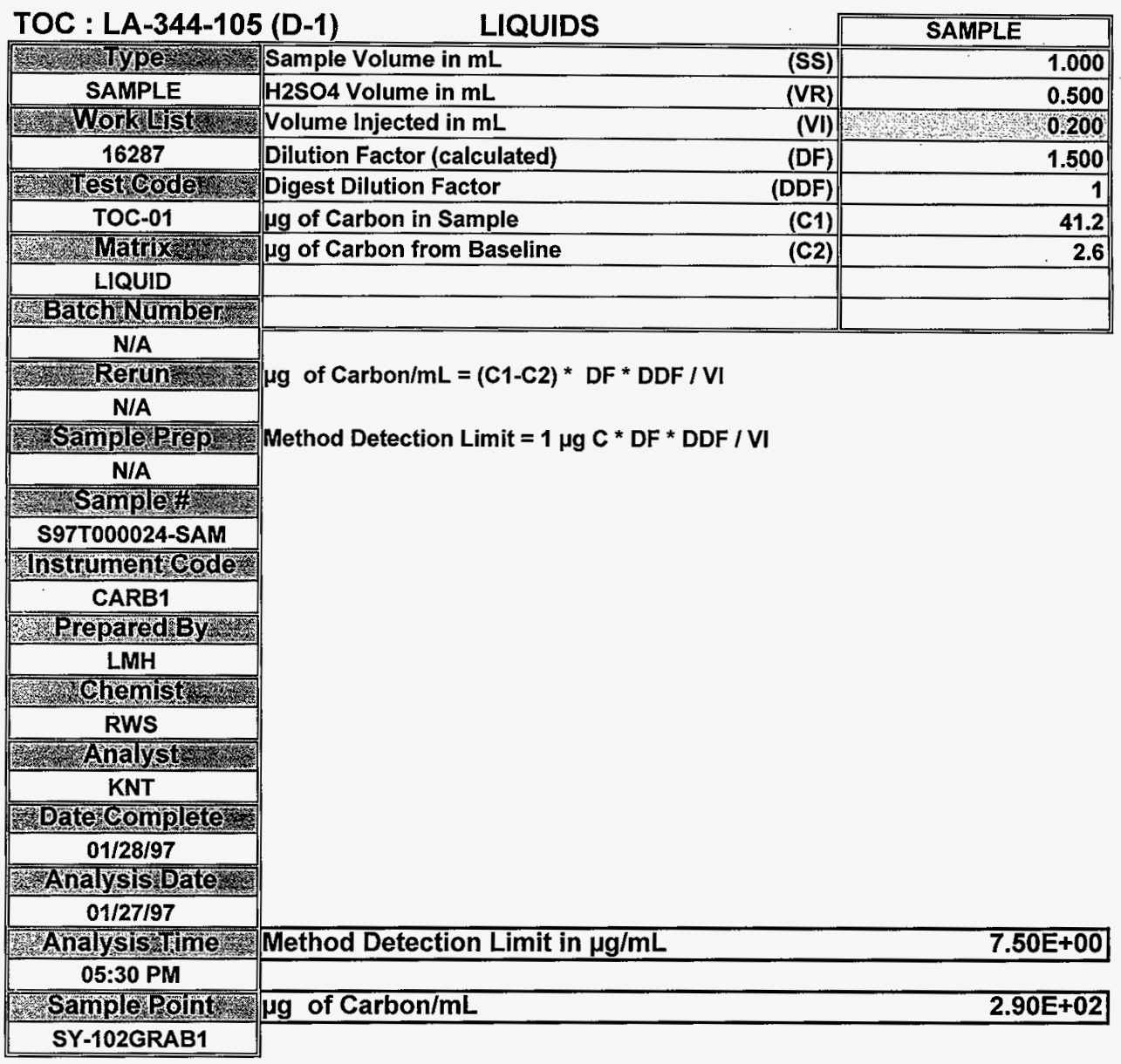

\begin{tabular}{|c|c|c|c|}
\hline Data Entered By: & $\mathrm{LMH}$ & Date: & $01 / 28 / 97$ \\
\hline Signature of Chemist: & & Date: & $02 / 03 / 97$ \\
\hline
\end{tabular}




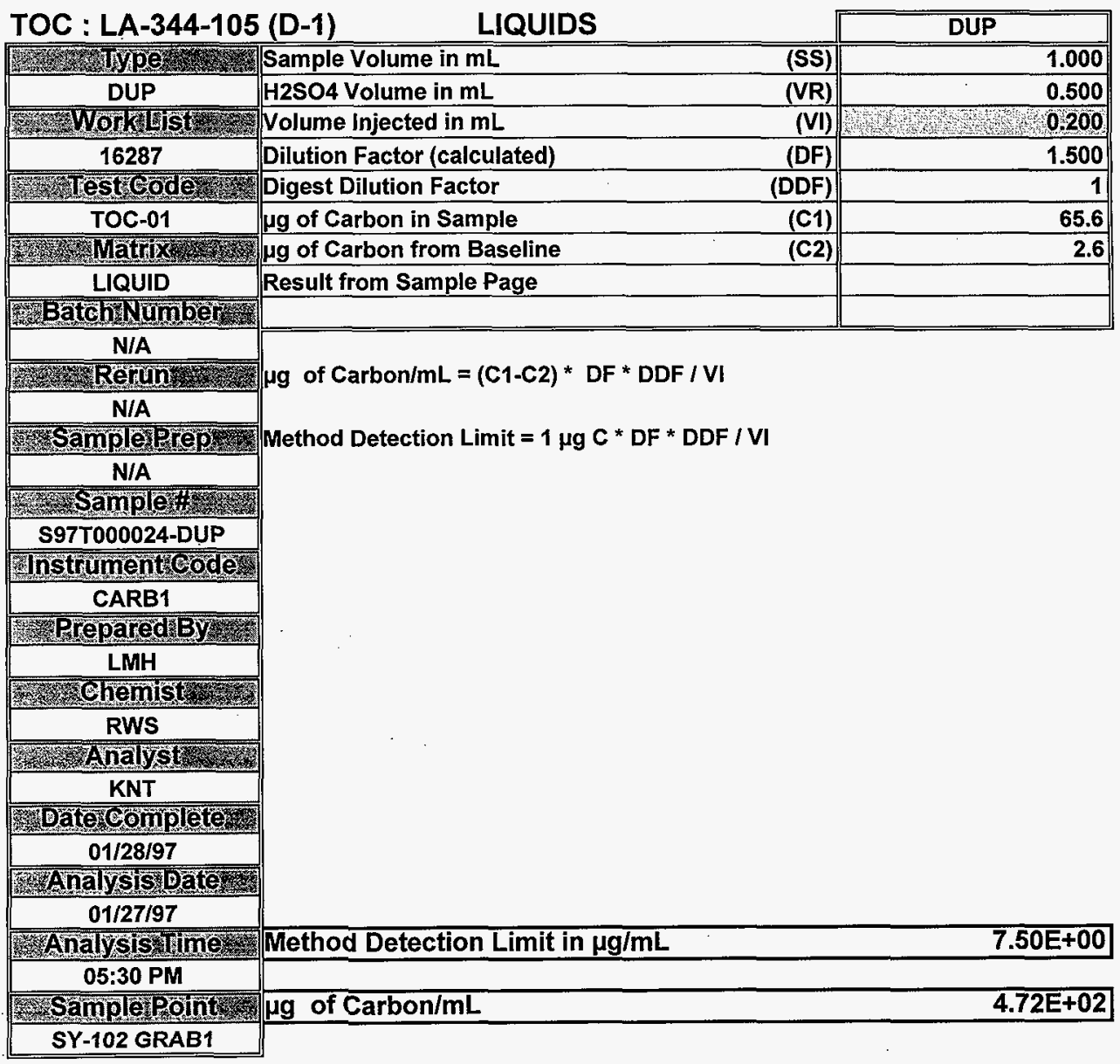

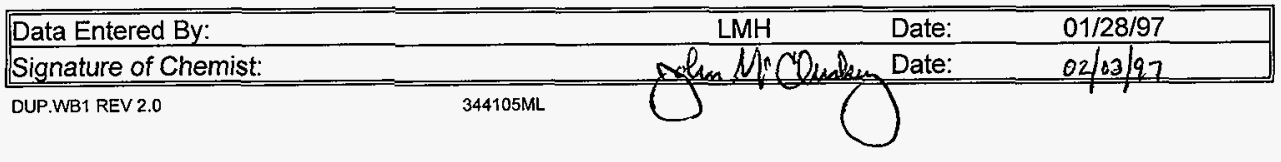




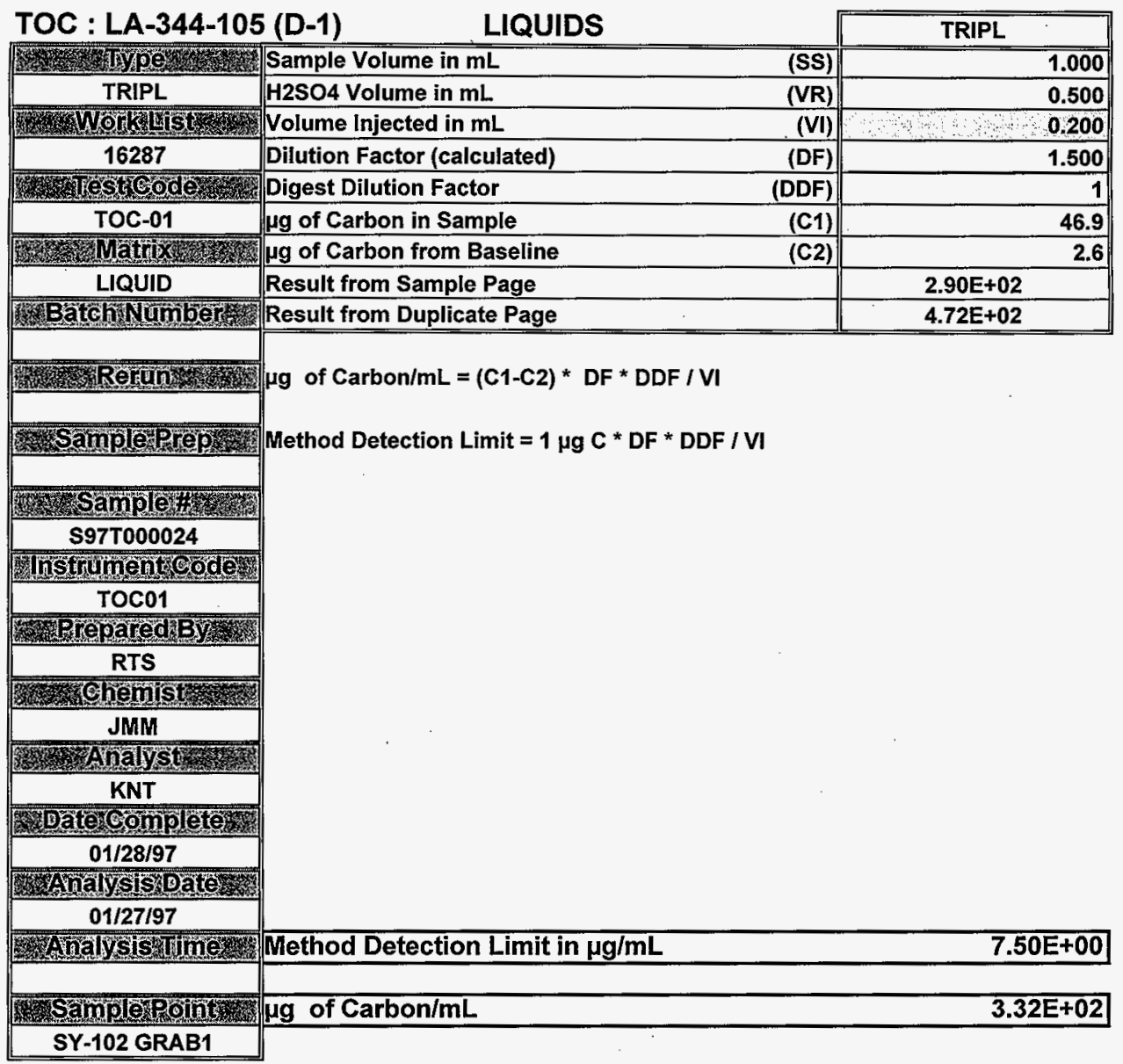

\begin{tabular}{|llll|}
\hline Data Entered By: & RTS & Date: & 01/28/97 \\
\hline Signature of Chemist: & & O2/03/97 \\
\hline TRIPL.WB1 REV 1.0 & & &
\end{tabular}




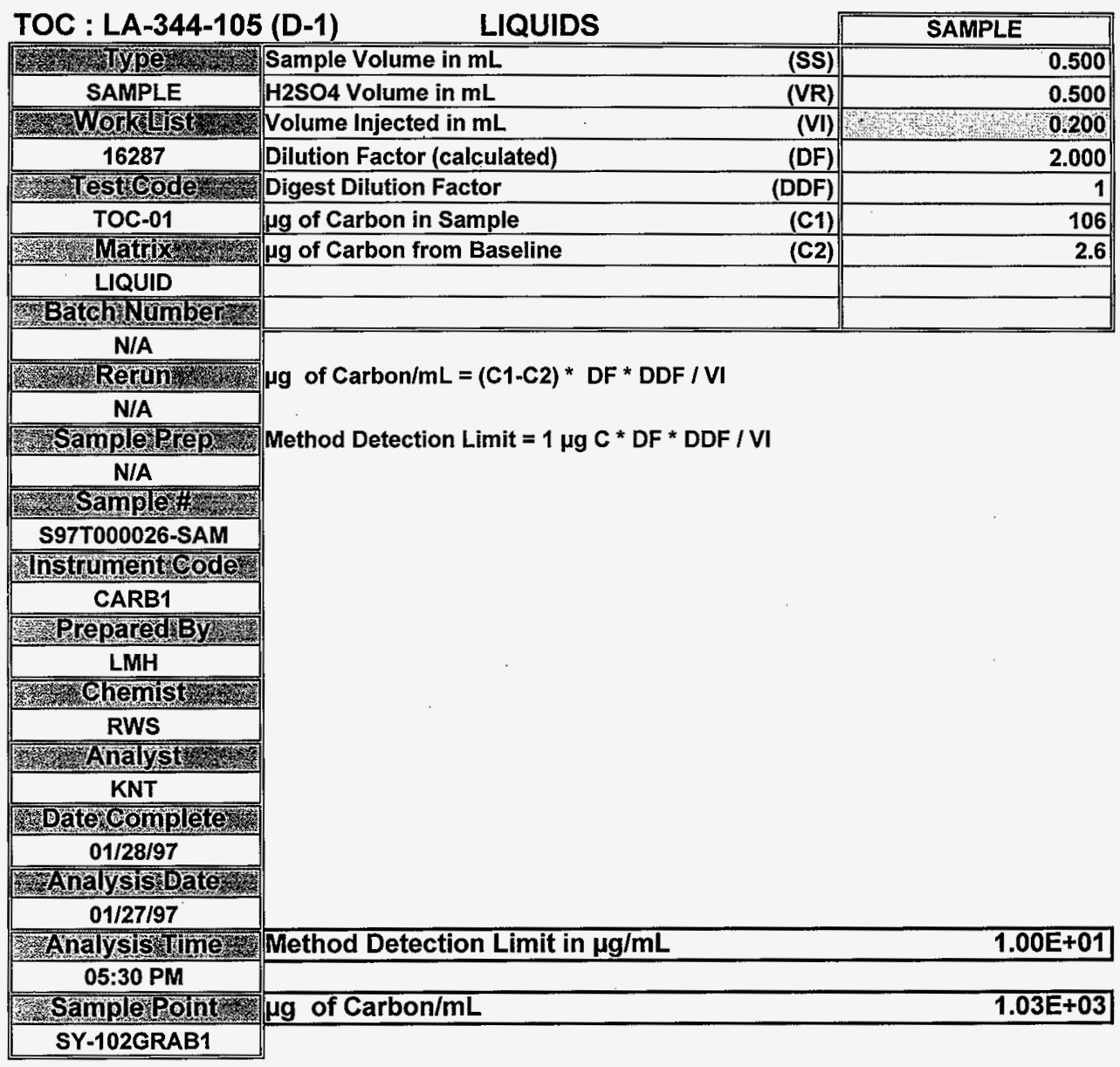

\begin{tabular}{|c|c|c|c|}
\hline Data Entered By: & $\mathrm{LMH}$ & Date: & $01 / 28 / 97$ \\
\hline Signature of Chemist: & 014 & Date: & $02 \log 67$ \\
\hline
\end{tabular}




\begin{tabular}{|c|c|c|}
\hline TOC : LA-344-105 & LIQUIDS & DUP \\
\hline Y) & Sample Volume in $\mathrm{mL}$ & 0.500 \\
\hline DUP & H2SO4 Volume in $\mathrm{mL}$ & 0.500 \\
\hline 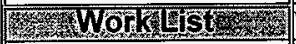 & Volume Injected in $\mathrm{mL}$ & 02200 \\
\hline 16287 & Dilution Factor (calculated) & 2.000 \\
\hline Testeode & Digest Dilution Factor & 1 \\
\hline TOC-01 & $\mu \mathrm{g}$ of Carbon in Sample & 103.8 \\
\hline NThatrix & $\mu g$ of Carbon from Baseline & 2.6 \\
\hline LIQUID & Result from Sample Page & \\
\hline Batchyombor & & \\
\hline N/A & & \\
\hline STrun & Hg of Carbon $/ \mathrm{mL}=(\mathrm{C} 1-\mathrm{C} 2){ }^{*} \mathrm{DF} * \mathrm{DDF} / \mathrm{VI}$ & \\
\hline N/A & & \\
\hline Sampleprep & Method Detection Limit $=1 \mu \mathrm{g} \mathrm{C}$ * DF * DDF $/$ VI & \\
\hline N/A & & \\
\hline 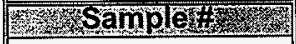 & & \\
\hline S97T000026-DUP & & \\
\hline Instrument $\mathrm{Code}$ & & \\
\hline CARB1 & & \\
\hline Prepared & & \\
\hline LMH & & \\
\hline Whemist & & \\
\hline RWS & & \\
\hline 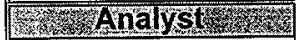 & & \\
\hline KNT & & \\
\hline Datecompleter & & \\
\hline $01 / 28 / 97$ & & \\
\hline Analysis & & \\
\hline $01 / 27 / 97$ & & \\
\hline Analysismine & Method Detection Limit in $\mu \mathrm{g} / \mathrm{mL}$ & $1.00 \mathrm{E}+01$ \\
\hline 05:30 PM & & \\
\hline Samplepront & $\mu \mathrm{g}$ of Carbon $/ \mathrm{mL}$ & $1.01 E+03$ \\
\hline SY-102 GRAB1 & & \\
\hline
\end{tabular}

\begin{tabular}{|llll|}
\hline Data Entered By: & LMH & Date: & $01 / 28 / 97$ \\
\hline Signature of Chemist: & 344105ML & & $02 / 03 / 97$ \\
\hline DUP.WB1 REV 2.0 & & \\
\hline
\end{tabular}


HNF-SD-WM-DP-227, REV. 0

TOC : LA-344-105 (D-1)

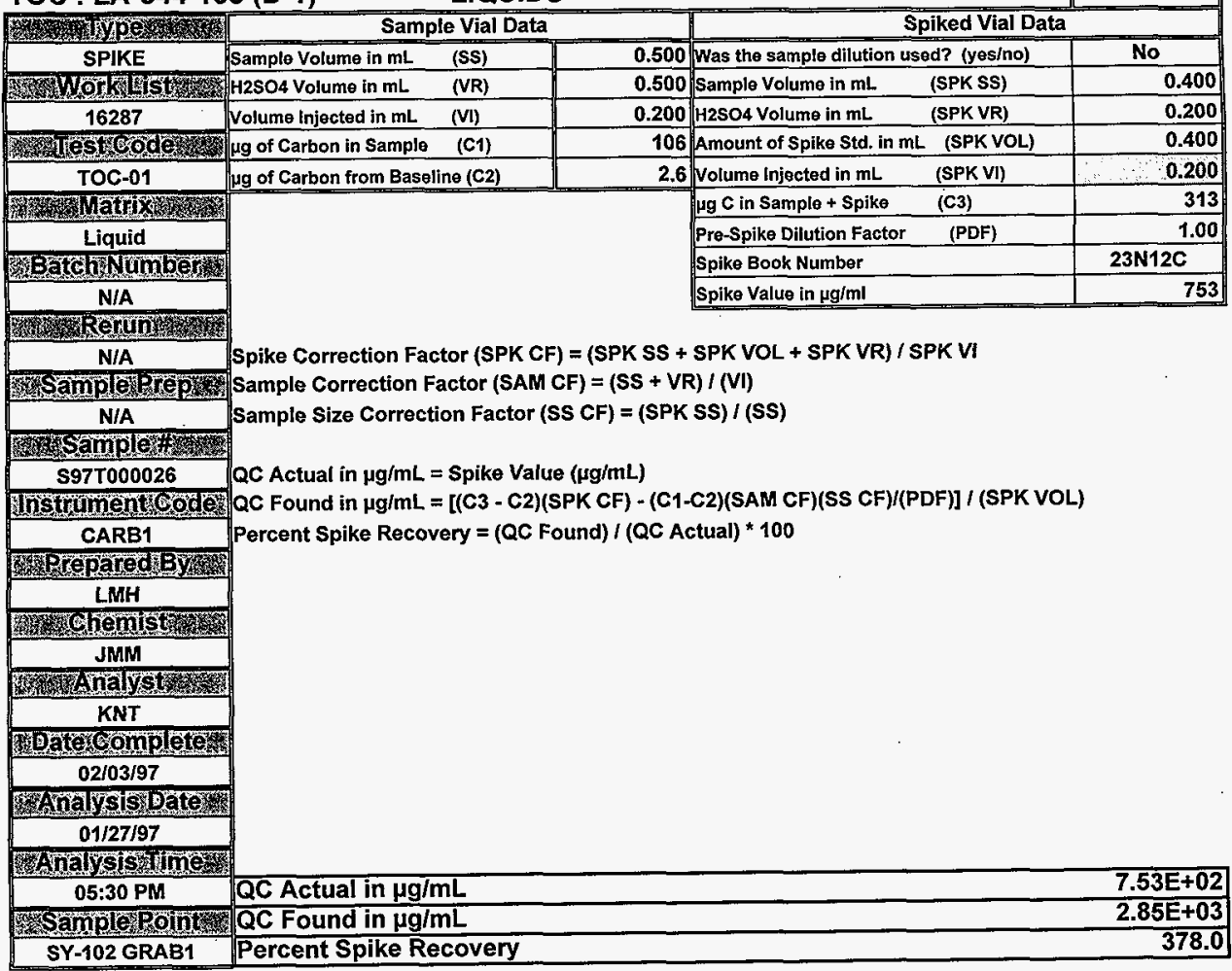

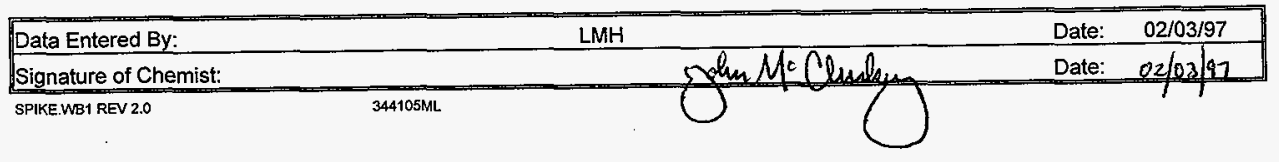


HNF-SD-WM-DP-227, REV. 0

\begin{tabular}{|c|c|c|}
\hline TOC : LA-344-105 & LIQUIDS & SAMPLE \\
\hline 10 & Sample Volume in $\mathrm{mL}$ & 0.200 \\
\hline SAMPLE & $\mathrm{H} 2 \mathrm{SO}_{4}$ Volume in $\mathrm{mL}$ & 2.000 \\
\hline 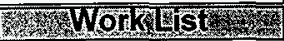 & Volume Injected in $\mathrm{mL}$ & 0200 \\
\hline 16287 & Dilution Factor (calculated) & 11.000 \\
\hline STestedo & Digest Dilution Factor & 1 \\
\hline TOC-01 & ug of Carbon in Sample & 105.3 \\
\hline Wrin & $\mu \mathrm{g}$ of Carbon from Baseline & 2.6 \\
\hline LIQUID & & \\
\hline BatchNomber & & \\
\hline N/A & & \\
\hline Shrun & ug of Carbon $/ \mathrm{mL}=(C 1-C 2) * D F * D D F / V 1$ & \\
\hline N/A & & \\
\hline Sampleprep & Method Detection Limit $=1 \mu \mathrm{g} \mathrm{C}{ }^{*} \mathrm{DF}$ * DDF $/ \mathrm{VI}$ & \\
\hline N/A & & \\
\hline Samplestits & & \\
\hline S97T000027-SAM & & \\
\hline Instrument Coder & & \\
\hline CARB1 & & \\
\hline Spreparedigy & & \\
\hline LMH & & \\
\hline Shemist & & \\
\hline RWS & & \\
\hline 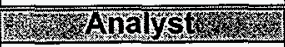 & & \\
\hline KNT & & \\
\hline Datecomplete & & \\
\hline $01 / 28 / 97$ & . & \\
\hline Analysispate & & \\
\hline $01 / 27 / 97$ & & \\
\hline Analysiswime & Method Detection Limit in $\mu \mathrm{g} / \mathrm{mL}$ & $5.50 E+01$ \\
\hline 05:30 PM & & \\
\hline Sampleoint & $\mu \mathrm{g}$ of Carbon $/ \mathrm{mL}$ & $5.65 \mathrm{E}+03$ \\
\hline SY-102GRAB1 & & \\
\hline
\end{tabular}

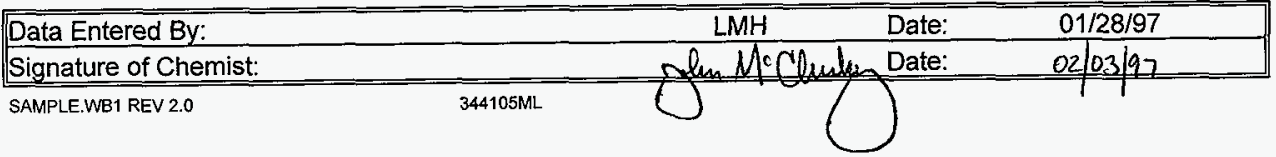




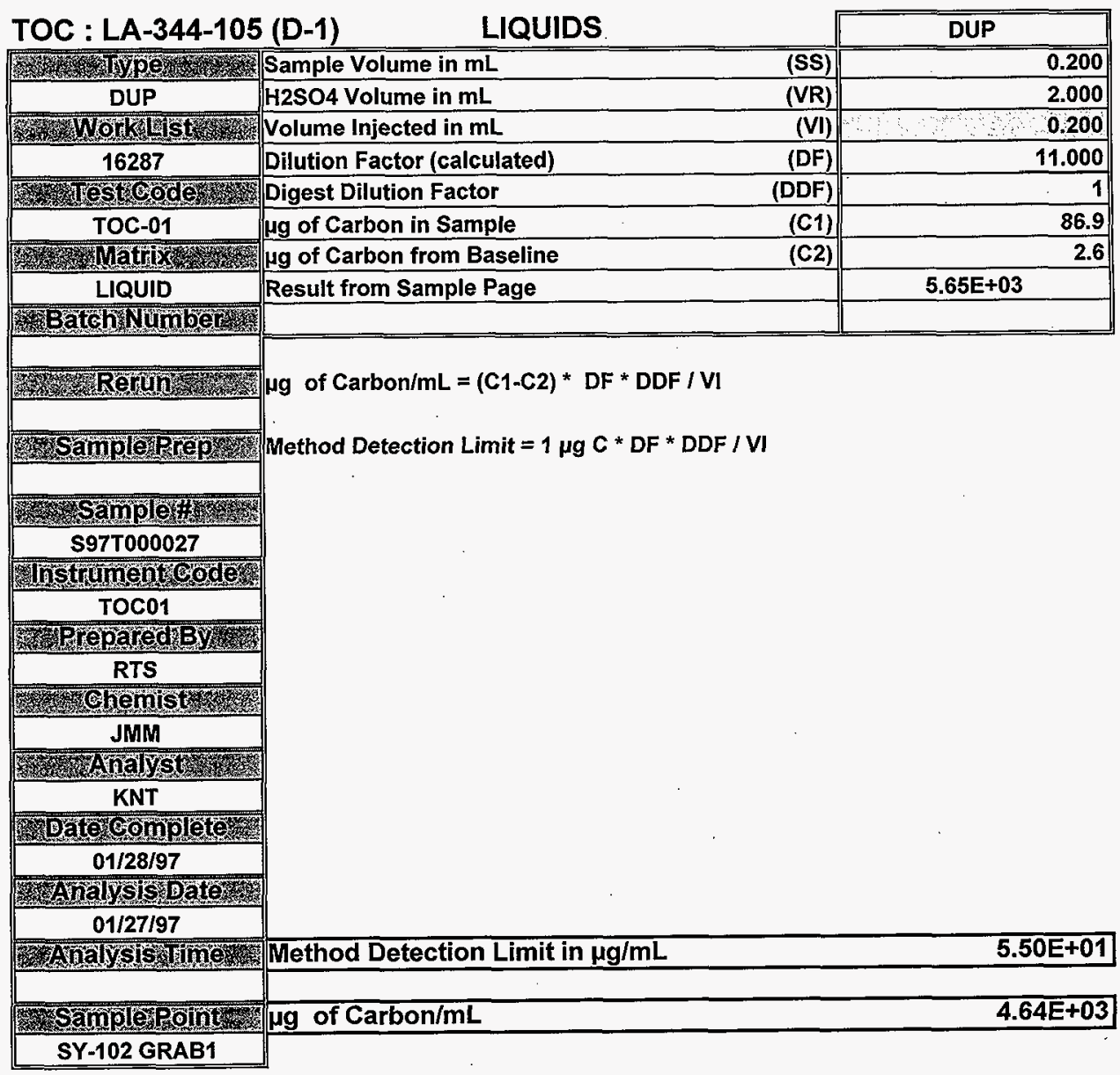

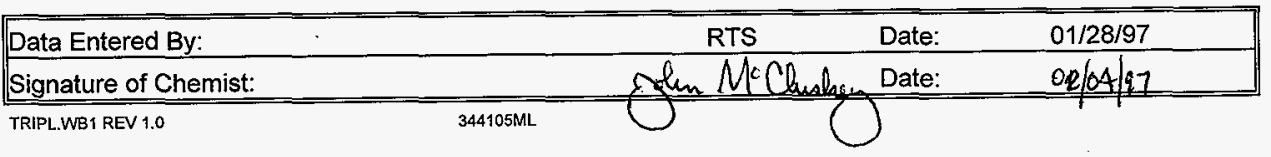




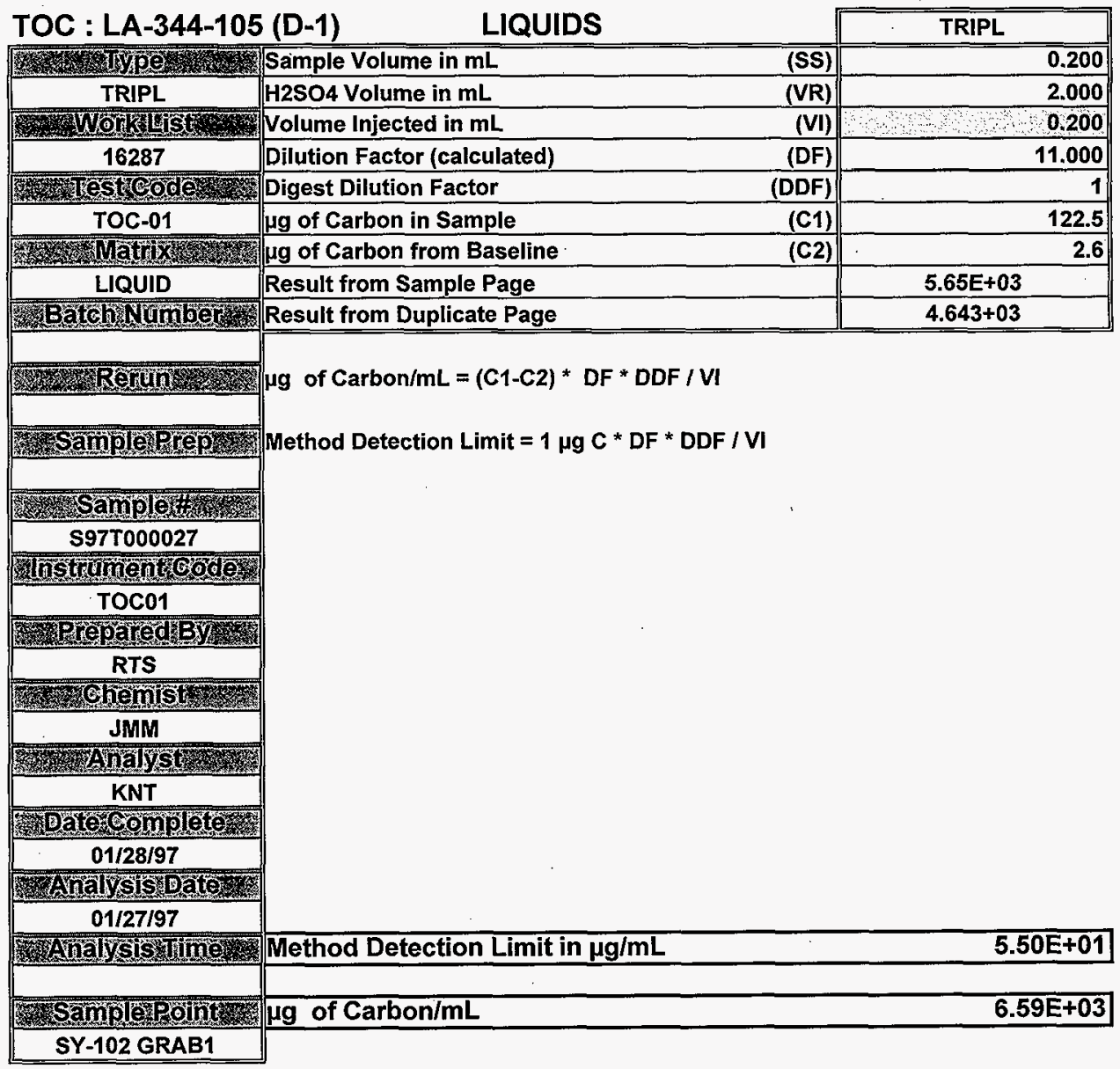

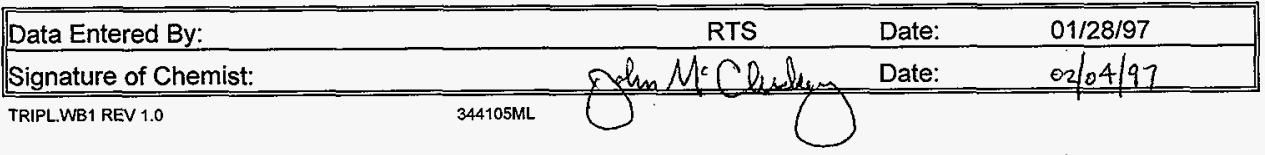




\section{LABCORE Completed Worklist Report for Worklist\# 16286}

Analyst: dgg

Instrument: TIC01

Method: LA - 342-100Rev/Mod $E-\not$

Worklist Comment: SY-102 Grab. Std: TICSTD 1.0ml.

new

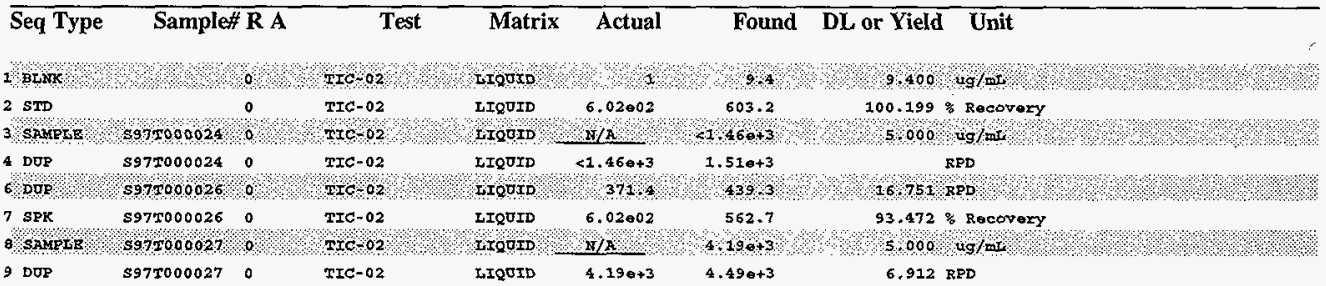

\section{Comments Section:}

Comments for sample\# S97T000024 and test TIC-02 .

HAD A PROBLEM WITH CELL TURNING GREEN AND USED SMALLER

SAMPLE SIZES AS A RESULT. SAMPLE COLOR IS A YELLOW LIQUID.

Comments for sample\# S97T000027 and test TIC-02 .

SAMPLE IS A BROWN SLUDGE AND COULD NOT PIPETTE.

\section{Final page for worklist\# 16286}

Analyst Signature Date
Snalyst Signature Date
Sq7T 000026 rejected due to poor QC.
Sampue was reanalyzed on worklist
16384.




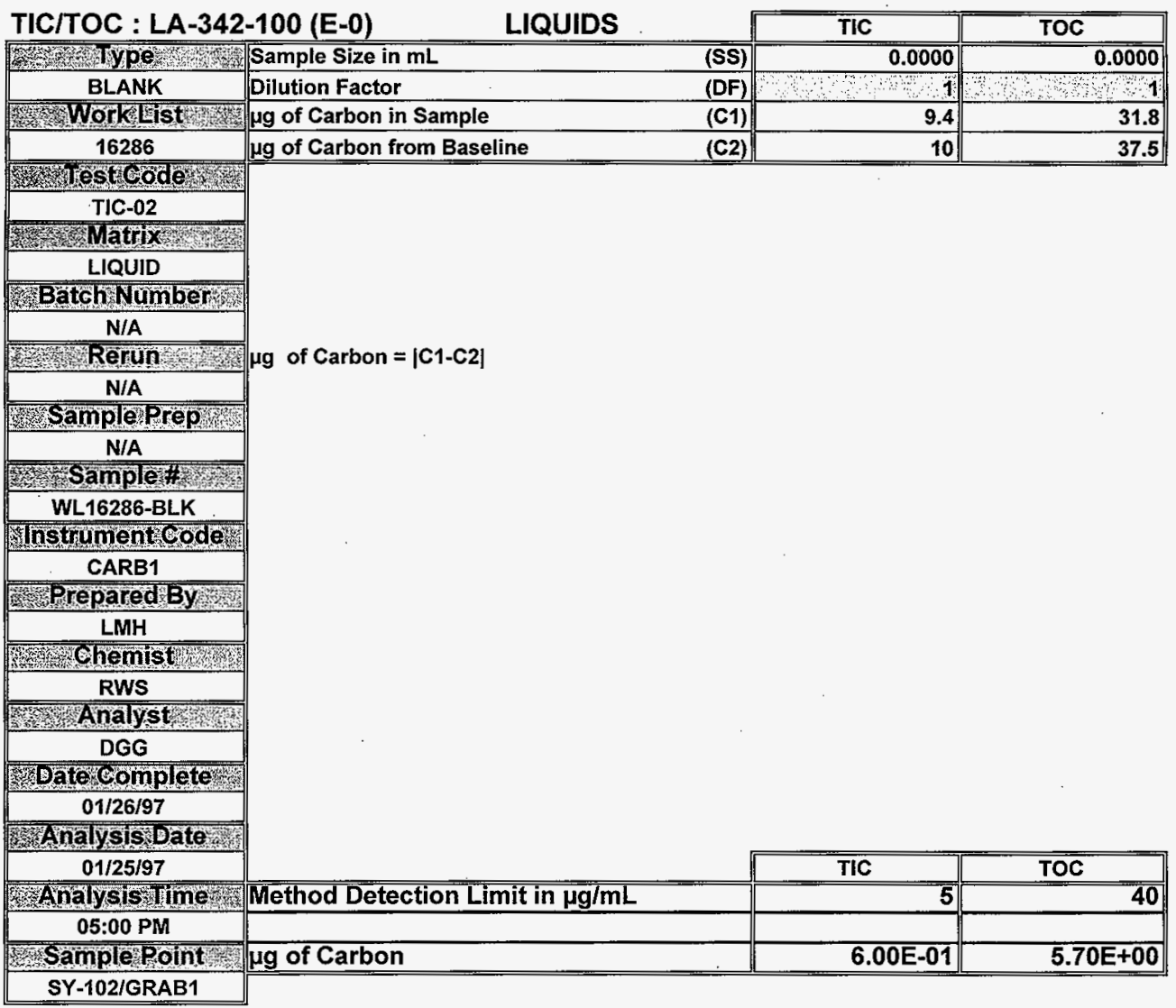

\begin{tabular}{|c|c|c|}
\hline Data Entered By: & $\mathrm{LMH}$ & $01 / 26 / 97$ \\
\hline Signature of Chemist: & Ru scindedu & Date: $1 / 27 / 97$ \\
\hline
\end{tabular}

\section{1}




\begin{tabular}{|c|c|c|c|}
\hline \multirow{2}{*}{\multicolumn{2}{|c|}{ TIC/TOC : LA-342-100 (E-0) LIQUIDS }} & \multirow[b]{2}{*}{ TIC } & \multirow[b]{2}{*}{ TOC } \\
\hline & & & \\
\hline 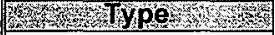 & Sample Size in $\mathrm{mL}$ & 1.0000 & 0.2000 \\
\hline STANDARD & Dilution Factor & 1 & 1 \\
\hline Work Wist & Final Coulometer Reading in $\mu \mathrm{g}$ & 603.2 & 612.2 \\
\hline 16286 & $\mu \mathrm{g}$ of Carbon from Baseline & 10 & 37.5 \\
\hline TestCode & Standard Book Number & 25N12A & $24 \mathrm{~N} 12 \mathrm{~F}$ \\
\hline TIC-02 & Standard Value $(\mu \mathrm{g} / \mathrm{ml})$ & 602 & 3000 \\
\hline WMatrix & \multirow{22}{*}{$\begin{array}{l}\text { QC Actual in } \mu \mathrm{g} / \mathrm{mL}=\text { Standard Value }(\mu \mathrm{g} / \mathrm{mL}) \\
\text { QC Found in } \mu \mathrm{g} / \mathrm{mL}=(C 1-\mathrm{C} 2)^{*} \mathrm{DF} / \mathrm{SS} \\
\text { QC Found in } \mu \mathrm{g} / \mathrm{mL} \text { for TIC }=5 \text { if } \mathrm{C} 1<\mathrm{C2} \\
\text { QC Found in } \mu \mathrm{g} / \mathrm{mL} \text { for TOC }=40 \text { if } \mathrm{C} 1<\mathrm{C} 2\end{array}$} & & \\
\hline LIQUID & & & \\
\hline 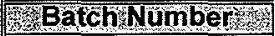 & & & \\
\hline N/A & & & \\
\hline Rerun & & & \\
\hline N/A & & & \\
\hline Sampleprep & & & \\
\hline N/A & & & \\
\hline Sample\# & & & \\
\hline WL16286-STD & & & \\
\hline Instrüment Code & & & \\
\hline CARB1 & & & \\
\hline Arepared By & & & \\
\hline LMH & & & \\
\hline Chemist & & & \\
\hline RWS & & & \\
\hline R. Analyst & & & \\
\hline DGG & & & \\
\hline Date complete & & & \\
\hline $01 / 26 / 97$ & & & \\
\hline Analysis Date & & & \\
\hline $01 / 25 / 97$ & & TIC & TOC \\
\hline Analysis & Method Detection Limit in $\mu \mathrm{g} / \mathrm{mL}$ & 5 & 40 \\
\hline 05:00 PM & QC Actual in $\mu \mathrm{g} / \mathrm{mL}$ & $6.02 E+02$ & $3.00 E+03$ \\
\hline Sample Point & QC Found in $\mu \mathrm{g} / \mathrm{mL}$ & $5.93 E+02$ & $2.87 E+03$ \\
\hline SY-102/GRAB1 & Percent Standard Recovery & 98.5 & 95.8 \\
\hline
\end{tabular}

\begin{tabular}{|c|c|c|c|}
\hline Data Entered By: & $\mathrm{LMH}$ & Date: & $01 / 26 / 97$ \\
\hline Signature of Chemist: & ive sanoedk & Date: & $1 / 27 / 97$ \\
\hline
\end{tabular}


HNF-SD-WM-DP-227, REV. 0

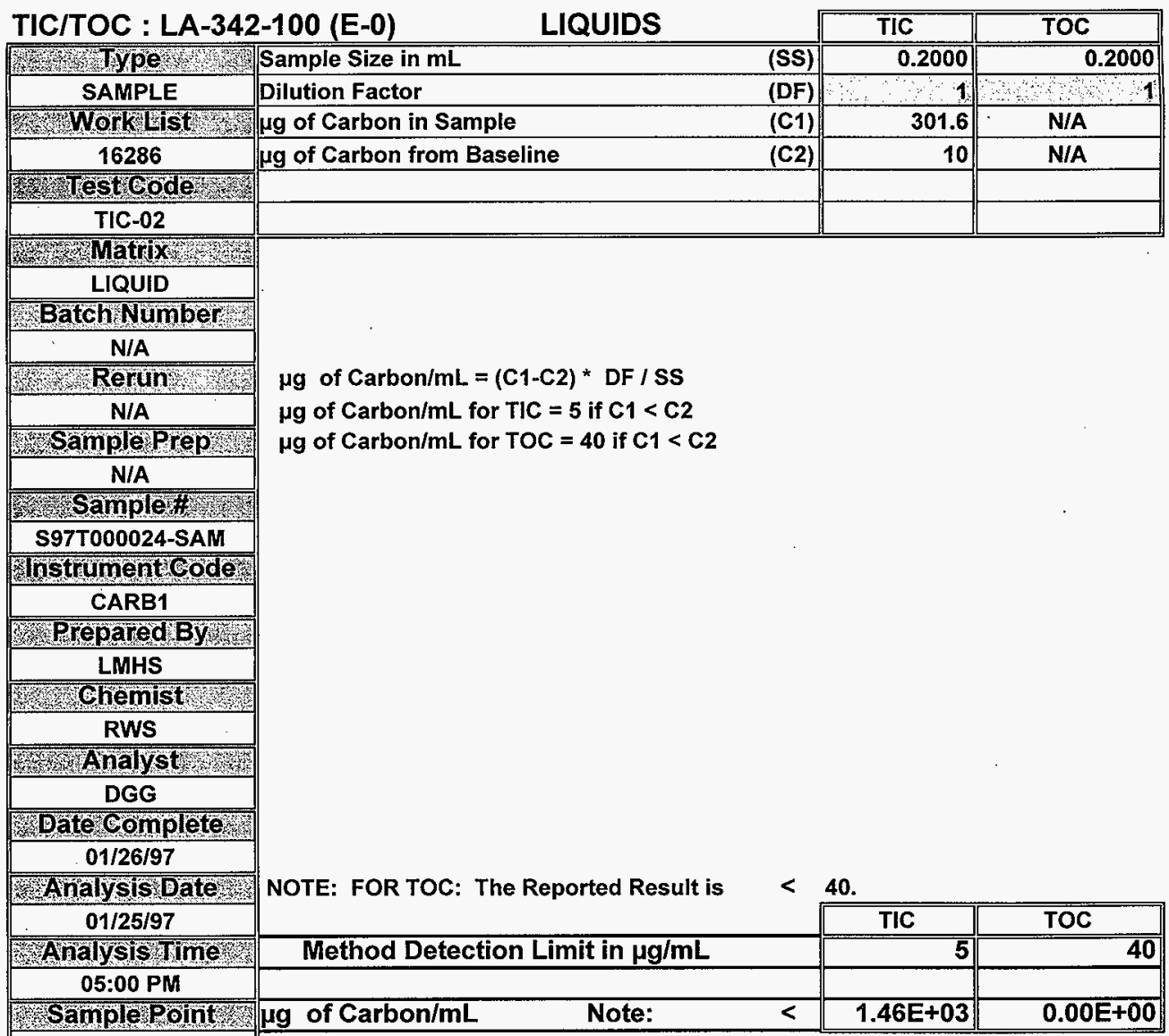

SY-102/GRAB

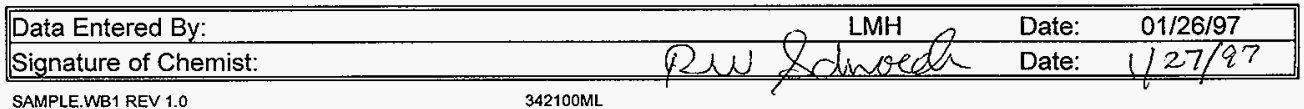

\section{3}




\begin{tabular}{|c|c|c|c|}
\hline TIC/TOC : LA-342 & $2-100(E-0)$ & TIC & TOC \\
\hline 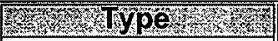 & Sample Size in $\mathrm{mL}$ & 0.2000 & N/A \\
\hline DUP & Dilution Factor & - 12 & +1 \\
\hline 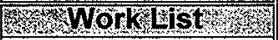 & $\mu \mathrm{g}$ of Carbon in Sample & 311.1 & N/A \\
\hline 16286 & pg of Carbon from Baseline & 10 & N/A \\
\hline Westeoder & Known $\mu \mathrm{g}$ of C from Original Sample & & \\
\hline TIC-02 & & & \\
\hline Satrix & \multirow{22}{*}{$\begin{array}{l}\mu g \text { of Carbon } / \mathrm{mL}=\left(C_{1}-C_{2}\right) * D F / S S \\
\mu g \text { of Carbon } / \mathrm{mL} \text { for TIC }=5 \text { if } \mathrm{C} 1<\mathrm{C} 2 \\
\mu \mathrm{g} \text { of Carbon } / \mathrm{mL} \text { for TOC }=40 \text { if } \mathrm{C} 1<\mathrm{C} 2\end{array}$} & & \\
\hline LIQUID & & & \\
\hline BatchNumber & & & \\
\hline N/A & & & \\
\hline 2 Rerun & & & \\
\hline N/A & & & \\
\hline Sampleprep & & & \\
\hline N/A & & & \\
\hline SW Sample & & & \\
\hline S97T000024-DUP & & & \\
\hline Snstrumentedede & & & \\
\hline CARB1 & & & \\
\hline PreparedBy & & & \\
\hline LMH & & & \\
\hline Whist & & & \\
\hline RWS & & & \\
\hline 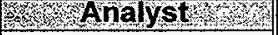 & & & \\
\hline DGG & & & \\
\hline Datercomplete & & & \\
\hline $01 / 26 / 97$ & & & \\
\hline Analysis bate & & \multirow{2}{*}{$\begin{array}{l}\text { ERR } \\
\text { TIC }\end{array}$} & \\
\hline $01 / 26 / 97$ & & & TOC \\
\hline Analys is Timo & Method Detection Limit in $\mu \mathrm{g} / \mathrm{mL}$ & 5 & 40 \\
\hline 05:00 PM & & & \\
\hline Sample Point & ERR ${ }^{\star \star \star \star \star \star}$ & $1.51 E+03$ & ERR \\
\hline
\end{tabular}

\begin{tabular}{|c|c|c|c|}
\hline Data Entered By: & $\ldots \mathrm{MH}$ & Date: & $01 / 26 / 97$ \\
\hline Signature of Chemist: & revesoweoth & Date: & $1 / 27 / 97$ \\
\hline
\end{tabular}

\section{4:}




\begin{tabular}{|c|c|c|c|}
\hline TIC/TOC : LA-342 & $2-100(E-0)$ & TIC & TOC \\
\hline $3 y_{2}$ & Sample Size in $\mathrm{mL}$ & 0.2000 & N/A \\
\hline SAMPLE & Dilution Factor & 11 & m \\
\hline Work & $\mu \mathrm{g}$ of Carbon in Sample & 371.4 & N/A \\
\hline 16286 & $\mu g$ of Carbon from Baseline & 10 & N/A \\
\hline 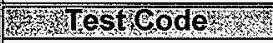 & & & \\
\hline TIC-02 & & & \\
\hline X & \multirow{22}{*}{$\begin{array}{l}\mu g \text { of Carbon } / \mathrm{mL}=(C 1-C 2) * D F / S S \\
\mu g \text { of Carbon } / \mathrm{mL} \text { for } \mathrm{TIC}=5 \text { if } \mathrm{C} 1<\mathrm{C} 2 \\
\mu \mathrm{g} \text { of Carbon } / \mathrm{mL} \text { for TOC }=40 \text { if } \mathrm{C} 1<\mathrm{C} 2\end{array}$} & & \\
\hline LIQUID & & & \\
\hline Batch Numbers & & & \\
\hline N/A & & & \\
\hline 26rin Rerun & & & \\
\hline N/A & & & \\
\hline Sample Prep & & & \\
\hline N/A & & & \\
\hline Whampleth & & & \\
\hline S97T000026-SAM & & & \\
\hline Listrument Code & & & \\
\hline CARB1 & & & \\
\hline Srepared By & & & \\
\hline LMH & & & \\
\hline Chemist & & & \\
\hline RWS & & & \\
\hline Analyst & & & \\
\hline DGG & & & \\
\hline Date complete & & & \\
\hline $01 / 26 / 97$ & & & \\
\hline Analysis Date & & \multirow{2}{*}{$\begin{array}{l}\text { ERR } \\
\text { TIC }\end{array}$} & \\
\hline $01 / 26 / 97$ & & & TOC \\
\hline Analysis Time & Method Detection Limit in $\mu \mathrm{g} / \mathrm{mL}$ & 5 & 40 \\
\hline \multicolumn{4}{|l|}{ 05:00 PM } \\
\hline Samplepoint & ERR $* * \star * *$ & $1.81 \mathrm{E}+03$ & ERR \\
\hline SY-102/GRAB1 & & & \\
\hline
\end{tabular}

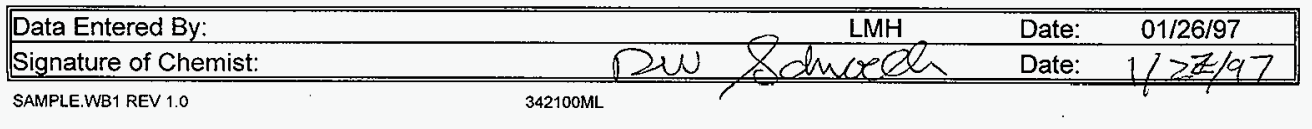




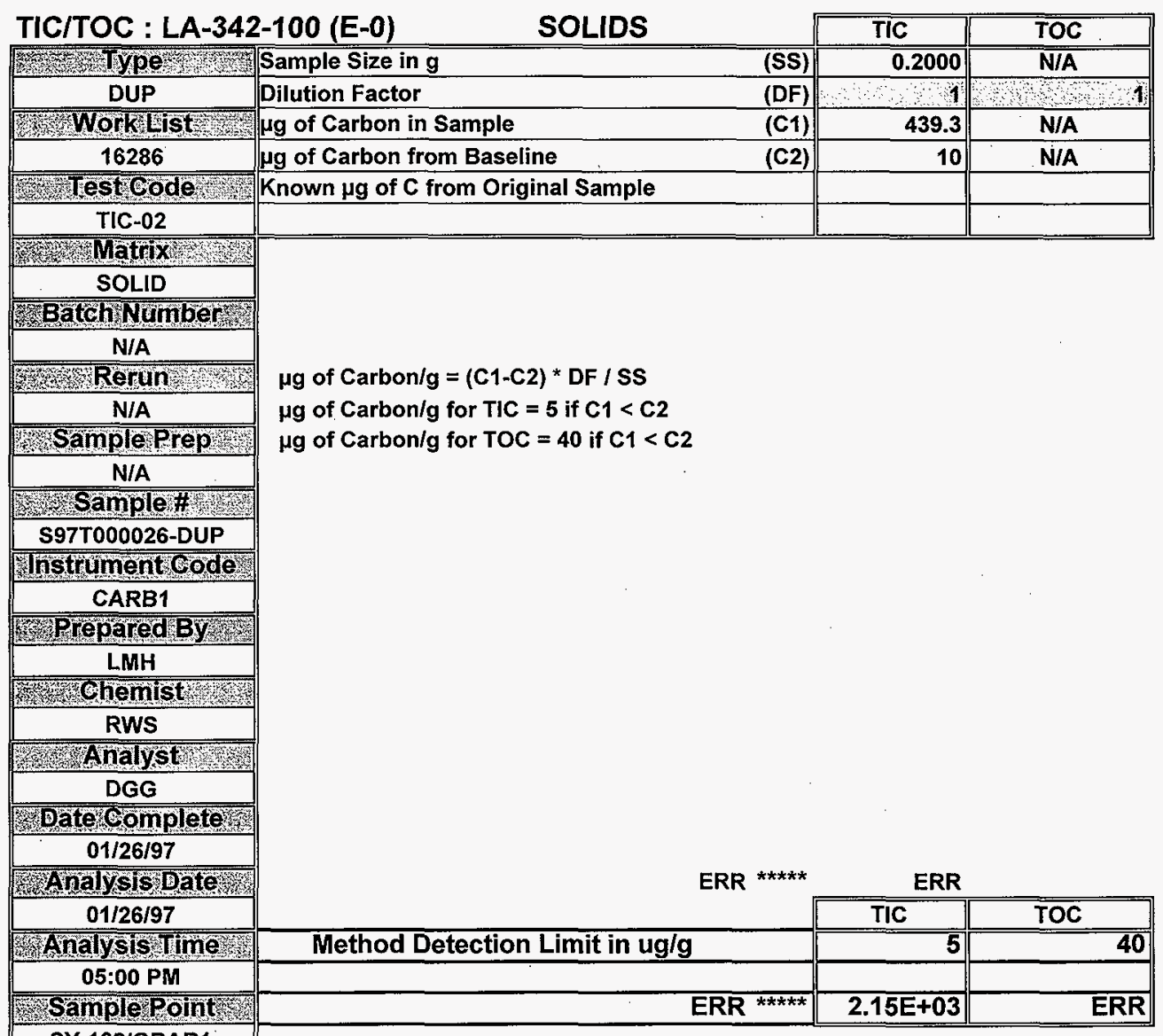

\begin{tabular}{|c|c|c|c|c|}
\hline Data Entered By: & & LMH $\Omega$ & Date: & $01 / 26 / 97$ \\
\hline Signature of Chemist: & Re & Sdiveses & Date: & $1 / 27 / 97$ \\
\hline
\end{tabular}




\section{TIC/TOC : LA-342-100 (E-0) LIQUIDS}

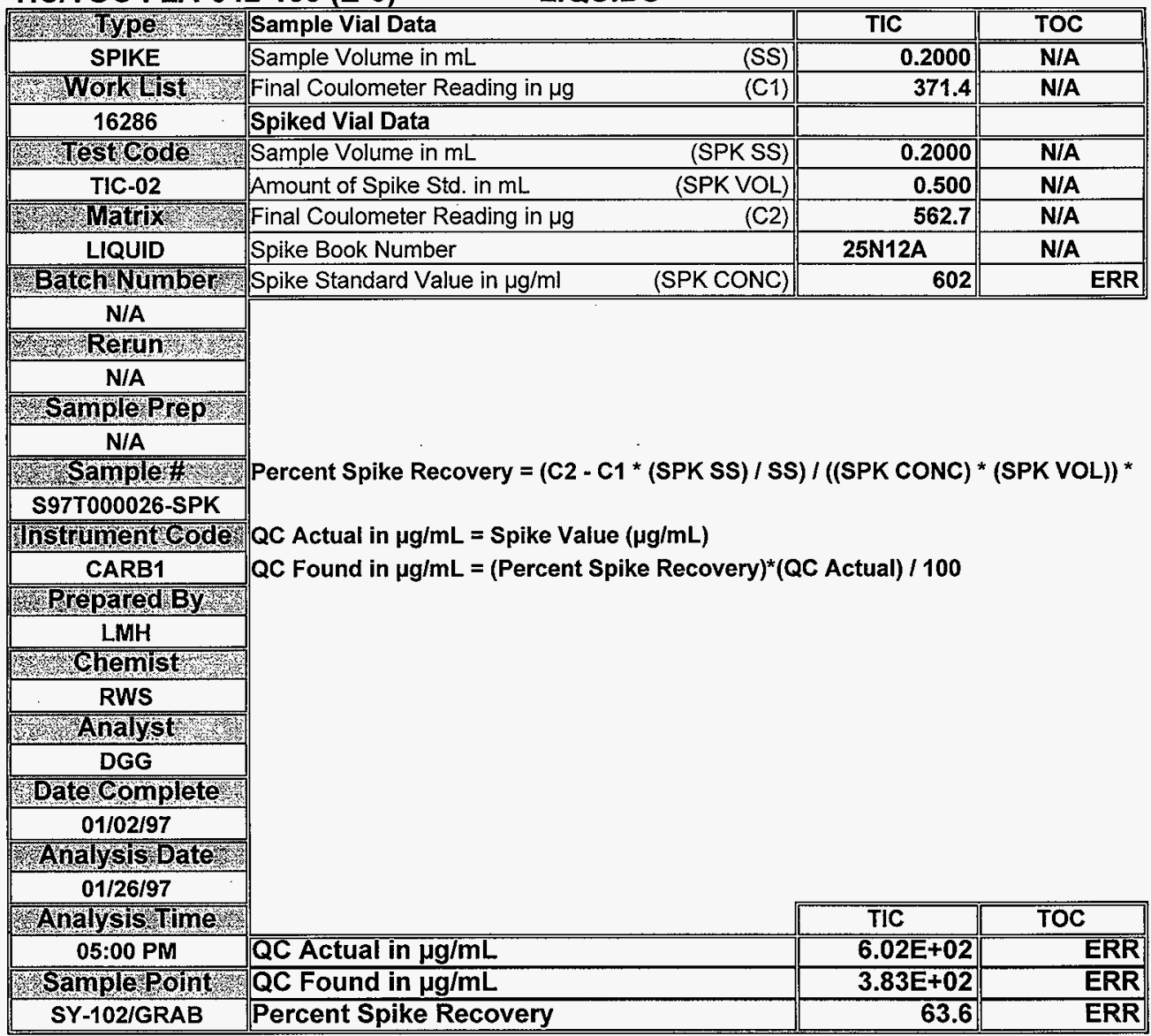




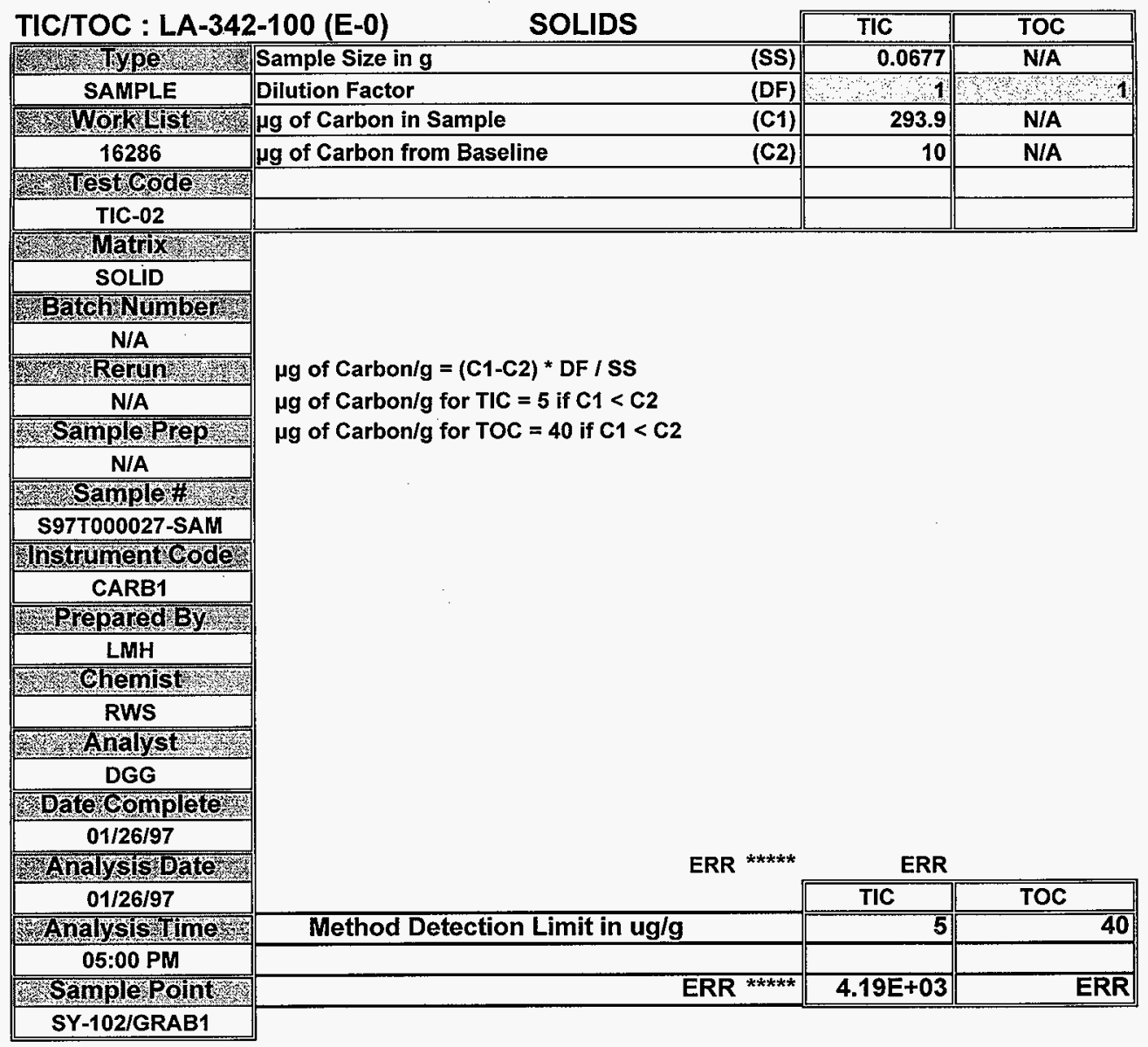

\begin{tabular}{|c|c|c|}
\hline Data Entered By: & LLMH & Date: $\quad 01 / 26 / 97$ \\
\hline Signature of Chemist: & RU Xcholed & Date: $/ / 27 / 97$ \\
\hline SAMPLE.WB1 REV 1.0 & \multicolumn{2}{|c|}{ SAMPLE.WB1 REV 1.0} \\
\hline \multicolumn{3}{|c|}{138} \\
\hline I:134210016286.WB1 & 12:19:48 AM & \\
\hline
\end{tabular}




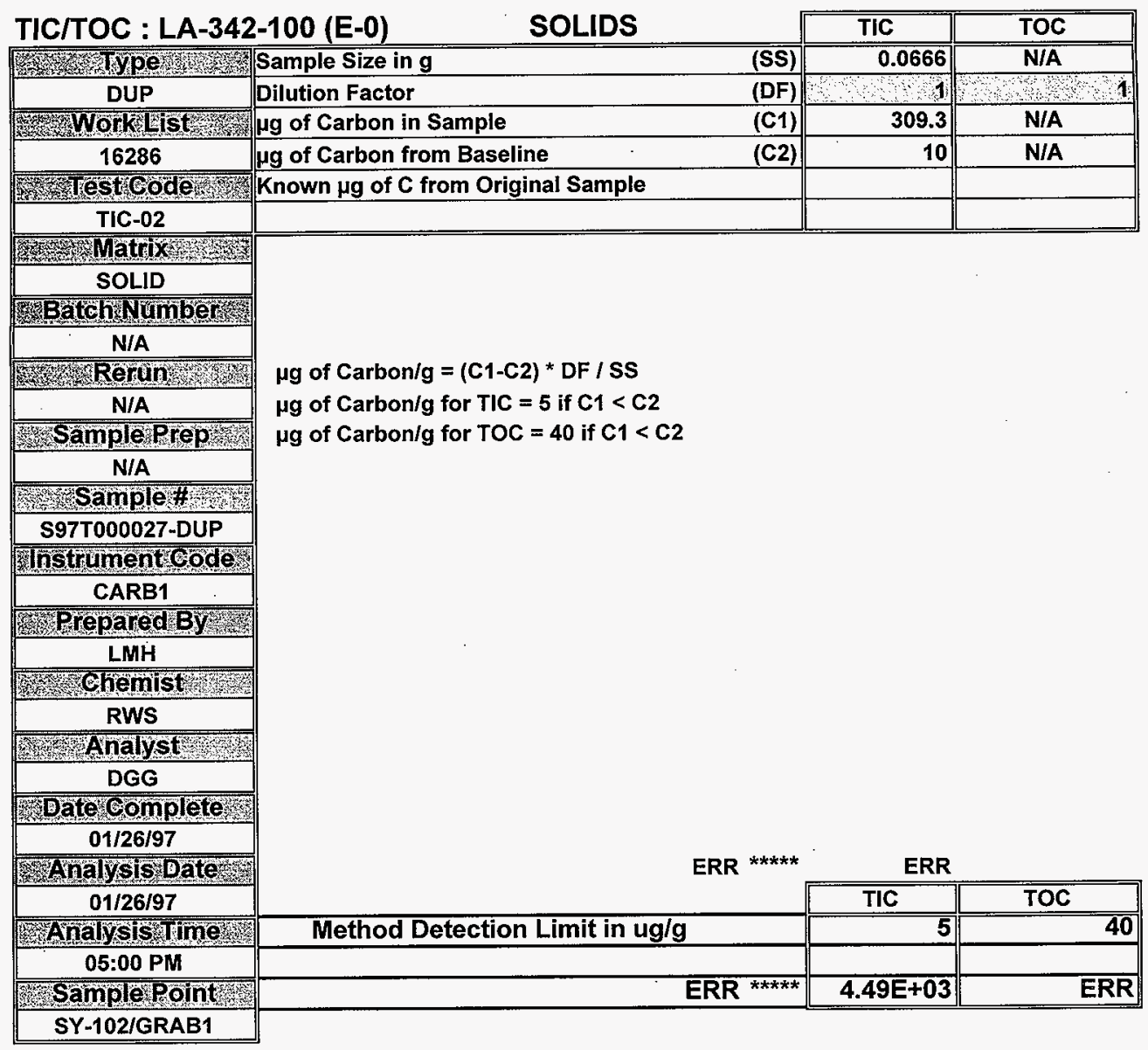

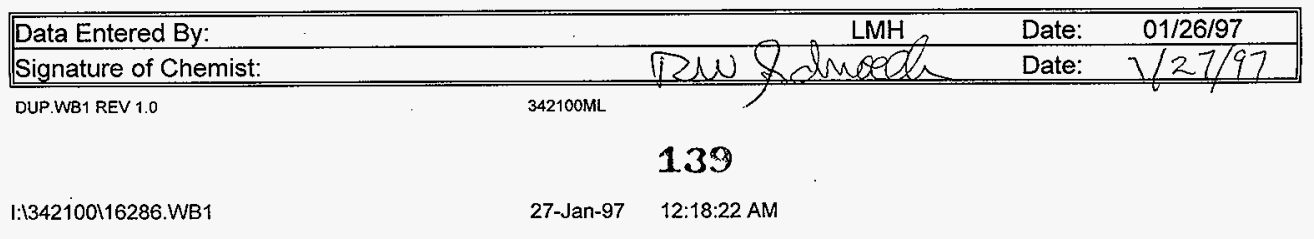




\section{LABCORE Data Entry Template for Worklist\# 16286}

\section{Analyst: \\ QP 8 Instrument: TIC01}

Method: LA-342-100 Rev/Mod E-O

Worklist Comment: SY-102 Grab. Std: TICSTD 1.0ml.

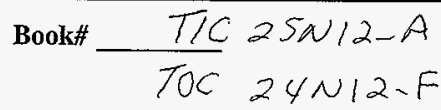

new

S Type Sample\# R A Test Matrix Group\# Project

1 BLNK

TIC-02 LIQUID

2 STD

TIC-02 LIQUID

3 SAMPLE

S97T000024 0

TIC-02 LIQUID

97000022 SY-102 GRAB1

Analytes Requested: TIC-02

4 DUP

$$
\text { S97T0000240 }
$$

TIC-02 IIQUID

5 SAMPLE

S97T000026 0 TIC-02 LIQUID

Analytes Requested: TIC-02

97000022 SY -102 GRAB1

6 DUP

S97T000026 O TIC-02 LIQUID

. 7 SPK

S97T000026 0

TIC-02 LIQUID

8 SAMPLE

S97T000027 0

TIC-02 LIQUID

Analytes Requested: TIC-02

97000022 SY-102 GRAB1

9 DUP

S97T000027 0 TIC-02 LIOUID

\section{Final page for worklist \# 16286}
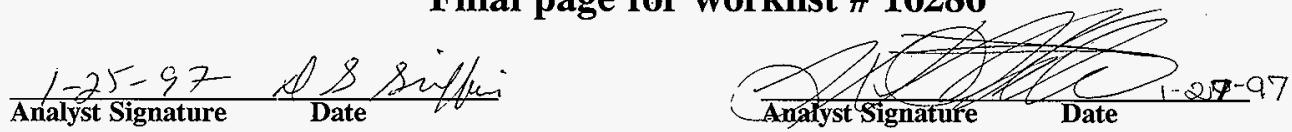

Data Entry Comments:

\section{LAd a bad problean with}

GRePN. Went to smaller sample sizes.

SAmale 4 is $5977000024+26$ are yellow 410,5977000027 is a BRownsladget $S=$ Worklist Slot Number, $R=$ Replicate Number, $A=$ Aliquot Code.

EnNat pipette. 
TIC- TOTAL INORGANIC CARBON ANALYSIS REPORT TICTOC REV 2.0

$\ll$ BLANK ANALYSIS $\gg>$

Sample: BASELINE

Sample Size = 1 uL

Dil Factor $=1$

Blank ID \# = BASELINE

Blank Value $=\mathrm{N} / \mathrm{A}$
Date: $01 / 25 / 97$

Time: $09: 22: 47$

$\begin{array}{crcc}=\text { Reading }===\text { Analysis } \text { Time }==0 \text { Coulometer }=== & \text { Difference }== \\ 1 & 0.51 & 0.40 & 0.00 \\ 2 & 1.01 & 0.80 & 50.00 \\ 3 & 1.51 & 1.30 & 38.46 \\ 4 & 2.00 & 1.90 & 31.58 \\ 5 & 2.50 & 2.50 & 24.00 \\ 6 & 3.00 & 3.00 & 16.67 \\ 7 & 3.50 & 3.50 & 14.29 \\ 8 & 4.00 & 4.00 & 12.50 \\ 9 & 4.50 & 4.50 & 11.11 \\ 10 & 5.00 & 5.00 & 10.00 \\ 11 & 5.50 & 5.40 & 7.41 \\ 12 & 6.00 & 5.80 & 6.90 \\ 13 & 6.50 & 6.20 & 6.45 \\ 14 & 7.00 & 6.70 & 7.46 \\ 15 & 7.50 & 7.10 & 5.63 \\ 16 & 8.00 & 7.60 & 6.58 \\ 17 & 8.50 & 8.00 & 5.00 \\ 18 & 9.00 & 8.50 & 5.88 \\ 19 & 9.50 & 8.80 & 3.41 \\ 20 & 10.00 & 9.30 & 5.38 \\ 21 & 10.50 & 9.60 & 3.13 \\ 22 & 11.00 & 10.00 & 4.00\end{array}$

BLANK VALUE $=10$ micrograms carbon

Sample Run By:

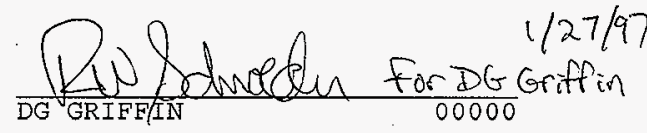


HNF-SD-WM-DP-227, REV. 0

TOC- TOTAL ORGANIC CARBON ANALYSIS REPORT

TICTOC REV 2.0

$\ll$ BLANK ANALYSIS $\gg>$

Sample: BASELINE

Sample Size = 1 uL

Dil Factor $=1$

Blank ID \# = BASELINE

Blank Value $=\mathrm{N} / \mathrm{A}$
Date: $01 / 25 / 97$

$==$ Read 1

$\begin{array}{rr}1 & 0.51 \\ 2 & 1.01 \\ 3 & 1.50 \\ 4 & 2.00 \\ 5 & 2.50 \\ 6 & 3.00 \\ 7 & 3.50 \\ 8 & 4.00 \\ 9 & 4.50 \\ 10 & 5.00 \\ 11 & 5.50 \\ 12 & 6.00 \\ 13 & 6.50 \\ 14 & 7.00 \\ 15 & 7.50 \\ 16 & 8.00 \\ 17 & 8.50 \\ 18 & 9.00 \\ 19 & 9.50 \\ 20 & 10.00 \\ 21 & 10.50 \\ 22 & 11.00\end{array}$

Time $===\begin{gathered}\text { Coulometer }==== \\ 0.20\end{gathered}$

0.70

3.40

10.50

17.70

23.30

27.10

28.70

30.00

30.70

31.40

32.40

33.20

33.70

34.30

34.70

35.30

35.70

36.10

36.60

37.10

37.50

Time: 09:42:45

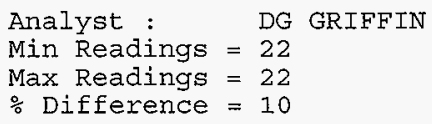

- Difference $=10$

Difference $==$
0.00
71.43
79.41
67.62
40.68
24.03
14.02
5.57
4.33
2.28
2.23
3.09
2.41
1.48
1.75
1.15
1.70
1.12
1.11
1.37
1.35
1.07

BLANK VALUE $=37.5$ micrograms carbon

BLANK FACTOR $=37.5 / 10.99799=+3.41 \mathrm{E}+00 \quad \mathrm{ug} / \mathrm{min}$ Carbon

$\ll<$ WARNING - BLANK VALUE EXCEEDS $1.5 \mathrm{ug} / \mathrm{min}$ Carbon!!!!! $>>>$ 
TIC- TOTAL INORGANIC CARBON ANALYSIS REPORT TICTOC REV 2.0

Sample: STD 25N12-A

Sample Size $=1000 \mathrm{uL}$ Dil Factor $=1$ Blank ID \# = Blank Value $=.909 \mathrm{ug} /$ minute $\mathrm{C}$
Time: $10: 38: 45$

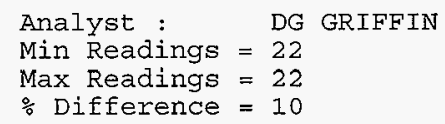

$\begin{array}{crcc}\text { Reading }===\text { Analysis } & \text { Time }===\text { Coulometer }==0 \% \text { Difference }== \\ 1 & 0.51 & 0.00 & 0.00 \\ 2 & 1.01 & 0.60 & 100.00 \\ 3 & 1.51 & 18.00 & 96.67 \\ 4 & 2.00 & 111.00 & 83.78 \\ 5 & 2.50 & 258.90 & 57.13 \\ 6 & 3.00 & 397.00 & 34.79 \\ 7 & 3.50 & 493.50 & 19.55 \\ 8 & 4.00 & 551.50 & 10.52 \\ 9 & 4.50 & 582.90 & 5.39 \\ 10 & 5.00 & 593.30 & 1.75 \\ 11 & 5.50 & 596.90 & 0.60 \\ 12 & 6.00 & 598.00 & 0.18 \\ 13 & 6.50 & 598.90 & 0.15 \\ 14 & 7.00 & 599.40 & 0.08 \\ 15 & 7.50 & 599.90 & 0.08 \\ 16 & 8.00 & 600.50 & 0.10 \\ 17 & 8.50 & 601.00 & 0.08 \\ 18 & 9.00 & 601.40 & 0.07 \\ 19 & 9.50 & 601.90 & 0.08 \\ 20 & 10.00 & 602.30 & 0.07 \\ 21 & 10.50 & 602.80 & 0.08 \\ 22 & 11.00 & 603.20 & 0.07\end{array}$

USER INPUT BLANK VALUE

BLANK VALUE $=9.997169$ micrograms carbon

BLANK FACTOR $=9.997169 / 10.99799=$

+9.1E-01 ug/min Carbon

SAMPLE RESULTS:

$\begin{array}{llll}(603.2-9.996225)(1) /(1000) & = & +5.932 \mathrm{E}-01 & \mathrm{~g} / \mathrm{L} \text { Carbon } \\ (603.2-9.996225)(1) /(1000)(12)= & +4.943 \mathrm{E}-02 \text { Molar Carbon }\end{array}$

Sample Run By:

\begin{tabular}{ll}
\hline DG GRIFFIN & 00000
\end{tabular} 
TOC- TOTAL ORGANIC CARBON ANALYSIS REPORT TICTOC REV 2.0

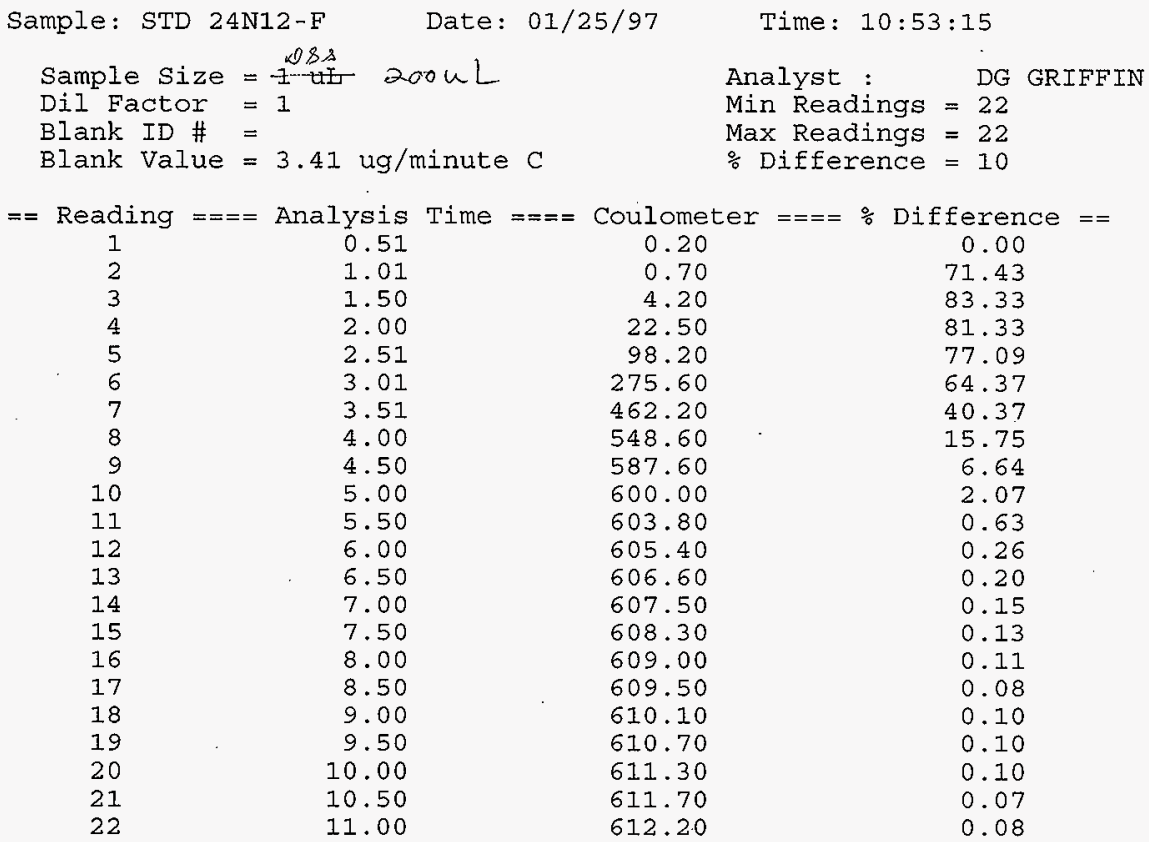

USER INPUT BLANK VALUE

BLANK VALUE $=37.50313$ micrograms carbon

BLANK FACTOR $=37.50313 / 10.99799=$

$+3.4 \mathrm{E}+00 \mathrm{ug} / \mathrm{min}$ Carbon

SAMPLE RESULTS:
$(612.2-37.50272)(1) /(1+D \sigma \sigma$
$(612.2-37.50272)$

$(612.2-37.50272)(1) /(1)(12)=$

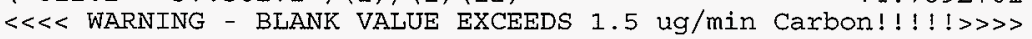




\section{TIC- TOTAL INORGANIC CARBON ANALYSIS REPORT TICTOC REV 2.0}

Sample: BLANK

$$
\begin{aligned}
& \text { Sample Size }=1 \mathrm{uL} \\
& \text { Dil Factor }=1 \\
& \text { Blank ID \# }= \\
& \text { Blank Value }=.909 \mathrm{ug} / \text { minute } \mathrm{C}
\end{aligned}
$$

Date: $01 / 25 / 97$

Time: $11: 14: 00$

$\begin{array}{crcc}\text { Reading }===\text { Analysis } \text { Time }===\text { Coulometer }===0 & \text { Difference }== \\ 1 & 0.51 & 0.30 & 0.00 \\ 2 & 1.01 & 0.70 & 57.14 \\ 3 & 1.51 & 1.30 & 46.15 \\ 4 & 2.00 & 1.70 & 23.53 \\ 5 & 2.50 & 2.30 & 26.09 \\ 6 & 3.00 & 2.80 & 17.86 \\ 7 & 3.50 & 3.20 & 12.50 \\ 8 & 4.00 & 3.70 & 13.51 \\ 9 & 4.50 & 4.10 & 9.76 \\ 10 & 5.00 & 4.60 & 10.87 \\ 11 & 5.50 & 4.90 & 6.12 \\ 12 & 6.00 & 5.30 & 7.55 \\ 13 & 6.50 & 5.70 & 7.02 \\ 14 & 7.00 & 6.10 & 6.56 \\ 15 & 7.50 & 6.50 & 6.15 \\ 16 & 8.00 & 6.90 & 5.80 \\ 17 & 8.50 & 7.30 & 5.48 \\ 18 & 9.00 & 7.70 & 5.19 \\ 19 & 9.50 & 8.20 & 6.10 \\ 20 & 10.00 & 8.60 & 4.65 \\ 21 & 10.50 & 8.90 & 3.37 \\ 22 & 11.00 & 9.40 & 5.32\end{array}$

USER INPUT BLANK VALUE

BLANK VALUE $=9.997169$ micrograms carbon

BLANK FACTOR $=9.997169 / 10.99799=$

+9.1E-01 ug/min Carbon

SAMPLE RESULTS:

$\begin{array}{llll}(9.4-9.997946)(1) /(1) & = & <5.00 \mathrm{~g}-3 \mathrm{~g} / \mathrm{L} \text { Carbon } \\ (9.4-9.997946)(1) /(1)(12)= & <4.17 \text { E-4 Molar Carbon }\end{array}$

Sample Run By:

$\overline{\text { DG GRIFFIN }} 00000$ 
TOC- TOTAL ORGANIC CARBON ANALYSIS REPORT TICTOC REV 2:0

Sample: BIANK

$$
\begin{aligned}
& \text { Sample Size }=1 \mathrm{uL} \\
& \text { Dil Factor }=1 \\
& \text { Blank ID \# }= \\
& \text { Blank Value }=3.41 \mathrm{ug} / \text { minute C }
\end{aligned}
$$

Date: $01 / 25 / 97$

Time: $11: 55: 45$

$\begin{array}{crcc}\text { Reading }===\text { Analysis } \text { Time }==0 & \text { Coulometer }==0 & \text { Difference }== \\ 1 & 0.51 & 1.40 & 0.00 \\ 2 & 1.01 & 2.20 & 36.36 \\ 3 & 1.51 & 7.50 & 70.67 \\ 4 & 2.00 & 15.00 & 50.00 \\ 5 & 2.50 & 20.30 & 26.11 \\ 6 & 3.00 & 22.80 & 10.96 \\ 7 & 3.51 & 23.90 & 4.60 \\ 8 & 4.00 & 24.70 & 3.24 \\ 9 & 4.50 & 25.50 & 3.14 \\ 10 & 5.00 & 26.00 & 1.92 \\ 11 & 5.50 & 26.50 & 1.89 \\ 12 & 6.00 & 27.00 & 1.85 \\ 13 & 6.50 & 27.60 & 2.17 \\ 14 & 7.00 & 27.90 & 1.08 \\ 15 & 7.50 & 28.50 & 2.11 \\ 16 & 8.00 & 28.90 & 1.38 \\ 17 & 8.50 & 29.40 & 1.70 \\ 18 & 9.00 & 29.90 & 1.67 \\ 19 & 9.50 & 30.40 & 1.64 \\ 20 & 10.00 & 30.90 & 1.62 \\ 21 & 10.50 & 31.30 & 1.28 \\ 22 & 11.00 & 31.80 & 1.57\end{array}$

USER INPUT BLANK VALUE

BLANK VALUE $=37.50313$ micrograms carbon

BLANK FACTOR $=37.50313 / 10.99799=+3.4 \mathrm{E}+00 \quad \mathrm{ug} / \mathrm{min}$ Carbon

SAMPLE RESULTS:
$(31.8-37.50334)(1) /(1)=$
$<5.00 \quad \mathrm{E}-3 \mathrm{~g} / \mathrm{L}$ Carbon
$(31.8-37.50334)(1) /(1)(12)=$
$<4.17 \quad \mathrm{E}-4$ Molar Carbon
$\ll<$ WARNING - BLANK VALUE EXCEEDS $1.5 \mathrm{ug} / \mathrm{min}$ Carbon!!!!! $\gg>>$ 
TIC- TOTAL INORGANIC CARBON ANALYSIS REPORT

TICTOC REV 2.0

Sample: S97T000024

$$
\begin{aligned}
& \text { Sample Size }=1 \mathrm{uL} \\
& \text { Dil Factor }=1 \\
& \text { Blank ID\# }= \\
& \text { Blank Value }=.909 \mathrm{ug} / \text { minute } \mathrm{C}
\end{aligned}
$$

Date: $01 / 25 / 97$
Time: $13: 38: 57$

$$
\begin{array}{ll}
\text { Analyst : } & \text { DG GRIFFIN } \\
\text { Min Readings }= & 22 \\
\text { Max Readings }= & 22 \\
\% \text { Difference }= & 10
\end{array}
$$

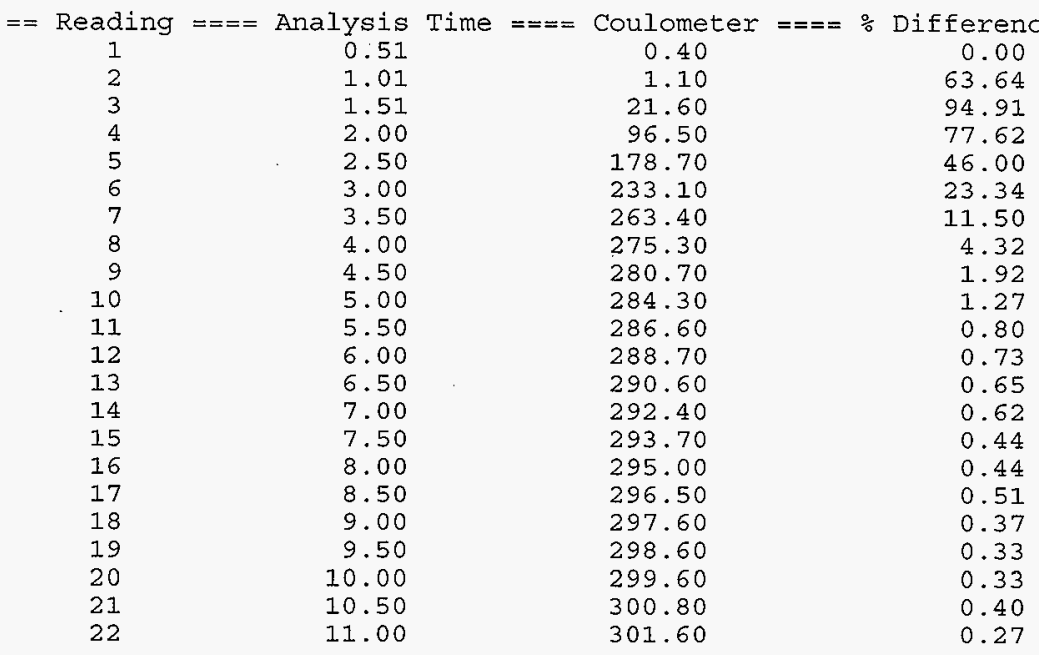

USER INPUT BLANK VALUE

BLANK VALUE $=9.997169$ micrograms carbon

BLANK FACTOR $=9.997169 / 10.99799=\quad+9.1 \mathrm{E}-01 \mathrm{ug} / \mathrm{min}$ Carbon

SAMPLE RESULTS:

$(301.6-9.995504)(1) /(1)=$

$(301.6-9.995504)(1) /(1)(12)=$
$+2.916 \mathrm{E}+02 \mathrm{~g} / \mathrm{L}$ Carbon

$+2.430 \mathrm{E}+01$ Molar Carbon

Sample Run By:

$\overline{\text { DG GRIFFIN } 00000}$


TIC- TOTAL INORGANIC CARBON ANALYSIS REPORT TICTOC REV 2.0

Sample: S97T000024DUP Date: 0I/25/97

Sample Size $=1 \mathrm{uL}$
Dil Factor $=1$
Blank ID \# $=$
Blank Value $=.909 \mathrm{ug} /$ minute C
Time: $13: 54: 08$

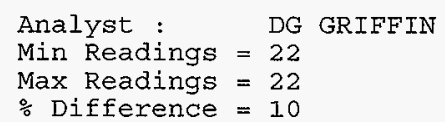

$==$ Reading $===$ Analysis Time $===$

Coulometer $====\frac{\circ}{0}$

Difference $==$

0.51

1.01

1.00

2.00

1.50

2.00

2.50

3.00

3.50

4.00

4.50

21.60

97.00

180.10

235.90

266.40

279.20

285.30

5.00

5.50

6.00

6.50

7.00

7.50

8.00

8.50

9.00

9.50

10.00

10.50

289.10

292.50

294.80

296.80

299.00

301.10

302.80

304.20

306.00

307.00

308.40

0.00

50.00

90.74

77.73

46.14

23.65

11.45

4.58

2.14

1.31

1.16

0.78

0.67

0.74

0.70

0.56

0.46

0.59

0.33

0.45

0.48

309.90

0.39

USER INPUT BLANK VALUE

BLANK VALUE $=9.997169$ micrograms carbon

BLANK FACTOR $=9.997169 / 10.99799=+9.1 \mathrm{E}-01 \quad \mathrm{ug} / \mathrm{min}$ Carbon SAMPLE RESULTS:

$(311.1-9.998667)(1) /(1)=$

$+3.011 \mathrm{E}+02$

$\mathrm{g} / \mathrm{L}$ Carbon

$+2.509 \mathrm{E}+01$ Molar Carbon

Sample Run By: 
HNF-SD-WM-DP-227, REV. 0

TIC- TOTAL INORGANIC CARBON ANALYSIS REPORT

TICTOC REV 2.0

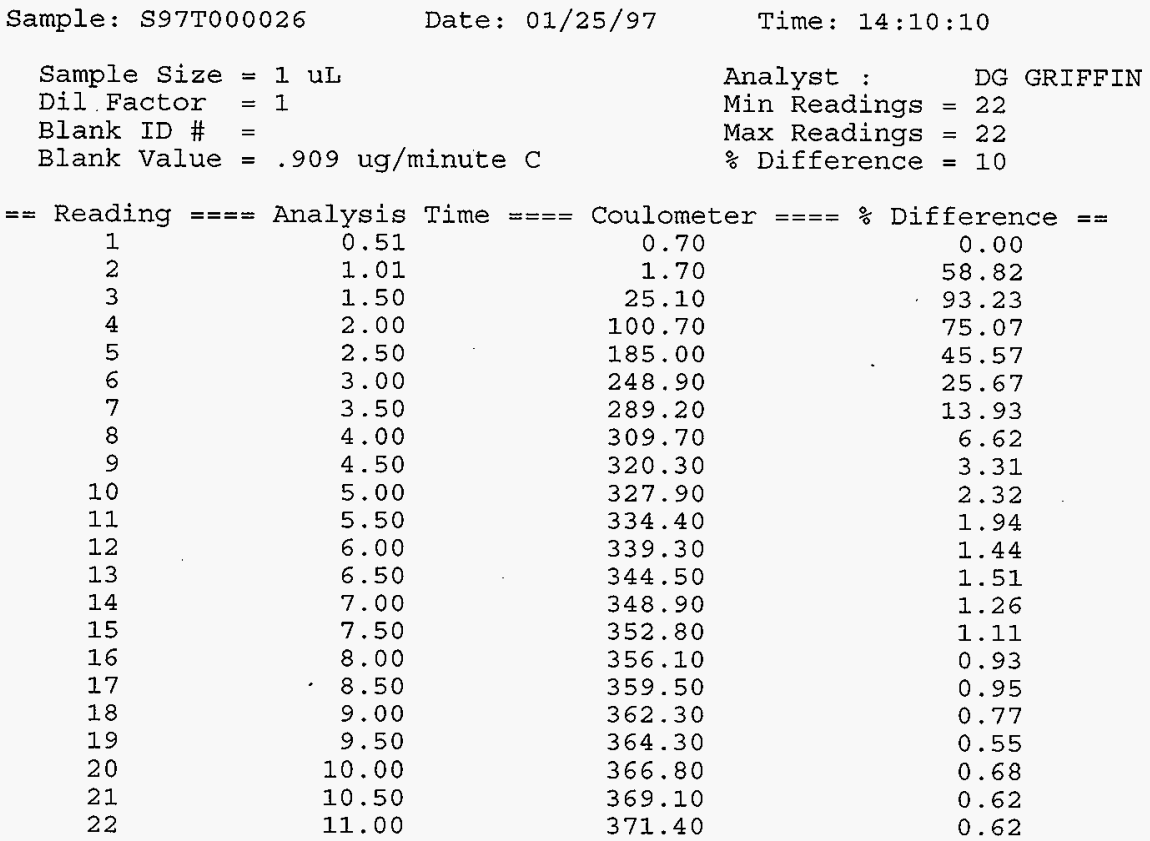

USER INPUT BLANK VALUE

BLANK VALUE $=9.997169$ micrograms carbon

BLANK FACTOR $=9.997169 / 10.99799=+9.1 \mathrm{E}-01 \quad \mathrm{ug} / \mathrm{min}$ Carbon SAMPLE RESULTS:
$(371.4-9.996282$
(1) $/(1)=$
$+3.614 \mathrm{E}+02$
g/L Carbon
$(371.4-9.996282)(1) /(1)(12)=$
$+3.012 \mathrm{E}+01$ Molar Carbon

Sample Run By:

$\overline{\text { DG GRIFFIN · }} 00000$


TIC- TOTAL INORGANIC CARBON ANALYSIS REPORT TICTOC REV 2.0

Sample: S97T000026DUP Date: 01/25/97 Time: 15:12:10

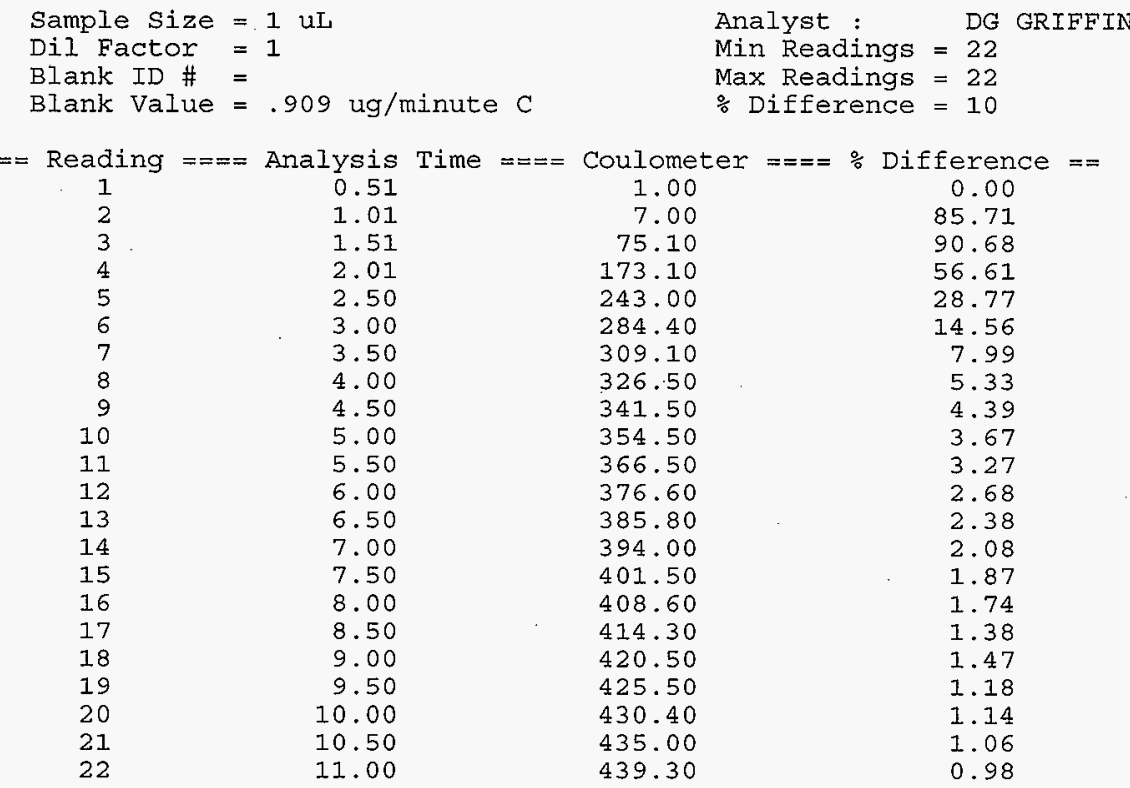

USER INPUT BLANK VALUE

BLANK VALUE $=9.997169$ micrograms carbon

BLANK FACTOR $=9.997169 / 10.99799=+9.1 \mathrm{E}-0 \mathrm{I} \mathrm{ug} / \mathrm{min}$ Carbon

SAMPLE RESULTS:

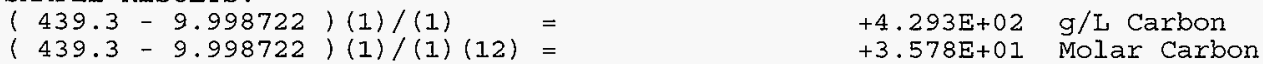

Sample Run By:

$\overline{D G}$ GRIFFIN 00000 
Sample: S97T000026SPK Date: 01/25/97

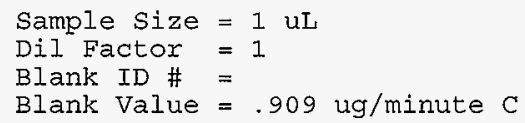

Time: $15: 51: 57$

$$
\begin{array}{ll}
\text { Analyst : } & \text { DG GRIFFIN } \\
\text { Min Readings }= & 22 \\
\text { Max Readings }= & 22 \\
\% \text { Difference }= & 10
\end{array}
$$

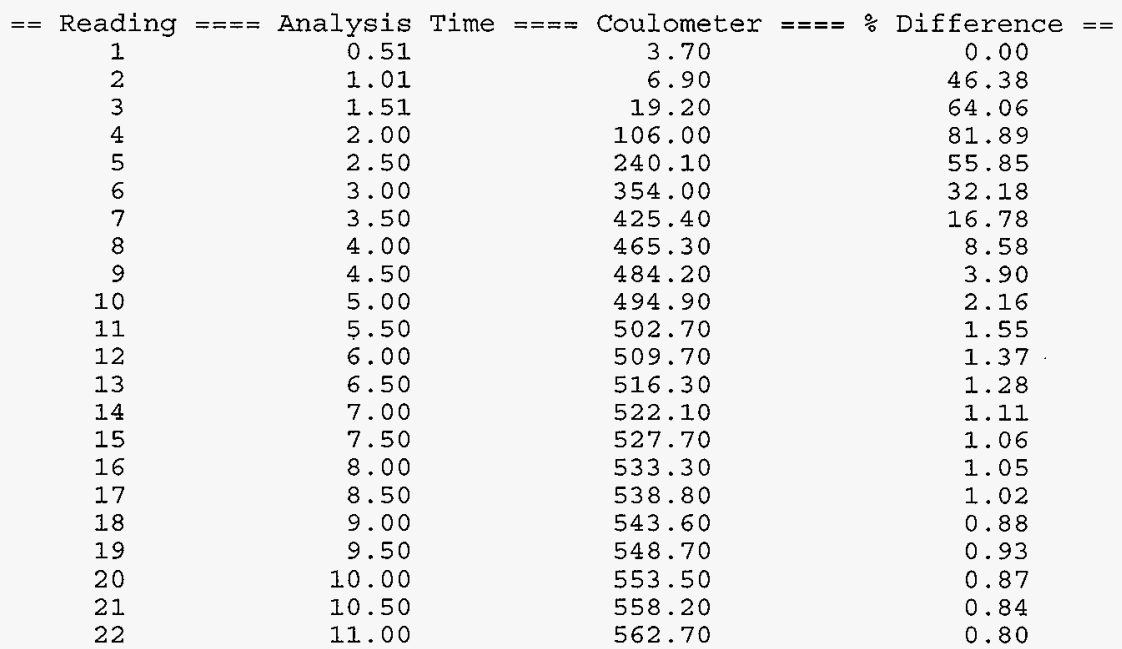

USER INPUT BLANK VALUE

BLANK VALUE $=9.997169$ micrograms carbon

BLANK FACTOR $=9.997169 / 10.99799=+9.1 \mathrm{E}-01 \mathrm{ug} / \mathrm{min}$ Carbon

SAMPLE RESULTS:

$\begin{array}{llll}(562.7-9.997003)(1) /(1) & = & +5.527 \mathrm{E}+02 & \mathrm{~g} / \mathrm{L} \text { Carbon } \\ 562.7-9.997003)(1) /(1)(12)= & & +4.606 \mathrm{E}+01 & \text { Molar Carbon }\end{array}$


HNF-SD-WM-DP-227, REV. 0

TIC- TOTAL INORGANIC CARBON ANALYSIS REPORT

TICTOC REV 2.0

Sample: 597 T000027

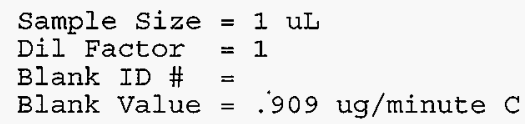

Time: $16: 12: 15$

$\begin{array}{ll}\text { Analyst : } & \text { DG GRIFFIN } \\ \text { Min Readings }= & 22 \\ \text { Max Readings }= & 22 \\ \% \text { Difference }= & 10\end{array}$

$\begin{array}{crcc}\text { Reading }===\text { Analysis Time }==0 & \text { Coulometer }=== & \text { Difference }== \\ 1 & 0.51 & 4.00 & 0.00 \\ 2 & 1.01 & 7.10 & 43.66 \\ 3 & 1.51 & 14.30 & 50.35 \\ 4 & 2.00 & 50.00 & 71.40 \\ 5 & 2.50 & 103.40 & 51.64 \\ 6 & 3.00 & 153.70 & 32.73 \\ 7 & 3.50 & 189.70 & 18.98 \\ 8 & 4.00 & 211.70 & 10.39 \\ 9 & 4.50 & 224.50 & 5.70 \\ 10 & 5.00 & 233.20 & 3.73 \\ 11 & 5.50 & 240.50 & 3.04 \\ 12 & 6.00 & 246.40 & 2.39 \\ 13 & 6.50 & 251.90 & 2.18 \\ 14 & 7.00 & 257.30 & 2.10 \\ 15 & 7.50 & 262.40 & 1.94 \\ 16 & 8.00 & 267.30 & 1.83 \\ 17 & 8.50 & 271.70 & 1.62 \\ 18 & 9.00 & 276.50 & 1.74 \\ 19 & 9.50 & 280.80 & 1.53 \\ 20 & 10.00 & 284.90 & 1.44 \\ 21 & 10.50 & 289.20 & 1.49 \\ 22 & 11.00 & 293.90 & 1.60\end{array}$

USER INPUT BLANK VALUE

BLANK VALUE $=9.997169$ micrograms carbon

BLANK FACTOR $=9.997169 / 10.99799=+9.1 \mathrm{E}-01 \mathrm{ug} / \mathrm{min}$ Carbon

SAMPLE RESULTS:

$\begin{array}{rlll}(293.9-9.995338)(1) /(1) & & +2.839 \mathrm{E}+02 & \mathrm{~g} / \mathrm{I} \text { Carbon } \\ 293.9-9.995338)(1) /(1)(12)= & & +2.366 \mathrm{E}+01 & \text { Molar Carbon }\end{array}$


HNF-SD-WM-DP-227, REV. 0

TIC- TOTAL INORGANIC CARBON ANALYSIS REPORT

TICTOC REV 2.0

Sample: S97T000027DUP Date: 01/25/97

Time: $16: 51: 32$

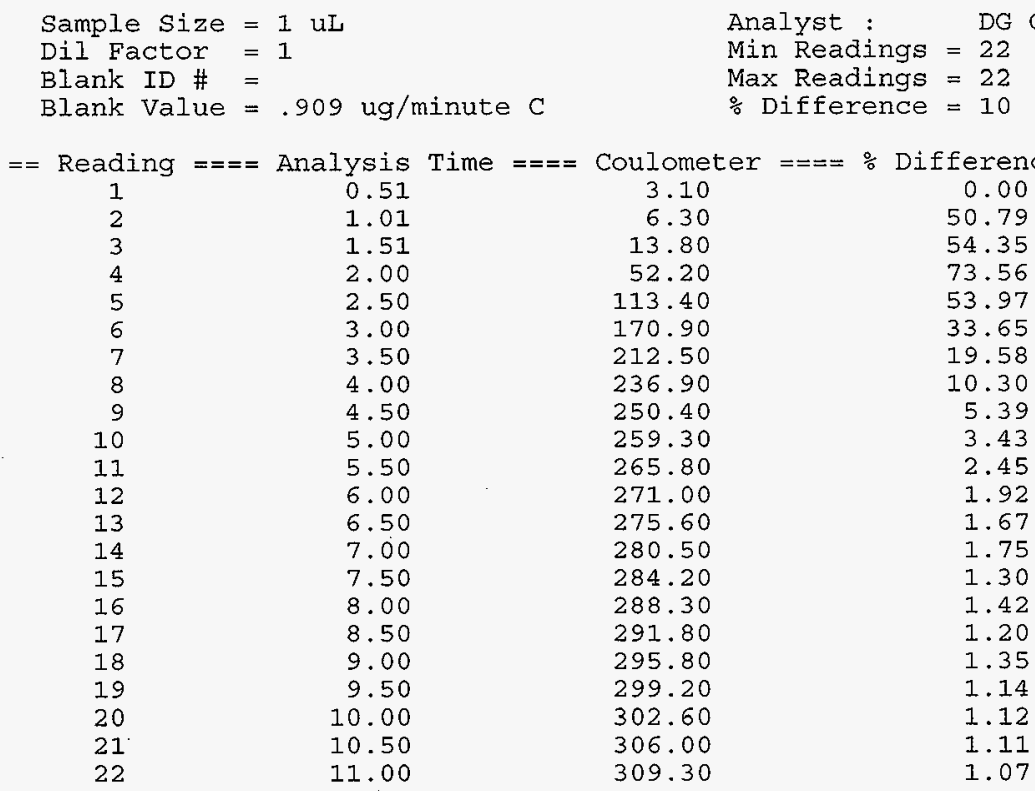

USER INPUT BLANK VALUE

BLANK VALUE $=9.997169$ micrograms carbon

BLANK FACTOR $=9.997169 / 10.99799=$

+9.1E-01 ug/min Carbon

SAMPLE RESULTS:

$(309.3-9.997169)(1) /(1)(12=$

$+2.993 \mathrm{E}+02 \mathrm{~g} / \mathrm{L}$ Carbon

$+2.494 \mathrm{E}+01$ Molar Carbon

Sample Run By: 


\section{LABCORE Completed Worklist Report for Worklist\# 16384}

Analyst: slh

Instrument: TIC01

Book\# TIC 25N12A

Method: $\angle A-342-100$ Rev/Mod E-O

Worklist Comment: SY-102 Grab. Run per procedure. Std: 1.0ml.

new

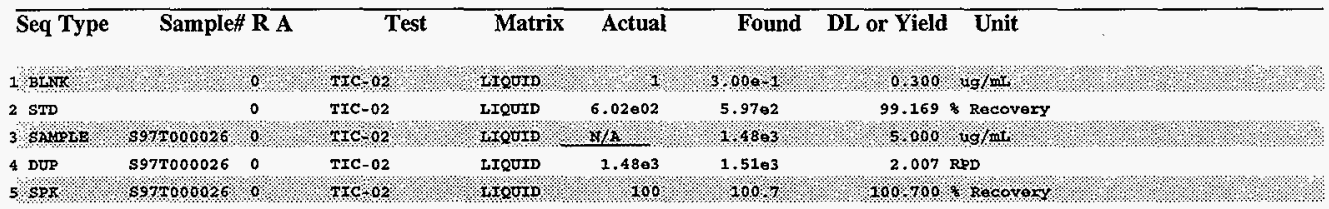

\section{Comments Section:}

Comments for sample\# S97T000026 and test TIC-02

HAD TO GO TO SMALLER SAMPLE SIZES DUE TO CELL TURNING GREEN

AND COLOR OF SAMPLE WAS YELLOW LIQUID.

\section{Final page for worklist\# 16384}

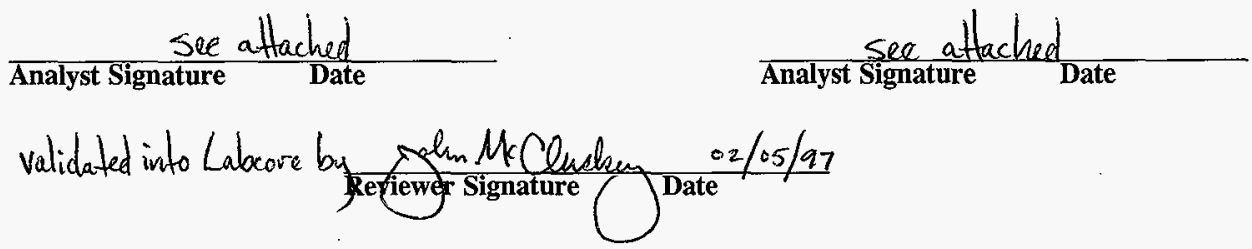




\section{LABCORE Data Entry Template for Worklist\# 16384}

Analyst: $\quad$ slh Instrument: TIC01 _ Book\# TIE 25N RA

Method: LA-342-100 Rev/Mod E-O

Worklist Comment: SY-102 Grab. Run per procedure. Std: $1.0 \mathrm{ml}$. new

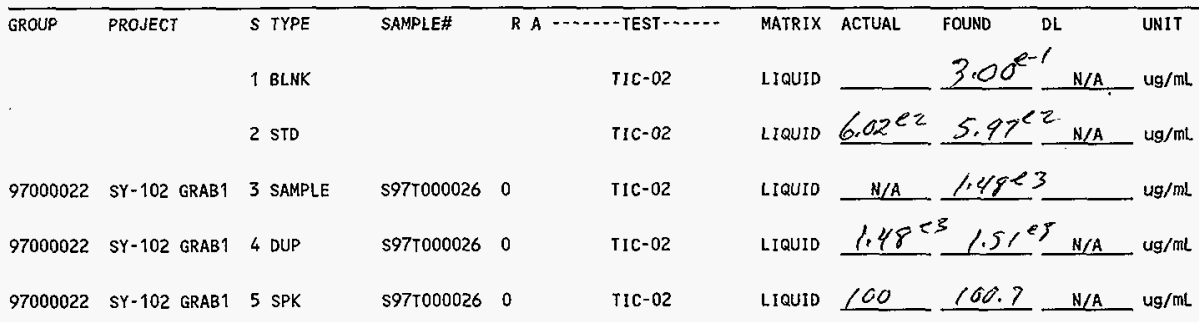

\section{Final page for worklist \# 16384}

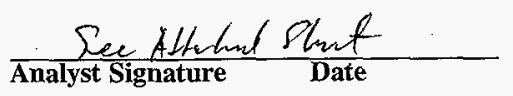

$\frac{2 / f\left(\frac{f}{8} \quad\right.}{\text { Analyst Signature }} \frac{\Sigma-3-97}{\text { Date }}$

Data Entry Comments:

Units shown for QC (SPK \& STD) moy not reflect the actual units. $D L=$ Detection Limit, $S=$ Worklist Slot Number, $R=$ Replicate Number,$A=$ Aliquot Code. 


Analyst: SLH Instrument: TIC01
Method: LA-342-100 Rev/Mod $\frac{E-O}{\text { Book\# }}$
Worklist Comment: SY-102 Grab. Run per procedure. Std: $1.0 \mathrm{ml}$.

\begin{tabular}{|c|c|c|c|c|c|c|c|}
\hline$s$ & Type & Sample\# & R A & Test & Matrix & Group\# & Erofect \\
\hline 1 & BLNK & . & & TIC-02 & LIQUID & & \\
\hline 2 & STD & & & TIC- 02 & LIQUID & & \\
\hline 3 & SAMPLE & $\begin{array}{c}\text { S97T000026 } \\
\text { Analytes Reque }\end{array}$ & $\begin{array}{l}0 \\
\text { egted: }\end{array}$ & $\begin{array}{l}\text { TIC-02 } \\
=\text { TIC-02 }\end{array}$ & LIQUID & 97000022 & SY-102 GRABI \\
\hline 4 & DUP & S97T000026 & 0 & TIC-02 & LIQUID & & . \\
\hline 5 & SPK & S97T000026 & 0 & TIC-02 & LIQUID & & \\
\hline
\end{tabular}

\title{
Final page for worklist \# 16384
}

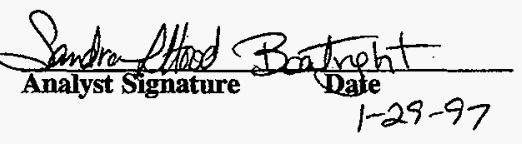

\author{
Analyst Signature Date
}

Data Entry Comments:

$S=$ Worklist Slot Number, $R=$ Replicate Number, $A=$ Aliquot Code. 


\section{TIC- TOTAL INORGANIC CARBON ANALYSIS REPORT} TICTOC REV 2.0

Sample: STD 2

Sample size $=1000 \mathrm{uL}$

Dil Factor $=1$

Blank ID \# =

Blank Value $=.9 \mathrm{ug} / \mathrm{minute} \mathrm{C}$
Time: $11: 02: 18$

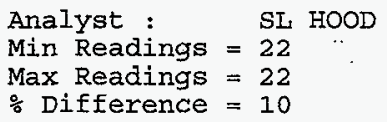

$==$ Reading $====$ Analysis Time $====$ Coulometer $====$ Difference $==$

$\begin{array}{lr}1 & 0.51 \\ 2 & 1.01 \\ 3 & 1.50 \\ 4 & 2.00 \\ 5 & 2.50 \\ 6 & 3.00 \\ 7 & 3.50 \\ 8 & 4.00 \\ 9 & 4.50 \\ 10 & 5.00 \\ 11 & 5.50 \\ 12 & 6.00 \\ 13 & 6.50 \\ 14 & 7.00 \\ 15 & 7.50 \\ 16 & 8.00 \\ 17 & 8.50 \\ 18 & 9.00 \\ 19 & 9.50 \\ 20 & 10.00 \\ 21 & 10.50 \\ 22 & 11.00\end{array}$

0.20

1.10

1.70

2.50

19.30

79.20

178.10

294.30

398.50

481.30

536.30

568.90

587.10

595.70

600.00

602.40

603.80

604.70

605.30

606.00

606.50

507.00
0.00

81.82

35.29

32.00

87.05

75.63

55.53

39.48

26.15

17.20

10.26

5.73

3.10

1.44

0.72

0.40

0.23

0.15

0.10

0.12

0.08

0.08

USER INPUT BLANK VALUE

BLANK VALUE $=9.899835$ micrograms carbon

BLANK FACTOR $=9.899835 / \cdot 10.99982=+9.0 \mathrm{E}-01 \mathrm{ug} / \mathrm{min}$ Carbon

SAMPLE RESULTS:
$(607-9.898077)($
$(1) /(1000)=$
$+5.971 \mathrm{E}-01$
g/L Carbon
$(607-9.898077)(1) /(1000)(12)=$ 
TIC- TOTAL INORGANIC CARBON ANALYSIS REPORT

TICTOC REV 2.0

$\ll<$ BLANR ANALYSIS 》>

Sample: BASE 2

Sample Size $=1$ uI

Dil Factor $=1$

Blank ID \# = BASE. 2

Blank value $=\mathrm{N} / \mathrm{A}$
Date: $01 / 29 / 97$

Time: $08: 10: 24$

$==$ Reading $====$ Analysis Time $====$ Coulometer $====\frac{\circ}{6}$ Difference $==$

$\begin{array}{rrrr}1 & 0.51 & 0.10 & 0.00 \\ 2 & 1.01 & 0.50 & 80.00 \\ 3 & 1.51 & 1.20 & 58.33 \\ 4 & 2.01 & 2.00 & 40.00 \\ 5 & 2.50 & 2.90 & 31.03 \\ 6 & 3.00 & 3.40 & 14.71 \\ 7 & 3.50 & 3.90 & 12.82 \\ 8 & 4.00 & 4.30 & 9.30 \\ 9 & 4.50 & 4.80 & 10.42 \\ 10 & 5.00 & 5.20 & 7.69 \\ 11 & 5.50 & 5.50 & 5.45 \\ 12 & 6.00 & 5.90 & 6.78 \\ 13 & 6.50 & 6.30 & 6.35 \\ 14 & 7.00 & 6.70 & 5.97 \\ 15 & 7.50 & 7.10 & 5.33 \\ 16 & 8.00 & 7.50 & 5.06 \\ 17 & 8.50 & 7.90 & 5.95 \\ 18 & 9.00 & 8.40 & 3.45 \\ 19 & 9.50 & 8.70 & 3.33 \\ 20 & 10.00 & 9.00 & 5.26 \\ 21 & 10.50 & 9.50 & 4.04 \\ 22 & 11.00 & 9.90 & \end{array}$

BLANK VALUE $=9.9$ micrograms carbon

BLANK FACTOR $=9.9 / 10.99969=$

+9.0E-01 ug/min Carbon

Sample Run By:

SL HOOD 00002 
HNF-SD-WM-DP-227, REV: 0

TIC- TOTAL INORGANIC CARBON ANALYSIS REPORT

TICTOC REV 2.0

Sample: BLK 2

$$
\begin{aligned}
& \text { Sample Size }=1 \mathrm{uL} \\
& \text { Dil Factor }=1 \\
& \text { Blank ID \# }= \\
& \text { Blank Value }=.9 \mathrm{ug} / \text { minute } \mathrm{C}
\end{aligned}
$$

Date: $01 / 29 / 97$

Time: $12: 48: 33$

$$
\begin{aligned}
& \text { Analyst } \quad \text { SL HOOD } \\
& \text { Min Readings }=22 \\
& \text { Max Readings }=22 \\
& \text { \% Difference }=10
\end{aligned}
$$

$\begin{array}{cccc}\text { Reading }===\text { Analysis Time }==0 & \text { Coulometer }==0 & \text { Difference }== \\ 1 & 0.51 & 0.00 & 0.00 \\ 2 & 1.01 & 0.30 & 100.00 \\ 3 & 1.50 & 1.10 & 72.73 \\ 4 & 2.00 & 1.60 & 31.25 \\ 5 & 2.50 & 2.30 & 30.43 \\ 6 & 3.00 & 2.70 & 14.81 \\ 7 & 3.50 & 3.40 & 20.59 \\ 8 & 4.00 & 3.70 & 8.11 \\ 9 & 4.50 & 4.40 & 15.91 \\ 10 & 5.00 & 4.80 & 8.33 \\ 11 & 5.50 & 5.20 & 7.69 \\ 12 & 6.00 & 5.7 .0 & 8.77 \\ 13 & 6.50 & 6.10 & 6.56 \\ 14 & 7.00 & 6.50 & 6.15 \\ 15 & 7.50 & 6.90 & 5.80 \\ 16 & 8.00 & 7.30 & 5.48 \\ 17 & 8.50 & 7.80 & 6.41 \\ 18 & 9.00 & 8.10 & 3.70 \\ 19 & 9.50 & 8.40 & 3.57 \\ 20 & 10.00 & 8.90 & 5.62 \\ 21 & 10.50 & 9.30 & 4.30 \\ 22 & 11.00 & 9.60 & 3.13\end{array}$

USER INPUT BIANK VAIUE

BLANK VALUE $=9.899835$ micrograms carbon

BLANK FACTOR $=9.899835 / 10.99982=+\quad+9.0 \mathrm{E}-01 \quad \mathrm{ug} / \mathrm{min}$ Carbon SAMPLE RESULTS:
$(9.6-9.896429)(1) /(1)$
$<5.00$
$\mathrm{E}-3 \mathrm{~g} / \mathrm{L}$ Caxbon
$(9.6-9.896429)(1) /(1)(12)=$
$<4.17$ E-4 Molar Carbon

Sample Run BY:

$\overline{\text { SL HOOD. }} 00002$


Sample: S97T00026

Sample Size $=1 \mathrm{uL}$

Dil Factor $=1$

Blank ID \# =

Blank Value $=.9 \mathrm{ug} /$ minute $\mathrm{C}$
Time: 13:08:15

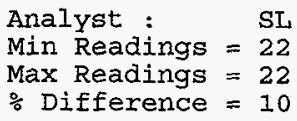

Analyst :

Min Readings $=22$

Max Readings $=22$

$\div$ Difference $=10$

$\begin{array}{crcc}\text { Reading }===\text { Analysis Time }=== & \text { Coulometer }==0 & \text { Difference }== \\ 1 & 0.51 & 0.00 & 0.00 \\ 2 & 1.01 & 0.00 & 0.00 \\ 3 & 1.51 & 0.00 & 0.00 \\ 4 & 2.01 & 0.00 & 0.00 \\ 5 & 2.51 & 0.80 & 100.00 \\ 6 & 3.00 & 13.70 & 94.16 \\ 7 & 3.50 & 38.60 & 64.51 \\ 8 & 4.00 & 68.70 & 43.81 \\ 9 & 4.50 & 98.80 & 30.47 \\ 10 & 5.00 & 121.90 & 18.95 \\ 11 & 5.50 & 135.50 & 10.04 \\ 12 & 6.00 & 144.40 & 6.16 \\ 13 & 6.50 & 148.90 & 3.02 \\ 14 & 7.00 & 151.80 & 1.91 \\ 15 & 7.50 & 153.60 & 1.17 \\ 16 & 8.00 & 154.60 & 0.65 \\ 17 & 8.50 & 155.20 & 0.39 \\ 18 & 9.00 & 155.70 & 0.32 \\ 19 & 9.50 & 156.30 & 0.38 \\ 20 & 10.00 & 156.80 & 0.32 \\ 21 & 10.50 & 157.30 & 0.32 \\ 22 & 11.00 & 158.10 & 0.51\end{array}$

USER INPUT BIAANK VALUE

BLANK VALUE $=9.899835$ micrograms carbon

BLANK FACTOR $=9.899835 / 10.99982=+9.0 \mathrm{E}-01 \quad \mathrm{ug} / \mathrm{min}$ Carbon

SAMPLE RESULTS:
$(158.1-9.898956)$
$(1) /(1)=$
$+1.482 \mathrm{E}+02$
g/L Carbon
$(158.1-9.898956$
$(1) /(1)(12)=$
$+1.235 E+01$
Molar Carbon

Sample Run By:

SL HOOD 00002

\section{ss. $100 \mathrm{ml}$ - \\ .10999}


TIC- TOTAL INORGANIC CARBON ANALYSIS REPORT TICTOC REV 2.0.

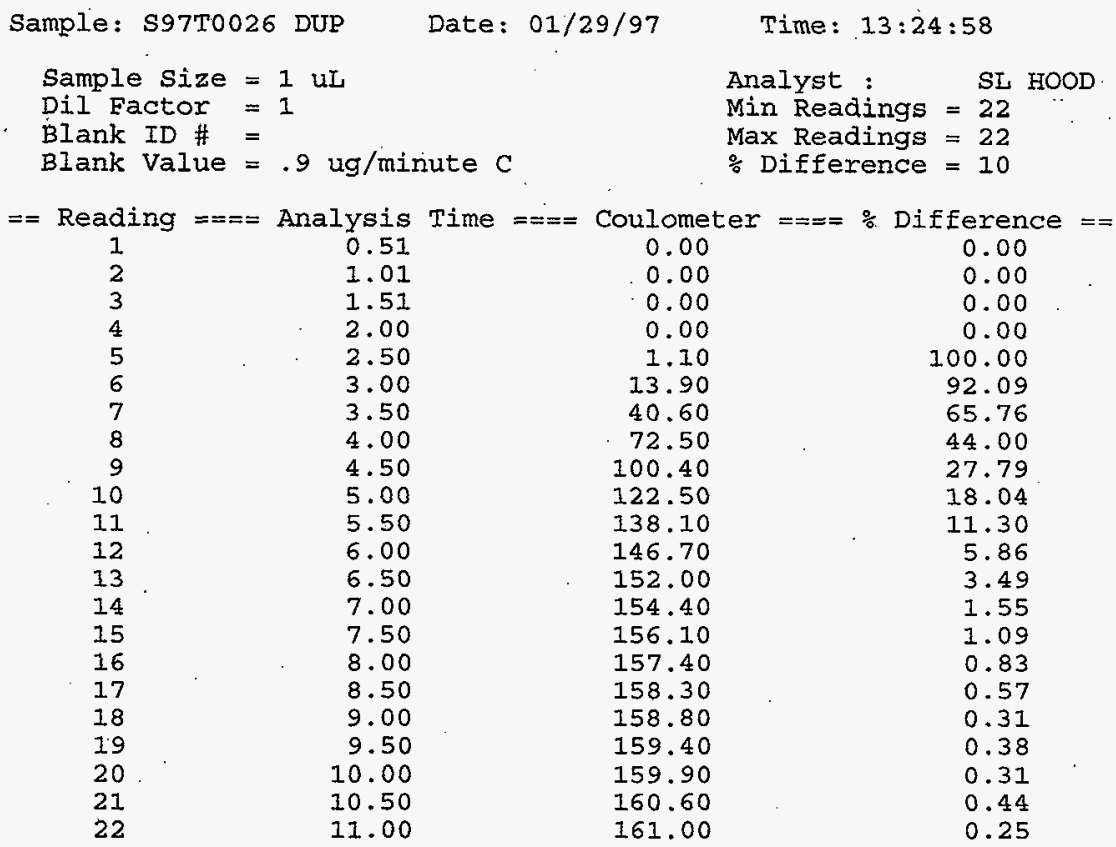

USER INPUT BLANK VALUE

BLANK VALUE $=9.899835$ micrograms carbon

BLANK FACTOR $=9.899835 / 10.99982=+9.0 \mathrm{E}-01 \quad \mathrm{ug} / \mathrm{min}$ Carbon

SAMPIE RESULTS:

$\begin{array}{ll}(161-9.898956)(1) /(1) & +1.511 \mathrm{E}+02 \mathrm{~g} / \mathrm{L} \text { Carbon } \\ (161-9.898956)(1) /(1)(12)= & +1.259 \mathrm{E}+01 \text { Molar Carbon }\end{array}$

Sample Run By:

ss $\begin{gathered}.100 \mathrm{ml}= \\ .1097 \mathrm{~g} .\end{gathered}$

SI HOOD 00002

161 


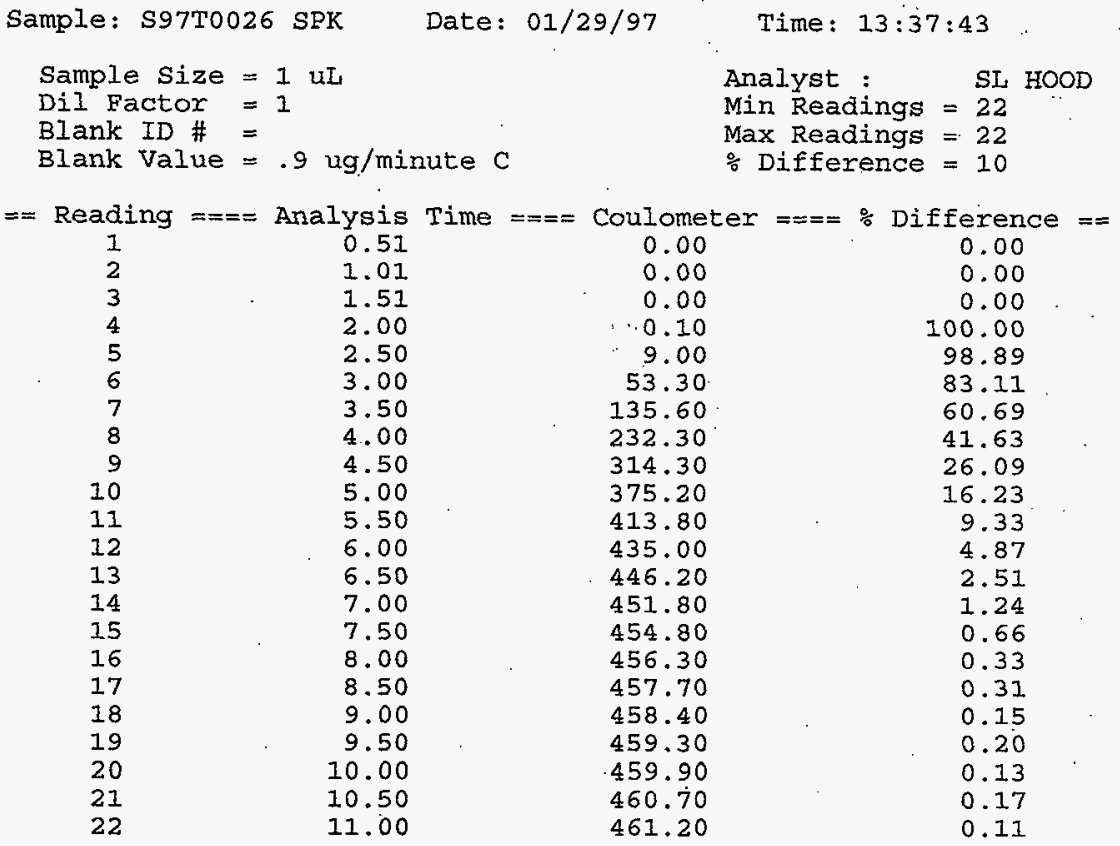

USER INPUT BLANK VALUE

BLANK VALUE $=9.899835$ micrograms carbon

BLANK FACTOR $=9.899835 / 10.99982=+9.0 \mathrm{E}-0.1 \mathrm{ug} / \mathrm{min}$ Carbon

SAMPLE RESULTS :

$(461.2-9.897308)(1) /(1)=$

$+4.513 \mathrm{E}+02 \mathrm{~g} / \mathrm{L}$ Carbon

$+3.761 \mathrm{E}+01$ Molar Carbon

Sample Run $\dot{B} y$ :

$\overline{\text { SL HOOD }} 00002$

ss $\quad .100=$

$500 \mathrm{mll} 25 N / 2+\mathrm{A}$ 


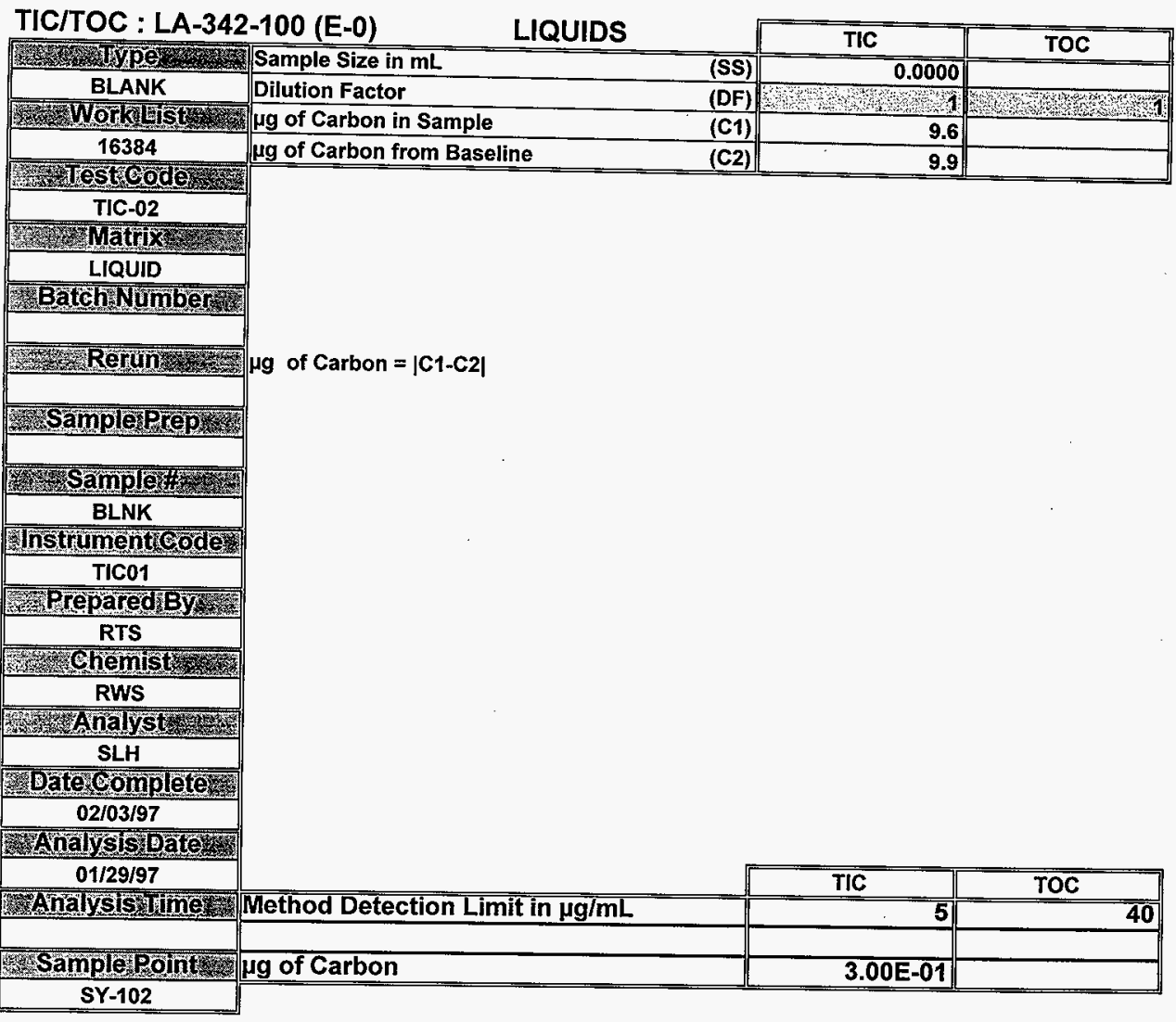

Data Entered By:

Signature of Chemist:

BLANK.WB1 REV 1.0

$342100 \mathrm{ML}$

RTS 


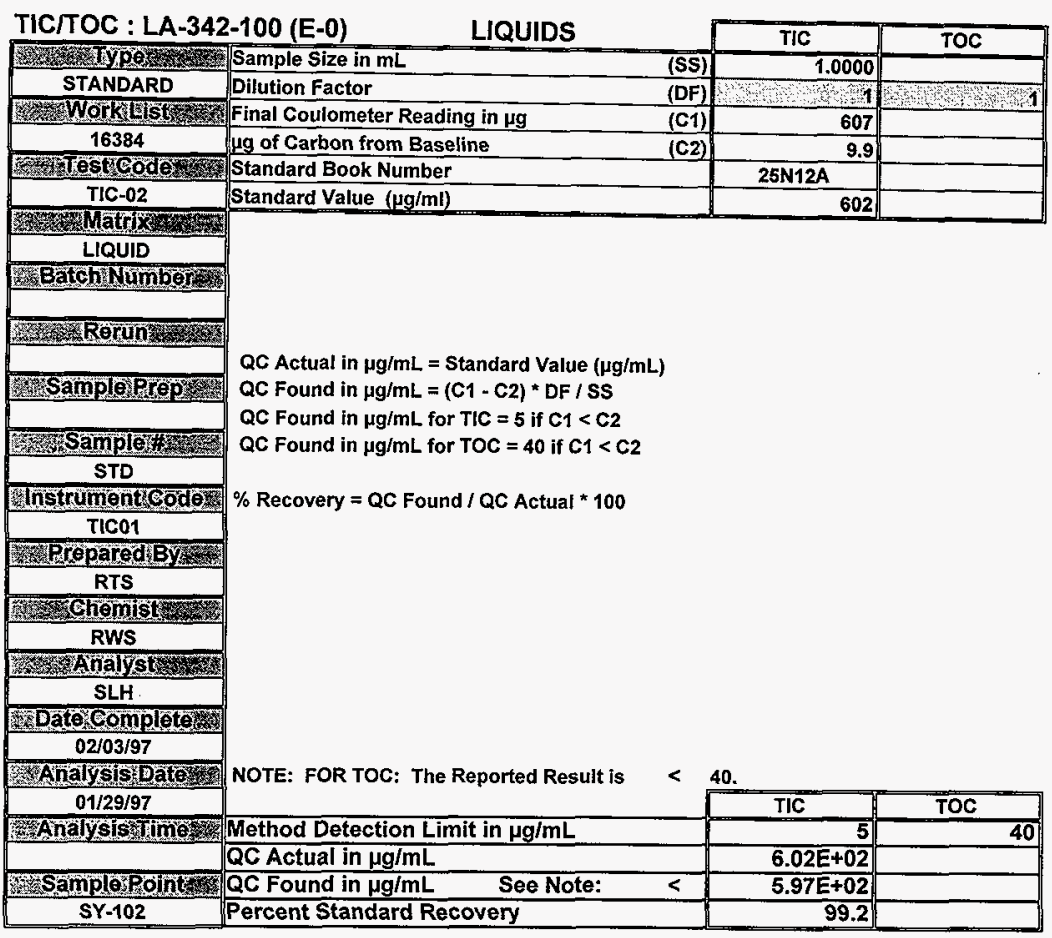

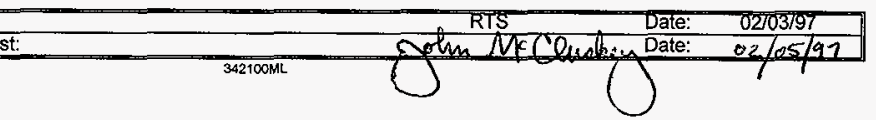




\begin{tabular}{|c|c|c|c|c|}
\hline TIC/TOC & & & & \\
\hline & LIQUIDS & & TIC & TOC \\
\hline 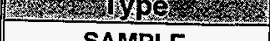 & Sample Size in $\mathrm{mL}$ & (SS) & 0.1000 & \\
\hline SAMPLE & Dilution Factor & (DF) & 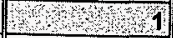 & 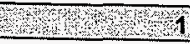 \\
\hline Work List & ug of Carbon in Sample & (C1) & 158.1 & \\
\hline 16384 & $\mu \mathrm{g}$ of Carbon from Baseline & (C2) & 9.9 & \\
\hline Testrode & & & & \\
\hline TIC-02 & & & & \\
\hline$\frac{{ }_{2}}{\text { LIQUID }}$ & & & & \\
\hline Batch Noinber & & & & \\
\hline Hering & $\mu \mathrm{g}$ of Carbon $/ \mathrm{mL}=\left(\mathrm{C}_{1}-\mathrm{C} 2\right) * \mathrm{DF} / \mathrm{SS}$ & & & \\
\hline & $\mu \mathrm{g}$ of Carbon $/ \mathrm{mL}$ for $\mathrm{TIC}=5$ if $\mathrm{C} 1<\mathrm{C} 2$ & & & \\
\hline Sampletep & $\mu \mathrm{g}$ of Carbon $/ \mathrm{mL}$ for TOC $=40$ if $\mathrm{C} 1<\mathrm{C} 2$ & & & \\
\hline 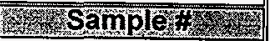 & & & & \\
\hline $597 T 000026$ & & & & \\
\hline Instrumentcode & & & & \\
\hline TIC01 & & & & \\
\hline PreparedBy & & & & \\
\hline RTS & & & & \\
\hline Chemist & & & & \\
\hline RWS & & & & \\
\hline 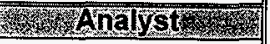 & & & & \\
\hline SLH & & & & \\
\hline Datecomplete & & & & \\
\hline $02 / 03 / 97$ & & & & \\
\hline A nalysis Dater & NOTE: FOR TOC: The Reported Result is & $<$ & 40. & \\
\hline $01 / 29 / 97$ & & & TIC & TOC \\
\hline Analysis 1 mo & Method Detection Limit in $\mu \mathrm{g} / \mathrm{mL}$ & & 5 & 40 \\
\hline Samplerotht & $\mu \mathrm{g}$ of Carbon $/ \mathrm{mL}$ & $<$ & $1.48 E+03$ & \\
\hline SY-102 GRAB & & & & \\
\hline
\end{tabular}

\begin{tabular}{|c|c|c|c|}
\hline Data Entered By: & RTS & Date: & $02 / 03 / 97$ \\
\hline Signature of Chemist: & & Date: & $02 / 05 / 97$ \\
\hline
\end{tabular}




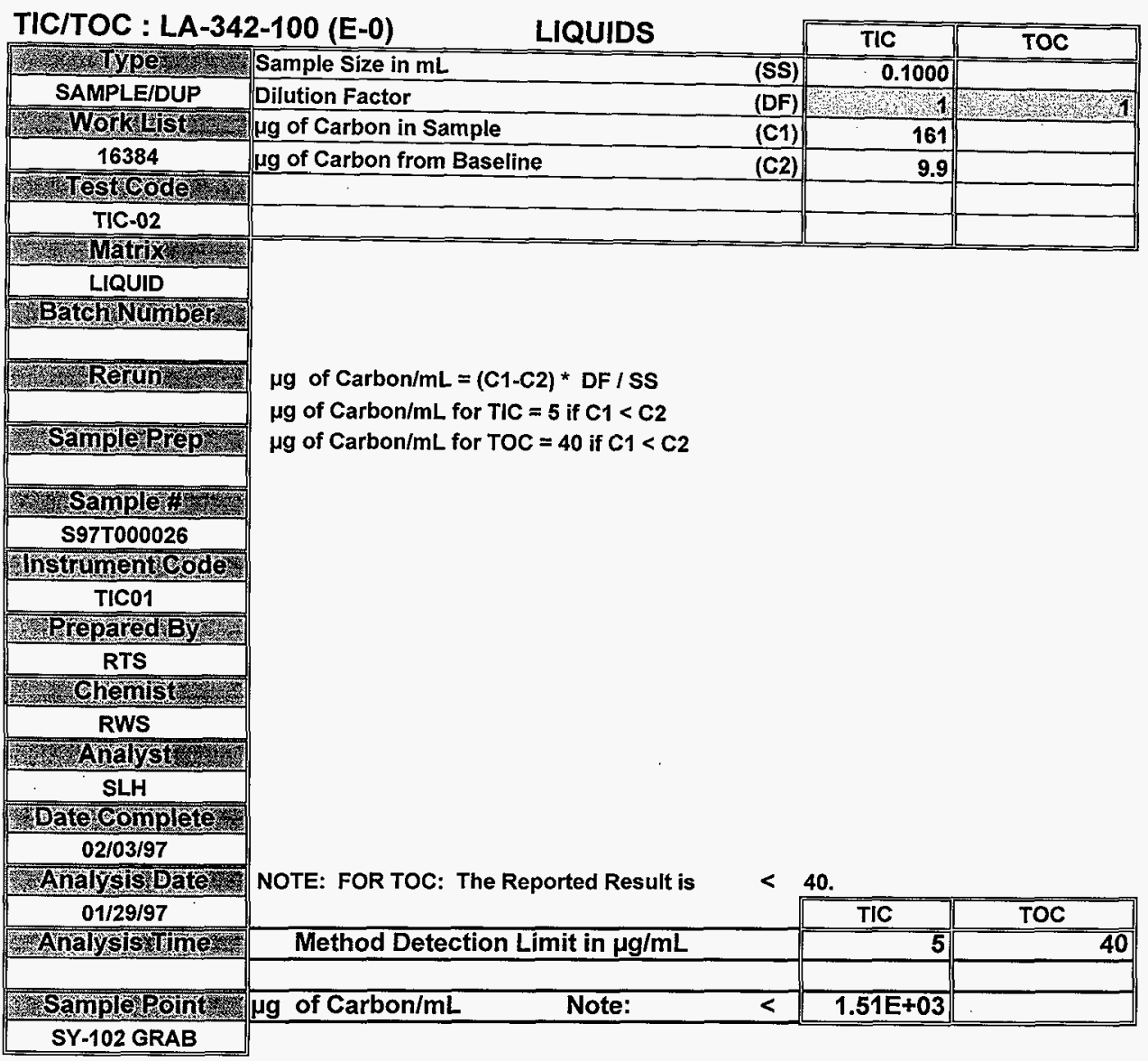

\begin{tabular}{|c|c|c|c|}
\hline Data Entered By: & RTS & Date: & $02 / 03 / 97$ \\
\hline Signature of Chemist: & $5 \ln M^{c} C l$ & Date: & $02 / 05 / 97$ \\
\hline
\end{tabular}


HNF-SD-WM-DP-227, REV. 0

TIC/TOC : LA-342-100 (E-0)

LIQUIDS

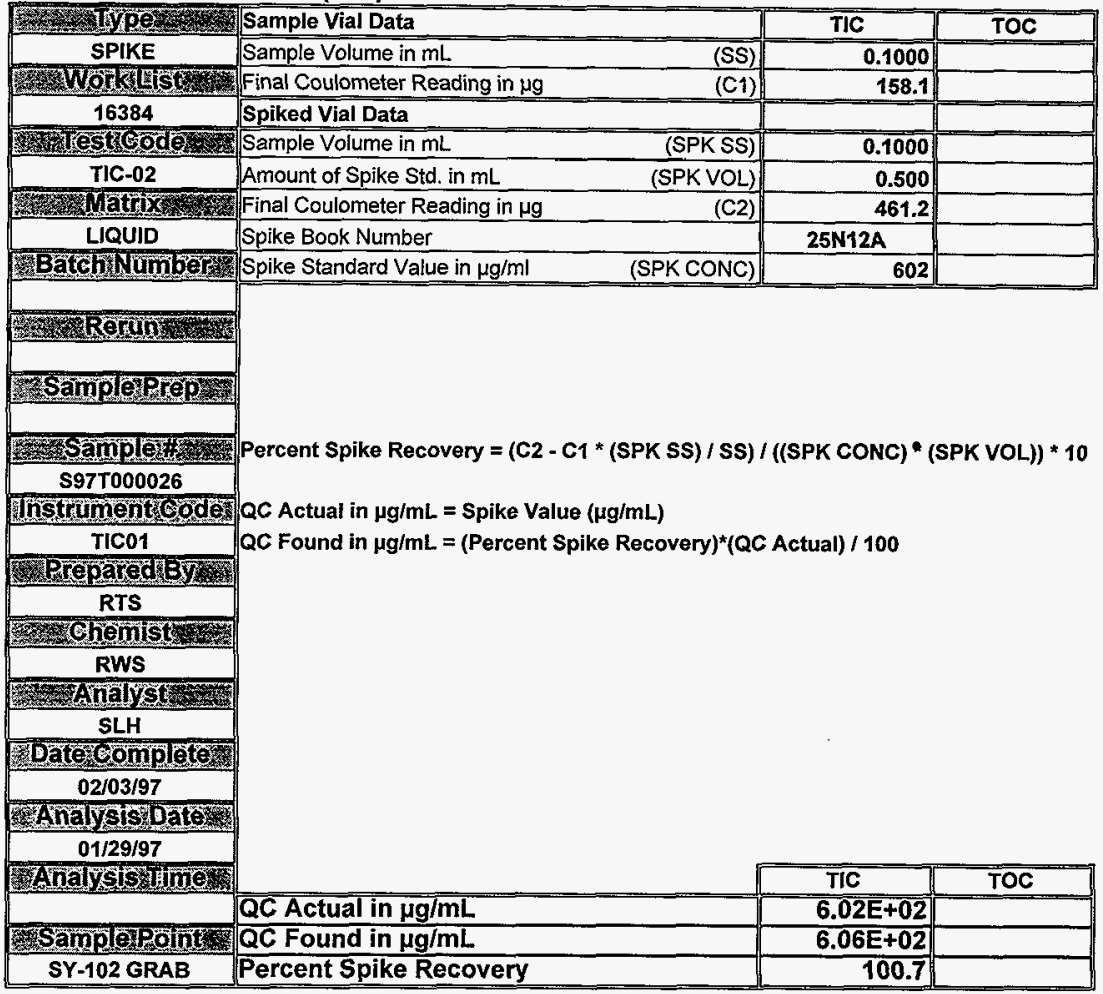

\begin{tabular}{|c|c|c|c|}
\hline Data Entered By: & RTS & Date: & $02 / 03 / 97$ \\
\hline Signature of Chemist: & & Date: & $02 / 05 / 97$ \\
\hline
\end{tabular}


HNF-SD-WM-DP-227, REV. 0

RADIOCHEMICAL ANALYSES 
HNF-SD-WM-DP-227, REV. 0

THIS PAGE INTENTIONALLY LEFT BLANK 


\section{LABCORE Completed RadChem Report for Worklist\#: 16280}

Analyst: rga

Method:
Instrument: GEA03

Rev/Mod

Worklist Comment: Sample size is on traveler. Std (TNKFUS) $1.0 \mathrm{ml}$. new

Book\#

\begin{tabular}{|c|c|c|c|c|c|c|c|c|c|c|}
\hline Seq Type & Sample & $\mathbf{R A}$ & & Test & Matrix & Actual & Found & DL or Yield & Unit & \\
\hline 1510 & & 6 & DEER & 8000.02 & 210010 & $66 \% 2103$ & $6.67<03$ & 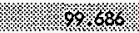 & 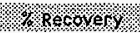 & ere \\
\hline 1 STD & & 0 & QGEA-01 & COSO-02E & LIQUID & 1 & 2.36 & 2.360 & $\%$ Ct Error & \\
\hline 1 $\mathrm{s}$ ro & & 0. & acter $=0$ & Ks 357.22 & Youro & $3.842-2.03$ & $3 \% 96-03$ & 1.02 .020 & 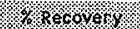 & \\
\hline 1 STD & & 0 & DGEA-01 & CS13702E & LIQUID & 1 & 3.07 & 3.070 & \% ct Error & \\
\hline $28 \mathrm{kX}$ & & \%o: & OLE 01 & 405002 & $-\alpha \%$ o & 1 & $25 s 3=5$ & & $14 \pi / w_{1}$ & \\
\hline 2 BLNK & & 0 & DGEA-01 & cs 13702 & LIQUID & 1 & $<5.62 \mathrm{e}-5$ & & $\mathrm{uCi} / \mathrm{mL}$ & \\
\hline 3. SARPLE & $\$ 971000025$ & 0 & 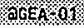 & $\operatorname{tos} 0.02$ & Orouro & $M / A$ & $3053=04$ & $3043=0 B 6$ & 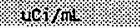 & \\
\hline 3 SAMPLE & s97ro00025 & 0 & DGEA-01 & COSO-02E & LIQUID & $N / A$ & $n / a$ & $0.0 \mathrm{e}+000$ & $\%$ ct. Error & \\
\hline 3. SAMP LE & 597000025 & 10 & SGEA $=01$ & CS1 3702 & 1Quido & 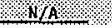 & $12230=+01$ & $000+00 \%$ & 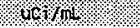 & \\
\hline 3 SAMPLE & s971000025 & 0 & aGEA-01 & CS13702E & LIQU1D & N/A & $\therefore \quad 0.170$ & $0.0 \mathrm{e}+000$ & \% ct. Error & \\
\hline $40 \%$ & 5971000025 & 20. & 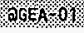 & coso 02. & Whouro & $3.04=\%$ & $3522-4$ & & $8 p 0 \%$ & \\
\hline 4 DUP & 5977000025 & 0 & ФGEA-01 & COSO-OZE & LIQUID & 1 & $n / a$ & & $\%$ ct Error & \\
\hline $4 \mathrm{pup}$ & 5921000025 & 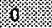 & ato $5=01 \%$ & rs $513 \%$ 2 & $\times 1 \% u$ ro & $1 / 23<+13$ & $1 / 22 \cdot 04$ & 0. $316 \%$ & RPJ & \\
\hline 4 DUP & $\$ 971000025$ & 0 & QGEA-01 & CS13702E & LIQUID & 1 & 0.170 & 0.170 & \% Ct Error & \\
\hline 5 SAMPEI & 597100028 & 0 & as & $6060 \% 2$ & 81010 & NHA & $8.855-04$ & $385 \cdot 5-006$ & $10 / 1 / m 4=$ & \\
\hline 5 SAMPLE & s971000028 & 0 & AGEA-01 & $\cos 0-02 E$ & LIQUID & N/A & $n / a$ & $0.0 \mathrm{e}+000$ & $\%$ ct. Error & \\
\hline 5. SAMPER & 897000028 & 0 & $\mathrm{agcS}$ & ss 13702 & WOUID & N/A & $3.7000+00$ & $000-000$ & anckit & \\
\hline 5 SAMPLE & $597 T 000028$ & 0 & DGEA-01 & CS13702E & LIQUID & $N / A$ & 0.160 & $0.0 \mathrm{e}+000$ & \% ct. Error & \\
\hline $6 \%$ & 5971000028 & 0 & 2a & 606002 & 140010 & $8.86-4$ & $405=3$ & /. & $B D O=$ & \\
\hline 6 DUP & $\$ 977000028$ & 0 & QGEA-01 & COSO-02E & LIQUID & 1 & $n / a$ & & \% ct Error & \\
\hline 6.0ur & 597600028 & 0 & asc $=01$ & $\operatorname{CSA} 37.02$ & Trouro & $3.700+01 \%$ & $3.65-01$ & $1.361 \%$ & $\mathrm{RPO}: 0$. & \\
\hline 6 DUP & S97T000028 & 0 & QGEA-01 & CS13702E & LIQUID & 1 & 0.170 & 0.170 & \% Ct Error & \\
\hline 7 SARP UL & $59700002 \%$ & 0. & 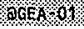 & $0050 \% 02$ & 4040 & $\sqrt[N]{2 / A}=$ & $5.310-03$ & $0.00+000$ & $101 \%$ & \\
\hline 7 SAMPLE & $\$ 971000029$ & 0 & QGEA-01 & $\cos 0-02 E$ & LIQUID & N/A & 37.1 & $0.0 \mathrm{e}+000$ & $\%$ ct. Error & \\
\hline $7 \mathrm{SPHOLE}$ & 8971000029 & 0 & $2 \mathrm{ag} / \mathrm{S}-0$ & $\operatorname{cs} 13702$ & 10410 & N/za & $2.450-107$ & 000000 & $00 \% \%$ & \\
\hline 7 SAMPLE & S97T000029 & 0 & AGEA-01 & CS13702E & LIQUIO & $N / A$ & 0.400 & $0.0 \mathrm{e}+000$ & $\%$ ct. Error & \\
\hline 8000 & S971000029 & 0 & $26650 \%$ & $0050 \% 02$ & 10010 & 5,312003 & 1005802 & $685 \% 55$ & 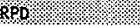 & \\
\hline 8 DUP & 5971000029 & 0 & QGEA-01 & COSO-02E & LIQUID & 1 & 20.8 & 20.800 & $\%$ Ct Error & \\
\hline 80.0 & 597000029 & o. & 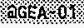 & $\operatorname{cs} 13702$ & . 10410 & $2.45-01$ & $2.22-01 \%$ & $1.232 \%$ & & \\
\hline 8 DUP & S971000029 & 0 & QGEA-01 & CS13702E & LIQUID & 1 & 0.400 & 0.400 & \% ct Error & \\
\hline
\end{tabular}

\section{Final page for worklist\# 16280}

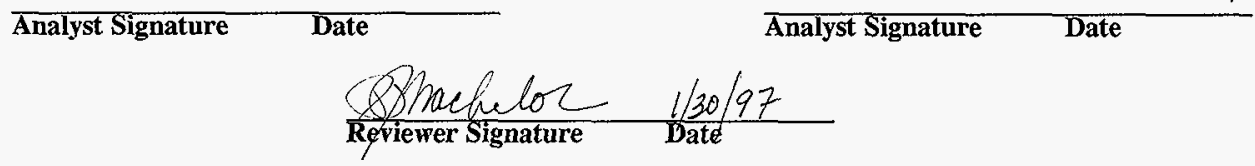

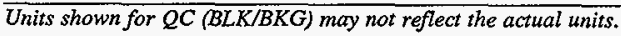




\section{LABCORE Data Entry Template for Worklist\# 16280}

Analyst: R6A Instrument: GEA00 \#3 Book\#25856

Method: LA-548-121 Rev/Mod $\underset{+}{\mathbb{Z}-0}$

Worklist Comment: Sample size is on traveler. Std (TNKFUS) 1.0ml. new

\begin{tabular}{llllll}
\hline$S$ Type & Sample\# & T Test & Matrix & Group\# & Project
\end{tabular}

1 STD @GEA-01 LIQUID

2 BINK @GEA-01 LIQUTD

3 SAMPLE \$97T000025 O @GEA-01 LIQUID 97000022 SY-102 GRAB1

Analytes Requested: CO60-02, CO60-02E, CS13702, CS13702E

4 DUP S97T000025 0 @GEA-01 LIQUID

5 SAMPLE S97T000028 0 @GEA-01 LIQUID 97000022 SY-102 GRAB1

Analytes Requested: CO60-02, CO60-02E, CS13702, CS13702E

6 DUP S97T000028 O @GEA-01 LIQUID

7 SAMPLE S97T00Q029 $0 \quad$ QGEA-01 LIQUID 97000022 SY-102 GRABI

Analytes Requested: CO60-02, CO60-02E, CS13702, CS13702E

8 DUP S97T0000290 @GEA-01 LIQUID

\section{Final page for worklist \# 16280}

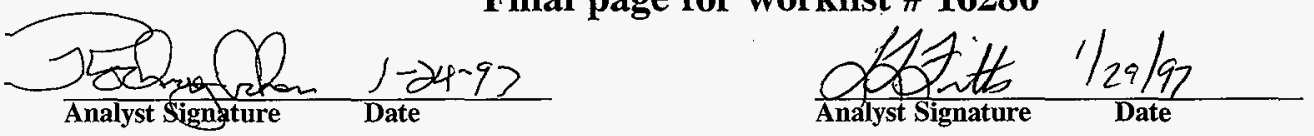

Data Entry Comments:

$S=$ Worklist Slot Number, $R=$ Replicate Number, $A=$ Aliquot Code. 
HNF-SD-WM-DP-227, REV. 0

16280

WL16280-STD

$1.00000 \mathrm{E}-03 \mathrm{~L}$

$1.00000 \mathrm{E}+00$

Sample Size:

Dilution Factor:

$\gg>>>>>>>>$ COUNT INFORMATION $<<<<<<<<<<~$

Detector ID:

File Number:

Geometry:

Count Time:

Real Time:

Dead Time:

\section{GEA3}

dka300: [spec.GEA3]3g3446.cnf

42

$000: 50: 00.00 \mathrm{sec}$

$0 \quad 00: 50: 02.75 \mathrm{sec}$

$0.1 \%$

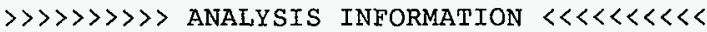

Sample Count Time:

27-JAN-1997 08:22:06.29

Decayed to:

Standard Deviations:

Analysis Library:

Analyst:

$27-J A N-199708: 22: 06.29$

2

ENVGEA

SCZ

Background Subtract: DKA300:[SPEC.GEA3]3GBACK

$\gg>>>>>>>$ CALIBRATION INFORMATION $<<<<<<<<<~$

Date of last energy calibration: 11-MAR-1994 11:47:01.11

Date of last efficiency calibration: 15-MAR-1994 10:28:40.20

$* * * * * * * * * * * * * * * * * * * * * * * * * * * * * * * * * * * * * * * * * * * * * * * * * * * * * * * * * * * * * * * * * * * * * * * * * * * * * * * *$

Post-NID Peak Search Report

Area FWHM Channel Left Pw \%Err Fit Nuclides Activity

$\mathrm{uCi} / \mathrm{L}$

$0 \quad 661.66 *$

5276

$1.68 \quad 1323.49$

1316

14

3.1

CS -137

5.96

1173.22

4315

1.972346 .71

2338

18

3.3

3736

$2.04 \quad 2665.29 \quad 2657$

$17 \quad 3.4$

$\mathrm{CO}-60$

6.81

$\mathrm{CO}-60$

6.54 
Total number of lines in spectrum Number of unidentified lines

3

\section{uumber of unides tified lines}

Nuclide Type :

Nuclide
Co-60
CS -137

$\begin{array}{rr}\text { Hlife } & \text { Decay } \\ 5.27 \mathrm{Y} & 1.000 \\ 30.00 Y & 1.000\end{array}$

Wtd Mean Uncorrected

Total Activity :

$$
\mathrm{uCi} / \mathrm{L}
$$

$6.671 \mathrm{E}+00$

$5.963 \mathrm{E}+00$

$-------$

1. $263 \mathrm{E}+01$
Wtd Mean

Decay Corr $\mathrm{uCi} / \mathrm{L}$

$6.671 \mathrm{E}+00$

$5.963 \mathrm{E}+00$

$-----\cdots-1$

$1.263 \mathrm{E}+01$
$100.00 \%$

Grand Total Activity : $1.263 E+01$

1. $263 E+01$

Flags: "K" = Keyline not found

" $\mathrm{E} "$ = Manually edited

"M" = Manually accepted

"A" = Nuclide specific abn. limit 
Minimum Detectable Activity Report Sample ID : WL16280-STD

Nuclide

$\mathrm{BE}-7$
$\mathrm{NA}-22$
$\mathrm{NA}-24$
$\mathrm{~K}-40$
$\mathrm{CR}-51$
$\mathrm{MN}-54$
$\mathrm{CO}-56$
$\mathrm{CO}-57$
$\mathrm{CO}-58$
$\mathrm{FE}-59$
$\mathrm{SE}-75$
$\mathrm{SR}-85$
$\mathrm{Y}-88$
$\mathrm{NB}-94$

ZRNB-95

RU-103

RURH-106

AG- $108 \mathrm{~m}$

$\mathrm{CD}-109$

AG-110M

$\mathrm{SN}-113$

$\mathrm{TE}-123 \mathrm{~m}$

$\mathrm{SB}-124$

$\mathrm{SB}-125$

TE $-125 \mathrm{~m}$

$I-131$

CS -134

$\mathrm{BA}-140$

LA -140

CEPR-144

$\mathrm{EU}-152$

EU-154

EU-155

HG-203

TL -208

BI -212

PB-212

BI -214

$\mathrm{PB}-214$

RA-224

RA- 226

AC -228

$\mathrm{TH}-228$

TH -229

PA-233

UTH-233

PA-234M

TH-234

U-235

NP -237

NP -239

\section{Bckgnd}

Sum

304.

45.

16.

135.

336.

219.

215.

385 .

201.

244 .

388 .

224 .

8.

251.

178.

255.

183.

162.

348 .

308 .

289.

410 .

158.

331 .

371.

313.

170 .

218.

9.

377.

16.

45 .

376 .

388 .

413.

174.

467.

223.

343.

478.

445.

263.

351 .

341 .

364 .

399.

245.

305.

439.

367.

367.

HNF-SD-WM-DP-227, REV. 0

Page : 3

Acquisition date : 27-JAN-1997 08:22:06

\section{Energy (keV)}

477.59

1274.53

1368.55

1460.75

320.08

834.83

846.76

122.06

810.78

1099.25

264.66

514.01

1836.06

871.09

724.18

497.08

621.93

722.94

88.03

657.76

391.69

159.00

602.73

427.89

109.27

364.48

604.70

537.31

1596.21

133.51

1408.01

1274.51

105.31

279.20

277.36

727.18

238.63

609.31

351.92

240.99

186.10

911.21

84.37

88.47

312.17

245.34

1001.03

63.29

185.71

86.48

106.12
MDA
$(u C i / L)$

5.7400E-01

$5.2738 \mathrm{E}-02$

$3.4174 \mathrm{E}-02$

9.5202E-01

$4.5412 \mathrm{E}-01$

$8.0835 \mathrm{E}-02$

8.1200E-02

$3.3973 \mathrm{E}-02$

$7.5957 \mathrm{E}-02$

$1.9140 \mathrm{E}-01$

7. 3125E-02

$5.6343 \mathrm{E}-02$

$2.9353 \mathrm{E}-02$

$8.9887 \mathrm{E}-02$

$1.4789 \mathrm{E}-01$

$6.1748 \mathrm{E}-02$

$1.1580 \mathrm{E}+00$

$6.7801 \mathrm{E}-02$

$1.0683 \mathrm{E}+00$

8.2690E-02

$7.7258 \mathrm{E}-02$

3. $6041 \mathrm{E}-02$

$5.3210 \mathrm{E}-02$

$1.9316 \mathrm{E}-01$

$1.0857 \mathrm{E}+01$

$6.0134 \mathrm{E}-02$

5. $5365 \mathrm{E}-02$

$2.2726 \mathrm{E}-01$

$2.9603 \mathrm{E}-02$

$5.0856 \mathrm{E}-01$

$1.6477 \mathrm{E}-01$

1. $5302 \mathrm{E}-01$

$1.4588 \mathrm{E}-01$

$5.4587 E-02$

$7.2324 \mathrm{E}-01$

9. $6460 \mathrm{E}-01$

9.9855E-02

1. $3922 \mathrm{E}-01$

$2.4752 \mathrm{E}-01$

$1.1210 \mathrm{E}+00$

9.8767E-01

3. $5904 \mathrm{E}-01$

$3.3076 \mathrm{E}+00$

$1.5346 \mathrm{E}-01$

$1.2122 \mathrm{E}-01$

$3.8408 \mathrm{E}+01$

$1.6944 \mathrm{E}-01$

$2.0758 \mathrm{E}+00$

5. $9625 \mathrm{E}-02$

$3.2953 \mathrm{E}-01$

1. $3689 E-01$ 
HNF-SD-WM-DP-227, REV. 0

Minimum Detectable Activity Report (continued)

Acquisition date : $27-J A N-199708: 22: 06$

Nuclide

Bckgnd

Energy

Sum

(keV)

MDA

$\mathrm{PU}-239$

AM-241

412 .

332 .

129.30

59.54

74.67

$A M-243$

362 .

$4.6264 \mathrm{E}+02$

$3.8219 \mathrm{E}-01$

9.3836E-02 
Worklist \#:

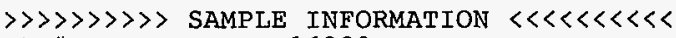

Sample ID:

Sample Size:

Dilution Factor:

16280

WL16280-BLNK

$5.00000 \mathrm{E}-04 \mathrm{~L}$

$1.00000 \mathrm{E}+00$

$>>>>>>>>>$ COUNT INFORMATION $<<<<<<<<<$

Detector ID:

File Number:

Geometry:

Count Time:

Real Time:

Dead Time:

\section{GEA3}

dka 300: [ spec. GEA3 ] 3g3464. cnf

42

$000: 50: 00.00 \mathrm{sec}$

$000: 50: 00.43 \mathrm{sec}$ $0.0 \%$
HNF-SD-WM-DP-227, REV. 0

Removed by:

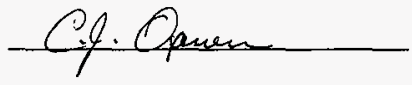

Verified by:

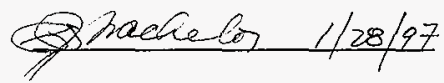

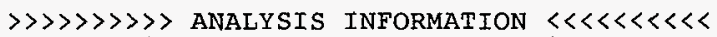

Sample Count Time:

27-JAN-1997 $09: 32: 45.18$

Decayed to:

Standard Deviations:

Analysis Library:

27-JAN-1997 09:32:45.18

Analyst:

ENVGEA

CJO

Background Subtract:

DKA300: [ SPEC.GEA3] 3GBACK

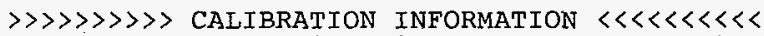

Date of last energy calibration: 11-MAR-1994 11:47:01.11

Date of last efficiency calibration: 15-MAR-1994 10:28:40.20

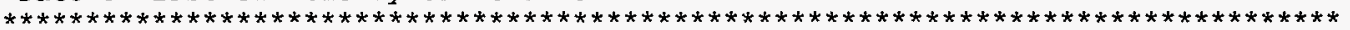

Post-NID Peak Search Report

It Energy

$0 \quad 611.05$

$0 \quad 645.56$

$0661.66 *$
Area FWHM Channel

70

15

25
$6.74 \quad 1222.28$

1.091291 .30

$1.46 \quad 1323.50$
Left Pw 을

1212

1289

1317 $\begin{array}{ll}24 & 47.3\end{array}$

$6 \quad 63.2$

13115.2
Fit Nuclides Activity $\mathrm{uCi} / \mathrm{L}$

CS -137

$5.620 E-02$ 
Total number of lines in spectrum Number of unidentified lines

Number of lines tentatively identified by NID
3 2

1

HNF-SD-WM-DP-227, REV. 0

$33.33 \%$

Nuclide Type :

$$
\begin{aligned}
& \text { Wtd Mean wtd Mean } \\
& \text { Uncorrected Decay Corr Decay Corr 2-Sigma } \\
& \text { Nuclide Hlife Decay uCi/L uCi/L 2-Sigma Error oError Flags }
\end{aligned}
$$

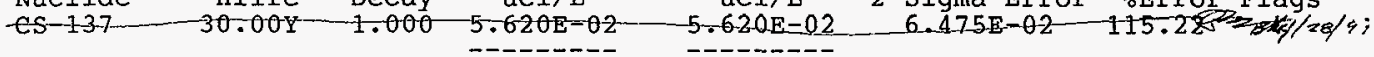

$$
\begin{aligned}
& \text { Total Activity : } 5.620 \mathrm{E}-02 \quad 5.620 \mathrm{E}-02 \\
& \text { Grand Total Activity : } 5.620 \mathrm{E}-02 \quad 5.620 \mathrm{E}-02 \\
& \text { Flags: "K" = Keyline not found } \\
& \text { "E" = Manualiy edited } \\
& \text { "M" = Manually accepted } \\
& \text { "A" = Nuclide specific abn. Iimit }
\end{aligned}
$$


Minimum Detectable Activity Report Sample ID : WL16280-BLNK

Nuclide

$\mathrm{BE}-7$

$\mathrm{NA}-22$

NA -24

$\mathrm{K}-40$

CR-51

MN-54

$\mathrm{CO}-56$

$\mathrm{CO}-57$

$\mathrm{CO}-58$

$\mathrm{FE}-59$

$\mathrm{CO}-60$

$\mathrm{SE}-75$

$\mathrm{SR}-85$

$\mathrm{Y}-88$

NB -94

ZRNB-95

$\mathrm{RU}-103$

RURH-106

AG $-108 \mathrm{~m}$

CD-109

AG-110M

$\mathrm{SN}-113$

TE-123m

SB-124

SB-125

$\mathrm{TE}-125 \mathrm{~m}$

$I-131$

CS-134

BA- 140

LA -140

CEPR-144

EU- 152

EU-154

EU-155

HG-203

TL-208

BI-212

PB- 212

BI-214

PB-2 14

RA-224

RA-226

$\mathrm{AC}-228$

TH -228

TH-229

$\mathrm{PA}-233$

UTH -233

$\mathrm{PA}-234 \mathrm{M}$

TH-234

U-235

NP-237

\section{Bckgnd \\ Sum}

34.

8.

144 .

45 .

18.

21 .

65.

13.

16.

12 .

55.

48 .

1 .

14.

18.

21.

29.

18.

63.

26.

43.

97.

24 .

42.

62 .

58 .

17.

33.

3.

80.

8.

82 .

58.

54 .

19.

83.

57.

51 .

74 .

83.

36 .

76.

64 .

62 .

65.

11 .

57.

83 .

70 .
HNF-SD-WM-DP-227, REV. 0

Page : 3

Acquisition date : 27-JAN-1997 09:32:45

Energy

(keV)

477.59

1274.53

1368.55

1460.75

320.08

834.83

846.76

122.06

810.78

1099.25

1332.50

264.66

514.01

1836.06

871.09

724.18

497.08

621.93

722.94

88.03

657.76

391.69

159.00

602.73

427.89

109.27

364.48

604.70

537.31

1596.21

133.51

1408.01

1274.51

105.31

279.20

277.36

727.18

238.63

609.31

351.92

240.99

186.10

911.21

84.37

88.47

312.17

245.34

1001.03

63.29

185.71

86.48
MDA

( $\mathrm{UCi} / \mathrm{L}$ )

$3.8273 E-01$

$4.4921 \mathrm{E}-02$

$4.7352 \mathrm{E}-02$

1. $9634 \mathrm{E}+00$

$3.3414 \mathrm{E}-01$

$4.6541 \mathrm{E}-02$

$5.0472 \mathrm{E}-02$

$2.7801 \mathrm{E}-02$

$3.8239 \mathrm{E}-02$

$9.8728 \mathrm{E}-02$

$5.5309 \mathrm{E}-02$

$5.5142 \mathrm{E}-02$

$5.2294 \mathrm{E}-02$

$2.0984 \mathrm{E}-02$

$4.2951 \mathrm{E}-02$

9. $3948 E-02$

3. $5792 \mathrm{E}-02$

9. $1780 \mathrm{E}-01$

4. $4700 \mathrm{E}-02$

$9.1132 \mathrm{E}-01$

$4.7927 \mathrm{E}-02$

$5.9844 \mathrm{E}-02$

3. 505 IE-02

4. 1895E-02

1. $3699 \mathrm{E}-01$

$8.9054 \mathrm{E}+00$

$5.1715 \mathrm{E}-02$

$3.4603 \mathrm{E}-02$

1. $7551 \mathrm{E}-01$

3. $2218 \mathrm{E}-02$

4. $6679 \mathrm{E}-01$

2. $2643 \mathrm{E}-01$

$1.3083 E-01$

1. $3652 \mathrm{E}-01$

4.2140E-02

5. $2363 \mathrm{E}-01$

6. $3941 \mathrm{E}-01$

8. $4282 \mathrm{E}-02$

1. $4030 \mathrm{E}-01$

$1.9163 \mathrm{E}-01$

8.8511E-01

8. $5538 \mathrm{E}-01$

2. $6667 \mathrm{E}-01$

3. $0729 \mathrm{E}+00$

$1.3286 \mathrm{E}-01$

$1.0035 E-01$

3. $1009 \mathrm{E}+0 \mathrm{I}$

7. 1992E-02

$1.7879 \mathrm{E}+00$

5. $1812 \mathrm{E}-02$

$2.8838 \mathrm{E}-01$ 
Minimum Detectable Activity Report (continued) HNF-SD-WM-DP-227, REV. 0 Page : 4 Sample ID : WL16280-BLNK

Acquisition date : 27-JAN-1997 09:32:45

Nuclide

NP-239

PU-239

AM-241

$A M-243$
Bckgnd

Sum

79.

54.

54 .
Energy

(kev)

106.12

129.30

59.54

74.67

$$
\begin{gathered}
M D A \\
(u C i / L)
\end{gathered}
$$

$1.2712 \mathrm{E}-01$

3. $9621 \mathrm{E}+02$

$3.0949 \mathrm{E}-01$

$7.2491 \mathrm{E}-02$ 
$* *^{4} * * * * * * * * * * * * * * * * * * * * * * * * * * * * * * * * * * * * * * * * * * * * * * * * * * * * * * * * * * * * * * * * * * * * * * * * * * * * *$

* 222-S Laboratory Counting Room 27-JAN-1997 11:21:49.97

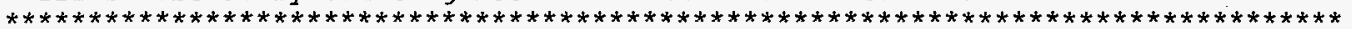
Worklist \#:

$>>>>>>>>$ SAMPLE INFORMATION $<<<<<<<<<~$

Sample ID:

Sample Size:

Dilution Factor:

$>>>>>>>>$ COUNT INFORMATION $<<<<<<<<<~$

Detector ID:

File Number:

Geometry:

Count Time:

Real Time:

Dead Time:
16280

S97T0025-SAM

$5.00000 \mathrm{E}-04 \mathrm{~L}$

1. $00000 \mathrm{E}+00$

GEA3

dka $300:$ [spec.GEA3] 3g3448.cnf

43

$000: 50: 00.00 \mathrm{sec}$

0 00:53:09.75 sec

$5.9 \%$

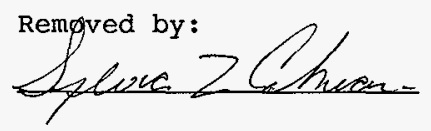

Verified by :

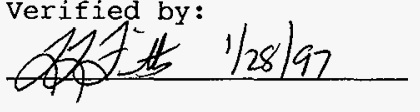

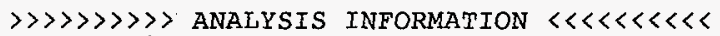

Sample Count Time:

Decayed to:

Standard Deviations:

Analysis Library:

Analyst:

27-JAN-1997 10:27:58.67

27-JAN-1997 $10: 27: 58.67$

2

ENVGEA

CJO

Background Subtract: DKA300: [ SPEC.GEA3] 3GBACK

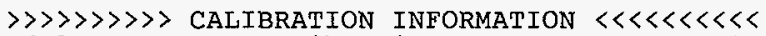

Date of last energy calibration: 11-MAR-1994 11:47:01.11

Date of last efficiency calibration: 15-MAR-1994 10:11:20.05

$* * * * * * * * * * * * * * * * * * * * * * * * * * * * * * * * * * * * * * * * * * * * * * * * * * * * * * * * * * * * * * * * * * * * * * * * * * * * * * * *$

Post-NID Peak Search Report

It Energy Area FWHM Channel Left Pw $\frac{\circ}{2}$ r Fit Nuclides Activity $\mathrm{uCi} / \mathrm{L}$

$\begin{array}{rrrrrrrrrr}0 & 661.61 * & 1393595 & 1.70 & 1323.40 & 1315 & 17 & 0.2 & C S-137 & 1.228 \mathrm{E}+04 \\ 0 & 1322.24 & 473 & 2.80 & 2644.85 & 2637 & 19 & 20.7 & \end{array}$


Sunmary of Nuclide Activity

Sample ID : S97T0025-SAM

Total number of lines in spectrum Number of unidentified lines

Number of lines tentatively identified by NID
HNF-SD-WM-DP-227, REV. 0

Page : 2

Acquisition date : 27-JAN-1997 10:27:58

Nuclide Type :
Nuclide CS -137
Hlife $30.00 \mathrm{Y}$
Wtd Mean
Wtd Mean
Uncorrected
Decay uCi/L
$1.000 \quad 1.228 \mathrm{E}+04$
Total Activity : $1.228 \mathrm{E}+04$ uCi/I 2-Sigma Error \%Error Flags
$1.228 \mathrm{E}+04$
$1.228 \mathrm{E}+04$
Decay Corr Decay Corr 2-Sigma
Grand Total Activity : $1.228 \mathrm{E}+04$
$1.228 \mathrm{E}+04$
Flags: " $\mathrm{K}$ " = Keyline not found
"M" = Manually accepted
"E" = Manually edited
"A" = Nuclide specific abn. limit 
Minimum Detectable Activity Report Sample ID : S97T0025-SAM

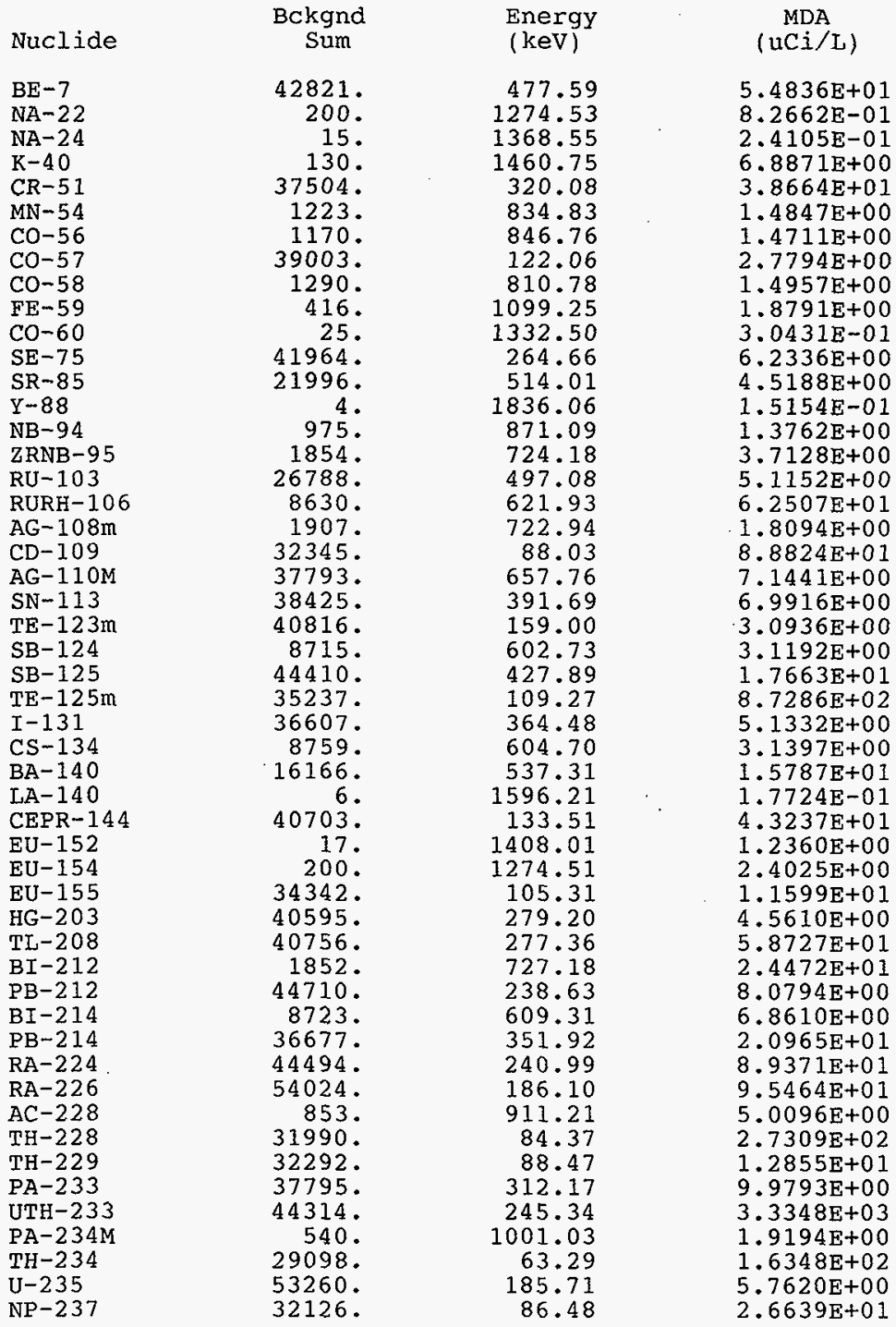

HNF-SD-WM-DP-227, REV. 0 Page : 3

Acquisition date : $27-J A N-1997$ 10:27:58

$5.4836 \mathrm{E}+01$

01

$6.8871 \mathrm{E}+00$

$3.8664 \mathrm{E}+01$

$2,7794 \mathrm{E}+00$

$1.4957 \mathrm{E}+00$

$1.8791 \mathrm{E}+00$

$3.0431 E-01$

1. $5154 \mathrm{E}-01$

$1.3762 \mathrm{E}+00$

$3.7128 \mathrm{E}+00$

$5.1152 \mathrm{E}+00$

$8.8824 \mathrm{E}+01$

$7.1441 \mathrm{E}+00$

$6.9916 \mathrm{E}+00$

$3.0936 \mathrm{E}+00$

$3.1192 \mathrm{E}+00$

$5.1332 \mathrm{E}+00$

$3.1397 \mathrm{E}+00$

1. $5787 \mathrm{E}+01$

1. $2360 \mathrm{E}+00$

$2.4025 \mathrm{E}+00$

1. $1599 \mathrm{E}+01$

$4.5610 \mathrm{E}+00$

$5.8727 \mathrm{E}+01$

. $4472 \mathrm{Et} 1$

$6.8610 \mathrm{E}+00$

$2.0965 \mathrm{E}+01$

$8.9371 \mathrm{E}+01$

9. $5464 \mathrm{E}+01$

$5.0096 \mathrm{E}+00$

1.2855 +01

$9.9793 \mathrm{E}+00$

$3.3348 \mathrm{E}+03$

1. $9194 \mathrm{E}+00$

$5.7620 \mathrm{E}+00$

$2.6639 \mathrm{E}+01$ 
Minimum Detectable Activity Report (continued)

HNF-SD-WM-DP-227, REV. $0_{\text {Page }: ~} 4$ Sample ID : S97T0025-SAM

Acquisition date : 27-JAN-1997 10:27:58

Nuclide

\section{Bckgnd}

Sum

NP-239

$\mathrm{PU}-239$

AM-241

$\mathrm{AM}-243$
34327 .

40605 .

28367 .

30701 .

\section{Energy}

(keV)

106.12

129.30

59.54

74.67

\section{MDA \\ ( UCi/L)}

1. $1003 \mathrm{E}+01$

3. $7414 \mathrm{E}+04$

$2.6642 \mathrm{E}+01$

$7.4381 \mathrm{E}+00$ 


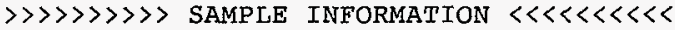

Worklist \#:

Sample ID:

Sample Size:

Dilution Factor:

$\gg \gg \gg \gg \gg \gg$ COUNT

Detector ID:

File Number:

Geometry:

Count Time:

Real Time:

Dead Time:
16280

S97T025-DUP

$5.00000 \mathrm{E}-04 \mathrm{~L}$

$1.00000 \mathrm{E}+00$
Removed by:

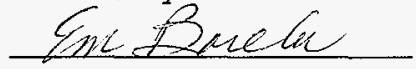

Verified by:

GEA3

NI $3 \mathrm{AC}: 1 \cdot \mathrm{cnf} \cdot 323^{4} 49 . \mathrm{Cof}$

$000: 50: 00.00 \mathrm{sec}$

$0 \quad 00: 53: 08.58 \mathrm{sec}$

$5.9 \%$

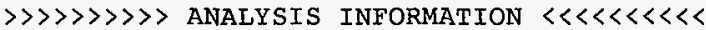

Sample Count Time:

27-JAN-1997 $11: 35: 32.21$

Decayed to:

Standard Deviations:

Analysis Library:

Analyst:

27-JAN-1997 $11: 35: 32.21$

2

ENVGEA

EMB

Background Subtract: DKA300: [SPEC.GEA3] 3GBACK

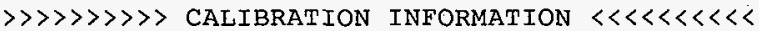

Date of last energy calibration: 11-MAR-1994 11:47:01.11

Date of last efficiency calibration: 15-MAR-1994 10:11:20.05

$* * * * \star * * * * * * * * * * * * * * * * * * * * * * * * * * * * * * * * * * * * * * * * * * * * * * * * * * * * * * * * * * * * * * * * * * * * * * * * * * *$

Post-NID Peak Search Report

Energy

Area FWHM Channel Left Pw $\%$ Err

Fit Nuclides Activity

$u \mathrm{Ci} / \mathrm{L}$

$661.61 * 1386482$

$1.70 \quad 1323.40$

1315

$17 \quad 0.2$

1322.21

531

$2.72 \quad 2644.79$

2637

$22 \quad 19.3$

CS-137

$1.222 \mathrm{E}+04$ 
Summary of Nuclide Activity

Sample ID : S97T025-DUP
HNF-SD-WM-DP-227, REV. 0

Page : 2

Acquisition date : 27-JAN-1997 11:35:32
Total number of lines in spectrum

Number of unidentified lines

2

Number of lines tentatively identified by NID

1

$150.00 \%$

Nuclide Type :

Nuclide

CS- 137
Wtd Mean

Uncorrected

HIife $30.00 Y \quad 1.000$

$\mathrm{uCi} / \mathrm{L}$

1.222E+04

Total Activity : $1.222 \mathrm{E}+04$
Wtd Mean

Decay Corr Decay Corr 2-Sigma $\mathrm{uCi} / \mathrm{L}$

1. $222 \mathrm{E}+04$

1. $222 \mathrm{E}+04$

Grand Total Activity : $1.222 \mathrm{E}+04$

$1.222 \mathrm{E}+04$

Flags: " $\mathrm{K}$ " = Keyline not found

" $\mathrm{E}$ " = Manually edited

"M" = Manually accepted

"A" = Nuclide specific abn. limit 
Minimum Detectable Activity Report Sample ID : S97T025-DUP

\begin{tabular}{|c|c|c|c|}
\hline Nuclide & $\begin{array}{l}\text { Bckgnd } \\
\text { Sum }\end{array}$ & $\begin{array}{l}\text { Energy } \\
\text { (kev) }\end{array}$ & $\begin{array}{c}\text { MDA } \\
(\mathrm{uCi} / \mathrm{L})\end{array}$ \\
\hline $\mathrm{BE}-7$ & 42671 . & 477.59 & $5.4740 \mathrm{E}+01$ \\
\hline$N A-22$ & 198. & 1274.53 & $8.2255 \mathrm{E}-01$ \\
\hline$N A-24$ & 17. & 1368.55 & $2.5698 \mathrm{E}-01$ \\
\hline $\mathrm{K}-40$ & 155. & 1460.75 & $7.5187 \mathrm{E}+00$ \\
\hline$C R-51$ & 37057. & 320.08 & $3.8433 \mathrm{E}+01$ \\
\hline$M N-54$ & 1223. & 834.83 & $1.4849 \mathrm{E}+00$ \\
\hline $\mathrm{CO}-56$ & 1129. & 846.76 & $1.4450 \mathrm{E}+00$ \\
\hline $\mathrm{CO}-57$ & 38590 . & 122.06 & $2.7647 \mathrm{E}+00$ \\
\hline $\mathrm{CO}-58$ & 1319. & 810.78 & $1.5128 \mathrm{E}+00$ \\
\hline $\mathrm{FE}-59$ & 434 . & 1099.25 & $1.9209 \mathrm{E}+00$ \\
\hline $\mathrm{CO}-60$ & 28. & 1332.50 & $3.2248 \mathrm{E}-01$ \\
\hline SE-75 & 41137. & 264.66 & $6.1719 \mathrm{E}+00$ \\
\hline$S R-85$ & 22214 . & 514.01 & $4.5411 \mathrm{E}+00$ \\
\hline $\mathrm{Y}-88$ & 7. & 1836.06 & $2.0105 \mathrm{E}-01$ \\
\hline $\mathrm{NB}-94$ & 1015 . & 871.09 & $1.4042 \mathrm{E}+00$ \\
\hline ZRNB-95 & 1871. & 724.18 & $3.7301 \mathrm{E}+00$ \\
\hline$R U-103$ & 27036 . & 497.08 & $5.1388 \mathrm{E}+00$ \\
\hline RURH-106 & 8261 . & 621.93 & $6.1157 \mathrm{E}+01$ \\
\hline$A G-108 m$ & 1865 & 722.94 & $1.7891 \mathrm{E}+00$ \\
\hline$C D-109$ & 31937. & 88.03 & $8.8263 \mathrm{E}+01$ \\
\hline$A G-110 \mathrm{M}$ & 37745 . & 657.76 & $7.1396 \mathrm{E}+00$ \\
\hline $\mathrm{SN}-113$ & 38250 . & 391.69 & $6.9757 \mathrm{E}+00$ \\
\hline$T E-123 m$ & 40496 . & 159.00 & $3.0815 \mathrm{E}+00$ \\
\hline$S B-124$ & 8545 . & 602.73 & $3.0886 \mathrm{E}+00$ \\
\hline$S B-125$ & 44250 . & 427.89 & $1.7631 \mathrm{E}+01$ \\
\hline$T E-125 m$ & 34795 . & 109.27 & $8.6737 \mathrm{E}+02$ \\
\hline$I-131$ & 36433 . & 364.48 & $5.1210 \mathrm{E}+00$ \\
\hline CS-134 & 8582 . & 604.70 & $3.1078 \mathrm{E}+00$ \\
\hline$B A-140$ & 15721. & 537.31 & $1.5568 \mathrm{E}+01$ \\
\hline$L A-140$ & & 1596.21 & $2.0466 \mathrm{E}-01$ \\
\hline CEPR-144 & 40632 . & 133.51 & $4.3199 \mathrm{E}+01$ \\
\hline $\mathrm{EU}-152$ & 13. & 1408.01 & $1.0794 \mathrm{E}+00$ \\
\hline EU-154 & 198. & 1274.51 & $2.3921 \mathrm{E}+00$ \\
\hline$E U-155$ & 34295 . & 105.31 & $1.1592 \mathrm{E}+01$ \\
\hline $\mathrm{HG}-203$ & 40383 . & 279.20 & $4.5490 \mathrm{E}+00$ \\
\hline TL-208 & 40332 . & 277.36 & $5.8421 \mathrm{E}+01$ \\
\hline$B I-212$ & 1833. & 727.18 & $2.4344 \mathrm{E}+01$ \\
\hline $\mathrm{PB}-212$ & 44822 . & 238.63 & $8.0895 \mathrm{E}+00$ \\
\hline BI-214 & 8642 . & 609.31 & $6.8291 \mathrm{E}+00$ \\
\hline $\mathrm{PB}-214$ & 36464 . & 351.92 & $2.0900 \mathrm{E}+01$ \\
\hline$R A-224$ & 44840 . & 240.99 & $8.9718 \mathrm{E}+01$ \\
\hline$R A-226$ & 54371. & 186.10 & $9.5771 \mathrm{E}+01$ \\
\hline$A C-228$ & 784. & 911.21 & $4.8046 \mathrm{E}+00$ \\
\hline $\mathrm{TH}-228$ & 31941 . & 84.37 & $2.7288 \mathrm{E}+02$ \\
\hline $\mathrm{TH}-229$ & 31870 . & 88.47 & $1.2771 \mathrm{E}+01$ \\
\hline $\mathrm{PA}-233$ & 37371 . & 312.17 & $9.9232 \mathrm{E}+00$ \\
\hline $\mathrm{UTH}-233$ & 43933. & 245.34 & $3.3204 \mathrm{E}+03$ \\
\hline$P A-234 M$ & 576 . & 1001.03 & $1.9816 \mathrm{E}+00$ \\
\hline $\mathrm{TH}-234$ & 28600 . & 63.29 & $1.6208 E+02$ \\
\hline $\mathrm{U}-235$ & 53713. & 185.71 & $5.7864 \mathrm{E}+00$ \\
\hline NP -237 & 31982 . & 86.48 & $2.6579 \mathrm{E}+01$ \\
\hline
\end{tabular}

HNF-SD-WM-DP-227, REV. 0

Page : 3

Acquisition date : 27-JAN-1997 11:35:32

$$
(u C i / L)
$$

$5.4740 \mathrm{E}+01$

$8.2255 \mathrm{E}-01$

$2.5698 \mathrm{E}-01$

$7.5187 \mathrm{E}+00$

$1.4450 \mathrm{E}+00$

$2.7647 \mathrm{E}+00$

$1.5128 \mathrm{E}+00$

$1.9209 \mathrm{E}+00$

$3.2248 \mathrm{E}-01$

$2.0105 \mathrm{E}-01$

$1.4042 \mathrm{E}+00$

$3.7301 \mathrm{E}+00$

$5.1388 \mathrm{E}+00$

$8.8263 \mathrm{E}+01$

$7.1396 \mathrm{E}+00$

$6.9757 \mathrm{E}+00$

$3.0815 \mathrm{E}+00$

8. $6737 \mathrm{E}+02$

$5.1210 \mathrm{E}+00$

$3.1078 \mathrm{E}+00$

$1.5568 \mathrm{E}+01$

$2.0466 \mathrm{E}-01$

$4.3199 \mathrm{E}+01$

$1.1592 \mathrm{E}+01$

$4.5490 \mathrm{E}+00$

$5.8421 E+01$

$2.4344 \mathrm{E}+01$

$2.0900 \mathrm{E}+0.1$

$8.9718 \mathrm{E}+01$

$9.5771 \mathrm{E}+01$

$4.8046 \mathrm{E}+00$

$2.7288 \mathrm{E}+02$

$3.3204 \mathrm{E}+03$

$1.9816 \mathrm{E}+00$

$1.6208 \mathrm{E}+02$

$2.6579 \mathrm{E}+01$ 
Minimum Detectable Activity Report (continued) HNF-SD-WM-DP-227, REV. 0 Page : 4 Sample ID : S97T025-DUP

Acquisition date : 27-JAN-1997 11:35:32

Nuclide

$\mathrm{NP}-239$

$\mathrm{PU}-239$

AM-241

$\mathrm{AM}-243$
Bckgnd

sum

34417 .

40254 .

28193.

30572 .
Energy

(keV)

106.12
129.30
59.54
74.67
MDA

( $\mathrm{UCi} / \mathrm{L})$

1. $1017 \mathrm{E}+01$

3. $7252 \mathrm{E}+04$

2. $6561 \mathrm{E}+01$

$7.4225 \mathrm{E}+00$ 


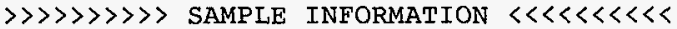

Worklist \#:

Sample ID:

Sample Size:

Dilution Factor:

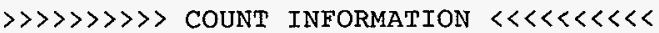
Detector ID:

File Number:

Geometry:

Count Time:

Real Time:

Dead Time:
16280

S97T028-SAM

$1.00000 \mathrm{E}-03 \mathrm{~L}$

$2.10000 \mathrm{E}+01$

GEA3

dka $300:[$ spec.GEA3] 3g3450.cnf

42

$000: 50: 00.00 \mathrm{sec}$

$000: 53: 21.73 \mathrm{sec}$

$6.3 \%$
HNF-SD-WM-DP-227, REV. 0

Removed by:

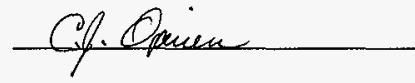

verified by:

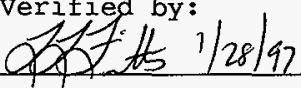

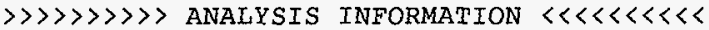

Sample Count Time:

Decayed to:

Standard Deviations:

Analysis Library:

Analyst:

Background Subtract:
27-JAN-1997 $12: 39: 39.06$

27-JAN-1997 $12: 39: 39.06$

2

ENVGEA

EMB

DKA300: [ SPEC.GEA3] 3GBACK

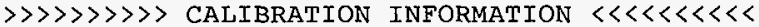

Date of last energy calibration: 11-MAR-1994 11:47:01.11

Date of last efficiency calibration: 15-MAR-1994 10:28:40.20

$\star * * * * * * * * * * * * * * * * * * * * * * * * * * * * * * * * * * * * * * * * * * * * * * * * * * * * * * * * * * * * * * * * * * * * * * * * * * * * * * *$

Post-NID Peak Search Report
It Energy

$0 \quad 661.61 * 1558165$

$0 \quad 1322.02$

01460.86 *

19
Area FWHM Channel

Left Pw \%Err

$1.71 \quad 1323.41$

$2.68 \quad 2644.40$

1315

2635
2.482922 .20
$17 \quad 0.2$

$22 \quad 18.2$

18150.7
Fit

Nuclides

CS -137

$\mathrm{K}-40$
Activity $\mathrm{uCi} / \mathrm{L}$

$3.698 \mathrm{E}+04$

6.97 
Summary of Nuclide Activity

Sample ID : S97T028-SAM
HNF-SD-WM-DP-227, REV. 0

Acquisition date : 27-JAN-1997 12:39:39

Total number of lines in spectrum 3

Number of unidentified lines

Number of lines tentatively identified by NID $2 \quad 66.67 \%$

Nuclide Type :

Nuclide

Wtd Mean wtd Mean

Uncorrected Decay Corr

K-40-1.28EF09Y $1.000-6.967 \mathrm{E}+0$
$\mathrm{uCi} / \mathrm{L}$

CS -137 $30.00 Y$ 1.000

$3.698 \mathrm{E}+04$

6. $967 \mathrm{E}+00$

$3.698 \mathrm{E}+04$

Decay Corr 2-Sigma
2-Sigma Error $\%$ Eror $10.50 \mathrm{E}+00$ 150.71 $0.006 \mathrm{E}+04$ 0.16 Total Activity : $3.699 \mathrm{E}+04 \quad 3.699 \mathrm{E}+04$

Grand Total Activity : $3.699 \mathrm{E}+04$

$3.699 E+04$

Flags: " $\mathrm{K} "=$ Keyline not found " $\mathrm{E} "$ = Manually edited

"M" = Manually accepted

"A" = Nuclide specific abn. limit 
Minimum Detectable Activity Report Sample ID : S97T028-SAM

\begin{tabular}{|c|c|c|c|}
\hline Nuclide & $\begin{array}{c}\text { Bckgnd } \\
\text { Sum }\end{array}$ & $\begin{array}{l}\text { Energy } \\
\text { (keV) }\end{array}$ & $\begin{array}{c}M D A \\
(\mathrm{uCi} / \mathrm{L})\end{array}$ \\
\hline 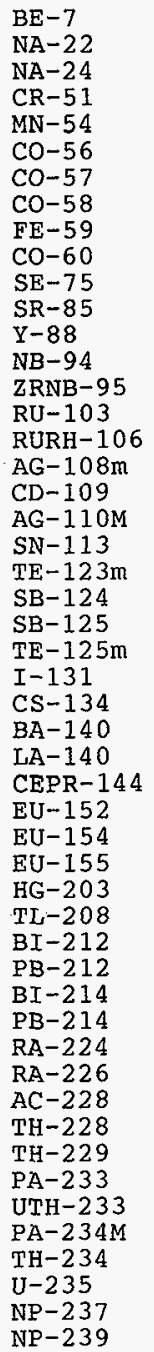 & 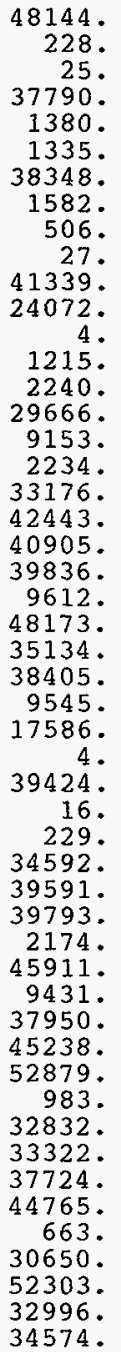 & $\begin{array}{r}477.59 \\
1274.53 \\
1368.55 \\
320.08 \\
834.83 \\
846.76 \\
122.06 \\
810.78 \\
1099.25 \\
1332.50 \\
264.66 \\
514.01 \\
1836.06 \\
871.09 \\
724.18 \\
497.08 \\
621.93 \\
722.94 \\
88.03 \\
657.76 \\
391.69 \\
159.00 \\
602.73 \\
427.89 \\
109.27 \\
364.48 \\
604.70 \\
537.31 \\
1596.21 \\
133.51 \\
1408.01 \\
1274.51 \\
105.31 \\
279.20 \\
277.36 \\
727.18 \\
238.63 \\
609.31 \\
351.92 \\
240.99 \\
186.10 \\
911.21 \\
84.37 \\
88.47 \\
312.17 \\
245.34 \\
1001.03 \\
63.29 \\
185.71 \\
86.48 \\
106.12\end{array}$ & $\begin{array}{l}1.5180 \mathrm{E}+02 \\
2.4903 \mathrm{E}+00 \\
8.8577 \mathrm{E}-01 \\
1.0121 \mathrm{E}+02 \\
4.2659 \mathrm{E}+00 \\
4.2481 \mathrm{E}+00 \\
7.1170 \mathrm{E}+00 \\
4.4775 \mathrm{E}+00 \\
5.7900 \mathrm{E}+00 \\
8.8554 \mathrm{E}-01 \\
1.5859 \mathrm{E}+01 \\
1.2272 \mathrm{E}+01 \\
4.4067 \mathrm{E}-01 \\
4.1539 \mathrm{E}+00 \\
1.1007 \mathrm{E}+01 \\
1.3992 \mathrm{E}+01 \\
1.7216 \mathrm{E}+02 \\
5.2816 \mathrm{E}+00 \\
2.1920 \mathrm{E}+02 \\
2.0380 \mathrm{E}+01 \\
1.9309 \mathrm{E}+01 \\
7.4625 \mathrm{E}+00 \\
8.7140 \mathrm{E}+00 \\
4.8919 \mathrm{E}+01 \\
2.2179 \mathrm{E}+03 \\
1.3991 \mathrm{E}+01 \\
8.7238 \mathrm{E}+00 \\
4.2870 \mathrm{E}+01 \\
4.1464 \mathrm{E}-01 \\
1.0914 \mathrm{E}+02 \\
3.3986 \mathrm{E}+00 \\
7.2390 \mathrm{E}+00 \\
2.9391 \mathrm{E}+01 \\
1.1573 \mathrm{E}+01 \\
1.4905 \mathrm{E}+02 \\
7.1501 \mathrm{E}+01 \\
2.0785 \mathrm{E}+01 \\
1.9014 \mathrm{E}+01 \\
5.6574 \mathrm{E}+01 \\
2.2912 \mathrm{E}+02 \\
2.2611 \mathrm{E}+02 \\
1.4587 \mathrm{E}+01 \\
6.7156 \mathrm{E}+02 \\
3.1839 \mathrm{E}+01 \\
2.5905 \mathrm{E}+01 \\
8.54199 \mathrm{E}+03 \\
5.8545 \mathrm{E}+00 \\
4.3704 \mathrm{E}+02 \\
1.3667 \mathrm{E}+01 \\
6.5655 \mathrm{E}+01 \\
2.7927 \mathrm{E}+01\end{array}$ \\
\hline
\end{tabular}

Acquisition date : $27-J A N-1997$ Page: $12: 39: 39$ 
Minimum Detectable Activity Report (continued) HNF-SD-WM-DP-227, REV. 0 Page : 4 Sample ID : S97T028-SAM Acquisition date : 27-JAN-1997 12:39:39

Nuclide

Bckgnd

Energy

( $\mathrm{keV}$ ) MDA

$$
\text { PU-239 }
$$

$\mathrm{AM}-241$

$\mathrm{AM}-243$

39162 .

30583 .

32020 .

129.30

59.54

74.67

( $\mathrm{uCi} / \mathrm{L}$ )

$9.4666 \mathrm{E}+04$

$7.7039 \mathrm{E}+01$

$1.8528 \mathrm{E}+01$ 


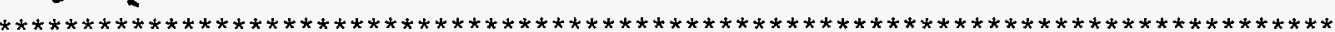

* 222-S Laboratory Counting Room 27-JAN-1997 14:45:20.85

$* * * * * * * * * * * * * * * * * * * * * * * * * * * * * * * * * * * * * * * * * * * * * * * * * * * * * * * * * * * * * * * * * * * * * * * * * * * * * * * *$

Worklist \#:

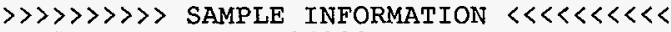

Sample ID:

Sample Size:

Dilution Factor:

$\gg \gg>>>>>>$ COUNT

Detector ID:

File Number:

Geometry:

Count Time:

Real Time:

Dead Time:
16280

S97T28-DUP

$1.00000 \mathrm{E}-03 \mathrm{~L}$

$2.10000 \mathrm{E}+01$

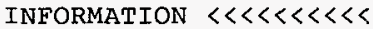

GEA3

dka300:[spec. GEA3] 3g3451. cnf

42

$000: 50: 00.00 \mathrm{sec}$

$0 \quad 00: 53: 18.67 \mathrm{sec}$

$6.2 \%$
HNF-SD-WM-DP-227, REV. 0

Remgved by:

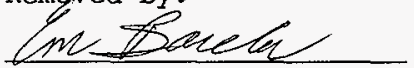

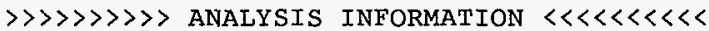

Sample Count Time:

Verified by:

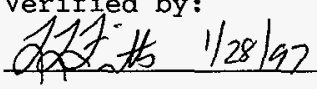

Decayed to:

Standard Deviations:

Analysis Library:

Analyst:

Background Subtract:
27-JAN-1997 $13: 51: 23.31$

27-JAN-1997 $13: 51: 23.31$

2

ENVGEA

C.JO

DKA300: [SPEC. GEA3 ] 3GBACK

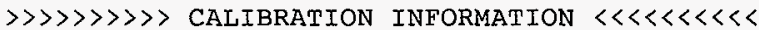

Date of last energy calibration: 11-MAR-1994 11:47:01.11

Date of last efficiency calibration: 15-MAR-1994 10:28:40.20

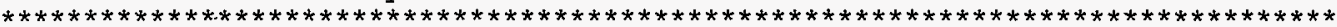

Post-NID Peak Search Report

It Energy Area FWHM Channel Left Pw $\%$ Err Fit Nuclides Activity $\mathrm{uCi} / \mathrm{L}$

$\begin{array}{llllllll}0 & 661.62 * & 1537989 & 1.71 & 1323.41 & 1315 & 18 & 0.2\end{array}$

$16 \quad 21.0$

CS-137

$3.651 \mathrm{E}+04$

Cs-137 
Summary of Nuclide Activity

Sample ID : S97T28-DUP
HNF-SD-WM-DP-227, REV. 0

Page : 2

Acquisition date : $27-J A N-199713: 51: 23$

Total number of lines in spectrum 2

Number of unidentified lines

Number of lines tentatively identified by NID $1 \quad 50.00 \%$

Nuclide Type :

\begin{tabular}{|c|c|c|c|c|c|c|c|}
\hline \multirow[t]{2}{*}{$\begin{array}{l}\text { Nuclide } \\
\text { CS-137 }\end{array}$} & $\begin{array}{l}\text { Hlife } \\
30.00 Y\end{array}$ & $\begin{array}{l}\text { Decay } \\
1.000\end{array}$ & $\begin{array}{c}\text { Uncorrected } \\
\text { uCi/L } \\
3.651 \mathrm{E}+04\end{array}$ & $\begin{array}{c}\text { Decay Corr } \\
\text { uCi } / \mathrm{L} \\
3.651 \mathrm{E}+04\end{array}$ & \multirow[t]{2}{*}{$\begin{array}{c}\text { Decay Corr } \\
\text { 2-Sigma Error } \\
0.006 \mathrm{E}+04\end{array}$} & \multirow[t]{2}{*}{$\begin{array}{c}2 \text {-Sigma } \\
\text { \% Error } \\
0.17\end{array}$} & Flags \\
\hline & Potal Act & ity & $3.651 \mathrm{E}+04$ & $3.651 \mathrm{E}+04$ & & & \\
\hline
\end{tabular}

Grand Total Activity : $3.651 \mathrm{E}+04 \quad 3.651 \mathrm{E}+04$

Flags: "K" $=$ Keyline not found

"E" = Manually edited

"M" = Manually accepted

"A" = Nuclide specific abn. limit 
Minimum Detectable Activity Report Sample ID : S97T28-DUP

\begin{tabular}{|c|c|c|c|}
\hline Nuclide & $\begin{array}{l}\text { Bckgnd } \\
\text { Sum }\end{array}$ & $\begin{array}{l}\text { Energy } \\
(\mathrm{keV})\end{array}$ & $\begin{array}{c}\mathrm{MDA} \\
(\mathrm{uCi} / \mathrm{L})\end{array}$ \\
\hline 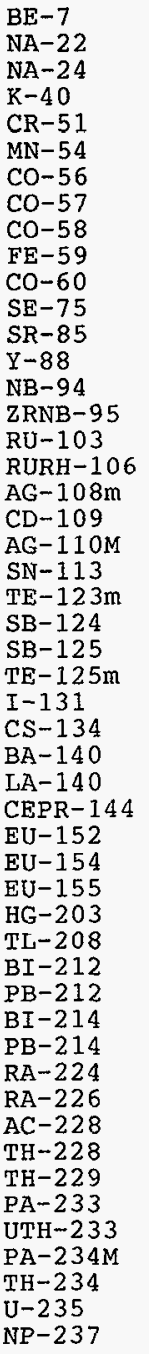 & 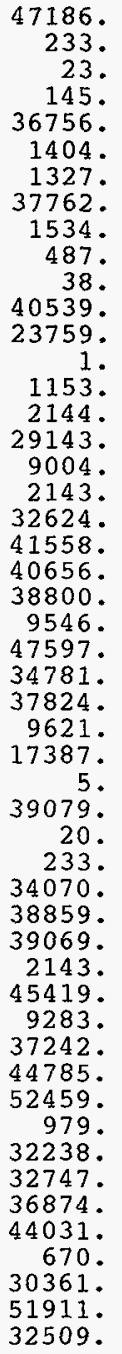 & $\begin{array}{r}477.59 \\
1274.53 \\
1368.55 \\
1460.75 \\
320.08 \\
834.83 \\
846.76 \\
122.06 \\
810.78 \\
1099.25 \\
1332.50 \\
264.66 \\
514.01 \\
1836.06 \\
871.09 \\
724.18 \\
497.08 \\
621.93 \\
722.94 \\
88.03 \\
657.76 \\
391.69 \\
159.00 \\
602.73 \\
427.89 \\
109.27 \\
364.48 \\
604.70 \\
537.31 \\
1596.21 \\
133.51 \\
1408.01 \\
1274.51 \\
105.31 \\
279.20 \\
277.36 \\
727.18 \\
238.63 \\
609.31 \\
351.92 \\
240.99 \\
186.10 \\
911.21 \\
84.37 \\
88.47 \\
312.17 \\
245.34 \\
1001.03 \\
63.29 \\
185.71 \\
86.48\end{array}$ & $\begin{array}{l}1.5028 \mathrm{E}+02 \\
2.5185 \mathrm{E}+00 \\
8.5064 \mathrm{E}-01 \\
2.0720 \mathrm{E}+01 \\
9.9817 \mathrm{E}+01 \\
4.3027 \mathrm{E}+00 \\
4.2366 \mathrm{E}+00 \\
7.0624 \mathrm{E}+00 \\
4.4102 \mathrm{E}+00 \\
5.6835 \mathrm{E}+00 \\
1.0505 \mathrm{E}+00 \\
1.5704 \mathrm{E}+01 \\
1.2192 \mathrm{E}+01 \\
2.2033 \mathrm{E}-01 \\
4.0469 \mathrm{E}+00 \\
1.0770 \mathrm{E}+01 \\
1.3868 \mathrm{E}+01 \\
1.7075 \mathrm{E}+02 \\
5.1730 \mathrm{E}+00 \\
2.1736 \mathrm{E}+02 \\
2.0166 \mathrm{E}+01 \\
1.9250 \mathrm{E}+01 \\
7.3648 \mathrm{E}+00 \\
8.6843 \mathrm{E}+00 \\
4.8625 \mathrm{E}+01 \\
2.2068 \mathrm{E}+03 \\
1.3884 \mathrm{E}+01 \\
8.7588 \mathrm{E}+00 \\
4.2626 \mathrm{E}+01 \\
4.4779 \mathrm{E}-01 \\
1.0866 \mathrm{E}+02 \\
3.7791 \mathrm{E}+00 \\
7.3137 \mathrm{E}+00 \\
2.9169 \mathrm{E}+01 \\
1.1466 \mathrm{E}+01 \\
1.4769 \mathrm{E}+02 \\
7.0991 \mathrm{E}+01 \\
2.0673 \mathrm{E}+01 \\
1.8864 \mathrm{E}+01 \\
5.6015 \mathrm{E}+01 \\
2.2797 \mathrm{E}+02 \\
2.2521 \mathrm{E}+02 \\
1.4555 \mathrm{E}+01 \\
6.6546 \mathrm{E}+02 \\
3.1563 \mathrm{E}+01 \\
2.5611 \mathrm{E}+01 \\
8.4715 \mathrm{E}+03 \\
5.8887 \mathrm{E}+00 \\
4.3497 \mathrm{E}+02 \\
1.3616 \mathrm{E}+01 \\
6.5169 \mathrm{E}+01\end{array}$ \\
\hline
\end{tabular}

Acquisition date : 27-JAN-1997 $13: 51: 23$

HNF-SD-WM-DP-227, REV. 0

(1)


Minimum Detectable Activity Report (continued)

HNF-SD-WM-DP-227, REV. 0 Page : 4

Sample ID : S97T28-DUP

Acquisition date : 27-JAN-1997 13:51:23

Nuclide

Bckgnd

Energy

sum

(keV)

$$
\begin{gathered}
\mathrm{MDA} \\
(\mathrm{uCi} / \mathrm{L})
\end{gathered}
$$

$\mathrm{NP}-239$

PU-239

34115 .

38251 .

106.12

129.30

59.54

74.67

$2.7740 \mathrm{E}+01$

$9.3558 \mathrm{E}+04$

$7.6553 \mathrm{E}+01$

1. $8301 \mathrm{E}+01$

31239 . 
$-1$

* 222-S Laboratory Counting Room

27-JAN-1997 16:03:17.89

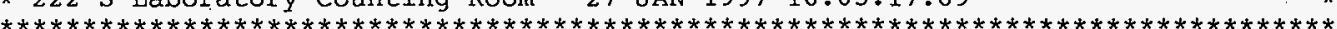
$>>>>>>>>>$ SAMPLE INFORMATION $<<<<<<<<<<~$

Worklist \#:

Sample ID:

Sample Size:

Dilution Factor:

$\gg \gg \gg \gg \gg \gg>$ COUNT

Detector ID:

File Number: .

Geometry:

Count Time:

Real Time:

Dead Time:
16280

S97T029-SAM

2.50000E-04 L

2. $10000 \mathrm{E}+01$
HNF-SD-WM-DP-227, REV. 0

Removed by:

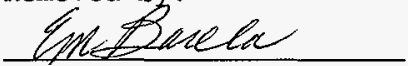

Verified by:

GEA3

dka300: [ spec.GEA3]3g3453.cnf

42

$000: 50: 00.00 \mathrm{sec}$

0 00:50:32.48 sec

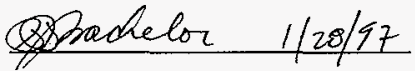

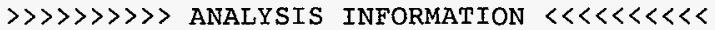

Sample Count Time: 27-JAN-1997 15:11:58.57

Decayed to: 27-JAN-1997 15:11:58.57

Standard Deviations: 2

Analysis Library:

Analyst:

ENVGEA

EMB

Background Subtract:

DKA $300:$ [ SPEC.GEA3] 3GBACK

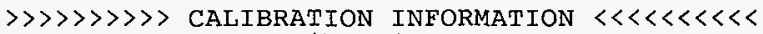

Date of last energy calibration: 11-MAR-1994 11:47:01.11

Date of last efficiency calibration: 15-MAR-1994 10:28:40.20

$* * * * * * * * * * * * * * * * * * * * * * * * * * * * * * * * * * * * * * * * * * * * * * * * * * * * * * * * * * * * * * * * * * * * * * * * * * * * * * * *$

Post-NID Peak search Report

It Energy Area FWHM Channel Left Pw $\%$ Err Fit Nuclides Activity

$\mathrm{uCi} / \mathrm{L}$

\begin{tabular}{|c|c|c|c|c|c|c|c|c|}
\hline $\begin{array}{r}59.34 \\
104.95 \\
122.89\end{array}$ & $\begin{array}{r}13520 \\
228 \\
733\end{array}$ & $\begin{array}{l}1.26 \\
0.95 \\
1.61\end{array}$ & $\begin{array}{l}119.22 \\
210.39 \\
246.27\end{array}$ & $\begin{array}{l}114 \\
209 \\
243\end{array}$ & $\begin{array}{rr}10 & 3.3 \\
5 & 92.0 \\
8 & 43.4\end{array}$ & & $\begin{array}{l}\mathrm{CO}-57 \\
\mathrm{HU}-154\end{array}$ & $5.123 E+03$ \\
\hline $\begin{array}{c}661.60 \star \\
723.67 \\
873.22 \\
996.48 \\
1004.87 \\
1173.75 \\
1274.38 \\
1332.34 \\
1460.60 *\end{array}$ & $\begin{array}{r}258248 \\
162 \\
64 \\
57 \\
75 \\
47 \\
140 \\
34 \\
16\end{array}$ & $\begin{array}{l}1.69 \\
1.84 \\
2.05 \\
2.35 \\
2.35 \\
2.41 \\
2.15 \\
2.29 \\
1.96\end{array}$ & $\begin{array}{l}1323.38 \\
1447.50 \\
1746.61 \\
1993.16 \\
2009.93 \\
2347.77 \\
2549.10 \\
2665.05 \\
2921.68\end{array}$ & $\begin{array}{l}1315 \\
1441 \\
1743 \\
1988 \\
1988 \\
2343 \\
2542 \\
2661 \\
2912\end{array}$ & $\begin{array}{rr}17 & 0.4 \\
16 & 33.1 \\
9 & 44.4 \\
34 & 41.2 \\
34 & 40.6 \\
15 & 63.0 \\
16 & 16.9 \\
10 & 45.5 \\
19 & 164.7\end{array}$ & 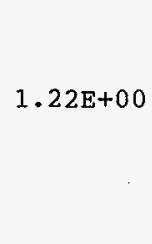 & $\begin{array}{l}\text { CS-137 } \\
\text { EU-154 } \\
\text { EU-154 } \\
\text { EU-154 } \\
\text { EU-154 } \\
\text { CO-60 } \\
\text { EU-154 } \\
C O-60 \\
\mathrm{~K}-40\end{array}$ & $\begin{array}{l}2.452 \mathrm{E}+04 \\
70.7 \\
54.6 \\
63.2 \\
48.7 \\
6.25 \\
57.7 \\
5.00 \\
23.8\end{array}$ \\
\hline
\end{tabular}


Total number of lines in spectrum 12 Number of unidentified Iines Number of lines tentatively identified by NID $12 \quad 100.00 \%$ Nuclide Type :

\begin{tabular}{|c|c|c|c|c|c|c|}
\hline \multirow[t]{2}{*}{$\begin{array}{l}\text { Nuclide } \\
\text { K-40 } \\
\mathrm{EO}-57 \\
\mathrm{CO}-60 \\
\mathrm{CS}-137 \\
\mathrm{EU}-154 \\
\mathrm{AM}-241\end{array}$} & $\begin{array}{r}\text { Hlife } \\
1.28 \mathrm{E}+09 \mathrm{Y} \\
27.8 .80 \mathrm{D} \\
5.27 \mathrm{Y} \\
30.00 \mathrm{Y} \\
8.59 \mathrm{Y} \\
432.20 \mathrm{Y}\end{array}$ & $\begin{array}{l}\text { Decay } \\
1.000 \\
1.000 \\
1.000 \\
1.000 \\
1.000 \\
1.000\end{array}$ & $\begin{array}{c}\text { Wtd Mean } \\
\text { Uncorrected } \\
\text { uCi/L } \\
2.380 \mathrm{~L}+01 \\
2.290 \mathrm{E}+01 \\
5.311 \mathrm{E}+00 \\
2.452 \mathrm{E}+04 \\
5.788 \mathrm{E}+01 \\
5.123 \mathrm{E}+03\end{array}$ & $\begin{array}{c}\text { Wtd Mean } \\
\text { Decay Corr } \\
\text { uCi/L } \\
2.380 \mathrm{E}+01 \\
2.290 \mathrm{E}+01 \\
5.311 \mathrm{E}+00 \\
2.452 \mathrm{E}+04 \\
5.788 \mathrm{E}+01 \\
5.123 \mathrm{E}+03\end{array}$ & $\begin{array}{c}\text { Decay Corr } \\
\text { 2-Sigma Error } \\
3.921 \mathrm{E}+01 \\
0.993 \mathrm{E}+01 \\
1.968 \mathrm{E}+00 \\
0.001 \mathrm{E}+05 \\
0.744 \mathrm{E}+01 \\
0.169 \mathrm{E}+03\end{array}$ & $\begin{array}{l}\text { 2-Sigma } \\
\% \text { Error Flags } \\
164.70 \text {, } \\
-43.38 \text { En }(28) 1 / 28 \\
37.06 \\
0.40 \\
12.85 \\
3.30\end{array}$ \\
\hline & Total Act & vity & $2.975 \mathrm{E}+04$ & $2.975 E+04$ & & \\
\hline Grand & Total Act & vity & $2.975 E+04$ & $2.975 \mathrm{E}+04$ & & \\
\hline Flags: & $\begin{array}{l}\mathrm{K}^{\prime \prime}=\text { Keyli } \\
\mathrm{E}^{\prime \prime}=\text { Manua }\end{array}$ & $\begin{array}{l}\text { e not } \\
\text { ly edit }\end{array}$ & $\begin{array}{l}\text { found } \\
\text { ted }\end{array}$ & $\begin{array}{l}\text { "M" = Manu } \\
\text { "A" = Nucl }\end{array}$ & $\begin{array}{l}\text { lly accepted } \\
\text { de specific abr }\end{array}$ & n. Iimit \\
\hline
\end{tabular}


- Minìmum Detectable Activity Report Sample ID : S97T029-SAM

\begin{tabular}{|c|c|c|c|}
\hline Nuclide & $\begin{array}{c}\text { Bckgnd } \\
\text { Sum }\end{array}$ & $\begin{array}{l}\text { Energy } \\
(\mathrm{kev})\end{array}$ & $\begin{array}{c}\mathrm{MDA} \\
(\mathrm{uCi} / \mathrm{L})\end{array}$ \\
\hline $\mathrm{BE}-7$ & 7755 . & 477.59 & $2.4370 \mathrm{E}+02$ \\
\hline NA- 22 & 135 . & 1274.53 & $7.6709 \mathrm{E}+00$ \\
\hline$N A-24$ & 9. & 1368.55 & $2.1126 \mathrm{E}+00$ \\
\hline$C R-51$ & 6108. & 320.08 & $1.6276 \mathrm{E}+02$ \\
\hline$M N-54$ & 76 & 834.83 & $4.0004 \mathrm{E}+00$ \\
\hline $\mathrm{CO}-56$ & 63. & 846.76 & $3.6857 \mathrm{E}+00$ \\
\hline $\mathrm{CO}-58$ & 64. & 810.78 & $3.6085 \mathrm{E}+00$ \\
\hline$F E-59$ & 22 & 1099.25 & $4.8508 \mathrm{E}+00$ \\
\hline $\mathrm{SE}-75$ & 6925 . & 264.66 & $2.5963 \mathrm{E}+01$ \\
\hline$S R-85$ & 3904 . & 514.01 & $1.9770 \mathrm{E}+01$ \\
\hline$Y-88$ & 3. & 1836.06 & $1.5265 \mathrm{E}+00$ \\
\hline NB-94 & 92. & 871.09 & $4.5599 \mathrm{E}+00$ \\
\hline ZRNB-95 & 202 & 724.18 & $1.3235 \mathrm{E}+01$ \\
\hline $\mathrm{RU}-103$ & 4771 . & 497.08 & $2.2446 \mathrm{E}+01$ \\
\hline RURH-106 & 1409 . & 621.93 & $2.7022 \mathrm{E}+02$ \\
\hline$A G-108 m$ & 211 & 722.94 & $6.4877 \mathrm{E}+00$ \\
\hline$C D-109$ & 5800 . & 88.03 & $3.6662 \mathrm{E}+02$ \\
\hline$A G-110 M$ & 6863. & 657.76 & $3.2781 \mathrm{E}+01$ \\
\hline$S N-113$ & 6813. & 391.69 & $3.1521 \mathrm{E}+01$ \\
\hline$T E-123 \mathrm{~m}$ & 6753. & 159.00 & $1.2290 \mathrm{E}+01$ \\
\hline $\begin{array}{l}S B-124 \\
S B-125\end{array}$ & 1470. & $\begin{array}{l}602.73 \\
427.89\end{array}$ & $\begin{array}{l}1.3632 \mathrm{E}+01 \\
7.8353 \mathrm{E}+01\end{array}$ \\
\hline $\begin{array}{l}\mathrm{SB}-125 \\
\mathrm{TE}-125 \mathrm{~m}\end{array}$ & 6162 . & 109.27 & $3.7153 \mathrm{E}+03$ \\
\hline$I-131$ & 6317. & 364.48 & $2.2695 \mathrm{E}+01$ \\
\hline CS-134 & 1447 . & 604.70 & $1.3589 \mathrm{E}+01$ \\
\hline$B A-140$ & 2819. & 537.31 & $6.8657 \mathrm{E}+01$ \\
\hline $\mathrm{LA}-140$ & 7 & 1596.21 & $2.1932 \mathrm{E}+00$ \\
\hline CEPR-144 & 6839. & 133.51 & $1.8183 \mathrm{E}+02$ \\
\hline EU-152 & 6. & 1408.01 & $8.2519 \mathrm{E}+00$ \\
\hline EU-155 & 6333. & 105.31 & $5.0304 \mathrm{E}+01$ \\
\hline $\mathrm{HG}-203$ & 6481 . & 279.20 & $1.8730 \mathrm{E}+01$ \\
\hline$T L-208$ & 6627 . & 277.36 & $2.4330 \mathrm{E}+02$ \\
\hline$B I-212$ & 86 . & 727.18 & $5.6788 \mathrm{E}+01$ \\
\hline $\mathrm{PB}-212$ & 7800. & 238.63 & $3.4269 \mathrm{E}+01$ \\
\hline BI -214 & 1431 . & 609.31 & $2.9627 \mathrm{E}+01$ \\
\hline PB-2 14 & 6270 . & 351.92 & $8.9392 \mathrm{E}+01$ \\
\hline$R A-224$ & 7763. & 240.99 & $3.7958 \mathrm{E}+02$ \\
\hline$R A-226$ & 9063 . & 186.10 & $3.7443 \mathrm{E}+02$ \\
\hline $\mathrm{AC}-228$ & 76 . & 911.21 & $1.6222 \mathrm{E}+01$ \\
\hline $\mathrm{TH}-228$ & 6032 . & 84.37 & $1.1514 \mathrm{E}+03$ \\
\hline $\mathrm{TH}-229$ & 5768 . & 88.47 & $5.2987 \mathrm{E}+01$ \\
\hline $\mathrm{PA}-233$ & 5933. & 312.17 & $4.1093 \mathrm{E}+01$ \\
\hline UTH -233 & 7534 . & 245.34 & $1.4017 \mathrm{E}+04$ \\
\hline $\mathrm{PA}-234 \mathrm{M}$ & 43. & 1001.03 & $5.9888 \mathrm{E}+00$ \\
\hline $\mathrm{TH}-234$ & 5512 . & 63.29 & $7.4133 \mathrm{E}+02$ \\
\hline $\mathrm{U}-235$ & 8923. & 185.71 & $2.2581 \mathrm{E}+01$ \\
\hline $\mathrm{NP}-237$ & 6068 . & 86.48 & $1.1262 \mathrm{E}+02$ \\
\hline NP -239 & 6268 . & 106.12 & $4.7550 \mathrm{E}+01$ \\
\hline$P U-239$ & 6870 . & 129.30 & $1.5860 \mathrm{E}+05$ \\
\hline$A M-243$ & 5555 . & 74.67 & $3.0870 \mathrm{E}+01$ \\
\hline
\end{tabular}

HNF-SD-WM-DP-227, REV. 0

Acquisition date : 27-JAN-1997 15:11:58

$2.4370 \mathrm{E}+02$

$1.6276 \mathrm{E}+02$

$4.0004 \mathrm{E}+00$

$3.6857 \mathrm{E}+00$

$3.6085 \mathrm{E}+00$

$4.8508 \mathrm{E}+00$

$2.5963 \mathrm{E}+01$

$4.5599 \mathrm{E}+00$

$1.3235 \mathrm{E}+01$

$2.2446 \mathrm{E}+01$

$2.702 \cdot 2 \mathrm{E}+02$

$6.4877 \mathrm{E}+00$

$3.6662 \mathrm{E}+02$

.

$1.2290 \mathrm{E}+01$

$1.3632 \mathrm{E}+01$

$7.8353 \mathrm{E}+01$

$3.7153 \mathrm{E}+03$

$6.8657 \mathrm{E}+01$

$2.1932 \mathrm{E}+00$

$1.8183 E+02$

$8.2519 \mathrm{E}+00$

$5.0304 \mathrm{E}+01$

$1.8730 \mathrm{E}+01$

.

$3.4269 \mathrm{E}+01$

$2.9627 \mathrm{E}+01$

$8.9392 \mathrm{E}+01$

$3.7958 \mathrm{E}+02$

$3.7443 \mathrm{E}+02$

$1.6222 \mathrm{E}+01$

.

$4.1093 \mathrm{E}+01$

$1.4017 \mathrm{E}+04$

$5.9888 \mathrm{E}+00$

$7.4133 \mathrm{E}+02$

$2.2581 \mathrm{E}+01$

1.72628+02

$3.0870 \mathrm{E}+01$ 
GHF-SD-WIMEDP-227, REV. 0

* 222-S Laboratory Counting Room 27-JAN-1997 16:58:54.17

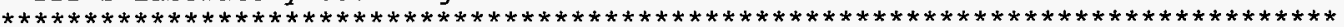

Worklist \#:

Sample ID:

Sample Size:

Dilution Factor:

$\gg \gg \gg \gg \gg \gg \gg \gg$ COUNT INFORMATION $<<<<<<<<<<~$

Detector ID:

File Number:

Geometry:

Count Time:

Real Time:

Dead Time:

16280

S97T029-DUP

2.50000E-04 L

$2.10000 \mathrm{E}+01$

GEA3

42

$000: 50: 00.00 \mathrm{sec}$

$000: 50: 32.14 \mathrm{sec}$

$1.1 \%$

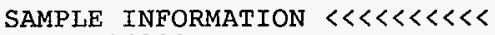

Removed by:

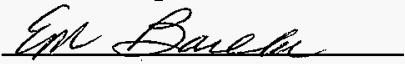

dka 300: [ spec.GEA3] 3g3454.cnf verified by:

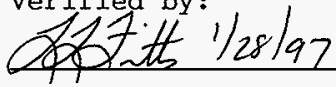

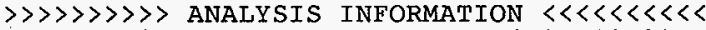

Sample Count Time: 27-JAN-1997 16:07:43.21

Decayed to:

Standard Deviations: 2

Analysis Library:

Analyst:

27-JAN-1997 $16: 07: 43.21$

ENVGEA

EMB

Background Subtract: DKA300:[SPEC.GEA3]3GBACK

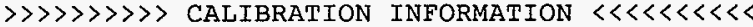

Date of last energy calibration: 11-MAR-1994 11:47:01.11

Date of last efficiency calibration: 15-MAR-1994 10:28:40.20

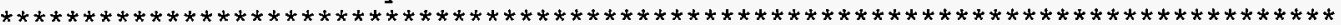

Post-NID Peak Search Report

It Energy

Area FWHM Channel Left Pw $\%$ Err

Fit Nuclides Activity $\mathrm{uCi} / \mathrm{L}$

$0 \quad 59.30$

122.80

$12308 \quad 1.23$

119.15

$\begin{array}{lll}560 & 1.07 & 246.09\end{array}$

$\begin{array}{lll}114 & 11 & 3.8\end{array}$

$\begin{array}{lll}243 & 7 & 50.6\end{array}$

$\mathrm{AM}-241$

$\mathrm{CO}-57$

EU-154

$661.59 *$

255072

1.691323 .36

1315

$\begin{array}{ll}17 & 0.4\end{array}$

CS-137

723.16

135

$1.24 \quad 1446.48$

1441

1129.4

872.64

$1.24 \quad 1745.45$

1741

1166.6

995.68

$52 \quad 1.601991 .55$

1985

1562.3

1004.41

$\begin{array}{lll}68 & 2.40 & 2009.03\end{array}$

2003

1350.3

$1119.95 *$

$\begin{array}{lll}20 & 1.78 & 2240.14\end{array}$

2235

$10 \quad 91.8$

1173.24

$\begin{array}{lll}70 & 1.39 & 2346.75\end{array}$

2341

$12 \quad 33.8$

1274.44

1332.42

$\begin{array}{lll}123 & 1.26 & 2549.22\end{array}$

2542

1428.0

$\begin{array}{lll}78 & 1.52 & 2665.21\end{array}$

2660

1026.1

EU-154

$\mathrm{EU}-154$

$\mathrm{EU}-154$

EU-154

4. $664 \mathrm{E}+03$

17.5

37.1

$2.422 \mathrm{E}+04$

58.7

41.7

58.3

43.7

$\mathrm{CO}-60$

9.34

EU-154

50.6

$\mathrm{CO}-60$

11.5 
Summary of Nuclide Activity

Sample ID : S97T029-DUP
HNF-SD-WM-DP-227, REV. 0

Acquisition date : 27-JAN-1997 16:07:43

Total number of lines in spectrum

11

Number of unidentified lines

Number of lines tentatively identified by NID 11

$100.00 \%$

Nuclide Type :

\begin{tabular}{|c|c|c|c|c|c|c|}
\hline $\begin{array}{l}\text { Nuclide } \\
\text { CO-57 } \\
\text { CO-60 } \\
\text { CS-137 } \\
\text { EU-154 } \\
\text { AM-241 }\end{array}$ & $\begin{array}{r}\text { Hlife } \\
271.80 \mathrm{Y} \\
5.27 \mathrm{Y} \\
30.00 Y \\
8.59 Y \\
432.20 Y\end{array}$ & $\begin{array}{r}\text { Decay } \\
-1.000 \\
1.000 \\
1.000 \\
1.000 \\
1.000\end{array}$ & $\begin{array}{c}\text { Wtd Mean } \\
\text { Uncorrected } \\
\text { uCi/L } \\
1.751 \mathrm{E}+01 \\
1.049 \mathrm{E}+01 \\
2.422 \mathrm{E}+04 \\
5.116 \mathrm{E}+01 \\
4.664 \mathrm{E}+03\end{array}$ & $\begin{array}{c}\text { Wtd Mean } \\
\text { Decay Corr } \\
\text { uCi/L } \\
1.751 \mathrm{E}+01 \\
1.049 \mathrm{E}+01 \\
2.422 \mathrm{E}+04 \\
5.116 \mathrm{E}+01 \\
4.664 \mathrm{E}+03\end{array}$ & $\begin{array}{c}\text { Decay Corr } \\
2-\text { Sigma Error } \\
0.806 \mathrm{E}+01 \\
0.218 \mathrm{E}+01 \\
0.001 \mathrm{E}+05 \\
0.896 \mathrm{E}+01 \\
0.176 \mathrm{E}+03\end{array}$ & $\begin{array}{l}2 \text {-Sigma } \\
\text { \%Error Flags } \\
50.62 \text { of } 1 / 2819 \\
20.77 \\
0.40 \\
17.51 \\
3.77\end{array}$ \\
\hline & Total AC & ity : & $2.896 \mathrm{E}+04$ & $2.896 \mathrm{E}+04$ & & \\
\hline
\end{tabular}

Grand Total Activity : $2.896 \mathrm{E}+04 \quad 2.896 \mathrm{E}+04$

Flags: "K" = Keyline not found

"M" = Manually accepted

"E" = Manually edited

"A" = Nuclide specific abn. limit 
Minimum Detectable Activity Report Sample ID : S97T029-DUP

Nuclide

Bckgnd

BE-7
$\mathrm{NA}-22$
$\mathrm{NA}-24$
$\mathrm{~K}-40$
$\mathrm{CR}-51$
$\mathrm{MN}-54$
$\mathrm{CO}-56$
$\mathrm{CO}-58$
$\mathrm{FE}-59$
$\mathrm{SE}-75$
$\mathrm{SR}-85$
$\mathrm{Y}-88$
$\mathrm{NB}-94$

ZRNB-95

$\mathrm{RU}-103$

RURH-106

AG- $108 \mathrm{~m}$

CD-109

AG- $110 \mathrm{M}$

$\mathrm{SN}-113$

TE-123m

$\mathrm{SB}-124$

$\mathrm{SB}-125$

$\mathrm{TE}-125 \mathrm{~m}$

$\mathrm{I}-131$

CS-134

$\mathrm{BA}-140$

LA -140

CEPR-144

EU-152

EU-155

HG -203

TL-208

$\mathrm{BI}-212$

PB-212

BI-214

PB-214

$\mathrm{RA}-224$

$\mathrm{RA}-226$

$\mathrm{AC}-228$

$\mathrm{TH}-228$

TH-229

$\mathrm{PA}-233$

UTH-233

$\mathrm{PA}-234 \mathrm{M}$

$\mathrm{TH}-234$

$\mathrm{U}-235$

$\mathrm{NP}-237$

NP -239

PU-239

AM-243

HNF-SD-WM-DP-227, REV. 0

Page : 3

Acquisition date : 27-JAN-1997 16:07:43

Energy
(kev)

477.59

1274.53

1368.55

1460.75

320.08

834.83

846.76

810.78

1099.25

264.66

514.01

1836.06

871.09

724.18

497.08

621.93

722.94

88.03

657.76

391.69

159.00

602.73

427.89

109.27

364.48

604.70

537.31

1596.21

133.51

1408.01

105.31

279.20

277.36

727.18

238.63

609.31

351.92

240.99

186.10

911.21

84.37

88.47

312.17

245.34

1001.03

63.29

185.71

86.48

106.12

129.30

74.67
MDA

( UCi/L)

$2.4003 E+02$

$8.0952 \mathrm{E}+00$

$1.7578 \mathrm{E}+00$

$7.4959 \mathrm{E}+01$

$1.6260 \mathrm{E}+02$

$3.3120 \mathrm{E}+00$

$3.8384 \mathrm{E}+00$

$3.6034 \mathrm{E}+00$

$5.7755 \mathrm{E}+00$

$2.5781 \mathrm{E}+01$

1.9515E+0 1

$1.2464 \mathrm{E}+00$

$4.6839 \mathrm{E}+00$

1. $2680 \mathrm{E}+01$

$2.2287 \mathrm{E}+01$

$2.6747 \mathrm{E}+02$

$6.2366 \mathrm{E}+00$

$3.6713 \mathrm{E}+02$

3. $2924 \mathrm{E}+01$

$3.1023 \mathrm{E}+01$

1. $2250 \mathrm{E}+01$

1. $3216 \mathrm{E}+01$

$7.8627 \mathrm{E}+01$

$3.6935 \mathrm{E}+03$

$2.2507 \mathrm{E}+01$

1. $3234 \mathrm{E}+01$

$6.8084 \mathrm{E}+01$

$2.0305 E+00$

$1.7981 \mathrm{E}+02$

1. $2591 \mathrm{E}+01$

4. $9796 \mathrm{E}+01$

$1.8773 \mathrm{E}+01$

2. $3981 \mathrm{E}+02$

$5.2605 \mathrm{E}+01$

3. $3764 \mathrm{E}+01$

$2.8841 \mathrm{E}+01$

$8.9398 \mathrm{E}+01$

$3.7309 E+02$

$3.7443 \mathrm{E}+02$

1. $3933 \mathrm{E}+01$

1. $1281 \mathrm{E}+03$

$5.2874 \mathrm{E}+01$

4. $1411 \mathrm{E}+01$

1. $3892 \mathrm{E}+04$

$6.7029 \mathrm{E}+00$

$7.2187 \mathrm{E}+02$

$2.2660 \mathrm{E}+01$

$1.1216 \mathrm{E}+02$

$4.7357 \mathrm{E}+01$

1. $5725 \mathrm{E}+05$

$3.0683 \mathrm{E}+01$ 


\section{LABCORE Completed RadChem Report for Worklist\#: 16361}

Analyst: scl

Method:
Instrument: $\mathrm{AB} 10$

Book\#

Worklist Comment: RR\#2 SY-102. Use 0.100-10-1mL. sac

\begin{tabular}{|c|c|c|c|c|c|c|c|c|c|}
\hline Seq Type & Sample & $\mathbf{R A}$ & & Test & Matrix & Actual & DL or Yield & Unit & \\
\hline $1 \mathrm{stp}$ & & 0 & $0 \mathrm{SR90001}$ & $\mathrm{SR} 900 \mathrm{O}$ & LIOUID & $1668 \mathrm{~B} 03$ & $166 \mathrm{~B} 3$ & I Rocovoy & \\
\hline 1 STD & & 0 & GSR90-01 & SR90-01C & IIOOID & 100 & $9.07 \mathrm{E}+01$ & \& Recovery & \\
\hline $1 \%$ & & 0 & 6sRoOo1 & $8 R 9001 R$ & mounp & 1,00 & $166 \mathrm{~B}+00$ & $10 t, B_{r}-0$ & \\
\hline 2 BLANK & & 0 & GSR90-01 & SR90-01 & LIQUID & 1 & $<2.39 \mathrm{E}-4$ & $\mathrm{uCi} / \mathrm{mL}$ & \\
\hline $2 \%$ & & 0 & $8 s_{90} 01$ & $8 R 90-01 \mathrm{C}$ & Irouth & 100 & $8,88 E+01$ & 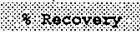 & \\
\hline 2 BLNK & & 0 & ESR90-01 & SR90-01B & IIQUID & 1.00 & $5.00 \mathrm{E}+02$ & $\mathrm{uCi} / \mathrm{mL}$ & \\
\hline 3 BसHK/BRG & & 0 & 6sR90001 & SR 9001 & HoOID & १1 & $1028 \times 00$ & BMKS/BKG & \\
\hline 4 SAMPLE & s97T000025 & 0 & OSR90-01 & $S R 90-01$ & IIOUID & $\mathrm{N} / \mathrm{A}$ & $434.00-006$ & $\mathrm{uCi} / \mathrm{mL}$ & \\
\hline $45 \mathrm{APHS}$ & 8979000025 & 0 & $6 \$ R 9001$ & sp90-010 & ItoutD & N/a & $8,943+01$ & K Recovery & 1 \\
\hline 4 SAMPLE & $\$ 97 T 000025$ & 0 & QSR90-01 & SR90-018 & IIQUID & N/A & $3.99 \mathrm{~B}+01$ & $\& \mathrm{Ct} \cdot \mathrm{Brror}$ & \\
\hline $5 \% 001 \%$ & 8917000025 & 0 & $6 \mathrm{SR} 9001$ & 1829001 & Louro & $9,291-4$ & $4,542-3$ & $\mathrm{RPD} / 1 / 4$ & \\
\hline 5 DOP & S97T000025 & 0 & OSR90-01 & SR90-01C & IIQUID & 100 & $9.05 \mathrm{E}+01$ & \& Recovery & \\
\hline $500 \mathrm{P}$ & $\$ 997000025$ & 0 & OSR90101 & sr90.011 & 110UTD & 1100 & $2612+01$ & $x_{10} x_{-1}$ & \\
\hline 6 SAMPLE & $597 T 000028$ & 0 & QSR90-01 & SR90-01 & IIQUID & $\mathrm{N} / \mathrm{A}$ & $438.00-006$ & $\mathrm{uCi} / \mathrm{mL}$ & \\
\hline 6 SAPYI & 8975000028 & 0 & $0 s 00001$ & sRso-o1C & 110010 & $\mathrm{~N} / \mathrm{A}$ & $8.81,-01$ & K Recovony & \\
\hline 6 SAMPLE & $\$ 977000028$ & 0 & OSR90-01 & SR90-01E & LIOUID & $\mathrm{N} / \mathrm{A}$ & $0.00+000$ & \& Ct. Exror & \\
\hline $7 \mathrm{pOP}$ & 597000028 & 0 & $8 s k 901$ & SR90\%01 & L 10410 & $7665-3$ & $126 \mathrm{z}-3$ & $\mathrm{BPD} / \mathrm{l}$ & \\
\hline $7 \mathrm{DOP}$ & $\$ 975000028$ & 0 & OSR90-01 & SR90-01C & IIOUID & 100 & $8.788+02$ & \& Recovery & \\
\hline $7 \mathrm{pup}$ & 5970000028 & 0 & 650000 & SR90 012 & IIONDP & 1,00 & $9006 \mathrm{z}+00$ & 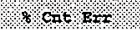 & \\
\hline
\end{tabular}

Final page for worklist\# 16361

Analyst Signature

Date

Analyst Signature

Datè

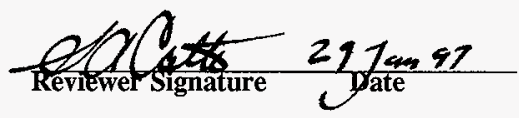

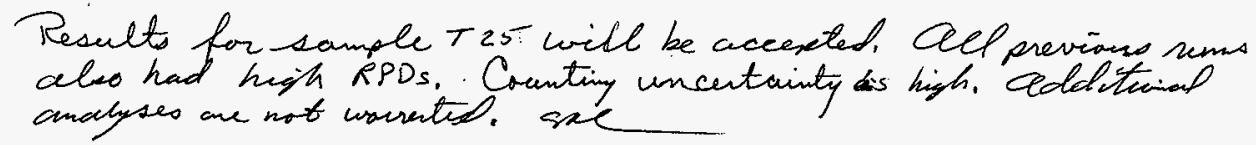

Units shown for $Q C(B L K / B K G)$ may not reflect the actual units. 


\section{LABCORE Data Entry Template for Worklist\# 16361}

Analyst: $\quad$ S.Lai Instrument: $\mathrm{AB} 00 \longrightarrow$ Book\# 25056

Method: LA-220-101 Rev/Mod D-1

Worklist Comment: RR\#2 SY-102. Use 0.100-10-1mL. sac

\begin{tabular}{lllllll}
\hline S Type Sample\# R A & Test & Matrix & Group\# & Project
\end{tabular}

1 STD QSR90-01 LIQUID

2 BLNK QSR90-01 LIQUID

3 BLNK/BKG QSR90-0I LIQUID

4 SAMPLE S97T000025 0 @SR90-01 LIQUID 97000022 SY-102 GRAB1

Analytes Requested: SR90-01, SR90-01C, SR90-01E

5 DUP S97T000025 O @SR90-01 LIQUID

6 SAMPLE S97T000028 0 QSR90-01 LIQUID 97000022 SY-102 GRABI

Analytes Requested: SR90-01, SR90-01C, SR90-01E

7 DUP S97T000028 O QSR90-0I LIQUID

\section{Final page for worklist \# 16361}

$\frac{\text { Sue Liv } 1-28-9]}{\text { Analyst Signature }}$

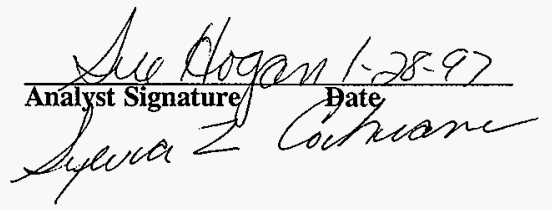

Data Entry Comments: 
WORKBOOK PAGE: STD1

Sr-89/90 : LA-220-101 (D-1), 102 (E-3), 104 (D-1) LIQUIDS

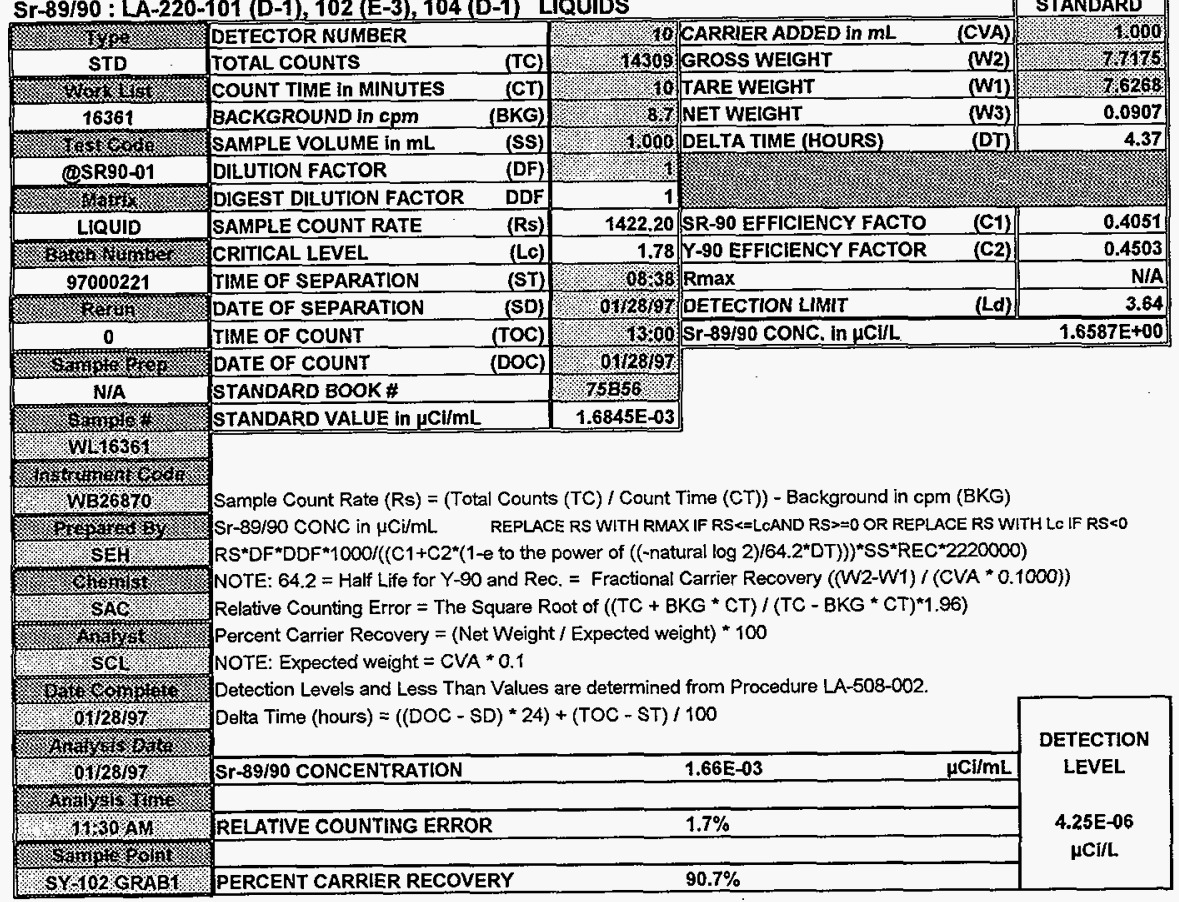

\begin{tabular}{|c|c|c|c|}
\hline Analyst: & SCL & Date: & $28-\operatorname{Jan}-97$ \\
\hline Signature of Chemist: & SAC & Date: & $2 \operatorname{Ln} 97$ \\
\hline
\end{tabular}


WORKBOOK PAGE; BLANK2

LA-220-101 / D-1 Sr-89/90 : LA-220-101 (D-1), 102 (E-3), 104 (D-1)

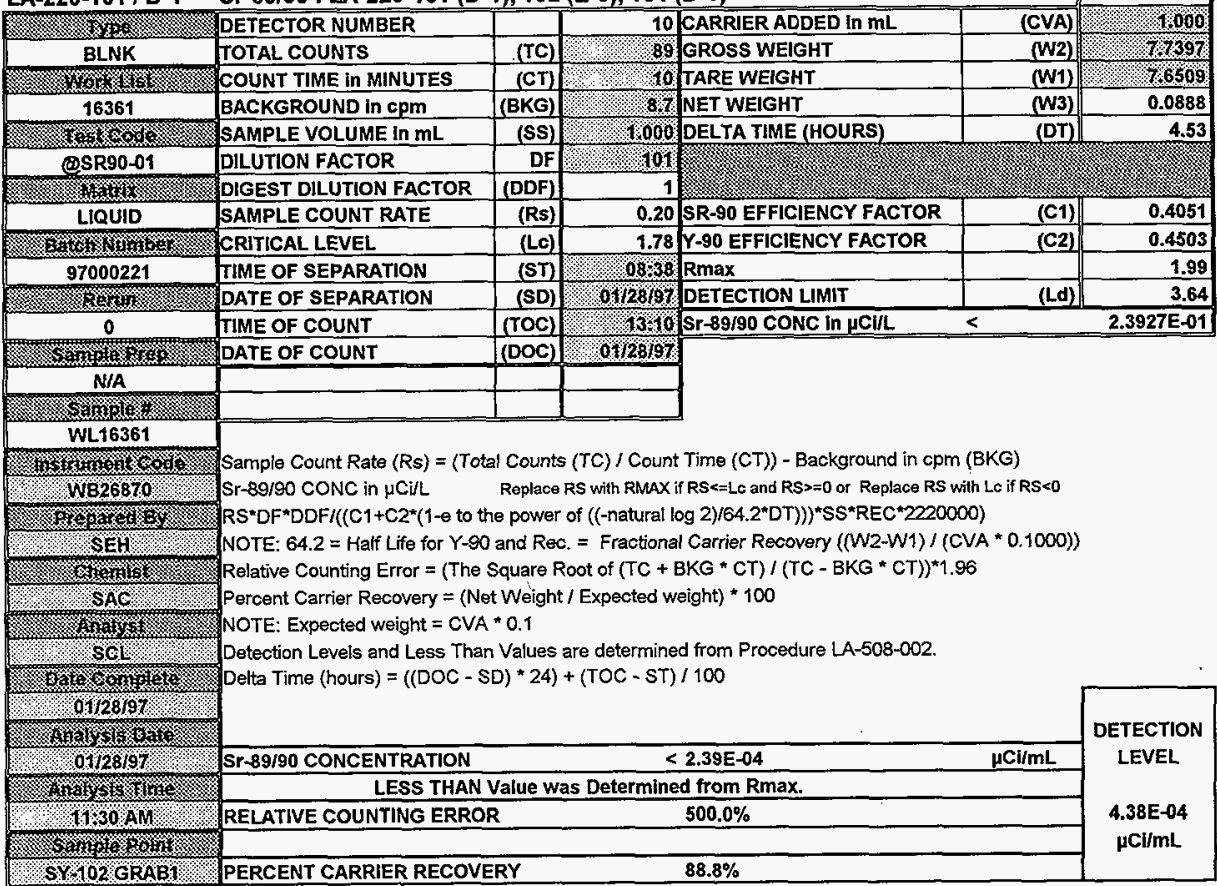

\begin{tabular}{|c|c|c|c|}
\hline Analyst: & SCL & Date: & 28-Jan-97 \\
\hline Signature of Chemist: & SAC & Date: & 217.17 \\
\hline
\end{tabular}


WORKBOOK PAGE: SAM4

Sr-89/90 : LA-220-101 (D-1), 102 (E-3), 104 (D-1)

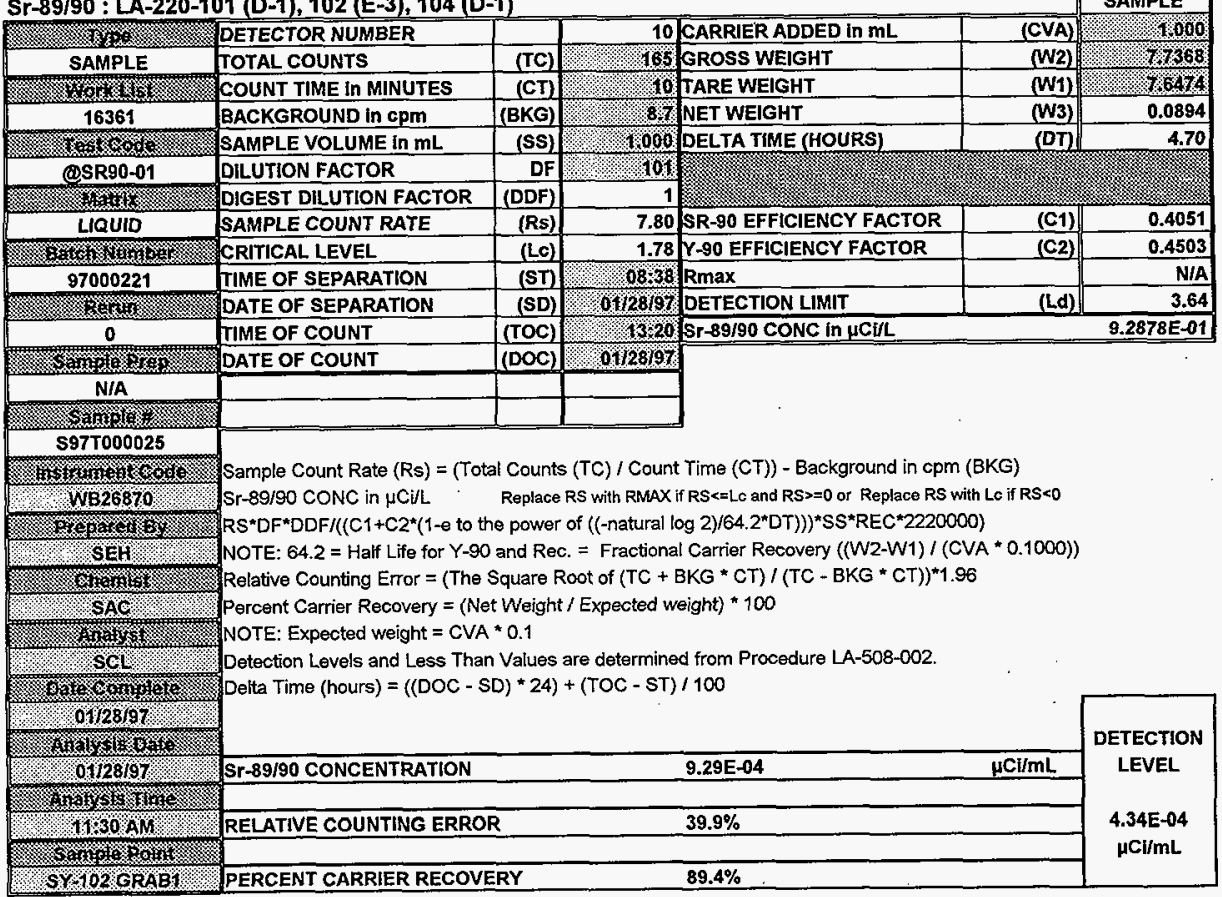

\begin{tabular}{|c|c|c|c|}
\hline Analyst: & SCL & Date: & $28-\operatorname{Jan}-97$ \\
\hline Signature of Chemist: & SAC & Date: & 1.17 \\
\hline
\end{tabular}


WORKBOOK PAGE: DUPS

Sr-89/90: LA-220-101 (D-1), 102 (E-3), 104 (D-1)

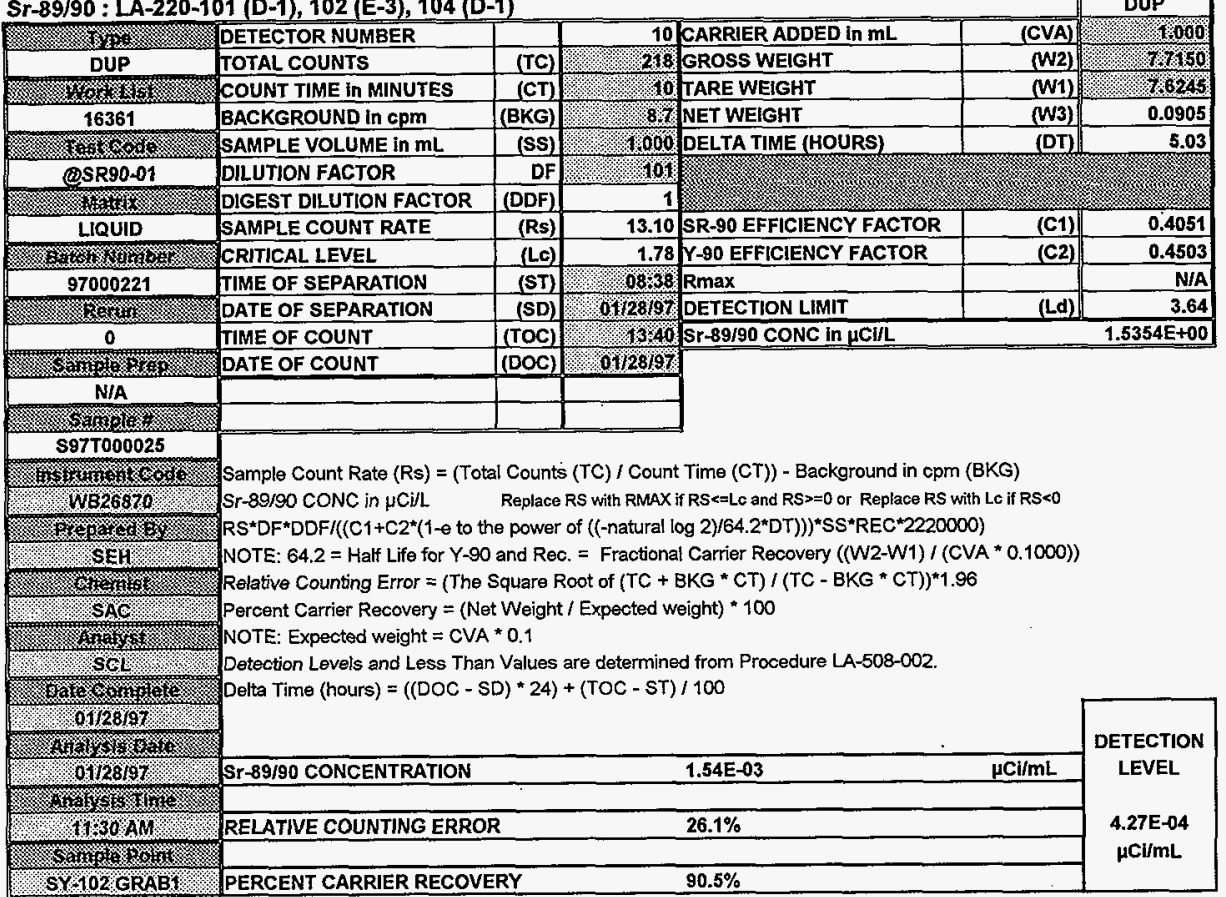

\begin{tabular}{|c|c|c|c|}
\hline Analyst: & SCL & Date: & 28-Jan-97 \\
\hline Signature of Chemist: & SAC & Date: & 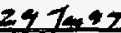 \\
\hline
\end{tabular}


WORKBOOK PAGE: SAM6

Sr-89/90 : LA-220-101 (D-1), 102 (E-3), 104 (D-1)

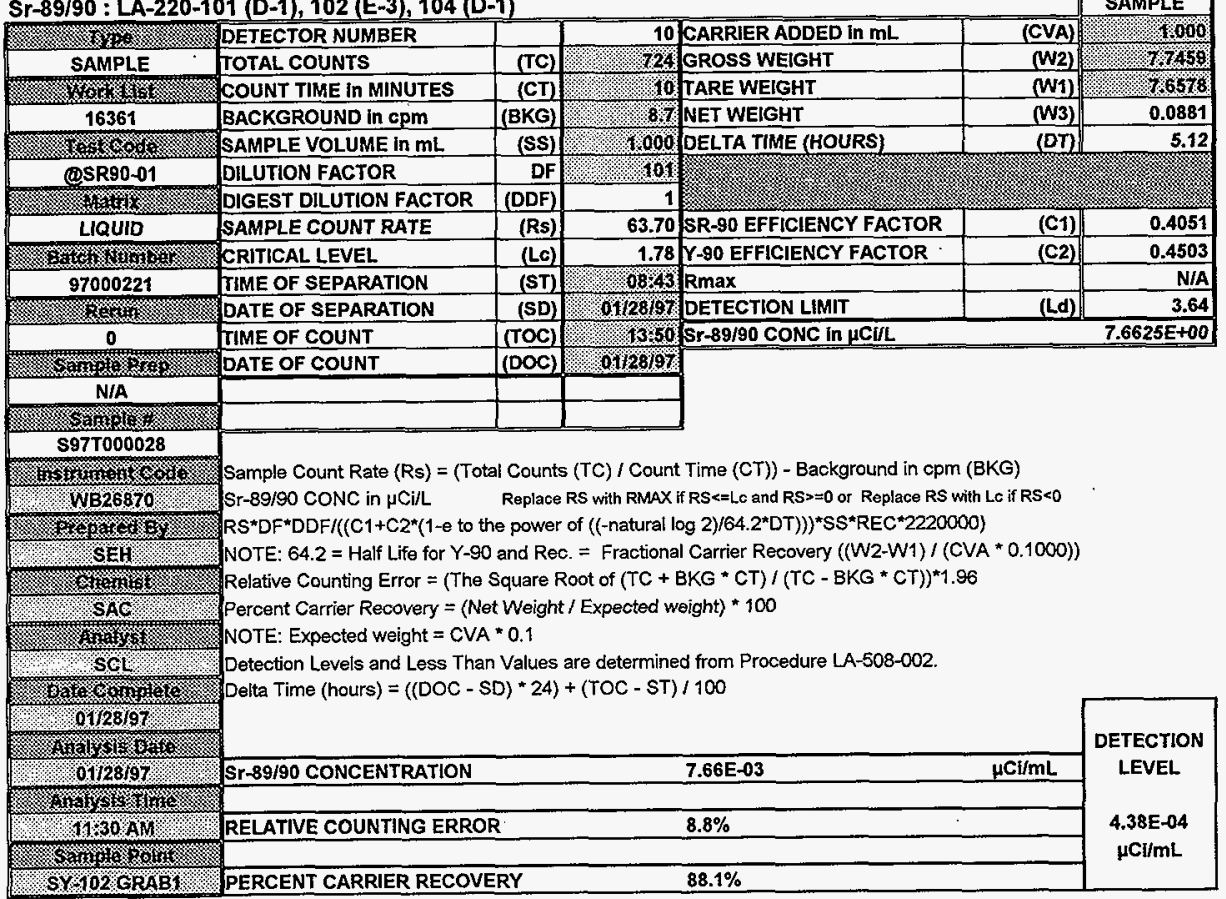

\begin{tabular}{|c|c|c|c|}
\hline Analyst: & SCL & Date: & 28-Jan-97 \\
\hline Signature of Chemist: & SAC & Date: & 2421 \\
\hline
\end{tabular}

\section{8}


WORKBOOK PAGE: DUP7

Sr-89/90 : LA-220-101 (D-1), 102 (E-3), 104 (D-1)

\begin{tabular}{|c|c|c|c|c|c|c|}
\hline \multicolumn{6}{|c|}{ 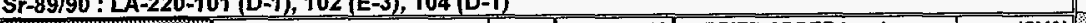 } & ON. \\
\hline 28 & DETECTOR NUMBER & & 10 & CARRIER ADDED in $\mathrm{mL}$ & (CVA) & 2.4000 \\
\hline DUP & TOTAL COUNTS & (TC) & 690 & GROSS WEIGHT & (W2) & $\% 1009$ \\
\hline xys & COUNT TIME in MINUTES & (CT) & 8 & TARE WEIGHT & (W1) & 186231 \\
\hline 16361 & BACKGROUND in cpm & (BKG) & $8 \%$ & NET WEIGHT & (W3) & 0.0878 \\
\hline smong & SAMPLE VOLUME in $\mathrm{mL}$ & (SS) & $x 000$ & DELTA TIME (HOURS) & (DT) & 5.37 \\
\hline (OSR90-01 & DILUTION FACTOR & DF & 0104 & & & \\
\hline 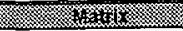 & DIGEST DILUTION FACTOR & (DDF) & 1 & & & \\
\hline LIQUID & SAMPLE COUNT RATE & (Rs) & 60.30 & SR-90 EFFICIENCY FACTOR & (C1) & 0.4051 \\
\hline 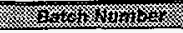 & CRITICAL LEVEL & (LC) & 1.78 & Y-90 EFFICIENCY FACTOR & (C2) & 0.4503 \\
\hline 97000221 & TIME OF SEPARATION & (ST) & 0873 & Rmax & & N/A \\
\hline 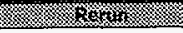 & DATE OF SEPARATION & (SD) & $01+2819$ & DETECTION LIMIT & (Ld) & 3.64 \\
\hline 0 & TIME OF COUNT & (TOC) & 101405 & $\mathrm{Sr}-89 / 90 \mathrm{CONC}$ in $\mathrm{\mu CI} / \mathrm{L}$ & & $7.2589 \mathrm{E}+00$ \\
\hline (1) & DATE OF COUNT & (DOC) & 012899 & & & \\
\hline \multicolumn{7}{|l|}{ N/A } \\
\hline \multicolumn{7}{|l|}{ 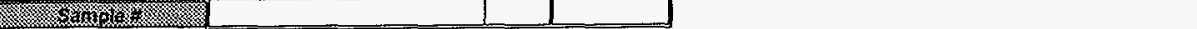 } \\
\hline S97T000028 & \\
\hline 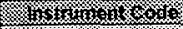 & \multicolumn{6}{|c|}{ Sample Count Rate $(R s)=($ Total Counts $(T C) /$ Count Time $(C T))-$ Background in cpm (BKG) } \\
\hline W WB26870 & ST-89/90 CONC in $\mu \mathrm{Ci} / \mathrm{L}$ & \multicolumn{5}{|c|}{ Replace RS with RMAX if RS $<=L C$ and RS $>0$ or Replace RS with Lc if RS $<0$} \\
\hline 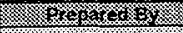 & \multicolumn{6}{|c|}{$\mathrm{RS}^{*} \mathrm{DF}{ }^{*} \mathrm{DDF} /\left(\left(\mathrm{C} 1+\mathrm{C} 2^{*}\left(1-\oplus \text { to the power of }\left((- \text { natural } \log 2) / 64.2^{*} \mathrm{DT}\right)\right)\right)^{*} S S^{*} \mathrm{REC}^{*} 2220000\right)$} \\
\hline WSEH & \multicolumn{6}{|c|}{ NOTE: $64.2=$ Half Life for $Y-90$ and Rec. = Fractional Carrier Recovery $((W 2-W 1) /(C V A * 0.1000))$} \\
\hline 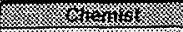 & \multicolumn{6}{|c|}{ Relative Counting Error $=(\text { The Square Root of }(T C+B K G * C T) /(T C-B K G * C T))^{* 1.96}$} \\
\hline 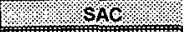 & \multicolumn{6}{|c|}{ Percent Carrier Recovery $=($ Net Weight $/$ Expected weight $) * 100$} \\
\hline (x) & \multicolumn{6}{|c|}{ NOTE: Expected weight $=$ CVA $* 0.1$} \\
\hline $\mathrm{sol}, \mathrm{H}$ & \multirow{2}{*}{\multicolumn{6}{|c|}{$\begin{array}{l}\text { Detection Levels and Less Than Values are determined from Procedure LA-508-002. } \\
\text { Delta Time (hours) }=((D O C-S D) * 24)+(T O C-S T) / 100\end{array}$}} \\
\hline 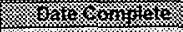 & & & & & & \\
\hline $0,01 / 28 / 97$ & \multicolumn{5}{|c|}{ Delta Time (hours) $=((\mathrm{DOC}-\mathrm{SD}) \times 24)+(10 \mathrm{C}-\mathrm{SI}) / 700$} & \multirow{4}{*}{$\begin{array}{l}\text { DETECTION } \\
\text { LEVEL }\end{array}$} \\
\hline 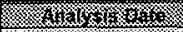 & & & & & & \\
\hline 01128197 & \multicolumn{3}{|l|}{ Sr-89/90 CONCENTRATION } & $7.26 \mathrm{E}-03$ & $\mu \mathrm{Cl} / \mathrm{mL}$ & \\
\hline 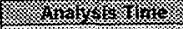 & & & & & & \\
\hline m.1. $150 \mathrm{AM}$ & \multicolumn{3}{|l|}{ RELATIVE COUNTING ERROR } & $9.1 \%$ & & \multirow{3}{*}{$\begin{array}{l}\text { 4.39E-04 } \\
\mu \mathrm{CCI} / \mathrm{mL}\end{array}$} \\
\hline 8 济 & \multirow{2}{*}{\multicolumn{3}{|c|}{ PERCENT CARRIER RECOVERY }} & & & \\
\hline SY 102 ERABS & & & & $87.8 \%$ & & \\
\hline
\end{tabular}

\begin{tabular}{|c|c|c|c|}
\hline Analyst: & SCL & Date: & 28-Jan-97 \\
\hline Signature of Chemist: & SAC & Date: & 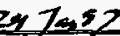 \\
\hline
\end{tabular}




\section{LABCORE Completed RadChem Report for Worklist\#: 16278}

Analyst: jmv

Instrument: $\mathrm{AB} 15$

Book\#

Method: $\angle A-953-103 \mathrm{Rev} / \mathrm{Mod} B-\mathcal{O}$

Worklist Comment: Determine sample size using ludlum.Std:(TNKFUS) 1.0ML. new

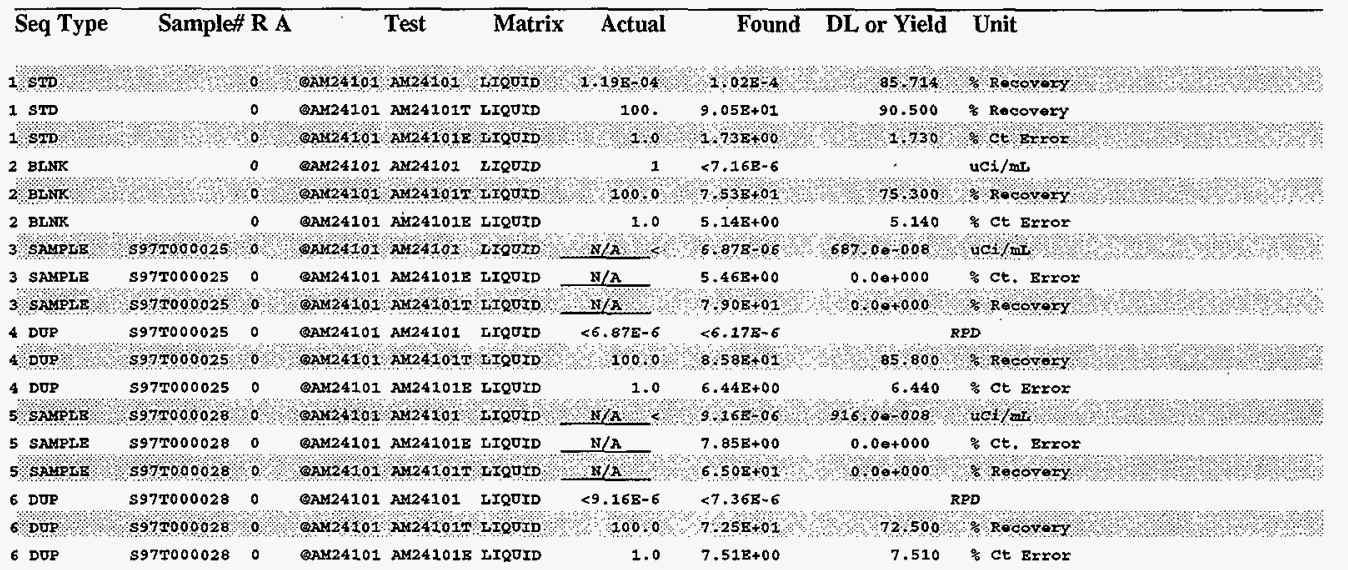

\section{Final page for worklist\# 16278}

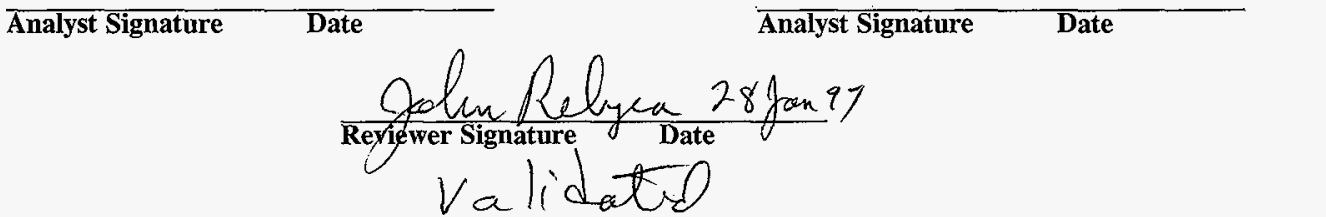


HNF-SD-WM-DP-227, REV. 0

01/27/97 08:30 A-0004-1

\section{LABCORE Data Entry Template for Worklist\# 16278}

Page: 1

\section{Analyst:}

Instrument: AM01

Book\#

Method: LA-953-103 Rev/Mod

Worklist Comment: Determine sample size using ludlum.Std:(TNKFUS) 1.0ML. new

S Type Sample\# R A Test Matrix

1 STD

GAM24101 IIQUID

2 BLNK

CAM24101 LIQUID

3 SAMPLE

s97T000025 0

AAM24101 IIQUID

97000022 SY-102 GRABI Analytes Requested: AM24101, AM24101E, AM24101T

4 DUP

S97T000025 0

QAM24101 LIQUID

5 SAMPLE

S97T000028 O OAM24101 LIQUID

97000022 SY-102 GRAB1

Analytes Requested: AM24101, AM24101E, AM24101T

6 DUP

S97T000028 O CAM24101 LIQUID

\section{Final page for worklist \# 16278}

Analyst Signature Date

Aralyst Signature

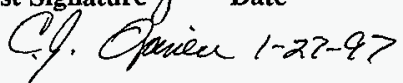

\section{Data Entry Comments:}

$S=$ Worklist Slot Number, $R=$ Replicate Number, $A=$ Aliquot Code. 


\section{LABCORE Data Entry Template for Worklist\# 16278}

Analyst: $\quad$ Smo Instrument: AM01 \& $\$ 5-$ Book\# 62356

Method: LA-953-103 Rev/Mod

Worklist Comment: Determine sample size using ludlum.Std:(TNKFUS) 1.0ML. new

S Type Sample\# R A Test Matrix Group\# Project

1 STD

@AM24101 LIQUID

2 BLNK

@AM24101 LIQUID

3 SAMPLE

\$97T000025 0

@AM24101 LIQUID

97000022 SY-102 GRAB1

Analytes Requested: AM24101, AM24101E, AM24101T

4 DUP S97T0000250 @AM24101 LIQUID

5 SAMPLE

S97T000028 0 @AM24101 LIQUID

97000022 SY-102 GRAB1 im! Analytes Requested: AM24101, AM24101E, AM24101T

6 DUP

S97T0000280 @AM24101 LIQUID

7 SAMPLE

S97T0000290@@AM24101 LIQUID Analytes Requested: AM24101, AM24101E, AM24101T

97000022 SY-102 GRAB1, $1-10-10-10$

8 DUP

$597 \mathrm{~T} 0000290$

@AM24101 LIQUID

\section{Final page for worklist \# 16278}

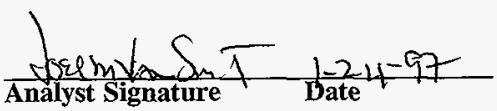

Analyst Signature Date

Data Entry Comments:

39729

Wreps

DIgess

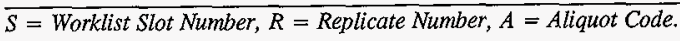


WORKBOOK PAGE: STD1

Am 241 and Cm 243/244: LA-953-103 (B-0)

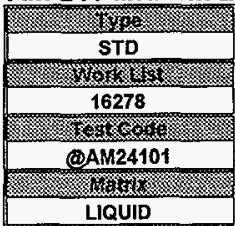

Date Counted

Sample Volume in $\mathrm{mL}$ (SS)

Sample D.F.

Tracer Volume in $\mathrm{mL}$

Digest D.F.

Tracer Book No.

Am-243 Tracer Value (dpm/mL)

Detector Number

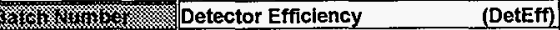

(DF)

LIQUID

I. 1 AN:25 97 Am 241 AEA Frac.

17o00 Am 243 AEA Frac.

Cm 243/244 AEA Frac.

0.200 Total AT Counts

(DDF)

7.uou AT Count TIme (min)

$125 \mathrm{~B} 43$

Background in cpm 1027 Am $241 \mathrm{cpm}$

16 Am $243 \mathrm{cpm}$

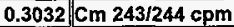

97000130

( \&

0

Standard Book No

$62 \mathrm{~B} 56$

AEA Count Time

1.186E-04 Am $241 \mathrm{\mu Cl} / \mathrm{L}=$

$\mathrm{Cm} 243 / 244 \mu \mathrm{Cl} / \mathrm{L}=$

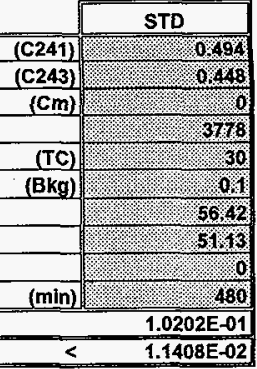

Sink

N/A

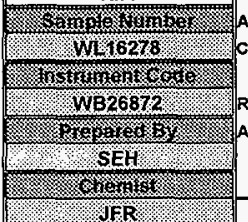

Am-241 $\mu C i L=(C 241$ *Am-243 Tracer Value * SPKV * DF * DDF * $(1000 \mathrm{~mL} / \mathrm{L})) /($ C243 * SS * $(2220000 \mathrm{dpm} / \mu C \mathrm{Ci}))$

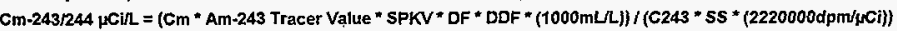

Relative Counting Error = Square Root of $[(1 /(\mathrm{Am}-243 \mathrm{cpm}$ * $\mathrm{min}))+(1 /(\mathrm{Am}-241 \mathrm{or} \mathrm{Cm}-243 / 244 \mathrm{cpm}$ * $\min ))]{ }^{*} 1.96$ * 100

Am 243 Tracer Recovery = (Total AT Counts / TC - Bkg) * (1/DetEff) * C243 * $100 /$ Am-243 Tracer Value * SPKV

Aitis

$\mathrm{Mv}$

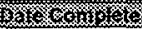

01127197.

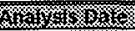

01124.97

\begin{tabular}{|ll|}
\hline Am $241 \mu \mathrm{Cl} / \mathrm{mL}$ & $=$ \\
\hline & $1.02 E-04$ \\
Relative Counting Error $=$ & $1.7 \%$ \\
\hline NOTE: Cm-243/244 Result is a LESS THAN Value. \\
\hline $\mathrm{Cm} 243 / 244 \mu \mathrm{CI} / \mathrm{mL}$ & $<1.14 \mathrm{E}-05$ \\
Relative Counting Error $=$ & $100.0 \%$ \\
\hline Am 243 Tracer Recovery $=$ & $90.5 \%$ \\
\hline
\end{tabular}

DETECTION

LEVELS

in $\mu \mathrm{Cl} / \mathrm{mL}$

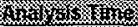

$10.05 \mathrm{PM}$

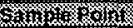

SY 102 GRABA

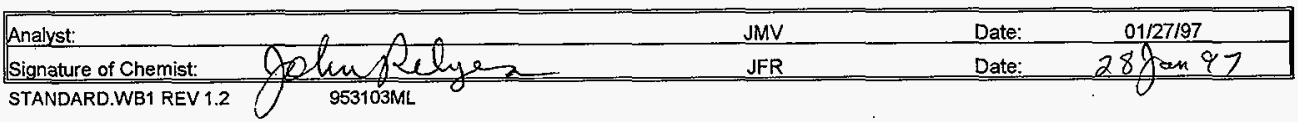




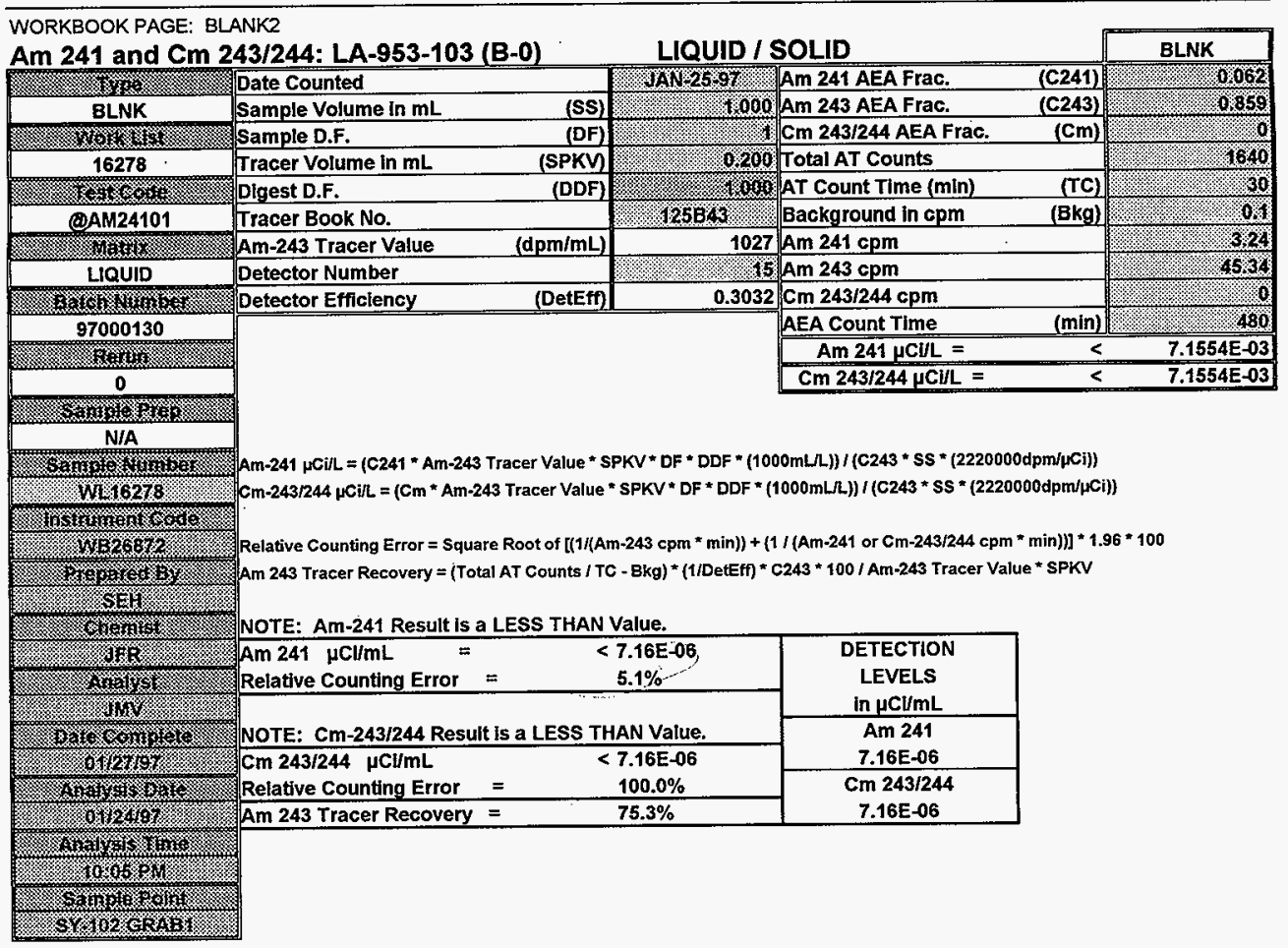

\begin{tabular}{|c|c|c|c|}
\hline Analyst: & JMV & Date: & $01 / 27 / 97$ \\
\hline Signature of Chemist: & JFR & Date: & $28 \tan 97$ \\
\hline
\end{tabular}


HNF-SD-WM-DP-227, REV. 0

WORKBOOK PAGE: SAM3

Am 241 and $C m$ 243/244: LA-953-103 (B-0)

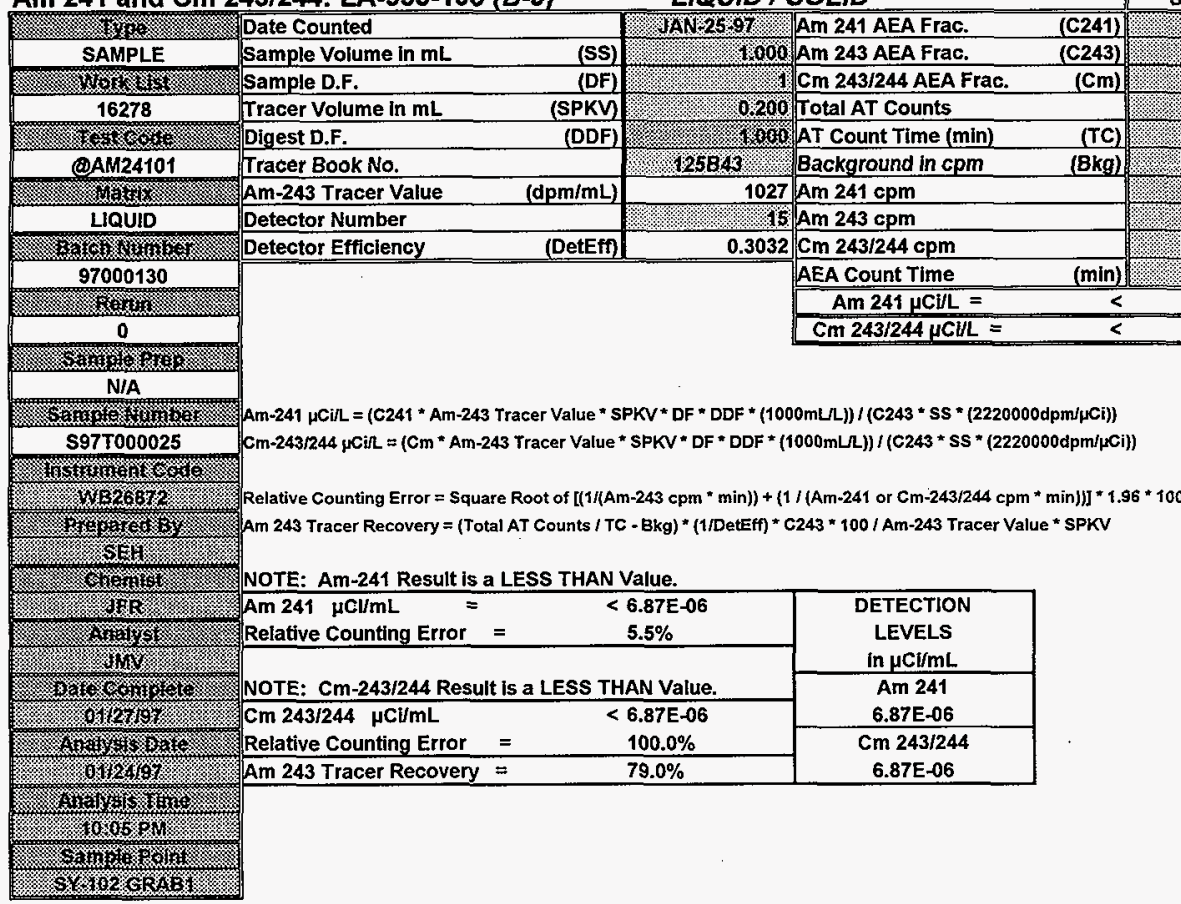

\begin{tabular}{|c|c|c|c|}
\hline Analyst: & JMV & Date: & $01 / 27 / 97$ \\
\hline Signature of Chemist: & JFR & Date: & $2 \times \operatorname{ran} 97$ \\
\hline
\end{tabular}




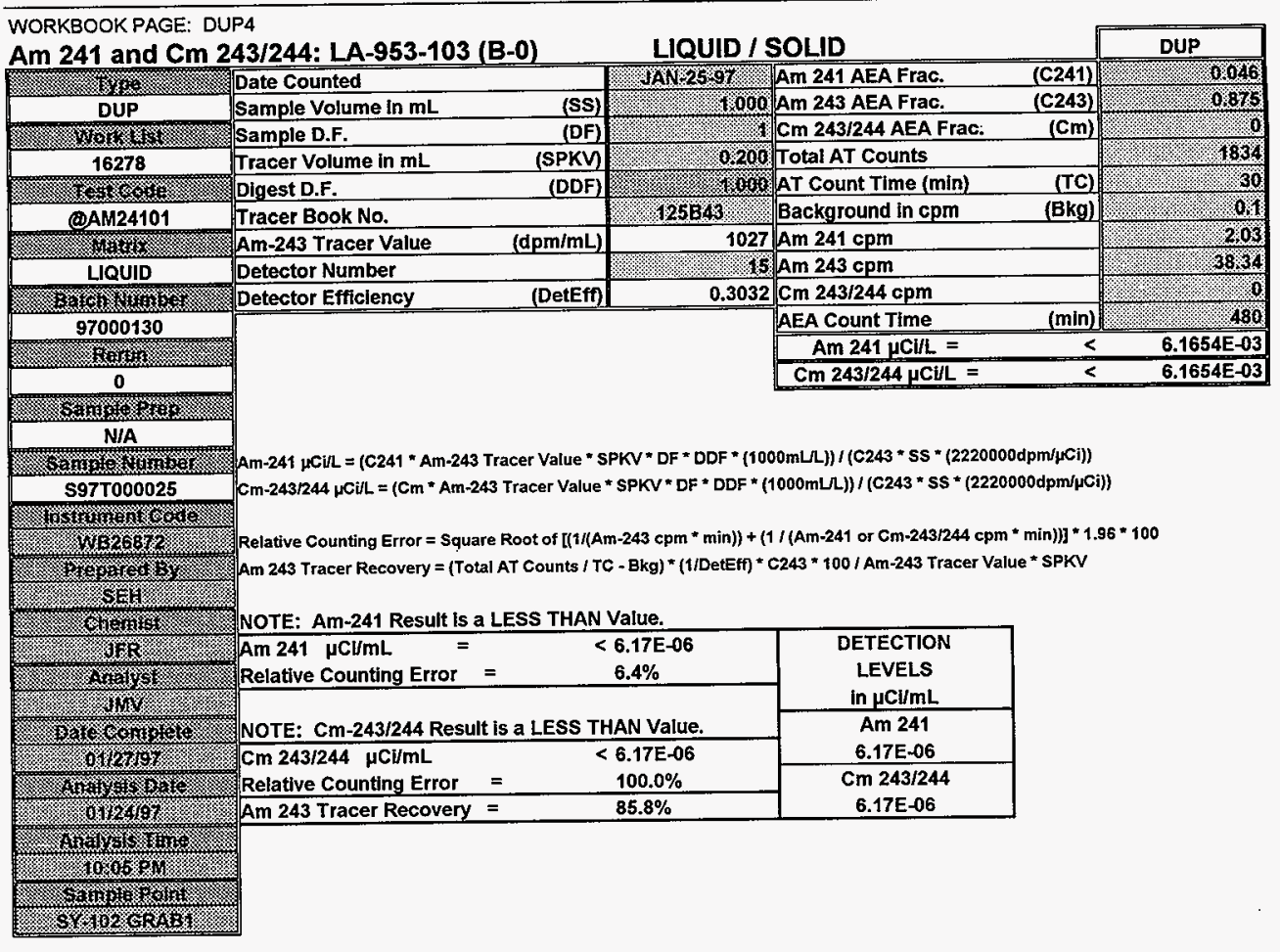

\begin{tabular}{|c|c|c|c|}
\hline Analyst: & JMV & Date: & $01 / 27 / 97$ \\
\hline Signature of Chemist: & JFR & Date: & $28 \tan 77$ \\
\hline
\end{tabular}


WORKBOOK PAGE: SAM5

Am 241 and $\mathrm{Cm}$ 243/244: LA-953-103 (B-O)

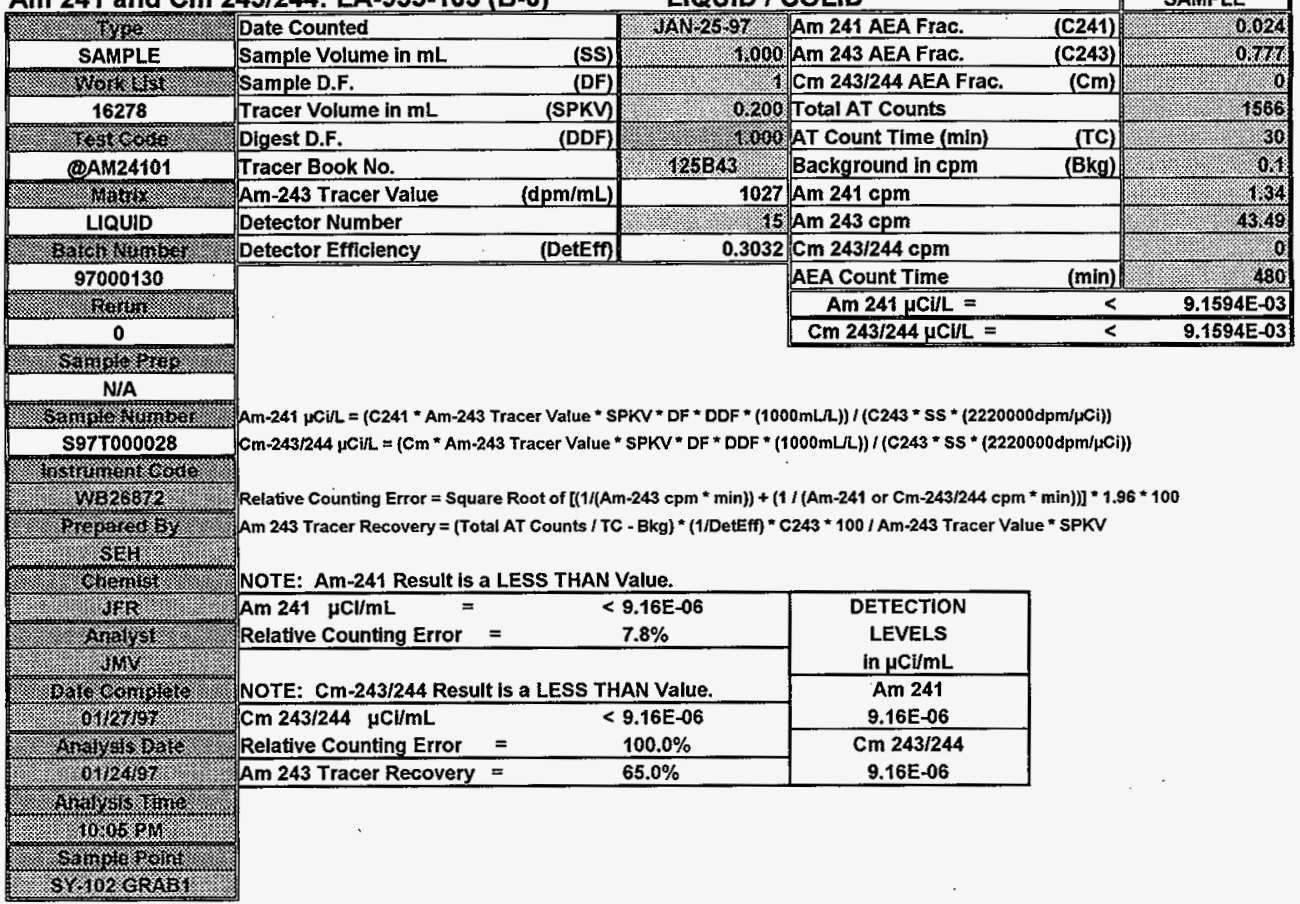

\begin{tabular}{|c|c|c|c|}
\hline Analyst: & JMV & Date: & $01 / 27 / 97$ \\
\hline Signature of Chemist: & JFR & Date: & $30 \operatorname{lom} 9$ \\
\hline
\end{tabular}


WORKBOOK PAGE: DUPG

Am 241 and Cm 243/244: LA-953-103 (B-0)

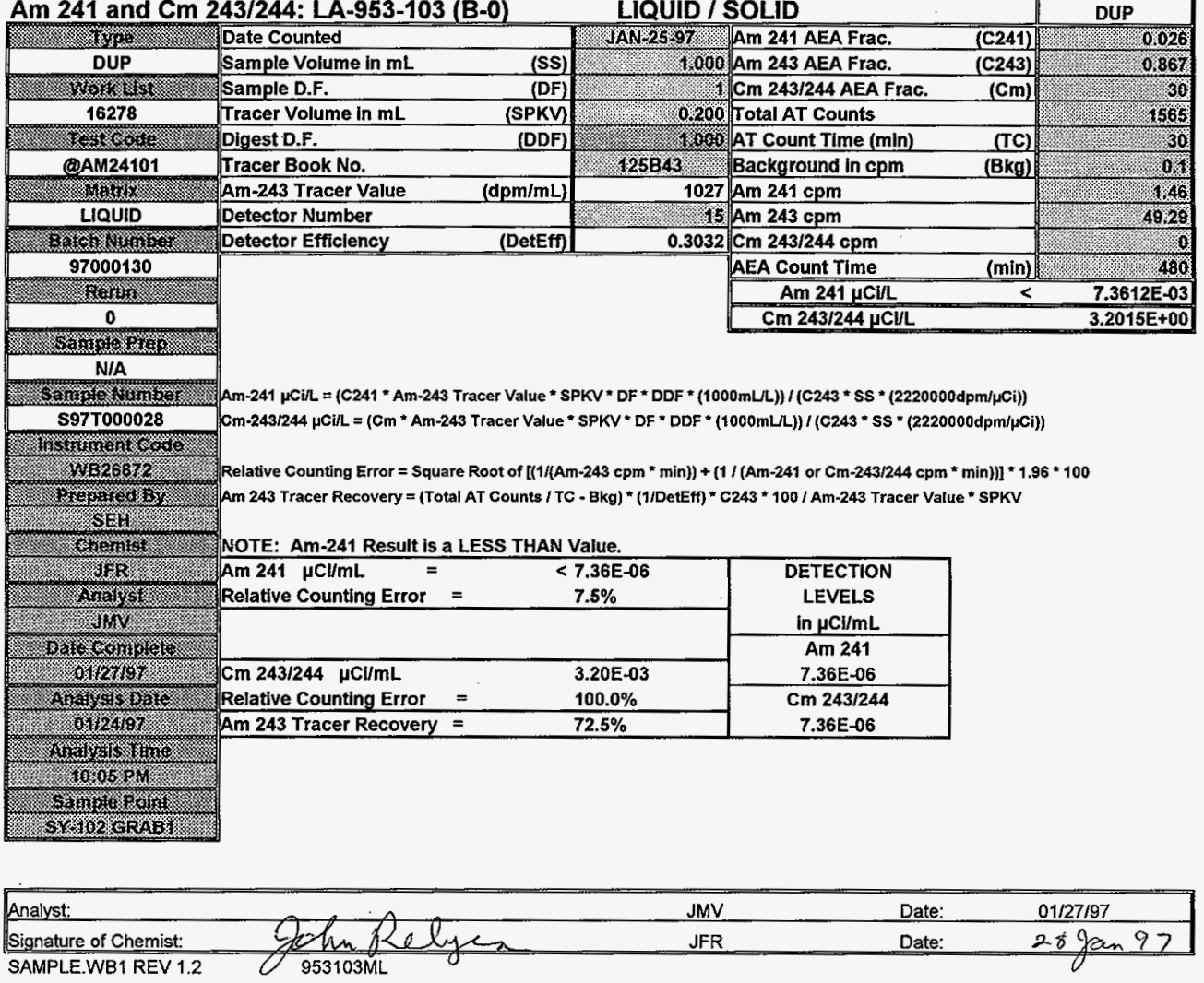


Westinghouse Hanford Co. A I $\begin{array}{lllllllll} & \mathrm{H} & \mathrm{A} & \mathrm{E} & \mathrm{N} & \mathrm{E} & \mathrm{R} & \mathrm{G} & \mathrm{Y}\end{array}$
Rev. 2.02
A N A I Y S I S

\title{
DATA REDUCTION REPORT
}

SAMPLE

WL16278-STD-AM

File ID: 7a7670.CNF

\author{
Counted on: \\ $1 / 25 / 97 @ 14: 19$ \\ Detector: \\ AEA 7 \\ Geometry number: 1 \\ Count time: \\ 28803. Sec
}

PEAK ANALYSIS

\begin{tabular}{|c|c|c|c|c|c|c|c|c|}
\hline Peak & Peak & height & Peak & center & & $\mathrm{HM}$ & Tau & \\
\hline ID & Initial & Final & Initia & 1 Final & Initial & Final & Initial & Final \\
\hline 1 & 1352.8 & 1352.8 & & 301.061 & 18.000 & 12.1 & .000 & \\
\hline 2 & 1215.0 & 1215.0 & 174 & 255 & 000 & 11. & 00 & \\
\hline $\begin{array}{l}3 \\
4 ?\end{array}$ & 21.1 & 21.1 & $\begin{array}{l}170.289 \\
154.677\end{array}$ & $\begin{array}{l}170.197 \\
153.639\end{array}$ & $\begin{array}{r}110.000 \\
6.000\end{array}$ & & & \\
\hline
\end{tabular}

PEAK RESULTS
Peak Error Limit: $30 \%$

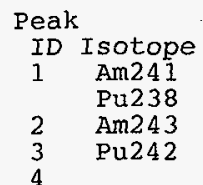

Totals :

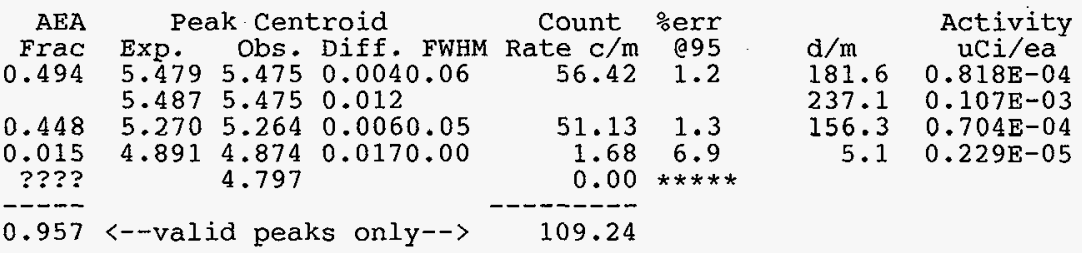

$$
\begin{gathered}
\text { DETECTOR CALIBRATION } \\
\text { Energy (MEV) }=4.091+(0.0046) * \text { Channel } \\
\text { Energy range }(\mathrm{MeV}): 4.091 \text { TO } 6.446 \\
\text { Efficiency }= \\
\text { TOTAL COUNT DATA: }
\end{gathered}
$$

Item

Raw spectrum

Smoothed

Composite fit

Residuals

Total
54817.0
54817.0
52441.9
2375.1

: Recovery

100.000

100.000

95.667

4.333

Analyzed by:

$\overline{V R}$ 
1 Legend: $\quad$ Raw $=\ldots$ Spectrum 7a7670.CNF Modeled Peaks $=1,2, \ldots$, etc $\quad$ Display Max.: 10104.5

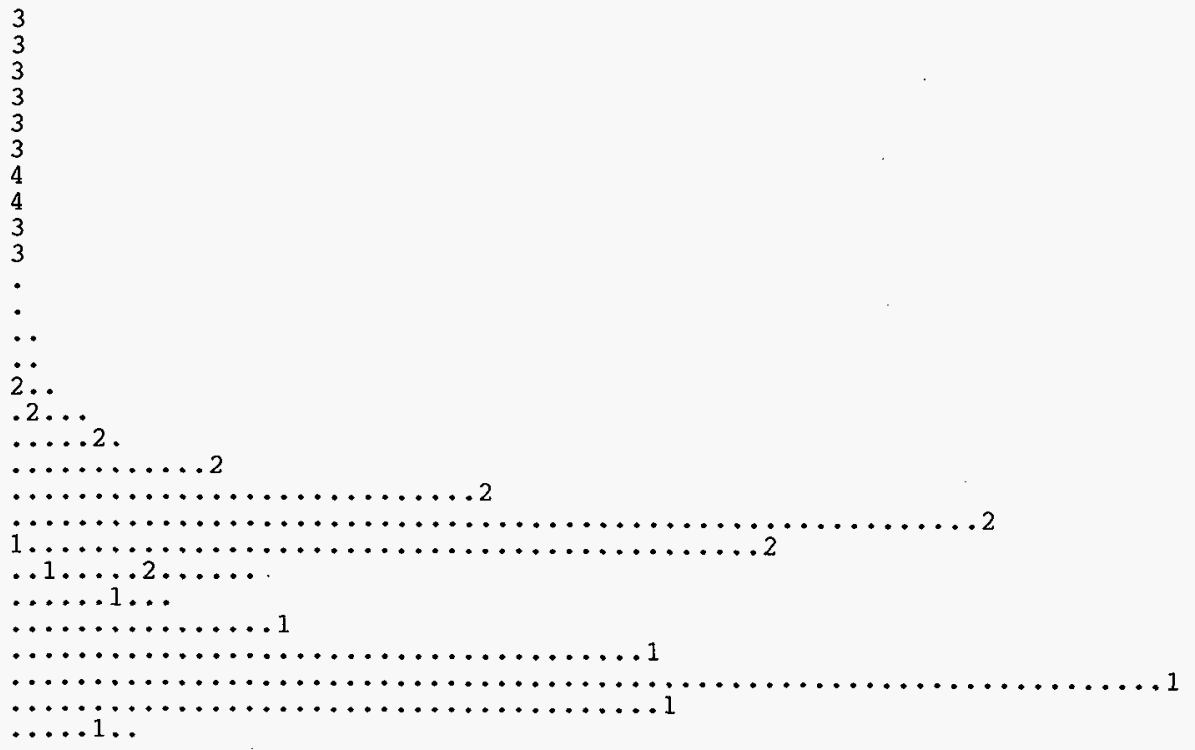


HNF-SD-WM-DP-227, REV. 0

Raw Data Dump for AEA Spectrum: 7a7670.CNF

\begin{tabular}{|c|c|c|c|c|c|c|c|c|c|c|}
\hline 1 & 0 & & & & & & & & & \\
\hline $1 \frac{1}{1}$ & 0. & $\begin{array}{l}0 . \\
0 .\end{array}$ & $\begin{array}{l}0 . \\
0 .\end{array}$ & $\begin{array}{l}0 . \\
0 .\end{array}$ & 0. & 0. & 0. & 0. & 0. & 0. \\
\hline 21 & 3. & 2. & 3. & 0. & $\frac{1}{3}$. & 3. & 7. & 3. & 3. & 2. \\
\hline 31 & 3. & 2 . & 2 . & 5. & 4. & 1. & 2 . & 4. & 3. & 5. \\
\hline 41 & 2 . & 4. & $\frac{1}{2}$. & 2. & 6. & 1. & 7. & 2. & 3. & 0. \\
\hline $\begin{array}{l}51 \\
61\end{array}$ & 4. & $\begin{array}{l}5 . \\
6 .\end{array}$ & 3. & $\begin{array}{l}7 . \\
4 .\end{array}$ & $\begin{array}{l}5 . \\
0 \text {. }\end{array}$ & $\frac{1}{4}$. & $\begin{array}{l}2 . \\
2\end{array}$ & $\begin{array}{l}6 . \\
2\end{array}$ & $\begin{array}{l}4 \\
8\end{array}$ & 3. \\
\hline 71 & 6 . & 1. & 9. & 2 . & 4. & 4. & 7. & 4. & 3. & 7. \\
\hline 81 & 8 . & 3 . & 3. & 3. & 3. & 3. & 8 . & 4. & 6. & 4. \\
\hline 91 & 5. & 2 . & 7. & 10. & 11. & 7. & 4. & 7. & 5. & 9. \\
\hline 101 & 5. & 4. & 6. & 6. & 3. & 7. & 10. & 6. & 10. & 3. \\
\hline 11 & 5. & 9. & 6. & 7. & 6. & 8. & 9. & 4. & 9. & 16. \\
\hline & 11. & 7. & 2 . & 7. & 10. & 11. & 3. & 5. & 8 . & 13. \\
\hline & 6. & 6. & 10. & 7. & 5. & 8. & 10. & 17. & 4. & 6. \\
\hline & 7. & 14. & 8. & 9. & 10. & 11. & 12. & 13. & 14. & 8. \\
\hline & 14. & 10. & 16. & 17. & 16. & 13. & 10. & 20. & 14. & 19. \\
\hline & 16. & 13. & 13. & 16. & 13. & 13. & 24. & 20. & 15. & 22 . \\
\hline & 18. & 21. & 13. & 18. & 15. & 23. & 16. & 17. & 29. & 19. \\
\hline & 24 . & 25 . & 27 . & 28 . & 21. & 20. & 27. & 27 . & 33 . & 34. \\
\hline & 32 . & 38. & 23. & 32. & 42 . & 50. & 45. & 37. & 35. & 44. \\
\hline & $\begin{array}{l}54 . \\
58\end{array}$ & 39. & $\begin{array}{l}49 . \\
58\end{array}$ & $\begin{array}{l}50 . \\
71 .\end{array}$ & 47. & $\begin{array}{l}52 . \\
67\end{array}$ & $\begin{array}{l}58 . \\
85\end{array}$ & 44. & 60. & 56. \\
\hline & 95. & 99. & 102. & 112. & 120 & 118. & 146. & 163. & $137^{\circ}$ & 152. \\
\hline & 180. & 203. & 174. & 188. & 240 . & 231. & 250 . & 317. & 284 . & 333. \\
\hline & 354. & 408 . & 431. & 516 . & 569. & 637. & 667 & 771 . & 924. & 990. \\
\hline & 1060. & 1180 . & 1204 . & 1326 . & 1341. & 1319 . & 1182 . & 1098. & 990. & 780 . \\
\hline & 674. & 520 & 420 & 327. & 282 & 281 & 261 & 230 & 175 . & 175. \\
\hline & 167. & 184. & 161. & 196. & 181. & 209. & 147 . & 200. & 194. & 222 . \\
\hline & 226 . & 227 & 245. & 259. & 313. & 362 . & 401 & 487 . & 519. & 565 . \\
\hline & 636 & 712 . & 786 & 848 & 1002 . & 1108. & 1191. & 1325 . & 1372 . & 1483 . \\
\hline & 1447 . & 1436. & 1328 . & 1200 . & 1061 . & 862 & 723. & 631 . & 490 & 422 . \\
\hline & 339. & 277 & 183. & 176 & 117 & 98. & 61. & 31. & 26 & 17. \\
\hline & 13 & 8. & 6. & 2 & 1. & 0. & 2 . & 2. & 1. & 0. \\
\hline & 0. & 1. & 1. & 0 . & 1. & 0 . & 0 . & 0 . & 0 . & 0 . \\
\hline & 0. & 0 . & 0. & 0. & 0 . & 2. & 1. & 2. & 0 . & . \\
\hline & 3. & 1. & 1. & 0 & 0 . & 1. & 0 . & 2. & 1. & I. \\
\hline & 0 . & 0 . & 2. & 1. & 0 . & 0 . & 0 . & 1. & 0 . & 0. \\
\hline & 0. & 0 . & 0. & 0 . & 0. & 0. & 0 . & 0. & 0. & . \\
\hline & 0 . & 0. & 0. & 0 . & 0. & 0. & 0 & 0. & 0 . & 0 . \\
\hline & 0 . & 0 . & 0 . & 0 . & 0 . & 0 . & 0 . & 0. & 0 . & 0. \\
\hline & 0 . & 0 . & 0. & 0 . & 0. & 0 & 0 . & 0. & 0 . & 0 . \\
\hline & 0. & 0 . & 0. & 0 . & 0 . & 0. & 0 . & 0 . & 0 . & 0 . \\
\hline & 0 . & 0 . & 0. & 0 . & 0 . & 0 . & 0 & 0. & 0 & \\
\hline & 1. & 0 . & 0. & 0 . & 0 . & 0. & 1. & 0. & 0 . & 0. \\
\hline & 0 . & 0 . & 0. & 0 . & 0. & 0. & 0 . & 0. & 0 . & 0. \\
\hline & 0. & 0. & 0. & 0. & 0 . & 0. & 0. & 0. & 0 . & \\
\hline & 0 . & 0 . & 0 . & 0 . & 0 . & 0 . & 0 . & 0 . & 0 . & 0. \\
\hline & 0 . & 1. & 0. & 0 . & 0 . & 1. & 0 . & 0 . & 0 . & 0. \\
\hline & 0. & 0. & 1. & . & 0. & 0. & 0. & 0. & 0. & 0 . \\
\hline 49 & 0. & 0 . & 0 . & 0 . & 0 . & 0. & 0 . & 0 . & 0 . & 0 . \\
\hline & & & & & & & & & & \\
\hline
\end{tabular}


Westinghouse Hanford Co.

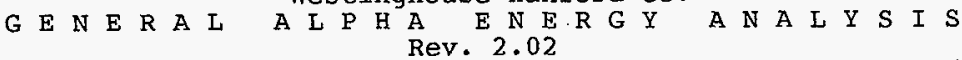

DATA REDUCTION REPORT

SAMPLE

WL16278-BLK-AM

File ID: $8 \mathrm{a} 8870 . \mathrm{CNF}$

Counted on: $\quad 1 / 25 / 97 @ 14: 19$

Detector:

AEA 8

Geometry number: 1

Count time: 28800. Sec

PEAK ANALYSIS

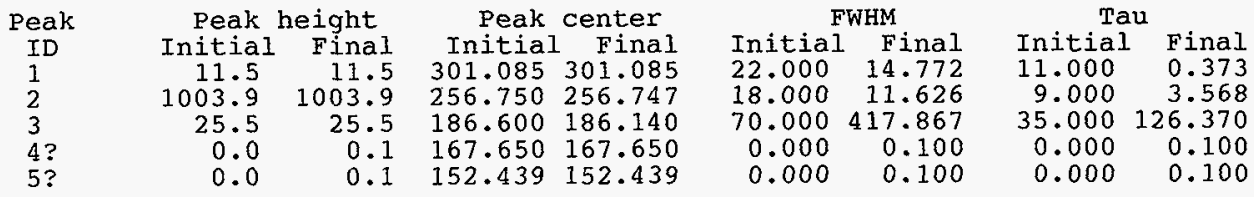

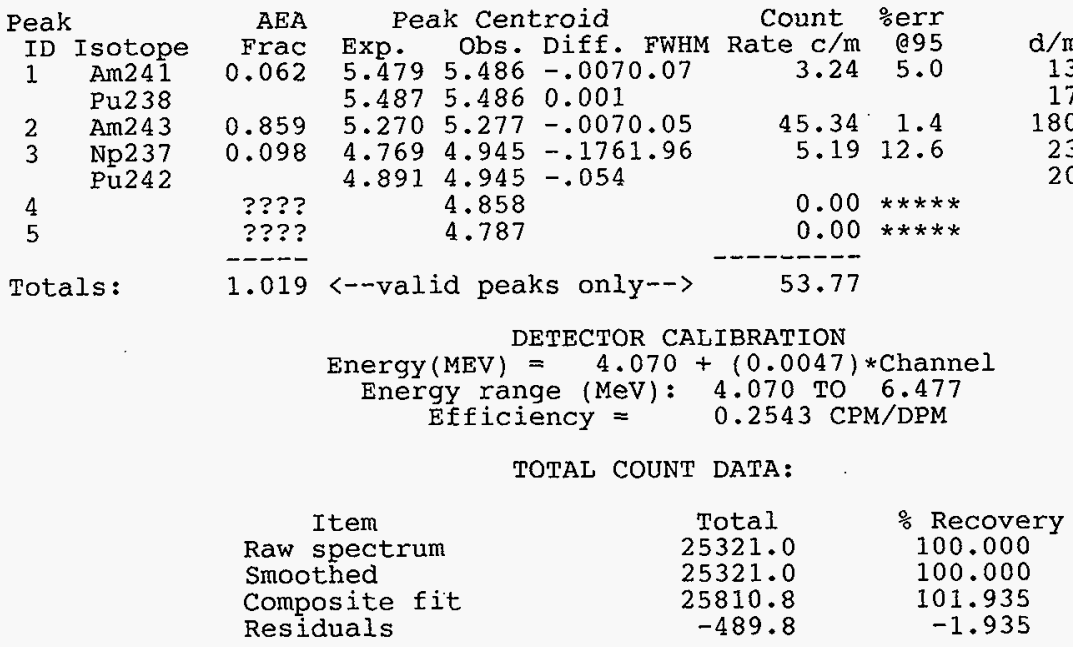

Analyzed by: 

1

1

1

$3 \ldots 2 \ldots$

$3 \ldots \ldots 2$.

$3 \ldots \ldots \ldots \ldots \ldots+\ldots \ldots+\ldots$

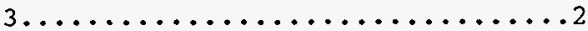

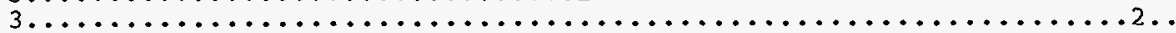

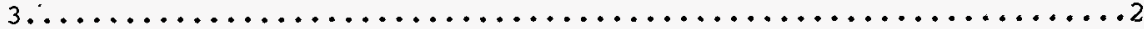

$1 \ldots \ldots \ldots \ldots \ldots 2$.

$2 \ldots$ 
Räw Data Dump for AEA Spectrum: 8 a8870.CNF

HNF-SD-WM-DP-227, REV. 0

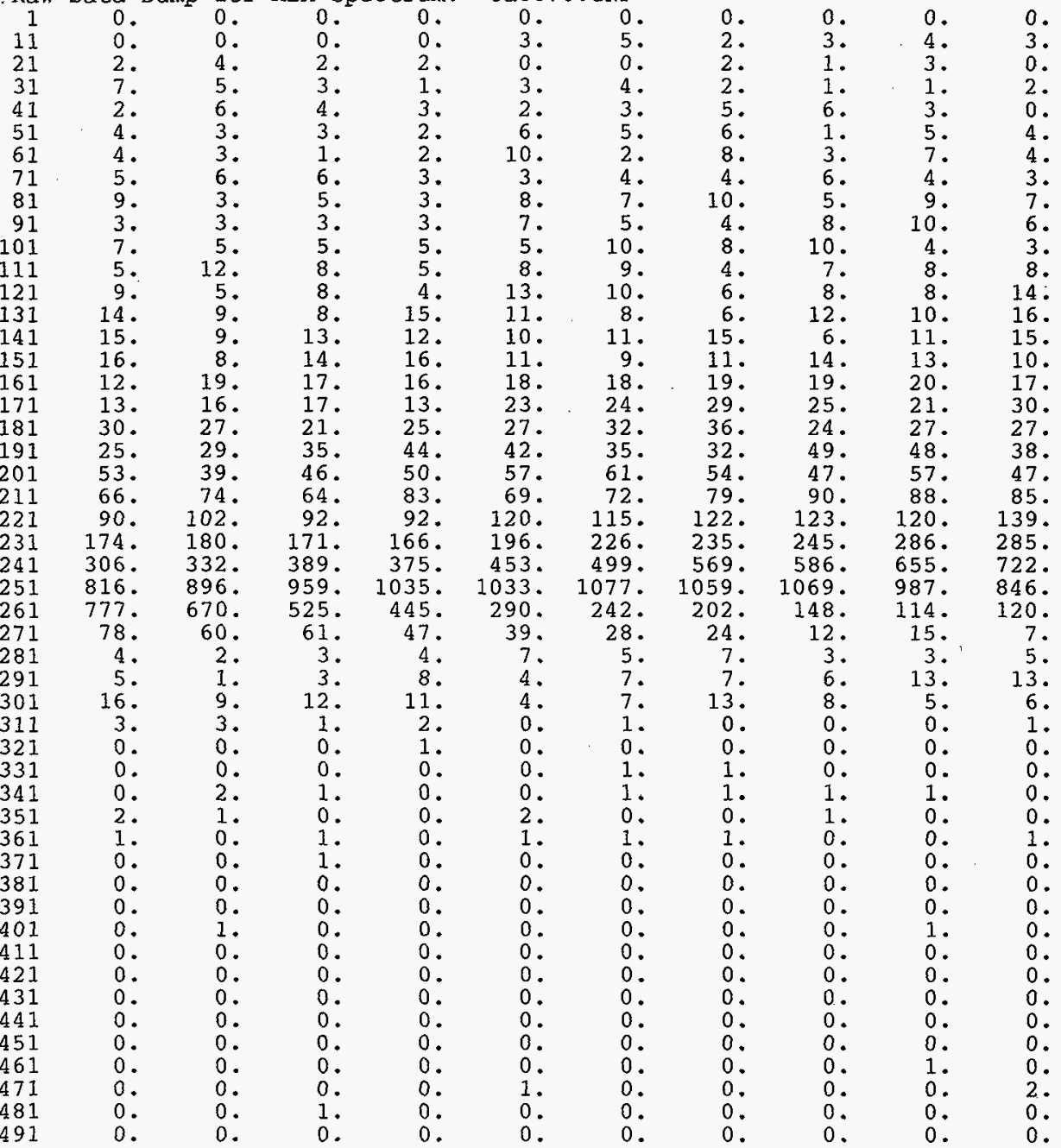

511

0 . 0 . 
Westinghouse Hanford $\mathrm{Co}$.

G E N E R L A L P HA ENERGY ANAIY S I $S$ Rev. 2.02

DATA REDUCTION REPORT

SAMPLE

S97T25-SAM-AM

File ID: 9 a9682.CNF

Counted on: 1/25/97 @14:20

Detector:

AEA9

Geometry number: 1

count time:

28803. Sec

PEAK ANALYSIS

Peak
ID
$\frac{1}{2}$

Peak

ID Isotope

1 Pu238 Am2 41

$2 \mathrm{Am} 243$

Totals :
Peak height Initial Final $\begin{array}{ll}79.4 & 79.4\end{array}$ $1674.0 \quad 1674.0$

\begin{abstract}
Peak center Initial Final 303.021303 .021 $256.970 \quad 256.969$
\end{abstract}$$
\begin{array}{cc}
\multicolumn{2}{c}{\text { Tau }} \\
\text { Initial } & \text { Final } \\
7.000 & 2.869 \\
6.000 & 2.397
\end{array}
$$

PEAK RESULTS

Peak Error Limit: $30 \%$

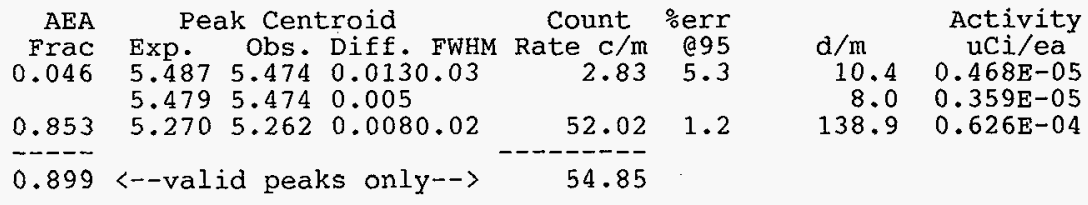

\section{DETECTOR CALIBRATION}

$$
\begin{aligned}
& \text { Energy (MEV) }=4.080+(0.0046) * \text { Channel } \\
& \text { Energy range (MeV): } 4.080 \text { To } 6.435 \\
& \text { Efficiency }=0.3783 \mathrm{CPM} / \mathrm{DPM} \\
& \text { TOTAL COUNT DATA: }
\end{aligned}
$$

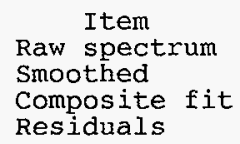

$$
\begin{array}{r}
\text { Total } \\
29278.0 \\
29278.0 \\
26331.7 \\
2946.3
\end{array}
$$

Analyzed by: Recovery
100.000
100.000
89.937
10.063 
1 Legend: Raw $=\ldots$.. Modeled Peaks $=1,2, \ldots$ etc $\quad$ Display Max.: 9000.1 
HNF-SD-WM-DP-227, REV. U

\begin{tabular}{|c|c|c|c|c|c|c|c|c|c|c|}
\hline & & & & & & & & & & \\
\hline 1 & 0. & 0 . & 0 . & 0 . & 0 . & 0 . & 0. & 0. & 0. & 0. \\
\hline 11 & 0. & 0 . & 0. & 0. & 0 . & 2. & 3. & 1. & 2 . & 1. \\
\hline 21 & 1 . & 2. & 1 . & 1 . & 1 . & 0. & 0. & 1. & 1. & 0 . \\
\hline 31 & 1 . & 0 . & 2 . & 4. & 1. & 4. & 2 . & 4. & 1 . & 3. \\
\hline 41 & 0. & 1. & 1. & 3. & i. & 0. & 0. & 4. & 0. & 1. \\
\hline 51 & 4. & 6. & 1 . & 4. & 1. & 5. & 2 . & 2 . & 5. & 2 . \\
\hline 61 & 2. & 5. & 2 . & 2 . & 7. & 1. & 4. & 1. & 4. & 1. \\
\hline 71 & 2. & 2. & 0 . & 1. & 5. & 3. & 1. & 5. & 3. & 2 . \\
\hline 81 & 1. & 3. & 0. & 2. & 2 . & 4. & 1. & 3 . & 1 . & 3. \\
\hline 91 & 4 . & 2 . & 2. & 7. & 4. & 1. & 2 . & 3. & 2 . & 1. \\
\hline 101 & 3. & 3. & 2 . & 0. & 2 . & 2. & 1. & 7. & 6. & 0. \\
\hline 111 & 3. & 5: & 3 . & 2. & 6. & 3 . & 4. & 5. & 2. & 2. \\
\hline 121 & 5. & 3. & 3. & 2 . & 2 . & 4. & 4. & 3. & 2 . & 3. \\
\hline 131 & 4. & 2 . & 2 . & 11. & 2 . & 6. & 7. & 6. & 4. & 5. \\
\hline 141 & 2. & 6. & 9. & 7. & 7. & 7. & 5. & 7. & 5. & 10. \\
\hline 151 & 11. & 3. & 9. & 7. & 4. & 13. & 6. & 6. & 10. & 10. \\
\hline 161 & 8. & 5. & 9. & 10. & 6. & 10. & 10. & 11. & 7. & 6. \\
\hline 171 & 13. & 11. & 10. & 9. & 10. & 13. & 8. & 10. & 6. & 13. \\
\hline 181 & 14. & 14. & 20. & 15. & 17. & 13. & 10. & 21. & 15. & 16. \\
\hline $\begin{array}{l}191 \\
201\end{array}$ & $\begin{array}{l}20 . \\
24 .\end{array}$ & $\begin{array}{l}16 . \\
22 .\end{array}$ & $\begin{array}{l}21 . \\
20 .\end{array}$ & $\frac{18}{26}$ & $\frac{17}{20^{\circ}}$ & 20. & 22. & 16. & $\frac{19}{26}$ & 18. \\
\hline 211 & 42 . & 34. & 38. & 42. & 49 . & 51. & 57. & 57. & 49. & $66^{\circ}$. \\
\hline 221 & 67 & 84. & 84. & 80. & 79. & 103. & 99. & 109. & 101. & 110. \\
\hline 231 & 137. & 148 & 140. & 152. & 214. & 203. & 211. & 228 . & 230 . & 245 . \\
\hline 241 & 297. & 304 & 340 & 408 & 509. & 554 . & 639. & 700 & 766 . & 816 . \\
\hline 251 & 960. & 1136. & 1214 . & 1458 . & 1666 , & 1761 . & 1951. & 1899. & 1544 . & 1179. \\
\hline 261 & 814. & 511. & 353 & 243 & 201 . & 178 & 146 & 130 & 133 & 124. \\
\hline 271 & 121. & 79. & 55. & 51. & 39. & 20. & 26. & 8. & 16. & 10. \\
\hline 281 & 8. & 8. & 12. & 14. & 10. & 12. & 17. & 15. & 23. & 18. \\
\hline 291 & 22 . & 30 . & 31. & 30. & 40. & 44. & 44. & 64. & 61. & 75. \\
\hline 301 & 64. & 94. & 89. & 93. & 69. & 63. & 40. & 30. & 26. & 27. \\
\hline 311 & 11. & 20. & 16. & 12. & 7. & 5. & 4. & 1. & 0 & 0. \\
\hline 32$]$ & 0. & 1. & 0. & 0 & 1. & 0. & 2. & 1. & 1. & 2 . \\
\hline 33 & 1. & 0 . & 1. & 1. & 0 . & 1. & 1. & 0. & 1. & 3. \\
\hline 341 & 1. & 0 . & 3. & 1. & 1. & 5. & 3. & 1. & 4. & 2. \\
\hline 35 & 6. & 2. & 3. & 2. & 2. & & 3. & 2. & 4. & 2 . \\
\hline & 4. & 8. & 1. & 3. & 3. & 0 & 3. & 1. & 1. & 1. \\
\hline 371 & 0. & 0 . & 1. & 0 . & 0. & 0. & 0 . & 0. & 0. & 0 . \\
\hline 381 & 0. & 0 . & 0. & 0. & 0. & 0. & 0. & 0. & 0. & 0. \\
\hline & 0. & 0 . & 0. & 0. & 0. & 0. & 0. & 0. & 0. & 0 . \\
\hline 40 & 0. & 0 . & 0. & 0. & 0. & 0. & 0. & 0. & 0 . & 0 . \\
\hline 41 & 0. & 0 . & 0. & 0. & 1. & 0. & 0 . & 0. & 0. & 0 . \\
\hline & 0. & 0. & 1. & 0. & 0. & 1. & 0 . & 0. & 0 . & 0 . \\
\hline 43 & 0. & 1. & 1. & 0. & 0 . & 0 . & 1. & 0. & 0. & 0 . \\
\hline 441 & 0. & 0 . & 0. & 0 . & 0. & 0 . & 0. & 0. & 0 . & 0 . \\
\hline 45 & 1. & 0. & 0. & 0. & 0 . & 0. & 0. & 0. & 0. & 1. \\
\hline 46 & 0. & 0 . & 0. & 1. & 0. & 0. & 2. & 0. & 2 & 1. \\
\hline & 1. & 1. & 1. & I. & 0 . & 0. & 1. & 0. & 0. & 0. \\
\hline 48 & 0. & 0 . & 0. & 0. & 0. & 0 . & 0. & 0. & 0. & 0 . \\
\hline & 0. & 0. & 0. & 0. & 0. & 0. & 0. & 0. & 0. & 0 . \\
\hline & 0. & 0. & & & & & & & & \\
\hline
\end{tabular}




G E N E R A I A L P H A E N E R G Y A N A L Y I S
Rev. 2.02
DATA REDUCTION REPORT
SAMPLE
S97T25-DUP-AM
File ID: 10 al085.CNF

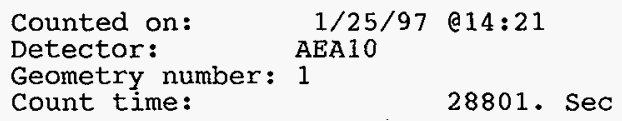

PEAK ANALYSIS

\begin{tabular}{|c|c|c|c|c|c|c|c|}
\hline Peak & Peak & height & Peak center & & & Tau & \\
\hline $\begin{array}{l}\text { ID } \\
1 \\
2\end{array}$ & $\begin{array}{c}\text { Initial } \\
64.2 \\
365.1\end{array}$ & $\begin{array}{r}\text { Final } \\
64.2 \\
1365.1\end{array}$ & $\begin{array}{cr}\text { Initial } & \text { Final } \\
304.478 & 304.478 \\
258.391 & 258.391\end{array}$ & $\begin{array}{l}\text { Initial } \\
12.000 \\
12.000\end{array}$ & $\begin{array}{l}\text { Final } \\
5.873 \\
4.627\end{array}$ & $\begin{array}{l}\text { Initial } \\
6.000 \\
6.000\end{array}$ & $\begin{array}{l}\text { Final } \\
2.615 \\
2.322\end{array}$ \\
\hline
\end{tabular}

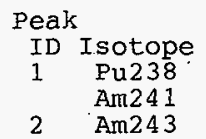

Totals :

\section{PEAK RESULTS \\ Peak Error Limit: $30 \%$}

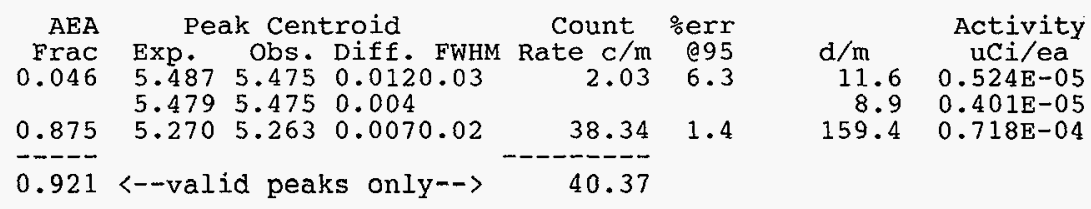

DETECTOR CALIBRATION

$$
\begin{gathered}
\text { Energy (MEV) }=4.074+(0.0046) * \text { Channel } \\
\text { Energy range }(\mathrm{MeV}): 4.074 \mathrm{TO} 6.429 \\
\text { Efficiency }= \\
\text { TOTAL COUNT DATA: }
\end{gathered}
$$

Item

Raw spectrum Smoothed Composite fit Residuals

$\begin{array}{rc}\text { Total } & \% \text { Recovery } \\ 21035.0 & 100.000 \\ 21035.0 & 100.000 \\ 19377.9 & 92.122 \\ 1657.1 & 7.878\end{array}$

Analyzed by: 
1 Eegend: $\quad$ Raw $=\ldots$ Spectrum 1 Modeled Peaks $=1,2, \ldots$ etc $\quad$ Display Max.: $\quad 8347.8$

$\dot{2}$.

$\ldots 2$.

$\ldots \ldots \ldots \ldots \ldots+\ldots \ldots+2$

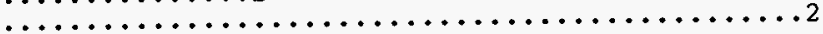

$\ldots \ldots 2 \ldots \ldots$

1

$\ldots 1$

$\because 1$ 
Raw Data Dump for AEA Spectrum: 10a1085.CNF

HNF-SD-WM-DP-227, REV. 0

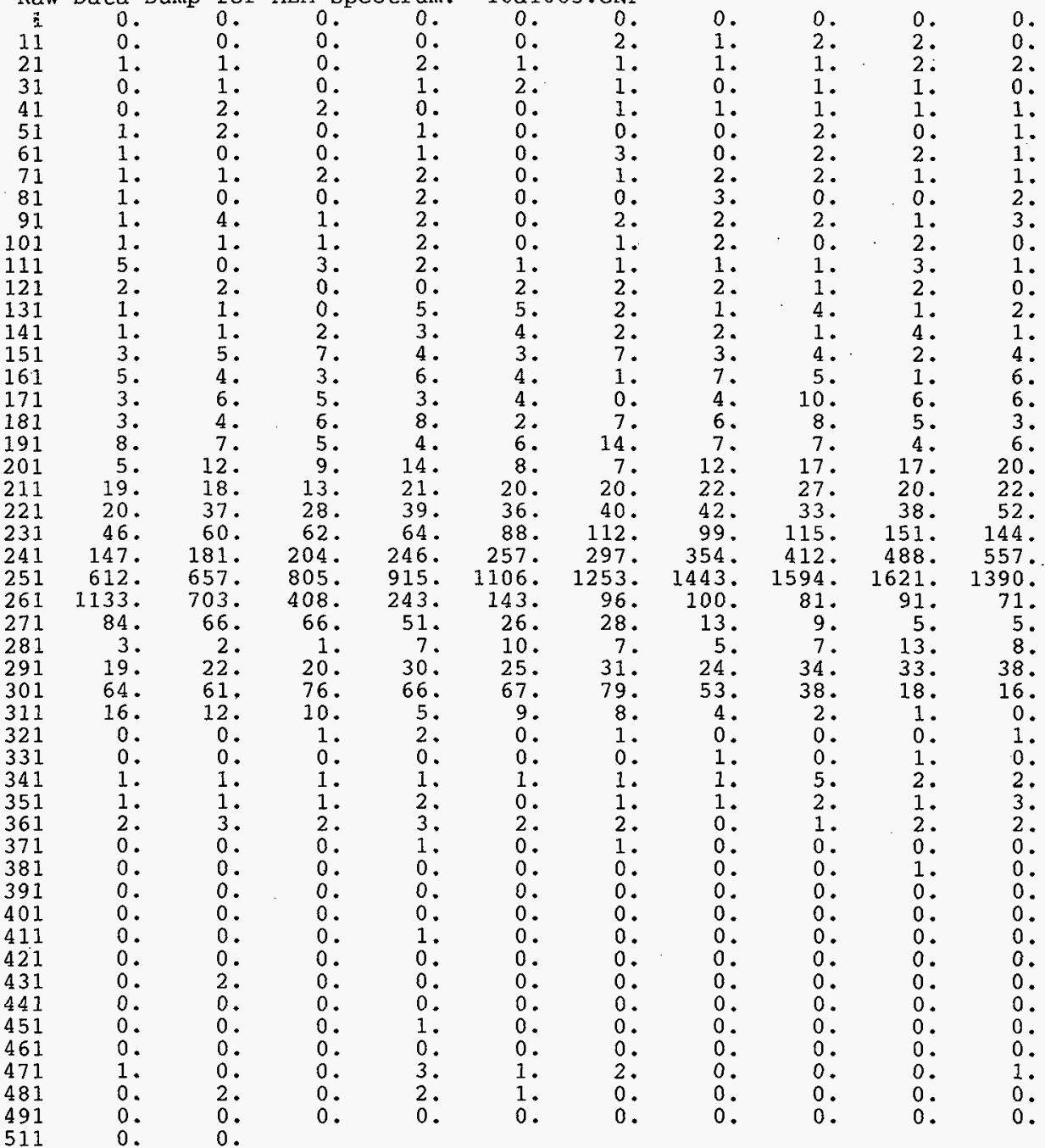


G E N E R A L A L P H A E N E R G Y A N A L Y S I S

DATA REDUCTION REPORT

SAMPLE

S97T28-AM

File ID: 11a1110.CNF

$\begin{array}{ll}\text { Counted on: } & 1 / 25 / 97 \text { @14:21 } \\ \text { Detector: } & \text { AEA11 } \\ \text { Geometry number: } 1 & \\ \text { Count time: } & \end{array}$

PEAK ANALYSIS

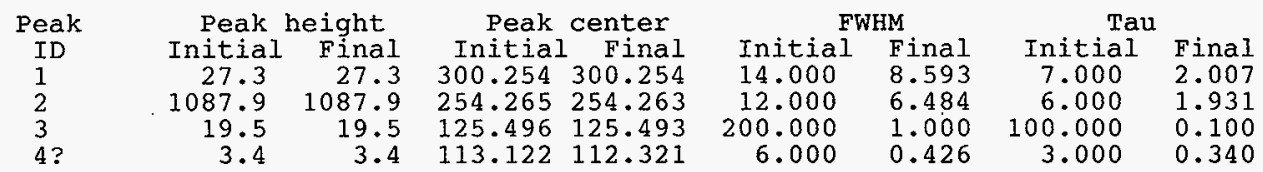

$\begin{array}{lc}\text { Peak } \\ \text { ID } & \text { Isotope } \\ 1 & \text { Am2 } 21 \\ & \text { Pu238 } \\ 2 & \text { Am2 } 43 \\ 3 & \text { Np237 } \\ 4 & \end{array}$

Totals :

\section{PEAK RESULTS \\ Peak Error Limit: $30 \%$}

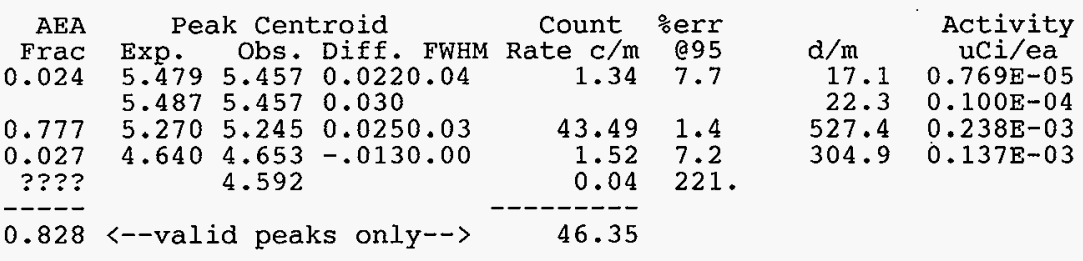

TOTAL COUNT DATA:

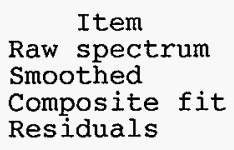

$\begin{array}{cc}\text { Total } & \% \text { Recovery } \\ 26860.0 & 100.000 \\ 26860.0 & 100.000 \\ 22273.7 & 82.925 \\ 4586.3 & 17.075\end{array}$

Analyzed by: 

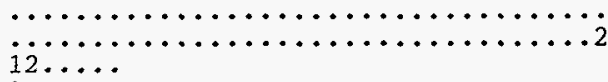
Raw Data Dump for AEA Spectrum: 11a1110.CNF

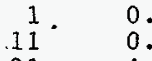

21

$\begin{array}{lll}31 & 4 . & 6 . \\ 41 & 4 . & 5 .\end{array}$

519.10.

61

71

81

101

111

121

131

141

151

161

171

181

191

201

211

221

231

241

251

261

271

281

291

301

311

321

331

341

351

361

371

381

391

401

411

421

431

441

451

461

471

481

491

511
0.
0.
0.
6.
5.
0.
6.

10.9.

14.

7.14 .

10. 16 .

10. 20 .

8. 15.

18. 18.

26 . 22 .

25. 40 .

31.37 .

43.34.

$53 . \quad 34$.

$\begin{array}{ll}68 . & 82 . \\ 84 . & 92 .\end{array}$

135. 151 .

186.228.

$4190^{\circ} \quad 441$.

210 . 148 .

$34 . \quad 19$.

7.4 .

11. 24.26

32.27

2. 0.

I. 0 .

0.1 .

1. 0.

0.1 .

0.0 .

$0 . \quad 0$.

0.0 .

0.0.

0.0.

$\begin{array}{ll}0 . & 0 . \\ 0 . & 0 .\end{array}$

0.0 .

0.0.

1. 0 .

0 . 0 .

$\begin{array}{ll}0 . & 0 . \\ 0 . & 0 .\end{array}$ \begin{tabular}{cccc}
\multicolumn{2}{c}{ Spectrum: } & 11 al110. CNF \\
0. & 0. & 0. & 0. \\
0. & 0. & 0. & 5.
\end{tabular}

$\begin{array}{lll}0 . & 0 . & 0 . \\ 2 . & 3 . & 3 .\end{array}$

4. 7.10 .

4. 6.63 .

9.5 .14$.

8. 12 .

8.11 .

4.

14.

11 .

22 .

12 .

11.

16.

30.
21.

35 .

49.

59.

68.

93.

150.138.

206

464 .

1197 .

140 .

28.

26.

3.

0 .

1 .

0 .

1.

0 .

0 .

0 .

0.

0 .

1.

0 .

0 .

0 .

0 .

0 .

249.

$594.560^{\circ}$.

$1174^{\circ} 1186$.

$100 . \quad 101$.

20 .

20.

22.20.

22 .

2 .

0 .

0 .

1.

1.

0 .

0 .

1.

0 .

0.

0 .

0 .

0 .

0 .

1 .

0 .

11.

3.

0 .

0 .

0 .

0 .

0 .

0 .

0 .

0 .

0 .

0 .

0 .

0 .

0 .

0 .

0 .

1134 .

0.

0.

HNF-SD-WM-DP-227, REV. 0

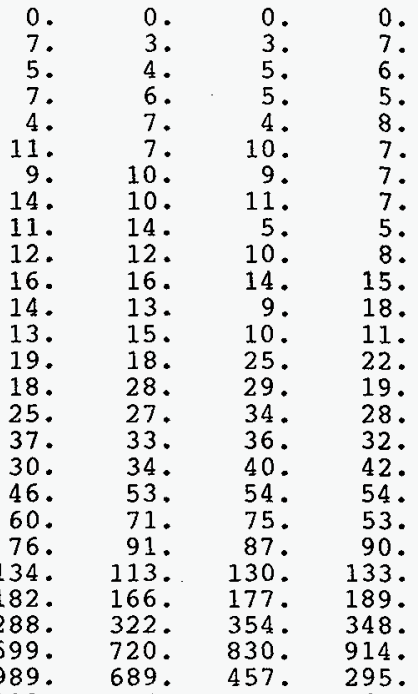

102 .

$\begin{array}{rrrr}5 . & 5 . & 7 . & 5 . \\ 12 . & 10 . & 15 . & 16 .\end{array}$

75 .

57 .

67.

36. 27. 25. 24 .

15. 2 . 8.7 .

0.0 .0$.

1. 0.00.

0

3.

0 .

1

2

0

0 .

0 .

3.

0 .

0 .

0 .

0.

0 .

0.

0 .

0 .

0 .

0 .

0 .

0 .

0 .

1.

0 .

0 .

1.

1.

0 .

0 .

0 .

0 .

0 .

0 .

0 .

0 .

0 .

0 .

0 .

0.

0.

0 .

1.

1.

0.

0 .

0 .

0. 
Westinghouse Hanford Co. G E N E R A L A L P H A E E R R Y A N A L Y S I S

DATA REDUCTION REPORT

SAMPLE

S97T28-DUP-AM

File ID: 12 a1258.CNF

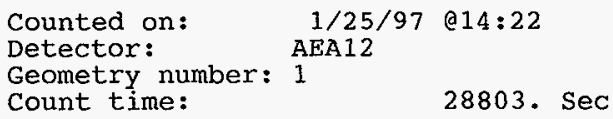

PEAK ANALYSIS

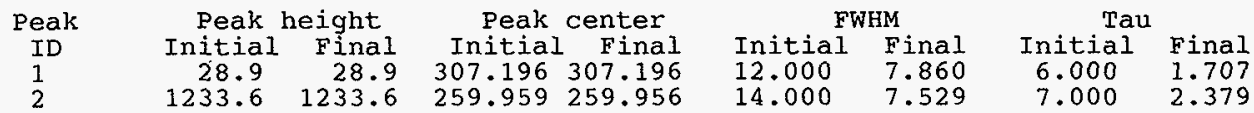

PEAK RESULTS
Peak Error Limit: $30 \%$

Peak

ID Isotope

1 Pu238

Am241

$2 \operatorname{Am} 243$

Totals:

\begin{tabular}{|c|c|c|c|c|c|}
\hline $\begin{array}{r}\text { AEA } \\
\text { Frac } \\
.026\end{array}$ & 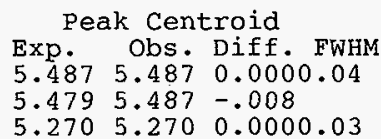 & $\begin{array}{r}\text { Count } \\
\text { Rate } \mathrm{c} / \mathrm{m} \\
1.46\end{array}$ & $\begin{array}{r}\text { oerr } \\
\text { @95 } \\
7.4 \\
1.3\end{array}$ & $\begin{array}{r}\mathrm{d} / \mathrm{m} \\
5.1 \\
3.9 \\
125.9\end{array}$ & $\begin{array}{l}\text { Activity } \\
\text { uCi/ea } \\
0.231 \mathrm{E}-05 \\
0.177 \mathrm{E}-05 \\
0.567 \mathrm{E}-04\end{array}$ \\
\hline 892 & $<--v a l i d$ peaks & 50.75 & & & \\
\hline
\end{tabular}

DETECTOR CALIBRATION

Energy (MEV) $=4.074+(0.0046) *$ Channel
Energy range $(\mathrm{MeV}): 4.074 \mathrm{TO} 6.429$
Efficiency $=$
$0.3954 \mathrm{CPM} / \mathrm{DPM}$

TOTAL COUNT DATA:

Item

Raw spectrum

Smoothed

Composite fit

Residuals

Total
27301.0
27302.7
24362.6
2938.4

\% Recovery

100.000

100.006

89.237

10.763

Analyzed by: 
1 Segend Spectrum 12a1258.CNF

1 Legend: Raw $=\ldots$ Modeled Peaks $=1,2, \ldots$, etc
HNF-SD-WM-DP-227, REV. 0 Display Max.: 
Raw Data Dump for AEA Spectrum: 12a1258.CNF

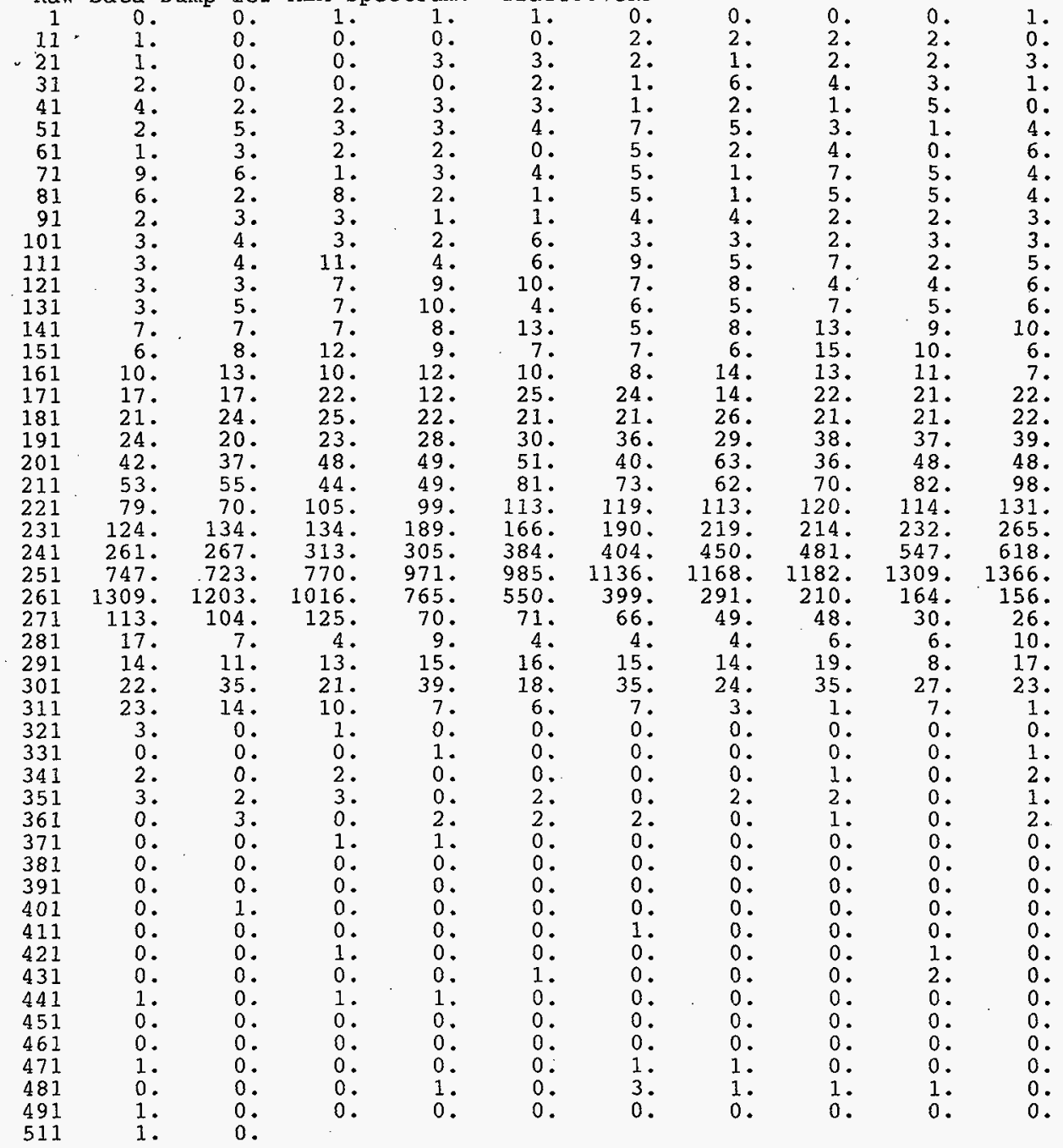




\section{LABCORE Completed Worklist Report for Worklist\# 16279}

Analyst: jmv

Instrument: $\mathrm{AB} 14$

Book\#

Method: $24-943-128$ Rev/Mod $B-0$

Worklist Comment: Determine sample size using ludlum.Std:(TNKFUS) 1.0ml. new

Seq Type Sample\# RA Test Matrix Actual Found DL or Yield Unit

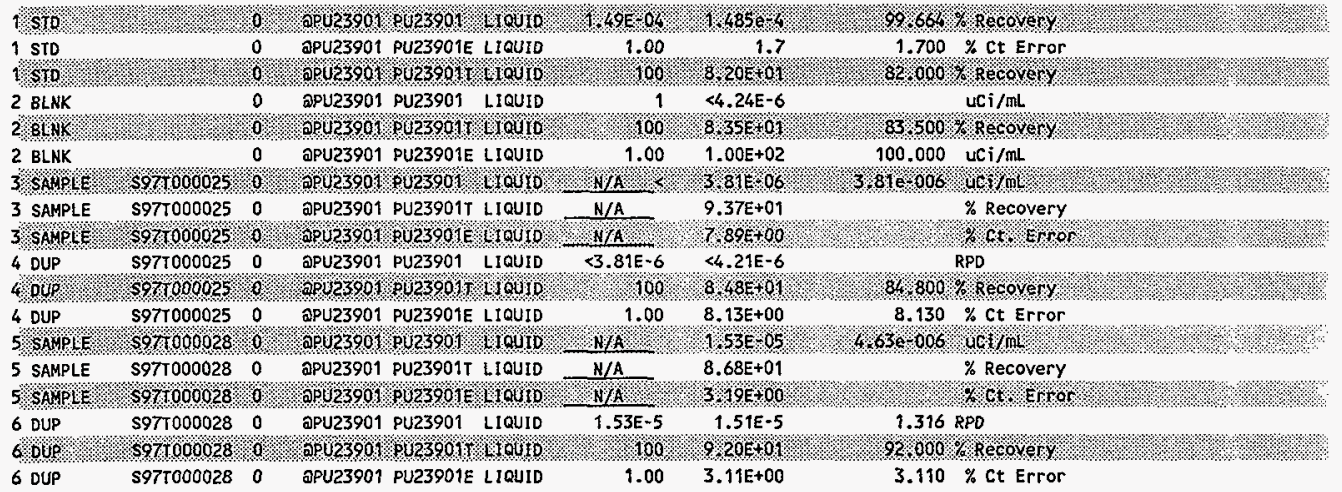

\section{Final page for worklist\# 16279}

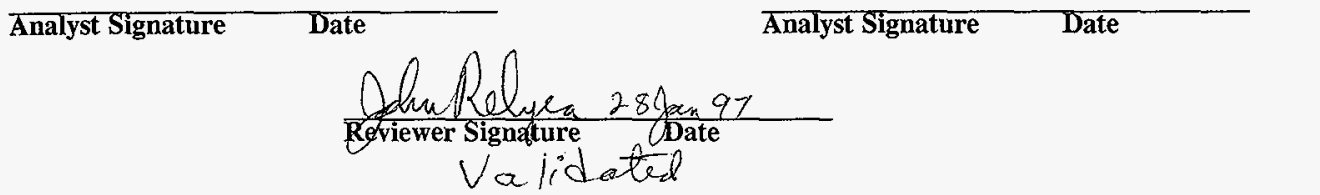


HNF-SD-WM-DP-227, REV. 0

01/27/97 13:34

A-0004-1

\section{LABCORE Data Entry Template for Worklist\# 16279}

Page: 1

Analyst:

Instrument: PU01

Book\#

Method: LA-943-128 Rev/Mod

Worklist Comment: Determine sample size using ludlum.Std:(TNKFUS) 1.0ml. new

S Type Sample\# R A Test Matrix Group\# Project

1 STD

GPU23901 LIQUID

2 BLNK

@PU23901 IIQUID

3 SAMPLE

S97T000025 O QPU23901 LIQUID

97000022 SY-102 GRABI

Analytes Requested: PU23901, PU23901E, PU23901T

4 DUP S97T0000250 QPU23901 LIQUID

5 SAMPLE S97T000028 0 QPU23901 LIQUID 97000022 SY 102 GRAB1

Analytes Requested: PU23901, PU23901E, PU23901T

6 DUP S97T000028 $\quad$ EPU23901 IIQUID

\section{Final page for worklist \# 16279}

Analyst Signature Date

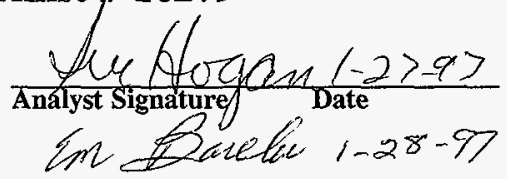

Data Entry Comments:

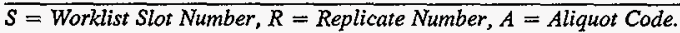




\title{
LABCORE Data Entry Template for Worklist\# 16279
}

Analyst: $J_{M V}$ Instrument: PU01 \#14 Book\# l.2B56

Method: LA-943-128 Rev/Mod

Worklist Cormment: Determine sample size using ludlum.Std:(TNKFUS) 1.0ml. new

\begin{tabular}{|c|c|c|c|c|c|c|c|}
\hline$S$ & Type & Sample\# & R A & Test & Matrix & Group\# & Project \\
\hline 1 & STD & & & $@$ PU23901 & LIQUID & & \\
\hline 2 & BLNK & & & $@$ @U23901 & LIQUID & & \\
\hline \multirow[t]{2}{*}{3} & SAMPLE & S97T000025 & 0 & $@$ @U23901 & LIQUID & 97000022 & SY-102 GRABI \\
\hline & & Analytes Requ & ested: & PU23901 & , PU23901E, & PU23901T & \\
\hline 4 & DUP & S97T000025 & 0 & @PU23901 & LIQUID & & \\
\hline \multirow[t]{2}{*}{5} & SAMPLE & S972000028 & 0 & $@$ @PU23901 & LIQUID & 97000022 & SY-102 GRAB 1 \\
\hline & & Analytes Requ & ested: & : PU23901 & , PU23901E, & PU23901T & \\
\hline 6 & DUP & $597 T 000028$ & 0 & @PU23901 & LIQUID & & \\
\hline \multirow[t]{2}{*}{7} & SAMPLE & S97T000029 & 0 & @PU23901 & LIQUID & 97000022 & SY-102 GRAB1 \\
\hline & & Analytes Requ & ested: & : PU23901 & , PU23901E, & PU23901T & \\
\hline & DUP & S97T000029 & 0 & @PU23901 & LIQUID & & \\
\hline
\end{tabular}

\section{Final page for worklist \# 16279}

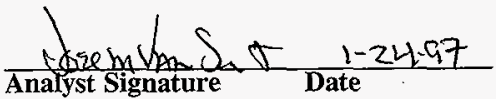

Analyst Signature Date

\author{
Analyst Signature Date
}

Data Entry Comments:

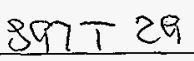

Weeds

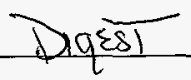

$S=$ Worklist Slot Number, $R=$ Replicate Number, $A=$ Aliquot Code. 
HNF-SD-WM-DP-227, REV. 0

WORKBOOK PAGE: STD 1

Pu 238 and 239/240 : LA-943-128 (B-0)

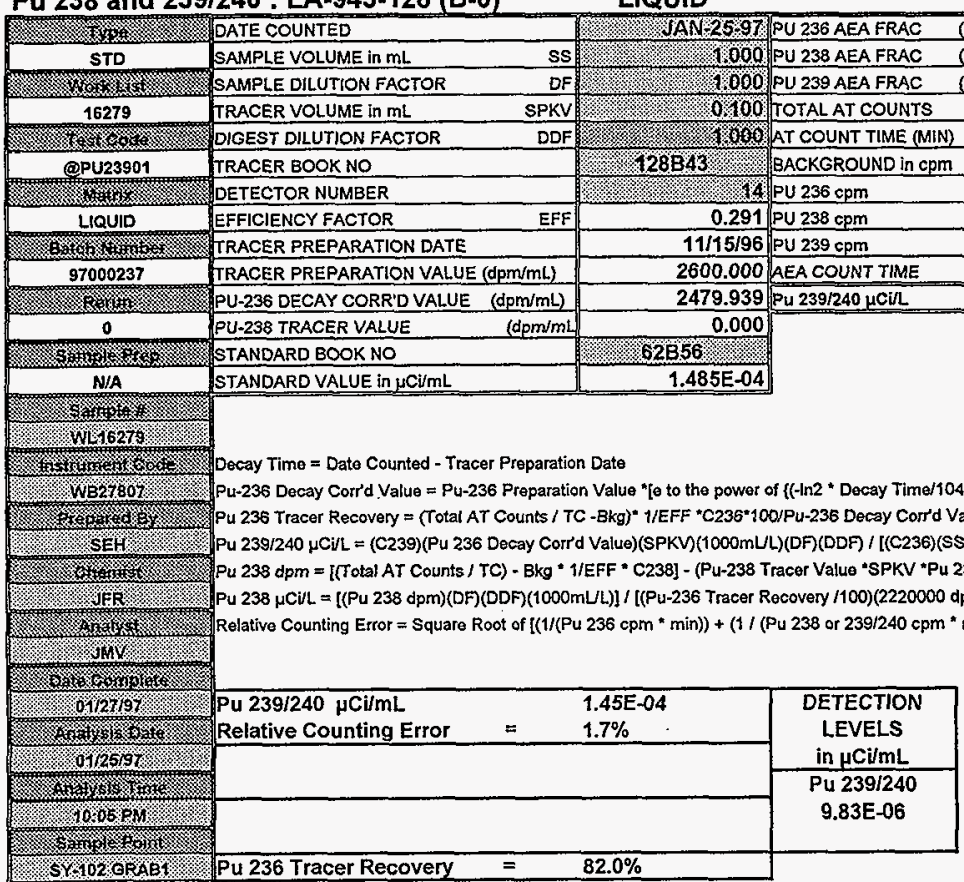

\begin{tabular}{|c|c|c|c|}
\hline Analyst: & JMV & Date: & $27-\operatorname{Jan}-97$ \\
\hline Signature of Chemist: & $\mathrm{JFR}$ & Date: & $8 \lambda$ \\
\hline
\end{tabular}


WORKBOOK PAGE: BLANK2

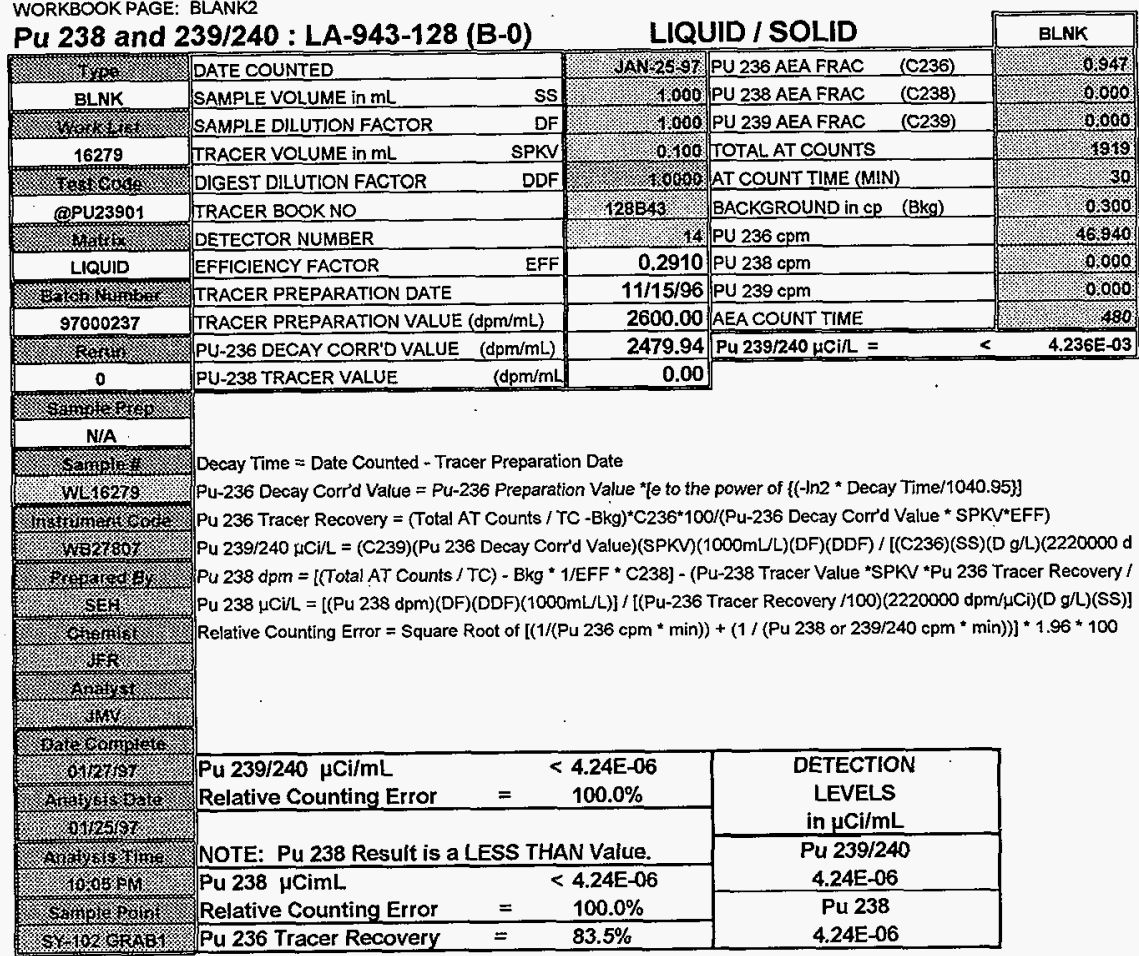

\begin{tabular}{|c|c|c|c|}
\hline Analyst: & JMV & Date: & 27-Jan-97 \\
\hline Signature of Chemist: & & & $\tan \theta$ \\
\hline
\end{tabular}

\section{1}




\section{WORKBOOKPAGE: SAM3}

\begin{tabular}{|c|c|c|c|c|}
\hline \multicolumn{4}{|c|}{ Pu 238 and $239 / 240:$ LA-943-128 (B-0) } & SAMPLE \\
\hline 23 & DATE COUNTED & $9 \mathrm{NL}_{2} 697$ & PU 236 AEA FRAC & \\
\hline SAMPLE & SAMPLE VOLUME in mL & $10 \% 000$ & PU 238 AEA FRAC & \\
\hline 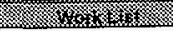 & SAMPLE DILUTION FACTOR & 4000 & PU 239 AEA FRAC & \\
\hline 16279 & TRACER VOLUME in $\mathrm{mL}$ & 10300 & TOTAL AT COUNTS & \\
\hline 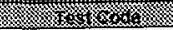 & DIGEST DILUTION FACTOR & 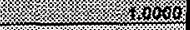 & AT COUNT TIME (MIN) & \\
\hline (9PU23901 & IRACER BOOK NO & 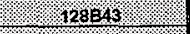 & BACKGROUND in cPm & \\
\hline 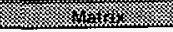 & DETECTOR NUMBER & 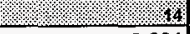 & PU $236 \mathrm{cpm}$ & \\
\hline LIQUID & EFFICIENCY FACTOR & 0.291 & PU $238 \mathrm{cpm}$ & \\
\hline 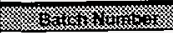 & TRACER PREPARATION DATE & $11 / 15 / 96$ & PU $239 \mathrm{cpm}$ & \\
\hline 97000237 & TRACER PREPARATION VALUE (dpm/mL) & 2600.000 & AEA COUNT TIME & \\
\hline 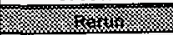 & PU-236 DECAY CORR'D VALUE (dpm/mL) & 2479.939 & Pu $239 / 240 \mu \mathrm{Ci} / \mathrm{L}=$ & 3.81 \\
\hline 0 & PU-238 TRACER VALUE (dpm/mL) & 0.000 & & \\
\hline 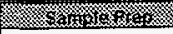 & \\
\hline N/A & & & & \\
\hline 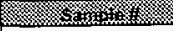 & \multirow{2}{*}{\multicolumn{4}{|c|}{$\begin{array}{l}\text { Decay Time = Date Counted }- \text { Tracer Preparation Date } \\
\text { Pu-236 Decay Corrd Value }=\text { Pu-236 Preparation Valtue *le to the power of }\{(-\operatorname{tn} 2 * \text { Decay Time/1040.95 }\}\end{array}$}} \\
\hline S97T000025 & & & & \\
\hline 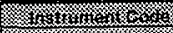 & \multirow{5}{*}{\multicolumn{4}{|c|}{ 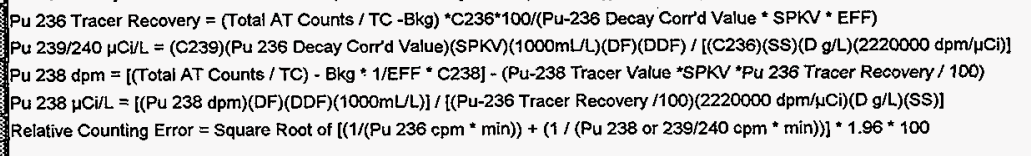 }} \\
\hline 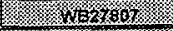 & & & & \\
\hline (3) & & & & \\
\hline 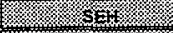 & & & & \\
\hline 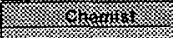 & & & & \\
\hline 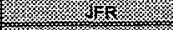 & & & & \\
\hline 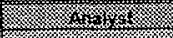 & & & & \\
\hline $10 \%$ mx & & & & \\
\hline 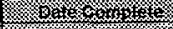 & & & & \\
\hline \% & $\mathrm{Pu} 239 / 240 \mu \mathrm{Ci} / \mathrm{mL}$ & 3.81E-06 & DETECTION & \\
\hline 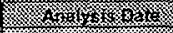 & Relative Counting Error & $7.9 \%$ & LEVELS & \\
\hline 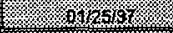 & \multirow{2}{*}{\multicolumn{2}{|c|}{ NOTE: Pu 238 Result is a LESS THAN Value. }} & in $\mu \mathrm{Ci} / \mathrm{mL}$ & \\
\hline 24 & & & Pu 239/240 & \\
\hline 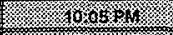 & Pu $238 \mu \mathrm{CimL}<$ & 3.81E-06 & $3.81 E-06$ & \\
\hline 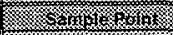 & Relative Counting Error & $10.8 \%$ & Pu 238 & \\
\hline 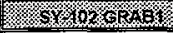 & Pu 236 Tracer Recovery & $93.7 \%$ & 3.81E-06 & \\
\hline
\end{tabular}

\begin{tabular}{|c|c|c|c|}
\hline Analyst: & JMV & Date: & 27-Jan-97 \\
\hline Signature of Chemist: & JFR & Date: & $x \tan 97$ \\
\hline
\end{tabular}


WORKBOOKPAGE: DUP4

Pu 238 and 239/240 : LA-943-128 (B-0)

\begin{tabular}{|c|c|c|c|}
\hline $3 \cos 2$ & DATE COUNTED & NAN-2567 & PU 236 AEA FRAC \\
\hline DUP & SAMPLE VOLUME in mL & 1.0\%, & PU 238 AEA FRAC \\
\hline 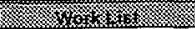 & SAMPLE DILUTION FACTOR & 10000 & PU 239 AEA FRAC \\
\hline 16279 & TRACER VOLUME in mL & 0.600 & TOTAL AT COUNTS \\
\hline 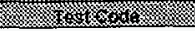 & DIGEST DILUTION FACTOR & 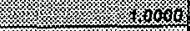 & AT COUNT TIME (MIN) \\
\hline @PU23901 & TRACER BOOK NO & 128844 & BACKGROUND in $\mathrm{cpm}$ \\
\hline (in & DETECTOR NUMBER & 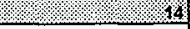 & PU $236 \mathrm{cpm}$ \\
\hline LIQUID & EFFICIENCY FACTOR & 0.291 & PU $238 \mathrm{cpm}$ \\
\hline Sath & TRACER PREPARATION DATE & $11 / 15 / 96$ & PU $239 \mathrm{cpm}$ \\
\hline 97000237 & IRACER PREPARATION VALUE (dpm/mL) & 26.00 .000 & AEA COUNT TIME \\
\hline (3. & PU-236 DECAY CORR'D VALUE (dpm/mL) & 2479.939 & Pu $239 / 240 \mu \mathrm{Ci} / \mathrm{L}=$ \\
\hline 0 & PU-238 TRACER VALUE $\quad$ (dpm/mL) & 0.000 & \\
\hline 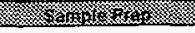 & \multirow{12}{*}{\multicolumn{3}{|c|}{ 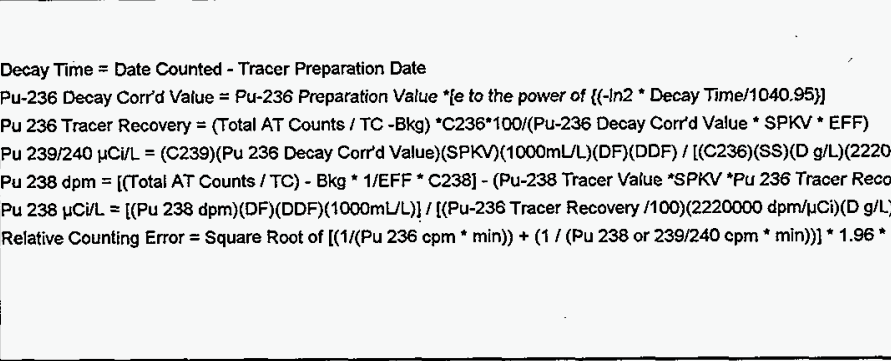 }} \\
\hline N/A & & & \\
\hline 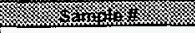 & & & \\
\hline S97T000025 & & & \\
\hline 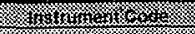 & & & \\
\hline 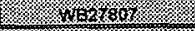 & & & \\
\hline (n) & & & \\
\hline (n) & & & \\
\hline 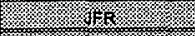 & & & \\
\hline ris & & & \\
\hline 3. & & & \\
\hline 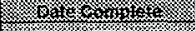 & & & \\
\hline (3) & $\mathrm{Pu} 239 / 240 \mu \mathrm{Ci} / \mathrm{mL}$ & $4.21 E-06$ & \multirow{3}{*}{$\begin{array}{l}\text { DETECTION } \\
\text { LEVELS } \\
\text { in } \mu \mathrm{Ci} / \mathrm{mL} \\
\end{array}$} \\
\hline 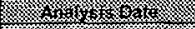 & Relative Counting Error & $8.1 \%$ & \\
\hline 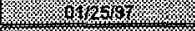 & \multirow{2}{*}{\multicolumn{2}{|c|}{ NOTE: Pu 238 Result is a LESS THAN Value. }} & \\
\hline 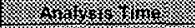 & & & \multirow{2}{*}{$\begin{array}{c}\text { Pu } 239 / 240 \\
4.21 E-06\end{array}$} \\
\hline (10.05 & Pu $238 \mu \mathrm{CimL}$ & 4.21E-06 & \\
\hline 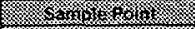 & Relative Counting Error & $10.0 \%$ & \multirow{2}{*}{$\begin{array}{c}\text { Pu } 238 \\
4.21 E-06\end{array}$} \\
\hline S. & Pu 236 Tracer Recovery & $84.8 \%$ & \\
\hline
\end{tabular}

\begin{tabular}{|c|c|c|c|}
\hline Analyst: & JMV & Date: & 27-Jan-97 \\
\hline Signature of Chemist: & JFR & Date: & $28 \operatorname{xan} 97$ \\
\hline
\end{tabular}


WORKBOOK PAGE: SAM5
PU 238 and $239 / 240:$ LA-943-128 (B-0)

SAMPLE
16279
@PU23901
LIQUID
97000237
0
0

DATE COUNTED

SAMPLE VOLUME in $\mathrm{mL}$ SAMPLE DILUTION FACTOR TRACER VOLUME in $\mathrm{mL}$ DIGEST DILUTION FACTOR TRACER BOOK NO DETECTOR NUMBER EFFICIENCY FACTOR TRACER PREPARATION DATE TRACER PREPARATION VALUE (dpm/mL) PU-236 DECAY CORR'D VALUE (dpm/mL) PU-238 TRACER VALUE
LIQUID / SOLID JAN 25.97\% PU 236 AEA FRAC 1:000. PU 238 AEA FRAC 1.000 PU 239 AEA FRAC 0.100. TOTAL AT COUNTS *60.0. LT COUNT TIME (MIN) 128843 BACKGROUND in com 4 PU $236 \mathrm{cpm}$ 0.291 Pu $238 \mathrm{cpm}$ $11 / 15 / 96$ PU $239 \mathrm{cpm}$ 2600.000 AEA COUNT TIME

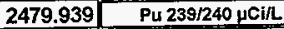
0.000

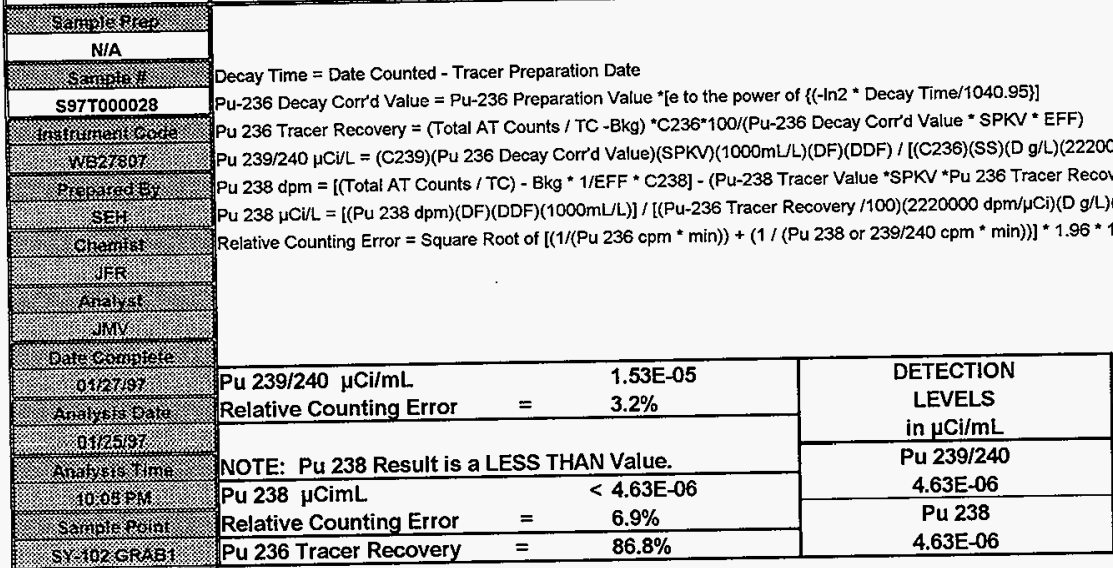

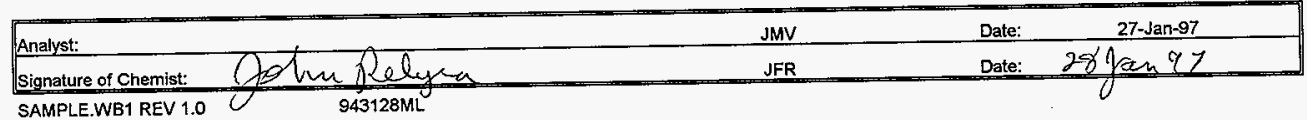


HNF-SD-WM-DP-227, REV. 0

WORKBOOK PAGE: DUP6

\section{Pu 238 and 239/240 : LA-943-128 (B-0)}

$\frac{\text { DUP }}{16279}$

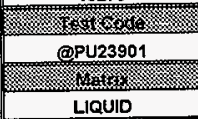

(2) 97000237

$\frac{0}{0}$

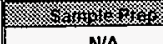

N/A

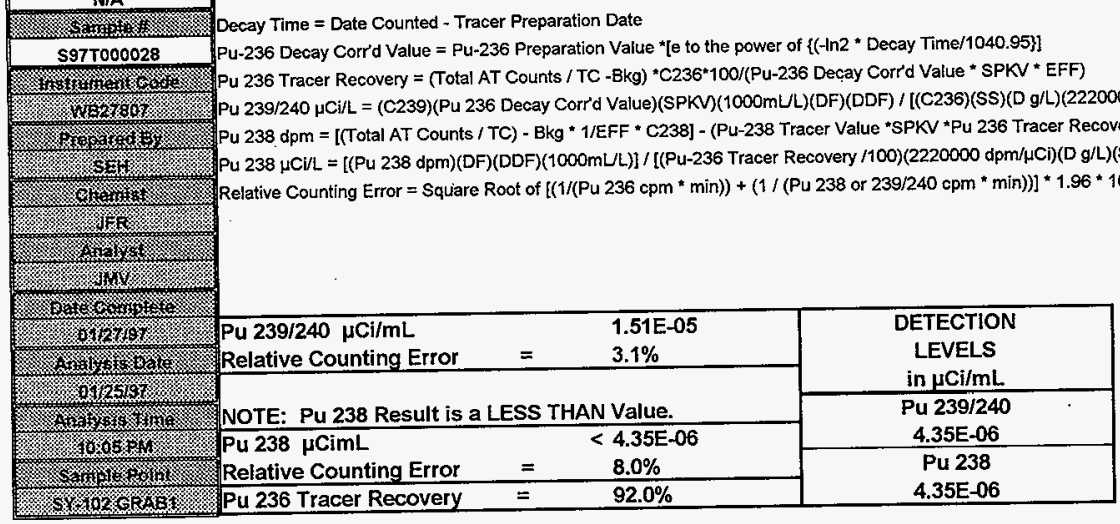

\begin{tabular}{|c|c|c|c|}
\hline Analyst: & JMV & Date: & 27-Jan-97 \\
\hline Signature of Chemist: & JFR & Date: & $28 x \operatorname{sen} 97$ \\
\hline
\end{tabular}




\title{
G E N E R A I \\ Westinghouse Hanford Co. \\ A L P H A E N E R G Y A N A L Y S I S \\ Rev. 2.02 \\ DATA REDUCTION REPORT \\ SAMPLE \\ WL16279-STD-PU \\ File ID: 13a1333.CNF
}

\author{
Counted on: $\quad 1 / 25 / 97$ a $14: 23$ \\ Detector: \\ Geometry number: 1 \\ count time: 28804. Sec
}

PEAK ANALYSIS

\begin{tabular}{|c|c|c|c|c|c|c|c|}
\hline Peak & Peak & neight & Peak center & & & Tar & \\
\hline ID & Initial & Final & Initial Final & Initial & Final & Initial & Final \\
\hline 1 & 1949.5 & 1949.5 & $361.803 \quad 361.803$ & 10.000 & 2.622 & 5.000 & 1.168 \\
\hline $2 ?$ & 38.5 & 18.5 & 302.653 & 32.000 & 9.359 & 16.000 & 207 \\
\hline $3 ?$ & 40.2 & 40.2 & 287 & .000 & & 000 & \\
\hline $4 ?$ & 13.7 & 13.7 & 270.425 & 10.000 & & .000 & \\
\hline 5 & 2853.0 & 853 & 229.390 & 10.000 & 80 & 5.000 & \\
\hline
\end{tabular}

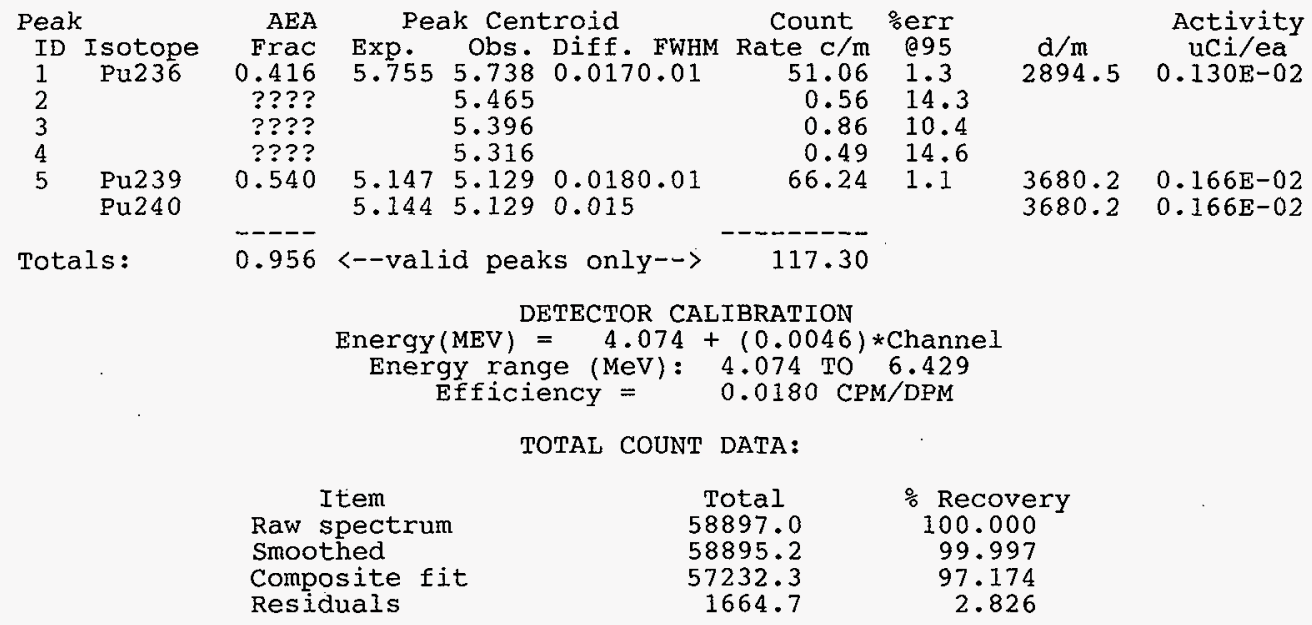

Analyzed by:

PEAK RESULTS

Peak Error Limit: $30 \%$ 
HNF-SD-WM-DP-227, REV. 0

1 Zegend: $\quad$ Raw $=\ldots$ Modeled Peaks $=1,2, \ldots$, etc $\quad$ Display Max.: 18577.3

$\dot{5}$.

.....5.

.................

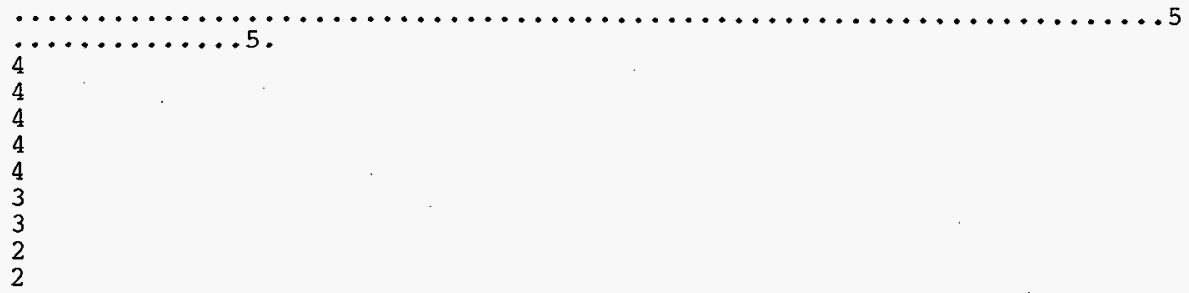

1

$\ldots 1$

$\ldots \ldots \ldots \ldots 1 \ldots$

$\ldots \ldots \ldots \ldots \ldots \ldots \ldots \ldots \ldots \ldots$

$\ldots \ldots \ldots \ldots \ldots \ldots \ldots \ldots \ldots \ldots \ldots \ldots \ldots \ldots$

$\cdots$ 
HNL-SU-WN-UF-ZZ/, HEV. U

Raw Data Dump for AEA Spectrum: 13a1333.CNF

\begin{tabular}{|c|c|c|c|c|c|c|c|c|c|c|}
\hline & & & & & & & & & & \\
\hline 11 & $\begin{array}{l}0 . \\
0 .\end{array}$ & $\begin{array}{l}0 . \\
0 .\end{array}$ & $\begin{array}{l}0 . \\
0 .\end{array}$ & $\begin{array}{l}0 . \\
0 .\end{array}$ & $\begin{array}{l}0 . \\
1 .\end{array}$ & $\begin{array}{l}1 . \\
0 .\end{array}$ & $\begin{array}{l}0 . \\
1 .\end{array}$ & $\begin{array}{l}0 . \\
0 .\end{array}$ & $\begin{array}{l}0 . \\
0 .\end{array}$ & $\begin{array}{l}0 . \\
0 .\end{array}$ \\
\hline 21 & 2 . & 3. & 0 . & 2 . & 2 . & 1 . & 0 . & 2 . & 1. & 0 . \\
\hline 31 & 1. & 0 . & 1. & 0 . & 0. & 0. & 2 . & 1. & 0. & 1. \\
\hline 41 & 1. & 2. & 2 . & 1 . & 1. & 3. & 0 . & 4. & 3. & 0 . \\
\hline 51 & 0 . & 2 . & 0 . & 3. & 2 . & 0 . & 1. & 1. & 2 . & 0 . \\
\hline 61 & 2. & 2 . & 2. & 8. & 5. & 7. & 5. & 10. & 9. & 8. \\
\hline 71 & 7. & 2 . & 5. & 4. & 2 . & 3. & 1. & 0 & 1. & 0 . \\
\hline 81 & 0. & 2 . & 1. & 0 . & 1 . & 2 . & 0 . & 0. & 0 . & 2 . \\
\hline 91 & 1. & 2 . & 1 . & 3. & 1 . & 0 . & 3. & 2 . & 2 . & 3. \\
\hline 101 & 0. & 3. & 2 . & 1. & 1. & 5. & 2 . & 2 . & 3. & 0. \\
\hline 111 & 1. & 5. & 3. & 1 . & 1 . & 1 . & 1. & 0. & 3. & 1 . \\
\hline 121 & 1 . & 0 . & 1. & 4. & 3. & 2 . & 2 . & 3 . & 3. & 3 . \\
\hline 131 & 1. & 4. & 1 . & 0 . & 1. & 4. & 1 . & 1. & 3. & 1. \\
\hline 141 & 3 . & 2 . & 2 . & 3. & 4. & 1 . & 1 . & 2 . & 3. & 2 . \\
\hline 151 & 5. & 0. & 1. & 4. & 3. & 7. & 5. & 5. & 10. & 3. \\
\hline 161 & 3. & 6. & 6. & 7. & 4. & 3 . & 8. & 3. & 9. & 9. \\
\hline 171 & 6. & 6. & 13. & 6. & 7. & 7. & 13. & 8. & 12 . & 20. \\
\hline 181 & 11. & 14. & 11. & 22 . & 12 . & 12. & 23. & 11. & 25 . & 22 . \\
\hline 191 & 31 . & 36. & 34 . & 39. & 30. & 34 . & 39 . & 42 . & 50 . & 66. \\
\hline 201 & 50 & 61. & 86 & 74. & 82. & 104 & 119 & 120 & 145 . & 183. \\
\hline 211 & 189 & 211 . & 254 & 314 . & 390. & 437 & 538. & 636. & 789. & 803. \\
\hline 221 & 930. & 1001. & 1099. & 1371. & 1632. & 2021 . & 2497. & 3015 . & 3442 . & 3483. \\
\hline 231 & 3113. & 2108 . & 875 & 258 & 46. & 7 & 4 & 1 . & 3. & 2 \\
\hline 241 & 2. & 1 . & 3. & 2 & 3. & 2 . & 1. & 2 . & 2 . & 5. \\
\hline 251 & 7 . & 1 . & 5. & 6. & 9. & 9 . & 5. & 4 . & 5 . & 7. \\
\hline 261 & 6. & 1. & 6. & 6. & 10. & 10. & 7. & 11. & 13. & 20. \\
\hline 271 & 24 . & 9. & 12. & 6. & 9. & 7. & 9. & 6. & 6. & 10. \\
\hline 281 & 16. & 20. & 22. & 34. & 32. & 47. & 36. & 52. & 51. & 28. \\
\hline 291 & 14. & 9. & 12 . & 7. & 15. & 7. & 11. & 12. & 15. & 16. \\
\hline 301 & 18. & 21. & 21 . & 19. & 16. & 15. & 9 . & 6. & 7. & 7 \\
\hline 311 & 6. & 8. & 8. & 12 . & 13. & 15. & 12. & 11. & 11. & 12. \\
\hline 321 & 18. & 12 . & 11. & 25 . & 30. & 21 . & 37. & 28 . & 42 . & 31 . \\
\hline 331 & 51. & 50. & 51. & 58. & 54 . & 61. & 80. & 71. & 107. & 98. \\
\hline 341 & 120. & 127. & 168 & 194 . & 229. & 280 . & 343 & 426 . & 526 . & 626 . \\
\hline 351 & 794. & 952 . & 1032 . & 984 . & 830 . & 807 . & 1033. & 1203 . & 1429 . & 1752 . \\
\hline 36 & 2120 . & 2454 . & 2292 . & 1689. & 731. & 196. & 35 . & 6 & 0 & 0 . \\
\hline 37 & 1. & 0. & 0 . & 0. & 0 . & 0 . & 0 . & 0 . & 0 . & 0 . \\
\hline 38 & 0 . & 0 . & 0 . & 0 . & 0. & 0 . & 0 . & 0. & 0 . & 0. \\
\hline 39 & 0 . & 0. & 0. & 0 . & 0 . & 0 . & 0 & 0. & 0 . & 0 . \\
\hline 40 & 0 . & 0. & 0. & 0 . & 0. & 0. & 0. & 0 & 0. & 0. \\
\hline 41 & 0 . & 0 . & 0 . & 0 . & 1 . & 0 . & 1. & 0 & 1 . & 1. \\
\hline 42 & 1 . & 0 . & 1. & 0 & 0 . & 0. & 0 . & 0 & 0 & 0 \\
\hline 43 & 1. & 2. & 1. & 1. & 0. & 0. & 0 & 0 & 0 . & 0 . \\
\hline 44 & 1. & 0 . & 0 . & 0 . & 0. & 0. & 1. & 0 & 0 . & 0. \\
\hline 45 & 0 . & 1. & 1. & 0 . & 0. & 0. & 0 . & 0 & 2. & 1 . \\
\hline & 1 . & 0 & 1 & 0 . & 2 & 1 & 3 & 1 & 2 . & 2 . \\
\hline & 9. & 4. & 2 . & 5. & 6. & 7. & 3 & 4 & 6. & 2. \\
\hline 48 & 2 & 1 . & 0 & 0 . & 0. & 0. & 0 & 1 & 0 & 0 \\
\hline 49 & 0 & 1 . & 0 . & 0 . & 0 . & 1. & 3 & 1 . & 0 . & 1. \\
\hline & 0 . & 0. & & & & & & & & \\
\hline
\end{tabular}


Westinghouse Hanford Co.

Rev. 2.02

DATA REDUCTION REPORT

SAMPLE

WL16279-BLK-PU

File ID: 14 a1416.CNF

Counted on:

$1 / 25 / 97 @ 14: 25$

Detector:

Geometry number: 1

Count time:

28800. Sec

PEAK ANALYSIS

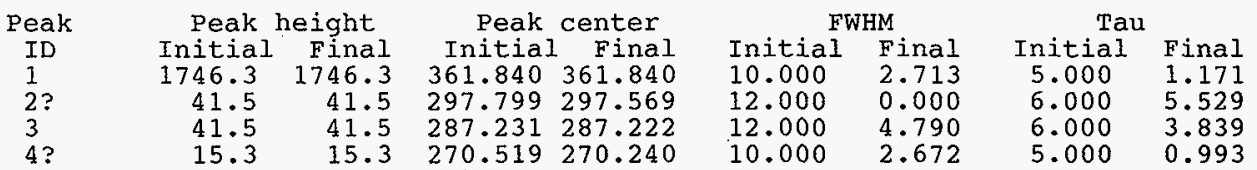

\section{PEAK RESULTS \\ Peak Error Limit: $30 \%$}

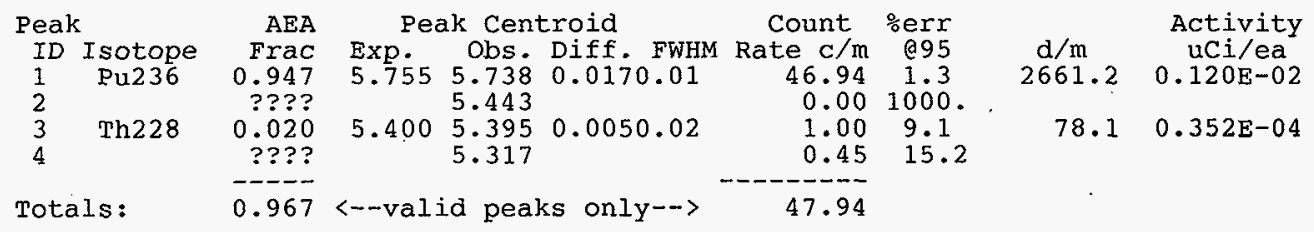

TOTAL COUNT DATA:

Item

Raw spectrum

Smoothed

Composite fit

Residuals

$$
\begin{array}{r}
\text { Total } \\
23803.0 \\
23802.0 \\
23229.3 \\
573.7
\end{array}
$$

Recovery
100.000
99.996
97.590
2.410

Analyzed by: 
1 Legend: Raw $=\ldots$ Modeled Peaks $=1,2, \ldots$, etc $\quad$ Display Max.: 8833.5 


\begin{tabular}{|c|c|c|c|c|c|c|c|c|c|}
\hline 0. & 0. & 0 . & 0 . & 0 & 0. & 0 & 0. & & \\
\hline 1. & 0. & 0. & 1. & 0. & 1. & 0. & 0. & 0 . & 0 \\
\hline $\begin{array}{l}0 . \\
0 .\end{array}$ & 0. & ${ }_{0 .}^{0}$ & $\begin{array}{l}0 . \\
0 .\end{array}$ & O. & ${ }_{0 .}^{0}$ & 1. & ${ }_{0}^{1 .}$ & 0. & \\
\hline 0 . & 0. & 0. & 0. & 1. & 0. & 1. & 2 . & 0 : & 1 \\
\hline 1. & 0. & 0. & 1. & 0. & 0. & 1. & 1 . & 2. & \\
\hline 3. & 1. & 5. & 5. & 2. & 2. & 6. & 6. & 4 . & \\
\hline 0. & 3. & 2. & 4. & 4. & 1. & 2. & 0. & 0. & \\
\hline 0. & 0. & 0. & 0 . & 0. & 0. & 0. & 0. & 2 . & \\
\hline 0. & 0. & 1. & 0 . & 0. & 0. & 0. & 1. & 0. & \\
\hline 4. & 0. & 0. & 0 . & 0. & 0. & 1. & 1. & 1. & \\
\hline 0. & ${ }_{1}^{0 .}$ & & $\begin{array}{l}0 . \\
0 .\end{array}$ & 1. & . & : & 0. & : & \\
\hline 0. & 0. & 0. & 1. & 1. & 0 & 1. & 10 & 0 & \\
\hline 0. & 0. & 0. & 2. & 1. & 1. & 0. & 1. & 0 . & \\
\hline 1. & 0. & 1. & 1. & 0. & 0. & 1. & 2 . & 0. & \\
\hline 1. & 0. & 1. & 2. & 0. & 2. & 1. & 0. & 0 . & \\
\hline 4. & 1. & 0. & 1 . & 0. & 2. & 0. & 0. & 1. & \\
\hline$\frac{1}{2}$. & 0. & 1. & $\frac{1}{2}$. & 0. & 2. & 1. & 0. & 0. & \\
\hline 2. & . & 0. & 2. & 0. & $0_{0}^{\circ}$ & : & 2. & 1. & \\
\hline 1 . & 3. & 1. & 2 . & 2. & 4. & 4. & 2. & 3. & \\
\hline 1. & 4. & 5. & 6. & 2 . & 6. & 9. & 5. & 10. & \\
\hline 7. & 11. & 7. & 1. & 2. & 4. & 0. & 1. & 2. & \\
\hline 4. & 1. & 1. & 4. & 2. & 3. & 0. & 0. & 2. & \\
\hline 4. & 7. & 7. & 6. & 7. & 7. & 4. & 8. & 3. & 3. \\
\hline $51^{\circ}$ & $50^{5}$ & 4. & 8. & 11. & $7{ }^{\circ}$ & 8. & 15. & 21. & 18. \\
\hline 21. & 20. & $\frac{11}{22}$ & $28^{\circ}$ & $39^{8}$ & $46^{\circ}$ & $3^{6}$ & $0^{\circ}$ & ${ }_{45}^{6}$ & 18. \\
\hline 15. & 12. & 5. & 6. & 8. & 11: & 7. & $190^{\circ}$ & $\begin{array}{l}45 . \\
15 .\end{array}$ & \\
\hline 8. & 13. & 8. & 16. & 14. & 13. & 13. & 9. & 6. & 12 \\
\hline 13. & 16. & 10. & 8. & 9. & 16. & 13. & 17. & 18. & 28 \\
\hline 23. & 18. & 21. & 30. & 30. & 25. & 29. & 46. & 44. & 38 \\
\hline 51. & 61. & 56. & 63. & 70. & 80. & 86. & 98. & 99. & 99 \\
\hline 127. & 128. & 163. & 223. & 221. & 264. & 308. & 395. & 494. & 595 \\
\hline $\begin{array}{r}121 \\
1902\end{array}$ & $8360^{8}$ & $\begin{array}{r}947 . \\
\end{array}$ & 876. & 842. & $\begin{array}{l}7577^{\circ} \\
212\end{array}$ & $849^{\circ}$ & 1004. & 1369. & 1590 \\
\hline 0 & 0. & 0. & 0. & 0. & 212. & ${ }^{29}$ & 0. & 0. & 0 \\
\hline 0. & 0. & 0. & 0. & 0. & 0. & 0. & 0. & 0. & \\
\hline & 0. & 0. & 0. & 0. & 0. & 1. & 0 . & 0. & \\
\hline 0 . & 0. & 0. & 0. & 0. & 0. & 0. & 0. & 0 . & \\
\hline 0. & 0. & 0. & 0. & 0. & 1. & 0. & 0. & 0. & \\
\hline 1. & 0. & 0. & 1. & 1. & 0. & 0. & 0. & 1. & \\
\hline 0. & 0. & 0 . & 0. & 0. & 0. & 1. & 0. & 0. & \\
\hline${ }_{0}^{0}$. & 3. & 0. & ${ }_{0}^{0}$. & ${ }^{0}$. & 0. & ${ }_{0}^{0}$ & ${ }_{0}^{0}$ & ${ }^{0}$. & \\
\hline 0 . & 0. & 0. & 0. & 3. & 0. & 2. & 3 & $\frac{1}{0}$ & \\
\hline 5 & 3. & 3. & 7 & 6. & 4. & 2. & 6. & 3. & \\
\hline 0 . & 1. & 1. & 1. & 0 . & 0 . & 0. & 0. & 0. & \\
\hline 1 & 0. & 0. & 0. & 0 . & 1. & 0. & 1. & 1. & \\
\hline
\end{tabular}


G E N E R A L

Westinghouse Hanford Co.

A I P H A E N E R G Y A N A I Y S I S

Rev. 2.02

DATA REDUCTION REPORT

SAMPLE

S97T25-SAM-PU

File ID: 15 a1525.CNF

$\begin{array}{ll}\text { Counted on: } & 1 / 25 / 97 @ 14: 26 \\ \text { Detector: } & \\ \text { Geometry number: } 1 & \\ \text { Count time: } & 28801 . \text { Sec }\end{array}$

PEAK ANALYSIS

\begin{tabular}{|c|c|c|c|c|c|c|c|}
\hline Peak & Peak & height & Peak center & & & Tar & \\
\hline ID & Initial & Final & Initial Final & Initial & Final & Initial & Final \\
\hline 1 & 1763.4 & 1763.4 & $361.946 \quad 361.946$ & 10.000 & 3.008 & 5.000 & 1.292 \\
\hline 2 & 17.3 & & 302.958 & 14.000 & 5.162 & 7.000 & 406 \\
\hline 3 & 34 & 34 & 287 . & 100 & 405 & 000 & \\
\hline $4 ?$ & $\cdot 1$ & 50 & 268.563 & 8.000 & 4.205 & 4.000 & 0.666 \\
\hline 5 & & 50 & 230.171230 .149 & 12.000 & 3.877 & 6.000 & \\
\hline
\end{tabular}

\section{PEAK RESULTS \\ Peak Error Limit: $30 \%$}

Peak

ID Isotope

$1 \mathrm{Pu} 236$

$2 \quad \mathrm{Pu} 238$

Am2 21

3 Th228

4
5

$5 \quad$ Pu239

Pu240

Totals:

\begin{abstract}
AEA
Frac

0.938

0.014

0.012

????

0.026
\end{abstract}

$-\cdots---$

0.990
Peak Centroid

$\begin{array}{lll}\text { Exp. } & \text { Obs. Diff. FWHM } \\ 5.755 & 5.739 & 0.0160 .01 \\ 5.487 & 5.468 & 0.0190 .02 \\ 5.479 & 5.468 & 0.011 \\ 5.400 & 5.398 & 0.0020 .01 \\ & 5.309 & \\ 5.147 & 5.133 & 0.0140 .02 \\ 5.144 & 5.133 & 0.011\end{array}$

$\langle--v a l i d$ peaks only-->

DETECTOR CALIBRATION

$$
\begin{array}{cc}
\text { Energy (MEV) }=4.074+(0.0046) * \text { Channel } \\
\text { Energy range }(\mathrm{MeV}): 4.074 \mathrm{TO} 6.429 \\
\text { Efficiency }= & 0.0180 \mathrm{CPM} / \mathrm{DPM}
\end{array}
$$

TOTAL COUNT DATA:

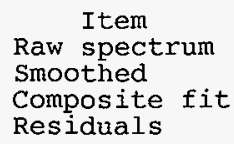

Residuals
Composite fit
Activity $\mathrm{uCi} / \mathrm{ea}$

$2751.8 \quad 0.124 \mathrm{E}-02$

$54.1 \quad 0.244 \mathrm{E}-04$

$41.4 \quad 0.187 \mathrm{E}-04$

$50.0 \quad 0.225 E-04$

$73.3 \quad 0.330 \mathrm{E}-04$

$73.3 \quad 0.330 \mathrm{E}-04$
$\%$ Recovery

100.000

100.000

99.904

0.096

$\begin{array}{rc}\text { Total } & \% \text { Recovery } \\ 24828.0 & 100.000 \\ 24828.1 & 100.000 \\ 24804.3 & 99.904 \\ 23.7 & 0.096\end{array}$

Analyzed by: 
1 Leğend: $\quad$ Raw $=\ldots$ Modeled Peaks $=1,2, \ldots$, etc $\quad$ Display Max.: 9325.2

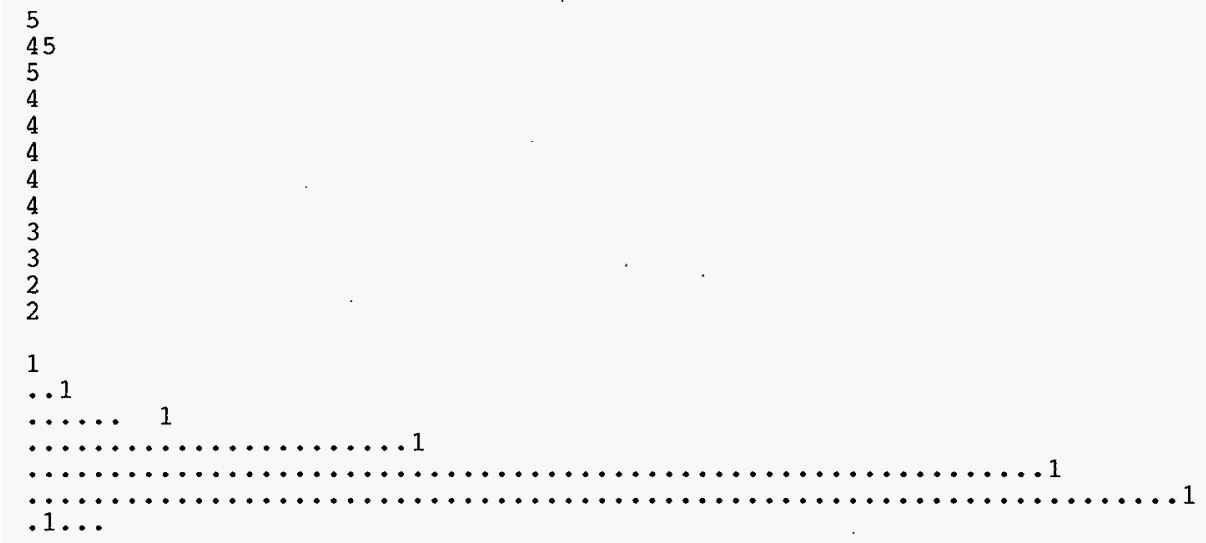


Raw Data Dump for AEA Spectrum: 15a1525.CNF

HNF-SD-WM-DP-227, REV. 0

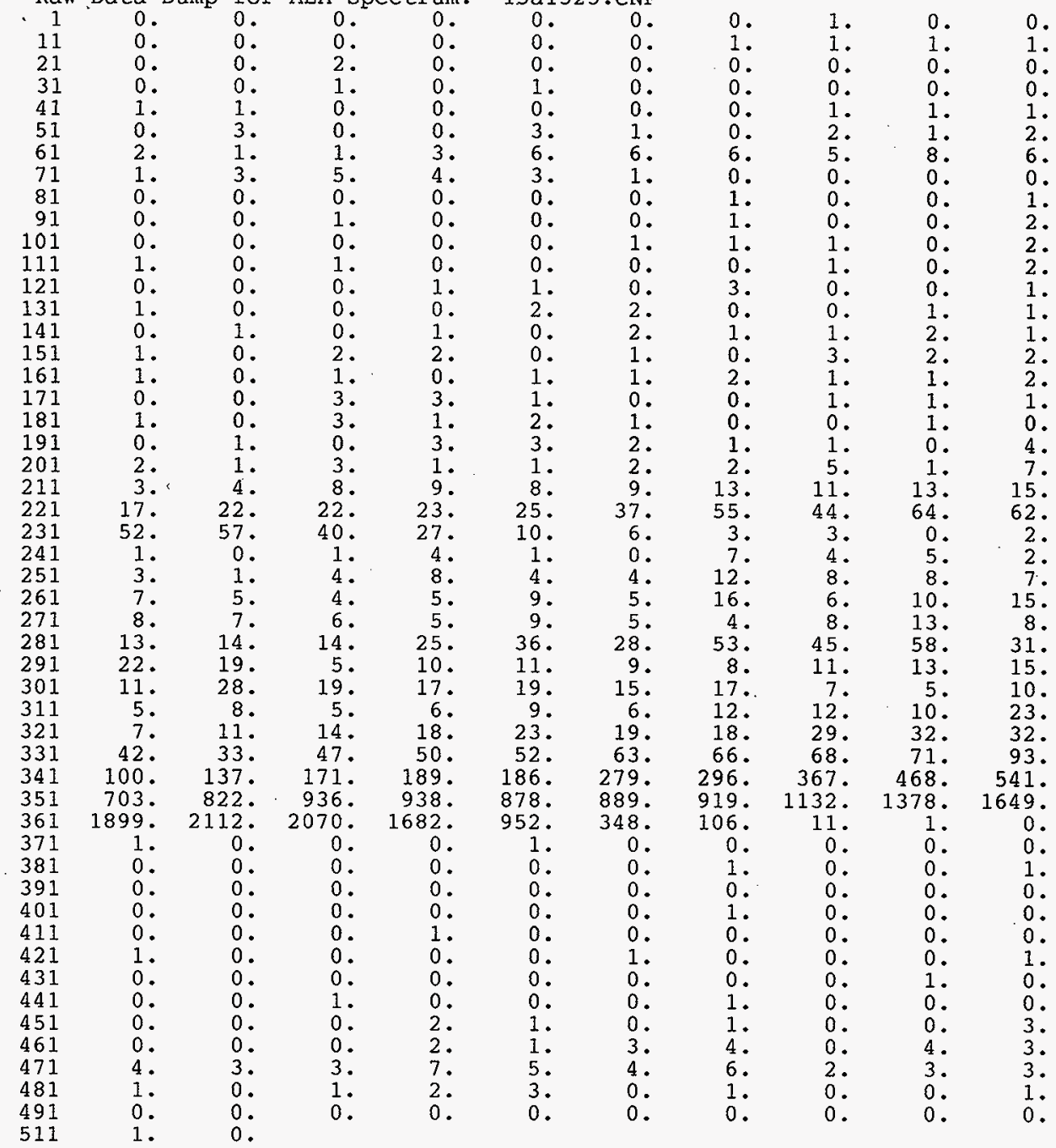


HNF-SD-WM-DP-227, REV. 0

G E N E R A L

Westinghouse Hanford Co.

A L P H A E N E R G Y A N A L Y S I S

Rev. 2.02

DATA REDUCTION REPORT

SAMPLE

S97T25-DUP-PU

File ID: $16 a 1658 . \mathrm{CNF}$

Counted on: $\quad 1 / 25 / 97$ @14:26

Detector:

Geometry number: 1

Count time: 28800. Sec

PEAK ANALYSIS

\begin{tabular}{|c|c|c|c|c|c|c|c|c|}
\hline Peak & Peak & zeight & Peak & center & & & Tal & \\
\hline ID & Initial & Final & Initial & Final & Initial & Final & Initial & Final \\
\hline 1 & 1987.2 & 1987.2 & 361.909 & 361.909 & 10.000 & 2.691 & 5.000 & 1.193 \\
\hline 2 & 15.5 & 15.5 & 304.049 & 303.715 & 10.000 & 5.308 & 5.000 & 014 \\
\hline 3 & & 33.0 & 286.791 & 286.602 & 000 & 20 & 000 & \\
\hline $\begin{array}{l}4 ? \\
5\end{array}$ & $\begin{array}{r}9.6 \\
53.0\end{array}$ & $\begin{array}{r}9.6 \\
53.0\end{array}$ & $\begin{array}{l}268.580 \\
228.550\end{array}$ & $\begin{array}{l}268.277 \\
228.529\end{array}$ & $\begin{array}{l}10.000 \\
12.000\end{array}$ & $\begin{array}{l}3.442 \\
3.398\end{array}$ & $\begin{array}{l}000 \\
000\end{array}$ & $\begin{array}{l}107 \\
11\end{array}$ \\
\hline
\end{tabular}

Peak

ID Isotope

$1 \quad$ Pu236

$2 \quad$ Pu238

Am2 21

3 Th 228

4
5

Totals:

\section{PEAK RESULTS \\ Peak Error Limit: $30 \%$}

AEA

Frac

0.938

0.014

0.011

????

0.022

0.986

Peak Centroid Exp. Obs

$$
\begin{array}{lll}
5.755 & 5.739 & 0.0160 .01 \\
5.487 & 5.471 & 0.0160 .02
\end{array}
$$$$
5.4795 .471
$$$$
5.4005 .392 \quad 0.0080 .01
$$

5.308

$$
5.144 \quad 5.125 \quad 0.0190 .02
$$

--valid

peaks only-->

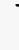

Count serr

DETECTOR CALIBRATION
Energy $(\mathrm{MEV})=4.074+(0.0046) *$ Channel
Energy range (MeV): 4.074 To 6.429 Efficiency $=0.0180 \mathrm{CPM} / \mathrm{DPM}$

TOTAL COUNT DATA:

Item

Raw spectrum

Smoothed

Composite fit

Residuals
Total

26851.0

26850.8

26697.6

153.4
\% Recovery

100.000

99.999

99.429

0.571
Activity uci/ea

2975.2 $0.134 \mathrm{E}-02$

$62.3 \quad 0.281 \mathrm{E}-04$

$47.7 \quad 0.215 \mathrm{E}-04$

$50.1 \quad 0.226 \mathrm{E}-04$

$69.0 \quad 0.311 \mathrm{E}-04$

Analyzed by: 


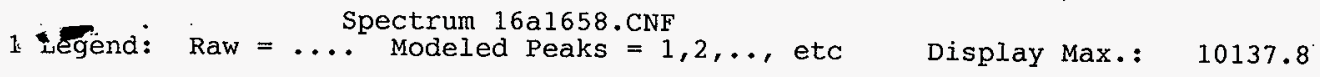

$\ldots 1$

… I

$\ldots \ldots \ldots \ldots \ldots \ldots \ldots \ldots$

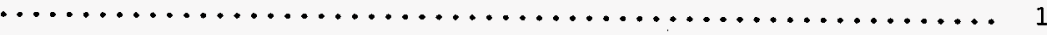

i..... 
Raw Data Dump for AEA Spectrum: 16a1658.CNF

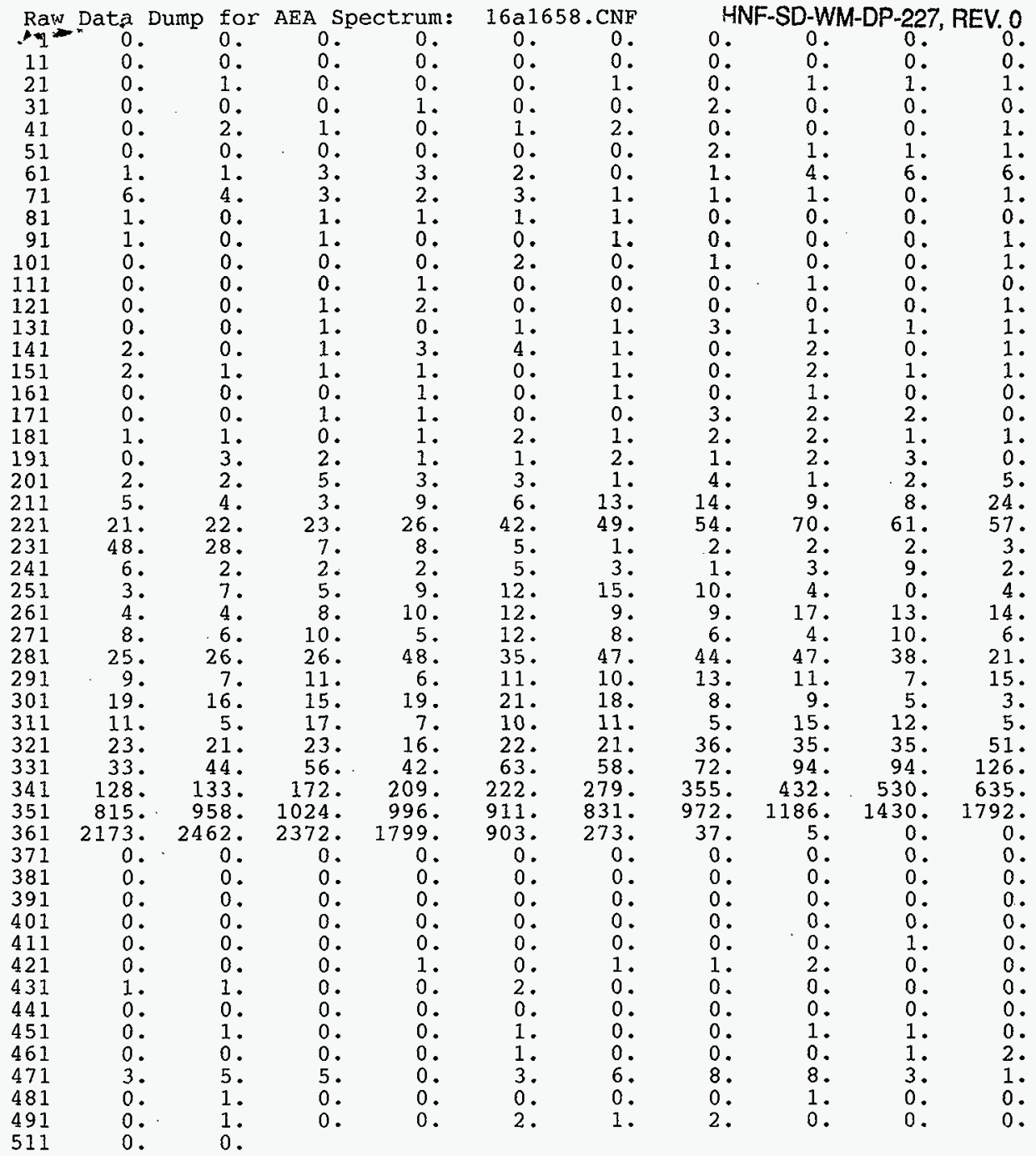


Westinghouse Hanford Co.

\section{DATA REDUCTION REPORT}

SAMPLE

S97T28-SAM-PU

File ID: $5 a 5087 . \mathrm{CNF}$

Counted on:

Detector:

Geometry number: 1

count time:
$1 / 25 / 97 @ 14: 10$

AEA 5

1

PEAK ANALYSIS

Peak height Initial Final 1711.01711 .0

$32.4 \quad 32.4$

$31.6 \quad 31.6$

$243.4 \quad 243.4$
Peak center Initial Final $362.757 \quad 362.757$ $308.302 \quad 307.098$ 289.645288 .748 230.547230 .538

\begin{tabular}{lr}
\multicolumn{2}{c}{ FWHM } \\
Initial & Final \\
16.000 & 8.947 \\
32.000 & 28.239 \\
12.000 & 6.906 \\
18.000 & 8.788
\end{tabular}

28805. Sec

$\begin{array}{lc}\text { Peak } \\ \text { ID } \text { Isotope } \\ 1 & \text { Cm243 } \\ & \text { Pu236 } \\ 2 & \text { Am241 } \\ & \text { Pu238 } \\ 3 & \text { Th228 } \\ 4 & \text { Pu239 } \\ & \text { Pu240 }\end{array}$

Totals:

\section{PEAK RESULTS \\ Peak Error Limit: $30 \%$}

\begin{tabular}{rr}
\multicolumn{2}{c}{ Tau } \\
Initial & Final \\
8.000 & 3.405 \\
16.000 & 16.066 \\
6.000 & 1.537 \\
9.000 & 3.608
\end{tabular}

\begin{tabular}{|c|c|c|c|c|c|c|c|}
\hline $\mathrm{AEA}$ & $\mathrm{Pe}$ & $k$ Cen & roid & count & \%err & & Activity \\
\hline $\begin{array}{r}\text { Frac } \\
0.834\end{array}$ & Exp. & Obs. & $\begin{array}{l}\text { Diff. FWHM } \\
0.0270 .04\end{array}$ & $\begin{array}{r}\text { Rate } \mathrm{c} / \mathrm{m} \\
65.26\end{array}$ & $\begin{array}{l}\text { C95 } \\
1.1\end{array}$ & $\begin{array}{l}\mathrm{d} / \mathrm{m} \\
200.8\end{array}$ & $\begin{array}{c}\text { uCi/ea } \\
0.905 E-04\end{array}$ \\
\hline 0.022 & & 5.496 & -.0170 .13 & 1.74 & 11.4 & & \\
\hline $\begin{array}{l}0.020 \\
0.114\end{array}$ & $\begin{array}{l}5.400 \\
5.147 \\
5.144\end{array}$ & $\begin{array}{l}5.412 \\
5.144 \\
5.144\end{array}$ & $\begin{array}{l}-.0120 .03 \\
0.0030 .04 \\
0.000\end{array}$ & $\begin{array}{l}1.53 \\
8.95\end{array}$ & 8. & $\begin{array}{r}4 \\
20 \\
20\end{array}$ & \\
\hline
\end{tabular}

$0.990<--v a l i d$ peaks only--> 77.48

$$
\begin{gathered}
\text { DETECTOR CALIBRATION } \\
\text { Energy (MEV) }=4.083+(0.0046) * \text { Channel } \\
\text { Energy range }(\mathrm{MeV}): 4.083 \text { TO } 6.439 \\
\text { Efficiency }=
\end{gathered}
$$

TOTAL COUNT DATA:

$\begin{array}{lrc}\quad \text { Item } & \text { Total } & \% \text { Recovery } \\ \text { Raw spectrum } & 37570.0 & 100.000 \\ \text { Smoothed } & 37570.0 & 100.000 \\ \text { Composite fit } & 37195.2 & 99.002 \\ \text { Residuals } & 374.8 & 0.998\end{array}$

Analyzed by: 
1 . Legend: Raw $=\ldots$ Modeled Peaks $=1,2, \ldots$ etc $\quad$ Display Max.: 12169.2

4

$\ldots 4$

$\ldots \ldots$.

$\ldots \ldots 4$

3

3

3

3

3

2

2

2

2 .

$\ldots 1$

$\ldots \ldots \ldots 1$

$\ldots \ldots \ldots \ldots \ldots \ldots \ldots \ldots \ldots \ldots \ldots \ldots \ldots$

$\ldots \ldots \ldots \ldots \ldots \ldots \ldots \ldots \ldots \ldots \ldots \ldots \ldots \ldots \ldots \ldots \ldots \ldots \ldots \ldots 1 \ldots \ldots$

$\ldots \ldots \ldots \ldots \ldots \ldots$

i 
Raw Data Dump for AEA Spectrum: 5a5087.CNF

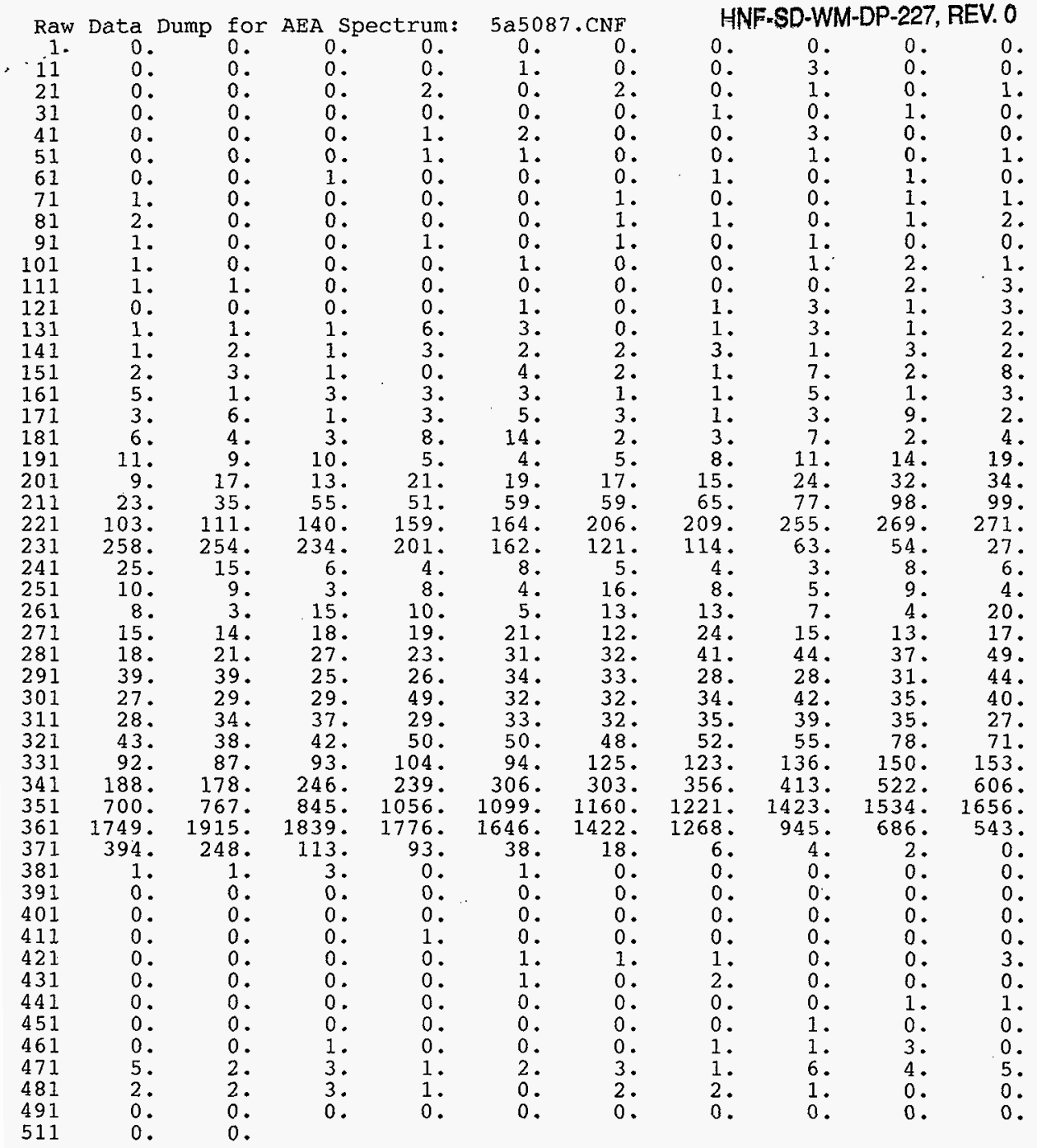


Westinghouse Hanford Co.

G E N E R A A L P H A E N E R G A N A L Y S I S

DATA REDUCTION REPORT

SAMPLE

S97T28-DUP-PU

File ID: $6 a 6906 . C N F$

$\begin{array}{ll}\text { Counted on: } & 1 / 25 / 97 @ 14: 17 \\ \text { Detector: } & \text { AEA6 }\end{array}$

Geometry number: 1

Count time: 28805. Sec

PEAK ANALYSIS

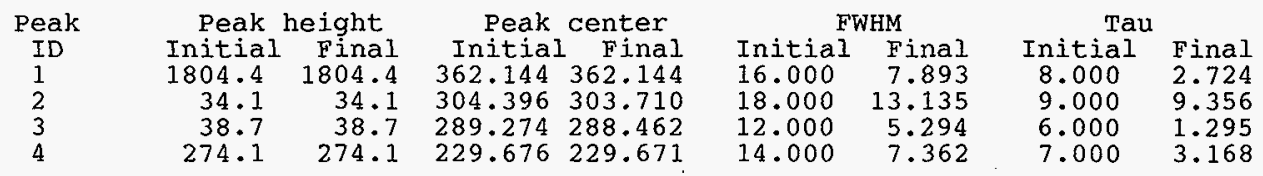

\section{PEAK RESULTS \\ Peak Error Limit: $30 \%$}

Peak
$\begin{array}{cc}\text { ID } & \text { Isotope } \\ 1 & \text { Cm243 } \\ & \text { Pu236 } \\ 2 & \text { Am241 } \\ & \text { Pu238 } \\ 3 & \text { Th228 } \\ 4 & \text { Pu239 } \\ & \text { Pu240 }\end{array}$

Totals :
AEA

Frac

0.837

0.015

0.020

0.113

$--. .-$

0.985 Peak Centroid Count \%err
Exp. Obs. Diff. FWHM Rate $\mathrm{c} / \mathrm{m}$ a 95

$$
\begin{array}{lllll}
5.779 & 5.746 & 0.0330 .04 & 69.67 & 1.1
\end{array}
$$

$5.7555 .746 \quad 0.009$

$5.479 \quad 5.4770 .0020 .06$

$5.4875 .477 \quad 0.010$

$5.400 \quad 5.407-.0070 .02$

$5.1475 .137 \quad 0.0100 .03$

$\begin{array}{lll}5.144 & 5.137 & 0.007\end{array}$

$1.26 \quad 11.5$

1.698 .2

$9.38 \quad 2.9$

$\langle--v a l i d$ peaks only-->

82.00

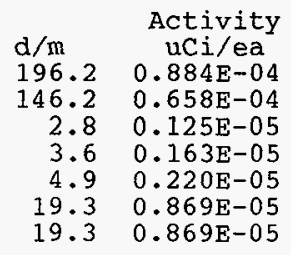

DETECTOR CALIBRATION
Energy $(\mathrm{MEV})=4.080+(0.0046) *$ Channel Energy range (MeV): 4.080 TO 6.435 Efficiency $=0.4864 \mathrm{CPM} / \mathrm{DPM}$

TOTAL COUNT DATA:

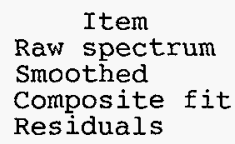

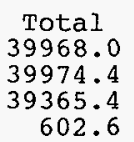

\% Recovery

100.000

100.016

98.492

1.508

Analyzed by: 
1 Lagend: Raw $=$ Spectrum $6 a 6906 . \mathrm{CNF}$ HNF-SD-WM-DP-227, REV. 0

1 Lagend: Raw $=\ldots$ Modeled Peaks $=1,2, \ldots$, etc $\quad$ Display Max.: 12204.3

.... 4

$\ldots \ldots . \ldots 4$

$\ldots .4$

3

3

3

3

3

2

2

2

.1

$\ldots \ldots 1$

........ 1

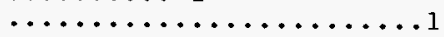

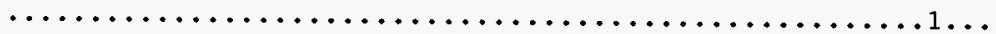

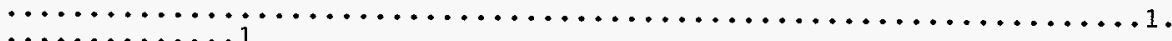

$\ldots \ldots \ldots \ldots 1$ 
Raw Data Dump for AEA Spectrum: 6a6906.CNF

I 0.00 .0 .0 .000$.

11

21

31

41

51

\section{1}

71

91

101

111

121

131

141

151

161

171

181

191

201

211

221

231

241

251

261

271

281

291

301

311

321

331

341

351

361

371

381

391

401

411

421

431

441

451

461

471

481

491

511

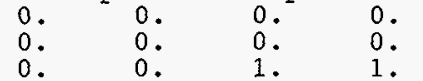

0.1 .01$.

0 .

2 .

0.1 .

1. $\quad 1$.

2 . 1 .

0.1 .

4.1 .

$2 . \quad 0$.

0.1.

2.3.

4. 6.

2. $\quad 1$.

6.10

14.27.

$\begin{array}{ll}118 . & 150 . \\ 301 . & 246 .\end{array}$

$\begin{array}{rr}301 . & 246 . \\ 8 . & 9 .\end{array}$

6.7 .

8. 11.

14. 21 .

23.29.

35.44 .

22.46.

36.28 .

94. 101 .

253 . 224 .

850.936.

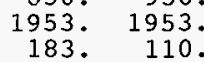

0.0.

$\begin{array}{ll}0 . & 0 . \\ 1 . & 0 .\end{array}$

1. 1.

0.0.

0.0.

$0 . \quad 0$.

1.0 .

1.2 .

5.1.

1. $\quad 1$.
40.36 .

45.43.
1.0 .

1.0 .

1. 0 .

1.1 .

$0 . \quad 0$.

$1 . \quad 0$.

1.

0 .

2 .

1.

5 .

2 .

7.

3.

6.

32 .

156.

221 .

967.132

9.3 . 8 .

7.

9.

15.

33.

31 .

38 .

28 .

54.

121 .

291.

346.

1882.

60.

60.

0 .

0 .

0 .

0 .

1 .

1 .

0 .

2 .

2.

0 .

1 .

0.

1.

1 .

3 .

1 .

1.

1.

2 .

2 .

2.

11.

17.

63.
194.
167.

7.

17.

34 .

27.

51 .

26.

$143^{\circ}$

1104.

1846 .

26 .

0.

0 .

0 .

0 .

0 .

0.

0 .

2 .

2 .

11 .

18.

44.

29.

38.

32 .

59.

133.

368 .

1199.

1613.

14.

0 .

0 .

0 .

1.

0.

0.

1.

3 .
0.

2.

4.

0 .

1.

8.

21 .

21.

HNF-SD-WM-DP-227, REV. 0

$\begin{array}{llll}0 . & 0 . & 0 . & 0 . \\ 1 . & 0 . & 0 . & 1 . \\ 1 . & 1 . & 0 . & 1 . \\ 2 . & 0 . & 0 . & 0 . \\ 0 . & 2 . & 1 . & 0 . \\ 0 . & 0 . & 0 . & 0 . \\ 0 . & 1 . & 2 . & 0 . \\ 1 . & 1 . & 0 . & 2 . \\ 0 . & 3 . & 0 . & 2 . \\ 1 . & 0 . & 0 . & 0 . \\ 0 . & 1 . & 3 . & 1 . \\ 0 . & 0 . & 1 . & 0 . \\ 2 . & 2 . & 5 . & 2 . \\ 1 . & 0 . & 0 . & 4 . \\ 7 . & 1 . & 5 . & 2 . \\ 7 . & 1 . & 4 . & 4 . \\ 8 . & 2 . & 9 . & 4 . \\ 4 . & 3 . & 8 . & 2 . \\ 7 . & 7 . & 6 . & 16\end{array}$

14.18

6. 16.

32. 30.33 .40$.

76. 99. 116. 106.

201. 266. 306. 296. 299.

88. 67. 39. 28. 14.

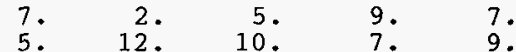

16. 16.212 .213 .20 .

16. 12. 15. 24. 21 .

37. 50.52 .55 .52 .

25. 30.39 .37 .42 .

49.31 .38 .32 .31 .

27.27 .39 .43 .45 .

63. 66. 74. 63. 100 .

143. 163. 190. 175. 225.

412. 495. 559. 645. 741.

1305. 1441. 1591. 1688. 1826 .

$\begin{array}{rrrrr}19 . & 1007 . & 777 . & 544 . & 328 . \\ 5 . & 4 . & 0 . & 0 . & 1 . \\ 0 . & 0 . & 0 . & 0 . & 0 .\end{array}$

$0 . \quad 0 . \quad 0 . \quad 0.000$.

0.0 .01 .00$.

$\begin{array}{lllll}0 . & 0 . & 0 . & 0 . & 0 .\end{array}$

2. 1.

1

0 .

0 .

0 .

2 .

0 .

1.

0 .

0.

2.

3.

1.

0 .

0.

0.

0 .

1 .

2.

0.

0 .

5.

0 . 


\section{DISTRIBUTION SHEET}

\begin{tabular}{|c|c|c|c|}
\hline \multirow{2}{*}{$\begin{array}{l}\text { To } \\
\text { Distribution }\end{array}$} & \multirow{2}{*}{\multicolumn{2}{|c|}{ From Production Planning \& Control }} & Page 1 of 1 \\
\hline & & & Date: $08 / 14 / 97$ \\
\hline \multirow{2}{*}{\multicolumn{3}{|c|}{$\begin{array}{l}\text { Project Title/Work Order } \\
\text { HNF-SD-WM-DP-227, Rev. } 0 \text { "Waste Compatibility Safety Issues and } \\
\text { Final Results for Tank 241-SY-102 Grab Samples" }\end{array}$}} & EDT N0.: EDT-620417 \\
\hline & & & ECN NO.: $N / A$ \\
\hline & MSIN & $\begin{array}{l}\text { Text With } \\
\text { al1 Attach }\end{array}$ & $\begin{array}{l}\text { EDT/ECN } \\
\text { ONLY }\end{array}$ \\
\hline
\end{tabular}

DE\&S Hanford, Inc.

J. E. Meacham

S7-14 $\quad X$

U.S. Department of Energy, RL

C. A. Babel

$\$ 7-54 \quad x$

Lockheed Mart in Hanford Corp.

J. N. Appe]

G3-21

K. G. Carothers

$\mathrm{R} 2-11$

T. J. Kelley

S7-21

M. J. Kupfer

H5 -49

J. B. Schaffer

R2-12

R. D. Schreiber

$\mathrm{R} 2-12$

L. A. Stauffer

R2-11

J. A. Voogd

H5-03

A. E. Young

R1-10

$x$
$x$
$x$
$x$
$x$
$x$
$x$

Lockheed Martin Services, Inc.

Centra? Files

A3-88 $\quad 1$

Los Alamos Technical Assocjates

M. T. Ellsworth

T6-06

$X^{*}$

Pacific Northwest National Laboratory

S. J. Harris

$K 7-22$

K. L. Silvers

$\mathrm{Kg}-08$

$X$

Waste Management of Hanford. Inc.

D. C. Hetzer

$56-31$

J. L. Nuzum

T6-06

T6-04

K. L. Powel1

T6-04

C. M. Seide1

T6-03

$x$

LTIC

$\mathrm{H} 5-27$

$X$

SGN Eurisys Services Corp.

B. A. Higley

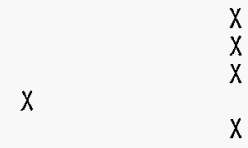

Defense Nuclear Federal Safety Board

Rich Tontodonato

625 Indiana Ave. N. W.

$X$

Washington D.C. 20004

* Needs only releasing paperwork, not a copy of the released document. 